\title{
UCLA
}

Cotsen Institute of Archaeology Press

Title

The Desert Fayum Reinvestigated

Permalink

https://escholarship.org/uc/item/49t6s1b9

ISBN

978-1-938770-09-8

\section{Publication Date}

2017-03-01

\section{Data Availability}

The data associated with this publication are within the manuscript.

Peer reviewed 


\section{The

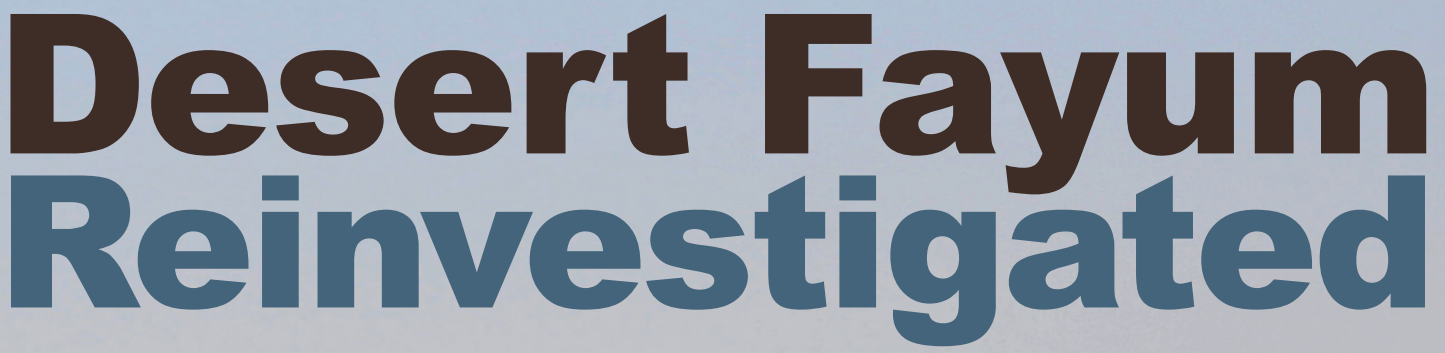

The Early to Mid-Holocene Landscape Archaeology of the Fayum North Shore, Egypt
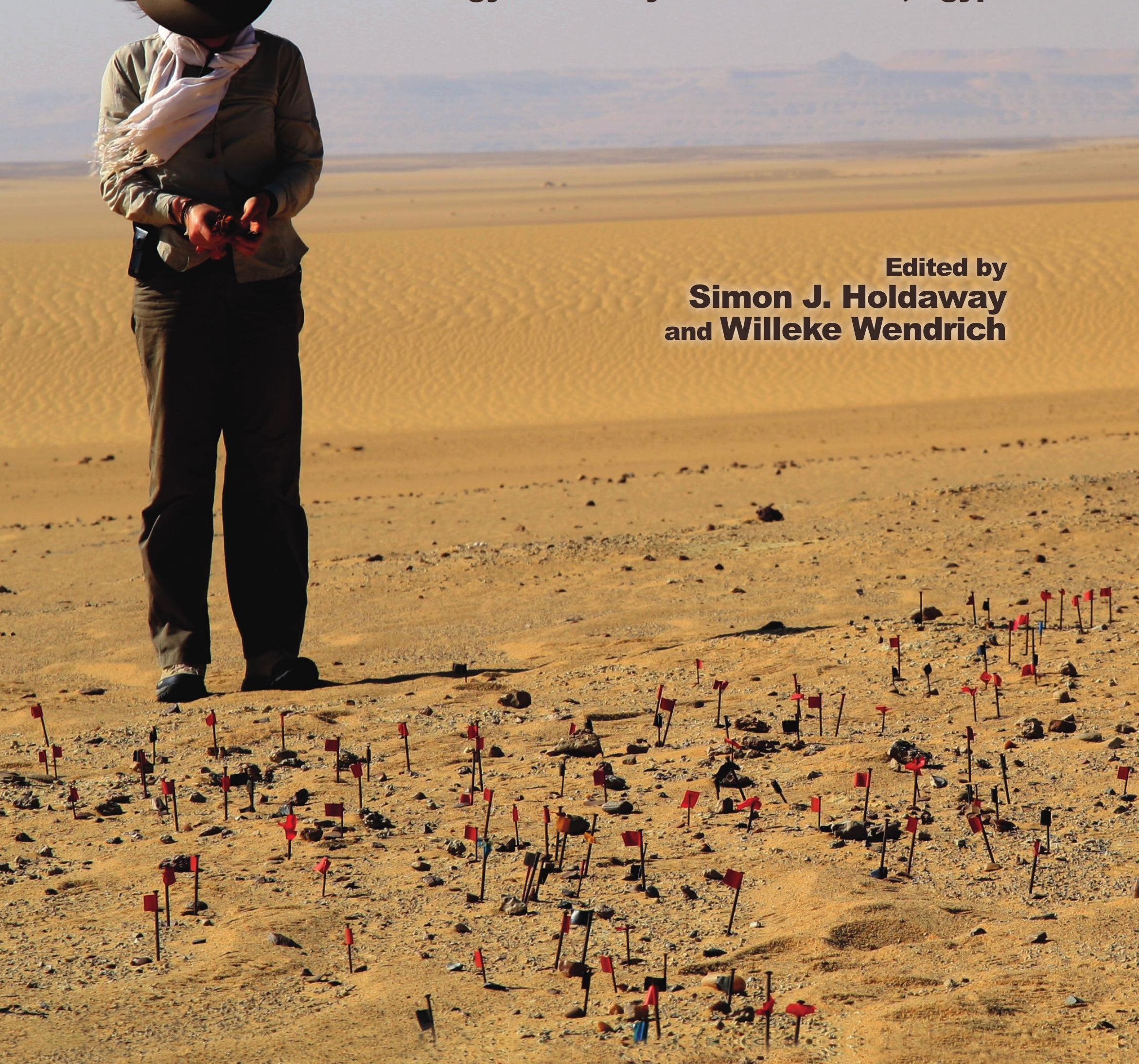


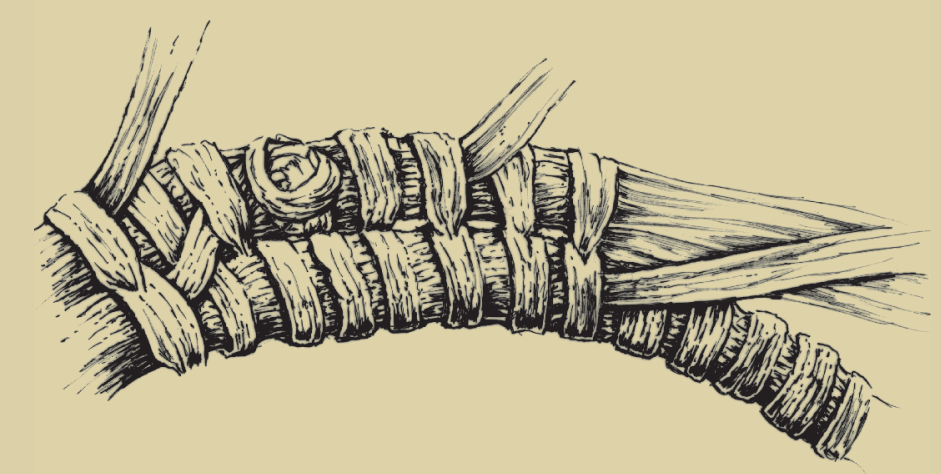




\section{The Desert Fayum
Reinvestigated}

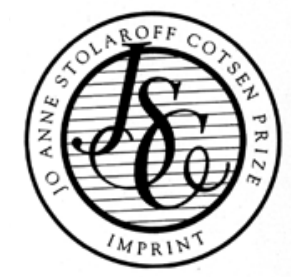

The Jo Anne Stolaroff Cotsen Prize Imprint honors outstanding studies in archaeology to commemorate a special person whose appreciation for scholarship was recognized by all whose lives she touched. 


\section{UCLA COTSEN INSTITUTE OF ARCHAEOLOGY PRESS Monumenta Archaeologica}

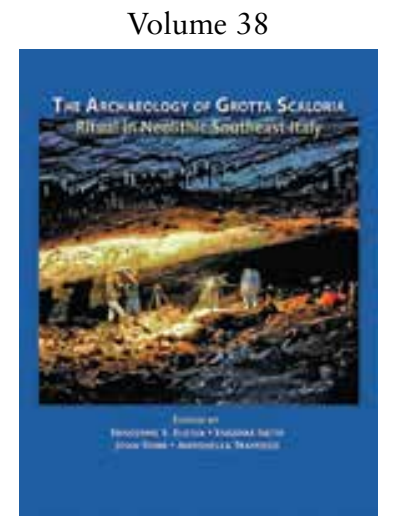

The Archaeology of Grotta Scaloria: Ritual in Neolithic Southeast Italy

Edited by Ernestine S. Elster, Eugenia Isetti, John Robb, and Antonella Traverso
Volume 37

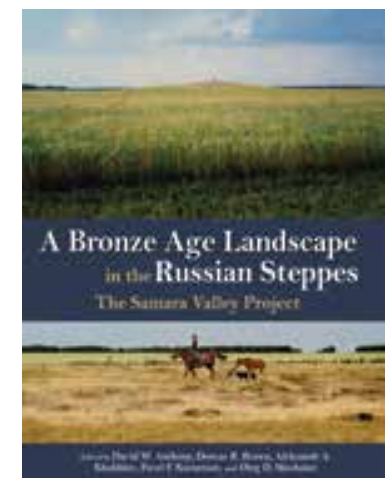

A Bronze Age Landscape in the Russian Steppes: The Samara Valley Project Edited by David W. Anthony, Dorcas R. Brown, Aleksandr A. Khokhlov, Pavel F. Kuznetsov, and Oleg D. Mochalov
Volume 36

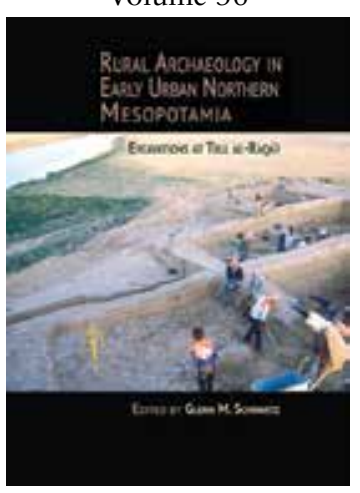

Rural Archaeology in Early Urban Northern Mesopotamia: Excavations at Tell al-Raqa'i Edited by Glenn M. Schwartz
Volume 35

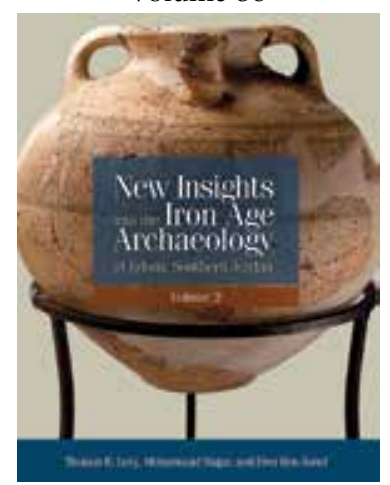

New Insights into the Iron Age Archaeology of Edom, Southern Jordan

By Thomas E. Levy, Mohammad Najjar, and Erez Ben-Yosef

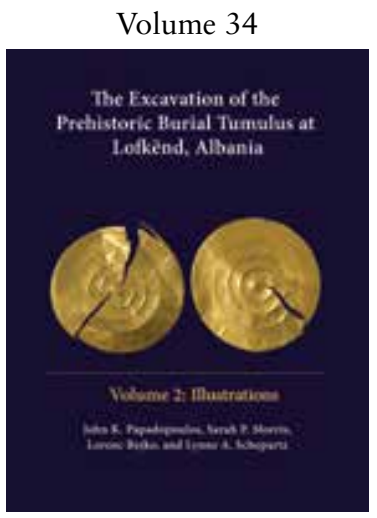

The Excavation of the Prehistoric Burial Tumulus at Lofkënd, Albania

By John K. Papadopoulos, Sarah P. Morris, Lorenc Bejko, and Lynne A. Schepartz
Volume 33

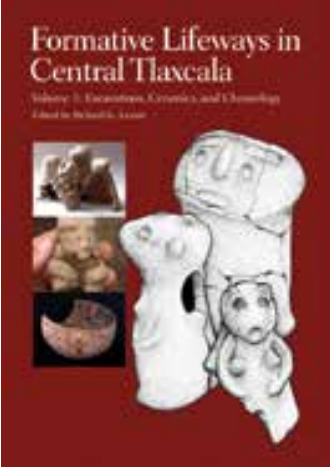

Formative Lifeways in Central Tlaxcala: Volume 1: Excavations, Ceramics, and Chronology Edited by Richard G. Lesure
Volume 32

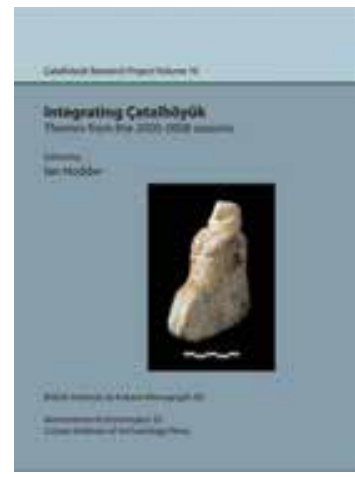

Integrating Çatalhöyük: The 2000-2008 Seasons

(Çatal Research Project Volume 10) Edited by Ian Hodder 


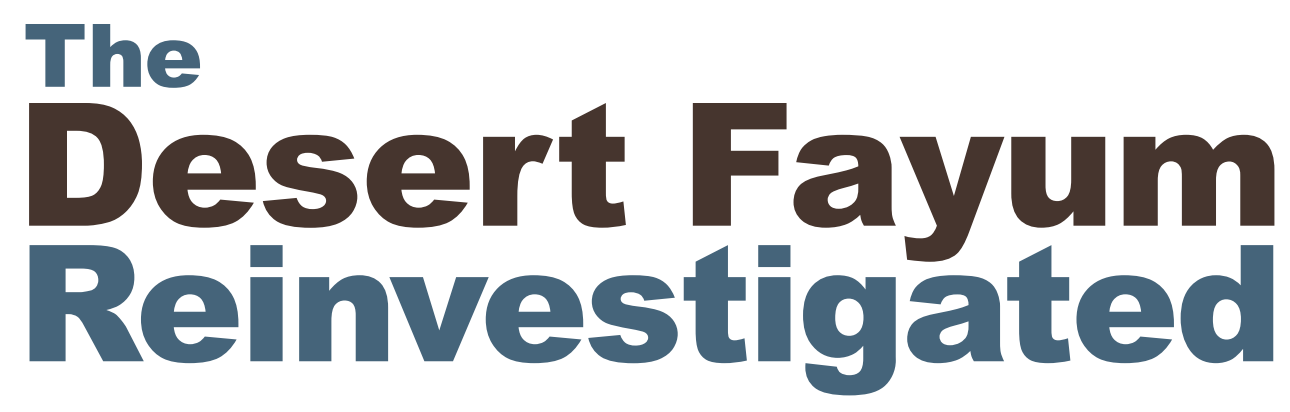

The Early to Mid-Holocene Landscape Archaeology of the Fayum North Shore, Egypt

\author{
Edited by \\ Simon J. Holdaway \\ and Willeke Wendrich
}

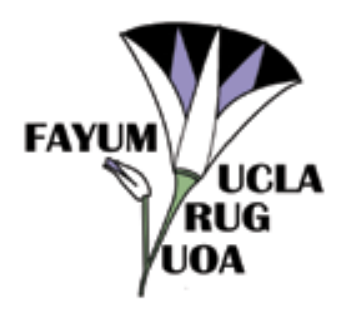

UCLA COTSEN INSTITUTE OF ARCHAEOLOGY PRESS 
The Cotsen Institute of Archaeology Press is the publishing unit of the Cotsen Institute of Archaeology at UCLA. The Cotsen Institute is a premier research organization dedicated to the creation, dissemination, and conservation of archaeological knowledge and heritage. It is home to both the Interdepartmental Archaeology Graduate Program and the UCLA/Getty Master's Program in the Conservation of Archaeological and Ethnographic Materials. The Cotsen Institute provides a forum for innovative faculty research, graduate education, and public programs at UCLA in an effort to positively impact the academic, local and global communities. Established in 1973, the Cotsen Institute is at the forefront of archaeological research, education, conservation and publication, and is an active contributor to interdisciplinary research at UCLA.

The Cotsen Institute of Archaeology Press specializes in producing high-quality academic volumes in nine different series, including Monumenta Archaeologica, Monographs, World Heritage and Monuments, Cotsen Advanced Seminars, and Ideas, Debates, and Perspectives. Through a generous endowment by Lloyd E. Cotsen, longtime Institute volunteer and benefactor, the Press makes the fruits of archaeological research accessible to scholars, professionals, students, and the general public. Our archaeological publications receive critical acclaim in both academic communities and the public at large.

\author{
The Cotsen Institute of Archaeology at UCLA \\ Willeke Wendrich, Director \\ Aaron A. Burke, Editor-in-Chief \\ Randi Danforth, Publications Director \\ Deidre Whitmore, Director of the Digital Archaeology Lab
}

EDITORIAL BOARD

Willeke Wendrich

Lothar von Falkenhausen

Sarah P. Morris

John K. Papadopoulos

Jeanne E. Arnold

Gregson Schachner

Charles Stanish

Richard G. Lesure

Aaron A. Burke

Randi Danforth

Edited by Peg Goldstein

Designed by Sally Boylan

Cover design by Sally Boylan

Index by Matthew White

\author{
Africa (Ex officio member) \\ East Asia \\ Mediterranean Basin \\ Mediterranean Basin \\ North America-Pacific Coast \\ North America-Southwest \\ South America-Andes \\ South America-Mesoamerica \\ West Asia-Near East \\ Ex officio member
}

On front cover: Colored markers used to indicate the locations of different types of artifacts during survey in the Fayum Basin. Photograph by Joshua J. Emmitt.

On back cover: View from inside one of the looting holes, while geomorphology data is being recorded. Photograph by Emily Cole.

\title{
Library of Congress Cataloging-in-Publication Data
}

Names: Holdaway, Simon (Simon John), author. I Wendrich, Willeke, author.

Title: The desert Fayum reinvestigated : early to mid-Holocene landscape archaeology of the Fayum north shore, Egypt / Simon J. Holdaway and Willeke Wendrich.

Other titles: Monumenta archaeologica (Los Angeles, Calif.) ; v. 39.

Description: Los Angeles : UCLA Cotsen Institute of Archaeology Press, 2017.

| Series: Monumenta archaeologica ; volume 39 | Includes bibliographical references and index.

Identifiers: LCCN 2017004635

Subjects: LCSH: Landscape archaeology--Egypt-- Fayyūm (Province) I

Archaeological geology--Egypt-- Fayyūm (Province) I Fayyūm (Egypt :

Province)--Antiquities. I Excavations (Archaeology)--Egypt-- Fayyūm

(Province) I Paleogeography--Holocene.

Classification: LCC GN776.42.E3 H65 2017 I DDC 932/.011--dc23

$\mathrm{LC}$ record available at https://lccn.loc.gov/2017004635

eBook ISBN 978-1-938770-50-0

Copyright (C2017 Regents of the University of California

All rights reserved. Printed in the United States of America 


\section{Contents}

List of Figures $\quad$ vii

List of Tables $\quad$ xi

Acknowledgments $\quad$ xiii

Editors Biographies $\quad$ xv

Contributors $\quad$ xvii

$\begin{array}{ll}\text { Chapter 1: Landscape Archaeology of the Desert Fayum } & 1\end{array}$

Willeke Wendrich and Simon J. Holdaway

Chapter 2: The Fayum in the Context of Northeast Africa 9

Rebecca Phillipps, Simon J. Holdaway, and Willeke Wendrich

Chapter 3: Approaches to Paleoenvironment and Landscape Use 17

Rebecca Phillipps, Simon J. Holdaway, Rebecca Ramsay, Willeke Wendrich, and Joshua J. Emmitt

Chapter 4: The L Basin Archaeological Record

Simon J. Holdaway, Rebecca Phillipps, Annelies Koopman, Veerle Linseele, and Willeke Wendrich

Chapter 5: The K Basin Archaeological Record

Willeke Wendrich, Simon J. Holdaway, Rebecca Phillipps, and Joshua J. Emmitt

Chapter 6: Kom K

Willeke Wendrich, Rebecca Phillipps, Simon J. Holdaway, Veerle Linseele, Joshua J. Emmitt, and John M. Marston

Chapter 7: The Desert Fayum Reinvestigated: The Evidence Considered

Simon J. Holdaway, Willeke Wendrich, and Rebecca Phillipps

Chapter 8: The Desert Fayum Reinvestigated: The Fayum in Context

Simon J. Holdaway, Rebecca Phillipps, and Willeke Wendrich

References Cited

Index 


\section{List of Figures}

Figure 1.1. Known archaeological sites within the Fayum Depression, organized by period.

Figure 2.1. Conceptual model based on Smith's (2001) low-level food producers using mobility estimates.

Figure 3.1. Early to Middle Holocene models of lake level fluctuations ( $m$ asl) for Lake Qarun, the Fayum Depression.

Figure 3.2. E29G1 (Z1) overlain with the digitized geomorphology from Wendorf and Schild (1976:Figure 97) and contemporary DSM-derived 1-m contours.

Figure 3.3. Ten-m, 18-m, 20-m, and 24-m contours from DSM 1-m data.

Figure 3.4. The wadis, basins, and Neolithic lake edge (18 $\mathrm{m}$ asl) identified by Caton-Thompson and Gardner (1934).

Figure 3.5. Reclassification of basin slope values based on the Parsons (1988) nine-unit model with an inset of the Z Basin north-south profile.

Figure 3.6. The simulated extent of Lake Qarun inundation when lake levels are increased to $4 \mathrm{~m}$ and 7 $\mathrm{m}$ asl, showing exposure of the north shore basins.

Figure 3.7. Surface sediment types identified at XB11.

Figure 3.8. Colored nails used to mark the location of different types of artifacts during transect survey.

Figure 3.9. Transect C4T5 showing surface sediment types mapped with a total station and stored in a GIS.

Figure 3.10. C4T5 surface sediment types overlain with total artifact density derived from the provenance of all objects greater than or equal to $20 \mathrm{~mm}$ in maximum dimension (artifact $/ \mathrm{m}^{2}$ ).

Figure 3.11. Survey corridors placed evenly across the project concession.
Figure 3.12. Density of complete flakes, based on high-visibility surfaces only, and the flake-to-core ratio from these same surfaces, for corridor transects with sufficient artifacts to allow the calculations.

Figure 3.13. Hearths and grindstone density (number/ $\mathrm{m}^{2}$ ) located within the corridor surveys.

Figure 3.14. Examples of flaked stone artifacts and a flaked cobble from the Fayum.

Figure 4.1. L1 Basin showing the location of E29H1 together with the transects surveyed, faunal collection squares, areas sampled for artifact analysis, and topography.

Figure 4.2. Sediment types identified at E29H1.

Figure 4.3. Stratigraphic profile west to east across E29H1.

Figure 4.4. E29H1 stone artifact density (number $/ \mathrm{m}^{2}$ ) overlaying the sediment types.

Figure 4.5. E29H1 hearth showing charcoal.

Figure 4.6. E29H1 bone density number/ $\left(\mathrm{m}^{2}\right)$ overlaying the sediment types.

Figure 4.7. A tree-map plot showing the relative proportions of objects by material for L1 transects and E29H1 areas in the inset box.

Figure 4.8. L1 Basin hearth density $\left(\mathrm{m}^{2}\right)$ overlaid with dated hearths coded by age class and showing the calibrated radiocarbon determinations.

Figure 4.9. Calibrated radiocarbon determinations from E29H1 plotted against the IntCal13 atmospheric data.

Figure 4.10. Calibrated ages from L1 hearths plotted against the IntCal13 atmospheric data.

Figure 4.11. L1 and E29H1 calibrated hearth ages plotted against the IntCal13 atmospheric data. 
Figure 4.12. Grinding stone density (number $/ \mathrm{m}^{2}$ ) from E29H1 and L1 overlaying areas surveyed and destroyed.

Figure 4.13. Hypothetical reduction sequence of an Egyptian flint cobble for flaking.

Figure 4.14. Mean core dimensions (in $\mathrm{mm}$ ) oriented relative to the largest core scar.

Figure 4.15. Original cobble volume estimates for analyzed assemblages in $\mathrm{mm}^{3}$.

Figure 4.16. Flake-to-core ratios in order for all assemblages in L1 and E29H1.

Figure 4.17. Ordered flake-to-core ratio divided by estimated cobble volume, calculated from unifacial and bifacial cores with more than 50 percent cortex.

Figure 4.18. Cortex ratio results for analyzed assemblages.

Figure 4.19. Mean platform thickness plotted against mean exterior platform angle for the analyzed assemblages.

Figure 5.1. K Basin Corridor 4, K1, and C4 transects with the location of Kom $\mathrm{K}$ and the Upper K Pits and the probable location of the Lower K Pits.

Figure 5.2. Tree-map plots showing the relative densities of complete flakes, broken flakes, cores, tools, bone, ostrich eggshell, and ceramic in K1 transects on desert pavement.

Figure 5.3. Hearth $\mathrm{K} 1 \mathrm{H} 20$ with in excess of 1,500 hearthstones.

Figure 5.4. Calibrated ages from K1 hearths plotted against the IntCal13 calibration curve.

Figure 5.5. K1 hearth density (per $\mathrm{m}^{2}$ ) overlaid with dated hearths coded by age classes.

Figure 5.6. Kom IV identified by Caton-Thompson, eastern edge, looking southwest and showing the effect of modern-day development and the destruction of the Kom to the south.

Figure 5.7. Identified C4T13 sediment surfaces overlaid with complete flake density $\left(\mathrm{m}^{2}\right)$.

Figure 5.8. Stratified deposits to the east of C4T13, excavated as Trenches 2 and 3.

Figure 5.9. Transects and extensions used to obtain a sample of flaked stone artifacts to analyze in $\mathrm{K} 1$, with flaked stone artifact density.

Figure 5.10. Mean $\mathrm{K} 1$ oriented core dimensions in comparison to the E29H1 and L1 assemblages.

Figure 5.11. Flake-to-core ratio for the $\mathrm{K} 1$ and $\mathrm{C} 4$ transects, with sufficient flakes and cores to enable calculation.

Figure 5.12. Mean complete flake platform thickness against mean exterior platform angle.
Figure 5.13. Basketry-lined Silo 12.

Figure 5.14. Basketry-lined Silo 1.

Figure 5.15. Distribution of the Upper K Pits identified by Caton-Thompson and Gardner in 1926 and additional pits discovered in 2004, as well as other features that may represent recent excavation.

Figure 5.16. A mechanically excavated large trough that destroyed the newly discovered K Pits.

Figure 5.17. Schematic overview of the western Upper K Pits.

Figure 5.18. Trench UKP01.

Figure 5.19. Pit 67 during reexcavation.

Figure 5.20. Lid of Pit 68 with the crushed shell deposit.

Figure 5.21. Pit 68; Chambardia sp. shell scoop and first of two tamarisk sticks.

Figure 5.22. Pit 68; second tamarisk stick and base of basketry lining.

Figure 5.23. Pit 68; cross-section of lid and basket.

Figure 5.24. Feature 72, interpreted as a preparation area in UKP05.

Figure 5.25. Overview of Pits 68, 69, and 73, with Features 70 and 72.

Figure 5.26. Feature 74 in UKP06, plan and cross-section. Figure 5.27. Pit 75 in Trench UKP07.

Figure 5.28. Cross-section of the lid of Pit 75.

Figure 5.29. Plan and cross-section of Pit 75 in Trench UKP07.

Figure 5.30. Surface of trench UKP09, with basket fragment in situ.

Figure 5.31. Pit 69 in Trench UKP09.

Figure 5.32. Cross-section of the fill of Pit 69 in Trench UKP09.

Figure 5.33. Feature 70 in Trench UKP09.

Figure 5.34. Discolored outline of the decayed basketry lining of Pit 71.

Figure 5.35. Lid of Pit 71 with an embedded, near-complete ceramic vessel.

Figure 5.36. Remains of the badly preserved straw basketry lining of Pit 71.

Figure 5.37. Feature 79 in Trench UKP31.

Figure 5.38. Outside of the finely coiled basket fragment found in Trench UKP09.

Figure 5.39. Inside of the finely coiled basket fragment found in Trench UKP09.

Figure 5.40. Construction drawing of the insertion of new grass winders in the finely coiled basketry.

Figure 5.41. Close-up of the winders (active system) of a basket fragment.

Figure 5.42. Finely coiled basket found in 1926 . 
Figure 5.43. Lining of Pit 30, excavated by CatonThompson in 1926.

Figure 5.44. Basketry lining of Pit 68, excavated in 2004 and 2005.

Figure 5.45. Construction drawing of how straw bundles are split off to form the winder that fastens the coils together.

Figure 5.46. Detail of the straw coiled lining of Pit 68 [04.0003].

Figure 5.47. Radiocarbon determinations from Pit 68 and Pit 75 and the two older determinations from Libby (1952).

Figure 6.1. Kom K 5 x 5-m collection square locations with $10-\mathrm{cm}$ contours showing the year of collection.

Figure 6.2. Magnetic anomaly survey of Kom K with 10-cm topographic contours superimposed.

Figure 6.3. Excavation trenches overlaid onto a map of magnetic anomalies and the approximate location of Caton-Thompson and Gardner's excavations.

Figure 6.4. Overview of Kom $\mathrm{K}$ excavations with $\mathrm{K}$ ridge in the background.

Figure 6.5. KK02 hearth set showing the extent of the excavation units.

Figure 6.6. Northern part of Trench KK04 with the heat retainers of Hearth 130 in the foreground.

Figure 6.7. KK04 Hearth 130, a large hearth with stone and ceramic heat retainers.

Figure 6.8. KK05, one of two circular pits with rounded bottoms.

Figure 6.9. Overview of KK06, looking south.

Figure 6.10. KK07 half section of excavated unfired clay vessel.

Figure 6.11. KK07 (front) and KK08 (back), looking north.
Figure 6.12. KK07 western profile of excavation trench. Figure 6.13. Radiocarbon determinations from the Kom $\mathrm{K}$ hearths plotted against the IntCal13 calibration curve.

Figure 6.14. Stone artifact density for collection squares where artifacts were recorded.

Figure 6.15. Mean complete flake platform thickness against mean exterior platform angle.

Figure 6.16. Density of ceramics recorded in analyzed Kom K sample squares.

Figure 6.17. Kom $\mathrm{K}$ sherd with parallel incised line decoration.

Figure 6.18. Incomplete ostrich eggshell bead.

Figure 6.19. Complete ostrich eggshell beads.

Figure 6.20. Bone bead.

Figure 6.21. One of two fragmentary bone rings found in Kom $\mathrm{K}$.

Figure 6.22. One of the three pieces of worked ostrich eggshell found at Kom K.

Figure 6.23. Faunal proportions based on NISP from excavated trenches at Kom K.

Figure 6.24. Caprid pellets from Kom K hearth [07.0019].

Figure 7.1. Radiocarbon determinations from hearths from sample locations in the Fayum with ages between 10,000 and $5500 \mathrm{cal} \mathrm{BP}$.

Figure 7.2. Summed probability plot for radiocarbon determinations from the Fayum north shore.

Figure 7.3. North of L Basin showing recent destruction as of December 2014.

Figure 7.4. Ostrich eggshell density $\left(\mathrm{m}^{2}\right)$ in transects from all areas with corridors and survey areas.

Figure 7.5. Ceramic density $\left(\mathrm{m}^{2}\right)$ in transects from all areas with corridors and survey areas. 


\section{List of Tables}

Table 3.1. Slope Change Calculations for the North Shore Basins Identified by Caton-Thompson and Gardner (1934).

Table 3.2. Surface Types Used to Characterize Survey Transects.

Table 3.3. Artifact, Bone, and Ceramic Categories Recorded in Transects.

Table 3.4. Densities of Artifacts, Fauna, and Ceramics in Corridor Transects as Number of Objects per Square Meter.

Table 3.5. Variables and Values Recorded for Grinding Stones.

Table 3.6. Variables Recorded for Hearths, 2012.

Table 4.1. Frequency of Materials Identified in the 2008 Survey at E29H1.

Table 4.2. Mean Clast Size for Artifacts from Three Sedimentary Units at E29H1.

Table 4.3. Density (per $\mathrm{m}^{2}$ ) of Artifacts and Fauna over $20 \mathrm{~mm}$ in Maximum Length in the Area Surrounding E29H1.

Table 4.4. Nonparametric Tests Comparing Density Measures for Different Object Types in Different Sediments for Each Transect.

Table 4.5. Maximum and Minimum Elevations in Meters above Sea Level for Hearths Excavated at E29H1.

Table 4.6. E29H1 Hearths That Were Excavated and Provided Sufficient Charcoal for a Radiocarbon Determination.

Table 4.7.Excavated L1 Hearths ThatProvided Sufficient Charcoal for a Radiocarbon Determination.

Table 4.8. L1 Grinding Stone Lithology and Shape.

Table 4.9. E29H1 Grinding Stone Lithology and Shape.

Table 4.10. Numbers of $3 \times 3-\mathrm{m}$ Squares Analyzed.
Table 4.11. Proportions of Faunal Elements with Different Colors from the Collected Trenches.

Table 4.12. List of Animal Taxa from the Different Trenches at E29H1.

Table 4.13. Shell Species Identification from E29H1.

Table 4.14. List of Animal Taxa from the Different Trenches at E29H1.

Table 4.15. Means and Standard Deviations (SD) for Cores from E29H1 and L1 Analyzed Assemblages.

Table 4.16. Core Form Frequency and Proportions.

Table 4.17. Eight Experimental Cobbles Reduced to Determine the Effect of Flake Removal on Core Size.

Table 4.18. Ratio of Mean Core Axis Dimensions for Core Types.

Table 4.19. Mean Complete Flake Length to Flake Width Ratios.

Table 4.20. Core Proportions with Proportion of Cortex Present by Assemblage.

Table 4.21. Cortex Proportion on Cores with Different Forms.

Table 4.22. Mean Complete Flake Dimensions for All Flakes and Those with Different Cortex Proportions.

Table 4.23. Volume Ratio Values for E29H1 and L1 Assemblages.

Table 4.24. Tool Type Frequencies in the Analyzed Assemblages.

Table 5.1. K1 Transect Object Density by Surface Type.

Table 5.2. Excavated K1 Hearths That Provided Sufficient Charcoal for a Radiocarbon Determination.

Table 5.3. K1 Grinding Stone Frequency by Raw Material and Form.

Table 5.4. Shape and Lithology of Grinding Stones Found in Corridor 4. 
Table 5.5. Flaked Stone Artifact Core Dimensions from the K1 Transects.

Table 5.6. K1 Flaked Stone Artifact Core Form Frequency and Proportion.

Table 5.7. K1 Ratio of Mean Core Axis Oriented Dimensions for Core Types.

Table 5.8. K1 Core Frequency by Core Type and Cortex Proportions.

Table 5.9. K1 Complete Flake Dimensions by Cortex Proportion.

Table 5.10. K1 Tool Type Frequency by Flake Class.

Table 5.11. Upper K Pits, Summary of Pits and Other Features from the Previous and Current Excavations.

Table 5.12. Basketry Finds from the K Pits from the 1926, 2004, and 2005 Excavations.

Table 5.13. Surface Areas $\left(\mathrm{cm}^{2}\right)$ and Volumes $\left(\mathrm{cm}^{3}\right)$ of the Upper K Pits Ceramic Vessels Organized by Shape, with Either a Restricted or Unrestricted Opening.

Table 5.14. Radiocarbon Determinations from K Pits.

Table 5.15. Diameters of Features from the Lower K Pits Compared with Similar Features from the Upper $\mathrm{K}$ Pits and Kom K.

Table 6.1. Characteristics of Wood Type Identification for Charcoal from Kom K Hearths.

Table 6.2. Radiocarbon Determination for Charcoal from Kom K Hearths.

Table 6.3. Feature Numbers and Units for Hearths Excavated at Kom K Discussed in Text.
Table 6.4. Stone Artifact Frequency by Technological Category and Artifact Density from Kom K Surface Sample Squares.

Table 6.5. Frequency of Analyzed Stone Artifacts by Technological Class Analyzed from Kom K Trench KK05.

Table 6.6. Frequency of Analyzed Stone Artifacts by Technological Class from Kom K Trench KK08.

Table 6.7. Frequency of Analyzed Stone artifacts by Technological Class from Kom K Trench KK07.

Table 6.8. Core Type Frequencies for Kom K Analyzed Assemblages.

Table 6.9. Core Type Mean Dimensions (with Standard Deviations) for Analyzed Kom K Assemblages.

Table 6.10. T-test Comparisons between the Three Most Common Core Forms in the Surface and Excavated Assemblages.

Table 6.11. Core Type Axial Dimension Ratios for Kom K Surface and Excavated Assemblages for the Most Common Worked Core Types.

Table 6.12. Core Type Frequencies by Cortex Proportion for Surface and Excavated Assemblages.

Table 6.13. Complete Flake Dimensions with Different Proportions of Dorsal Cortex.

Table 6.14. Retouched Tools from Kom K from Surface and Excavated Contexts Combined.

Table 6.15. List of Identified Faunal Taxa (Numbers of Identified Specimens, NISP) by Trench at Kom K.

Table 6.16. Nile Perch Sizes from Kom K. 


\section{Acknowledgments}

T

he work reported in this volume was conducted from 2003 to 2012 in a collaborative project involving colleagues from the University of California-Los Angeles in the United States, Rijksuniversiteit Groningen in the Netherlands, and the University of Auckland in New Zealand. A large number of individuals contributed to the fieldwork conducted in successive seasons.

First, exploration of the Lake Qarun north shore, in 2003, was undertaken by Willeke Wendrich, Hans Barnard, Mohsen Kamel, Noriyuki Shirai, Amber Myers, Mayyada Ahmed Naguib, and Nahlah Mohamed Ahmed. In 2004 the team was expanded to include Maria Gatto, Tonnie de Wit, Eric Wells, Kandace Pansire, and Rebecca Phillipps, and in 2005 Johanna Then, Justin Eichelberger, Erica Ellis, and Miguel Alvarez. Excavations at the Upper K Pits occurred during the 2004 and 2005 seasons, led by Willeke Wendrich and Maria Gatto.

In 2006 and 2007, excavations were undertaken at Kom K. Trench supervisors were Emily Cole, Rebecca Phillipps, Angela Susak, Carrie Zarnoch, Jessica Martinez, and Melina Goddard, assisted by Nevine Moussa, Charlene Collazzi, Rebekah Merriman, Leigh Winiata, and Bethany Simpson.

Geoarchaeological research was performed by Lewis Owen (2003, 2004, 2008), Kimberly Le (2005, 2006), Ed Rhodes (2012), Marcus Thompson (2012), and Annelies Koopman (2007, 2008, 2010), with contributions by advisors Sjoerd Kluiving (2007, 2008), Erika Guttmann-Bond (2010), and Glen McDonald (2008, 2010). The survey at XB11 in 2004 was performed by Rebecca Phillipps with the assistance of Eric
Wells, Kandace Pansire, Tonnie de Wit, Maria Gatto, Noriyuki Shirai, Sander Droogsma, and Marianna Betti.

The intensive survey directed by Simon Holdaway and Rebecca Phillipps started in 2008. The survey team worked in 2008, 2009, 2010, and 2012 and consisted of Sam Lin, Melina Goddard, Natasha Phillips, Emma Holdaway, Shezani Nasoordeen, Thomas Barker, Tamzin Linnell, Kane Ditchfield, Joshua Emmitt, Stacey Palmer, Anna Stuart, Karl LaFavre, Ben Nigra, Matthew Barrett, Tara Copplestone, Rachel Scott, Samantha Lagos, Bethany Simpson, Anna Ietswaart, Paul van Pelt, Rinus Ormeling, Meryl King, Anne Austin, and Nagwan Bahaa el-Hadidi. Analysis of the lids of the Upper K Pits was performed by Paul van Pelt and Alexandra Winkels. Magnetometric survey was done by Thomasz Herbich, Artur Buszek, and Sebastian Pietrzak.

Archaeobotanical analysis of materials from the Upper K Pits and Kom K was directed by René Cappers, who was assisted by Mans Schepers, Ingrid Heijen, Pascal Flohr, and Jasmijn van der Veen. Fauna analysis was performed by Wim Van Neer and Veerle Linseele, while Kim Duistermaat recorded the personal ornaments.

The support staff for the team consisted of Reda Sayyed Hassan (Hamam), Fatma Mohamed Abd el Ghani, Abir Faruq Zaki, Nubi Sayed Hassan, Sayed Nubi Sayed, and Mohamed Nubi Sayed.

The project thanks the respective chief inspectors of the Fayum, Dr. Abdel Rahman el Ayedi and Mr. Ahmed Abd el-Aal, as well as former secretary general of the Supreme Council of Antiquities Dr. Zahi Hawass; Dr. 
Mustafa Amin; and their excellencies Dr. Mohamed Ibrahim and Dr. Mamdouh Eldamaty of the Ministry of State for Antiquities. We would also like to thank our colleagues at the Fayum Inspectorate and beyond who accompanied our work: Nuby Mohamed Ahmed, Mustafa Faisal Himeidi, Sabr Attiya Duwib, Ashraf Sobhy Rizqalla, Nahlah Mohamed Ahmed, Rehab Galal Darwish, Mohamed Ahmed Siddek, Mazhar Ezzat Abderrahim, Mustafa Mohamed Nureldin, Sayed Awad, Mohsen Helmi Badawy, Anwar Ahmad Abd-el Samad, Mahmoud Galal Mokhtar, Mohamed Ragaie Abdel-Hakim, and Mohamed Hussein Fathi.

Cooperation with the Egyptian Mineral Resources Authority for the geoarchaeological work was initiated and supported by Dr. Fekry Youssef Mohamed (chairman) and Dr. Bahaa G. Gayed (director general of planning and follow-up). The URU project also wants to express its enormous gratitude to Dr. Bahay Issawi, whose extensive knowledge and experience in the field has unfailingly supported the work of the URU team.

Illustrations were prepared by Briar Sefton, Matthew Barrett, Tim Mackrell, Matthew Hill, Seline McNamee,
Rebecca Ramsey, and Hans Barnard. We also would like to thank Dr. Alice Stevenson and Dr. Emma Libonati from the Petrie Museum, University College London, for providing information on museum holdings from the 1924 excavations of Gertrude CatonThompson and Elinor Gardner.

The prehistoric work of the URU Fayum Project has been supported financially by the National Geographic Society (8293-07, 8295-07, and 8413-08); the Royal Society of New Zealand Marsden Fund (UOA1106); the UCLA Council on Research; the Ahmanson Fund of the Cotsen Institute of Archaeology; the Faculty of Arts, University of Auckland; British Petroleum, which funded a protective fence in 2004; the Apache Oil Company, which provided multiple years of continuous support; and several individual donors, among whom we would like to thank especially Piers Litherland for his support of the 2004 and 2005 field schools, Deborah Arnold and Harris Bass for their many years of continued support, Tom Voytovich, Art Muir, and lastly the Steinmetz Family Fund, which supported UCLA graduate students with travel funds. 


\title{
Editors Biographies
}

\author{
Simon J. Holdaway \\ Professor of Archaeology Head of School of Social Sciences \\ University of Auckland
}

Simon J. Holdaway (PhD University of Pennsylvania, 1991) is professor of archaeology and head of the School of Social Sciences at the University of Auckland in New Zealand. He holds honorary chairs at Macquarie University, the University of Queensland (Australia), and the University of York (United Kingdom). He has research interests in hunter-gathering and low-level food production as well as stone artifact technology, geoarchaeology, and landscape archaeology. He has directed field projects in Egypt, Australia, and New Zealand, investigating the surface archaeology of arid regions, coastal and island archaeology, and historical archaeology. His latest books are $A$ Geoarchaeology of Aboriginal Landscapes in Semiarid Australia (CSIRO Publishing, 2014) and Finding Our Recent Past: Historical Archaeology in New Zealand. (New Zealand Archaeological Association, 2013).

\section{Willeke Wendrich}

Professor of Egyptian Archaeology and Digital Humanities

Joan Silsbee Chair in African Cultural Archaeology

University of California, Los Angeles

Willeke Wendrich (PhD Leiden University, the Netherlands, 1999) holds the Joan Silsbee Chair in African Cultural Archaeology and is professor of Egyptian archaeology and digital humanities in the Department of Near Eastern Languages and Cultures at the University of California, Los Angeles. She has worked for 30 years in Egypt and currently directs a project in the north of Ethiopia. Focusing on the "invisible material past," the project combines archaeology with an ethnoarchaeological study of the use of organic materials, ancient technology, and communities of practice. Wendrich is the faculty director of the Center for Digital Humanities and director of the Cotsen Institute of Archaeology at UCLA, as well as editor-in-chief of the online UCLA Encyclopedia of Egyptology, a worldwide cooperation of Egyptologists, archaeologists, linguists, art historians, geologists, and all other disciplines involved in research in Egypt. She chairs the board of the Institute for Field Research. Her latest books are Egyptian Archaeology (Wiley-Blackwell, 2010) and Archaeology and Apprenticeship, Body Knowledge, Identity, and Communities of Practice (University of Arizona Press, 2012). 


\section{Contributors}

Joshua J. Emmitt

University of Auckland

Simon J. Holdaway

University of Auckland

Annelies Koopman

University of Amsterdam

Veerle Linseele

University of Leuven
John M. Marston

Boston University

Rebecca Phillipps

University of Auckland

Rebecca Ramsay

University of Auckland

Willeke Wendrich

University of California, Los Angeles 


\section{1 \\ Landscape Archaeology of the Desert Fayum}

Willeke Wendrich and Simon J. Holdaway

The Northern Fayum desert as we know it, with all its diversified archaeological and physiographical features is probably doomed to vanish in a few years. The pressure of Egypt's teeming population, and her economic expansion, are bound before long to play their part in the reclamation of every acre of desert ground which, by irrigation, can be rendered life-supporting. The desert Fayum - the Wadi Rayan-the great tract of desert to the west, cannot possibly escape; Ptolemy's engineers with relatively small resources, led the way, and on an infinitely larger scale modern enterprise will follow [Caton-Thompson and Gardner 1934:12].

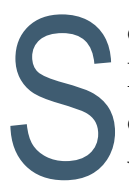
o wrote Gertrude Caton-Thompson and Elinor Gardner in 1934. As a consequence, our expectations of the possibility of reinvestigating this region with new research questions and techniques were not very high, but as it turned out, Caton-Thompson and Gardner's assessment was too negative. For more than 50 years after they wrote these words, the region saw only very moderate agricultural development on the north shore. Their comments are, however, all too poignant today. As documented here, the destruction of the desert landscape has increased exponentially, starting in the decade before our first survey and continuing year by year at an incredibly rapid pace. This volume therefore represents a combination of research and salvage archaeology reflecting in some cases the last fieldwork report on locations that have been destroyed.
The Fayum is a natural depression, separated from the Nile Valley by a ridge known as the Nile-Fayum divide (Sandford and Arkell 1929). This volume reports the results of work carried out in a portion of the depression, on the Fayum north shore, where we have a concession that extends from Qasr el-Sagha up to and including Karanis, an area of $676 \mathrm{~km}^{2}$ (Figure 1.1). The term north shore refers to the shoreline of Lake Qarun. The shoreline of the present-day lake features curved spits of land, and it was probably these that gave Lake Qarun its Arabic name (qarun means "horns"). The ancient Egyptian name for the lake was hnt $m r$ wr ("Lake of Great-Canal"), probably named after the village Mer-wer, possibly Gurob, from which the classical name of the Limne Moeris ( $\dot{\eta}$ Moígıs $\lambda$ í $\mu$ inj; "the Lake of Moeris") was derived (Cruz-Uribe 1992; Gardiner and Bell 1943). The great canal after which this village was named most probably was 
the artificially adapted branch of the Nile, at present known as the Bahr el-Yusuf. Influx of floodwaters from the Nile Valley, through a branch of the Nile that ran parallel to the main channel of the river at Dairut and entered the Fayum Basin at Hawara, caused fluctuation in lake levels at times during the Holocene. At present, the surface of the lake is on average $44 \mathrm{~m}$ below sea level (bsl). The lowest-lying remains from the Roman period are found at approximately $40 \mathrm{~m}$ bsl. Ptolemaic-period settlement remains occur at slightly higher elevations, although most are found outside the basin around sea level. The rationale for the location of these settlements is that they were built just outside, and thus on the higher rim, of the basin, while the agricultural fields were located at lower elevations, around $38 \mathrm{~m}$ bsl. From papyrological evidence, it is clear that there were many Ptolemaic and later settlements in the Fayum Basin itself as well, but these are rarely located or identified because they are usually underneath present-day towns and villages (Davoli 1998; Derda 2006).
There are very few Pharaonic remains in the Fayum Desert, with the exception of Kom IV, an Old Kingdom settlement in the L Basin area, and the Old and Middle Kingdom remains near Qasr el-Sagha, an area that falls outside our research concession. This results in a somewhat skewed image of activity in the Fayum Basin because there were extensive changes to the landscape during the Middle Kingdom, when the great push for agricultural expansion resulted in the construction of a system of canals and control of the influx of Nile water at the entrance of the Fayum near Hawara (Butzer 1976:37). The Middle Kingdom canals were an inspiration for the officials who, under Ptolemy II (285-246 $\mathrm{BCE})$, restored and expanded the system and were under instruction to make full use of the existing canals (Thompson 1999:119). The relative lack of Pharaonic remains is, however, a fair reflection of the dearth of activity in the increasingly arid region northeast of the lake. Three Greco-Roman villages are located in the research area: el-Qarah el-Hamra, discovered in 2003

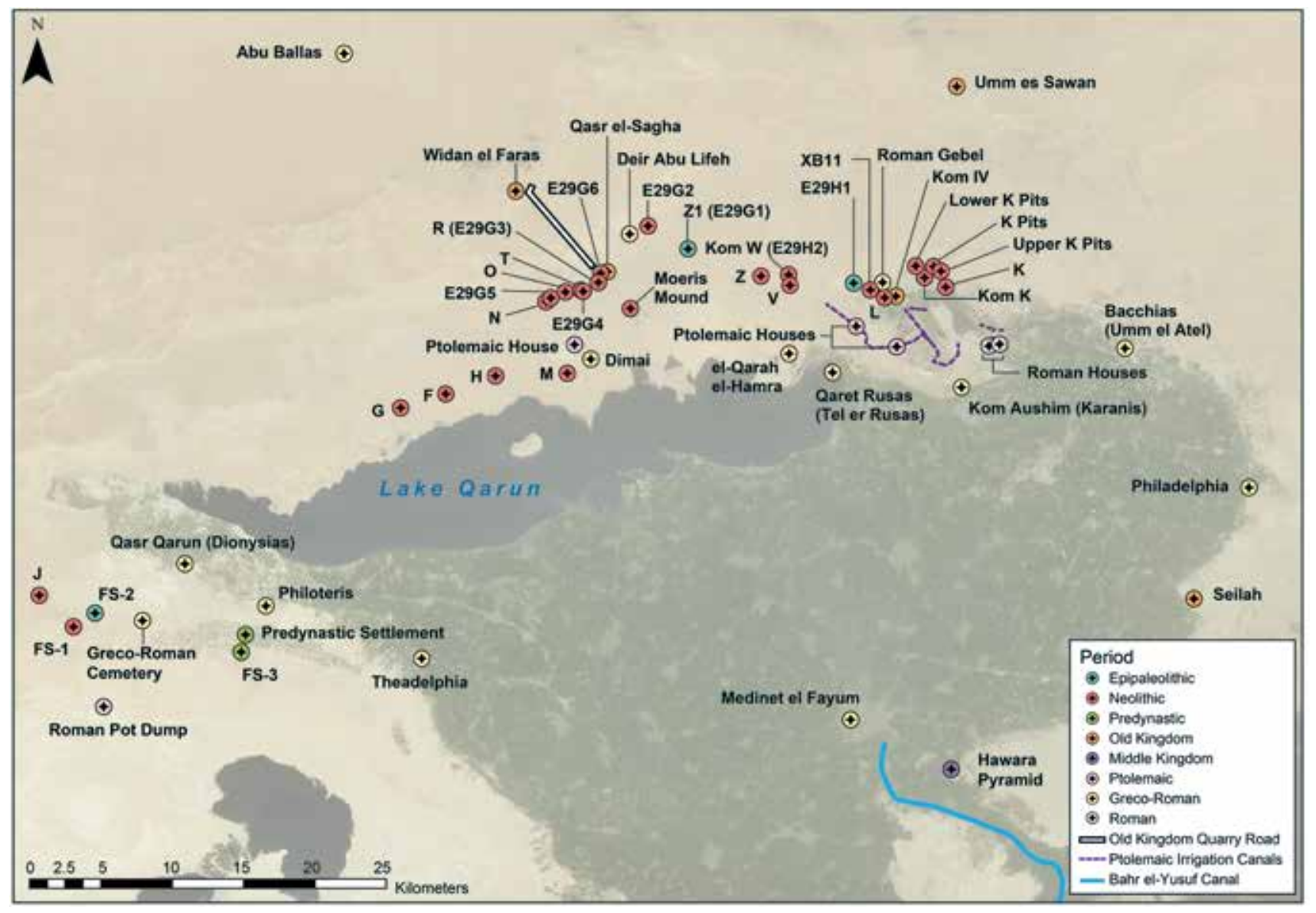

Figure 1.1. Known archaeological sites within the Fayum Depression, organized by period. 
on the north shore of the lake; Qaret Rusas, located on a peninsula at the lake's eastern side; and Kom Aushim/ Karanis, the largest settlement on the south side of a wadi, through which the main road from Cairo to the Fayum runs today (Figure 1.1). Of several small Greco-Roman settlements noticed in the 1920s, only scant surface scatters remain (Caton-Thompson and Gardner 1934).

While remains dating to historic periods are limited to the areas discussed above, at higher elevations there are (but sadly in some cases were) extensive surface and buried deposits of prehistoric remains. These are found at elevations between 0 and $35 \mathrm{~m}$ asl, mostly concentrated in a band several hundred meters from what have been interpreted as ancient lakeshores, visible to the north and northeast of the present-day lake. The region was until very recently arid, eroded, and deflated but was otherwise an intact ancient landscape because large parts of it were not built over with roads, settlements, or industry. However, this important area is today under increasing threat due to largescale development of clay mining, irrigation agriculture, road construction, and tourism. The most direct threats to the early to mid-Holocene remains come from large-scale land reclamation, roadwork, and irrigation projects, which result in canal systems cutting through the landscape, plowing of the surface, contamination of ancient remains with modern plant material, and problems for archaeologists wanting to gain access to land that is increasingly coming under cultivation.

We report here on the early to mid-Holocene remains from the eastern section of our concession, since it includes parts of the north shore that have been most subject to modern-day development. Two wellknown sites-Kom K, excavated by Caton-Thompson and Gardner, and E29H1, studied by Wendorf and Schild-are located in this area, and we report here on new work at both sites. However, in addition to single locations, we also report on extensive and intensive studies of the surface remains that are common in many parts of the north shore. Like Caton-Thompson and Gardner's original work, our focus is both on a geoarchaeological and a landscape approach to Fayum archaeology. While neither of these terms were current when Caton-Thompson and Gardner worked, their original study certainly had aspects of both, and we continue to admire how much their approach was ahead of its time in the consideration they gave to the geomorphic contexts of the prehistoric remains they discovered. We are also struck by the commitment they showed in dealing with Fayum archaeology at a spatial scale that has rarely been followed since in early to mid-Holocene studies in Egypt.

The Fayum is known for the earliest evidence of the use of domesticated wheat and barley in Egypt during the Neolithic but also for an expansion and intensification of agricultural activity during the Middle Kingdom and the Greco-Roman period. It was the combination of both of these aspects of Egyptian socioeconomy that interested us in the region. Under the auspices of the Ministry of State for Antiquities (MSA; previously the Supreme Council of Antiquities or SCA), the University of California, Los Angeles, Rijksuniversiteit Groningen in the Netherlands, and the University of Auckland in New Zealand, the URU Fayum Project was formed to work in the area. The main research objective of the URU Fayum Project is to interpret the land and water use of the region northeast of Lake Qarun by studying, analyzing, and interpreting the natural, anthropogenic, economic, and social processes that formed the present landscape and the archaeological remains that are part of it. The development of agriculture is a central theme in this study, but this is put in its environmental, economic, and social context by the study of natural processes and human activities related to subsistence, economy, procurement, production, consumption, ritual, and discard. In this volume, we consider evidence for landscape use in the Fayum during the early to mid-Holocene, a period that includes but is not limited to the appearance of domestic plants and animals.

\section{Project History}

The work on which this volume is based started in 2003, when we were granted a research concession for the region north of Lake Qarun, east of the line Qasr el-Sagha-Dimai. We initially embarked on a brief exploratory survey with a small team to check the potential of this area and the range of archaeological periods represented in the region. Neither the Pharaonic remains at Qasr el-Sagha nor the Greco-Roman remains at Dimai were included in the research area.

The 2003 season started with survey work in the western part of our concession, revisiting some of the excavations of Wendorf and Schild (1976). We then selected one particular area, the north shore of CatonThompson's Z Basin, for a systematic survey, surface recording, and excavation to obtain a good geomorphological sequence and overview of the surface scatter 
distribution along the slope of the lakeshore and a cross-section of the shoreline deposits.

Based on the results of the first season, the 2004 team was expanded. The prehistoric work during a period of six weeks continued the exploratory survey, involved an intensive study of and excavations at the Upper K Pits area, and involved surface collections at a number of locations. A magnetic survey of the unexcavated area around Kom W was undertaken.

In 2005 the team worked for a month recording the prehistoric remains, continuing the exploratory survey as well as excavation at the Upper K Pits area. Two localities in the X Basin were surveyed to better understand the local geomorphology where a large wadi ran into the ancient basin. At a trench excavated at Kom Aushim, the team explored the preservation of botanical remains; this will be reported on in a future volume.

During the 2006 season, a small-scale excavation was started at Kom K and Kom W, concentrating on the area outside the large 1924-1925 excavations. Surface collections of stone artifacts and ceramics were also made at Kom K and Kom W. Toward the end of the four-week season, a magnetic survey of the central parts at both locations demonstrated the potential for gaining important new information: Kom $\mathrm{K}$ displayed clear anomalies in the area that was supposed to be exhaustively excavated by Caton-Thompson, while at Kom W, results indicated that Caton-Thompson's strip-like trench excavations had left baulks between the excavated areas. In her 1934 publication, these baulks are shown as drawings but are never described, and from the published description it seemed that they were excavated after recording. However, the discovery that they remained intact indicated that the stratigraphy of the site was still available for examination.

Upon our arrival in Egypt in 2007, it appeared that the part of the Upper K Pits area where we had excavated in 2004 and 2005 was destroyed. Increased efforts were made to invite the SCA to protect the Kom $\mathrm{K}$ site, and to present a convincing case, excavations concentrated on this area. The SCA sent an investigative committee to inspect the area, and we provided evidence of the scientific value and cultural importance of archaeological remains that at first sight might not look particularly impressive.

Our efforts to bring the privately owned Kom K land under control of the SCA seemed to be successful. In 2008 work was originally planned to continue there, but upon our arrival in the region, it appeared that another very important area, a dense surface scatter with many hearths-noted by Caton-Thompson in the 1920s, published as site E29H1 by Wendorf and Schild (1976), and explored by the URU team as part of the 2005 survey-was being destroyed by large-scale agricultural development. After a discussion with the landowner, we gained his permission to survey for one month while agricultural activity was halted, and we decided to put most of our resources into this area. In addition, two trenches were excavated at Kom W, based on the 2006 magnetic survey results. It was deemed unwise to postpone this research because of the encroaching agricultural development and increased touristic and military traffic indicated by disturbance on the top of the mound. In discussion with the Fayum antiquities inspectorate, we decided to build a wire fence around the area, a measure that had been successful in protecting the eastern part of the Upper K Pits.

Upon our return in 2009 , we found that the fence had been destroyed and that Kom W had been damaged by a bulldozer and a large-format mining drill. The main damage was a large square hole with the approximate dimensions of our $5 \times 5-\mathrm{m}$ trench and a depth of almost $7 \mathrm{~m}$. We concentrated on cleaning up and recording the most important Kom W robber sections, while the intensive survey concentrated on sampling the wider concession area. Survey transects to the east and west of the Kom W area demonstrated the existence of further stratified deposits within the mostly deflated landscape. The first part of the season (before the arrival of the Neolithic survey team) concentrated on continuing survey, excavations, and field school training at Kom Aushim.

During the 2010 season we found further destruction at Kom W, with additional bulldozer activity and an enormous circular hole of roughly $4 \mathrm{~m}$ in diameter, drilled to a depth of approximately $15 \mathrm{~m}$. The drilling technique was the same as in the destruction of several Roman tombs in the rocky escarpment just above the agricultural land of the Fayum Basin proper. The intensive survey of the area around Kom W, including the recording of all hearths and grinding stones, was continued.

In 2011 political unrest in Egypt caused a delay in the issuance of the military clearance and a permit that allowed survey, conservation, and restoration, but not excavation. A brief period of survey of the area north and west of Kom $\mathrm{K}$ was undertaken to establish the 
potential for future work. Fall 2012 saw the return of the full URU team, with survey concentrated on the area north and west of Kom $\mathrm{K}$, following the same recording method as used in previous years.

Participants in the project, their home universities at the time of participation, the period of their involvement, and their tasks and responsibilities are listed on the website of the URU Fayum Project at http://www. archbase.com/fayum/participants.htm. For the work on the early and mid-Holocene published here, a large number of people have contributed their energy, time, and efforts. Some of the work was preparatory and is not directly reflected in the analysis and discussions presented below, or it is published elsewhere.

\section{Previous Studies and Theoretical Perspectives}

Gertrude Caton-Thompson and Elinor Gardner published their early work on the Fayum quite extensively for the time (Caton-Thompson 1926a, 1926b, 1927; Caton-Thompson and Gardner 1929, 1934; CatonThompson et al. 1936, 1937), and their studies were extended in work by Fred Wendorf and Romuald Schild (1976); Boleslav Ginter, Janusz Kozlowski, and others (Ginter et al. 1980; Kozlowski 1983; Kozlowski and Ginter 1989, 1993), Robert Wenke (1984; Wenke and Casini 1989; Wenke et al. 1983, 1988); Fekri Hassan (1986; Hassan et al. 2006, 2012); and Douglas Brewer (1987, 1989a,1989b). More recently, Noriyuki Shirai (2010) published a study focusing on stone tools from the Fayum north shore.

The present volume builds on the contributions of these scholars and adds to them by restudying locations that were previously recorded as well as analyzing the results of field recording in new areas. The majority of the work reported here was carried out in a series of field seasons in 2004-2005 and 2007-2012 and incorporates results from preliminary work conducted from 2003 to 2007. A detailed list of participants can be found in the acknowledgements.

The number of scholars who have studied the Fayum for nearly a century means that Fayum archaeology continues to feature in regional syntheses of Egyptian prehistory and particularly in models of the arrival of domestic plant and animal species from Southwest Asia (e.g., Wengrow 2006; Wenke 2009). Questions concerning the apparent late arrival of domesticated plants and animals into Egypt and the relative contribution to early Egyptian cultures from Southwest Asian and western desert peoples are prevalent in the literature. Linked to these concerns are analyses that seek to identify the time and place for the appearance of domestic species and stylistic traits in portable artifacts that might indicate the movement of people and/or ideas from external locations. Much debate continues to center around whether the appearance of domestic species in the Fayum is associated with sedentary settlement in villages similar in appearance to those found during the Pre-Pottery Neolithic and Pottery Neolithic periods in Southwest Asia or whether people were mobile, with an economy more similar to pastoralists thought to typify the desert regions. In the chapters that follow, we summarize previous studies and make comments on current theoretical models, including cultural connections, paleoeconomy, settlement, and mobility. But in designing our work in the Fayum, we paid particular attention to what can be said archaeologically about these topics.

An issue with some current interpretative models for the early to mid-Holocene periods in Egyptian archaeology is that a great deal is drawn from rather small sets of data. There is a tendency to single out particular sites or even particular artifact forms and to use the patterns apparent in these to construct wide-ranging synthetic models. While some of this of course relates to the range of materials that preserve in the archaeological record and in Egypt in particular, the materials and sites that were the focus of previous studies, it is also true that archaeologists working in Egypt have not always dealt well with the variability apparent in the archaeological record both spatially and among material types. In this volume we attempt to address this deficit by considering a range of remains from a range of contexts, concentrating on documenting the variability we see in the archaeological record.

We have adopted a different theoretical stance than many contemporary studies. A legacy of both culture historical approaches and processual functionalist approaches to archaeology in Egypt (and elsewhere) is a concern in developing behavioral models and in examining these in comparison to the archaeological record. Culture historical studies, for instance, seek to understand the movement of people or ideas as seen through artifact style similarities and changes across space and time. Processual studies emphasize the activities of past people as indicated by artifacts, assemblages, and sites. In this volume, we reverse the order of analysis and emphasize an archaeological approach 
rather than one that begins with explanatory models of behavior. Obviously the archaeological record reflects at some level the actions of people, but the relationship between this behavior and the material remains that we as archaeologists study is one that requires careful consideration. The archaeological record forms as a result of the complex interplay between human actions and environmental processes. This record therefore reflects the summation of the material remains of these interactions, represented by deposits that occur in a variety of locations. We as archaeologists need to pay attention to how these deposits are formed (e.g., Butzer 1971, 1982, 2011), and we initially focus our research on answering a series of simple questions related to the formation of this record: Why can we see this deposit here? How old is the deposit and why has it accumulated here (Brown 2008; Holdaway and Fanning 2014)? By answering these questions, we gain an understanding of why the archaeological record is patterned the way it is. As we illustrate in the chapters that follow, at times in the past, seemingly straightforward behavioral interpretations of archaeological patterns have paid scant attention to how these patterns are derived from the preservation and accumulation of material remains. When scholars sought to test behavioral models of record formation, the complex interplay of different processes received less attention than it should have, and as a consequence, incorrect inferences were drawn. In this volume we pay attention to questions of formation first. We are certainly interested in how people acted in the past, but by using a formational perspective we are able to provide interpretations of Fayum records that differ in significant ways from those expressed in previous studies.

\section{Organization of the Volume}

In chapter 2 we provide an introduction to the Fayum in relation to previous studies both in Egypt and in neighboring regions. We consider studies of paleoenvironment in relation to both regional environmental shifts and the local consequences of these shifts. We consider previous studies that have sought to explain the origins of the Neolithic in Egypt. A focus of the current volume is material culture studies, so we spend time reviewing how artifacts have been studied. We also discuss landscape archaeology as we apply it in the Fayum.

Chapter 3 develops our geoarchaeological approach, beginning with an assessment of changes in lake levels that have been proposed in a number of Fayum studies. We report new assessments of the north shore topography and its relationship to the lake basins that Caton-Thompson and Gardner identified based on an analysis of a satellite-derived digital surface model. We also consider paleoenvironmental reconstructions for the Fayum north shore, particularly the environments in and around the lake edge basins. Finally we consider the approach we developed to record the Fayum archaeological record at a landscape scale and we discuss the types of analyses we employ in subsequent chapters to analyze this record.

Chapter 4 begins the analysis of the archaeological materials that we recorded, concentrating on the record found in and around the site of E29H1, originally studied by Wendorf and Schild (1976). As we report, we were forced to undertake what amounted to rescue archaeology at this location before the site was destroyed by a short-lived agricultural development. We consider a surface archaeological record that is more extensive than that originally recorded by Wendorf and Schild (1976) adjacent to L Basin, as identified by Caton-Thompson and Gardner (1934). We report on the geomorphology of the sediments on which the archaeological materials rest and the impact this has on material density. We also report on the excavation and dating of a number of near-surface hearths. Grinding stones were located in the study area, and the distribution of these is analyzed. We also report on the composition and distribution of faunal remains in the region of E29H1. A large number of flaked stone artifacts were recorded from locations around L Basin, and we analyze these, concentrating on the abundant flakes and cores rather than the retouched tools.

Chapter 5 follows a similar format to chapter 4 and reports on surface material from around $\mathrm{K}$ Basin as defined by Caton-Thompson and Gardner (1934). We assess the geomorphic context in which the archaeological materials occur and the density of these materials. We also report on the excavation and dating of near-surface hearths similar to those investigated in $\mathrm{L}$ Basin. We did not analyze faunal material from surface locations in K Basin, but we do report on the distribution of grinding stones. Finally we report on the analysis of a large assemblage of flaked stone artifacts from different locations around the basin. This chapter includes a discussion of excavations we undertook at the Upper K Pits. Caton-Thompson and Gardner reported basket-lined pits in two locations (the Upper and Lower K Pits), and we reassess this early work 
in relation to our more recent studies. We relocated extant examples of the Upper K Pits, but between excavation seasons the remaining pits were destroyed by earthmoving equipment.

Chapter 6 reports the results of excavations at the site of Kom K. Kom K was originally excavated by Caton-Thompson and Gardner (1934), but their excavations were not extensive. We relocated the site and undertook excavations, showing that in situ material exists beneath deposits that have been disturbed by contemporary cultivation. We report on the results of these excavations, including excavation of the large number of hearths that we uncovered. The results of the analysis of faunal material from the site are reported, as well as the results of the analysis of a large number of flaked stone artifacts.

Chapter 7 discusses how integrating a number of data sources provides a landscape understanding of the early to mid-Holocene Fayum archaeological record. Here we pose and answer a series of questions related to the Fayum north shore archaeological record. We discuss issues connected with identifying archaeological sites in the Fayum, issues with identifying occupational phases in the Fayum, the significance of the paleoenvironmental history in the region, the nature of settlement and changes in this settlement over the period spanned by the record, what we can determine concerning the economic history of the peoples who occupied the Fayum, whether or not we can detect different cultural groups moving into the Fayum, and why the arrival of domestic species into the Fayum appears to be late compared to adjacent locations in Southwest Asia.

In the final chapter, we place the Fayum archaeological record in its regional context. We consider evidence from the Nile Delta, the Nile Valley, and the Egyptian Western Desert, considering first settlement systems and then socioeconomy. We assess the degree to which we can or should use the Fayum to create models for the wider eastern Sahara. Despite our ability to analyze a largely intact landscape across the Fayum north shore, the archaeological materials that remain suggest the use of a yet larger region. The record in the Fayum therefore tells only part of the historical record of the peoples who spent time in the region. We consider the current models from this perspective. 


\section{2 \\ The Fayum in the Context of Northeast Africa}

\section{Rebecca Phillipps, Simon J. Holdaway, and Willeke Wendrich}

The Fayum desert had been known for years as the source of beautifully flaked and typologically varied chert implements which were always to be had in dealers' shops [Caton-Thompson 1983:94].

gyptian archaeological evidence played an early role in explaining the development of agriculture in the Old World. Evidence uncovered by Caton-Thompson and Gardner (1934) was incorporated into early models (e.g., Braidwood 1960), as their work was contemporary with significant efforts in understanding the development of the Neolithic (Childe 1956). Childe's environmental prime mover as a stimulus for the domestication of plants and animals could be easily associated with the Fayum and ideas about post-Pleistocene drying current at the time. The use of the Fayum evidence in Braidwood's five village assemblages arguably sparked the incorporation of the Fayum, and by default Egypt, into the notion of the Neolithic package. Particular attributes of material culture, architecture, and subsistence marked the transition into the Neolithic. The identification of the location of such Neolithic packages and the way in which they were identified, in addition to the prime mover arguments, had a substantial impact on subsequent Egyptian Neolithic scholarship. Initially, early investigations into the origins of agriculture and civilization placed Egypt at the center of such developments (e.g.,
Elliot Smith 1932; Perry 1923). These ideas contrasted with Petrie's (1939) later argument for a "dynastic race" of migrants from Southwest Asia as the founders of Egyptian civilization, very much in keeping with culture historical interpretations of culture change. The migration of people would later feature as a key stimulus for culture change during the Holocene.

Further to the culture historical agenda, Baumgartel (1955:19) suggested that the origins of the Neolithic and Predynastic could not be the Sahara or Southwest Asia because no typological connection could be securely established; nor could they be an indigenous development because there was insufficient evidence to support a preceding "Mesolithic civilization." Rather Baumgartel (1955:49) suggested that the origin lay in southern Africa, and it was proposed that the Fayum Neolithic originated from Naqada I (about $450 \mathrm{~km}$ to the south of the Fayum), with which she saw a typological connection, although it is now known that the Naqada period postdates the Fayum Neolithic.

The demonstration that domesticated plant and animal species used in Egyptian agriculture were not indigenous to North Africa but in fact originated in 
Southwest Asia drove scholars to search for connections between the two regions and to question why agriculture appeared in Southwest Asia so much earlier than in Egypt, despite their geographic proximity. All these early ideas about the Neolithic in Egypt focused on two major themes. Why did the Egyptian Neolithic develop and where did it come from? As we illustrate below, the majority of subsequent research on the Neolithic of Egypt, and indeed the prehistory of the wider Northeast African region in general, has attempted to answer either or both of these questions.

\section{Environment}

Since Childe's (1956 [1936]) oasis hypothesis, based upon Pumpelly's (1908) original observations, environment has played a large part in explaining the domestication of plants and animals, although the exact climatic sequences and human behavior correlations that he posited have of course been reexamined and refined (e.g., Bar-Yosef 1998). In the case of North Africa, the idea, suggested by Childe, of a drying climate that forced people into areas with permanent water sources seemed to fit well with the geographic makeup of Northeast Africa. However, it was later established that this drying occurred during the mid-Holocene (Kröpelin et al. 2008), perhaps after significant socioeconomic change had already occurred, and was in fact preceded by an increase in temperature and humidity, propelled by global climatic change at the beginning of the Holocene, arguably the stimulus for agriculture in Southwest Asia.

Contemporary studies continue to suggest that changes in Holocene paleoclimate had an impact on human occupation of Egypt, particularly in the eastern Sahara, in regions away from permanent water sources (Kuper and Kröpelin 2006). Moisture reservoirs available in North Africa (for example, soil moisture, vegetation, and surface water sources) reached their maximum extent between 8000 and 5000 BP (Geb 2000:86). As a consequence of the northward movement of the Inter-Tropical Convergence Zone (ITCZ) and the southward movement of Mediterranean winter rainfall areas, environmental changes had significant implications for the inhabitability of some regions of North Africa.

Bubenzer and Reimer (2007) suggest that the locations of prehistoric occupations were often concentrated around drainage systems and water pools. Evidence of this may be found in the abandonment of the eastern Sahara in the mid-Holocene when the ITCZ retreated (Kuper 2006; Kuper and Kröpelin 2006; Wendorf and Schild 2001), leaving the Western Desert oases as the only permanent water sources. Analyses of radiocarbon determinations by Kuper and Kröpelin (2006) were thought to show evidence of continued occupation of only the oases after mid-Holocene aridity (e.g., McDonald 2001). However, recent reanalysis of the available radiocarbon determinations from the eastern Sahara, Fayum, and Nile Delta suggest that occupation in the eastern Sahara continued until later than previously supposed and that aridification occurred later and more gradually than previously reconstructed (Kröpelin et al. 2008). Reanalysis of the available radiocarbon data sets suggests that a generalized pattern of movement to remaining permanent water sources may be too simplified (Phillipps et al. 2012; Phillips 2013).

The Fayum was also thought to be affected by Holocene climate change, despite containing a permanent water source. Early and mid-Holocene occupations, equated to the Epipaleolithic and Neolithic periods, were thought to be separated by a hiatus due to the substantial reduction in lake level after the Epipaleolithic period but before reoccupation by Neolithic groups (Hassan 1986). The lack of continuity in the occupation of the Fayum was viewed as significant in explaining how agriculture developed during the Neolithic period. If the Fayum was abandoned, it is possible that the groups that reoccupied the area were of different origin and arrived in the Fayum with a completely new socioeconomic system, a notion that supports the idea of a rapid transition at the beginning of the Neolithic (Wenke 2009).

\section{Origins}

As noted above, interest in Egypt's Neolithic origins has persisted into the twenty-first century, but the majority of studies continue to debate the same origin issues introduced half a century or more ago (e.g., Shirai 2006; Wenke 2009; Zeder 2008). The influence of ideas put forward by Petrie and Baumgartel in the early twentieth century, for instance, can be seen in currently accepted models that suggest the diffusion of technology and ideas and/or migratory waves of people into the Nile Valley and Nile Delta (e.g., Close 2002; Zeder 2008). These movements of people and/or ideas, whether from the eastern Sahara or Southwest Asia, are thought to be motivated by climatic change during 
the mid-Holocene (e.g., Close 2002; Kozlowski and Ginter 1993; Lindstädter and Kröpelin 2004; Riemer 2007; Vermeersch 2006), much as Childe predicted, even though, as noted above, the details of the nature of these changes have shifted.

Movement from Southwest Asia, for instance, is linked to population expansion into peripheral areas, such as the Negev and Sinai, based on a pastoral subsistence during the PPNA and PPNB (Goring-Morris 1993). Droughts between 7500 and 7000 BP then forced people onto the Mediterranean coast and from there into the Nile Valley (Bar-Yosef 2002; Close 2002; Galili et al. 1993, 2002; Gopher and Gophna 1993; Goring-Morris 1993; Hassan 2002; Shirai 2005; Wengrow 2006). Either alternatively or as well, the presence of early domesticated caprids on the Egyptian Red Sea coast (Vermeersch et al. 1994) suggests the Red Sea as a route for the dispersal of Southwest Asian domesticates, which were then moved into the eastern Sahara (Close 2002; Kindermann et al. 2006). Alternatively, or again as well, movement into the Nile Valley may be linked with Mediterranean colonization, albeit with a late arrival in Egypt (e.g., Zeder 2008). Bar-Yosef (2002), for instance, suggests movement into Egypt via the Nile Delta. However, despite such hypothesized movements and evidence for the comparatively rapid Neolithic colonization of the Mediterranean Basin (e.g., Zeder 2008), it is still not certain that this expansion of domestic plants and animals included Egypt (Linseele et al. 2016; Wengrow 2006:25).

As noted above, for some researchers, environment stimulated the movement of people who inhabited the eastern Sahara into the Nile Valley during the early Holocene (e.g., Kuper and Kröpelin 2006). This model is supported by archaeological evidence based on typological similarities among stone artifacts, such as concave projectile points (Eiwanger 1988; Kindermann 2003:276; Warfe 2003:184). However, quite apart from the unresolved issues about what such artifact similarities mean in cultural terms, and therefore whether they should be used as a proxy for the identification of distinct groups of people, the stone artifact similarities do not extend to other forms of material culture. Pottery, for instance, is different in the eastern Sahara and Nile Valley locations, generally with highly decorated forms found in the eastern Saharan sites but not in the Fayum and only to a limited extent at Merimde Beni Salama. Some archaeologists argue for similarities between the black topped ware in the later phases of
Nabta Playa in the eastern Sahara and the Predynastic Badarian ceramics (e.g., Nelson et al. 2002), but like the stone artifact evidence, these claims do not address the issue of how much similarity is needed to establish a cultural or, in the sense of population movement, an ethnic connection. As Warfe (2003:177) summarizes the situation, archaeologists see Southwest Asia as the origin of domesticated species and therefore the subsistence regime of Neolithic Egypt but see North Africa (that is, the eastern Sahara) as the origin of Egyptian Neolithic "culture" (that is, material culture). Putting this in older, early-twentieth-century terminology, the Neolithic package came with village life from the Levant spurred on by climate change and/or population movement, while cultural influence came from the desert through the same combination of mechanisms. The current debates seem to move back and forth between these positions, each protagonist attempting to determine which direction was responsible for the origin of the more dominant set of influences. At the beginning of the twentieth century, evidence was slim and the need to explain the origins of the Neolithic great. Searching for the origins of a distinct entity like the Egyptian Neolithic made sense. In the twenty-first century, however, our understanding of the Neolithic has expanded greatly (Finlayson 2013). The Neolithic package that made sense when Braidwood sought examples of village life that included the Fayum is now known to take more than 10,000 years to develop, with individual components having quite different temporal and/or spatial trajectories (Zeder 2009). As a consequence, its status as a package at the very least takes on a quite different meaning from the way this term was used in the past. In addition, our understanding of the domestication process has changed quite dramatically, as has our understanding of the variability of the societies that used domesticates. It is no longer a revolution from one socioeconomy to another but rather a complex landscape, to use Smith's (2001) metaphor, where variability of economic pursuit and society is possibly more apparent than at any time before (cf. Richerson et al. 2001). Should debates about the Egyptian Neolithic therefore continue to use ideas developed to explain a less complex record, in the sense that there was a lot less data to talk about, or should new ideas with which to discuss a more variable North African record be developed? In the chapters that follow we report on studies of the early to mid-Holocene Fayum record that do indeed illustrate a more complex record than 
previously reported. In this way, our work adds to a sense of disquiet already apparent among archaeologists working in North Africa that this time period saw a much more regionally and chronologically diverse past than can be accommodated by models of influence either from Southwest Asia or the Western Desert.

\section{The Neolithic in Egypt}

A distinction is often made between the Neolithic of the eastern Sahara and that of the Nile Valley. Wenke (2009), for instance, suggests that the Saharan Neolithic originated with immigrants from the Nile Valley who moved out into the Sahara during the early Holocene, using cattle pastoralism to access areas made economically useful by the northward movement of the ITCZ. He contrasts this movement with the Neolithic in the Nile Valley, Nile Delta, and Fayum, where the economic system is based on the cultivation of cereals in addition to animal domesticates. The origins of this form of the Neolithic remain unclear, but Wenke (2009:179) suggests the presence of influences from Sudan, the Sahara, and Southwest Asia.

Wengrow (2006) divides the Egyptian Nile Valley Neolithic in two. The Upper Nile Valley contains evidence for a "primary pastoral community" influenced by the Khartoum Neolithic in Sudan (Wengrow 2003, 2006:26; Wengrow et al. 2014). Here he suggests that the evidence supports a socioeconomy reliant on cattle, sheep, and goat pastoralism. Occupation remains are ephemeral, but large numbers of human burials are present (Wengrow 2006:30). In contrast, the Lower Egyptian Neolithic in the Fayum and Nile Delta combines domesticated cereals and animals, although, as in Upper Egypt, no permanent domestic architecture is present, with the exception of the later phases of Merimde Beni Salama in the Delta (Wengrow 2006:63).

However, while these large-scale regional models tackle the big question of Neolithic origins in Egypt, they inevitably do so by subsuming much local variability. Thus the apparent uniformity portrayed by largescale models may actually relate to the relatively small numbers of regional case studies available. A small number of cases will always have restricted variability and will therefore appear to demonstrate marked regional differences.

Minimally, how people locally adapted to the potential offered by new introduced species is important for understanding how the Egyptian agricultural regime developed, which is in turn so critical to understanding how Pharaonic civilization developed. The issue of limited data revolves around the lack of detailed studies of specific locations, although of course some information is available in the published literature (e.g., Brunton and Caton-Thompson 1928; Caton-Thompson and Gardner 1934; Debono and Mortensen 1990; Eiwanger 1984, 1988, 1992; Wilson 2006; Wilson et al. 2014). This volume is designed to at least partially address the deficit by providing a detailed localized case study of the Fayum north shore.

\section{Alternative Approaches}

The alternative to either large-scale environmental models or population movement as explanations for Egypt's Neolithic origins is to focus more specifically on the localized variability of Egypt's key early Neolithic locations (e.g., Holdaway et al. 2010; Phillipps 2012; Wengrow et al. 2014; Wenke et al. 1988). This requires posing alternative questions addressed through a closer examination of how the archaeological record is used to reconstruct aspects of prehistoric human behavior, including social structure, economic practice, settlement pattern, long-distance interactions, and response to both large-scale and small-scale climatic and environmental change.

Holdaway et al. (2010) suggest the use of an alternative conceptual framework based on Smith's (2001) notion of low-level food producers to explain the variability among socioeconomies where wild food resources may still play an important role (Figure 2.1). They extend Smith's ideas to allow for consideration of settlement pattern, mobility, and social structure. While discussions of mobility in the context of Neolithic Egypt are not new (e.g., Close 2000; Marshall and Hildebrand 2002; Shirai 2010; Wengrow 2006), few studies have empirically demonstrated past human movement based on the "hard evidence" in the archaeological record (e.g., Close 2000; Holdaway et al. 2010). As well, archaeological theory is rarely discussed with reference to Egyptian archaeology, especially more recent archaeological theory dealing with the structure of the archaeological record (e.g., Bailey 1983, 2006, 2008; Holdaway and Wandsnider 2008; Lucas 2012; Murray 1999). Given that much of the prehistoric record in Egypt and the eastern Sahara occurs as surface scatters of stone artifacts and remains of hearths, or very shallow buried deposits often subject to post-depositional disturbance, it is surprising that neither the literature on taphonomy nor survey 


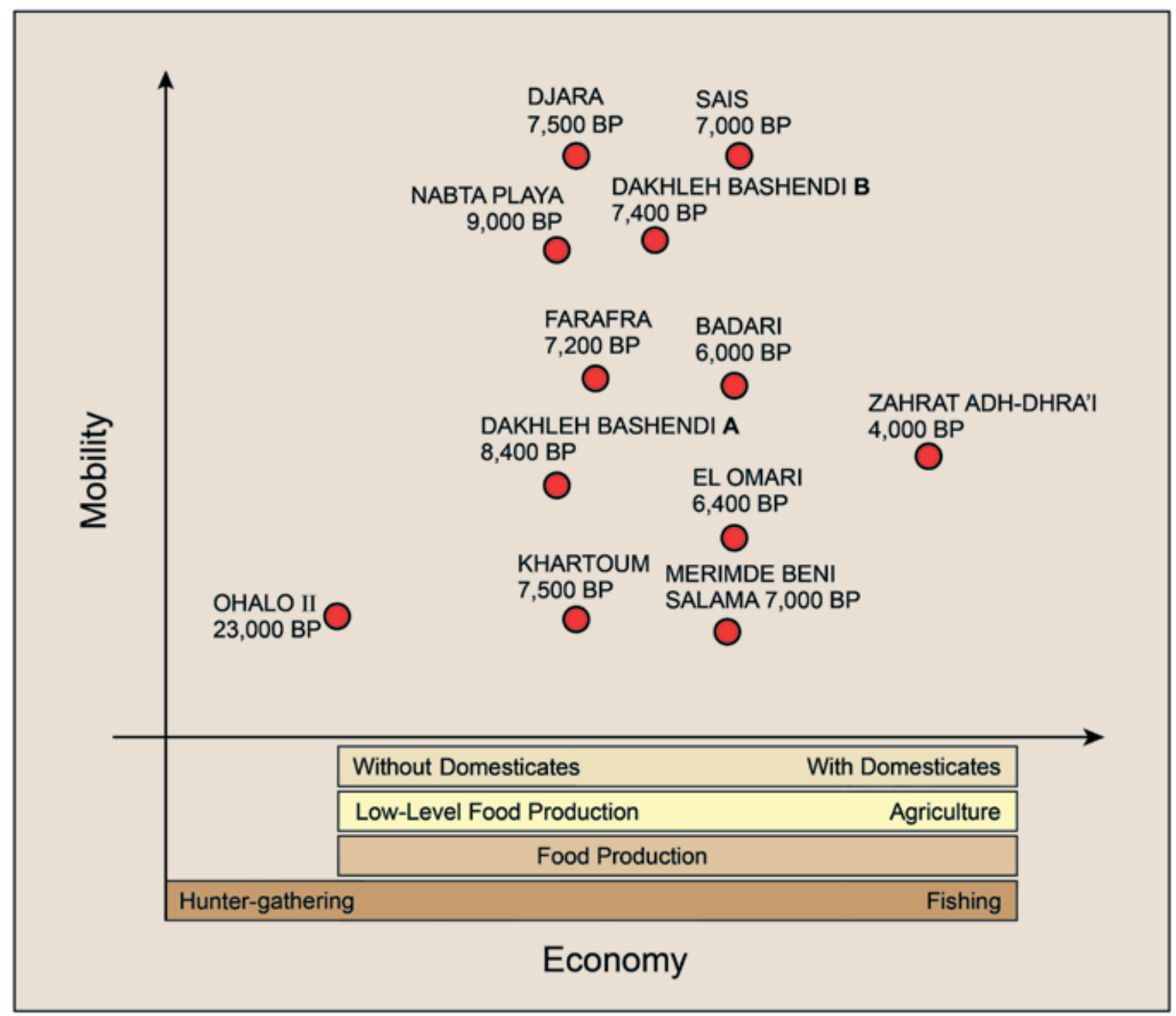

Figure 2.1. Conceptual model based on Smith's (2001) low-level food producers using mobility estimates.

methods in archaeology have had much impact in Egypt. Below we discuss research designs that are cognizant of the nature of the archaeological record and move away from broad regional pattern recognition to a focus on local variability in the Egyptian early to mid-Holocene record.

\section{Analysis of Material Culture}

Portable material culture analysis is critical to understanding socioeconomic change in Northeast Africa, particularly Egypt during the early to mid-Holocene. In some cases, a lack of other features, such as a built environment due to post-depositional processes or their absence, heightens this emphasis on portable material culture. Much of the analysis of material culture is focused on "culture" change in the sense that the presence and absence of portable material culture types are used to develop regional and supra-regional culture histories. The presence of flaked stone artifacts, particularly projectile points, bifacials, and some technological forms, is used to describe this culture change throughout a sequence of occupations, and with the later addition of pottery, this forms the basis for the definition of culture groupings and change through time. While the literature has alternative approaches to material culture analyses (e.g., Close 2000), these are 
rarely discussed; nor do they feature in major syntheses of Northeast African prehistory (e.g., Midant-Reynes 2000; Wengrow 2006; Wenke 2009).

Caton-Thompson and Gardner (1934) described the stone artifacts and pottery of the Fayum and associated them to particular periods based on typological features. The only instance where they did not accept typological features was in the case of the Fayum B, which was typologically similar to the Mesolithic of Europe or what is now referred to as the Epipaleolithic of the Levant (after Perrot 1966). Because she believed Lake Qarun was constantly receding, Caton-Thompson could not accept the typological features of this grouping, which should have indicated a Mesolithic "culture" preceding the Neolithic. Instead she argued that the Fayum $B$ was a period of culture regression that occurred later than the Neolithic (Fayum A) proper (Caton-Thompson 1928; Caton-Thompson and Gardner 1934:2).

Later typologies used in Egypt and North Africa applied techniques based on cumulative frequencies in stone tool types to North African assemblages, enabling the construction of culture historical sequences and documenting relationships between culture groupings. Tixier (1963) created a typology for the Epipaleolithic of the Maghreb, describing Moroccan, Algerian, Libyan, and Saharan assemblages for North Africa. The typology was subsequently modified and used in stylistic comparisons throughout North Africa (e.g., Chmielewska 1968; Hassan 1978; Hays 1975; Marks 1970; McBurney 1967; Mussi et al. 1984; Schild and Wendorf 1975; Schild et al. 1968; Wendorf and Schild 1984). The typology provided a method for comparisons between Egypt and the rest of North Africa based on tool type proportions (Schild and Wendorf 1975; Schild et al. 1968; Wendorf 1968; Wendorf and Schild 1976). Typological connections around the region enabled postulation of a range of theories surrounding the movement of populations from the Levant to North Africa, including the movement of populations from the Western Desert into the Nile Valley (Haaland 1984; Mussi et al. 1984:190; Phillips 1972; Vermeersch 1984:142; Wenke et al. 1988:37).

Following on from earlier typological connections, the notion of style versus function in stone artifact morphology was examined in the context of North Africa. Close (1978) highlighted the assumptions made by archaeologists regarding the role of style in assemblage variation. Her analysis of North African assemblages suggested that style and cultural tradition were ultimately responsible for variation in the assemblages analyzed from Tamar Hat (eastern Algeria), Haua Fteah (Cyrenaica), Wadi Halfa, Debeira West, and the Nile Valley. Close examined attributes that she defined as "functional" on backed bladelets, such as backing to protect the user's hand, considered to be a primarily functional tool attribute (Close 1978:226; Jelinek 1976:25). She argued that in many North African industries, backing may have consisted of specific "stylistic" variations (for example, Ouchtata backing, an extremely fine and regular retouch). In the Close study, stylistic explanation was confirmed by grouping assemblages together statistically using principal components analysis to derive a hierarchy of assemblage relationships (Close 1978:229).

In Egypt, material culture typology, apparently confirmed by studies like that of Close, was used as a proxy with which to trace the arrival of domesticated species into the Nile Valley (Eiwanger 1988; Haaland 1984; Hassan 1988; McDonald 1982; Wenke et al. 1988:37) since assemblages in the early Neolithic of Southwest Asia and early Neolithic sites in the Nile Delta appeared to contain some similar projectile point types, leading to the assumption of a similar "culture" (Caton-Thompson and Gardner 1934; Eiwanger 1979:34, Plate 4; Kuijt and Goring-Morris 2002:401, Figure 11). However, other typological comparisons of ground stone artifacts and flaked projectile points suggested connections between the Sahara, the Western Desert oases, and the Nile Valley during the early to mid-Holocene. No matter what the result of these different studies, any differences between assemblages that were detected were explained as representing minor regional variation (e.g., Midant-Reynes 1992:148). For the Fayum, such typological connections were considered critical to establishing the origins of the Neolithic. Wenke and Casini (1989:148), for instance, suggest that concave-based arrowheads "exhibit considerable stylistic expression" and so might serve as an indicator of a "cultural complex" that linked places in the Fayum with others in Egypt and the greater North African region.

Despite the relative lack of theoretical influence in Egyptian archaeology noted above, processual archaeology developed outside Egypt did lead some investigators to propose functional explanations for changes in flaked stone artifact assemblages from the early to mid-Holocene. It was always acknowledged that food production during the mid-Holocene required 
specialized tools for grain harvesting and processing, such as sickle blades and grinding stones to cut grasses and process grain (e.g., Bar-Yosef 1998:164; CatonThompson 1952; Caton-Thompson and Gardner 1934; Wenke et al. 1988). However, in the 1980s Wenke et al. (1988) developed this notion further based on survey data. They defined functionally specific locations based on the analysis of stone artifacts on the southwestern side of Lake Qarun (FS-1 and FS-2 in Figure 1.1). The distribution of sickle blades and grinding stones was used to characterize the economy and the likely nature of occupation (Wenke et al. 1988:33). Wenke et al. (1988:39) concluded that the majority of grinding stones and sickle blades occurred in instances with very few additional artifacts (for example, flakes, scrapers, and so on) and that these occurred within a narrow elevation band (although see the discussion in chapter 3). Although they highlighted the problems of associating the presence of an artifact with its place of use, these results seemed to correlate with the presumed location of wheat and barley habitats in narrow bands along the lake margin.

Changes in the uses of and access to raw material sources were also used in North Africa to examine spatial and temporal variability in socioeconomy. Wendorf and Schild (1984:96), for instance, suggested that decreasing numbers of backed bladelets from Epipaleolithic contexts compared to numbers in assemblages from the later Neolithic reflected a later dependence on agriculture. Fewer backed blades produced necessitated less flint raw material and therefore explained the increasing amounts of quartz, limestone, chalcedony, and other metamorphic stone in later assemblages compared to the number of flint artifacts. These authors also noted that access to flint may have become more restricted due to increased sedentism, with people either having less access to the source or access that was restricted due to the presence of other groups (Wendorf and Schild 1984:96).

Cagle (1995) examined the relationship between raw material types and technological variability in the Fayum, paying particular attention to the chert and flint outcrops above Qasr el-Sagha (Cagle 1995:2). He measured the completeness and complexity of reduction sequences for different chronological periods, considering distance to raw material source. Debitage analysis suggested that Neolithic reduction was both more complex and more complete than that undertaken in previous periods (Cagle 1995:7), a result that might be explained by some locally available raw material being underwater during the Neolithic (e.g., Wenke et al. 1988; see chapter 3 for more discussion of lake levels). Alternatively, the more diverse range of raw material during the Epipaleolithic, compared to the consistent use of homogenous, fine-grained cherts during the Neolithic, might reflect changes in the level of mobility (Cagle 1995:6).

In contrast, Close's (1999) analysis of raw material use at sites in the Egyptian Sahara suggested that there was no differential reduction of material based on distance to raw material resources. What Close termed "early Neolithic" sites at Nabta Playa (10,800 to 9800 cal BP; Wendorf and Schild 1998:100) were more than $40 \mathrm{~km}$ from the nearest flint source, while the site of El Gebal el Beid was less than $10 \mathrm{~km}$ from the source. Based on the size of the bladelets produced and the size of the discarded cores, Close (1999:27) suggested that there was greater concern for producing consistently sized blade blanks than for the conservation of raw material from distant sources. Cores were reduced in the same way regardless of the raw material type and were used until they could no longer produce the required blank size, at which point they were discarded (Close 1999:27).

\section{A Landscape Approach for the Fayum}

As discussed above, analyses of artifacts from prehistoric Egypt have to date focused largely on the form of highly curated objects, particularly stone projectile points, and have used these to make stylistic and in some instances functional interpretations. These artifacts are typically interpreted independently of their assemblage contexts, using a method that effectively requires that the entire style of a culture be found within a single artifact type or group of types. This can be critiqued on numerous levels, for in addition to falling short of culture historical aims, these methods do not explain why the artifact type is found in one particular deposit and not another, nor its relationship to other artifacts associated with it.

As discussed above, in Egypt, the typological method of analysis and interpretation is connected with the arrival of domesticated species from Southwest Asia into Egypt and the movement of populations out of the eastern Sahara. The interpretation that people moved from Southwest Asia and possibly the eastern Sahara into Egypt, bringing with them domesticated species, is based on the presence of different projectile point types 
(e.g., Caton-Thompson and Gardner 1934, Eiwanger 1979:34, Plate 4; Kuijt and Goring-Morris 2002:401, Figure 11). The difficulty with this line of reasoning is that it is an all-or-nothing interpretation. As with all diffusion models, change comes from the source and is delivered to the destination. No explanation is provided for variability at the destination sites. Diffusion from Southwest Asia cannot, for instance, explain the different settlement patterns and subsistence strategies that are apparent between Merimde Beni Salama and the Fayum.

A key issue is that typological approaches face a fundamental problem. They isolate individual artifact types from other material culture forms and treat each form as though it acted in society independently. This of course did not happen. Artifacts of different forms, manufactured from different materials, were used together. The analysis of one form gains meaning in relation to the analyses of other forms (Hodder 2012; Olsen 2010). However, this meaning is complicated by the structure of the archaeological record as a palimpsest. Artifact assemblages are the result of multiple individual events undertaken by multiple actors. They are therefore unlikely to reflect a single manufacturing set or single set of functions or even necessarily result from the activities of related peoples. The analyses that seek culture historical sequences or the function of artifact assemblages assume that group activity was highly structured, so that the majority of behavior produced similar outcomes. Unfortunately, there is little ethnographic support for this. Instead, ethnographic studies indicate that considerable variability is created through the variety of individuals who make and use material culture (e.g., Holdaway and Douglass 2012).

Typological studies look at the form or the function of an artifact type as the representation of culture, but material culture is not an end in itself; nor is it the "bearer of culture." There are alternative approaches to material culture. Portable material culture survives in the archaeological record and therefore is a useful medium through which to understand how people acted in the past. When the analytical focus is changed, it provides a useful proxy for a range of activities that people undertook. People as agents acted in different ways, in different places, and at different times, and of course not all people acted in the same way at the same time and place. Variability is therefore to be expected, and it is in fact this variability that reflects the existence of culture in the past.
Stone artifact analysis, for instance, provides the means to understand how people moved about the landscape, how often they moved, whether they left permanently, and where activities were concentrated (e.g., Douglass et al. 2008; Holdaway et al. 2010; Phillipps 2012; Phillipps and Holdaway 2016). These inferences in turn can be combined with economic information as well as paleoenvironmental reconstruction to indicate why people were drawn to and modified particular landscape settings. As indicated above, the Neolithic did not arrive as a distinct package, and people did not simply become Neolithic. Instead we should expect to see variability in socioeconomies as well as other aspects of society, contingent on the history of the particular location considered.

\section{Discussion}

In the following chapters we seek to develop an alternative to the current emphasis on regional model construction, concentrating instead on describing the regional variability-temporal, spatial, and materialthat we see in the early to mid-Holocene archaeological record from the Fayum north shore. We attempt to look at the whole record that has survived rather than select a part of it. We do not, for instance, analyze only stratified deposits. Instead we incorporate intensive studies of the rich surface deposits in the Fayum as well as both small-scale and larger-scale excavations. We analyze whole artifact assemblages, including the fragmented debitage and the so-called expediently produced artifacts, counteracting a long-standing bias toward what archaeologists perceive as stylistically significant tools. We report large-scale but intensive surveys together with excavation, applying the same standards to data recording to both surface and excavated material.

The analyses reported here are comparative. We seek out variability not conformity. We therefore define the spatial and chronological attributes of multiple assemblages, creating large data sets that enable us to utilize interferential statistics to help assess the validity of the patterns we detect.

In the following chapter we develop this approach first by considering the geomorphological setting of our study area. As Caton-Thompson and Gardner (1934) acknowledged, understanding the history of the Fayum is closely bound up with understanding the history of Lake Qarun, or ancient Lake Moeris. It is to understanding this significant topographic feature that we now turn. 


\title{
3 \\ Approaches to Paleoenvironment and Landscape Use
}

\author{
Rebecca Phillipps, Simon J. Holdaway, Rebecca Ramsey, \\ Willeke Wendrich, and Joshua J. Emmitt
}

It has been said that whoever works in the Faiyum is sooner or later drawn into the problem of Lake Moeris [Caton-Thompson and Gardner 1929:20].

n 1929 Caton-Thompson and Gardner commented on the importance of understanding the variation in lake levels of Lake Qarun, and so it is perhaps fitting that this volume includes a review of the relationship between the lake and terminal Pleistocene through mid-Holocene archaeology. Both these authors reviewed late-nineteenth- and early-twentieth-century work on the lake, and both their own work and the work of later twentieth-century authors was in turn reviewed by Wendorf and Schild (1976), as well as by Kozlowski and Ginter (1989) and Hassan (1986). The authors of the earlier studies recognized that modern-day Lake Qarun was once a more substantial body of water, evidenced by paleoshorelines together with lake sediment and shell deposits. The stratigraphic position of these deposits suggested times when the lake was higher and times when it was lower. What was not indicated directly, however, was the chronology of these lake advances and retreats. The late-twentieth-century authors cited above made attempts to develop such a chronology, but these were based on age determinations largely from datable materials in archaeological deposits rather than the shoreline deposits themselves. These studies included the critical assumption that the archaeological materials found on lake edge deposits reflected lake edge occupation. Therefore, age estimates for these materials could be used to provide a chronology for the deposition of the lake edge sediments. Under this assumption, variations in the ages and locations of hearths dating from the early to mid-Holocene seemed to indicate a sequence of lake advances and retreats, summarized in Figure 3.1. Archaeological materials were at times buried by sediments deposited as a result of the rising lake and then subsequently reexposed as a result of lake retreat. The heights above sea level (asl) of the sediments on which the datable archaeological material rested provided a means of establishing a chronostratigraphy that could be traced across both the northern and southwestern regions of the Fayum Basin (Hassan 1986; Kozlowski and Ginter 1989).

Lakes were certainly attractive to people in the past, but how people interacted with these lakes was variable, based on a large number of factors, such as lake edge morphology, cultural preferences, and particular use of lake resources. This is documented in present-day 


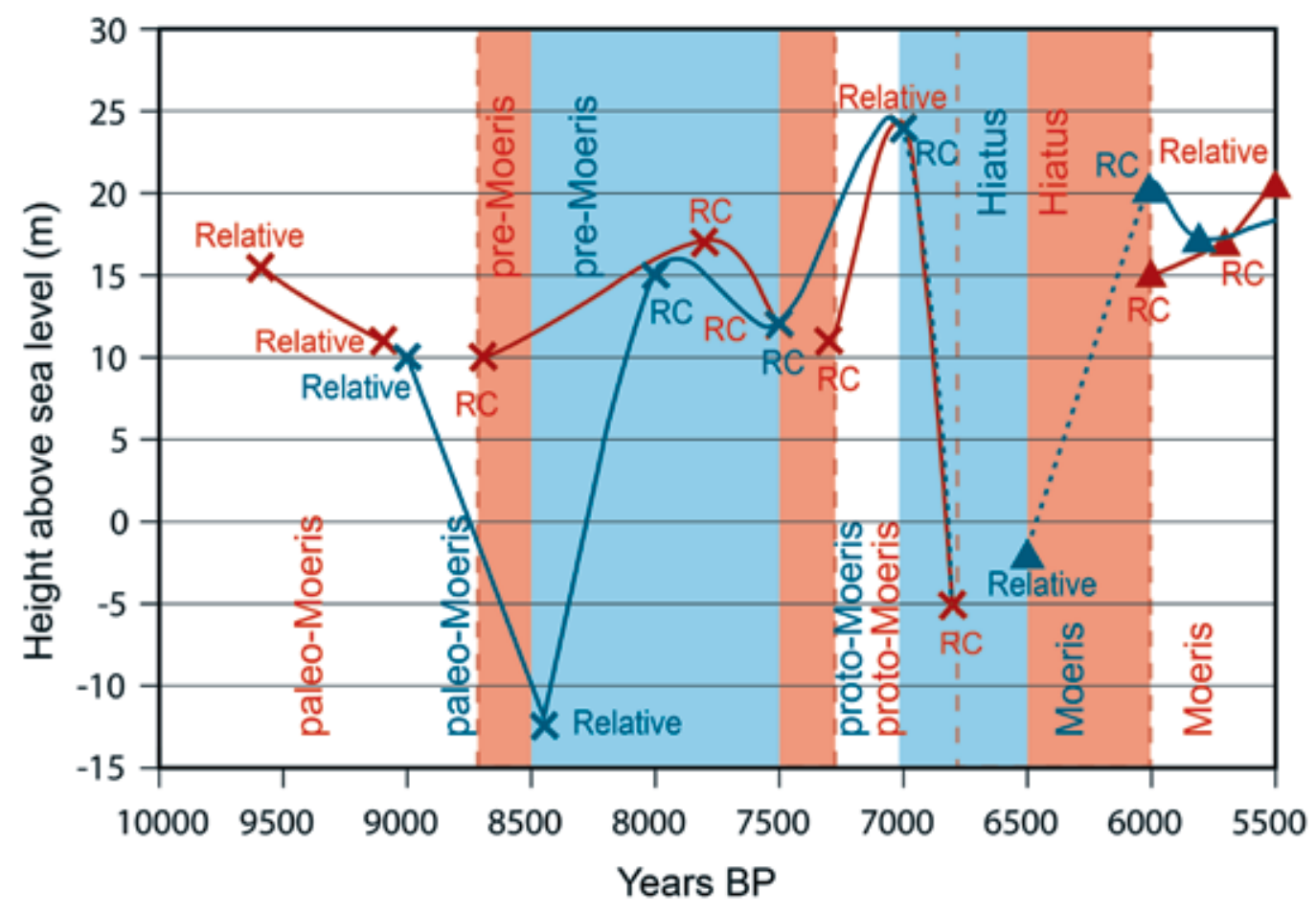

Wendorf and Schild 1976

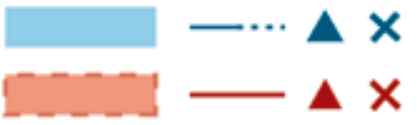

Figure 3.1. Early to Middle Holocene models of lake level fluctuations ( $\mathrm{m}$ asl) for Lake Qarun, the Fayum Depression. Blue: lake level changes from Wendorf and Schild (1976). Red: lake level changes from Hassan (1986). "RC" indicates a date from a radiocarbon determination. "Relative" indicates an age estimated from a stratified sample. Modified from Phillips 2013:Figure 5.7.

and historical ethnographic examples, and it was likely this way in the past (Bouquet 1990). People might have lived immediately adjacent to a water body, but they also might have lived some distance away while still utilizing its resources. How far people lived away from Lake Qarun is of course critical for the construction of a chronostratigraphy, because the published chronologies for changing lake levels require that the archaeological ages obtained from materials resting on the lake edge sediments relate to the times when these sediments were deposited. If, for instance, people lived away from the lake edge rather than directly adjacent to it, then a direct chronological relationship between the sediments and the archaeological materials would not exist. In the case of hearth construction, one could imagine a hearth being constructed on lake edge sediments deposited at a time predating the age of the hearth itself. The studies published in the later twentieth century all made the assumption that sediments could be dated by direct association with archaeological features, most often hearths that retained charcoal. Unfortunately, there is no independent proof that such an association existed-in other words, that people definitely and always occupied the ground immediately 
adjacent to the ancient Lake Qarun. Without this assumption, aspects of the currently accepted sequence of lake fluctuations must be called into question.

There is moreover a second problem with the currently accepted chronology for lake advances and retreats. The chronostratigraphic correlations on which lake levels and falls were based required that sediments from different locations in the northern and southern parts of the Fayum Basin could be related through their heights above sea level. Obtaining exact locations and associated heights of stratigraphic sections was of course difficult in the past, particularly in desert areas that lacked obvious geographic points of reference or topographic markers. With GPS technology combined with remotely obtained digital surface models (DSM), it is now possible to be much more certain about the location, and especially the elevation, of points on the surface of Earth. As part of the project reported here, a Worldview-2 stereo imagery pair (dated May 17, 2012) was obtained for the $142-\mathrm{km}^{2}$ project area. Initial processing produced a DSM (that is, all surfaces, including vegetation and human-constructed features) based on a 1-m pixel size with a vertical accuracy of $4 \mathrm{~m}$. This was improved by comparing the height values from 676 surveyed points with the DSM heights. An average difference of $2.886 \mathrm{~m}$ was indicated, and the DSM was then block shifted (that is, lowered) $2.88 \mathrm{~m}$ to compensate. The DSM was then used for height control in the orthorectification of the most vertical of each stereo pair. The pan image was used to pansharpen the multispectral image, providing a $0.5-\mathrm{m}$ resolution, natural-color image. As a result, we have high-resolution georectified contour maps of our Fayum north shore study region.

As we show below, using the new satellite-derived data sets, we were able to show errors in absolute height estimates for the Fayum stratigraphic sections recorded by previous researchers. For example, at E29G1 and E29H1, height estimates in the published contour maps are different by several meters compared to those obtained from satellite-derived DSM. These errors are cumulative in the sense that any error introduced in one study will lead to potentially false chronostratigraphic correlations in later studies. Thus errors in the heights attributed to E29G1 were accepted as correct by both Hassan (1986) and Kozlowski and Ginter (1993). Because of this, we have reservations about accepting the proposed chronostratigraphic correlations in these studies.
Two further problems have an impact on the current lake chronology. Firstly, there were limits on the quantities of observations that could be made in twentieth-century projects as a result of the technology available at the time. As we demonstrate below, the Fayum surface archaeological record is in places very dense, but it is also spatially extensive. With some notable exceptions, it is a record made up of many thousands of portable artifacts, by far the largest number being pieces of worked stone. Recording the precise spatial location and character of so many artifacts is a time-consuming endeavor even with the use of modern electronic survey technologies. With the exception of the work of Caton-Thompson and Gardner, who spent months in the Fayum, the field time of later twentieth-century projects was much reduced. This meant that only relatively small areas could be observed, often from disparate locations. As a consequence of our access to better technology and to longer periods in the field, we are able to demonstrate that what appeared to earlier researchers to be diagnostic locations for artifacts related to a particular time period turned out to be simply samples of a much larger distribution. As we describe below, modern technologies that allow us to rapidly acquire precise information on the location of large numbers of artifacts have given us a better understanding of both the distribution and the characteristics of portable artifacts across the north shore of the Fayum Basin. This has enabled us to correct some previous stratigraphic associations.

Secondly, the later twentieth-century studies paid relatively little attention to the condition of the surface archaeological materials. Essentially it was assumed that lake advances and retreats had little effect on portable artifacts and features exposed as a result of subsequent erosion, although Caton-Thompson and Gardner (1929) did comment on the consequences of this in relation to earlier studies of the Fayum lake levels. In our previous work, we demonstrated that it is possible to quantifiably assess how artifact assemblages are affected by water movement (e.g., Fanning and Holdaway 2001, 2004; Fanning et al. 2008). One of the remarkable aspects of the north shore of the Fayum archaeological record is the level of preservation of materials-this despite more than a century of collecting and modern-day agricultural development. Unfortunately, this may not continue for much longer, since the intensity of modern-day farming and 
road building has in places destroyed the surface (and buried) archaeological records. Despite this recent destruction, where the record is still intact, its condition needs to be assessed in relation to the proposed levels of lake inundation.

\section{Paleo-Lakes and Previous Research}

Caton-Thompson and Gardner (1929) proposed a Pleistocene lake that reached a high stand of 278 feet $(84.7 \mathrm{~m})$. Sea level was recorded at 147 feet, and therefore the equivalent of the Pleistocene lake level is $40.3 \mathrm{~m}$ asl. This lake receded and was replaced by a second lake rising to a maximum height of 206 feet

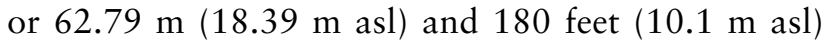
during the Neolithic. This second lake then progressively fell, with stages at 10 to $6 \mathrm{~m}, 4 \mathrm{~m}$, and $-2 \mathrm{~m}$ asl. Fayum A archaeological deposits with concave base projectile points thought to represent the Neolithic were associated with the 10-to-6-m stage. Settlements with the lower lake stages $(4 \mathrm{~m}$ and $-2 \mathrm{~m})$ were associated with artifacts of what Caton-Thompson and Gardner described as a Mesolithic group, Fayum B. Because Caton-Thompson and Gardner assumed that lake recession was a continuous process through time, their Fayum B was interpreted as postdating the Fayum A Neolithic, a change that Caton-Thompson suggested indicated a cultural "regression" (CatonThompson and Gardner 1934:2).

Wendorf and Schild (1976) disagreed with CatonThompson and Gardner's interpretation of a gradually lowering Holocene lake, suggesting instead a series of four lake advances with associated lake retreats. The oldest paleo-Moeris lake was identified by the presence of a diatomite layer at Area F, near E29G1 (Figure 3.2) in the west of the northern Fayum shore. Diatomites are the skeletal remains of algae (diatoms) that form deposits at the bases of lakes. Wendorf and Schild identified this deposit at a height of $10 \mathrm{~m}$ asl associated with the burning of aquatic vegetation but with no evidence for human occupation.

Cracks present in the surface of the diatomite deposit were interpreted to indicate exposure of the diatomite surface and therefore a lowering of the lake level. This, therefore, indicated that the once-high paleo-Moeris lake had receded. The age of these deposits was estimated at 9000 BP, but they were not directly dated. Whatever their age, their location was not correctly recorded. The contemporary DSM shows that the diatomite deposits are today at around $4 \mathrm{~m}$ asl.
At Area F, Wendorf and Schild described $3 \mathrm{~m}$ of stratified sand beneath a further $5.5 \mathrm{~m}$ of fine sand. Within this higher deposit were eight separate beds of what are described as "organogenic" powdery swamp sediments with traces of burning.

A few reworked stone artifacts occurred with the third through fifth layers (from the bottom). These artifacts possibly represent a single reworked occupation. A fragmentary human skull and numerous fish bones were also present [Wendorf and Schild 1976:163].

Significantly, these black organic sediments were traced from Area F to Areas A through E, where they could be associated with archaeological deposits. To establish a relationship between these deposits, accurate levels were needed. Figure 3.2 shows the contour intervals for the area around E29G1 published in Wendorf and Schild (1976:Figure 97) and the equivalent area with 1-m contours derived from the modern-day DSM. The slope indicated by the contours in these two diagrams is not the same. This casts doubt on the equivalence of deposits identified in Area $\mathrm{F}$ and Area A as described below.

Describing Area A, Trench 1, Wendorf and Schild (1976:167) state:

Unconformably above a unit of white, highly cemented, horizontally stratified, fine to medium-grained sand, is a yellow $(2.5 \mathrm{y}, 7 / 6)$, almost horizontally stratified, fine to medium grained sand with visible oxidation stains and with three thin $(1-2 \mathrm{~cm})$, organogenic, gray $(2.5 \mathrm{y}, 5 / 0)$ and dark gray $(2.5 \mathrm{y}, 4 / 0)$ banks of swamp sediments. The lowest, at an elevation of $15 \mathrm{~m}$ above sea level, contained numerous fishbones, chipped stone, and charcoal. A sample of the latter yielded a radiocarbon date of $6150 \mathrm{BC} \pm 130$ years $(1-4128)$. This level seemingly correlates with the lowest gray-colored, powdery level at Area F.

This date is used to indicate the age of the pre-Moeris lake, as indicated by the swampy deposits in Area F. However, the radiocarbon determination dates the archaeological materials, not the lake sediments. Therefore, the existence of a pre-Moeris lake remains at best equivocal. 


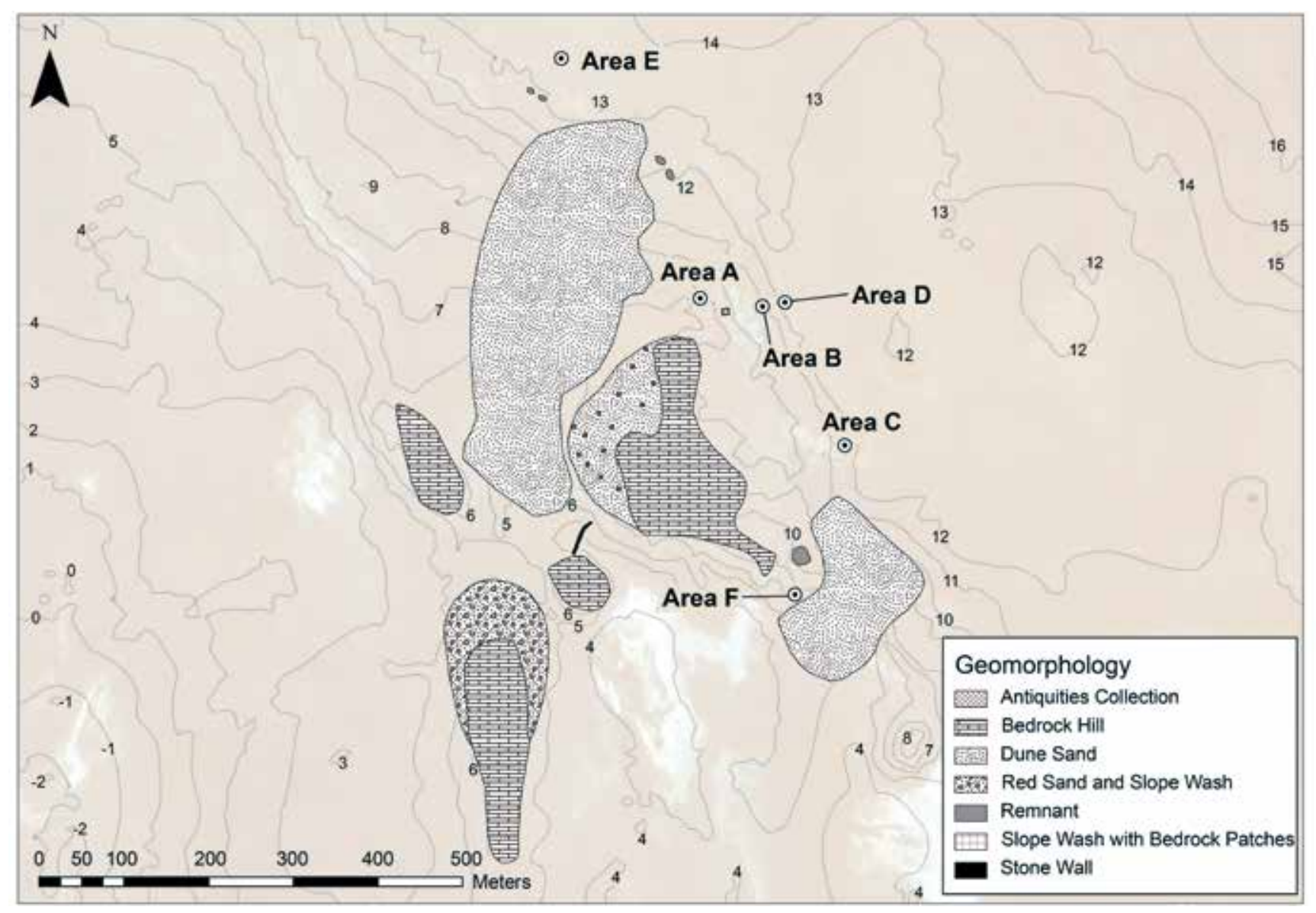

Figure 3.2. E29G1 (Z1) overlain with the digitized geomorphology from Wendorf and Schild (1976:Figure 97) and contemporary DSM-derived 1-m contours.

In Area E, Wendorf and Schild (1976) excavated an L-shaped trench to expose a concentration of flakes associated with fish bone and freshwater Pila ovata shells. One of the shells (a burned specimen) was used to obtain a radiocarbon date of $5190 \pm 120$ BC (I-4129). Wendorf and Schild used this date to propose a high lake stand at $19 \mathrm{~m}$ asl and compared it to deposits at E29G3, with dated archaeological deposits at $12 \mathrm{~m}$ asl in the range 5200 to $5600 \mathrm{BC}$. These lower deposits were thought to indicate a decline in lake level, with the 5190 BC shell date indicating a subsequent high lake stand that they termed a proto-Moeris lake.

Our DSM coverage did not extend to the location of E29G3, so we are unable to comment on lake level reconstructions based on heights from this location. However, it is important to note that the radiocarbon ages obtained do not necessarily date the lake sediments. As discussed above, for archaeological deposits to have a bearing on the lake levels, we must assume that the occupied regions were located immediately adjacent to the claimed rising and falling lake. Without this assumption, the reconstructed variations in height of the preand proto-Moeris lakes remain questionable.

Wendorf and Schild also worked further to the east at the site of E29H1, which we also studied, as described in chapter 4 . Here a belt of sandy sediments contained the oldest in a long sequence of lacustrine deposits, including what are described as loose swampy sediments. A 10- to 20-cm-thick archaeological deposit was described at an elevation of $15 \mathrm{~m}$ asl (Wendorf and Schild 1976:186). A radiocarbon date obtained on charcoal from this layer gave a date of $6120 \pm 115 \mathrm{BC}$ (I-4126). This is correlated with the date on Pila ovata from Area A at E29G1 to the west, described above, also associated with swampy deposits. Wendorf and Schild then correlated archaeological deposits from Area C at E29H1 at an elevation of $17 \mathrm{~m}$ asl, with the Area E date of 5190 BC from E29G1, suggesting 
that these higher deposits represented the proto-Moeris lake. The presence of swamps is highly dependent on local circumstances, which makes it difficult to associate swamp layers that occur in different localities as belonging to the same lake level, event, or time period.

Wendorf and Schild excavated a series of trenches across the slope at E29H1, the highest elevation being Trench 14. Sediments are described as water-laid (Wendorf and Schild 1976:199) and associated with an elevation of $24.5 \mathrm{~m}$ asl. This level was in turn associated with the highest level of the proto-Moeris lake; however, Wendorf and Schild suggest that the lake did not rest at this level for long, since there was no beach line evident. Trench 17 was excavated at the lowest elevation and revealed a soil-like deposit overlaid by a diatomite layer. This soil was associated with a lake recession, marking the change between the proto-Moeris and Moeris lakes.

Wendorf and Schild (1976) excavated two test trenches into Kom W to the southwest of CatonThompson and Gardner's original excavations. These also showed lacustrine deposits as well as archaeological materials suggesting that Kom W was occupied during a period of slowly rising lake levels. The maximum elevation of Kom W is given as $20 \mathrm{~m}$ asl. A radiocarbon date from the lowest archaeological materials in their Trench 1 returned $3860 \pm 115$ BC (I-4127).

According to the DSM, this height for the top of Kom $\mathrm{W}$ is accurate. However, the height estimates provided by Wendorf and Schild for E29H1 are not. Area A is placed close to the $15-\mathrm{m}$ contour while Area C rests adjacent to the $17-\mathrm{m}$ contour. The DSM places E29H1 between the 9-m and 12-m contours. These differences are significant because the succession of high and low lake levels is based on correlating deposits with specific heights. Trench 14, for instance, supposedly records the high Lake Qarun level. At $24 \mathrm{~m}$ it is higher than the highest point on Kom W. This would suggest that Kom W was indeed adjacent to, and at times inundated by, Lake Qarun. However, the highest deposits at E29H1, which were thought to be at $24 \mathrm{~m}$, are in fact around 15 $\mathrm{m}$ asl. As noted above, there are no direct dates on these sediments. Therefore it is difficult to determine whether the proposed order and timing of lake advances, including the Neolithic Lake Qarun, are in fact correct.

Work on the Fayum lakes subsequent to Wendorf and Schild followed a similar chronostratigraphic approach. Hassan (1986), for instance, working on the southwestern shoreline in the region investigated by
Wenke et al. (1983), observed diatomite deposits at a depth of 11 to $12 \mathrm{~m}$ asl, with what are described as terminal Paleolithic artifacts on the surface. Dates for the deposits come from archaeological materials that rest on the surface of the lake sediments. However, Hassan notes that the ages, calculated as a weighted average at $7715 \pm 45 \mathrm{BP}$, are younger than the ages estimated by Wendorf and Schild for the northern diatomite deposits. The ability to assign spatially separated sediments to a uniform lake chronology was not questioned.

The pre-Moeris deposits were identified by Hassan (1986) on the basis of similarities in grain size, color, and composition, particularly the presence of swampy carbonized material, and were correlated with the dated deposits identified by Wendorf and Schild (1976) (as well as Kozlowski and Ginter 1993, discussed below). The same is true of the proto-Moeris lake deposits that Hassan claimed were separated from pre-Moeris deposits by a disconformity. The Lake Qarun deposits on the southwestern shore were correlated with those on the northwestern shore on the basis of the presence of a pale brown sand as well as an association with Neolithic stone artifacts and heights at $17 \mathrm{~m}$ and $15 \mathrm{~m}$ asl. Radiocarbon dates were obtained from archaeological materials resting on the lake sediments. Therefore, following previous comments, these dated human activity rather than lake movements directly. Comparisons of heights of deposits with those identified by Wendorf and Schild are problematic for the reasons outlined above, and we have no means of independently determining if the heights used by Hassan are correct.

Kozlowski and Ginter (1993) reported on work conducted in the region of Qasr el-Sagha, reviewing their chronostratigraphic interpretations in relation to the earlier work by Wendorf and Schild (1976) and Hassan (1986). As in the earlier studies, they correlated diatomite deposits with the oldest Holocene lake (their lacustrine marls and diatomites LMD deposit). The top of this deposit indicated erosion and hence was interpreted to show lake recession, and this was followed by the deposition of gray hard silts (GHS), which marked a lake transgression. The top of the GHS deposits in turn showed evidence of further erosion, and therefore lake regression, with complex white sands and silts (CWSS) indicating a subsequent period of lake transgression. The top of the CWSS deposits indicated another period of erosion, with brown sands (BS) indicating another lake transgression. This final lake advance was associated with Middle Kingdom hydraulic works. 
Kozlowski and Ginter (1993) synthesized their results with those of Wendorf and Schild (1976) and Hassan (1986), making use of radiocarbon ages obtained from archaeological deposits associated with the lake sediment deposits they described. Pazdur (1983) reported these radiocarbon dates, indicating that the majority came from hearths rather than the sediments themselves. Kozlowski and Ginter (1993) agreed with the reconstruction of three pre-Neolithic lake transgressions but discussed different age estimates for the proto-Moeris lake and the subsequent recession before the Lake Moeris advance. A second area of disagreement concerned a proposed lake recession at the end of the Neolithic, proposed by Hassan and supported by Kozlowski and Ginter, and subsequent lake advances during the historic period. Wendorf and Schild argued for only a single lake advance during the Neolithic that continues into the historic period.

\section{Discussion}

Figure 3.3 highlights the $10-\mathrm{m}, 18-\mathrm{m}, 20-\mathrm{m}$, and $24-\mathrm{m}$ contours based on the DSM-derived 1-m contours. The locations of E29H1, E29G1, Kom W, and Kom $\mathrm{K}$ are indicated. Proposed lake heights between $18 \mathrm{~m}$ and $24 \mathrm{~m}$ submerge all these sites. Even the 18 -m contour means that the majority of areas that have surface archaeological deposits would be beneath the lake waters during some periods. While it is true that there have been late-twentieth- and twenty-first-century changes to the surface of the Fayum north shore, these effects are localized, with areas of significant surface remodeling limited to intensively worked farmlands to the south of the areas in which we are interested. As Figure 3.3 shows, the estimated high lake stands inundate very large areas, including the archaeological record deposited by the people inferred to have lived adjacent to the lake. Therefore it is unlikely that these

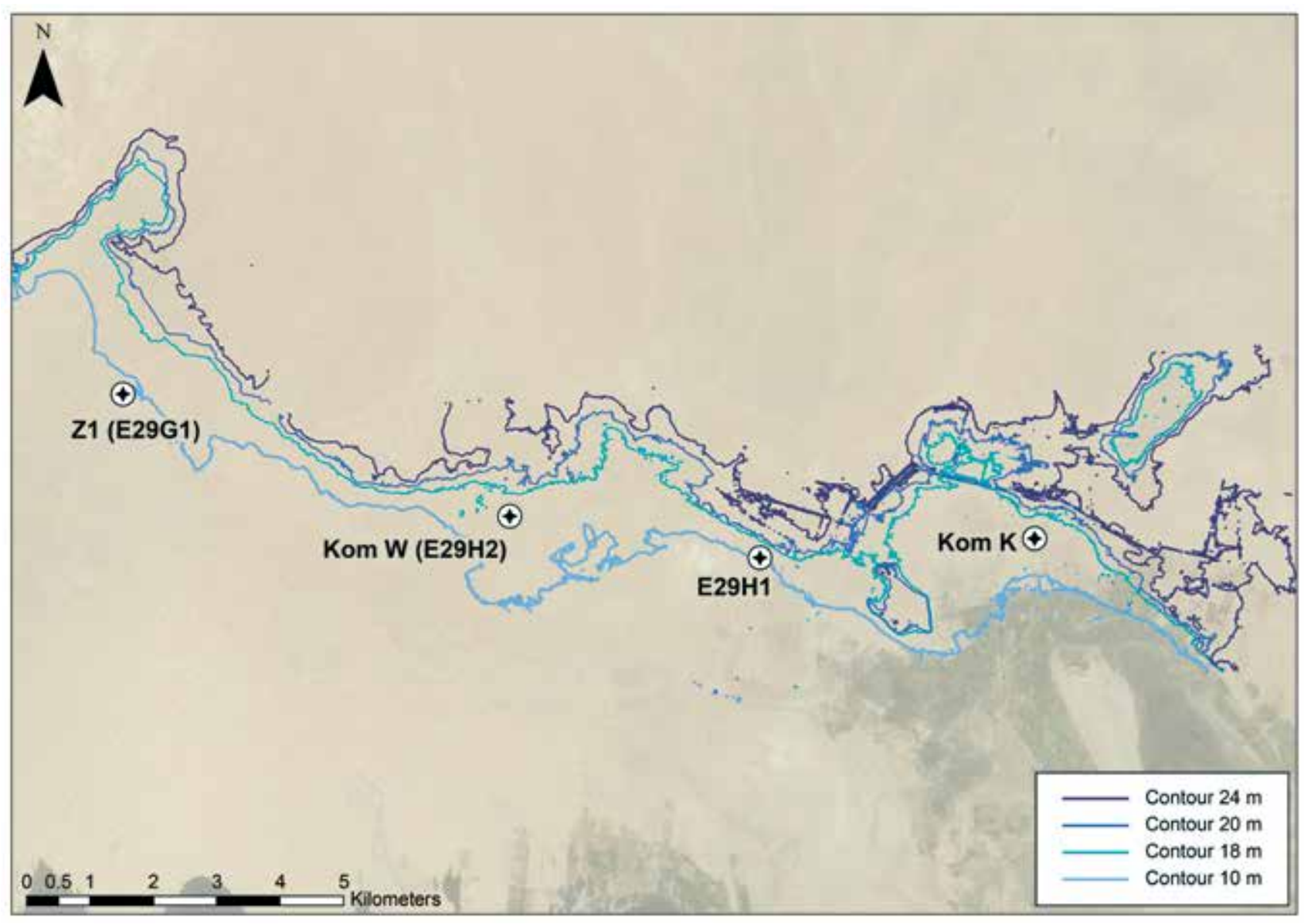

Figure 3.3. Ten-m, 18-m, 20-m, and 24-m contours from DSM 1-m data. Proposed lake advances above $10 \mathrm{~m}$ submerge the four sites indicated. 
lake level estimates can be correct. These archaeological deposits do indeed rest on lake-derived sediments, but the age of these sediments is not indicated by the radiocarbon ages obtained from cultural deposits except in the sense that ages so obtained provide a minimum age for the lake sediments. As we discuss in chapters 4 and 5, our results indicate that archaeological deposits are much more extensive than originally thought, including deposits much lower in elevation than those reported by later twentieth-century fieldwork.

As discussed above, one of the difficulties with working in the Fayum is the extent of the archaeological deposits made up largely of portable artifacts, an issue to which we now turn. The very richness of the surface deposits to some degree confounded earlier twentieth-century studies, leaving researchers unaware of the true artifact extent when they made their lake level reconstructions. This, combined with the difficulty of obtaining accurate heights, compromised the stratigraphic correlations they made in ways they were unable to detect. Our criticisms therefore detract not from the intent of this earlier work but simply from the interpretations derived from the results. Future work on lake level reconstruction should focus on developing independent, datable measures of lake level throughout the Holocene. We have obtained some relevant dating samples that are at the time of writing still being processed.

\section{Paleoenvironment}

As discussed at the beginning of this chapter, most considerations of Fayum paleoenvironment have concentrated on the relationship between archaeological deposits and changes in paleo-lake levels. There are, however, three other significant environmental drivers of interest in the Fayum during the early to mid-Holocene. First, as reviewed in chapter 2, radiocarbon determinations from other the eastern Saharan locations illustrate occupation, abandonment, and subsequent reoccupation of locations in the eastern Sahara, consistent with the northward shift of the Inter-Tropical Convergence Zone (ITCZ) (Hassan et al. 2001; Kindermann 2004; Kindermann et al. 2006; Kuper and Kröpelin 2006; Marshall and Hildebrand 2002; McDonald 2009; Wendorf et al. 2001). The Fayum shows periods of occupation and abandonment throughout the Holocene; however, the northern limit of the ITCZ did not reach the Fayum, so this periodicity may indicate the operation of other paleoenvironment drivers (Haynes 2001; Kuper and Kröpelin 2006; McCorriston 2006).

Second, as discussed above, Hassan (1984:58) correlates fluctuations in Lake Qarun with an increase in Nile levels that allowed mid-Holocene reoccupation of the Fayum. He links an apparent hiatus in the Fayum archaeological evidence between the Epipaleolithic and the Neolithic periods to the sudden decline in the level of Lake Qarun (Hassan 1997:Figure 1). However, recent paleoclimatic reconstructions suggest an increase in White and Blue Nile flow at approximately 6560 to $6250 \mathrm{cal} \mathrm{BP}$, about the time the Fayum appears to have been abandoned (Williams et al. 2010). Williams (2009:11) therefore suggests that times of lower rather than higher Nile flow and Nile incision were conducive to agriculture on the Nile floodplain, since this allowed swampy ground to drain. Determining the true, rather than assumed, relationship between the Lake Qarun shoreline and evidence of human occupation is obviously crucial if links to fluctuations in Nile levels and associated shoreline environments are to be made, as we discuss in more detail below.

The third environmental variable involves the southward movement of Mediterranean winter rains (Arz et al. 2003; Bryson 1992; Hassan et al. 2001; Kindermann et al. 2006; Phillipps et al. 2012). There is paleoclimatic evidence that the Fayum once received more winter rainfall from the eastern Mediterranean. Today, rainfall occurs in the southeastern Mediterranean associated with depressions and cold frontal troughs (Arz et al. 2003:119). Changes in Red Sea salinity in the early to mid-Holocene indicate a southward shift of Mediterranean winter cyclonic rainfall, while lower sea surface temperatures in the northern Red Sea and the eastern Mediterranean during the early to mid-Holocene provide evidence for the inflow of cold continental air masses during winter (Arz et al. 2003:119). The presence of these air masses during the mid-Holocene resulted in increased winter precipitation falling farther south than it now does, at a date that correlates with Neolithic settlement in the Fayum (Phillipps et al. 2012). This time period, 6700 to 5800 cal BP, is bracketed by the North Atlantic Bond Events 4 and 5 (Bond et al. 1997). Each of these produced drying in the Middle East and northern Africa. Thus the moist period indicated by the paleoclimatic record that existed between these events would have been a time of more benign, moister conditions on the Fayum north shore. 
The existence of benign climatic conditions circa 6700 to 5800 cal BP might be an example of what Williams (2009:11) calls "geological opportunism," a term introduced by Claudio Vita-Finzi (1978), in the sense that land was not sought out for cultivation until it was physically possible to do so. If so, then the Fayum paleoenvironment suggests occupation of a suitable habitat for cultivation when it became available. This reasoning is similar in some respects to that developed for the Sahel region by Marshall and Hildebrand (2002) but posits a different set of opportunities more suitable for the Southwest Asian domesticates. The period from 6700 to $5800 \mathrm{cal} \mathrm{BP}$ that Williams proposes correlates with the end of the period of Mediterranean dispersal of Southwest Asian plant and animal domesticates from Turkey into the northern Mediterranean Basin (Zeder 2008). This may indicate that northern Egyptian environmental conditions enabled the use of Southwest Asian domesticated species only in Egypt after people had begun moving around the Mediterranean Basin. However, while domestic species and associated technologies were exported, the introduction of Southwest Asian domesticates around the Mediterranean Basin, including Greece, Cyprus, Italy, Spain, and Portugal, also indicates local variability in environmental interaction by a range of Neolithic colonists (Finlayson 2004; Gkiastra et al. 2003; Perles 2001; Runnels 2003; Zeder 2008). In the following chapters, we investigate what effect local variability in environment, both natural and social, had on Fayum settlement. In Egypt the use of domesticated cereal species eventually led to the development of the décrue system of Nile flood manipulation, the economic foundation for dynastic civilization (Butzer 1976:20). In determining how this system developed, it is crucial to understand how people first used particular domestic plants and animals in different regional microenvironments. For Lower Egypt, the Fayum offers the best opportunity to undertake such an assessment.

The Fayum was apparently abandoned quite suddenly circa 6200 to 6000 cal BP (Wenke 2009; chapters 4-6 this volume), suggesting that Southwest Asian domestic species could be used in the Fayum only under certain conditions. Hassan (1986:497) discusses the problem of draining and irrigating lake margin fields as well as removing reed thickets to allow cultivation. Others have questioned the sustainability of agriculture in the Fayum due to the effect of lower sediment loads that flowed into Lake Qarun with the Nile flood and the amount of the lake edge that was actually covered by floodwaters (Bard 2008; Wenke 2009; Wenke et al. 1988). And as we have demonstrated, we do not know that occupation was concentrated on the paleo-lake shore margins. Increases in Nile floods, due to increases in summer precipitation in Ethiopia at around $6100 \mathrm{BP}$, actually decreased sediment load in the Nile Valley, suggesting that from this period, conditions were not optimal for Nile flood-based agriculture (Williams 2009). The nature of the connection between the Nile and the lake must be considered in relation to sediment deposition, but also salinity levels of the lake itself. As we discuss below, a great deal can be learned by considering the topography of the Fayum north shore, particularly the series of basins that Caton-Thompson identified, and this suggests a different paleoenvironment to that reconstructed in the older literature.

Kozlowski and Ginter (1993:333) report increased wadi activity during some of the lake recession periods and attribute this to the occurrence of what are described as "seasonal torrential rains in the desert." However, like the fluctuations in lake margin deposits, we lack independent means of establishing when this increased wadi activity occurred. Marshall and Hildebrand (2002:122) suggest that the Southwest Asian domesticates were confined to the Nile Valley because they were winter crops and thus were dependent on the availability of water from November to April. If so, then the presence of winter rains in the northern Nile Valley was crucial to the success of the Southwest Asian domestic grains, based on a winter rain growth cycle, and a collapse in use of these grains would have ensued if the winter rains retreated due to changes in the flow of cold air into the eastern Mediterranean discussed above. This may help explain the abandonment of the Fayum after 6000 BP. In addition, abandonment of the region might relate to environmental changes that had an impact on the availability of lacustrine resources.

If people were in fact targeting places that accumulated moisture derived from winter rains in which to plant crops, it is possible to predict the location of settlement and field systems. It is also possible to predict the nature of the settlement system, since occupation should differ from the earlier mobile occupations. Key requirements to testing such a model are good preservation of floral and faunal remains together with a spatially distributed archaeological record that permits 
an understanding of which parts of the landscape were targeted for use. In addition, datable archaeological materials are needed, since testing the paleoclimatic model requires the ability to understand when, as well as where, occupation occurred and when occupation duration reduced or ceased. Preservation of paleoenvironmental indicators is needed to test the veracity of the winter rain paleoclimatic reconstruction, as well as to test the nature of Nile inundation as suitable for early agriculture. Fortunately, these critical parts of the archaeological record are preserved in the Fayum, in a spatially extensive and varied set of deposits. As discussed, this record is both spatially extensive and in places quite dense. In the following chapters, we concentrate on determining the age and distribution of the record in the area adjacent to the basins CatonThompson and Gardner identified with the aim of understanding why archaeological materials are concentrated in these regions and not in others.

\section{Analysis of the Lake Basins Identified by Caton-Thompson and Gardner}

In addition to examining past lake levels in the Fayum, the high-resolution DSM, combined with a larger-coverage advanced space-borne thermal emission and reflection radiometer global digital elevation model (ASTER GDEM), allows examination of local topographic variability that helps indicate the potential ecosystems that developed around the lake edge. Topographic variability along the shoreline was observed by Caton-Thompson and Gardner (1934), and they identified a series of basins along the paleoshoreline. In the context of discussions of Kom W, Caton-Thompson and Gardner (1934:22) suggested that occupants during the early Neolithic were attracted to the $\mathrm{Z}$ Basin, then an inlet from the larger Lake Moeris. Later, when the lake level fell, the basins $(\mathrm{Z}, \mathrm{X}, \mathrm{K}, \mathrm{L}$, and $\mathrm{N})$ became lagoons, which CatonThompson and Gardner thought also attracted occupation (Figure 3.4). Both authors also noted the presence of wadis and, as mentioned above, Kozlowski and Ginter (1993:333) suggested that increased rainfall fed wadi activity during periods of lake recession.

The relationship between the intensity of Nile discharge and the position and intensity of monsoonal rainfall in the ITCZ affected the level of Lake Qarun as long as a permanent connection with the Nile existed. Local topographic variability along the lake edge therefore dictated the impact of lake level rise and fall on the amount of land surface exposed, subsequent vegetation growth, and animal habitats that the basins supported, both lacustrine and terrestrial. Examining topography is therefore a useful starting point from which to understand the impact lake rises and falls may have had on human interactions with the environment, particularly relating to subsistence strategies. The energy of the shoreline environment, including the impact of wind, was likely affected by local topography and may ultimately have affected geomorphological processes relating to sediment deposition and preservation of the archaeological record.

Six basins (Z, X, L, K, N, and U, the latter unnamed by Caton-Thompson) were identified by CatonThompson and Gardner (1934) and can be seen in the contemporary satellite imagery. Changes in the levels of Lake Qarun can be examined in relationship to the topography of potential shorelines, the connectedness of the basins to the main lake, and the exposure of land surface. Basin topography is an important variable for reconstructing the paleoenvironment. Figure 3.5 illustrates the topographic variability of the basins using a nine-unit hillslope model adapted to a lacustrine basin environment, although in our case only five of the nine classes were identified. The model identifies components of a hillslope profile based on the slope values and how sediments move within and between these components (Parsons 1988:28-29). The classes identified represent different erosional and depositional environments, with low slopes characteristic of depositional and high slopes producing erosional environments.

To assess the potential north shore basin paleoenvironments, we studied their topography using satellite-derived DSMs in combination with field checking. The area of each basin as defined by Caton-Thompson and Gardner's original boundaries was confirmed by comparing these with the contemporary satellite imagery ASTER GDEM in addition to the Worldview-2 stereo imagery. The ASTER GDEM Version 2 (Tachikawa et al. 2011) allows the construction of contours from a period before large-scale quarrying activity, agricultural development, and road building in the Fayum. This comparison also helped define basins where modern agricultural intervention made identifying individual basin topography difficult. Elevation values were extracted for each basin based on a mask of each basin area. These points were used to create a raster surface to a resolution of $30 \mathrm{~m}$, with values based on elevation. A slope function command in ESRI ArcGIS was used to derive slope for each cell, measuring the maximum 


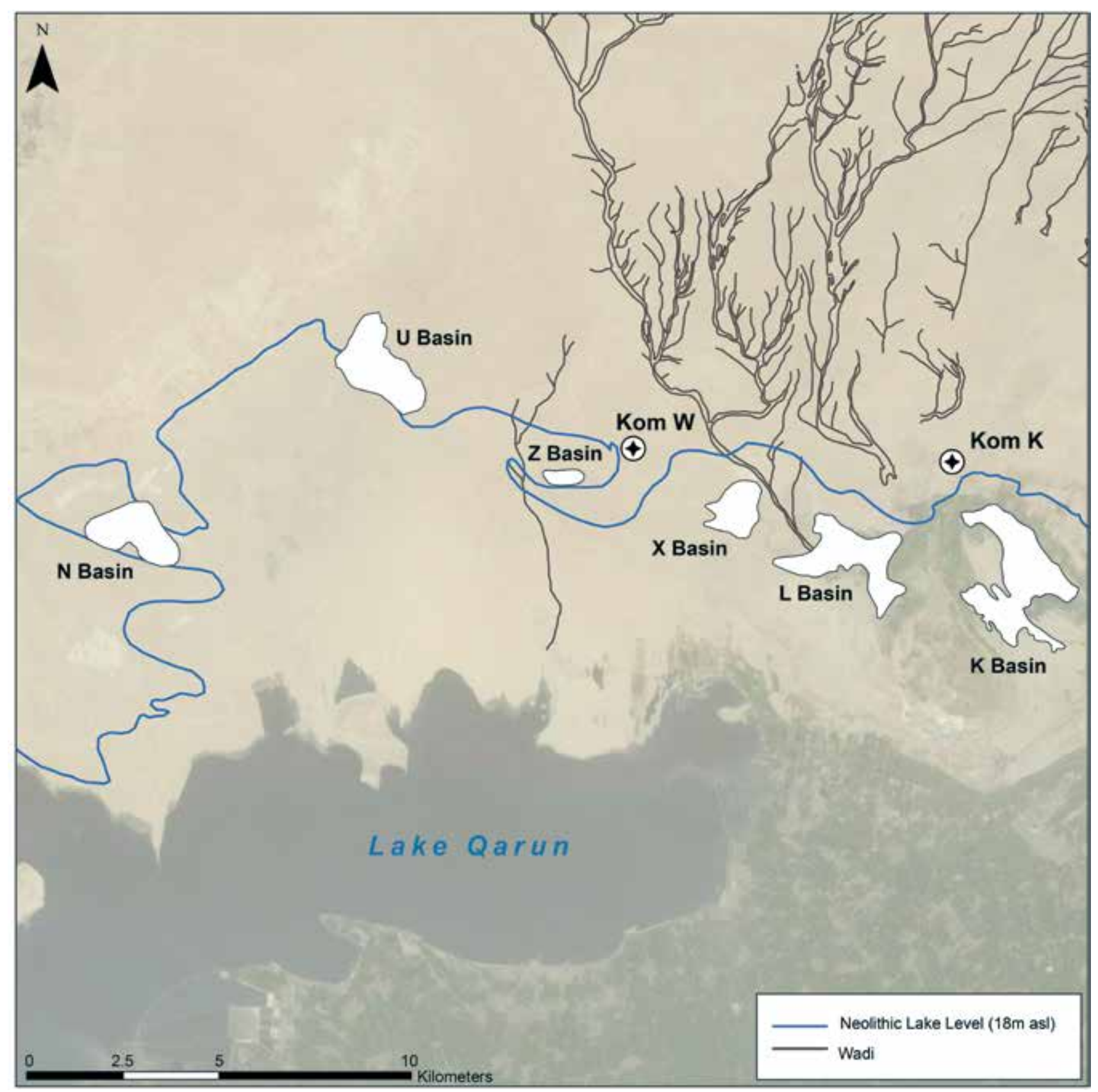

Figure 3.4. The wadis, basins, and Neolithic lake edge (18 m asl) identified by Caton-Thompson and Gardner (1934).

change in elevation between cells, and was displayed as change in slope between $0^{\circ}$ and $90^{\circ}$. The slope values were reclassified into a nine-unit hillslope model, based on slope angle following Parsons (1988:28). Lower slopes may receive more sediment deposition while steeper slopes will often be more prone to erosion. The surface areas of each basin were calculated based on Caton-Thompson and Gardner's definition.
Results indicate that $\mathrm{K}$ Basin is the largest $\left(6.06 \mathrm{~km}^{2}\right)$, followed by L Basin $\left(4.51 \mathrm{~km}^{2}\right)$, U Basin $\left(3.13 \mathrm{~km}^{2}\right)$, X Basin $\left(2.46 \mathrm{~km}^{2}\right), \mathrm{N}$ Basin $\left(2.45 \mathrm{~km}^{2}\right)$, and Z Basin $(1.10$ $\mathrm{km}^{2}$ ). Unfortunately, slope could not be investigated in detail for K Basin due to extensive modern agricultural intrusion. Comparisons between the remaining basins suggest that $\mathrm{Z}$ Basin (mean change of slope $65.07^{\circ}$ ) has the steepest gradient while $X$ Basin (mean change of 
slope $16.99^{\circ}$ ) is the shallowest (Table 3.1). The slope reclassification (Figure 3.5) shows the presence of only two classes, 1 and 2, in every basin except Z Basin, which contains five different classes (1 to 5).

Results of the analysis suggest differences between the six basins along the northern shoreline. $\mathrm{Z}$ Basin is small and steep, while X Basin is large and shallow. Regardless of the ultimate level of the lake, differences in the steepness of the basins likely affected lake inundation and subsequent retreat, whether annually or otherwise. In 1939 Ball suggested that the lake rose and fell between $2.5 \mathrm{~m}$ and $4.0 \mathrm{~m}$ annually during the Nile flood. Using recorded mid-Holocene archaeological remains as the absolute maximum lake level for the period, since otherwise, as discussed above, remains would be under the lake waters, Ball's (1939) suggestion can be used to examine the connectedness of the basins to the lake during a lower lake level or an annual retreat of the Nile inundation. The reconstructed high and low lake stands

Table 3.1. Slope Change Calculations for the North Shore Basins (Figures 3.5 and 3.6) Identified by Caton-Thompson and Gardner (1934).

\begin{tabular}{|l|l|}
\hline Basin & Degrees of Slope Change \\
\hline $\mathrm{Z}$ & 65.07 \\
\hline $\mathrm{N}$ & 32.36 \\
\hline $\mathrm{U}$ & 29.37 \\
\hline $\mathrm{K}$ & 19.48 \\
\hline $\mathrm{L}$ & 18.27 \\
\hline $\mathrm{X}$ & 16.99 \\
\hline
\end{tabular}

show that $\mathrm{Z}$ Basin and the $\mathrm{U}$ Basin are the only basins to remain connected to the main lake if the lake were to retreat $3 \mathrm{~m}$ during non-inundated periods (Figure 3.6). During the inundation or high lake stands, all basins were potentially connected to the lake. As a consequence, summed across all the basins, approximately $235.7 \mathrm{~km}^{2}$ of "irrigated" land surface would be exposed if the lake inundated and retreated $3 \mathrm{~m}$ annually. Given the slope of each basin, it is likely that Basins X, L, K, and $\mathrm{N}$ could receive sediment if it was available, but $\mathrm{U}$ Basin and especially $\mathrm{Z}$ Basin would be more prone to erosion due to steepness of slope.

In addition to sediment deposition and erosion, these results have implications for the types of vegetation and animal habitats that may have existed within the basins. Given the large shallow, and possibly unconnected, nature of the eastern basins $(\mathrm{K}, \mathrm{L}$, and $\mathrm{X})$, some fish species may not have survived in these environments. Catfish favor shallow, deoxygenated environments (Brewer 1987:461) and so might have lived in the shallow eastern basins if these were indeed connected to the lake. In contrast, the steepness and possibly higher-energy environment of $\mathrm{Z}$ Basin may have provided a more suitable environment for species such as Nile perch (Brewer 1991:291; Van Neer 2004).

If agriculture in the Fayum was indeed dependent on an annually receding lake level and exposure of irrigated land surface, a sufficient amount of land surface would need to be available, as in the main Nile Valley. A gentle slope gradient along the lakeshore would ensure sufficient annual exposure of moist sediment, producing an environment where crops could be

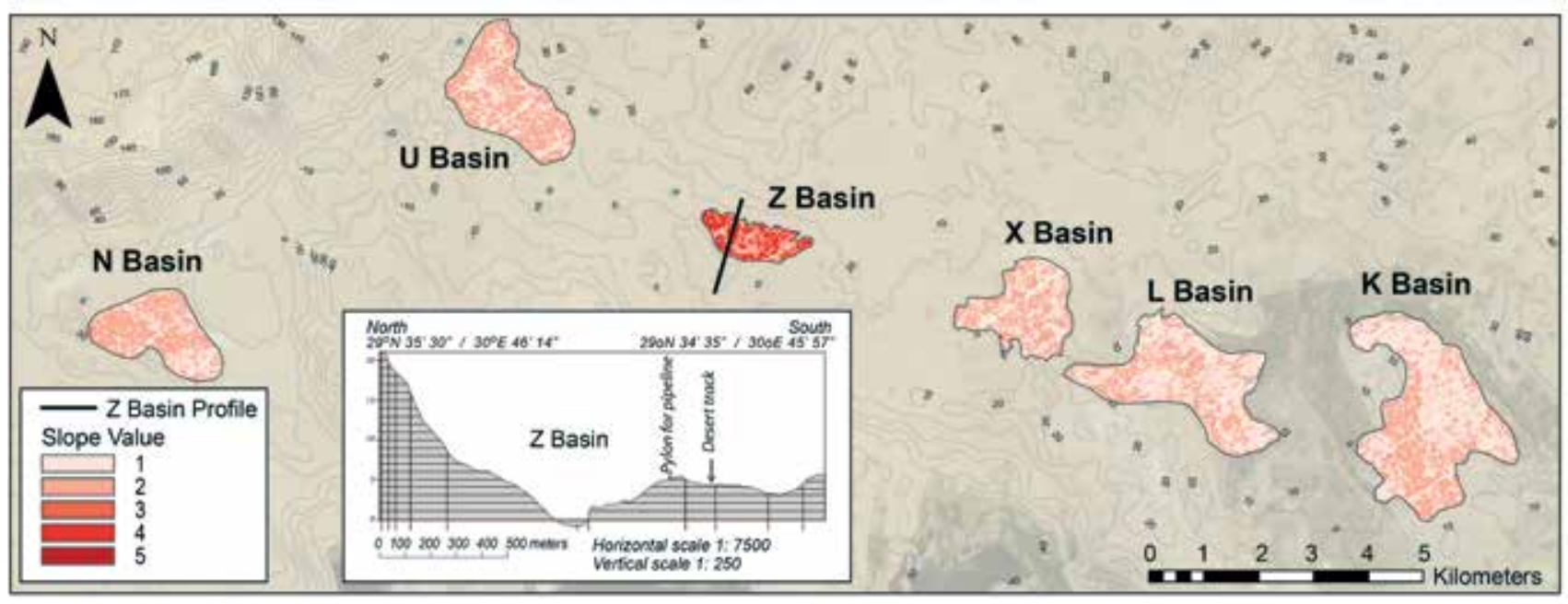

Figure 3.5. Reclassification of basin slope values based on the Parsons (1988) nine-unit model (see text for details) with an inset of the $\mathrm{Z}$ Basin north-south profile. 


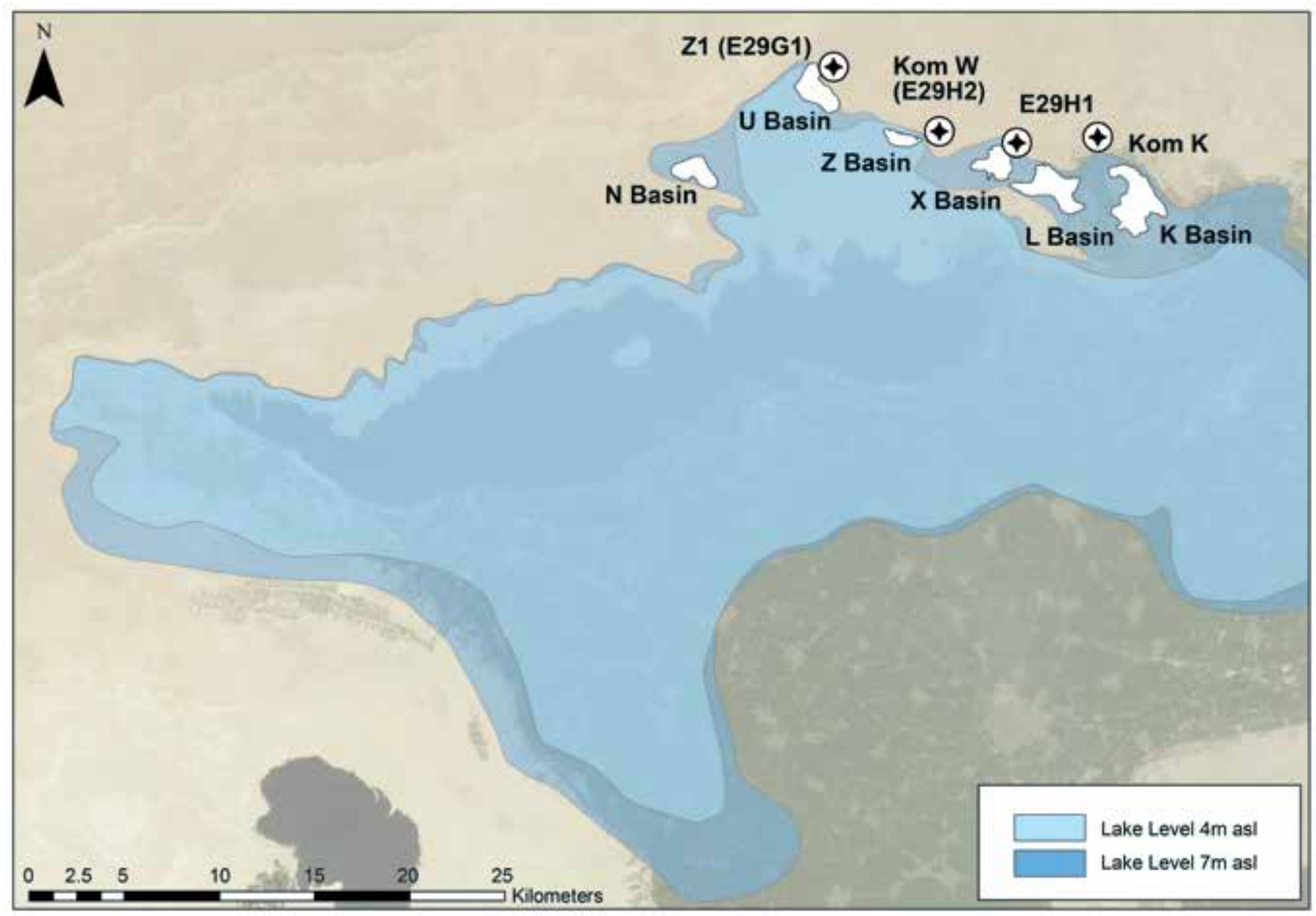

Figure 3.6. The simulated extent of Lake Qarun inundation when lake levels are increased to $4 \mathrm{~m}$ and $7 \mathrm{~m}$ asl, showing exposure of the north shore basins.

sown without the need for terracing steeper slopes. The results of the analysis of basin topography suggest that a much greater surface would be exposed in the eastern basins (X, $\mathrm{L}$, and $\mathrm{K}$ ) compared to the western basins $(\mathrm{Z}$ and $\mathrm{U})$. This suggests that if lake edge agriculture was practiced, it was more likely undertaken in the eastern basins. In addition, during periods of lower Nile flow, winter rainfall feeding the eastern basins through the wadis may have supplemented the water supply. If the lake did not inundate, or did not inundate to the degree suggested by Ball (1939), rainfall may have been the only source of freshwater for the eastern basins during low lake stands. This local variability in basin topography and the outcomes of likely human interaction with and reaction to a variable environment fits with William's (2009) notion of geological opportunism. The potential implications of the basin topography are considered in relation to the distribution of archaeological remains in the chapters that follow.

\section{Lake Basin Vegetation}

Today in the Fayum, native woody taxa are generally restricted to irrigation canal banks, the shore of Lake Qarun, and some desert areas. The shores of Lake Qarun today are dominated by tamarisk (Tamarix nilotica), reeds (Phragmites australis), and the salt-tolerant chenopodiaceous herb Sarcocornia, with tamarisk and reeds also found along canals. The deserts north of Lake Qarun contain only the chenopodiaceous woody shrub Haloxylon salicornicum. A pollen core from Lake Qarun spanning the modern period (circa AD 1650 to 1976) indicates that Chenopodiaceae/ Amaranthaceae and tamarisk are the primary terrestrial taxa contributing to the pollen assemblage (Mehringer et al. 1979:244). Tamarisk is the dominant native tree in the Fayum today, with Tamarix nilotica the only species identified in the area.

Lake Qarun has been strongly saline for the last 150 years (Flower et al. 2006). However, the freshwater 
paleo-lake likely had a greater diversity of marshland plants than is currently the case. Sedge (Cyperaceae), cattail (Typhaceae), and grass (Poaceae) pollen are present in a recently obtained lake core, suggesting that marshlands existed in the past (Hamdan et al. 2016). If rainfall was more frequent and substantial during the Early and early Middle Holocene (Hassan et al. 2012; Phillipps et al. 2012), this likely supported a greater density and diversity of woody taxa. Acacia was likely more common, and other native woody species found on canal banks in the Nile Valley (Zahran and Willis 2009:277-279) may also have been present. Of these, pollen from the families Capparaceae (Capparis), Chenopodiaceae (Suaeda), Euphorbiacae (Ricinus), Fabaceae (Faidherbia), and Salicaceae (Salix) was identified from the Lake Qarun pollen core (Hamdan et al. 2016).

\section{Understanding the Fayum \\ Archaeological Landscape}

As discussed above, as archaeologists, we are interested in how people used different parts of the landscape in relation to the potential these areas provided. In the remainder of this chapter, we describe the methods we adopted to understand the distribution of the archaeological record across this landscape and the types of processes that affected this distribution. The results allow us to compare the distribution of the archaeological record with our understanding of the Fayum paleoenvironment discussed above. In the text below, we describe how the material archaeological record may be interpreted as a proxy for landscape use.

\section{The Distribution of Archaeological Deposits}

One of the difficulties that all researchers face in the Fayum is the high density of artifacts that today rest on deflated surfaces. Because the artifacts are small in size and distributed across very large areas, estimating changes in artifact density can be a difficult task. For instance, it is very difficult to draw boundaries around high-density areas based on sight alone. Added to this are difficulties of surveying boundaries made without recourse to modern geopositioning systems and in the absence of permanent geographic reference points. Published observations on the location and size of sites, and even the existence of distinct sites, therefore need to be treated with these cautions in mind, much the way we indicated for the chronostratigraphic correlations discussed above. As described below, we developed methods to overcome these limitations by combining the use of modern survey technologies with a concern to locate each artifact precisely in space. We use these data to compare artifact density differences at different places in the north shore landscape.

As discussed in chapter 2, archaeologists sometimes identify sites as locations where particular sets of activities occurred, considering these to be the remains of occupations associated with particular functions. Interpretation then comes from comparing the distribution of different site types categorized on the basis of these activities across a landscape. However, as noted, it can be difficult to determine both the spatial extent and composition of deflated surface assemblages by eye. This makes it difficult to determine whether distinct, functionally discrete site types existed. One alternative is to use spatial units independent of the archaeology being investigated. In this way, we make no assumptions that concentrations of archaeological materials represent discrete aspects of a settlement system. For surface deposits, areas of erosion may be suitable to lay out such recording units if they can be shown to be unrelated to past activity. A record may then be taken of the distribution of archaeological features (for example, artifacts) within these spatial units as well as between them. In areas with later, spatially segregated land use, modern divisions such as field dividers are frequently used with the same intent (e.g., Cherry 2003), with the caveat that the divisions must be truly modern and not simply overlaid onto ancient field systems. In other regions, naturally occurring units may be used (e.g., Holdaway and Fanning 2008, 2014). However, in the Fayum such units, in the form of discrete eroded areas or modern field systems, did not exist in all the areas we wished to investigate. For this reason, we developed our own observation units, which are described in detail below.

Some archaeologists make a distinction between sites and non-sites (Bintliff 2000; Dunnell 1989; Ebert 1992:53; Kvamme 1998). Sites are places with intact remains of functional areas used in the past with a "pristine" archaeological context or in "primary" context (in situ; Schick 1986:7), whereas non-sites are places with material relocated through post-depositional processes in "secondary" or "derived" context (Schick 1986:7). Many archaeologists favor material considered to represent a site and disregard non-site material (Bintliff 2000:200). Sites and non-sites may be differentiated by the presence of buried material (in sites), on the basis of 
artifact density, or at times on arbitrary standards of perceived artifact disturbance. However, a number of studies discuss post-depositional processes that concentrate artifacts giving the appearance of sites, whether buried or not (Bintliff 2000:206; Clark and Schofield 1991:93; Gallant 1986:408; Redman and Watson 1970:285; Schofield 1991:5; Shackley 1978). Similarly, all sites have to some degree undergone modifications that might be thought of as disturbance. Therefore it is important to study what types of processes are responsible for the archaeological record as it exists today rather than to simply classify sites as in situ or not (Holdaway and Fanning 2008). Because at least part of the Fayum archaeological record was potentially affected by changes in lake level and the majority of the deposits are deflated, it is also important to determine how artifacts have moved since abandonment (e.g., Fanning and Holdaway 2001). It is also important to adopt methods of analysis that are not based on an assumption that all aspects of behavior are present undisturbed at single locations.

As discussed in chapter 1, the project area extends for approximately $20 \mathrm{~km}$ along the north Fayum lakeshore with an area of circa $142 \mathrm{~km}^{2}$. This represents approximately 30 percent of the shoreline of ancient Lake Qarun and the associated lake basins during the Early to Middle Holocene and is therefore of sufficient size to provide an indication of settlement variability on the lakeshore. As noted above, Caton-Thompson and Gardner (1934) identified six basins along the Fayum north shore, and these were used as a boundary for construction of our survey design, intended to determine differences in the distribution and nature of artifact assemblages across the north shore area as a whole, including the areas away from the basins north into the Fayum Basin.

Using a line built around the topographic outline of the basins as one survey boundary, we used a radiating design (discussed below) to determine artifact presence and density. Evenly spaced transects, orientated northsouth and east-west, were used to provide information on artifact density. Based on the distance between artifact concentrations established at E29H1 during a preliminary study conducted in 2004 and again in 2008 (reported in chapter 4), we defined transect survey units of $100 \mathrm{~m}$ in length, orientated north-south and eastwest to form a cross shape, with $10 \mathrm{~m}$ widths, giving a total area of approximately $2000 \mathrm{~m}^{2}$ for each transect unit. The actual area varied depending on where the transect boundaries were placed. Within each transect unit, stone, ceramic, bone, and shell objects were located in three-dimensional space, with a total station and the underlying surface recorded (for example, sand, diatomite, and so on), as described below.

We calculated coordinates representing the nodes of the survey unit shape in UTM on the project GIS before going into the field and transferring them to a differential GPS. This unit was used to locate the nodes in the field, the nodes marked with a rebar stake. The differential GPS was used to locate a foresight and backsight in the northeast and southwest corners of the survey unit, respectively, and these were used to establish a location for a Leica robotic total station. The total station was used to record the outline of the different sediment types found within the survey unit, as well as the true area of the transect, and to locate the position of each artifact with a maximum dimension greater than $20 \mathrm{~mm}$, a size limit that reflects the tendency of small objects to be moved by water flow (Fanning and Holdaway 2001).

This approach was initially developed at the site of XB11 (Figure 1.1) by one of us (Phillipps 2006). It is described here to illustrate the type of surface sediment observations we subsequently made at other locations. XB11 was identified as part of a preliminary pedestrian survey in 2004 as an extensive surface scatter of stone artifacts, bone, and ceramics. An intensive survey was conducted between November and December 2004. The position of every stone artifact, ceramic fragment, and bone within the survey area was recorded in three dimensions using a total station across an area of approximately $12,300 \mathrm{~m}^{2}$ (Figure 3.7 ). Part of the surface at XB11 was made up of consolidated fine-grained sands with calcified nodules of sand clast (Qfs). Some of the calcified nodules most likely represent mineralized vegetation, which was noted at other locations around the northern lakeshore in earlier studies (e.g., Caton-Thompson and Gardner 1934:14; Wendorf and Schild 1976:192). Where these were particularly concentrated, we labeled the surface as "root casts." At XB11, this surface formed an elongated ridge tending northwest-southeast elevated above other surfaces. A test trench revealed a maximum thickness of $1 \mathrm{~m}$ for this unit. Similar material extended to the south and west (Qfsb), although at a lower elevation, most likely representing the same geomorphological layer as Qfs. In depressions of this surface, accumulations of windblown sand were present. Qfsc represents another exposure of this surface. "Windblown sand" was the label used in subsequent surveys when such sand deposits dominated the surface. 
To the north of the ridge, a less compacted and less calcified sand layer (Qe) (relative to the sands of the ridge) was overlain by scattered clasts of angular cobbles and pebbles (referred to as concentrated gravel or gravel lag). To the south of the ridge, the third unit identified (Qcs) is characterized by an undulating surface of loose sand composed of moderately sorted, sub-angular to sub-rounded, coarse-grained sand (approximately $3 \mathrm{~mm}$ ) clasts in a fine-grained sand matrix overlain by calcified casts and artifacts. This surface was slightly solidified and approximately 2 to $5 \mathrm{~cm}$ thick. Elsewhere we referred to this type of sediment as windblown sand. Within this unit, another surface unit (Qcsb) was identified. It consisted of an undulating, loose, sandy surface of moderately sorted sub-angular to sub-rounded, coarse-grained sand clasts in a finegrained sand matrix overlain by calcified casts. As noted above, these are referred to as root casts. In this unit, the surface was more disturbed than the Qcs surface, which may be due to the movement of heavy vehicles excavating canals, referred to as modern intervention, as was a final surface unit, defined as fill and described as excavated sediment from trenches dug for the irrigation canals.

Sediment characterization in subsequent years followed the approach developed at XB11 and focused on recording different surfaces that might obscure artifacts. For instance, on some survey units, aeolian sand deposits formed ripples that exposed artifacts in the swales between small dunes (referred to as sand ripples). This obviously reduces artifact density. Table 3.2 provides a description of the surface codes and surface types identified. Sediment descriptions are based on the presence of sand, gravel, mud, fossilized shell, and gypsum. Modern intervention, such as the presence of vehicle tracks, was also recorded.

Artifacts within the survey units were identified according to class and material and were coded directly as such into the total station. Colored nails were used to identify the different types of artifacts, as illustrated in Figure 3.8. Table 3.3 provides a list of the different types of objects recorded.

The total station records were downloaded each night and used to construct GIS shape files for each material category for each survey unit. Figure 3.9 shows the survey unit C4T5 (Corridor 4, Transect 5), with the sediment types that were recorded. Figure 3.10 shows the same survey unit with both the surfaces and the artifact density indicated.

To determine changes in assemblage composition and density away from the basins and the paleoshoreline, transect survey units were located using a logarithmic scale at $100 \mathrm{~m}, 200 \mathrm{~m}, 400 \mathrm{~m}, 800 \mathrm{~m}, 1,600 \mathrm{~m}, 3,200 \mathrm{~m}$, and $6,400 \mathrm{~m}$. Up to eight blocks were sampled in each of the four transects, covering $64,000 \mathrm{~m}^{2}$, or approximately 1.2 percent of the total study concession area.

Table 3.2. Surface Types Used to Characterize Survey Transects.

\begin{tabular}{|c|c|c|}
\hline Windblown sand & Qcs, Qfsc & windblown sand (but not forming large ridges) \\
\hline Sand ripples & & $\begin{array}{l}\text { windblown sand forming sand ridges with intervening } \\
\text { swales }\end{array}$ \\
\hline \multicolumn{3}{|l|}{ Carbonate pebbles } \\
\hline Consolidated sands and calcified sand clast nodules & Qfsb & consolidated fine-grained sands \\
\hline Dune sand & & $\begin{array}{l}\text { windblown sand ridges that are substantially larger } \\
\text { than aeolian sand }\end{array}$ \\
\hline Concentrated gravel or gravel lag & Qe & unconsolidated mixture of rock fragments and pebbles \\
\hline Modern intervention & Qcsb, fill & any recent mechanical modification of the surface \\
\hline Root casts & Qfs & $\begin{array}{l}\text { calcified nodules most likely representing mineralized } \\
\text { vegetation }\end{array}$ \\
\hline Silt substrate & & unconsolidated fine-grained sands \\
\hline \multicolumn{3}{|l|}{ Tertiary outcrop } \\
\hline Vegetation & & plant material \\
\hline Wadi & & dry water channel \\
\hline
\end{tabular}




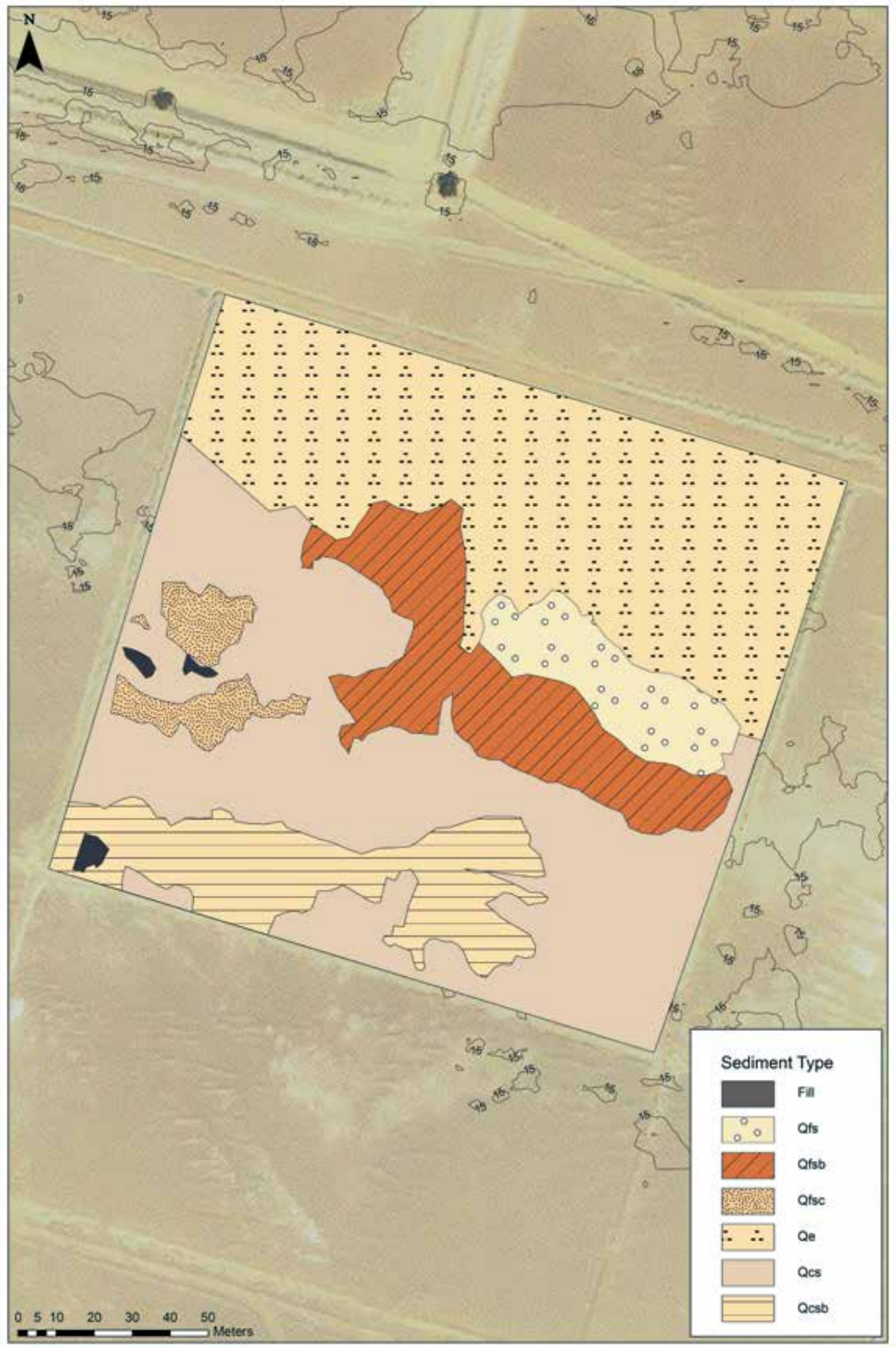

Figure 3.7. Surface sediment types identified at XB11. 


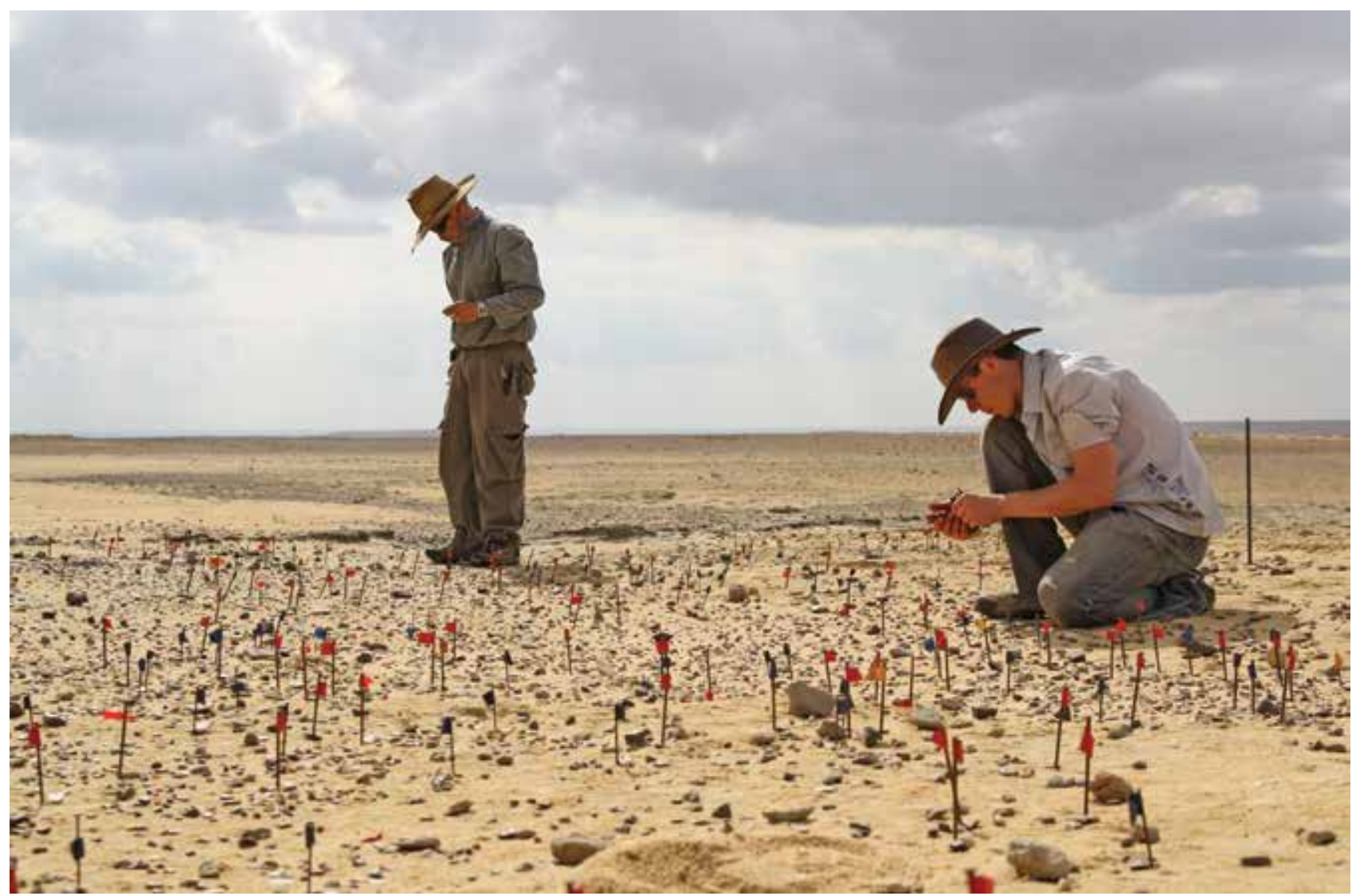

Figure 3.8. Colored nails used to mark the location of different types of artifacts during transect survey.

Table 3.3. Artifact, Bone, and Ceramic Categories Recorded in Transects.

\begin{tabular}{|l|l|}
\hline Bone & any bone fragment $>20 \mathrm{~mm}$ in length \\
\hline Broken flake & stone artifact $>20 \mathrm{~mm}$ in length that lacks a platform \\
\hline Complete flake & stone artifact $>20 \mathrm{~mm}$ in length that retains a platform \\
\hline Core & worked cobble with negative flake scars \\
\hline Tool & stone artifact $>20 \mathrm{~mm}$ in length with retouch \\
\hline Ceramic & ceramic fragment $>20 \mathrm{~mm}$ in length \\
\hline
\end{tabular}

Figure 3.11 shows the distribution of the survey corridors placed evenly across the project concession. Not all transects in Corridor 1 were surveyed, since it crossed the escarpment that marked the western boundary of the survey area. All corridors included survey units placed well below the Neolithic lake level estimated in previous studies (e.g., Hassan 1986; Wendorf and Schild 1976).

To account for differential visibility, densities of different artifact types were calculated for each of the survey transects by surface type (Table 3.4).
Artifact densities vary considerably according to sediment type. Windblown sand covers artifacts, reducing artifact densities. For this reason, artifact densities from high-visibility surfaces (consolidated sand and gravel surfaces) were used to compare transects in each of the corridors. Many transects contained either none or very few artifacts, even accounting for surface type. Artifacts were concentrated near the basins that Caton-Thompson and Gardner identified.

Figure 3.12 shows bar graphs of the density of complete flakes, based on high-visibility surfaces only, and 


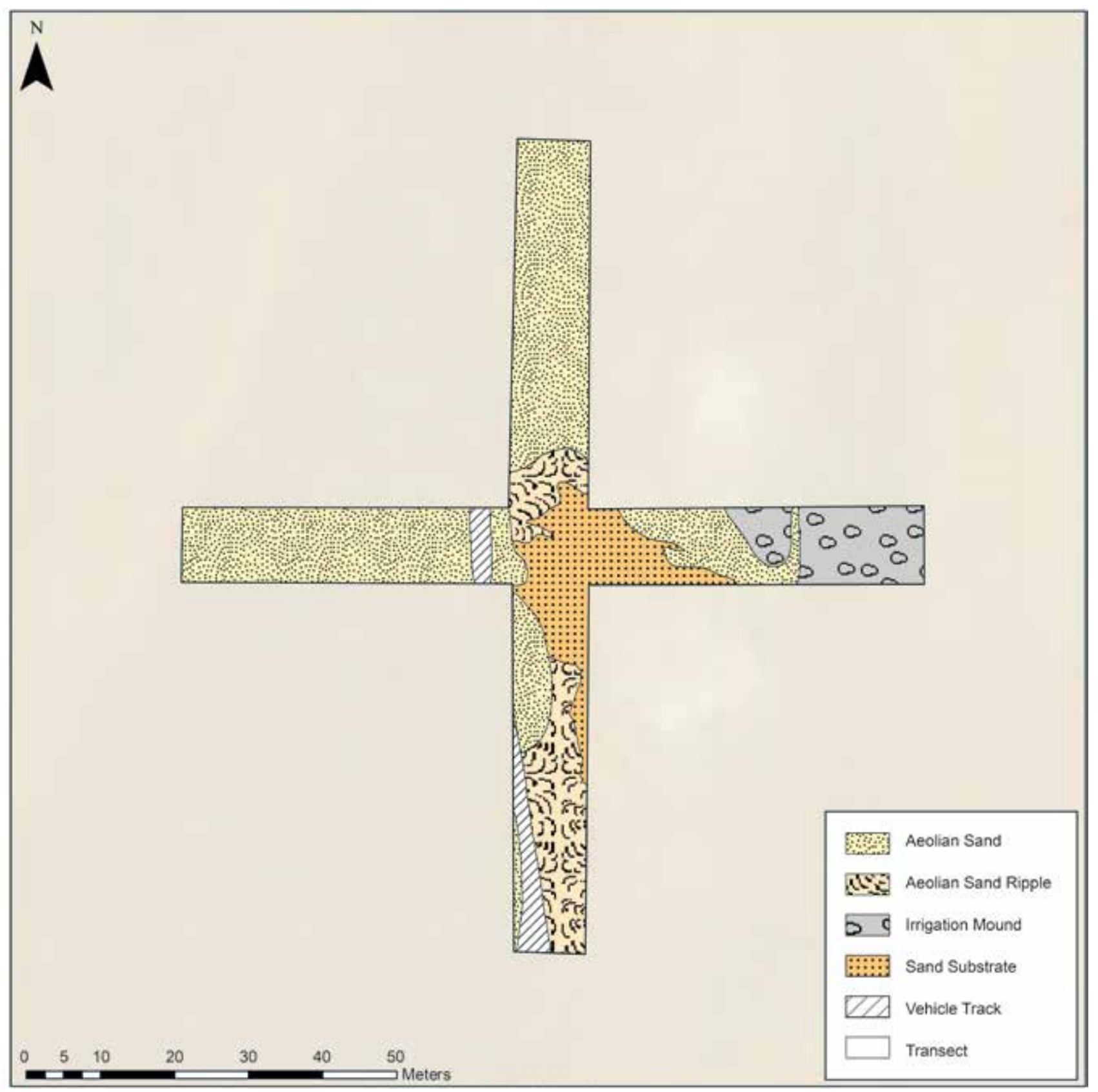

Figure 3.9. Transect C4T5 showing surface sediment types mapped with a total station and stored in a GIS.

the flake-to-core ratio from these same surfaces. The graphs are aligned to the nearest 5 -m contour, derived from the satellite DSM data discussed above. Both the plots of the flake-to-core ratio values and complete flake densities show a variable distribution, with low-density values both above and below the assumed lake edge across all the corridors. These distributions do not pattern relative to the contour heights. Transects with low flake-to-core ratio values, indicating a smaller number of flakes relative to the number of cores, occur at lower-contour heights except for C3T4. Much higher flaketo-core values occur in other transects at heights of 15 to $20 \mathrm{~m}$ asl. With the exception of C1T3 and C4T13, higher artifact densities tend to occur at relatively lower elevations. C1T1 shows artifacts present at $0 \mathrm{~m}$ asl, considerably lower than the lowest lake level estimates 


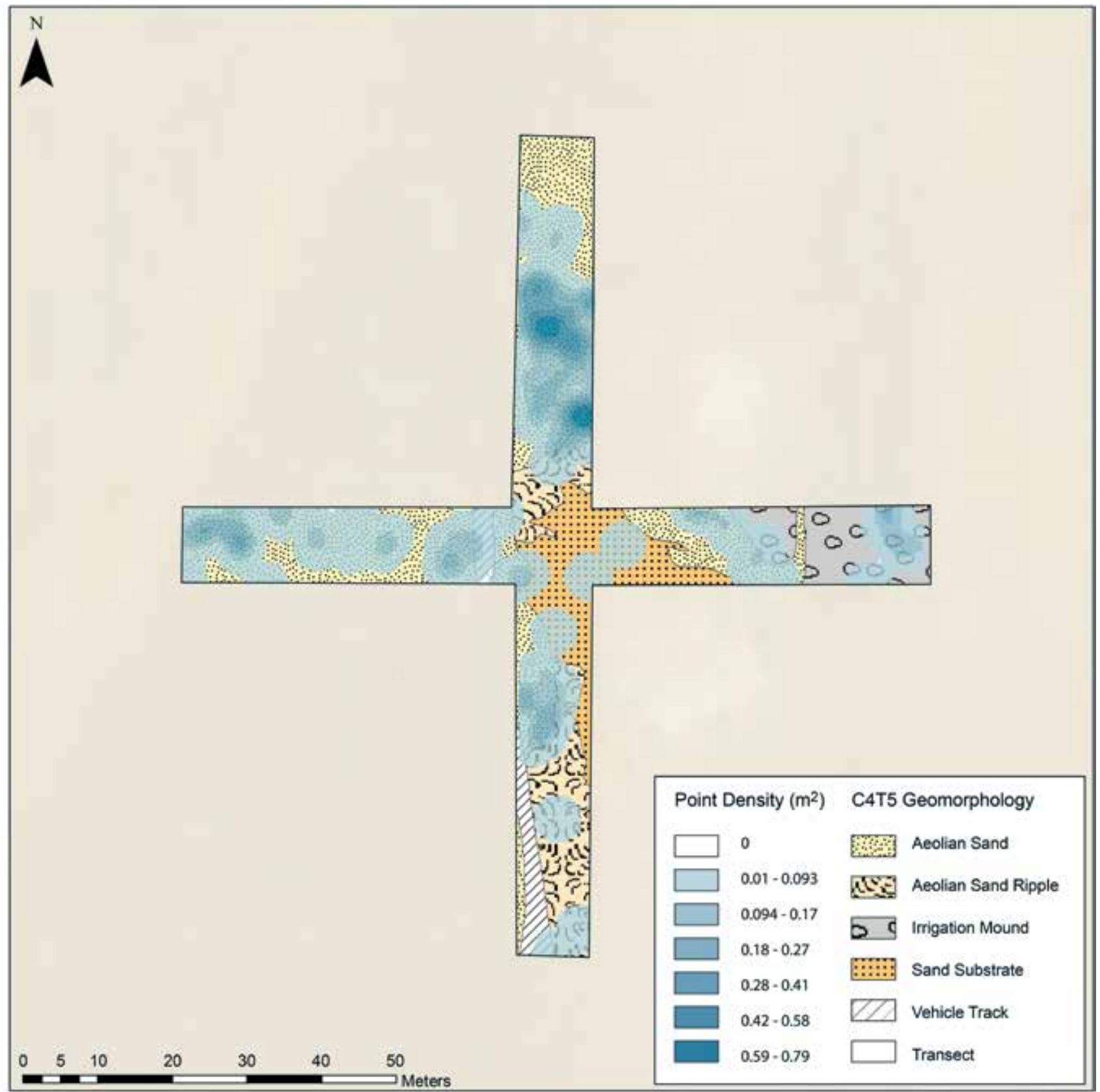

Figure 3.10. C4T5 surface sediment types overlain with total artifact density derived from the provenance of all objects greater than or equal to $20 \mathrm{~mm}$ in maximum dimension $\left(\operatorname{artifact} / \mathrm{m}^{2}\right)$.

presented in earlier studies. Similar results from the region around the site of E29H1, originally studied by Wendorf and Schild (1976), are discussed in chapter 4.

In addition to the intensive survey for portable artifacts, the corridors between the transect survey units were walked along a 100 -m-wide band north into the Fayum Basin. The locations of grinding stones and hearths were recorded using a handheld GPS unit with a precision of $+/-5 \mathrm{~m}$. The location of both hearths and grinding stones are shown in Figure 3.13. Both feature types are concentrated around the basins in a similar manner to the stone artifacts. In Corridor 3, isolated hearths and grinding stones were identified $1,700 \mathrm{~m}$ and $3,700 \mathrm{~m}$ north of those concentrated close to the basins. 


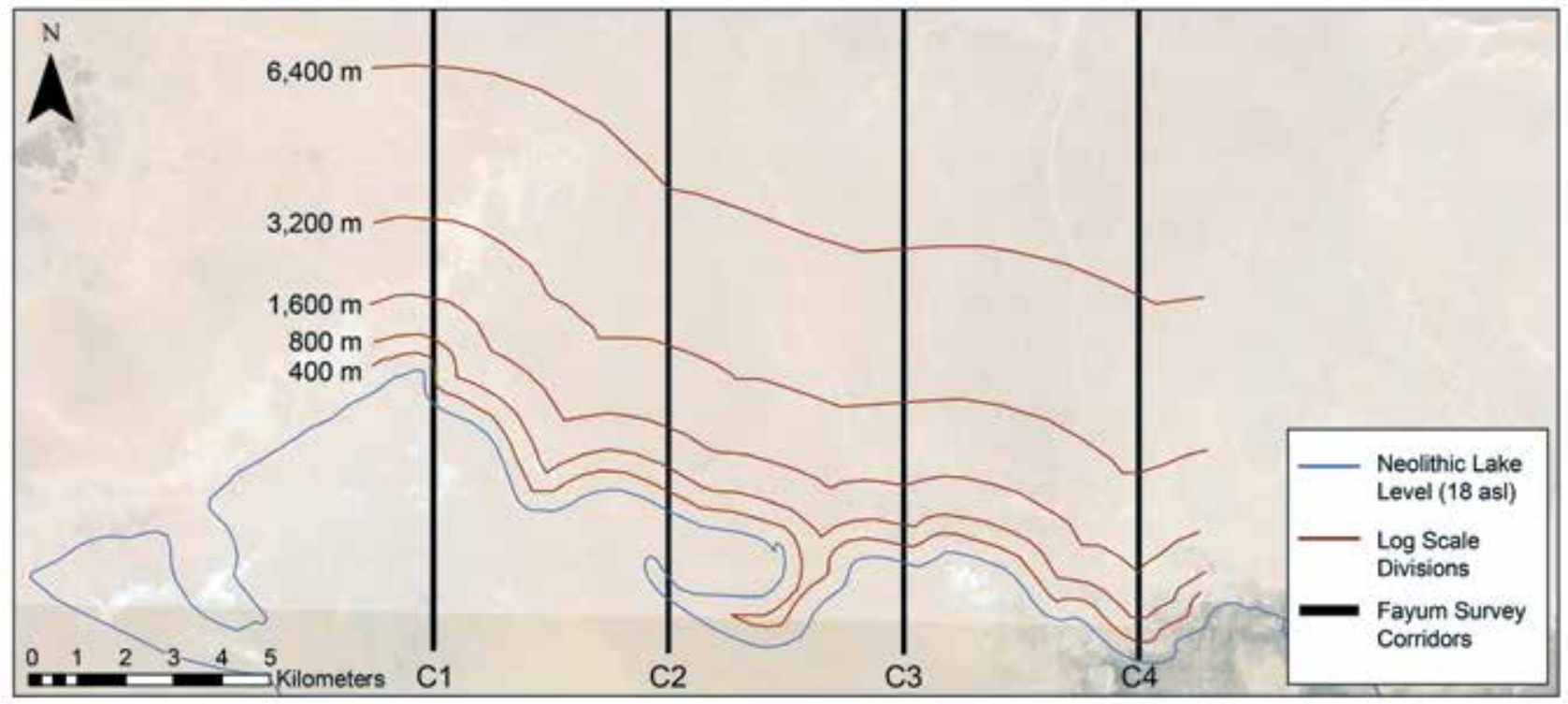

Figure 3.11. Survey corridors placed evenly across the project concession.

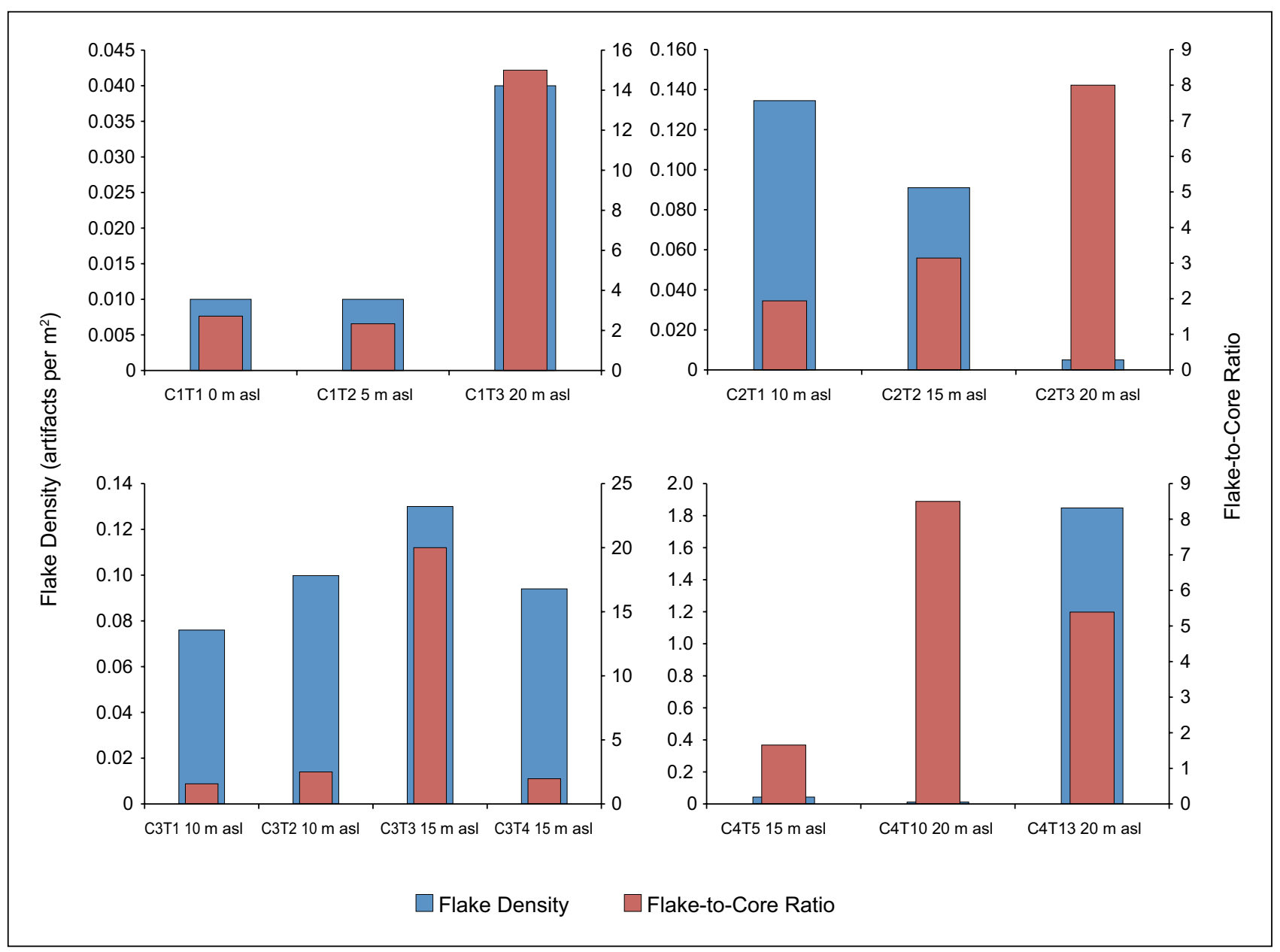

Figure 3.12. Density of complete flakes, based on high-visibility surfaces only, and the flake-to-core ratio from these same surfaces, for corridor transects with sufficient artifacts to allow the calculations. 
Table 3.4. Densities of Artifacts, Fauna, and Ceramics in Corridor Transects as Number of Objects per Square Meter.

\begin{tabular}{|c|c|c|c|c|c|}
\hline C1T1 & Dune Sand & & & & \\
\hline Complete flake & 0.01 & & & & \\
\hline Broken flake & 0 & & & & \\
\hline Core & 0 & & & & \\
\hline Tool & 0 & & & & \\
\hline Bone & 0.01 & & & & \\
\hline C1T2 & Dune Sand & & & & \\
\hline Complete flake & 0.01 & & & & \\
\hline Broken flake & 0 & & & & \\
\hline Core & 0 & & & & \\
\hline Tool & 0 & & & & \\
\hline Bone & 0.02 & & & & \\
\hline C1T3 & Dune Sand & Modern Intervention & Consolidated Sand & & \\
\hline Complete flake & 0 & 0 & 0.04 & & \\
\hline Broken flake & 0 & 0.01 & 0.01 & & \\
\hline Core & 0 & 0 & 0 & & \\
\hline Tool & 0 & 0 & 0 & & \\
\hline Bone & 0 & 0 & 0 & & \\
\hline C2T1 & Dune Sand & Consolidated Sand & Calcified Gravel & & \\
\hline Complete flake & 0 & 0.030 & 0.223 & & \\
\hline Broken flake & 0 & 0.028 & 0.062 & & \\
\hline Core & 0 & 0.072 & 0.060 & & \\
\hline Tool & 0 & 08 & 0.014 & & \\
\hline Bone & 0 & 0 & 0 & & \\
\hline $\mathrm{C} 2 \mathrm{~T} 2$ & Desert Pavement & Dune Sand & Aeolian Sand & Carbonate Pebbles & Tertiary Outcrop \\
\hline Complete flake & 0.091 & 0.031 & 0.013 & 0.221 & 0.023 \\
\hline Broken flake & 0.033 & 08 & 0 & 0.071 & 05 \\
\hline Core & 0.029 & 0.023 & 0 & 0.056 & 0.028 \\
\hline Tool & 0.010 & 0 & 0 & 0.011 & 05 \\
\hline Bone & 0 & 0 & 0 & 0 & 0 \\
\hline $\mathrm{C} 2 \mathrm{~T} 3$ & Desert Pavement & Aeolian Sand & Calcified Gravel & & \\
\hline Complete flake & 05 & 0 & 02 & & \\
\hline Broken flake & 01 & 0 & 0 & & \\
\hline Core & 01 & 0 & 0 & & \\
\hline Tool & 0 & 0 & 0 & & \\
\hline Bone & 0 & 0 & 0 & & \\
\hline
\end{tabular}




\begin{tabular}{|c|c|c|c|c|c|}
\hline $\mathrm{C} 2 \mathrm{~T} 4$ & Desert Pavement & Aeolian Sand & & & \\
\hline Complete flake & 0 & 0 & & & \\
\hline Broken flake & 0 & 0 & & & \\
\hline Core & 01 & 0 & & & \\
\hline Tool & 0 & 0 & & & \\
\hline Bone & 0 & 0 & & & \\
\hline C2T5 & Desert Pavement & Aeolian Sand & & & \\
\hline Complete flake & 03 & 0 & & & \\
\hline Broken flake & 0 & 0 & & & \\
\hline Core & 0 & 0 & & & \\
\hline Tool & 01 & 0 & & & \\
\hline Bone & 0 & 0 & & & \\
\hline C2T6 & Desert Pavement & Aeolian Sand & Vehicle Track & & \\
\hline Complete flake & 01 & 07 & 0 & & \\
\hline Broken flake & 0 & 0 & 0 & & \\
\hline Core & 0 & 0 & 0 & & \\
\hline Tool & 01 & 0 & 0 & & \\
\hline Bone & 0 & 0 & 0 & & \\
\hline C2T7 & Desert Pavement & Vehicle Track & & & \\
\hline Complete flake & 01 & 02 & & & \\
\hline Broken flake & 0 & 0 & & & \\
\hline Core & 0 & 0 & & & \\
\hline Tool & 0 & 0 & & & \\
\hline Bone & 0 & 0 & & & \\
\hline C2T9 & Desert Pavement? & Aeolian Sand & & & \\
\hline Complete flake & 06 & 04 & & & \\
\hline Broken flake & 0 & 0.032 & & & \\
\hline Core & 01 & 0 & & & \\
\hline Tool & 02 & 0 & & & \\
\hline Bone & 01 & 0 & & & \\
\hline C3T1 & Dune Sand & Aeolian Sand & & & \\
\hline Complete flake & 0.014 & 0.076 & & & \\
\hline Broken flake & 04 & 0.040 & & & \\
\hline Core & 02 & 0.048 & & & \\
\hline Tool & 01 & 05 & & & \\
\hline Bone & 01 & 0 & & & \\
\hline
\end{tabular}


Table 3.4. Densities of Artifacts, Fauna, and Ceramics in Corridor Transects as Number of Objects per Square Meter. Continued

\begin{tabular}{|c|c|c|c|c|c|}
\hline $\mathrm{C} 3 \mathrm{~T} 2$ & Dune Sand & Aeolian Sand & & & \\
\hline Complete flake & 01 & 0.100 & & & \\
\hline Broken flake & 0 & 0.020 & & & \\
\hline Core & 0 & 0.040 & & & \\
\hline Tool & 0 & 0.020 & & & \\
\hline Bone & 0 & 0 & & & \\
\hline C3T3 & Aeolian Sand & Rhizolith & & & \\
\hline Complete flake & 0.312 & 0.130 & & & \\
\hline Broken flake & 0.109 & 0.013 & & & \\
\hline Core & 0.055 & 0.013 & & & \\
\hline Tool & 0.014 & 0.019 & & & \\
\hline Bone & 0.043 & 0.039 & & & \\
\hline Ceramic & 0.024 & 0.026 & & & \\
\hline C3T4 & Consolidated Sand? & & & & \\
\hline Complete flake & 0.094 & & & & \\
\hline Broken flake & 0.044 & & & & \\
\hline Core & 0.048 & & & & \\
\hline Tool & 05 & & & & \\
\hline Bone & 05 & & & & \\
\hline Ceramic & 0.023 & & & & \\
\hline C3T5 & Aeolian Sand & Modern Intervention & Gravel & Vegetation & \\
\hline Complete flake & 0 & 0 & 0 & 0 & \\
\hline Broken flake & 0 & 0 & 0 & 0 & \\
\hline Core & 0 & 0 & 01 & 0 & \\
\hline Tool & 0 & 0 & 0 & 0 & \\
\hline Bone & 02 & 0 & 0 & 0 & \\
\hline C3T6 & Desert Pavement? & Vehicle Track & & & \\
\hline Complete flake & 0 & 0 & & & \\
\hline Broken flake & 0 & 02 & & & \\
\hline Core & 0 & 0 & & & \\
\hline Tool & 0 & 0 & & & \\
\hline Bone & 0 & 0 & & & \\
\hline C3T7 & Aeolian Sand & Gravel & Vehicle Track & Wadi & \\
\hline Complete flake & 0 & 0 & 0 & 0 & \\
\hline Broken flake & 0 & 0 & 0 & 0 & \\
\hline Core & 0 & 0 & 0 & 0 & \\
\hline Tool & 0 & 0 & 0 & 0 & \\
\hline Bone & 0 & 0 & 0 & 0 & \\
\hline
\end{tabular}




\begin{tabular}{|c|c|c|c|c|c|}
\hline C4T5 & Aeolian Sand & Aeolian Sand Ripple & Vehicle Track & Sand Substrate & Irrigation Mound \\
\hline Complete flake & 0.043 & 0 & 0 & 03 & 0.013 \\
\hline Broken flake & 0.019 & 03 & 0 & 0 & 04 \\
\hline Core & 0.026 & 0 & 0 & 0 & 04 \\
\hline Tool & 0 & 0 & 0 & 0 & 04 \\
\hline Bone & 0 & 0 & 0 & 0 & 04 \\
\hline Ceramic & 0.014 & 0 & 0 & 0 & 0 \\
\hline C4T6 & Desert Pavement & Vehicle Track & Road & & \\
\hline Complete flake & 0 & 0 & 0 & & \\
\hline Broken flake & 0 & 0 & 0 & & \\
\hline Core & 0 & 0 & 0 & & \\
\hline Tool & 0 & 0 & 0 & & \\
\hline Bone & 0 & 0 & 0 & & \\
\hline Ceramic & 0 & 0 & 0 & & \\
\hline C4T7 & Desert Pavement & Modern Intervention & & & \\
\hline Complete flake & 0 & 0 & & & \\
\hline Broken flake & 0 & 0 & & & \\
\hline Core & 0 & 0 & & & \\
\hline Tool & 0 & 0 & & & \\
\hline Bone & 0 & 0 & & & \\
\hline Ceramic & 0 & 0 & & & \\
\hline $\mathrm{C} 4 \mathrm{~T} 10$ & $\begin{array}{r}\text { Soft Desert } \\
\text { Pavement }\end{array}$ & Vehicle Track & & & \\
\hline Complete flake & 0.012 & 0.014 & & & \\
\hline Broken flake & 0 & 0 & & & \\
\hline Core & 01 & 0 & & & \\
\hline Tool & 0 & 0 & & & \\
\hline Bone & 0 & 0 & & & \\
\hline Ceramic & 0 & 0 & & & \\
\hline $\mathrm{C} 4 \mathrm{~T} 13$ & Desert Pavement & Soft Desert Pavement & $\begin{array}{r}\text { Modern } \\
\text { Intervention } \\
\end{array}$ & Calcite Pebbles & \\
\hline Complete flake & 1.848 & 2.726 & 0.013 & 5.232 & \\
\hline Broken flake & 0.530 & 0.784 & 0.011 & 1.509 & \\
\hline Core & 0.343 & 0.458 & 0 & 0.704 & \\
\hline Tool & 0.027 & 0.033 & 0 & 0.066 & \\
\hline Bone & 0 & 0 & 0 & 0 & \\
\hline Ceramic & 0.637 & 0.797 & 0 & 0 & \\
\hline
\end{tabular}




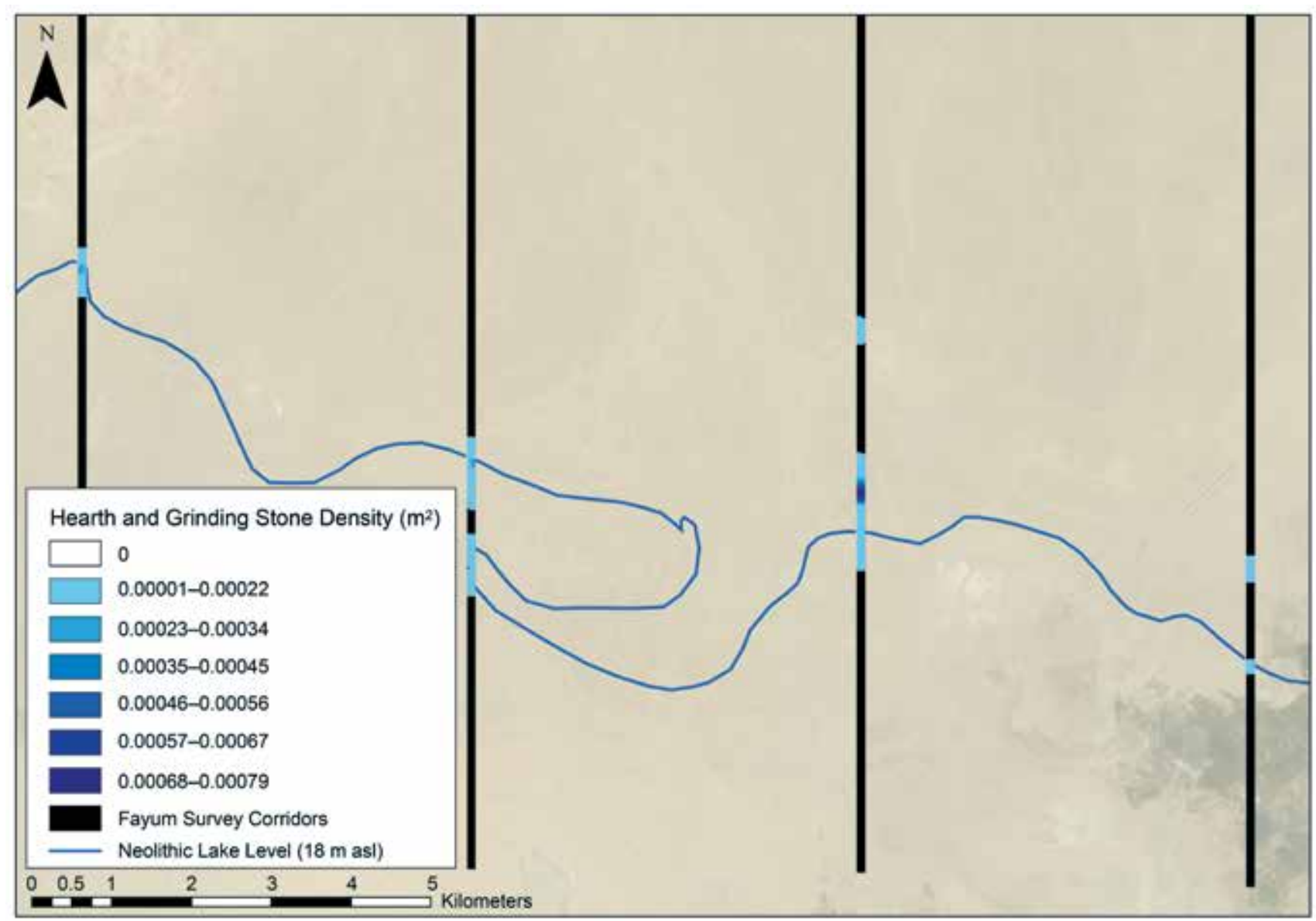

Figure 3.13. Hearths and grinding stones density $\left(\right.$ number $\left./ \mathrm{m}^{2}\right)$ located within the corridor surveys.

\section{Discussion}

As others have observed, artifacts are concentrated in a band close to the basins that Caton-Thompson and Gardner identified. However, these artifacts occur at a range of elevations, including points considerably lower in elevation than those identified by twentieth-century archaeologists working in the Fayum. Occasional artifacts are present in transects to the north but at concentrations that are well below those closer to the basins. Mapping of sediment types in all transects indicates that the absence of artifacts in those transects placed well north in the Fayum Basin cannot be accounted for by the presence of low-visibility surface types. Consolidated sands and gravels are present in the more distant transects, but there are few if any artifacts on these surfaces.

Hearths and grinding stones are also concentrated close to the basins, with occasional examples farther north. The spatial distribution of artifact density values and in the values of the flake-to-core ratios, with both measures showing considerable variability at different contour heights, is similar to the observations originally made by Caton-Thompson and Gardner, on the basis of which they defined the Fayum A and Fayum B industries. Lower flake-to-core ratio values, reflecting high numbers of cores relative to the number of flakes, sometimes occur at lower elevations. However, there is considerable local variation between the transects, a topic that is considered in more detail in chapter 4 . Artifact densities are in some instances highest around the $10-$ to $15-\mathrm{m}$ asl contours, although here as well, there is variability between the corridors.

Results of the survey along the corridors suggest that artifact concentrations are largely limited to areas around the basins that Caton-Thompson and Gardner identified and that the artifact assemblages around the basins may show interesting variability. For these reasons, our fieldwork concentrated on describing the 
artifact concentrations around $\mathrm{K}, \mathrm{L}$, and X Basins. We report on the first two of these in this volume (chapters 4 and 5).

\section{Landscape Approaches to the Analysis of Material Culture}

As discussed in chapter 2, our approach to material culture analysis is based on the study of variability using artifacts as proxies for a range of behaviors. This necessitated a number of methods designed to capture the variability both spatially and temporally. We review these methods here and apply them in chapters 4,5 , and 6 . We begin by describing the survey methods used to identify the distribution of portable material culture and features and then discuss the methods used to analyze different portable material culture types and features, beginning with flaked stone artifacts, grinding stones, hearths, ceramics, basketry, ornaments, faunal material, and floral material, including charcoal. The procedures for obtaining radiocarbon determinations are also discussed, along with details of the permit we received enabling the transportation of samples outside Egypt. Finally we discuss methods used in the excavations undertaken.

\section{Survey}

Conventional approaches to site survey face the issues of identifying sites in an environment where stratified deposits are rare and surface artifact scatters extensive. Early in the project, attempts to identify the location and extent of sites quickly ran into issues of observer bias. Without systematic recording of the distribution of portable material culture, the definition of sites becomes arbitrary and therefore it is very difficult to maintain consistency in recording. As discussed above, this is not a new problem, but one for which we had to develop a solution. As discussed in chapters 4 and 5, this involved the use of the transect crosses and a systematic application of these to assess the distribution of portable material culture across extensive surfaces that had not been damaged by contemporary agricultural development. An exception to this was our work at E29H1, which is described in detail in chapter 4.

We were also intent on using an approach to survey that differentiated between describing the distribution of material across the surface from the interpretation of this material. We noted that in surveys where sites are defined, these are frequently interpreted using functional labels. For example, resource extraction sites such as quarries are distinguished from occupation sites sometimes labeled as villages. Likewise, there are categories of site-like hunting stands or blinds and fishing camps. While there is of course good ethnographic information to indicate that activities like those indicated by the site names existed in the past, the identification of these activities is an exercise in archaeological inference and cannot be done based only on the location or composition of archaeological materials. Nor, given the way archaeological material accumulates, is there necessarily a direct relationship between the nature of artifacts found at one location and the activities that occurred there. Following Lucas (2012), we should not expect the archaeological record at a site to be a complete indication of all the activities that occurred. In the Fayum, our interest was to determine how the landscape was used through time, so we adopted approaches to surveying and the analysis of material culture that enabled us to understand such usage, including analyses designed to indicate what was missing as well as what was present. Survey identified some stratified sites but allowed us also to consider what information might be obtained from extensive surface artifact scatters.

\section{Flaked Stone Artifacts}

Because of the traditional focus on culture origins in North Africa, stone tool typologies have dominated artifact studies. However, recent discussion in lithic studies suggests that the role of tools and tool production is overemphasized in analyses (e.g., Holdaway and Douglass 2012). Instead of privileging certain stone tools, we employ complete assemblage analysis as a critical part of understanding not only the context of tools within stone artifact assemblages but also aspects of raw material reduction, use, and discard, and the movement of stone artifacts across landscapes. As many variables contribute to the assemblages archaeologists analyze, it is important to consider raw material, technology, function, reduction, and movement of artifacts. Examining complete assemblages permits the examination of a broader range of patterns of use and discard at single locations and across landscapes than analyses restricted to retouched tools.

The method of analysis reported in the following chapters uses established definitions for defining artifact classes. Divisions are made between flakes, cores, and tools (Holdaway and Stern 2004) (Figure 3.14). A flake is defined as the smaller detached piece from a 
raw material nodule that has a dorsal and ventral surface, a platform, and termination. A core is the remnant nodule. A tool is any artifact with one or more edges of regular retouch. Broken artifacts are also recorded, with divisions made between longitudinal and latitudinal breakage.

Quantifying patterns at a site or landscape level requires the spatial positions and attributes of artifacts and features to be measured consistently. Only through the repeated observation of records can patterns be observed and reliably compared between different locations. To enable this, we made use of electronic systems and software to record data. Locations were acquired with the aid of a robotic total station and differential GPS. Attributes recorded for individual objects of material culture using specially created data entry software ensured that the same set of attributes and associated values were available to record each object. Information was collated at the end of each day's fieldwork and entered into a GIS (ArcGIS) as the key strategic database for the project. With a relational design, the GIS allowed us to use multiple instruments at the same time to collect data and transfer it into a single GIS system.

Raw material has traditionally been an important aspect of stone artifact studies, especially for understanding the movement of people to raw material sources, trade and exchange, and economizing strategies (Kelly 1992). Understanding distance to source is particularly important, and sourcing studies have received much attention globally (Kuhn 2004). However, movement of raw material from a source to the location alone is not normally sufficient as a basis for the study of mobility, trade, and exchange,
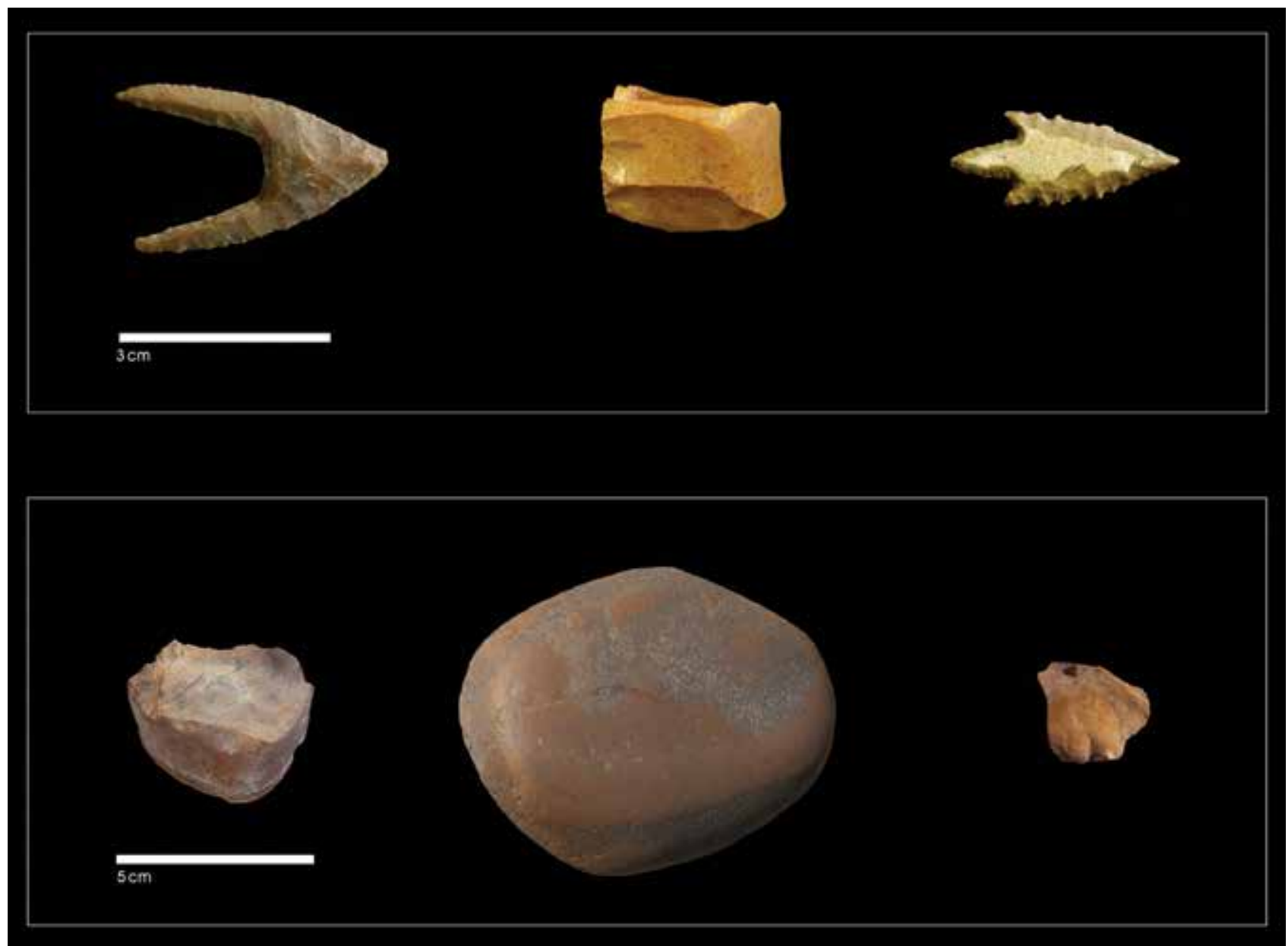

Figure 3.14. Examples of flaked stone artifacts and a flaked cobble from the Fayum. Top sequence, left to right: bifacial crescent point, microblade core, bifacial stemmed point. Bottom sequence, left to right: flake core, unworked flint cobble, dorsal surface of a flake. 
or economizing strategies. The observation of the distance to source acknowledges the movement of material from A to B but not the nature of activities that occurred between the source and the final resting place of the artifact. Recent reexamination of the use of stone artifacts in the study of mobility, economizing strategies, and trade is therefore based on close examination of the range of processes that lead to archaeological assemblage formation in addition to observations about raw material sources (Douglass et al. 2008; Holdaway et al. 2010; Kuhn 2004; Phillipps 2012).

Recent changes in thinking about the Egyptian Neolithic have prompted a revision of methods used to understand how people lived and interacted with their environment. Part of the shift away from typologically driven approaches is seen in the development of innovative methods for studying other aspects of the Neolithic (that is, not simply their origins, be this the migration of people or the diffusion of ideas). Methods for studying movement using stone artifact analysis have prompted a reconsideration of how prehistoric mobility is conceptualized and how it is assessed in the archaeological record. Mobility is of course simply the opposite of being sedentary, and therefore the reconstruction of mobility is no more interesting on its own than many other aspects of life. However, through understanding mobility, it is possible to consider wider aspects of landscape use, which of course influence a variety of past activities. Stone artifacts provide the means to reconstruct mobility and therefore provide a proxy for landscape use, as is illustrated here. Assessing mobility in this way also removes the need to assume that all aspects of the settlement system are available for study. From what is missing, it is possible to infer that people used parts of the landscape where the record is no longer extant or has not yet been studied. The methods employed to understand mobility (described below) are not dependent on knowing how artifacts were used but instead are based on their surface areas and volumes. These methods are particularly useful for assessing spatially extensive surface deposits where potentially the remains of several different occupations are combined.

Considerations of mobility using stone artifacts are frequently based on indirect proxy measures of mobility or sedentism. Usually these proxy measures rely on culture historical association or ethnographic analogy. However, these types of inferences can be separated from archaeological evidence of human movement based on direct proxy measures of artifact movement, such as distance to raw material source, refitting analyses, and other stone artifact analyses (e.g., Douglass et al. 2008; Turq et al. 2013). Close (2000) suggested that archaeologists should attempt to quantify the "hard evidence" for human movement or what is considered here to be direct proxy measures. However, the refitting analysis proposed by Close (2000) is not always a realistic goal for some assemblages, especially where repeated reoccupation of place may have occurred. Douglass et al. (2008) propose instead a method to identify the movement of stone artifacts based on complete assemblage analysis. This method was applied to stone artifact assemblages at Kom K and Kom W (Phillipps 2012) and will be discussed in chapters 4, 5, and 6 in the context of the $\mathrm{K}$ and $\mathrm{L}$ Basin areas.

To work stone, one must follow certain physical principles; otherwise conchiodal flake production will not ensue. However, even raw material from the same source varies somewhat in shape and consistency. Therefore, stone cannot be considered as plastic a medium as, say, clay. While people followed a set of actions to produce useful stone artifacts, they were not always able to produce products with uniform shapes. This was mitigated somewhat by the ability to produce very large numbers of stone artifacts relatively easily, assuming sufficient raw material (Holdaway and Douglass 2012). For this reason, analysis must deal with considerable variability among stone artifacts as well as large numbers of objects. Manufacture of stone artifacts creates products with clear landmarks. Flakes, for instance, have platforms as well as bulbs of percussion and can be oriented relative to the passage of force through the stone when the flake is struck (Holdaway and Stern 2004). Fragments of flakes are defined on the basis of these landmarks. (For example, proximal flakes retain a platform but no termination.) The dimensions of stone artifacts can be measured relative to these landmarks, and with the aid of small computers, very large numbers of observations can be taken relatively quickly. Holdaway and Stern (2004) illustrate how such dimensions are measured.

To analyze these observations, inferential statistics permit both central tendency and variance to be considered. In the analyses presented in chapters 4, 5, and 6 , we make use of both parametric and nonparametric 
tests obtained using IBM SPSS 22.0.0.0 software. Analysis of variance (ANOVA) and, at times, t-tests allow for the comparison of central tendency among different assemblages. In these tests, the dependent variable is one of the stone artifact dimensions (for example, length), and independent variables are used to divide the stone artifact groups that represent, for instance, different stages of manufacture. Core reduction removes material from a stone cobble, meaning that in many situations, flakes removed later in the reduction sequence will be smaller than those removed at the beginning of core reduction. Thus assemblages of stone artifacts may be divided in such a way that the independent variables relate in some way to flake production (for example, the presence or absence of cortex) and the values of the dependent variable assessed between these groups to test whether core reduction has occurred. Where a significant result for the ANOVA is obtained, we make use of post hoc Bonferroni t-tests to determine which pairs of assemblages are significantly different from each other. The SPSS software calculates the Bonferroni tests, with the overall error rate controlled by setting the error rate for each test to the experiment-wise error rate divided by the total number of tests, hence compensating for the number of tests undertaken. This adjusts the observed significance level for multiple comparisons and helps reduce the chance of falsely rejecting tests. Friedman's nonparametric test based on ranks is used as an alternative to ANOVA, and the Wilcoxon signed rank test is used as an alternative to the t-test. Spearman's rho based on rank order is used to assess the correlation between variables, since this does not require that the variables are normally distributed. Probability levels of 0.05 are used to assess significance throughout.

In the following chapters, we make use of statistics to test a variety of hypotheses. Significant results help us assess whether the patterns we see by grouping material culture observations in different ways are different from those we might expect by chance.

Although Caton-Thompson and Gardner recorded the presence of ground stone axes, we did not record additional examples of these in the material reported in this volume.

\section{Grinding Stones}

Grinding stones are numerous across intact parts of the Fayum north shore and were recorded as part of both the corridor surveys into the Fayum Basin described above and the transect surveys discussed in chapters 4, 5, and 6 . Each grinding stone was located using a GPS and was photographed. Morphology was described using variables that recorded lithology, shape, and completeness (Table 3.5). A great many of the grinding stones we recorded were fragments. The analyses undertaken were limited to morphology. Unfortunately we lacked the facilities to investigate for the presence of residues on the surfaces of the grinding stones we identified.

Table 3.5. Variables and Values Recorded for Grindstones.

\begin{tabular}{|c|c|}
\hline Variable & Values \\
\hline \multirow[t]{3}{*}{ Type } & lower \\
\hline & upper \\
\hline & unknown \\
\hline \multirow[t]{6}{*}{ Lithology } & conglomerate \\
\hline & limestone \\
\hline & pink limestone \\
\hline & sandstone \\
\hline & red granite \\
\hline & flint \\
\hline \multirow[t]{3}{*}{ Condition } & weathered \\
\hline & very weathered \\
\hline & broken \\
\hline \multirow[t]{3}{*}{ Latitudinal cross-section } & concave \\
\hline & convex \\
\hline & flat \\
\hline \multirow[t]{7}{*}{ Shape } & broken \\
\hline & circular \\
\hline & oval \\
\hline & rectangular \\
\hline & square \\
\hline & trapezoid \\
\hline & triangular \\
\hline \multirow[t]{6}{*}{ Working surface* } & eroded \\
\hline & eroded smooth section \\
\hline & pitted \\
\hline & pitted smooth \\
\hline & polished smooth \\
\hline & polished \\
\hline Shaping & yes/no \\
\hline
\end{tabular}

*The working surface variable was not recorded in 2008. 


\section{Hearths}

Hearths were recorded as part of the same survey used to locate grinding stones. We used variables to assess whether a hearth was still buried or scattered on the surface, and we noted the nature of the heat retainers (number, lithology) as well as the size and orientation of the hearth (Table 3.6). We excavated samples of hearths to obtain charcoal for age determinations and to determine the structure of hearths. The results of these excavations are described in chapters 4,5 , and 6 .

Table 3.6. Variables Recorded for Hearths, 2012.

\begin{tabular}{|l|l|}
\hline Variable & Value \\
\hline Type & buried \\
\hline & halo \\
\hline & remnant \\
\hline Number of heat retainers & scattered \\
\hline Heat retainer lithology & clay \\
\hline & clay, limestone bedrock \\
\hline & limestone bedrock \\
\hline & limestone bedrock, clay \\
\hline & limestone bedrock, ceramic \\
\hline & ceramic, limestone bedrock \\
\hline Orientation & E-W \\
\hline & N-S \\
\hline Charcoal observed & \\
\hline Length & \\
\hline Width & \\
\hline & \\
\hline & \\
\hline & \\
\hline &
\end{tabular}

\section{Ceramics}

Ceramic material was identified in some transects as fragments of vessels (sherds), and unlike other forms of material culture, these were collected and transported back to our field laboratory. The ceramics previously found in the Fayum were poorly fired and have a chaff temper, meaning that they are more fragile and in most cases more deteriorated than other material culture, such as stone artifacts (Caton-Thompson and Gardner 1934:35). The ceramics we identified conform to this, and most of the ceramic fragments we analyzed were heavily deteriorated. Many of the measurements and analyses taken on ceramics could not be completed in the field, unlike the measurements on stone artifacts. However, the locations of all ceramics were recorded with a total station.

\section{Basketry and Textiles}

One unique aspect of the Upper $\mathrm{K}$ Pits discovered in 1926 was the nature of the basketry that lined some of the pits. The forms and techniques used to make this basketry are described here, together with the material from which the baskets were made, where this could be identified. While preservation is sufficient to determine the nature of construction of the basketry, identification of the plant material used is dependent on preservation. As described in chapter 5, a SEM was used to examine the surface of some of the materials used in basketry construction.

\section{Personal Ornaments}

The positions of ostrich eggshell beads encountered during survey were recorded. In addition, beads excavated at Kom $\mathrm{K}$ were examined under a binocular microscope to record the presence and location of damage related to bead manufacture. Beads were made from ostrich eggshell, stone, and bone. Ornaments manufactured from shell were also described.

\section{Faunal Material}

Faunal samples were collected and analyzed from surface contexts at E29H1 and from the surface and buried deposits at Kom K. As with ceramics, analysis could not be undertaken in the field, so faunal remains were collected and analyzed in our field lab. Results of these analyses are presented in chapters 4, 5, and 6 . Bone preservation varied considerably, depending on the depositional context.

In addition, simple counts of bone elements were made as part of the surface surveys at E29H1 and the $\mathrm{L}$ and $\mathrm{K}$ Basin transects. Bone count density calculations are discussed in chapters 4,5 , and 6 .

One of the hearths from Kom K, discussed in chapter 6 , contained coprolites, the morphology of which was analyzed to determine the species from which they derived.

\section{Floral Material and Charcoal}

During excavation of Kom $\mathrm{K}$ and the near-surface hearths encountered during survey, charcoal was sampled both for radiocarbon dating and for wood species 
identification. Charcoal was identified at our field laboratory in the Fayum primarily with a stereomicroscope; however, a modified transmission microscope using an incident light source was used in some cases. At least 10 fragments of charcoal were observed for each sample where possible. Charcoal remains were identified by comparison with a local comparative collection and published wood atlases (Fahn et al. 1986; Neumann 1989; Neumann et al. 2001; Schweingruber 1990) and two wood anatomy websites (InsideWood 2004 onward; Schoch et al. 2004; Wheeler 2011).

Cappers (2006) outlines the methods used to identify sub-fossil plant remains from the Fayum. Samples analyzed were obtained from the Upper K Pits and from hearths excavated at Kom K.

\section{Radiocarbon Determinations}

A permit to remove samples for radiocarbon determinations was obtained from the Supreme Council of Antiquities in collaboration with the Egyptian Mining Resources Authority (EMRA). Charcoal samples were sent to Laboratoire de Datation par le Radiocarbon at the Institut Français d'Archéologie Orientale and the Keck Carbon Cycle AMS facility, University of California-Irvine, for dating. The rationale for collecting and submitting radiocarbon samples from particular features is discussed in the chapters that follow. Samples were selected from buried hearth features (including near-surface hearth features) where the anthropogenic association was felt to be certain. We do not consider dates from charcoal found in stratified deposits outside of these features since the origin of this charcoal may be uncertain.

The radiocarbon samples submitted for dating were subjected to an acid-base-acid (ABA) chemical cleaning to remove humic and fulvic acids (Olsson 1986). The procedure involved application of approximately $6 \mathrm{ml}$ of $1 \mathrm{~N}$ (normal) $\mathrm{HCl}$ for 30 minutes, followed by $1 \mathrm{~N} \mathrm{NaOH}$ for one hour and then $1 \mathrm{~N} \mathrm{HCl}$ for another 30 minutes, all at temperatures between 70 and $90^{\circ} \mathrm{C}$. The samples were then neutralized with deionized water and dried in a vacuum oven. Typically, several rinses were required to produce relatively clean samples. Approximately $2 \mathrm{mg}$ of dried sample were then placed in a quartz tube, along with cupric oxide to provide an oxygen source, plus silver wire to "getter" any impurities that might adversely impact the graphitization process. The tubes were sealed under vacuum using a gas torch and then combusted at $900^{\circ} \mathrm{C}$ for three hours to generate $\mathrm{CO}_{2}$ gas. The tubes were then placed on a vacuum line and the gaseous samples were cryogenically moved to vials containing an iron powder catalyst. The gaseous samples were converted into graphite via the hydrogen reduction method by heating to $550^{\circ} \mathrm{C}$ for three hours. The graphite was then packed into aluminum sample pellets and analyzed by the AMS spectrometer. Aliquots of the gaseous samples were collected from the vacuum line and analyzed separately for carbon-stable isotopes using a Fisons NA-1500NC elemental analyzer equipped with a Delta-Plus CFIRMS mass spectrometer. It was decided that gas bench extractions would be performed on relatively large samples only (that is, more than $0.7 \mathrm{mg} \mathrm{C}$ ). These ages were converted into calibrated (calendar) dates using OxCal 4.1.7 (Bronk Ramsey 2001, 2009), which incorporates the IntCal13 atmospheric curve (Reimer et al. 2013). Radiocarbon results are reported with the margin of error representing 68.2 percent. Individual calibrated ages for each sample, together with the details of the sample context, are discussed in chapters 4,5 , and 6 .

\section{Excavation Procedures}

Excavations took place where we encountered stratified deposits: the Upper K Pits area, Kom K, and stratified hearths in $\mathrm{L}$ Basin and $\mathrm{K}$ Basin. The excavation method was based on the Museum of London Archaeological Service (MOLAS) manual (1994), adapted for arid contexts. Excavation areas were defined as trenches-eight in Kom K and 41 in the Upper K Pits area-or as individual hearths in the otherwise deflated landscape (L Basin and K Basin). Stratigraphic units were identified, based on soil composition and color, starting with 0001 in each trench. In this publication, units are indicated in square brackets, preceded by the trench number and the abbreviated excavation area-for example, UKP [04.0003] for Unit 0003 in Trench 4 in the Upper K Pits area and KK [02.0032] for Unit 0032 in Trench 2 in Kom K. All units were described in detail in the excavation database and indicated on plans (scale 1:20), baulk drawings (scale 1:10), and annotated photographs. The relationships between units were defined in the database as formal relations (for example, "is directly over," "is directly under," "cuts," "is cut by," "fills," "is filled by," an so on). The relationships were also represented as a stratigraphic matrix, commonly known as a Harris matrix. 
Units were grouped in features with their own numbering sequences, such as the individual pits in the Upper K Pits area (Pits 1 to 82) and hearths in Kom K (Hearths 101 to 151 ).

Excavation was done mostly with trowels and brushes, but hard layers of evaporates (salts and gypsum) required the use of small hand picks and in some cases pickaxes. These rock-hard layers, 0.10 to $0.50 \mathrm{~m}$ thick, comprised multiple stratigraphic units and made it difficult to discern the unit boundaries in those areas. The soft sandy and ashy layers in Kom K provided another difficulty in discerning stratigraphic units because the boundaries were diffuse. Often the many-layered hearths in Kom K could be defined only in cross-section. For each unit we gave a description, measured the size and elevation, and noted whether there was a danger of contamination.

All excavated soil was sieved on 2-mm screens. The hearth and pit contents were collected in bulk and sieved back at the field lab in multilayered botanical sieves. The use of flotation was not necessary, but some of the compact layers, such as pit lids and layers compacted with evaporates, were dissolved in water to retrieve botanical and bone samples.

\section{Summary}

The most prominent landscape feature in the Fayum Basin is Lake Qarun, which has featured in a number of paleoenvironmental reconstructions of Early to Middle Holocene occupations over the years. There is a long history of studies aimed at correlating archaeological deposits with lake advances and retreats. However, these have assumed that human occupation was close to the lake and that surface hearths can therefore be used to date the age of the sediments on which the hearths rest. But without direct dating of sediments, chronostratigraphic correlations remain problematic. Added to this is the difficulty of conducting topographic survey when places of known location, particularly known height above sea level, are rare.

As we detail in the following chapters, our more extensive surveys benefitting from technologies like GPS cast doubt on some of the lake advances and retreats discussed in earlier studies. Lake edge sediments onto which flaked stone artifacts, hearths, grinding stones, pottery, personal ornaments, and floral and faunal remains were deposited certainly exist; however, the chronological relationship between these deposits is far from clear. As well, the nature of preservation of both portable material culture and hearths makes it unlikely that these deposits were repeatedly covered and exposed by lake waters.

Satellite-derived topographic data indicate that the six basins that Caton-Thompson and Gardner identified differ in both their extent and the steepness of the basin edge slopes. It seems likely that the hydrology of the basins differed, with those to the east being shallow, with little relief, and those to the west being deeper and considerably steeper. While we cannot be certain of lake level changes associated with the Nile inundation, the topography of the basins suggests that at least the eastern basins were filled from rain-fed wadis during times of low lake levels. This suggests that the eastern and western basins would have had a significantly different ecology and therefore different plant and animal life associations. Some indications of past vegetation can be gained by comparing archaeological charcoal assemblages with likely past vegetation communities.

One of the difficulties that previous researchers faced was the sheer volume of material deposited across the Fayum north shore. Very large numbers of flaked stone artifacts, grinding stones, hearths, ornaments, and pottery, together with large numbers of animal bones, are distributed across kilometers of what today is a largely undifferentiated landscape. In the past, the scale of these deposits meant that only limited observations could be made, meaning in turn that the true extent of the deposits was often underestimated. As described above, we developed new methods to deal with such extensive artifact scatters distributed in a way that defies site boundary attribution.

Technology also greatly aided our ability to deal with large numbers of portable artifacts. The typologies that dominated earlier studies used only a small portion of the available artifacts to trace relationships between assemblages based on manufacturing style. Assumptions about relationships between the ethnic groups assumed to have made these artifacts remain largely untested. Here we have outlined alternative methods based on a different set of observations related to how artifacts were moved. We are particularly interested in issues of sedentism and mobility because both relate to landscape use.

In the following chapters we report on work carried out in two of the basins originally identified by CatonThompson and Gardner- $\mathrm{L}$ and $\mathrm{K}$ - assessing the age and distribution as well as composition of the portable artifacts and features. 


\section{4 \\ The L Basin Archaeological Record}

\section{Simon J. Holdaway, Rebecca Phillipps, Annelies Koopman, Veerle Linseele, and Willeke Wendrich}

The sheltered northern shores of the L Basin, judging by the tools and sherds, had been attractive to man from palaeolithic to Roman times [Caton-Thompson and Gardner 1934:97].

n November 2008 we visited the site of E29H1 (Figure 1.1), originally described by Caton-Thompson and Gardner, who referred to it as unnamed Fayum B site on the edge of X Basin. Wendorf and Schild (1976:182) gave the site its current designation in the 1960s and described it as a spatially extensive distribution of flaked stone artifacts, faunal material, and hearths. What we discovered were irrigation canals and PVC tubing, as the site was being prepared for cultivation. Over the next five weeks, we attempted to obtain what information we could in what became an archaeological salvage project. In hindsight, we would of course have done things differently. In 2008 much of the methodology described in chapter 3 was yet to be designed. We had intended the 2008 season to be exploratory, determining how we might approach the landscape archaeology of the Fayum north shore. However, we were forced to abandon such plans and develop a salvage strategy on the fly.

Here we report the results of the studies made during the five-week period. We returned to the area in 2012 to find the cultivations abandoned but not before
E29H1 had been effectively destroyed. We were, however, able to undertake additional studies in the regions surrounding E29H1 and thereby provide some important contextual information on the material we analyzed in 2008. We report on these more recent studies here as well. In the years between 2005 and 2012 our ideas developed, particularly in the form of a growing skepticism concerning the relationship between archaeological deposits and paleo-lakeshores. Our observations concerning the state of preservation of both the artifact and hearth records at E29H1 were particularly influential. As described below, hearths at E29H1 date back to more than $9000 \mathrm{cal} \mathrm{BP}$ yet are preserved just below a thin surface layer of aeolian sand. Considering large numbers of relatively small flaked stone artifacts, which show no evidence of sorting due to water action, it seems difficult to believe that they were submerged beneath the series of paleo-lake advances and retreats proposed by the authors and discussed in chapter 3 .

We begin by considering the geomorphological setting of the deposits at E29H1, paying particular attention to the types of sediments on which we find the 
archaeological materials deposited. As discussed in chapter 3, previous analyses have sought to use the archaeological deposits as a means to construct chronostratigraphies for lake advances and retreats. Here we adopt a different approach, studying the geomorphic contexts in which we find the archaeological deposits. The sediments reflect a lacustrine history, but we do not begin by assuming that the archaeological materials that have accumulated on these sediments represent lake edge occupations, keeping an open mind on questions of landscape reconstruction and land use.

Flaked stone artifacts were once abundant at E29H1, and along with animal bones, these first drew the attention of archaeologists (Wendorf and Schild 1976:182). We were able to record the location of a very large number of these objects in 2008 but had the opportunity to analyze only a much smaller sample from a number of different parts of the location. We compare the results of the density calculations at E29H1 with those obtained from transects we surveyed in the immediate area in 2012 to understand how and where archaeological materials are preserved.

A number of hearths were excavated as part of the rescue archaeology at E29H1, and these are described, as are the radiocarbon determinations we obtained from charcoal in these hearths. We compare these results with a similar set obtained from hearths excavated in L Basin. Both the age and location of the hearths allow us to develop an impression of the chronology of landscape use.

Finally, we compare the results of a technological analysis of flaked stone artifacts that we recorded in more detail from different areas of E29H1 with similar technological analyses performed on a sample of artifacts recorded in 2012.

\section{Boundaries and Artifact Density}

As discussed in chapter 3, Epipaleolithic and Neolithic sites in the Fayum are most often identified as surface artifact scatters with a limited number and range of features. This is true for E29H1, where it is the distribution of flaked stone artifacts and animal bones that marks the extent of the site, with the only features being isolated heat retainer hearths. Defining site boundaries is therefore difficult and previous attempts have largely relied on impressions rather than actual measures of relative artifact density. Wendorf and Schild (1976:182), for instance, describe artifacts at E29H1 as occurring "Most commonly in an oval area
$300 \mathrm{~m}$ by $540 \mathrm{~m}$; however careful surface examination disclosed that the artifact-littered area consisted in reality of two groups, one inside the other."

In the associated figure, Wendorf and Schild illustrate an oval area $300 \mathrm{~m}$ long by $100 \mathrm{~m}$ wide, in which they describe several artifact concentrations with an additional scatter of "Terminal Paleolithic" (Epipaleolithic) artifacts together with "later artifacts." The Epipaleolithic artifacts had less weathering than the later artifacts, which are described as "heavily eolized" (1976:184) (that is, heavily abraded artifacts worn smooth via windblown sand). Our observations suggest that Wendorf and Schild may have meant to indicate that it was the rarely found Paleolithic artifacts that were heavily eolized rather than the later artifacts. Beyond the inner oval was an area of thinly scattered artifacts described as "composed entirely of later tools, grinding stones, and very few potsherds, all heavily wind polished." The second larger oval is illustrated in Wendorf and Schild (1976:Figure 121).

As noted in chapter 3, there are issues with mapping in places like the Fayum, particularly the problem of establishing real-world locations before the advent of GPS technology. The maps Wendorf and Schild (1976) published have a scale and a north arrow as well as contours but cannot otherwise be located in a coordinate system. Thus they effectively float in horizontal space, and while we have a general idea of the area Wendorf and Schild studied, we cannot determine the exact location. Wendorf and Schild were also limited in the time they had available to study in the Fayum, much like us in 2008, and therefore considered a relatively small area. Our additional work in 2012 permitted artifacts over a much larger area to be recorded, enabling us to get a better understanding of the distribution of artifacts in the area of the Fayum North shore likely to contain the material Wendorf and Schild identified as E29H1. In addition to estimating the distribution of artifacts, Wendorf and Schild collected artifacts from three areas (A to C in Wendorf and Schild 1976:Figure 121). We have not attempted to locate and reanalyze these collections.

\section{Object Distribution}

Figure 4.1 illustrates part of the Fayum north shore, indicating areas that we have intensively surveyed. As discussed above, we were able to work on the area under threat of farming in 2008 but were able to survey only across three fields. These fields were crossed by 
PVC irrigation pipelines, and each field was separated by an excavated channel that carried an underground water supply pipe. Areas around these three fields were already being cultivated, and we did not survey these areas, although we were able to take sediment samples from cores as well as make use of sections cut by the irrigation channels for geomorphological description. Some areas within the boundaries of the three fields we did survey were badly damaged before our arrival and so were excluded from consideration in 2008.

We were unable to return to the area in 2009 or 2010, and the political situation in 2011 prevented our work in Egypt. However, we did undertake additional fieldwork in 2012. By this time the farming activity had ceased and all farming infrastructure was abandoned. As noted above, cultivation had destroyed the archaeological record across a substantial area, more than 56 ha, indicated on Figure 4.1. Other areas surrounding the farm were also damaged by previous irrigation works, with substantial irrigation channels dug between fields. However, despite the destruction, including the ever-present tire tracks from vehicles, substantial areas of intact surface remained, and we surveyed these intensively.

Figure 4.1 shows the areas surveyed as well as the area destroyed by cultivation. To the east we were prevented from surveying further by the presence of disused military earthworks. To the northeast and to the south, the land surfaces were under cultivation and therefore were no longer accessible. The survey results stop in the northwest at the edge of the area we completed in 2012, and we plan to extend this area in future fieldwork seasons. To the southeast the area is both cultivated, although now abandoned, and covered with a diatomite surface. As discussed below, artifact densities markedly decline as this surface is reached.

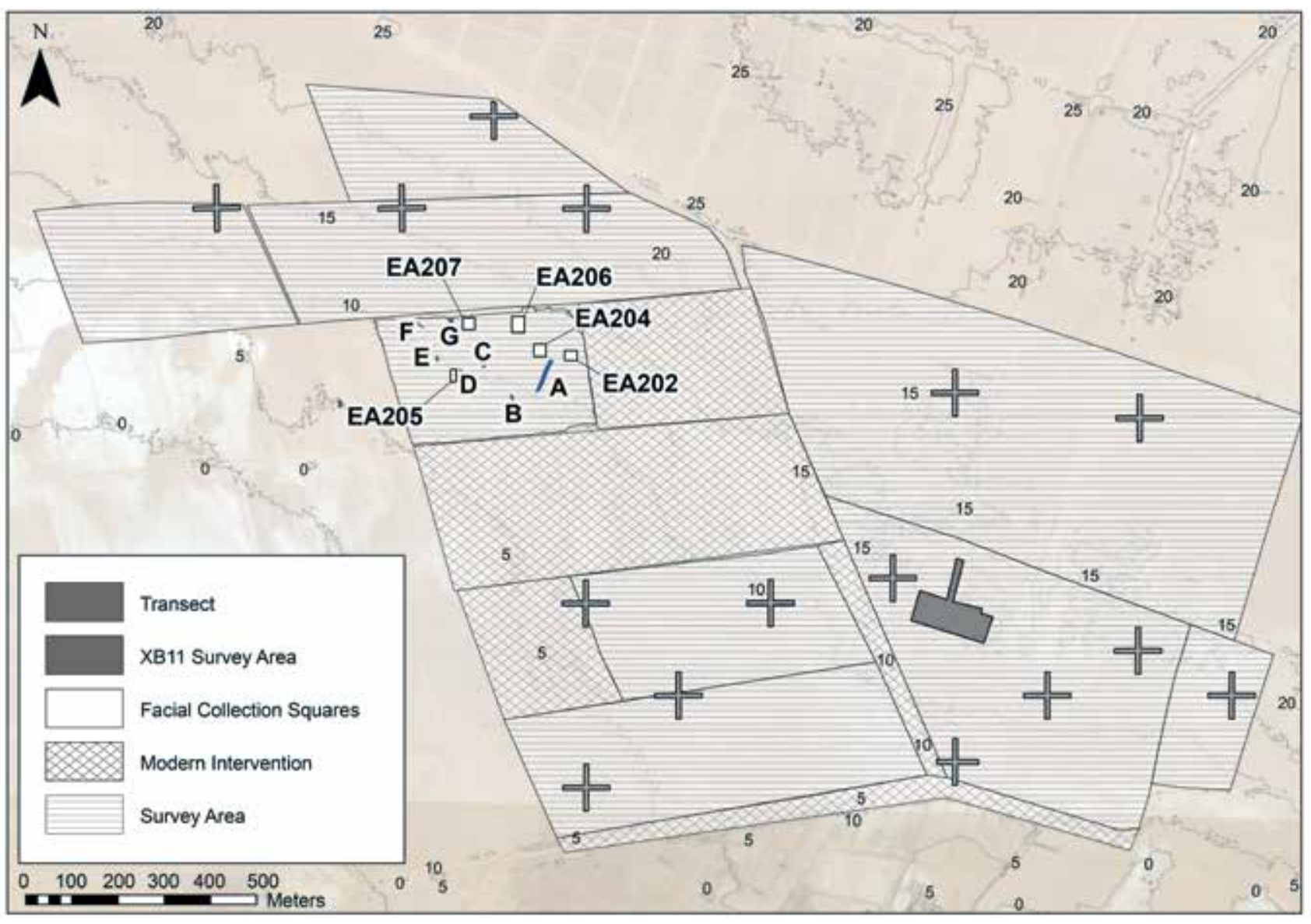

Figure 4.1. L1 Basin showing the location of E29H1 together with the transects surveyed, faunal collection squares (labeled EA202 and EA204 to EA207), areas sampled for artifact analysis (A to G), and topography (5-m contours). 
Our survey strategy followed that discussed in chapter 3. However, rather than corridors we surveyed blocks in areas where we could identify substantial intact surfaces. These are marked in Figure 4.1. Across these areas we walked in lines $10 \mathrm{~m}$ apart (as we did the length of the corridors), flagging likely hearths and grinding stones. A recording team followed the line of surveyors and assessed each hearth or grinding stone identification.

Figure 4.1 shows the areas surveyed. We identified 221 grinding stones in 2012, adding to the 67 identified in 2008. These grinding stones were distributed across an area of 156 ha surveyed in 2012 combined with the 10 ha surveyed in 2008, giving an overall density of 1.73 grinding stones per hectare. Hearths are less common than grinding stones and are more concentrated in parts of the survey area and absent from others. The 2012 survey identified 28 hearths while 38 were identified in 2008 , the majority of these located in the western field.

Figure 4.1 also shows the outline of transects we placed around the E29H1 sites for artifact survey. As discussed in chapter 3, these were approximately 2,000 $\mathrm{m}^{2}$, varying in area depending on the edges that were mapped with a total station. The densities of portable artifacts within these transects vary considerably (see below).

In 2008, in the face of the threat from agricultural development, we used a different strategy for recording portable artifacts. We had not adopted the cross-shaped transects that we used the next year for the corridor surveys but were aware of the difficulty of defining sites when faced with very large numbers of artifacts from our earlier work in Australia (e.g., Holdaway and Fanning 2008) and on the basis of a study of the site of XB11 (Phillipps 2006). We therefore sought to measure the location of individual artifacts to quantify artifact density. The PVC irrigation pipes strung across the three fields provided a convenient means of dividing each field into small, manageable units.

Using this approach, we located and logged more than 94,000 objects (Table 4.1). By far the most abundant materials were flaked stone artifacts (just under 80,000 ), followed by bone fragments (just under $14,000)$. Locating each item individually gave us the ability to quantify the density of materials. As well, 6,292 flaked stone artifacts from a variety of locations across E29H1 were analyzed using a suite of technological variables, also discussed below.
Table 4.1. Frequency of Materials Identified in the 2008 Survey at E29H1.

\begin{tabular}{|l|l|}
\hline Form & $\mathbf{n}$ \\
\hline Bone & 15,138 \\
\hline Button & 1 \\
\hline Islamic coin & 3 \\
\hline Lithic & 84,239 \\
\hline Ostrich eggshell & 3 \\
\hline Ceramic & 3 \\
\hline Shell & 953 \\
\hline Worked bone & 7 \\
\hline
\end{tabular}

Bones were logged individually to provide a measure of density, but we also excavated in selected areas to provide samples for identification (described below).

\section{Topography}

Figure 4.2 includes the three sediment types identified as part of a geomorphological study discussed below and in Koopman et al. $(2010,2016)$. The surfaces immediately to the south of the E29H1 irrigation fields represent damage from contemporary agriculture (Figure 4.1). Figure 4.3 illustrates the slope of the area that includes E29H1, showing the eastern edge of the basin that Caton-Thompson and Gardner identified as X Basin. Farther to the east, Caton-Thompson and Gardner identified another concentration of artifacts that they named Kom L. We discuss the nature of these deposits in the following chapter.

\section{Geomorphology}

Work conducted at E29H1 included an intensive study of the sediments both underlying the archaeological deposits and in the immediate vicinity (Koopman et al. 2010, 2016), which we summarize here. Results indicate that the sediments on which archaeological materials in this area rest derive from four types of sedimentary facies: fluvial $(2 \mathrm{a}-\mathrm{b})$, lacustrine $(3 \mathrm{a}-\mathrm{h})$, lacustrine reworked $(4 \mathrm{a}-\mathrm{c})$, and aeolian $(5 \mathrm{a}-\mathrm{b})$ deposits. In addition, there are bedrock outcrops to the northeast and southwest of E29H1. Similar sediments are found to the east of E29H1, although their description and analysis was not complete at the time of writing this volume.

Figure 4.3 shows that bedrock units $(1 \mathrm{a}-\mathrm{d})$ are made up of silty clay (T1c1d) or predominantly clayey silt (T1a, T1b1a-c), with gravel, pebbles, and sand 


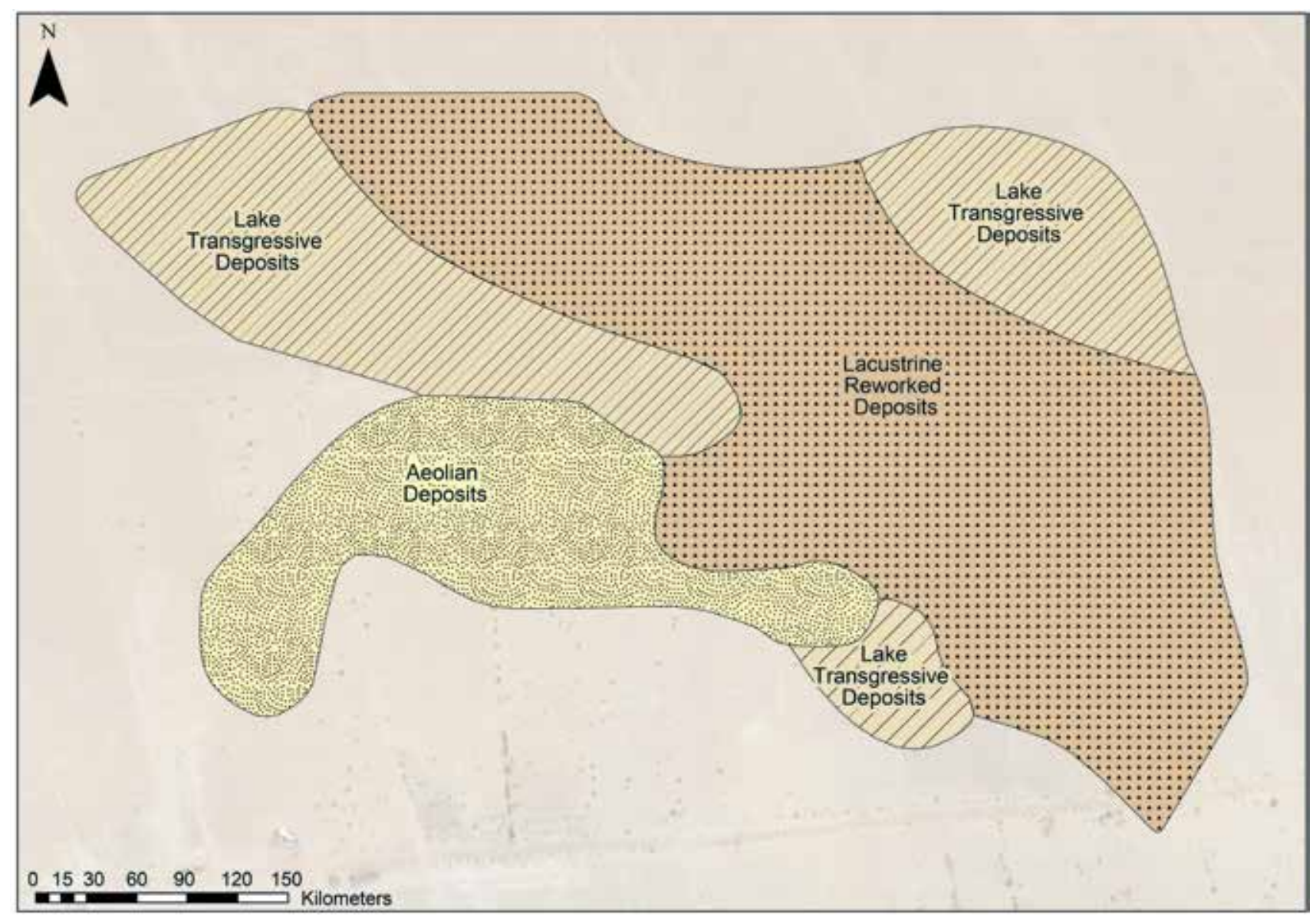

Figure 4.2. Sediment types identified at E29H1.

intercalations (T1b1b), and have a yellowish-brown hue. The units are extremely compacted, are predominantly calcareous, and often contain abundant gypsum crystals that are intertwined and vertically oriented (T1c1d). In the southwest, the bedrock units $1 \mathrm{a}-\mathrm{b}$ are exposed at the present-day surface as dome-shaped higher locations. The bedrock outcrops have a distinct surface cover, made of abundant fossilized marine oysters and marine corals, worked by wind erosion.

Units of moderately to poorly sorted, poorly silty sand, with predominantly orange hues and medium grains, are interpreted as sediments deposited in and along river courses $(2 \mathrm{a}-\mathrm{b})$. The sand is occasionally very calcareous and slightly organic. The deposits are low angular bedded, cross- to horizontally laminated, and occasionally indurated. The sediments contain occasional salt crusts (2a), abundant gypseous concretions, and crystals (2a), as well as traces of fossilized shells (2b). The fluvial deposits are unconformably stratified above the peneplain bedrock units $(1 \mathrm{a}-\mathrm{d})$ in both the lower southwest and higher northeast parts of the study area.

Units made of clayey silt to silty clay (3a), or predominantly moderately sorted, poorly silty sands ( $3 \mathrm{~b}-$ h), with light gray to pale yellow hues, are interpreted as formed inside a lacustrine depositional environment $(3 a-h)$. The sand deposits $(3 b-h)$ are predominantly fine $(3 b, 3 c, 3 g)$ to medium grained ( $3 d-f, 3 h)$, not organic, and relatively calcareous, but extremely calcareous in the lower southwest of the study area (3a). The compaction of the lacustrine deposits ranges from extremely indurated in the southwest (3a) to variably indurated in the higher northeast $(3 \mathrm{~b}-\mathrm{h})$. The finegrained lake deposits in the lower southwest of the study area (3a) are interpreted as sediment deposited in the deeper parts of the lake, similar to the unlaminated freshwater diatomite described elsewhere (Aleem 1958; Flower et al. 2012; Wendorf and Schild 1976). 


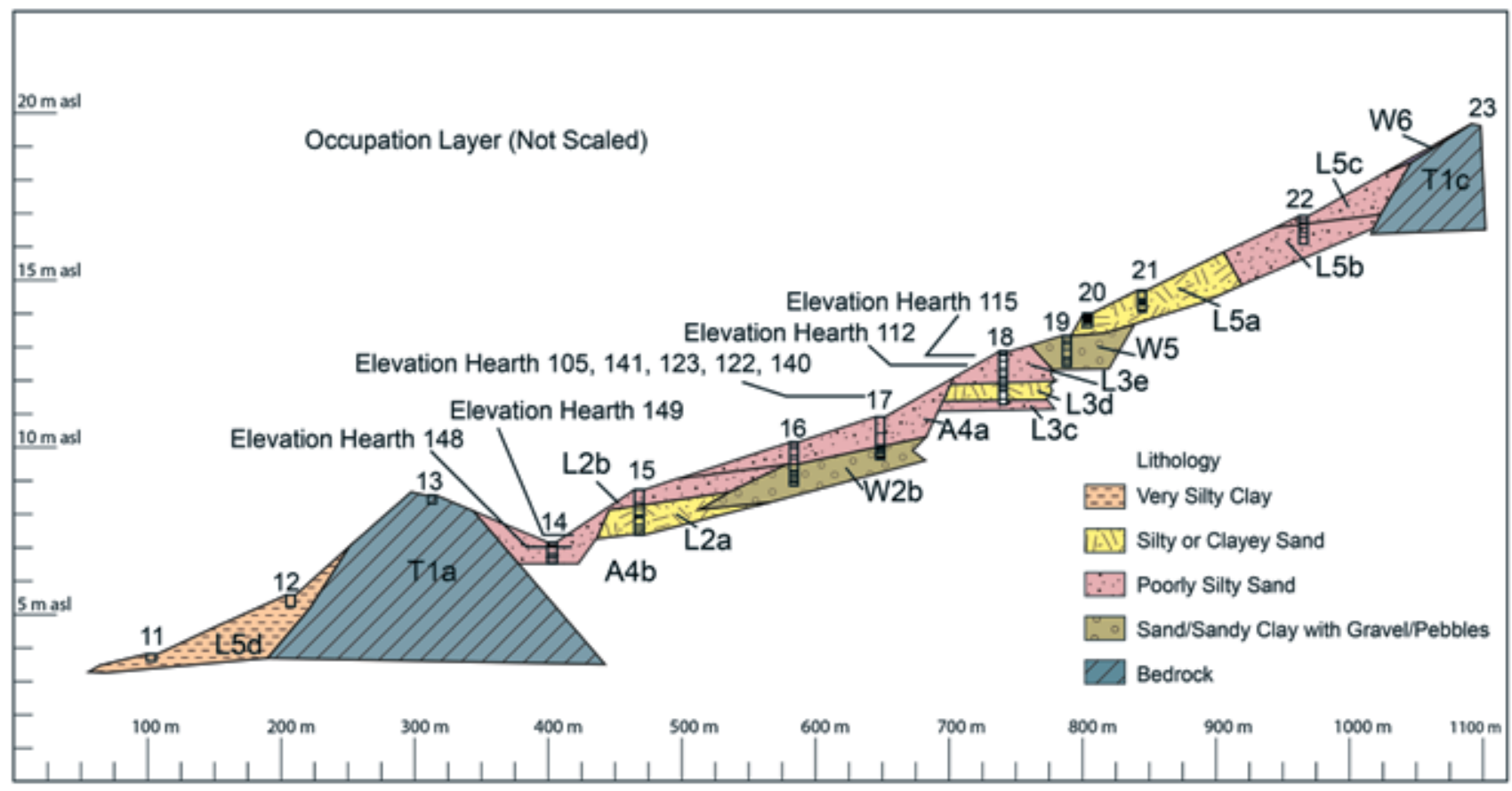

Figure 4.3. Stratigraphic profile west to east across E29H1.

The polygonal patterns of cracks where Unit $3 \mathrm{a}$ is currently exposed indicate subaerial conditions and prolonged desiccation after lacustrine wet deposition (Nichols 2006). The lacustrine units observed at higher elevations toward the northeast $(3 \mathrm{~b}-\mathrm{h})$ were deposited in the shallow parts of a water body, with occasional evidence for slight reworking of lake sediments (3e). Layers of abundant intact mollusks (3b, 3d) indicate littoral accumulations that may form because of clastic sediment starvation or by winnowing of finer sediment, perhaps due to variations in water levels (Talbot and Allen 1996:94). Pronounced yellow oxidation stains in irregular to vertical orientations, horizontal to wavy laminae (mm-scale) (3c, 3e), and a calcium carbonate crust $(3 \mathrm{~g})$ point to switches between wet and dry conditions during the deposition of Units 3c, $3 \mathrm{e}$, and $3 \mathrm{~g}$. Pronounced horizontal to wavy laminations on an mm-scale (3f, $3 \mathrm{~h}$ ) suggest wave-dominated shore activity due to wind action. The presence of root casts in exhumed hills provides evidence for vegetation along the shore during deposition of Units $3 \mathrm{f}$ and $3 \mathrm{~h}$, as root encrustations form by secondary precipitation of calcium carbonate and/or calcium sulfate around plant roots (Glennie 2005:179-181; Hugget 2007:15; Nichols 2006:126). The deepwater (3a) and shallow-water deposits $(3 \mathrm{~b}-\mathrm{h})$ bury the bedrock units $(1 \mathrm{a}-\mathrm{d})$ and the fluvial deposits $(2 \mathrm{a}-\mathrm{b})$ in the southwest.
Units made of very poorly sorted and heterogeneous mixtures of silt, clay, and sand, with gravel and/or pebbles, are interpreted as lacustrine reworked deposits $(4 a-c)$, with evidence for the action of ephemeral desert streams. The lithology of the deposits changes frequently along relatively short intervals, both vertically $(<15 \mathrm{~cm})$ and laterally $(<1 \mathrm{~m})$. The reworked deposits have variable hues and the sand component of the deposits is mostly very fine (4a) to fine grained ( $4 b$, $4 c)$. The deposits have predominantly high carbonate content, are slightly organic, are variably indurated, and contain occasional horizontally to wavy laminae (mm-scale). The abundance of white concretions, most probably gypsum or calcium, with small gypsum crystals and much oxidation staining, occurs predominantly in the east (4b) and suggests changes between wet and dry conditions. Patches of black silty deposits at the present-day surface and in the shallow subsurface (4b), with an abundance of lacustrine biota (fish bones and small mollusks) and charred plant material, indicate subaqueous swampy conditions and burning of vegetation in the east of the study area (4b). Abundant vertically oriented oxidized stripes (about $1 \mathrm{~cm} \times 1-2$ $\mathrm{mm})$, occasionally surrounded by gypsum crystals (4b), indicate oxidation of former plant roots. The lacustrine reworked deposits overlie and/or cut the earlier deposited lacustrine deposits $(3 \mathrm{a}-\mathrm{h})$ at several locations. 
Units of moderately sorted, poorly silty sands, with a predominantly yellow hue, are interpreted as windblown deposits $(5 \mathrm{a}-\mathrm{b})$. The sand deposits are predominantly fine $(5 \mathrm{~b})$ to medium $(5 \mathrm{a})$ grained, not calcareous, and variably indurated, with some evidence for salt accumulations. The aeolian deposits were observed in the center and southwest of the study area and cover parts of the lacustrine reworked deposits in the southwest $(4 a-b)$.

Figure 4.3 plots the stratigraphic profile constructed from a series of sections in a west-to-east direction roughly in line with the surface contours. Bedrock units are exposed at both ends of the section while the bulk of the flaked stone artifacts and hearths are concentrated on aeolian sediments, the northern part of aeolian sand Unit $5 \mathrm{a}$ and adjacent lacustrine sediments, the southeastern part of lake Unit $3 \mathrm{f}$, and the most southern edge of the reworked lake Unit 4a. Higher concentrations of flaked stone artifacts are found on aeolian deposits (Figure 4.4), and some higher concentrations of bone are founds on lacustrine reworked deposits (Figure 4.5). Hearths are most concentrated in the aeolian and adjacent lacustrine deposits and when excavated preserve sufficient charcoal from which to obtain a radiocarbon determination (Figure 4.6).

\section{Discussion}

These results suggest a relationship between the nature of the sediments onto which archaeological material has lagged and the quantity of archaeological material preserved. Aeolian deposits are associated with large concentrations of flaked stone artifacts. This could reflect a preference by people in the past to occupy these surfaces, but it may well also reflect localities where aeolian deposits have accumulated and through this process acted to preserve the artifacts.

As noted above and discussed in more detail below, samples of flaked stone artifacts from different parts of E29H1 were analyzed in detail, including

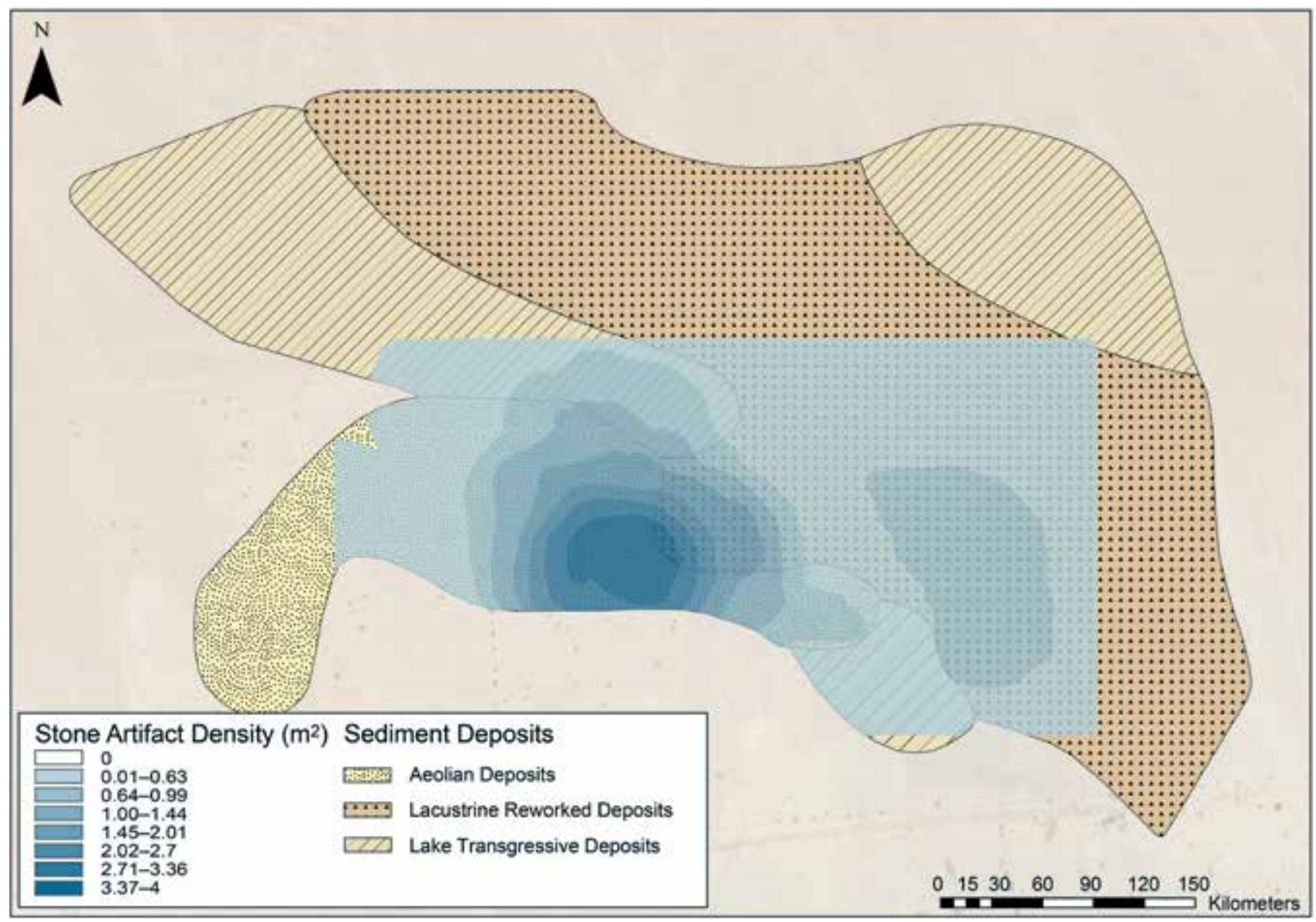

Figure 4.4. E29H1 stone artifact density $\left(\right.$ number $\left./ \mathrm{m}^{2}\right)$ overlaying the sediment types. 


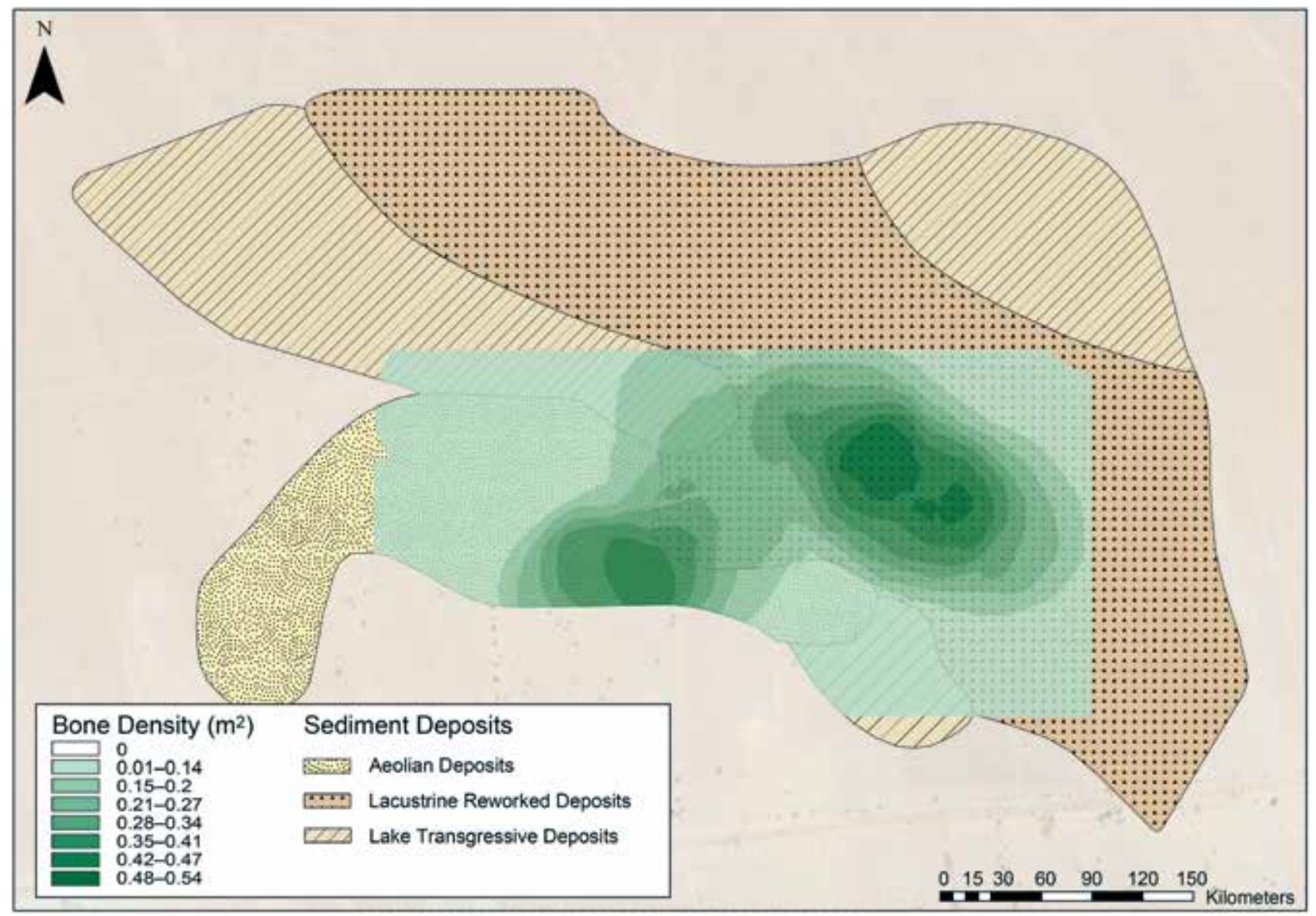

Figure 4.5. E29H1 bone density number/ $\left(\mathrm{m}^{2}\right)$ overlaying the sediment types.

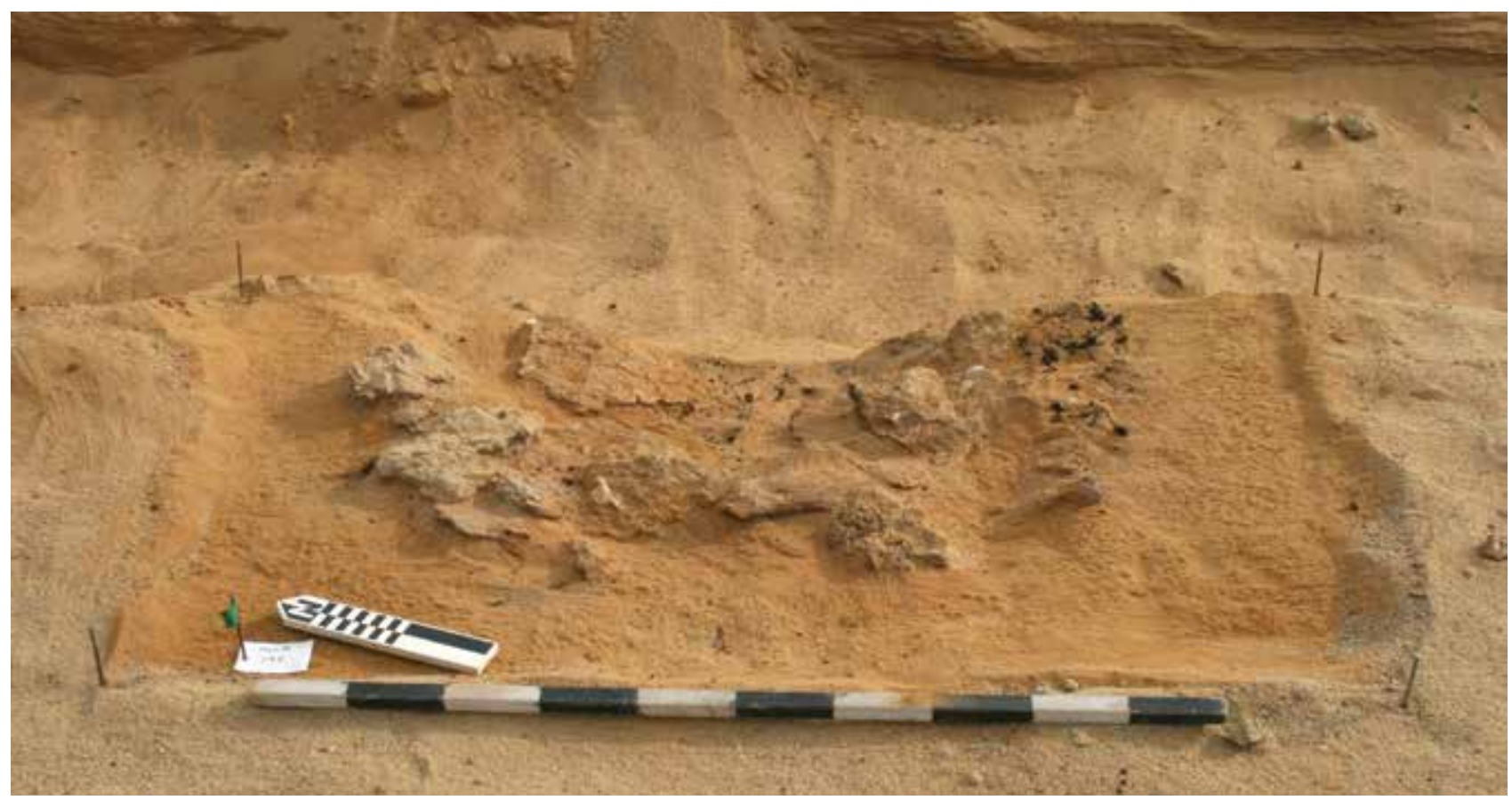

Figure 4.6. E29H1 hearth showing charcoal. 
Table 4.2. Mean Clast Size for Artifacts from Three Sedimentary Units at E29H1.

\begin{tabular}{|l|l|r|r|r|}
\hline \multicolumn{2}{|c|}{} & \multicolumn{1}{l|}{ N } & \multicolumn{1}{l|}{ Mean } & \multicolumn{1}{l|}{ SD } \\
\hline \multirow{4}{*}{ Length } & $5 \mathrm{a}$ & 2,966 & 31.03 & 7.83 \\
\cline { 2 - 6 } & $3 \mathrm{f}$ & 2,066 & 30.57 & 8.06 \\
\cline { 2 - 5 } & $4 \mathrm{a}$ & 825 & 28.29 & 5.91 \\
\hline \multirow{4}{*}{ Width } & $5 \mathrm{a}$ & 2,966 & 19.33 & 6.93 \\
\cline { 2 - 5 } & $3 \mathrm{f}$ & 2,066 & 19.46 & 8.70 \\
\cline { 2 - 5 } & $4 \mathrm{a}$ & 825 & 18.57 & 5.49 \\
\hline \multirow{4}{*}{ Thickness } & $5 \mathrm{a}$ & 2,966 & 9.53 & 5.57 \\
\cline { 2 - 5 } & $3 \mathrm{f}$ & 2,066 & 9.52 & 16.67 \\
\cline { 2 - 5 } & $4 \mathrm{a}$ & 825 & 7.48 & 4.45 \\
\hline
\end{tabular}

Note: a-axis length; b-axis width; c-axis thickness.

measurements of the $a, b$, and c clast axes (a-axis length, $b$-axis width, and c-axis thickness) (Table 4.2). Samples were obtained from artifacts that rest on the units described above. A comparison of the mean lengths of artifacts resting on these units indicates a significant difference between the size of flaked stone artifacts, here considered simply as clasts, among these units. ${ }^{1}$ Bonferroni post hoc comparisons between means for each case tested indicate that significance comes from the contrast with Unit $5 \mathrm{a}$, where clast size is significantly smaller compared to the other units. The post hoc comparisons are interesting because it is small clasts that are most susceptible to loss and thus are expected to be underrepresented on aeolian, compared to lacustrine, units. The direction of the significant difference confirms this expectation in that smaller artifacts represented by a lower clast mean are present on the lacustrine-derived units rather than on the aeolian unit. However, while it is expected that smaller artifacts might be obscured by the aeolian sediment, as shown in Figure 4.4, flaked stone artifact concentrations are in fact higher on this sediment type compared to those adjacent. This result therefore suggests that the aeolian unit and sediments immediately adjacent have preserved a more concentrated record of activities that left quantities of flaked stone artifacts despite visibility issues with smaller artifacts.

1 ANOVA for length test statistic $F=41.307$; degrees of freedom $(d f)=2,2,431.483$; probability $(p)<0.001$. Width $F=4.408$; $d f=2,243.685 ; p=0.012$. Thickness $F=12.673 ; d f=2$, $1,476.661 ; p<0.001$.

\section{Object Density}

Figure 4.1 shows the distribution of transects placed across the areas adjacent to E29H1. As discussed in chapter 3 , sediment types were mapped using a total station across each of these transects together with the location of artifacts, bones, and pieces of pottery. Using a GIS, it is possible to calculate the area of each sediment type and thereby the density of artifacts and faunal material on each of the different sediment types for each transect (Table 4.3). Densities vary due to object visibility and the differential distribution of objects both within and between transects.

Table 4.3 shows considerable variation between the density values for each object type by sediment type. For example, the mean density of complete flakes on desert pavement is 1.591 objects per square meter (excluding XB11 where complete flake was not recorded), but the associated standard deviation (not shown) is 1.414. The mean density for complete flakes on windblown sand is lower ( 0.515 objects per square meter) but equally variable (standard deviation 0.604). Comparing complete flake density across five of the transects that have common sediment types (L1T7, L1T8, L1T24, L1T25, and L1T40 complete flake density on desert pavement, modern intervention, and windblown sand) shows no systematic difference using Friedman's nonparametric equivalent to the two-way analysis of variance by ranks. ${ }^{2}$ However, within individual transects there are some systematic differences among the different object types when densities are compared among different sediment types. ${ }^{3}$ In transect L1T7, densities for desert pavement, sand ripples, and windblown sand have higher mean ranks than those for modern intervention, root clast, and silt. In general, where there are differences in artifact densities between the sediment types found within a transect, desert pavement and windblown sand have higher densities than other sediment types (Table 4.4).

It is likely that these differences reflect the differential visibility of objects. When there are sufficient numbers, objects are more visible on surfaces such as desert pavement and those surfaces with only limited amounts of sand cover (that is, windblown sand). Moreover, these results are consistent with the analysis of artifact densities in different sediment types from E29H1 discussed above. Like the results from transects, objects are more common on aeolian deposits compared to other sediment types at E29H1.

\footnotetext{
2 Test statistic $=2.8 ; d f 2 ; p=0.247$.

3 For example, L1T7. Friedman's two-way analysis of variance by ranks $=22.7 ; d f=5 ; p<0.001 ;$ Table 4.4 .
} 
Table 4.3. Density $\left(\right.$ per $\mathrm{m}^{2}$ ) of Artifacts and Fauna over $20 \mathrm{~mm}$ in Maximum Length in the Area Surrounding E29H1.

\begin{tabular}{|c|c|c|c|c|c|c|}
\hline L1T5 & Desert Pavement & Windblown Sand & & & & \\
\hline Complete flake & 7.327 & 0 & & & & \\
\hline Broken flake & 1.385 & 0 & & & & \\
\hline Core & 0.668 & 0 & & & & \\
\hline Tool & 0.216 & 0 & & & & \\
\hline Ostrich eggshell & 0.074 & 0 & & & & \\
\hline Bone & 1.292 & 0 & & & & \\
\hline Ceramic & 1.008 & 0.006 & & & & \\
\hline L1T7 & Desert Pavement & $\begin{array}{l}\text { Modern } \\
\text { Intervention }\end{array}$ & $\begin{array}{l}\text { Windblown } \\
\text { Sand }\end{array}$ & Sand Ripples & Silt Substrate & $\begin{array}{l}\text { Rhizolith } \\
\text { Mound }\end{array}$ \\
\hline Complete flake & 1.676 & 0.199 & 0.636 & 4.370 & 0.429 & 0.040 \\
\hline Broken flake & 0.465 & 0.022 & 0.156 & 0.942 & 0.086 & 0 \\
\hline Core & 0.345 & 0.027 & 0.143 & 0.721 & 0.043 & 0.040 \\
\hline Tool & 0.032 & 0.011 & 0.036 & 0.070 & 0 & 0 \\
\hline Ostrich eggshell & 0 & 0 & 0.001 & 0 & 0 & 0 \\
\hline Bone & 0.024 & 0 & 0.009 & 0.011 & 0 & 0 \\
\hline Ceramic & 0 & 0 & 0.001 & 0 & 0 & 0 \\
\hline L1T8 & Desert Pavement & $\begin{array}{l}\text { Modern } \\
\text { Intervention }\end{array}$ & $\begin{array}{l}\text { Windblown } \\
\text { Sand }\end{array}$ & & & \\
\hline Complete flake & 1.015 & 0.396 & 0.639 & & & \\
\hline Broken flake & 0.244 & 0.096 & 0.181 & & & \\
\hline Core & 0.133 & 0.051 & 0.126 & & & \\
\hline Tool & 0.015 & 0.013 & 0.016 & & & \\
\hline Ostrich eggshell & 0 & 0 & 0.001 & & & \\
\hline Bone & 0.005 & 0.016 & 0.041 & & & \\
\hline Ceramic & 0.002 & 0 & 0 & & & \\
\hline L1T11 & Desert Pavement & $\begin{array}{l}\text { Modern } \\
\text { Intervention }\end{array}$ & & & & \\
\hline Complete flake & 3.902 & 1.278 & & & & \\
\hline Broken flake & 0.448 & 0.111 & & & & \\
\hline Core & 0.366 & 0.080 & & & & \\
\hline Tool & 0.078 & 0.024 & & & & \\
\hline Ostrich eggshell & 0 & 0 & & & & \\
\hline Bone & 0.005 & 0.003 & & & & \\
\hline Ceramic & 0 & 0 & & & & \\
\hline L1T12 & Gypsum Sand & $\begin{array}{l}\text { Modern } \\
\text { Intervention }\end{array}$ & $\begin{array}{l}\text { Windblown } \\
\text { Sand }\end{array}$ & & & \\
\hline Complete flake & 0.010 & 0.005 & 0 & & & \\
\hline Broken flake & 0.003 & 0 & 0 & & & \\
\hline Core & 0.005 & 0 & 0 & & & \\
\hline Tool & 0.001 & 0 & 0 & & & \\
\hline Ostrich eggshell & 0 & 0 & 0 & & & \\
\hline Bone & 0 & 0 & 0 & & & \\
\hline Ceramic & 0 & 0 & 0 & & & \\
\hline
\end{tabular}




\begin{tabular}{|c|c|c|c|c|c|c|}
\hline L1T23 & $\begin{array}{l}\text { Modern } \\
\text { Intervention }\end{array}$ & Windblown Sand & Rhizolith & $\begin{array}{l}\text { Trampled } \\
\text { Soft Sand } \\
\end{array}$ & & \\
\hline Complete flake & 0.116 & 0.126 & 0.134 & 0.053 & & \\
\hline Broken flake & 0.039 & 0.031 & 0.024 & 0.010 & & \\
\hline Core & 0.004 & 0.024 & 0.024 & 0.005 & & \\
\hline Tool & 0.008 & 0.009 & 0 & 0.004 & & \\
\hline Ostrich eggshell & 0 & 0 & 0 & 0 & & \\
\hline Bone & 0.031 & 0.064 & 0.159 & 0.055 & & \\
\hline Ceramic & 0.019 & 0.040 & 0.041 & 0.014 & & \\
\hline L1T24 & Desert Pavement & $\begin{array}{l}\text { Modern } \\
\text { Intervention }\end{array}$ & $\begin{array}{l}\text { Windblown } \\
\text { Sand }\end{array}$ & & & \\
\hline Complete flake & 1.668 & 1.472 & 0.425 & & & \\
\hline Broken flake & 0.338 & 0.210 & 0.078 & & & \\
\hline Core & 0.234 & 0.315 & 0.069 & & & \\
\hline Tool & 0.094 & 0 & 0.039 & & & \\
\hline Ostrich eggshell & 0.010 & 0 & 0 & & & \\
\hline Bone & 1.905 & 3.049 & 0.616 & & & \\
\hline Ceramic & 0.003 & 0 & 0.003 & & & \\
\hline L1T25 & Desert Pavement & $\begin{array}{l}\text { Modern } \\
\text { Intervention }\end{array}$ & $\begin{array}{l}\text { Windblown } \\
\text { Sand }\end{array}$ & & & \\
\hline Complete flake & 0.164 & 0.143 & 0.165 & & & \\
\hline Broken flake & 0.027 & 0.024 & 0.034 & & & \\
\hline Core & 0.068 & 0.026 & 0.056 & & & \\
\hline Tool & 0.014 & 0.011 & 0.005 & & & \\
\hline Ostrich eggshell & 0 & 0 & 0 & & & \\
\hline Bone & 0.164 & 0.011 & 0.007 & & & \\
\hline Ceramic & 0 & 0.004 & 0 & & & \\
\hline L1T26 & Desert Pavement & $\begin{array}{l}\text { Modern } \\
\text { Intervention }\end{array}$ & $\begin{array}{l}\text { Vehicle } \\
\text { Track }\end{array}$ & $\begin{array}{l}\text { Water } \\
\text { Erosion }\end{array}$ & & \\
\hline Complete flake & 0.172 & 0.007 & 0.058 & 0 & & \\
\hline Broken flake & 0.050 & 0 & 0.007 & 0 & & \\
\hline Core & 0.016 & 0.004 & 0.018 & 0 & & \\
\hline Tool & 0.009 & 0 & 0.001 & 0 & & \\
\hline Ostrich eggshell & 0 & 0 & 0 & 0 & & \\
\hline Bone & 0 & 0 & 0.002 & 0 & & \\
\hline Ceramic & 0 & 0 & 0 & 0 & & \\
\hline L1T27 & Desert Pavement & $\begin{array}{l}\text { Modern } \\
\text { Intervention }\end{array}$ & & & & \\
\hline Complete flake & 0.023 & 0.007 & & & & \\
\hline Broken flake & 0.007 & 0.007 & & & & \\
\hline Core & 0.012 & 0 & & & & \\
\hline Tool & 0.004 & 0 & & & & \\
\hline Ostrich eggshell & 0 & 0 & & & & \\
\hline Bone & 0.001 & 0 & & & & \\
\hline Ceramic & 0 & 0 & & & & \\
\hline
\end{tabular}


Table 4.3. Density (per $\mathrm{m}^{2}$ ) of Artifacts and Fauna over $20 \mathrm{~mm}$ in Maximum Length in the Area Surrounding E29H1. Continued

\begin{tabular}{|c|c|c|c|c|c|c|}
\hline L1T28 & $\begin{array}{l}\text { Modern } \\
\text { Intervention }\end{array}$ & Windblown Sand & & & & \\
\hline Complete flake & 0.006 & 0.006 & & & & \\
\hline Broken flake & 0 & 0.001 & & & & \\
\hline Core & 0.003 & 0.001 & & & & \\
\hline Tool & 0 & 0.001 & & & & \\
\hline Ostrich eggshell & 0 & 0 & & & & \\
\hline Bone & 0 & 0 & & & & \\
\hline Ceramic & 0 & 0.005 & & & & \\
\hline L1T36 & Desert Pavement & $\begin{array}{l}\text { Modern } \\
\text { Intervention }\end{array}$ & $\begin{array}{l}\text { Vehicle } \\
\text { Track }\end{array}$ & Road & & \\
\hline Complete flake & 0 & 0 & 0.006 & 0 & & \\
\hline Broken flake & 0 & 0 & 0.001 & 0 & & \\
\hline Core & 0 & 0 & 0.001 & 0 & & \\
\hline Tool & 0 & 0 & 0 & 0 & & \\
\hline Ostrich eggshell & 0 & 0 & 0 & 0 & & \\
\hline Bone & 0 & 0 & 0 & 0 & & \\
\hline Ceramic & 0 & 0 & 0 & 0 & & \\
\hline L1T37 & $\begin{array}{l}\text { Modern } \\
\text { Intervention }\end{array}$ & Vehicle Track & & & & \\
\hline Complete flake & 0 & 0.002 & & & & \\
\hline Broken flake & 0 & 0.001 & & & & \\
\hline Core & 0 & 0.001 & & & & \\
\hline Tool & 0 & 0 & & & & \\
\hline Ostrich eggshell & 0 & 0 & & & & \\
\hline Bone & 0 & 0 & & & & \\
\hline Ceramic & 0 & 0 & & & & \\
\hline L1T38 & Desert Pavement & $\begin{array}{l}\text { Modern } \\
\text { Intervention }\end{array}$ & $\begin{array}{l}\text { Vehicle } \\
\text { Track }\end{array}$ & Road & & \\
\hline Complete flake & 0.018 & 0.002 & 0.005 & 0 & & \\
\hline Broken flake & 0 & 0.004 & 0.001 & 0 & & \\
\hline Core & 0.009 & 0 & 0.005 & 0 & & \\
\hline Tool & 0.009 & 0 & 0.001 & 0 & & \\
\hline Ostrich eggshell & 0 & 0 & 0 & 0 & & \\
\hline Bone & 0 & 0 & 0 & 0 & & \\
\hline Ceramic & 0 & 0.002 & 0 & 0 & & \\
\hline L1T40 & Desert Pavement & Windblown Sand & $\begin{array}{l}\text { Vehicle } \\
\text { Track }\end{array}$ & Road & & \\
\hline Complete flake & 1.537 & 0.727 & 10.231 & 0 & & \\
\hline Broken flake & 0.292 & 0.106 & 2.160 & 0 & & \\
\hline Core & 0.414 & 0.099 & 1.718 & 0 & & \\
\hline Tool & 0.027 & 0.015 & 0.236 & 0 & & \\
\hline Ostrich eggshell & 0 & 0.004 & 0.079 & 0 & & \\
\hline Bone & 0.053 & 0.099 & 0.550 & 0 & & \\
\hline Ceramic & 0 & 0 & 0 & 0 & & \\
\hline
\end{tabular}




\begin{tabular}{|l|l|l|l|l|l|l|}
\hline & $\begin{array}{l}\text { Fill (Equivalent } \\
\text { Xo Modern } \\
\text { Intervention) }\end{array}$ & $\begin{array}{l}\text { Qcs (Equivalent } \\
\text { to Desert } \\
\text { Pavement) }\end{array}$ & $\begin{array}{l}\text { Qcsb } \\
\text { (Equivalent } \\
\text { to Desert } \\
\text { Pavement) }\end{array}$ & $\begin{array}{l}\text { Qfsb } \\
\text { (Equivalent } \\
\text { to Rhizolith) }\end{array}$ & $\begin{array}{l}\text { Qfsc } \\
\text { (Equivalent to } \\
\text { Windblown } \\
\text { Sand) }\end{array}$ & $\begin{array}{l}\text { Qe } \\
\text { (Equivalent to } \\
\text { Windblown } \\
\text { Sand) }\end{array}$ \\
\hline Stone artifact & 1.42 & 1.06 & 0.21 & 0.51 & 6.40 & 1.23 \\
\hline Bone & 0.77 & 0.79 & 0.05 & 0.12 & 4.12 & 0.76 \\
\hline Ceramic & 0.05 & 0.03 & 0.01 & 0.02 & 0.01 & 0.04 \\
\hline
\end{tabular}

Note : Complete flake = complete and proximal flakes; broken flake = medial flakes, distal flakes, and angular fragments; core = artifact with negative flake scars; tool = artifact with retouch; bone = any faunal object; Ostrich eggshell = any fragment of ostrich eggshell; ceramic = any fragment of ceramic material. The labels L1T5 and so on name transects surveyed. Columns represent types of surface cover identified in each transect. Only sediment types with objects are shown. A different set of surface cover types was used in the XB11 study, where in addition, complete flakes were not distinguished from other stone artifact forms (Phillipps 2006).

Table 4.4. Nonparametric Tests Comparing Density Measures for Different Object Types in Different Sediments for Each Transect.

\begin{tabular}{|c|c|c|c|c|c|c|}
\hline Transect & $\begin{array}{l}\text { Test } \\
\text { Statistic }\end{array}$ & df & $\mathbf{P}$ & $\begin{array}{l}\text { Sediments with } \\
\text { Highest Density }\end{array}$ & & Test \\
\hline L1T7 & 22.7 & 5 & $<0.001$ & sand ripples & windblown sand & $\begin{array}{l}\text { Friedman's two-way analysis of } \\
\text { variance by ranks }\end{array}$ \\
\hline L1T8 & 5.769 & 2 & 0.056 & desert pavement & windblown sand & $\begin{array}{l}\text { Friedman's two-way analysis of } \\
\text { variance by ranks }\end{array}$ \\
\hline L1T23 & 7.373 & 3 & 0.061 & windblown sand & rhizolith & $\begin{array}{l}\text { Friedman's two-way analysis of } \\
\text { variance by ranks }\end{array}$ \\
\hline $\mathrm{L} 1 \mathrm{~T} 24$ & 5.615 & 2 & 0.06 & desert pavement & modern intervention & $\begin{array}{l}\text { Friedman's two-way analysis of } \\
\text { variance by ranks }\end{array}$ \\
\hline $\mathrm{L} 1 \mathrm{~T} 25$ & 1.826 & 2 & 0.401 & desert pavement & windblown sand & $\begin{array}{l}\text { Friedman's two-way analysis of } \\
\text { variance by ranks }\end{array}$ \\
\hline L1T26 & 6.421 & 2 & 0.04 & desert pavement & modern intervention & $\begin{array}{l}\text { Friedman's two-way analysis of } \\
\text { variance by ranks }\end{array}$ \\
\hline L1T27 & -1.826 & & 0.068 & \multicolumn{2}{|c|}{ desert pavement or modern intervention } & Wilcoxon signed rank test \\
\hline $\mathrm{L} 1 \mathrm{~T} 28$ & -0.736 & & 0.461 & \multicolumn{2}{|c|}{ windblown sand or modern intervention } & Wilcoxon signed rank test \\
\hline L1T38 & 0.737 & 2 & 0.692 & desert pavement & modern intervention & $\begin{array}{l}\text { Friedman's two-way analysis of } \\
\text { variance by ranks }\end{array}$ \\
\hline $\mathrm{L} 1 \mathrm{~T} 40$ & 9.333 & 2 & 0.009 & $\begin{array}{l}\text { modern } \\
\text { intervention }\end{array}$ & desert pavement & $\begin{array}{l}\text { Friedman's two-way analysis of } \\
\text { variance by ranks }\end{array}$ \\
\hline XB11 & 5.400 & 1 & 0.02 & $\begin{array}{l}\text { concentrated gravel } \\
\text { or gravel lag }\end{array}$ & & $\begin{array}{l}\text { Friedman's two-way analysis of } \\
\text { variance by ranks }\end{array}$ \\
\hline
\end{tabular}

Note: Boldface indicates significant results.

Using these results, it is possible to compare the densities of objects between transects by observing the differences on those sediments with the best visibility and preservation of objects, in effect providing a means to alleviate the impact of differential visibility due to surface type.

In Figure 4.7 the density of object classes from transects and the areas analyzed within the bounds of
E29H1 are compared using tree-map plots (Jadeja and Shah 2015; Wang et al. 2015). E29H1 has very much higher densities of objects than the L1 transects, necessitating a change of scale in Figure 4.7 for the E29H1 areas. Densities of material vary considerably across the area surveyed. However, while there is a significant correlation between the density of complete flakes and cores, the correlation between either of these objects and 
bone is not significant. ${ }^{4}$ This suggests that bone is not as well preserved in all locations as flaked stone artifacts, although if the density of all flaked stone artifacts is added together with the density of bone, the results are significant. When the XB11 artifacts were recorded, complete flakes and cores were not separately recorded in all cases. Bone is abundant at $\mathrm{E} 29 \mathrm{H} 1$ and in the transects to the southeast of the area surveyed. However, even when analysis is restricted to localities with significant bone concentrations, there is no statistically significant correlation with the density of flaked stone artifacts except when XB11 is included. ${ }^{5}$

4 Complete flake to core Spearman rho $=0.966 ; \mathrm{n}=17 ; p<0.001$. Bone to complete flake Spearman rho $=0.394 ; \mathrm{n}=17 ; p=0.118$. Bone to core Spearman rho $=0.406 ; \mathrm{n}=17 ; p=0.106$.

5 For example, E29H1-area bone to complete flake Spearman rho $=0.393 ; \mathrm{n}=7 ; p=0.383$. $\mathrm{L} 1$ transects bone to complete flake Spearman rho $=0.5 ; \mathrm{n}=5 ; p=0.391$.
Detailed faunal analyses were undertaken on relatively small samples of the bone material (as described below). The lack of an association between densities of bone and flaked stone artifacts raises the possibility that at least some of the bone is not derived from human activity but represents natural death assemblages connected with lake deposits. However, although the bone densities do not correlate statistically with flaked stone artifacts, like the flaked stone artifacts, they are not present in all locations. As indicated in Figure 4.7, bone is dense only in some parts of the areas we surveyed. Some areas equally lack flaked stone artifacts, but there are also areas that have relatively high densities of flaked stone but no or very little bone. The presence of these areas at least in part accounts for the insignificant correlation coefficients for flaked stone artifacts and bone. As

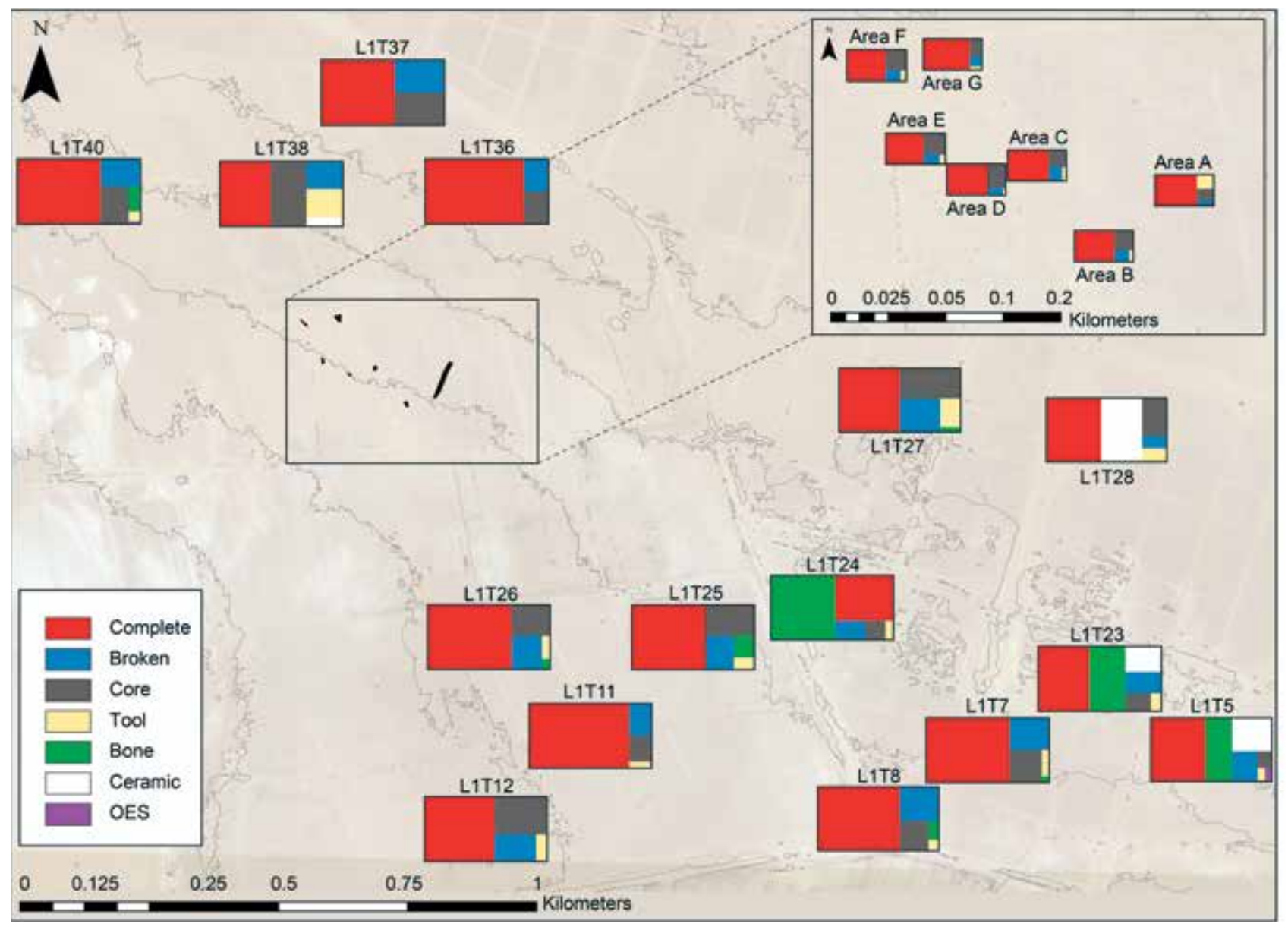

Figure 4.7. A tree-map plot (Jadeja and Shah 2015; Wang et al. 2015) showing the relative proportions of objects by material for L1 transects and E29H1 areas in the inset box. The size of the rectangle reflects the relative density of the material types in each of the study areas. "Complete," "broken," "core," and "tool" refer to flaked stone artifacts. "Complete" includes complete and proximal flakes while "broken" includes medial flakes, distal flakes, and angular fragments. "OES” refers to ostrich eggshell. 
far as we can determine, there are some places where flaked stone and bone objects are associated with the caveat that to some degree all the surface deposits are lagged surfaces. It is also true that in buried deposits, flaked stone artifacts are found with bone deposits, including quantities of fish bone (chapter 6). So it is possible that the differential density of bone relates to places where bone was deposited and places where it was not rather than simply to differential preservation.

\section{Discussion}

Flaked stone artifacts, bones, and to a lesser degree hearths and ceramics are abundant in and around the E29H1 locality studied by Wendorf and Schild. Modern-day agricultural development has truncated the distribution of archaeological objects, but based on the fieldwork we conducted in 2008 and 2012, it is likely that the area with high concentrations of objects is much more extensive than Wendorf and Schild originally mapped. Within the region that we surveyed, objects occur in markedly different densities. Highest concentrations are found on surfaces made up of concentrated gravel, in areas with a gravel lag, and in areas covered by a thin layer of yellow sand (presumably aeolian deposits), although not in regions covered by dunes. Areas of concentrated flaked stone artifacts are more spatially extensive than areas with concentrations of bone, accounting for the low overall correlation of stone and bone concentrations. Sediments that have accumulated at least some aeolian sand deposits seem to have preserved flaked stone artifact deposits more completely than other types of sediment. Features like grinding stones are more spatially extensive than areas with hearths, and we turn to a consideration of the hearth features in the next section.

The distribution of archaeological objects across the north shore of the Fayum cannot easily be divided into separate archaeological sites. Densities of artifacts differ and it seems likely that some of this variability reflects the nature of the sediments on which the artifacts are deposited. Sediments of different types have preserved artifact and faunal accumulations of different types. While the majority of sediments have a lacustrine origin, this does not indicate a close temporal association between deposition of archaeological materials and the existence of paleo-lakes.

\section{Hearths \\ E29H1 Hearths}

Figure 4.8 shows the density of hearths identified in L1

Basin. Table 4.6 lists hearths from E29H1 that were excavated and that provided sufficient charcoal for a radiocarbon determination.

Stratigraphically, the hearths are relatively simple, with a layer of fire-cracked rock found beneath a surface unit. In the majority of cases, it is the fire-cracked rock that has helped preserve small quantities of charcoal. Hearth 148 (E29H1 H148) is an exception, with substantial quantities of charcoal preserved. However, this hearth was found partially sectioned by a drainage ditch. Hearth 149 was identified close to Hearth 148 and has a roughly similar age but was much closer to the surface. Hearth 201 was also different, located in the eastern irrigation field, away from the main E29H1 hearth concentration. Excavation of Hearth 201 revealed a series of sand units on top of a clay-silt unit but with few fire-cracked rocks. The age returned is the youngest of those found at E29H1 but is close to ages from the L1 hearths discussed below.

Figure 4.9 emphasizes that the record of radiocarbon age determinations varies through time. Determinations from Hearths 105 through 141 seem to form a continuous set of ages, but it is important to compare the radiocarbon age with the calibration curve, plotted for each determination in Table 4.6 and for all determinations in Figure 4.9. There is a pronounced "flat spot" in the curve, meaning that the apparent similarity in ages for hearths from 9500 to 9000 cal BP may well hide discontinuities in the dates when E29H1 was occupied, albeit over a period measured in centuries rather than the millennial spread when all determinations are considered together.

The hearths are in general in quite shallow deposits. (Hearth 148 is an exception.) Hearth 115, for instance, with a radiocarbon determination of $9160 \pm 62 \mathrm{cal}$ $\mathrm{BP}$, has a maximum height of $12.84 \mathrm{~m}$ and a minimum height of $12.70 \mathrm{~m}$ asl. Therefore the height of the hearth deposit is $14 \mathrm{~cm}$. Depths for E29H1 hearths are given in Table 4.5. With the exception of Hearth 148 , the hearths were identified because of fire-cracked rock present on the surface. Thus the hearths are in effect shallow deposits immediately below the surface. Despite this, in a number of cases, charcoal with ages in excess of 9,000 calendar years was preserved. As noted above, the hearths are largely found in areas of aeolian sand and adjacent lake-derived sediments. As is 


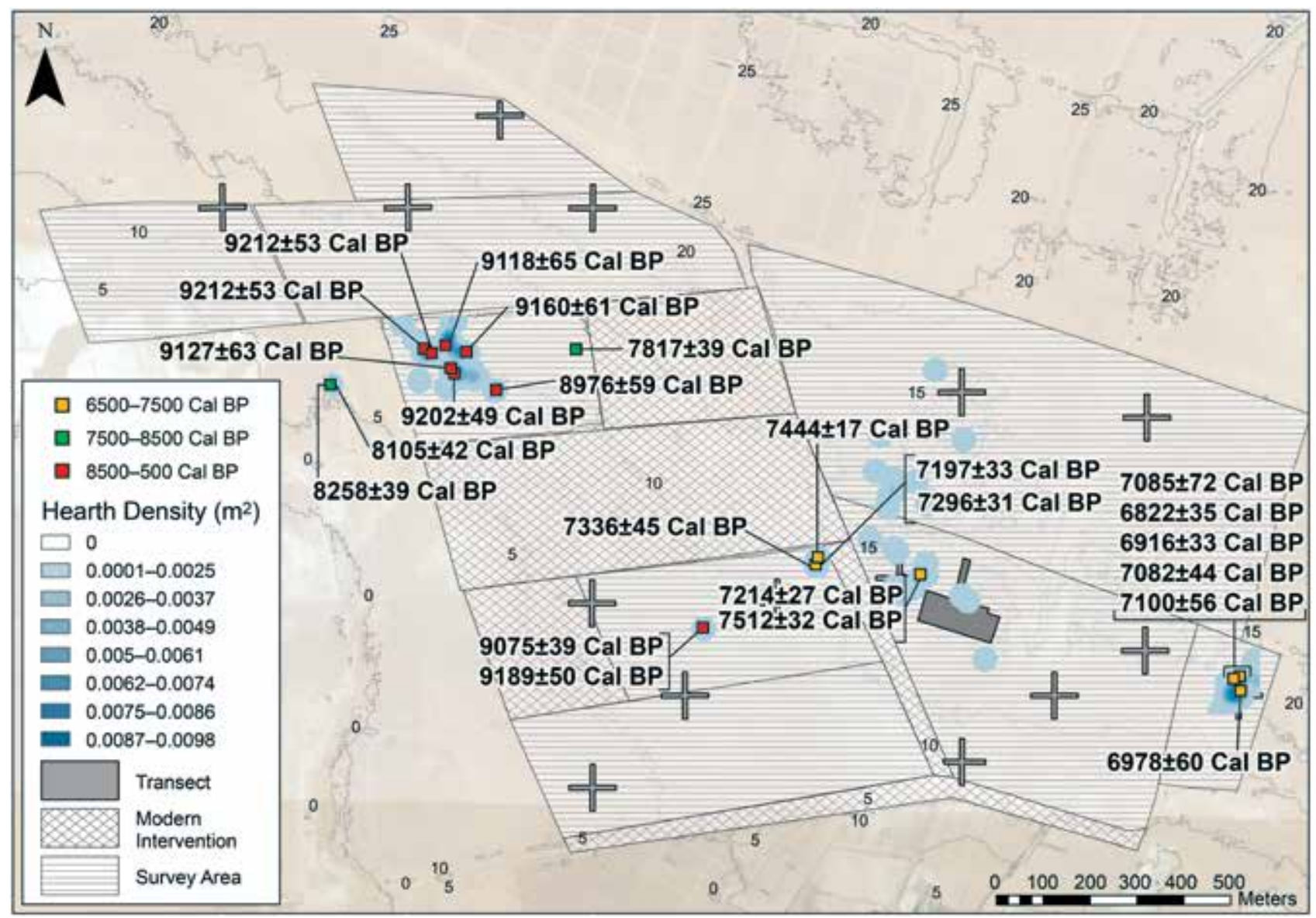

Figure 4.8. L1 Basin hearth density $\left(\mathrm{m}^{2}\right)$ overlaid with dated hearths coded by age class and showing the calibrated radiocarbon determinations.

the case in other parts of the world (e.g., Fanning et al. 2009), it is likely that the remaining hearth deposits in the Fayum are remnants of depressions dug by people in the past into which heat retainers were placed. The heat retainers remain today as fire-cracked rock, and in some instances they have served to protect charcoal deposits that can be used for radiocarbon dating.

Charcoal samples containing at least one fragment of wood charcoal larger than $2 \mathrm{~mm}$ were analysed to determine wood species, with a total weight of $319.4 \mathrm{~g}$. Less than 1 percent by weight and 2 percent by count could not be identified. Tamarisk was identified in every charcoal sample, with two other taxa identified in 16 percent of samples: a member of the Chenopodiaceae family, most likely Haloxylon salicornicum, and a monocotyledon wood, probably Phragmites australis. Large pieces of charcoal from large branches or even trunks of smaller trees were found, with diameters approaching $3 \mathrm{~cm}$. Assuming that cutting large branches takes more effort than gathering smaller-diameter wood, this may indicate some pressure on wood sources.

Table 4.5. Maximum and Minimum Elevations in Meters above Sea Level for Hearths Excavated at E29H1.

\begin{tabular}{|c|c|c|c|}
\hline Hearth & $\begin{array}{l}\text { Maximum } \\
\text { Elevation (m) }\end{array}$ & $\begin{array}{l}\text { Minimum } \\
\text { Elevation (m) }\end{array}$ & Depth (m) \\
\hline $\begin{array}{r}\text { E29H1 } \\
\text { H112 } \\
\end{array}$ & 12.56 & 12.40 & 0.16 \\
\hline 115 & 12.84 & 12.70 & 0.14 \\
\hline 122 & 11.56 & 11.48 & 0.08 \\
\hline 123 & 11.33 & 11.28 & 0.05 \\
\hline 140 & 11.55 & 11.49 & 0.06 \\
\hline 141 & 11.55 & 11.33 & 0.22 \\
\hline 148 & 7.36 & 7.18 & 0.18 \\
\hline
\end{tabular}

Note: Some hearth data was not available. 
Table 4.6. E29H1 Hearths That Were Excavated and Provided Sufficient Charcoal for a Radiocarbon Determination.

\begin{tabular}{|c|c|c|}
\hline Hearth Number & Area $m^{2}$ & $\begin{array}{l}\text { Radiocarbon Determination } \\
\text { Oxcal 4.2.2 Calibration (Bronk Ramsey 2009)* }\end{array}$ \\
\hline E29H1 H105 & 1.473 & $\begin{array}{l}\text { UCIAMS }-57215 \\
8245 \pm 20 \mathrm{bp} \\
9212 \pm 53 \mathrm{cal} \mathrm{BP} \\
7263 \pm 53 \mathrm{cal} \mathrm{BCE}\end{array}$ \\
\hline E29H1 H112 & 1.245 & $\begin{array}{l}\text { UCIAMS }-57216 \\
8175 \pm 30 \mathrm{bp} \\
9118 \pm 65 \mathrm{cal} \mathrm{BP} \\
7169 \pm 65 \mathrm{cal} \mathrm{BCE}\end{array}$ \\
\hline E29H1 H115 & 10.999 & $\begin{array}{l}\text { UCIAMS- } 57217 \\
8200 \pm 20 \mathrm{bp} \\
9160 \pm 61 \mathrm{cal} \mathrm{BP} \\
7211 \pm 61 \mathrm{cal} \mathrm{BCE}\end{array}$ \\
\hline E29H1 H122 & 10.170 & $\begin{array}{l}\text { UCIAMS- } 57218 \\
8185 \pm 20 \mathrm{bp} \\
9127 \pm 63 \mathrm{cal} \mathrm{BP} \\
7178 \pm 63 \mathrm{cal} \mathrm{BCE}\end{array}$ \\
\hline E29H1 H123 & 3.480 & $\begin{array}{l}\text { UCIAMS }-57219 \\
8235 \pm 20 \mathrm{bp} \\
9202 \pm 49 \mathrm{cal} \mathrm{BP} \\
7253 \pm 49 \mathrm{cal} \mathrm{BCE}\end{array}$ \\
\hline E29H1 H140 & 1.884 & $\begin{array}{l}\text { UCIAMS }-57220 \\
8050 \pm 20 \mathrm{bp} \\
8976 \pm 59 \mathrm{cal} \mathrm{BP} \\
7027 \pm 59 \mathrm{cal} \mathrm{BCE}\end{array}$ \\
\hline E29H1 H141 & 0.668 & $\begin{array}{l}\text { UCIAMS- } 57221 \\
8245 \pm 20 \mathrm{bp} \\
9212 \pm 53 \mathrm{cal} \mathrm{BP} \\
7263 \pm 53 \mathrm{cal} \mathrm{BCE}\end{array}$ \\
\hline E29H1 H148 & 0.986 & $\begin{array}{l}\text { UCIAMS- } 57188 \\
7305 \pm 25 \mathrm{bp} \\
8105 \pm 42 \mathrm{cal} \mathrm{BP} \\
6156 \pm 42 \mathrm{cal} \mathrm{BCE}\end{array}$ \\
\hline E29H1 H149 & 0.121 & $\begin{array}{l}\text { UCIAMS }-57222 \\
7430 \pm 20 \mathrm{bp} \\
8258 \pm 39 \mathrm{cal} \mathrm{BP} \\
6309 \pm 39 \mathrm{cal} \mathrm{BCE}\end{array}$ \\
\hline E29H1 H201 & & $\begin{array}{l}\text { UCIAMS }-57223 \\
6980 \pm 20 \mathrm{bp} \\
7817 \pm 39 \mathrm{cal} \mathrm{BP} \\
5868 \pm 39 \mathrm{cal} \mathrm{BCE}\end{array}$ \\
\hline
\end{tabular}

* Plotted with atmospheric data from Reimer et al. (2013). Error margins are 68.2 percent. 


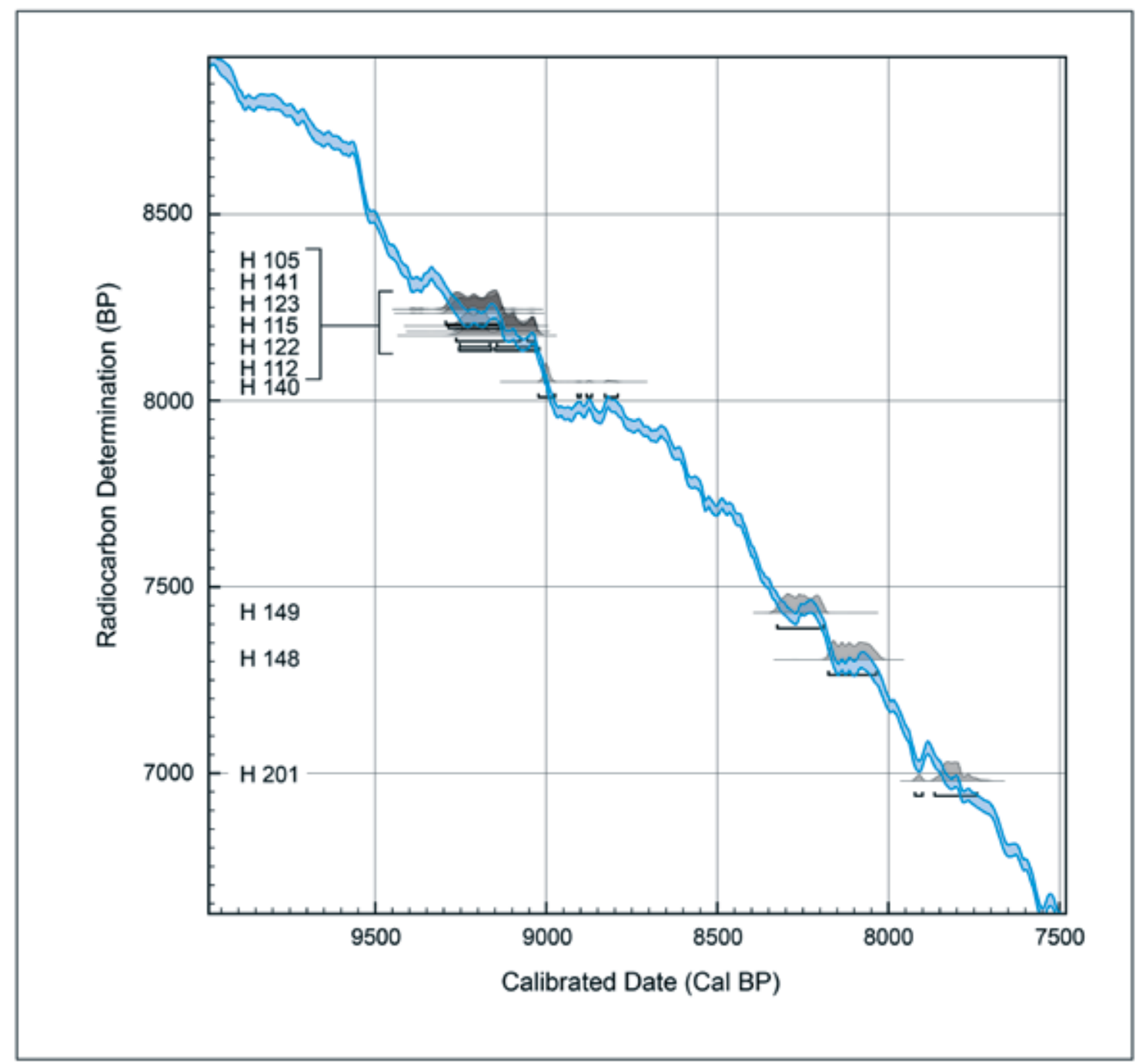

Figure 4.9. Calibrated radiocarbon determinations from E29H1 plotted against the IntCal13 atmospheric data.

\section{L1 Hearths}

Table 4.7 lists the ages of the hearths excavated in the areas to the east and south of E29H1. Like the E29H1 hearths, those from L1 are also shallow deposits. However, unlike those farther west, they have relatively fewer heat retainers. Counting hearthstones is a crude measure of hearth form, since one hearthstone may fracture into a number of fire-cracked rock pieces. These fire-cracked rock fragments are counted only if they are exposed on the surface. Comparing hearths from L1 and E29H1 also makes an arbitrary spatial division between hearths formed at different times. Nevertheless, some patterns that warrant consideration. The median number of fire-cracked rocks per hearth at E29H1 is 36, while for the L1 hearths it is
31. These are not significantly different. ${ }^{6}$ Some hearths have very large numbers of fire-cracked rock; L1H63 and L1H66 have 100 and 120, respectively, while Hearths 122 and 135 have 209 and 128 fire-cracked rocks. These hearths may be more deflated than the others and could represent fire-cracked rock from more than one hearth that is now indistinguishable. Hearths in both groups have different areas, although these are extremely variable. The median area for $\mathrm{L} 1$ hearths is $0.95 \mathrm{~m}^{2}$ while that for the E29H1 hearths is $1.39 \mathrm{~m}^{2}$. These are not significantly different. ${ }^{7}$

\footnotetext{
6 Chi-square with Yates continuity correction $=1.46 ; d f=1 ; p=$ 0.227 .

7 Chi-square with Yates continuity correction $=2.84 ; d f=1 ; p=$ 0.092 .
} 
Hearths from L1 are in general younger than those at E29H1, although the distribution of calibrated ages indicates some overlap between both sets of hearths. Figure 4.10 shows the calibrated ages of the L1 hearths, using only one determination from each hearth. L1H54, with a calibrated age around 9000 to $9500 \mathrm{BP}$, is close to the older E29H1 hearth ages.

Combining both sets of radiocarbon determinations indicates that the ages fall into three distinct groups, albeit with different spans (Figure 4.11). The oldest group of hearths is that from E29H1, with addition of the hearth mentioned above, and the hearths from L1 form a younger group, around 7000 BP. Hearth ages from E29H1 also form an intermediate group, between 7500 and $8500 \mathrm{BP}$. The distribution of hearths with age determinations is illustrated in Figure 4.8, where the hearth icons are colored to reflect the three groups of hearth ages illustrated in Figure 4.11. Although there is a spatial separation between the older and more recent hearths, those of intermediate age are present adjacent to the ancient hearths at E29H1. There is also an older hearth to the west of one of the concentrations of recent $\mathrm{L} 1$ hearths.

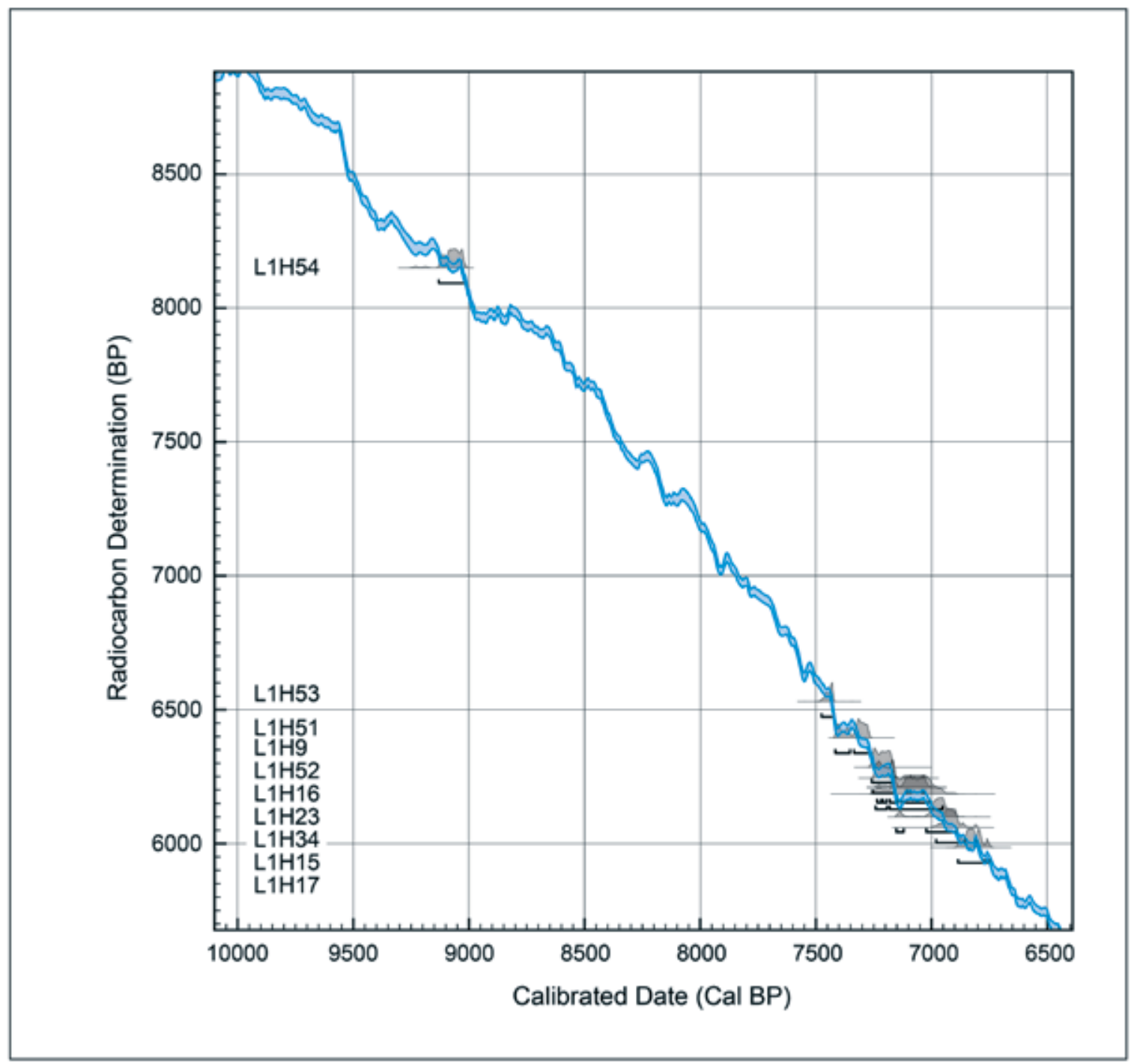

Figure 4.10. Calibrated ages from L1 hearths plotted against the IntCal13 atmospheric data. 
Table 4.7. Excavated L1 Hearths That Provided Sufficient Charcoal for a Radiocarbon Determination.

\begin{tabular}{|c|c|c|}
\hline Hearth & Area $\mathrm{m}^{2}$ & $\begin{array}{l}\text { Radiocarbon Determination } \\
\text { Oxcal 4.2.2 calibration (Bronk Ramsey (2009)* }\end{array}$ \\
\hline L1H17 & 1.24 & $\begin{array}{l}\text { UCIAMS } 122024 \\
5985 \pm 20 \mathrm{bp} \\
6822 \pm 35 \mathrm{cal} \mathrm{BP} \\
4873 \pm 35 \mathrm{cal} \mathrm{BCE}\end{array}$ \\
\hline L1H9 & 1.96 & $\begin{array}{l}\text { UCIAMS } 122030 \\
6285 \pm 20 \mathrm{bp} \\
7214 \pm 27 \mathrm{cal} \mathrm{BP} \\
5265 \pm 27 \mathrm{cal} \mathrm{BCE} \\
\text { UCIAMS } 122041 \\
6615 \pm 20 \mathrm{bp} \\
7512 \pm 32 \mathrm{cal} \mathrm{BP} \\
5563 \pm 32 \mathrm{cal} \mathrm{BCE}\end{array}$ \\
\hline L1H16 & 1.31 & $\begin{array}{l}\text { UCIAMS } 122031 \\
6210 \pm 20 \mathrm{bp} \\
7100 \pm 56 \mathrm{cal} \mathrm{BP} \\
5151 \pm 56 \mathrm{cal} \mathrm{BCE}\end{array}$ \\
\hline L1H51 & 0.37 & $\begin{array}{l}\text { UCIAMS } 122042 \\
6395 \pm 20 \mathrm{bp} \\
7336 \pm 45 \mathrm{cal} \mathrm{BP} \\
5387 \pm 45 \mathrm{cal} \mathrm{BCE}\end{array}$ \\
\hline L1H23 & 1.07 & $\begin{array}{l}\text { UCIAMS } 122032 \\
6185 \pm 50 \mathrm{bp} \\
7085 \pm 72 \mathrm{cal} \mathrm{BP} \\
5136 \pm 72 \mathrm{cal} \mathrm{BCE}\end{array}$ \\
\hline L1H52 & 0.78 & $\begin{array}{l}\text { UCIAMS } 122033 \\
6245 \pm 20 \mathrm{bp} \\
7296 \pm 31 \mathrm{cal} \mathrm{BP} \\
5347 \pm 31 \mathrm{cal} \mathrm{BCE} \\
\text { UCIAMS } 122043 \\
6365 \pm 20 \mathrm{bp} \\
7197 \pm 33 \mathrm{cal} \mathrm{BP} \\
5348 \pm 33 \mathrm{cal} \mathrm{BCE}\end{array}$ \\
\hline
\end{tabular}




\begin{tabular}{|c|c|c|}
\hline Hearth & Area $m^{2}$ & $\begin{array}{l}\text { Radiocarbon Determination } \\
\text { Oxcal 4.2.2 calibration (Bronk Ramsey (2009): }\end{array}$ \\
\hline L1H54 & 0.75 & $\begin{array}{l}\text { UCIAMS } 122034 \\
8150 \pm 20 \mathrm{bp} \\
9075 \pm 39 \mathrm{cal} \mathrm{BP} \\
7126 \pm 39 \mathrm{cal} \mathrm{BCE} \\
\text { UCIAMS } 122045 \\
8220 \pm 20 \mathrm{bp} \\
9189 \pm 50 \mathrm{cal} \mathrm{BP} \\
7240 \pm 50 \mathrm{cal} \mathrm{BCE}\end{array}$ \\
\hline L1H51 & 0.37 & $\begin{array}{l}\text { UCIAMS } 122042 \\
6395 \pm 20 \mathrm{bp} \\
7336 \pm 45 \mathrm{cal} \mathrm{BP} \\
5387 \pm 45 \mathrm{cal} \mathrm{BCE}\end{array}$ \\
\hline L1H53 & 1.92 & $\begin{array}{l}\text { UCIAMS } 122044 \\
6530 \pm 20 \mathrm{bp} \\
7444 \pm 17 \mathrm{cal} \mathrm{BP} \\
5495 \pm 17 \mathrm{cal} \mathrm{BCE}\end{array}$ \\
\hline L1H34 & 1.36 & $\begin{array}{l}\text { UCIAMS } 121796 \\
6100 \pm 25 \mathrm{bp} \\
6978 \pm 60 \mathrm{cal} \mathrm{BP} \\
5029 \pm 60 \mathrm{cal} \mathrm{BCE}\end{array}$ \\
\hline L1H15 & 1.58 & $\begin{array}{l}\text { UCIAMS } 121798 \\
6060 \pm 20 \mathrm{bp} \\
6916 \pm 33 \mathrm{cal} \mathrm{BP} \\
4967 \pm 33 \mathrm{cal} \mathrm{BCE} \\
\\
\text { UCIAMS } 121809 \\
6190 \pm 20 \mathrm{bp} \\
7082 \pm 44 \mathrm{cal} \mathrm{BP} \\
5133 \pm 44 \mathrm{cal} \mathrm{BCE}\end{array}$ \\
\hline
\end{tabular}

* Plotted with atmospheric data from Reimer et al. (2013).

\section{Discussion}

Studies in Australia indicate that once exposed, heat retainer hearths similar to those found in the Fayum are very susceptible to erosion, especially as the fire-cracked rock is dislodged (Fanning et al. 2009). In the Australian examples, the fire-cracked rock will eventually form a circle surrounding the hearth, described as a halo. Lacking the protection provided by the fire-cracked rock, the exposed hearth sediments are very susceptible to erosion and will not survive for long.

While there are environmental differences between Australia and Egypt, there are likely to be similarities in the erosive processes acting on hearths. For the hearths at $\mathrm{E} 29 \mathrm{H} 1$ to be preserved at all, it is unlikely that they were exposed for sufficient time for the fire-cracked rock to have been significantly moved at any time in the past. With the exception of Hearth 148, which was buried, the E29H1 hearths and those from L1 are close to the surface. Therefore the state of preservation of these features and the charcoal retrieved from within these features indicate something about the nature of the processes that affected the area through time. If, as has been suggested by a number of authors reviewed in chapter 3 , the area was subject to a series of lake inundations, then 


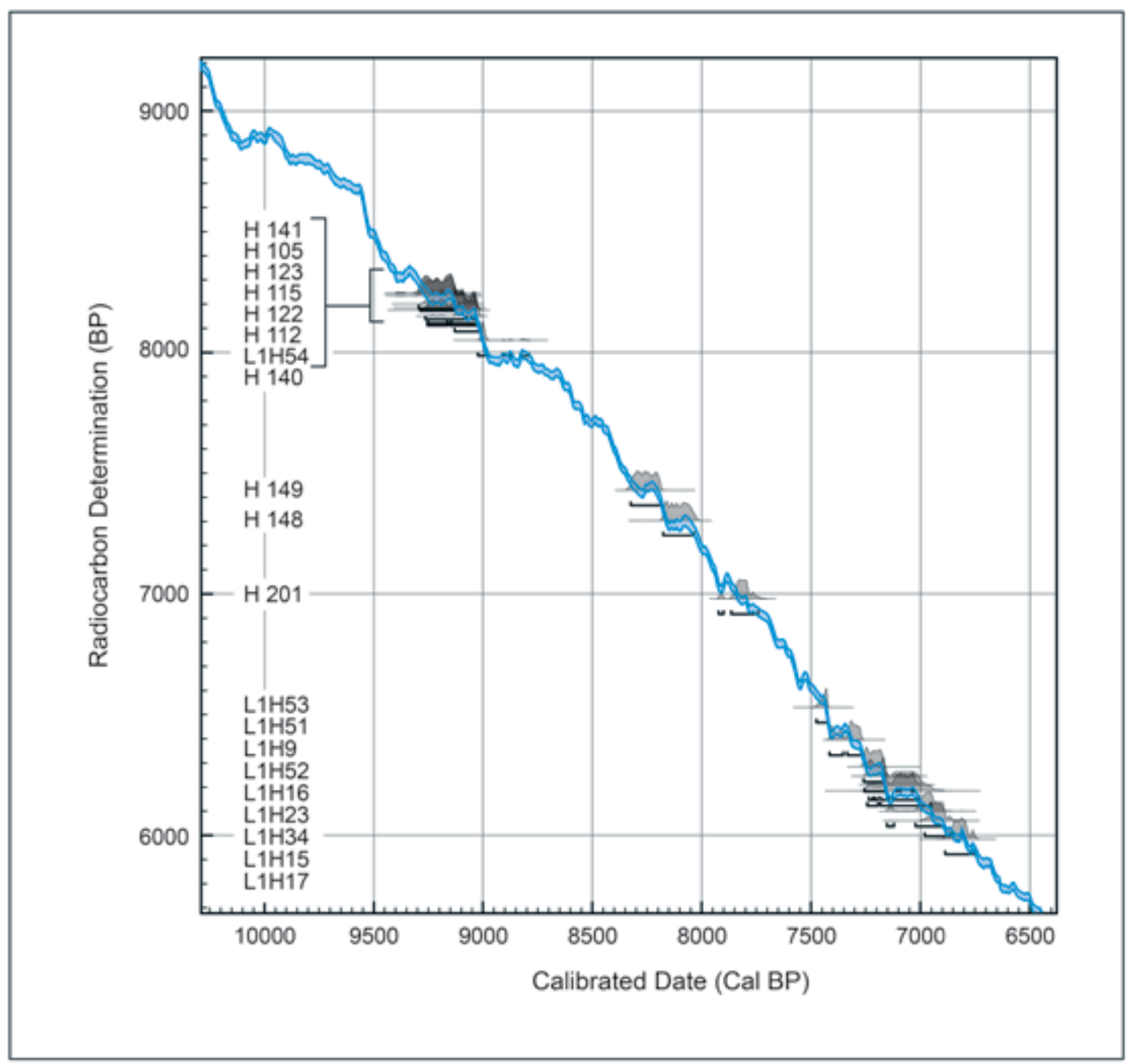

Figure 4.11. L1 and E29H1 calibrated hearth ages plotted against the IntCal13 atmospheric data.

the hearths remained sufficiently protected to retain their structure into the present. Moreover, if this process of inundation actually occurred, then not only were hearths of different ages protected but in addition, whatever protective sediments covered the hearths were subsequently removed by some unknown erosion process, exposing the hearths to a sufficient degree that they could be found. At the same time, this erosion process maintained the hearth deposits with relatively similar shallow depths. We have yet to discover where any such eroded material was subsequently deposited. It therefore seems hard to reconcile the nature of hearth preservation with scenarios that involve repeated inundation by lake waters.

As discussed in chapter 3, much of the evidence for lake level changes during the Holocene assumes that hearths were placed close to the ancient lake edge. However, the ages of the hearths we have dated help illustrate the difficulty with such an assumption. As illustrated in Figure 4.8, the concentration of older hearths at E29H1 is spatially bracketed by younger hearths of intermediate age. Two hearths dated to around 8100 cal BP are located between the $5-\mathrm{m}$ and $6-\mathrm{m}$ contours. Even allowing for changes in landscape heights since the early Holocene, the difference in height between these hearths, at around $6 \mathrm{~m}$, is substantial. Moreover, these hearths are adjacent to the older $\mathrm{E} 29 \mathrm{H} 1$ hearths, which are all located between the current-day 9-m and 11-m contours. Hearths with more recent ages, 7200 to 7400 cal BP, occur between the $12-\mathrm{m}$ and $14-\mathrm{m}$ contours. If indeed lake edges can be inferred from the position of these hearths, then the zone from $6 \mathrm{~m}$ to $14 \mathrm{~m}$ is likely to have seen significant erosion as lake levels rose and fell. This seems impossible to reconcile with the state of preservation of the hearths themselves. 
A simpler explanation for the preservation of the hearths is that they were not repeatedly inundated by lake waters, and in fact the lake edges, or as discussed in chapter 3 the lake basin edges, were always somewhat distant to the hearth concentrations that have survived. This might also help explain the relatively large-diameter tamarisk wood being accessed for firewood. The results of the sediment analyses at E29H1 reported above suggest that hearths are found in particular depositional contexts, particularly in areas that have accumulated aeolian sediment on top of ancient lake deposits. While hardly surprising, these results emphasize that despite the excellent preservation of surface and near-surface deposits over significant parts of the Fayum north shore, it is likely that the differential distribution of hearths reflects, at least in part, differential survival.

\section{Grinding Stones}

Thought of as substantial clasts in addition to manufactured artifacts, grinding stones are likely to survive in more contexts than are hearths. Figure 4.12 shows the density of grinding stones as well as the hatched areas surveyed in 2012. Table 4.8 summarizes the lithology and form of the grinding stones recorded. Most of the grinding stones are made from limestone, and most are oval or fragmented. Table 4.9 provides the same information for grinding stones from E29H1. Here grinding stones are also predominately made from limestone. However, the predominant shape is rectangular.

Figure 4.12 shows the distribution of grinding stones with different surfaces. Red dots represent concave grinding stones while green dots are flat grinding stones and yellow dots are grinding stones with a convex shape. E29H1 stones have predominantly flat surfaces while those from L1 are equally divided between flat and concave surfaces. However, beyond this, the distribution of the grinding stones with different surfaces shows no clear spatial pattern.

Grinding stones occur at a large range of elevations. The lowest are found in the far western edge of the area surveyed in 2012 and occur at elevations between $2 \mathrm{~m}$ and $3 \mathrm{~m}$. However, there are only two grinding stones at this elevation, and there is of course the possibility that these were moved to their present location at some point in the past. Many more grinding stones are found between the 5-m and 9-m contours in both the western and eastern areas surveyed. The E29H1 grinding stones are found at elevations above $9 \mathrm{~m}$. There is a single grinding stone above the 21-m contour band, with a much larger number below the 15 -m contour line.

Figure 4.12 shows the density of grinding stones, with darker shading indicating higher densities. While, as noted above, grinding stones are more widely distributed than hearths, grinding stone concentrations are highest close to areas with concentrations of hearths. In addition, some areas have higher densities of grinding stones but no hearths.

\section{Discussion}

Grinding stones have a spatially extensive distribution, much more so than the hearths. However, grinding stones occur more frequently in places where hearths are concentrated. We cannot know the age of the grinding stones, although a review of the literature indicates that grinding stones are reported from a number of North African early Holocene sites (Nasoordeen 2009). Grinding stones separated by raw material type and by surface attrition do not appear to have markedly different distributions. The grinding stones from E29H1 are often rectangular in form, while those from L1 are more oval or broken, but it is difficult to interpret the significance of these differences.

Grinding stones are relatively massive artifacts, although we have not quantified their weight. We have evidence that they have at times been collected together, particularly in recent times, to form makeshift shelters. This has definitely occurred at the site of Kom W, a site we intend to report on in a future volume, but there is no indication that the grinding stones at E29H1 and L1 have been similarly moved, with the possible exception of those at high and low elevations. If they have not moved, then their distribution may give a better indication of the extent of past human activity than, say, the hearths that are more subject to erosion. Excluding the areas we have not surveyed, the distribution of the grinding stones suggests human utilization of a more extensive area of the Fayum north shore than indicated by the hearths. However, like the flaked stone artifacts discussed below, the grinding stones are concentrated in an area between the 9-m and 14-m contour bands, in the same place as hearth concentrations. There are concentrations of grinding stones at $\mathrm{E} 29 \mathrm{H} 1$ and farther to the east in two L1 locations (Figure 4.12). Although artifacts seem to have spread farther, there is evidence that activity involved with artifact deposition and feature construction was concentrated in these areas. 


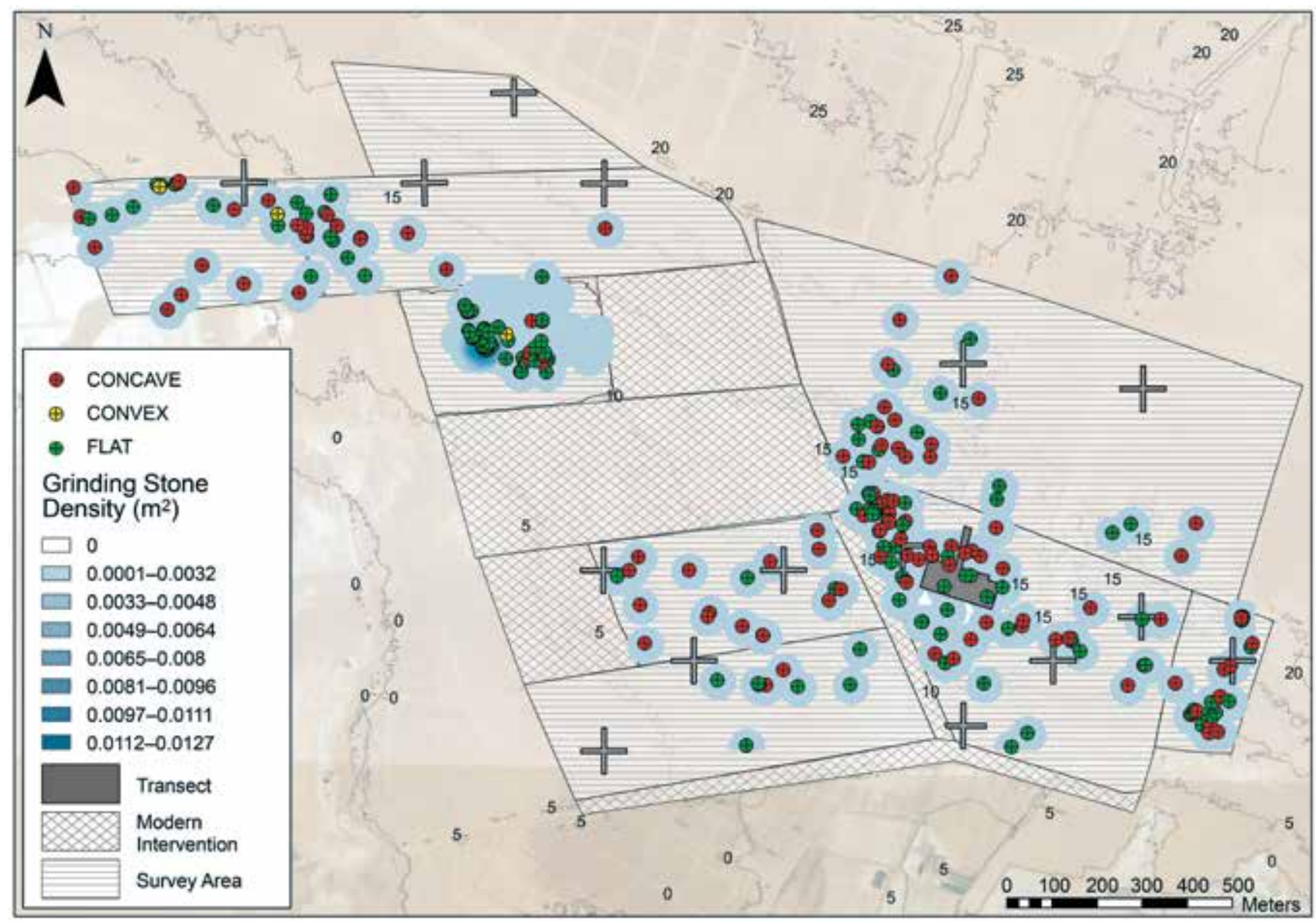

Figure 4.12. Grinding stone density $\left(\right.$ number $\left./ \mathrm{m}^{2}\right)$ from E29H1 and L1 overlaying areas surveyed and destroyed.

Table 4.8. L1 Grinding Stone Lithology and Shape.

\begin{tabular}{|l|r|r|r|r|r|r|r|r|r|}
\hline Raw Material & Broken & Circular & Oval & Rectangular & Square & Trapezoid & Trapezoidal & Triangular & Total \\
\hline Conglomerate & 42 & 16 & 25 & 0 & 0 & 0 & 1 & 1 & 85 \\
\hline Flint (?) & 0 & 0 & 1 & 0 & 0 & 0 & 0 & 0 & 0 \\
\hline Limestone & 80 & 9 & 55 & 4 & 2 & 2 & 0 & 1 & 153 \\
\hline Pink limestone & 3 & 0 & 0 & 0 & 0 & 0 & 0 & 0 & 3 \\
\hline Red granite & 1 & 0 & 0 & 0 & 0 & 0 & 0 & 0 & 1 \\
\hline Sandstone & 5 & 1 & 7 & 0 & 0 & 0 & 0 & 0 & 13 \\
\hline Total & 89 & 10 & 62 & 4 & 2 & 2 & 0 & 1 & 170 \\
\hline
\end{tabular}

Table 4.9. E29H1 Grinding Stone Lithology and Shape.

\begin{tabular}{|l|r|r|r|r|r|}
\hline Material & Circle & Oval & Rectangle & Square & Total \\
\hline Conglomerate & 0 & 1 & 0 & 0 & 1 \\
\hline Granite & 0 & 1 & 0 & 0 & 1 \\
\hline Limestone & 2 & 11 & 46 & 5 & 64 \\
\hline Sandstone & 1 & 0 & 0 & 0 & 1 \\
\hline Total & 3 & 13 & 46 & 5 & 67 \\
\hline
\end{tabular}

\section{Faunal Remains}

Animal bones and shells were collected from the surface in five different areas at E29H1 where concentrations were recorded: Trenches 202, 204, 205, 206, and 207. In addition, fauna was analyzed from excavated hearths. The collection was done in $3 \times 3-\mathrm{m}$ squares, which were first swept. Then the collected sediment was sieved through 2-mm meshes. In this way, relatively small faunal remains were recovered. More than 50 percent of 
the faunal remains were less than $2 \mathrm{~mm}$ in maximum length. Table 4.10 lists the squares analyzed in each of the trenches. All squares from Trench 204 were analyzed, while time constraints allowed only a selection of squares from the other trenches to be studied. The remains were studied at the field lab or in the storeroom, using a locally built reference collection, in combination with reference skeletons, mostly of fish, brought from the Royal Belgian Institute of Natural Sciences.

The trenches provide an indication of the distribution of faunal material across E29H1 as well as the relative degree of preservation. During collection and analyses of the bones, a pattern was noticed in the coloring they displayed, which can be correlated with their preservation state.

The bones usually have either a gray to white, a pink, or a (dark) red color. The grayish and especially the white bones were presumed to have rested directly on the surface, where they weathered due to exposure to the sun and wind. In contrast, the reddish bones were probably lying directly under the sand, where they were more protected, hence their relatively fresh appearance. Some bones were white on one side and reddish on the other. Pink bones are limited to Trench 205, where the species composition suggests that preservation is the poorest. There is a significant difference between the proportions of different colored bones from Trench 204 compared to the others, excluding pink bones from Trench 205 (Table 4.11). Trench 204 has significantly more gray to white bones, suggesting poorer preservation.

Traces related to butchery or food preparation were not recorded on any of the bones; nor were clear traces of burning identified. However, some of the gray-white coloring on the bones may be due to burning rather than surface weathering. The absence of traces related to food preparation is consistent with the use of the identified taxa as food. In early Holocene locations, such traces are often missing due to the techniques and tools used during food preparation, as well as to preservation conditions after the bones were abandoned. Fish bones in particular often retain no or few traces of food preparation.

Most of the fauna is assumed to reflect consumption refuse, apart from the very small taxa that are more

Table 4.10. Numbers of 3 x 3-m Squares Analyzed.

\begin{tabular}{|l|l|l|l|l|l|}
\hline Trench & 202 & 204 & 205 & 206 & 207 \\
\hline $\begin{array}{l}\text { Number of Squares } \\
\text { Analyzed }\end{array}$ & 24 & 102 & 36 & 37 & 36 \\
\hline
\end{tabular}

likely intrusive: frogs or toads, small lizards, snakes, the small passerines and perhaps other small birds, bats, and small rodents. As the fauna from E29H1 was retrieved from surface or very shallow deposits, the influence of differential preservation on species composition needs to be considered. Moreover, there may be admixture with later fauna, although the other archaeological material suggests that recent admixture is very limited.

Assemblages from Trenches 202, 204, 206, and 207 have a similar faunal composition, mostly consisting of fish. The predominant taxa are clariid catfish (Clariidae), tilapia, Nile perch (Lates niloticus), and Synodontis catfish (Synodontis sp.) (Table 4.12). Trench 202 is the only one where tilapia bones are more common than clariids. Tilapia have smaller, more fragile bones. Other taxa with similarly fragile bones, like amphibians and birds, are also more common in Trench 202, although the amphibians are unlikely to relate to human activity. Apart from Trench 202, all trenches yielded some pieces of the carapace or plastron of the soft-shell turtle (Trionyx triunguis). They are proportionally the most common in Trench 207. Soft-shell turtles seem to preserve particularly well when exposed to various weathering processes at the surface (Linseele et al. 2014). Bird bones are not common compared to those of fish and contain many unidentified remains. Nevertheless, at least 10 species are present; most of them are water birds. Mammal bones are not common, with hare, gazelle, and hartebeest identified. Five bones were attributed to sheep or goats. Two could be identified as goat, and others are from a bovid in the same size range. One of the caprid bones (unfused distal epiphysis of domesticated sheep from Trench 206) was submitted for radiocarbon dating. The sample returned a radiocarbon determination of $6684 \pm 43 \mathrm{BP}(7590$ to $7545 \mathrm{cal} \mathrm{BP} ; 5640$ to $5595 \mathrm{cal} \mathrm{BC})$. The age falls close to the youngest age range for the E29H1 and L1 hearths: 7200 to $7400 \mathrm{cal}$ BP (Linseele et al. 2016).

Table 4.11. Proportions of Faunal Elements with Different Colors from the Collected Trenches.

\begin{tabular}{|r|r|r|r|}
\hline & Gray-White & \multicolumn{1}{l|}{ link } & \multicolumn{1}{l|}{ Red } \\
\hline 202 & 371 & 0 & 335 \\
\hline 204 & 3,919 & 0 & 422 \\
\hline 205 & 36 & 263 & 38 \\
\hline 206 & 280 & 0 & 361 \\
\hline 207 & 211 & 0 & 153 \\
\hline
\end{tabular}


Table 4.12. List of Animal Taxa from the Different Trenches at E29H1.*

\begin{tabular}{|c|c|c|c|c|c|c|}
\hline Intrusive & 202 & 204 & 205 & 206 & 207 & Total \\
\hline Fossil shark tooth & 0 & 1 & 1 & 0 & 0 & 2 \\
\hline Fossil shell & 36 & 26 & 28 & 33 & 0 & 123 \\
\hline \multicolumn{7}{|l|}{ Terrestrial Snail } \\
\hline Helicidae & 18 & 0 & 1 & 6 & 4 & 29 \\
\hline \multicolumn{7}{|l|}{ Freshwater Snail } \\
\hline Bellamya unicolor & 13 & 50 & 1 & 24 & 4 & 92 \\
\hline Valvata nilotica & 5 & 0 & 177 & 6 & 0 & 188 \\
\hline Melanoides tuberculate & 9 & 9 & 171 & 54 & 2 & 245 \\
\hline Cleopatra bulimoides & 609 & 259 & 86 & 279 & 3 & 1,236 \\
\hline Lymnaea cf. natalensis & 24 & 10 & 15 & 1 & 0 & 50 \\
\hline Gyraulus costulatus & 120 & 55 & 192 & 32 & 1 & 400 \\
\hline Bulinus truncates & 207 & 69 & 780 & 147 & 8 & 1,211 \\
\hline \multicolumn{7}{|l|}{ Freshwater Bivalve } \\
\hline Corbicula consobrina & 2 & 0 & 0 & 14 & 0 & 16 \\
\hline Unidentified large bivalve & 0 & 0 & 0 & 1 & 0 & 1 \\
\hline Unidentified bivalve & 1 & 3 & 1 & 1 & 0 & 6 \\
\hline Unidentified gastropod & 40 & 68 & 98 & 38 & 33 & 277 \\
\hline Bird eggshell (not ostrich) & 0 & 0 & 0 & 48 & 0 & 48 \\
\hline \multicolumn{7}{|l|}{ Amphibian and Reptile } \\
\hline Toad (Bufonidae) & 30 & 7 & 0 & 5 & 1 & 43 \\
\hline Frog or toad (Batrachia) & 25 & 26 & 0 & 4 & 5 & 60 \\
\hline Small lizard & 1 & 0 & 0 & 0 & 0 & 1 \\
\hline Snake (Serpentes) & 0 & 1 & 0 & 0 & 0 & 1 \\
\hline Small Passeriformes & 1 & 0 & 0 & 0 & 0 & 1 \\
\hline Bats (Chiroptera) & 4 & 1 & 0 & 0 & 0 & 5 \\
\hline Small rodent & 6 & 4 & 0 & 0 & 0 & 10 \\
\hline \multicolumn{7}{|l|}{ Anthropogenic and Unidentified } \\
\hline Coelatura aegyptiaca & 0 & 0 & 0 & 1 & 0 & 1 \\
\hline \multicolumn{7}{|l|}{ Fish } \\
\hline Mullets (Mugilidae) & 2 & 1 & 0 & 0 & 0 & 3 \\
\hline Elephant-snout fishes (Mormyridae) & 1 & 1 & 0 & 0 & 0 & 2 \\
\hline Barbel family (Cyprinidae) & 3 & 8 & 0 & 1 & 1 & 13 \\
\hline Alestes/Brycinus & 0 & 1 & 0 & 0 & 0 & 1 \\
\hline Catfish 1 (Clarias sp.) & 0 & 0 & 0 & 1 & 0 & 1 \\
\hline Clariid catfish (Clariidae) & 168 & 874 & 201 & 416 & 185 & 1,844 \\
\hline Catfish 2, bagrid catfish (Bagrus sp.) & 0 & 22 & 1 & 3 & 1 & 27 \\
\hline Catfish 3 (Synodontis schall) & 0 & 2 & 0 & 2 & 0 & 4 \\
\hline Synodontis sp. & 23 & 185 & 1 & 25 & 4 & 238 \\
\hline Nile perch (Lates niloticus) & 60 & 254 & 7 & 40 & 55 & 416 \\
\hline tilapia (Tilapiini) & 354 & 335 & 4 & 88 & 59 & 840 \\
\hline Pufferfish (Tetraodon lineatus) & 9 & 37 & 83 & 28 & 18 & 175 \\
\hline
\end{tabular}




\begin{tabular}{|c|c|c|c|c|c|c|}
\hline Intrusive & 202 & 204 & 205 & 206 & 207 & Total \\
\hline Identified Fish & 620 & 1,720 & 297 & 604 & 323 & 3,564 \\
\hline Unidentified fish & 1,190 & 1,856 & 88 & 590 & 486 & 4,210 \\
\hline \multicolumn{7}{|l|}{ Reptiles } \\
\hline Soft-shell turtle (Trionyx triunguis) & 0 & 20 & 20 & 16 & 27 & 83 \\
\hline \multicolumn{7}{|l|}{ Birds } \\
\hline Great crested grebe (Podiceps cristatus) & 1 & 2 & 0 & 0 & 0 & 3 \\
\hline Stork (Ciconiidae) & 0 & 0 & 0 & 1 & 0 & 1 \\
\hline Duck (Anatidae) & 10 & 22 & 1 & 2 & 2 & 37 \\
\hline cf. duck (Anatidae) & 0 & 0 & 0 & 1 & 1 & 2 \\
\hline Goose (size Alopochen aegyptiaca) & 0 & 1 & 0 & 0 & 0 & 1 \\
\hline Goose (size Anser anser) & 1 & 0 & 0 & 0 & 0 & 1 \\
\hline Coot (Fulica atra) & 2 & 8 & 0 & 2 & 2 & 14 \\
\hline Common raven (Corvus corax) & 0 & 1 & 0 & 0 & 0 & 1 \\
\hline Rail (Rallidae) & 0 & 0 & 0 & 1 & 0 & 1 \\
\hline Identified Birds & 14 & 34 & 1 & 7 & 5 & 61 \\
\hline Unidentified birds & 64 & 114 & 6 & 28 & 52 & 264 \\
\hline Ostrich eggshell & 0 & 1 & 2 & 0 & 0 & 3 \\
\hline \multicolumn{7}{|l|}{ Wild Mammals } \\
\hline Hare (Lepus capensis) & 1 & 0 & 0 & 1 & 1 & 3 \\
\hline Hippopotamus (Hippopotamus amphibius) & 0 & 0 & 3 & 0 & 0 & 3 \\
\hline Dorcas gazelle (Gazella dorcas) & 1 & 3 & 2 & 2 & 1 & 9 \\
\hline Hartebeest (Alcelaphus buselaphus) & 2 & 2 & 0 & 0 & 0 & 4 \\
\hline Medium antelope & 0 & 0 & 5 & 0 & 0 & 5 \\
\hline \multicolumn{7}{|l|}{ Domesticated Mammals } \\
\hline Sheep (Ovis ammon f. aries) & 0 & 0 & 0 & 1 & 0 & 1 \\
\hline Goat (Capra aegagrus f. hircus) & 0 & 2 & 0 & 0 & 0 & 2 \\
\hline Sheep or goat & 1 & 1 & 0 & 0 & 0 & 2 \\
\hline \multicolumn{7}{|l|}{ Wild or Domesticated Mammals } \\
\hline Wolf or dog (Canis lupus [f. familiaris]) & 1 & 4 & 0 & 0 & 0 & 5 \\
\hline Unidentified carnivore & 1 & 1 & 0 & 0 & 1 & 3 \\
\hline Small bovid (teeth) & 11 & 11 & 120 & 14 & 7 & 163 \\
\hline Small bovid (other) & 1 & 14 & 1 & 11 & 1 & 28 \\
\hline Cattle or aurochs (Bos primigenius [f. Taurus]) & 0 & 0 & 3 & 0 & 0 & 3 \\
\hline Large bovid & 2 & 17 & 118 & 3 & 7 & 147 \\
\hline Bovid & 0 & 0 & 0 & 6 & 1 & 7 \\
\hline Very large mammal & 0 & 0 & 1 & 0 & 0 & 1 \\
\hline Identified Mammals & 21 & 55 & 253 & 38 & 19 & 386 \\
\hline Unidentified mammals & 258 & 266 & 1,382 & 156 & 98 & 2,160 \\
\hline Unidentified vertebrates & 284 & 1,346 & 918 & 86 & 269 & 2,903 \\
\hline Grand total (vertebrates) & 2,518 & 5,453 & 2,968 & 2,307 & 1,285 & 14,531 \\
\hline$\%$ vertebrates identified & 28.7 & 33.2 & 18.6 & 28.1 & 27.0 & 27.6 \\
\hline
\end{tabular}

*Numbers of identified specimens (NISP). 
None of the sheep or goat bones are present in Trench 205, the trench closest to the oldest E29H1 hearths. This raises the possibility that the faunal remains in Trench 205 are older than those in the other trenches, a possibility that would also explain their different color (pink bones predominate) and the markedly different species proportions. Some carnivore remains, as well as a cattle-size bovid were also present.

The fauna from Trench 205 is different than the faunal assemblages from the other trenches. The majority of the bones are pink in color, as noted above, and most are too fragmented and too weathered to allow for species identification. However, where this is possible, mammal bone predominates. Species identified include hippo, Dorcas gazelle, and a medium-size antelope, most likely hartebeest. In addition, wild or domestic bovids were identified among the remains. The trench yielded a large number of remains identified as small or large bovids. About 75 percent of these are teeth fragments. Fish are also present and consist mainly of pieces of the skull roof of clariid catfish (Clariidae) and jaws of pufferfish (Tetraodon fahaka), two types of elements that are particularly resistant to weathering. Trench 205 is the only sampling unit where frogs or toads are entirely missing. Bird bones are very rare in this part of E29H1, but the trench yielded ostrich eggshell fragments.

Table 4.13 lists the shell species found. As with the vertebrate fauna, Trench 205 has a different shell species composition than the other trenches. Cleopatra, a lacustrine species, is less common, and there are relatively more Bulinus, which inhabits a different type of aquatic environment than Cleopatra.

Fauna retrieved from the excavated hearths is listed in Table 4.14. The majority of the bones identified from the hearths are fish, with a small number of hearths indicating small or large unidentified bovids.

\section{Discussion}

Bone preservation varies, suggesting better preservation in the north and east and more prolonged exposure close to the area that preserves hearths at E29H1. Most of the trenches show large numbers of fish bones, with more variable quantities of mammal bone. However, mammal is present in higher proportions in Trench 205, where preservation is probably less complete.

Faunal preservation is variable, which may go some way to explaining the poor correlation between numbers of faunal elements and numbers of flaked stone artifacts. However, there is sufficient preservation to indicate that
Table 4.13. Shell Species Identification from E29H1.

\begin{tabular}{|c|c|c|c|c|c|}
\hline Trench & 202 & 204 & 205 & 206 & 207 \\
\hline Cleopatra bulimoides & 609 & 259 & 86 & 279 & 3 \\
\hline Bellamya unicolor & 13 & 50 & 1 & 24 & 4 \\
\hline Gyraulus costulatus & 120 & 55 & 192 & 32 & 1 \\
\hline Bulinus truncates & 207 & 68 & 780 & 147 & 8 \\
\hline Melanoides tuberculata & 9 & 9 & 171 & 54 & 2 \\
\hline Valvata nilotica & 5 & 0 & 177 & 6 & 0 \\
\hline Corbicula consobrina & 2 & 0 & 0 & 14 & 0 \\
\hline Caelatura aegyptiaca & 0 & 0 & 0 & 1 & 0 \\
\hline Large bivalve & 0 & 0 & 0 & 1 & 0 \\
\hline $\begin{array}{l}\text { Unidentified } 1= \\
\text { Lymnaea }\end{array}$ & 24 & 10 & 15 & 1 & 0 \\
\hline $\begin{array}{l}\text { Unidentified } 2= \\
\text { unidentified }\end{array}$ & 0 & 33 & 0 & 0 & 0 \\
\hline $\begin{array}{l}\text { Unidentified } 3= \\
\text { Bulinus }\end{array}$ & 0 & 1 & 0 & 0 & 0 \\
\hline $\begin{array}{l}\text { Unidentified } 4= \\
\text { Helicidae }\end{array}$ & 18 & 0 & 1 & 6 & 4 \\
\hline Unidentified 6 & 0 & 0 & 1 & 0 & 0 \\
\hline $\begin{array}{l}\text { Indeterminate } \\
\text { Gastropoda }\end{array}$ & 40 & 35 & 97 & 38 & 33 \\
\hline Indeterminate bivalve & 1 & 1 & 1 & 1 & \\
\hline $\begin{array}{l}\text { Indeteterminate } \\
\text { mollusk }\end{array}$ & 0 & 0 & 0 & 0 & \\
\hline Fossil shell & 36 & 26 & 28 & 33 & \\
\hline Ostrich eggshell & & 1 & 0 & 0 & \\
\hline Bird eggshell & & 0 & 0 & 48 & \\
\hline
\end{tabular}

a range of fauna is present. Fish species are consistent with those obtained from the Nile. Most fish are from shallow waters, especially represented by clariid catfish (Clariidae) and tilapia (Tilapiini) but including also fish from the Barbel family (Cyprinidae). Other species are typical of well-oxygenated water: bagrid catfish (Bagrus sp.), Synodontis catfish, and Nile perch (Lates niloticus). These species suggest access to shallow, near lake edge waters as well as deeper areas.

The identified mammal taxa are hare (Lepus capensis), hippopotamus, Dorcas gazelle (Gazella dorcas), and hartebeest (Alcelaphus buselaphus). Bovids, both small and large, are present particularly in Trench 205. However, this trench lacks evidence for sheep and goats, which are represented by small numbers of bones in the other trenches. One of these bones gave a radiocarbon age of around $7500 \mathrm{cal}$ BP. The age of this bone falls 
Table 4.14. List of Animal Taxa from the Different Trenches at E29H1.*

\begin{tabular}{|c|c|c|c|c|c|c|c|c|c|c|c|}
\hline & 105 & 112 & 115 & 122 & 123 & 140 & 141 & 148 & 149 & 201 & Total \\
\hline Cleopatra bulimoides & - & - & - & - & - & 1 & - & - & - & - & 1 \\
\hline Bulinus sp. & - & - & - & - & - & 1 & - & - & - & - & 1 \\
\hline Melanoides tuberculata & - & - & - & - & - & 1 & - & - & - & - & 1 \\
\hline Clariid catfish (Clariidae) & - & 2 & 8 & - & - & - & 1 & 1 & 15 & - & 27 \\
\hline Bagrid catfish (Bagrus sp.) & - & - & - & - & - & - & - & - & 1 & - & 1 \\
\hline Synodontis schall & - & - & - & - & - & - & - & - & - & 1 & 1 \\
\hline Nile perch (Lates niloticus) & - & - & - & - & - & 1 & - & - & - & 1 & 2 \\
\hline tilapia (Tilapiini) & - & - & - & - & - & - & 1 & 1 & 1 & 4 & 7 \\
\hline Unidentified fish & - & 5 & 1 & 1 & - & - & 6 & 16 & 33 & - & 62 \\
\hline Unidentified birds & - & 3 & - & - & - & - & - & - & - & - & 3 \\
\hline Small bovid & - & - & 1 & - & 1 & - & - & - & - & - & 2 \\
\hline Unidentified mammals & - & 5 & 21 & 3 & 13 & 7 & 1 & 7 & - & - & 57 \\
\hline
\end{tabular}

close to the range of ages obtained from the youngest of the L1 hearths, suggesting perhaps that it relates to activities centered slightly farther to the east of E29H1.

The frequency and species spectrum of wild game recorded for E29H1 is very similar to that of Predynastic sites in the Nile Valley of Upper Egypt, where it has been interpreted as evidence for opportunistic hunting close to the habitat of the animals that were preyed upon (Linseele et al. 2014).

\section{Flaked Stone Artifacts}

Flaked stone artifacts are the most abundant indication of human activity on the north shore of the Fayum. They are abundant on the surface, although they are also present in stratified deposits, as described in chapter 6, but they vary in density across the E29H1 and L1 areas. Flint cortical cobbles (for example, Figure 3.14) were used to manufacture the majority of the flaked stone artifacts, with the possible exception of some of the flaked stone tools discussed further below. Sources for the flint are not known, but potential sources are discussed by Shirai (2010).

We recorded flaked stone artifacts in three different ways. At E29H1 we used total stations to locate flaked stone artifacts in three-dimensional space to quantify their density. In 2012 we used the same technique to quantify the density of artifacts in the 2,000$\mathrm{m}^{2}$ transects but differentiated between flakes with platforms, broken flakes, cores, and retouched tools. Finally, in both 2008 and 2012, we analyzed spatially located samples of artifacts, recording technological and formal variables. The differential density of flaked stone artifacts is described above. Here we concentrate on understanding the manufacture, use, and abandonment of flaked stone artifacts as a proxy for understanding the range of behaviors associated with occupation on the Fayum north shore.

\section{Cores}

Cores are abundant in both the E29H1 assemblages and those from L1. Table 4.15 provides mean sizes for cores from each of the assemblages that were fully analyzed, while Table 4.16 gives the frequency and proportions of cores of different forms. Comparison of mean sizes indicates a significant difference among the assemblages. ${ }^{8}$ Post hoc Bonferroni tests indicate that significant differences occur between E29H1 Area A and L1T24 and the other assemblages for maximum length, width, and thickness. For core scar length, E29H1 Area E and L1T5 differ from the other assemblages. XB11 differs in length from E29H1 Areas B, D, and G and L1T24 and from all other assemblages except E29H1 Area A in core scar length.

Based on preliminary investigation of reduction of raw material at Kom K and Kom W, three basic flaking scenarios that present a useful way to conceptualize cobble reduction can be inferred (Phillipps 2012; Phillipps and Holdaway 2016). Cobbles can be flaked

\footnotetext{
8 ANOVA maximum length $F=12.09 ; d f=9,1,458 ; p<0.001$. Maximum width $F=6.57 ; d f=9,1,458 ; p<0.001$. Maximum thickness $F=6.79 ; d f=8,1,458 ; \mathrm{p}<0.001$. Core scar length $F=$ $7.89 ; d f=8,1,458 ; p<0.001$.
} 
Table 4.15. Means and Standard Deviations (SD) for Cores from E29H1 and L1 Analyzed Assemblages.

\begin{tabular}{|c|c|c|c|c|c|c|c|c|c|}
\hline & \multirow[b]{2}{*}{$\mathrm{n}$} & \multicolumn{2}{|c|}{$\begin{array}{l}\text { Maximum Length } \\
(\mathrm{mm})\end{array}$} & \multicolumn{2}{|c|}{$\begin{array}{l}\text { Maximum Width } \\
(\mathrm{mm})\end{array}$} & \multicolumn{2}{|c|}{$\begin{array}{l}\text { Maximum Thickness } \\
(\mathrm{mm})\end{array}$} & \multicolumn{2}{|c|}{$\begin{array}{l}\text { Core Scar Length } \\
(\mathrm{mm})\end{array}$} \\
\hline & & Mean & SD & Mean & SD & Mean & $\mathrm{SD}$ & Mean & SD \\
\hline E29H1 Area A & 51 & 41.67 & 9.85 & 29.67 & 8.04 & 17.41 & 5.70 & 23.61 & 8.22 \\
\hline E29H1 Area B & 184 & 33.62 & 8.25 & 23.28 & 6.33 & 16.76 & 4.79 & 22.58 & 8.10 \\
\hline E29H1 Area C & 183 & 35.58 & 8.37 & 26.38 & 15.94 & 16.77 & 4.90 & 23.33 & 8.62 \\
\hline E29H1 Area D & 223 & 33.90 & 7.20 & 25.44 & 5.77 & 16.19 & 5.10 & 23.86 & 7.70 \\
\hline E29H1 Area E & 230 & 36.11 & 8.20 & 25.38 & 6.75 & 17.94 & 5.05 & 25.08 & 7.37 \\
\hline E29H1 Area F & 123 & 35.53 & 7.46 & 26.80 & 5.94 & 16.47 & 4.71 & 22.50 & 6.42 \\
\hline E29H1 Area G & 87 & 33.87 & 7.45 & 25.33 & 6.13 & 16.56 & 5.95 & 22.18 & 6.27 \\
\hline L1T5 & 79 & 37.05 & 10.31 & 27.30 & 7.43 & 17.52 & 4.48 & 21.56 & 6.20 \\
\hline L1T24 & 93 & 43.84 & 20.39 & 31.41 & 15.07 & 19.92 & 8.10 & 23.32 & 9.75 \\
\hline XB11 & 213 & 32.58 & 11.51 & 23.47 & 11.41 & 17.99 & 9.05 & 27.36 & 7.48 \\
\hline
\end{tabular}

parallel to the long axis of the nodule or perpendicular to this axis if the nodule is an ellipsoid. In the first flaking scenario, unifacial reduction of a cobble parallel to its long axis occurs, while in strategy two, this flaking occurs perpendicular to the long axis. The third scenario involves reduction of an elliptical nodule by rotating the core (Figure 4.13). The positions of the major, semimajor, and semiminor axes of the core products are different in all three flaking scenarios.
Flaking scenario two results in unifacial cores, while flaking strategy three results in multiple cores. Using these assumptions about the flaking scenario, the original size of nodules can be reconstructed based on the remnant cores within an assemblage. However, it must be established on average how much of the long axis is lost in flaking sscenario two or potentially how much of all axes in flaking scenario three.

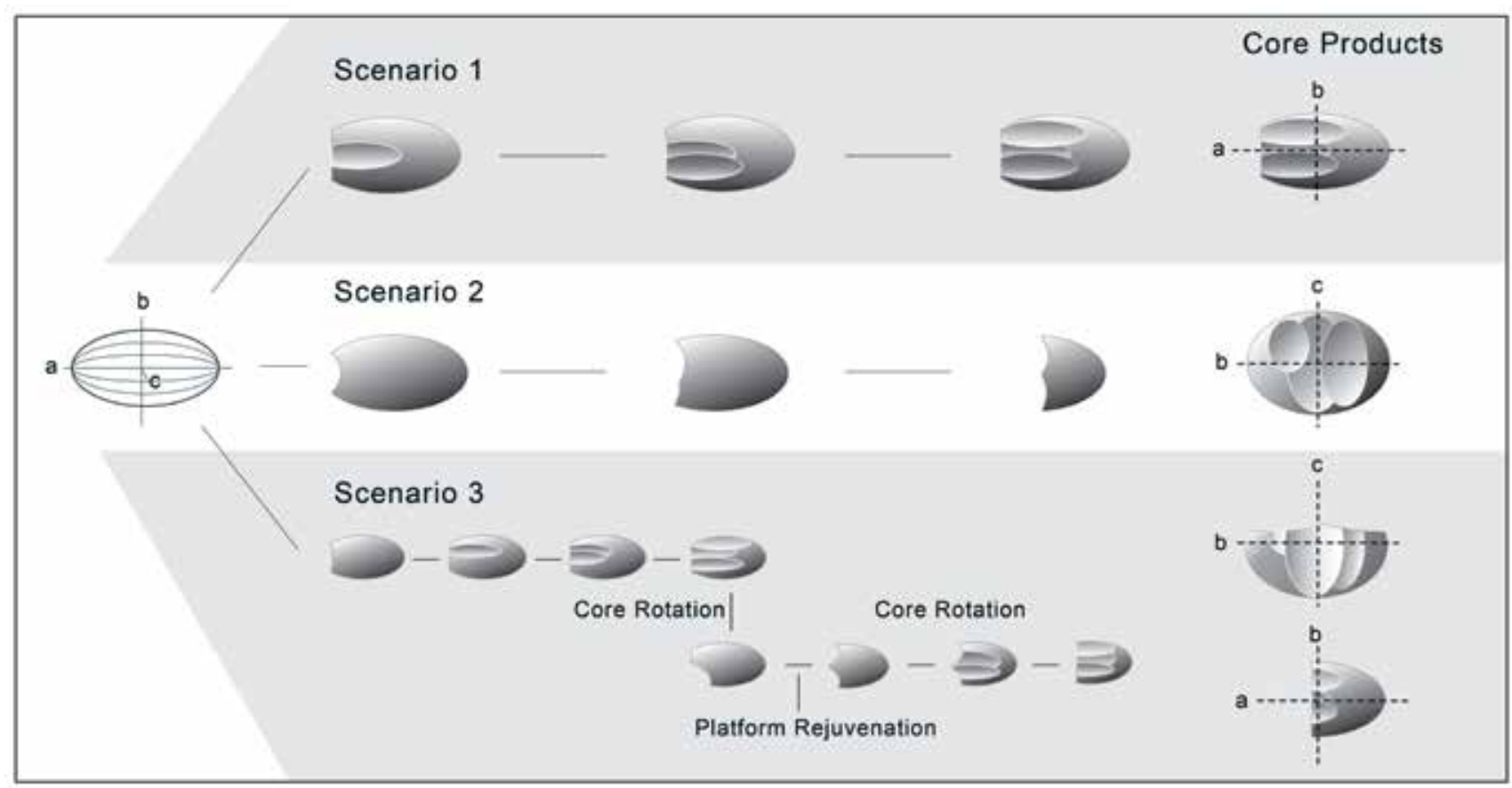

Figure 4.13. Hypothetical reduction sequence of an Egyptian flint cobble for flaking (reproduced from Phillipps and Holdaway 2016:Figure 4). 


\begin{tabular}{|c|c|c|c|c|c|c|c|c|c|c|}
\hline$\partial^{\theta}$ & $\begin{array}{l}\stackrel{0}{ } \\
\stackrel{2}{\sim}\end{array}$ & $\stackrel{\vartheta}{\stackrel{\gamma}{\sigma}}$ & $\underset{+}{\stackrel{\infty}{+}}$ & $\approx$ & $\stackrel{?}{\vec{\gamma}}$ & $\stackrel{\text { j. }}{\text {. }}$ & $\begin{array}{l}n \\
\stackrel{n}{v}\end{array}$ & $\begin{array}{l}\infty \\
\dot{\varphi}\end{array}$ & ஸे & ষ \\
\hline $\bar{\tau}$ & $\stackrel{\ominus}{ }$ & চ & $\infty$ & $\stackrel{\infty}{\wedge}$ & $\tilde{\sigma}$ & ๙ે & N & $\hat{m}$ & g & $\stackrel{m}{n}$ \\
\hline $\overrightarrow{0}$ & in & in & $n$ & $n$ & in & in & $n$ & in & in & in \\
\hline & $\hat{n}$ & ? & $\ddot{n}$ & $\vec{\infty}$ & $\dot{\sigma}$ & $\underset{\infty}{-}$ & $\begin{array}{l}\infty \\
\dot{\infty}\end{array}$ & & "n & $\nabla$ \\
\hline $\overrightarrow{3}$ & $\infty$ & $\underset{\sim}{\sim}$ & $\stackrel{\circ}{-}$ & $\stackrel{\infty}{\sim}$ & $a$ & $\stackrel{ }{-}$ & $\underset{\sim}{\sim}$ & in & 6 & $\infty$ \\
\hline & $n$ & in & in & n & in & in & v & in & $\ddot{v}$ & $\stackrel{n}{v}$ \\
\hline & $\underset{\dot{m}}{\dot{r}}$ & $\stackrel{m}{\sim}$ & $\ddot{\sigma}$ & $\begin{array}{l}0 \\
\stackrel{0}{0}\end{array}$ & $\stackrel{+}{\circ}$ & $\begin{array}{l}\infty \\
\underset{\sim}{N}\end{array}$ & $\begin{array}{l}m \\
m\end{array}$ & $\underset{+}{\stackrel{+}{d}}$ & $\stackrel{\sim}{\sim}$ & $\infty$ \\
\hline$\frac{\pi}{2}$ & $\underset{-}{\sigma}$ & $\stackrel{ \pm}{\sim}$ & $\tilde{n}$ & fo & $\stackrel{\sim}{\sim}$ & $\stackrel{\infty}{\sim}$ & ন & 9 & ป & $\bar{\infty}$ \\
\hline 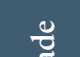 & in & in & n & in & in & in & in & in & in & in \\
\hline$\partial^{\theta}$ & & & $\stackrel{\sim}{\sim}$ & $\dot{\forall}$ & $\Lambda$ & & & & & \\
\hline$\frac{\mathscr{V}}{\widetilde{F}}$ & $\stackrel{n}{v}$ & $\hat{v}$ & in & $\stackrel{\sim}{\sim}$ & $\underset{r}{\sigma}$ & in & in & in & $\ddot{n}$ & \\
\hline & $\underset{\dot{\sim}}{\stackrel{\sigma}{N}}$ & $\begin{array}{l}0 \\
\dot{0} \\
\sim\end{array}$ & $\ddot{n}$ & $\stackrel{+}{\circ}$ & $\stackrel{+}{\dot{0}}$ & $\ddot{0}$ & $\stackrel{n}{\sim}$ & 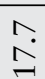 & $\stackrel{\text { }}{\text { d }}$ & $\begin{array}{l}\infty \\
\infty \\
\sim\end{array}$ \\
\hline & 二 & $\mathscr{\gamma}$ & $\stackrel{\infty}{\sim}$ & $\infty$ & ㅇ & $\hat{m}$ & $\stackrel{\circ}{-}$ & \pm & $\stackrel{\mathcal{I}}{\mathcal{H}}$ & 우 \\
\hline$\partial^{e}$ & & qे & $\underset{\infty}{\infty}$ & $\stackrel{n}{n}$ & $\dot{i n}$ & & $\stackrel{n}{\rightleftarrows}$ & ?ִ & & \\
\hline ฐ్ & in & $a$ & $n$ & i & $\stackrel{n}{\sim}$ & in & $\stackrel{0}{-}$ & in & in & \\
\hline & 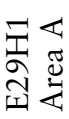 & 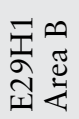 & 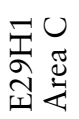 & 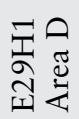 & 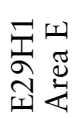 & 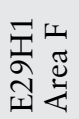 & 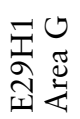 & $\underset{\Xi}{\stackrel{\Xi}{\Xi}}$ & $\stackrel{n}{\models}$ & $\vec{\nabla}$ \\
\hline
\end{tabular}


To determine the proportion of the cobble lost in flaking, we manufactured cores and flakes using cobbles obtained from different sources in the Fayum (Phillipps 2012; Phillipps and Holdaway 2016). In the experiment, the original nodule size, number of flakes produced through flaking per core, and resulting core attributes, including size, were recorded (Table 4.17).

Results suggest that nodule size is variable throughout the Fayum. For example, scenario 3 is similar to some of the raw material types identified at Kom K. This nodule was flaked unifacially and produced 21 flakes, reducing the long axis from $90 \mathrm{~mm}$ to $30 \mathrm{~mm}$. However, to reconstruct the approximate original nodule volume using core dimensions oriented relative to the core platform, the thickness, or the original nodule length, must be multiplied up to four times. This may suggest that on average (including cores with more cortex, 50 to 99 percent), the thickness should be multiplied by three to four times when performing calculations to approximate original nodule volume. While scenario 3 is listed as a core with only 1 to 50 percent cortex remaining, this core has around 50 percent cortex rather than 1 percent and has lost around 67 percent of its original length. IH1 and S1 are unifacial cores that still have 50 to 99 percent cortex but have both lost 31 percent of their original length. In contrast, GR is a multiple core with 1 to 50 percent cortex that has lost 48 percent of its length, and S2 and US are multiple cores that have 50 to 99 percent cortex remaining and have lost 15 percent and 46 percent of their lengths. This suggests that multiple cores initially lose length, but once they are rotated, all dimensions are reduced in a similar manner. In contrast, unifacially flaked cores lose long axis length very rapidly as the cortex is lost (that is, as the core is reduced).

Figure 4.14 displays the mean core lengths, widths, and thicknesses for each of the analyzed assemblages, where these dimensions were recorded, relative to the position of the longest remaining flake scar on the cores. The lines in the graph emphasize the changes in relative dimensions in the cores among the assemblages. For the E29H1 assemblages B, C, D, E, and G and for XB11, core length (that is, the length following the percussion axis of the longest flake scar) is greater than the corresponding width measurements taken orthogonally to length and thickness measurements taken orthogonally where these two meet. However, for E29H1 A and F, core widths are close to the same size as lengths. For the L1T24 and L1T5 assemblages, widths are considerably higher than mean lengths. These results suggest that cores were formed by working cobbles in different ways in some assemblages. The two L1 assemblages, for instance, have cores that are wide relative to their lengths, while those from E29H1, with the exception of $\mathrm{A}$ and $\mathrm{F}$, have cores that are long and wide relative to their thicknesses. This suggests a different orientation of flaking, with the E29H1 assemblages flaked parallel to the longest axis of the cobble, thereby reducing cobble thickness, while the cores from the $\mathrm{L} 1$ assemblages are flaked across the width of the cobble, thereby reducing cobble length. For most of the E29H1 assemblages, cobbles are reduced such that core length relative to the core flake scars remains relatively unchanged while the corresponding thickness measurement reduces, while for the L1 assemblages, and some of those from E29H1, it is width that remains relatively unchanged while thickness also reduces.

Ratios of the mean axis dimensions indicate relative size differences for cores of different types (Table 4.18). Cores from E29H1 and XB11 indicate that the axis parallel to the longest flake scar is large relative to both the axes orthogonal to this orientation. However, the two L1 assemblages and the G assemblage from $\mathrm{E} 29 \mathrm{H} 1$ show different results for unifacial cores and to some degree bifacial and multiple cores. In these cases, a number of ratios show that the largest differences are between core width and thickness. These results indicate, as do the results in Figure 4.14, that the L1 assemblages have cores formed from cobbles that are closest to scalene ellipsoids, with flaking progressing by striking along the shortest axis. The cobbles used at E29H1 also likely had a scalene ellipsoid shape but were flaked by striking along the longest axis. Unifacial and bifacial cores were flaked in this way until they were abandoned. However, some core forms were rotated in the sense that the core was rotated and new platforms were struck. This resulted in some bidirectional forms, struck from two platforms at opposite ends of the core, and more common multiple platform cores where several platforms were used. For these cores there is no simple relationship between platform location and cobble shape.

The differences in the core reduction strategies are reflected in flakes removed from the cores. Complete flakes, discussed in more detail below, produced from the L1 and XB11 assemblages are wide relative to their length, reflected in low values for the mean ratio of flake length divided by width (Table 4.19). Flakes from E29H1 are more "blade-like," in the sense that a number of assemblages have higher flake-length-to-flake-width ratios, although in no cases does this ratio exceed 2.0 in value. 


\begin{tabular}{|c|c|c|c|c|c|c|c|c|c|c|c|}
\hline 总离 & 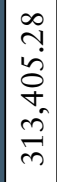 & 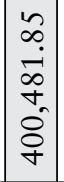 & $\mid \begin{array}{c}0 \\
\infty \\
1 \\
\hat{0} \\
0 \\
0 \\
0 \\
-1\end{array}$ & $\mid \begin{array}{l}\sigma \\
\dot{n} \\
\bar{\sigma} \\
\alpha \\
\hat{\sim}\end{array}$ & 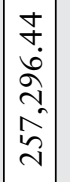 & 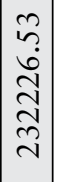 & $\mid \begin{array}{c}0 \\
\infty \\
\infty \\
\infty \\
\infty \\
m \\
\infty \\
\infty \\
n \\
n\end{array}$ & 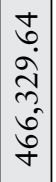 & & 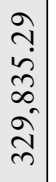 & $\begin{array}{l}R \\
\text { à } \\
\hat{n} \\
\sigma \\
\dot{d}\end{array}$ \\
\hline 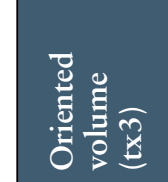 & $\begin{array}{l}0 \\
2 \\
\tilde{n} \\
0 \\
0 \\
\tilde{n} \\
\tilde{n}\end{array}$ & 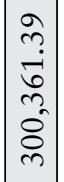 & $\left|\begin{array}{c}0 \\
+ \\
0 \\
0 \\
0 \\
\infty \\
\infty \\
1\end{array}\right|$ & $\mid \begin{array}{l}n \\
\dot{0} \\
\tilde{\alpha} \\
\hat{\partial} \\
\tilde{\nu}\end{array}$ & $\mid \begin{array}{c}n \\
\tilde{n} \\
i \\
\hat{\alpha} \\
\hat{\Omega} \\
\tilde{z}\end{array}$ & 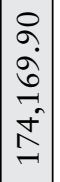 & 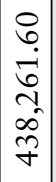 & 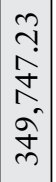 & & 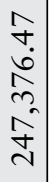 & 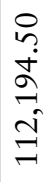 \\
\hline 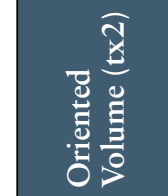 & 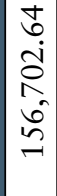 & 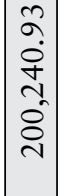 & $\begin{array}{l}n \\
\infty \\
\infty \\
n \\
\hat{n} \\
\hat{n}\end{array}$ & $\begin{array}{l}2 \\
\hat{n} \\
2 \\
\alpha \\
2 \\
n\end{array}$ & 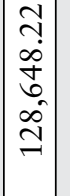 & $\mid \begin{array}{l}0 \\
\sim \\
n \\
= \\
ت \\
0 \\
= \\
=\end{array}$ & 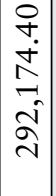 & 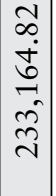 & & 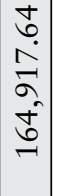 & $\begin{array}{l}\stackrel{9}{+} \\
\dot{0} \\
\stackrel{1}{1} \\
\text { i }\end{array}$ \\
\hline 总泀 & 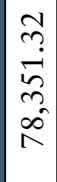 & 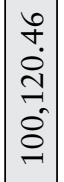 & 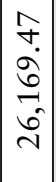 & $\left|\begin{array}{l}\infty \\
\alpha \\
\infty \\
\hat{\alpha} \\
\sigma \\
b\end{array}\right|$ & 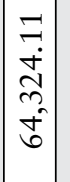 & $\left|\begin{array}{c}6 \\
6 \\
0 \\
0 \\
0 \\
\infty \\
n \\
n\end{array}\right|$ & $\mid \begin{array}{c}0 \\
1 \\
1 \\
\infty \\
0 \\
0 \\
0 \\
\\
-1\end{array}$ & $\begin{array}{c}\vec{J} \\
i \\
0 \\
0 \\
n \\
0 \\
=\end{array}$ & & $\begin{array}{c}1 \\
\infty \\
\infty \\
i n \\
n \\
0 \\
\infty \\
\infty\end{array} \mid$ & $\begin{array}{l}\text { ते } \\
\infty \\
\infty \\
n^{2} \\
\text { ñ }\end{array}$ \\
\hline 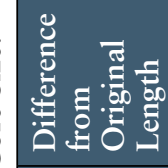 & 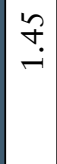 & $\mid$\begin{tabular}{c}
$n$ \\
$\stackrel{n}{-}$ \\
\hdashline
\end{tabular} & $n$ & $\begin{array}{l}\hat{a} \\
\dot{\lambda}\end{array}$ & $\begin{array}{l}\Omega \\
\text { ¿े }\end{array}$ & 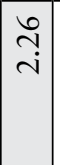 & $\stackrel{\overbrace{}}{\stackrel{\longrightarrow}{-}}$ & 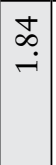 & & $\begin{array}{c}\mathbf{D} \\
\mathrm{i}\end{array} \mid$ & $\hat{0}$ \\
\hline 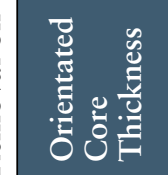 & 8 & 市 & @ & $\stackrel{n}{q}$ & ळे & f & $\infty$ & in & & & \\
\hline 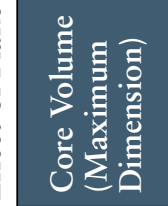 & $\begin{array}{l}\tilde{r} \\
\dot{0} \\
0 \\
\vdots \\
i \\
\infty\end{array}$ & 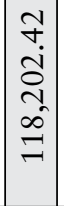 & 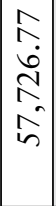 & $\left|\begin{array}{c}n \\
0 \\
\dot{d} \\
\dot{n} \\
0 \\
a \\
a\end{array}\right|$ & $\left|\begin{array}{c|}\infty \\
\dot{d} \\
\dot{\infty} \\
\dot{\infty} \\
\dot{\sigma} \\
\dot{\sigma}\end{array}\right|$ & $\left|\begin{array}{c}5 \\
\hat{\sigma} \\
\stackrel{0}{1} \\
\hat{\sigma} \\
\sigma\end{array}\right|$ & $\mid \begin{array}{c}1 \\
\vdots \\
0 \\
\hat{n} \\
\tilde{n} \\
2 \\
2\end{array}$ & 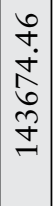 & & & \\
\hline 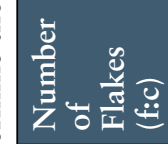 & 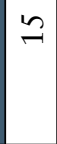 & 6 & $\vec{\sim}$ & $\vec{\sim}$ & 守 & q & 10 & $\sim$ & & $\tilde{v}$ & $\stackrel{+}{ \pm}$ \\
\hline نे & $\begin{array}{l}\stackrel{0}{2} \\
\sigma \\
1 \\
1 \\
n\end{array}$ & ○े & $\mid \begin{array}{c}0 \\
0 \\
0 \\
1 \\
1 \\
-1\end{array}$ & $\begin{array}{l}0 \\
\circ \\
\hat{1} \\
0 \\
i n\end{array}$ & $\mid \begin{array}{l}0 \\
0 \\
0 \\
1 \\
1\end{array}$ & $\mid \begin{array}{l}0 \\
8 \\
0 \\
1 \\
-1\end{array}$ & $\mid \begin{array}{c}o 0 \\
\partial \\
\hat{d} \\
i \\
i n\end{array}$ & $\begin{array}{l}\partial^{0} \\
\partial \\
\hat{\alpha} \\
i \\
n\end{array}$ & & & \\
\hline$\theta_{1}^{2}$ & $\bar{Z}$ & $\bar{z}$ & $\bar{z}$ & $\vec{n}$ & $\vec{\infty}$ & 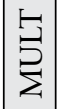 & 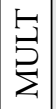 & $\begin{array}{l}\stackrel{5}{5} \\
\stackrel{3}{\Sigma}\end{array}$ & & & \\
\hline 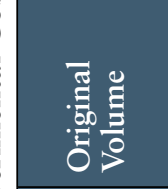 & 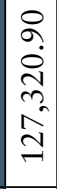 & 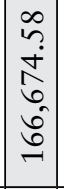 & 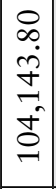 & 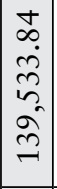 & 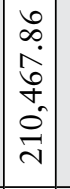 & 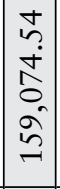 & $\mid \begin{array}{c}0 \\
\tilde{n} \\
\tilde{n} \\
0 \\
\infty \\
-\tilde{n} \\
\vec{n}\end{array}$ & $\begin{array}{l}q \\
\dot{q} \\
\vec{n} \\
\tilde{n} \\
\vec{n} \\
\vec{v}\end{array}$ & & 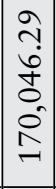 & $\begin{array}{l}R \\
\hat{\sigma} \\
\hat{\sigma} \\
\hat{0} \\
+\end{array}$ \\
\hline 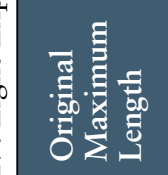 & $\infty$ & $\hat{O}$ & ㅇ & ন & \pm & $\tilde{a}$ & 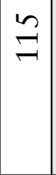 & $\hat{0}$ & & $\begin{array}{l}0 \\
\dot{0} \\
\dot{8} \\
0\end{array}$ & $\stackrel{\hat{o}}{\ominus}$ \\
\hline $8 \Omega$ & 急 & $\bar{n}$ & $\tilde{s}$ & 비 & 㕦 & 寽 & ஸี & $\tilde{D}$ & $\bar{z}$ & $\stackrel{\Xi}{\Xi}^{\Xi}$ & के \\
\hline
\end{tabular}




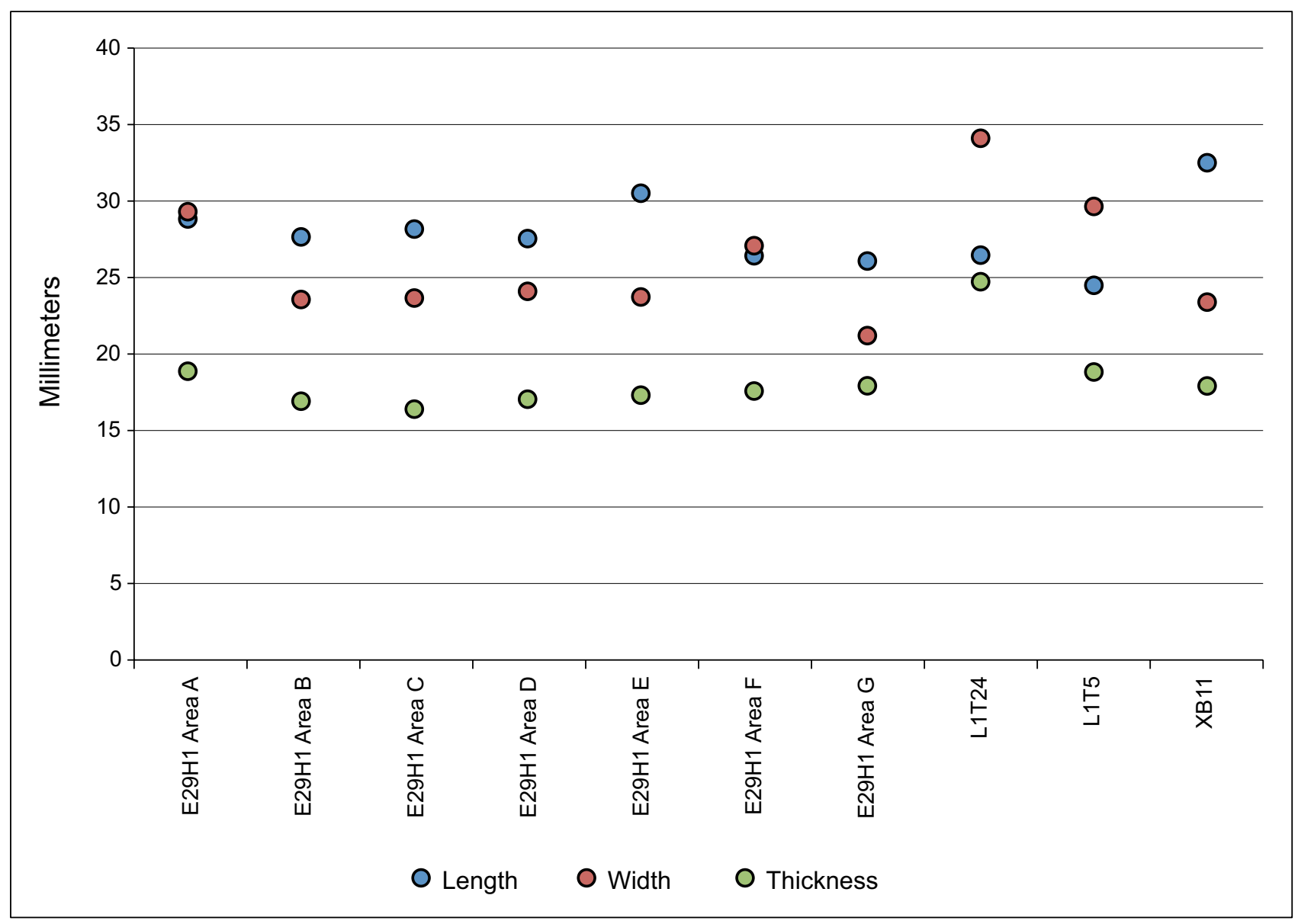

Figure 4.14. Mean core dimensions (in $\mathrm{mm}$ ) oriented relative to the largest core scar.

Table 4.18. Ratio of Mean Core Axis Dimensions for Core Types.

\begin{tabular}{|c|c|c|c|c|c|c|c|c|c|c|c|}
\hline & & E29H1 A & E29H1 B & E29H1 C & E29H1 D & E28H1 E & E29H1 F & E29H1 G & L1T24 & L1T5 & XB11 \\
\hline \multirow[t]{3}{*}{ Unifacial } & $A: B$ & 1.13 & 1.23 & 1.24 & 1.27 & 1.62 & 1.08 & 1.90 & 0.82 & 0.90 & 1.37 \\
\hline & A:C & 1.99 & 1.74 & 1.96 & 2.20 & 2.14 & 1.64 & 1.59 & 1.29 & 1.53 & 1.73 \\
\hline & $\mathrm{B}: \mathrm{C}$ & 1.83 & 1.56 & 1.74 & 1.76 & 1.59 & 1.63 & 1.28 & 1.73 & 1.76 & 1.27 \\
\hline \multirow[t]{3}{*}{ Bifacial } & A:B & 1.13 & 1.37 & 1.41 & 1.13 & 1.13 & 1.06 & 1.43 & 1.14 & 0.91 & 1.28 \\
\hline & A:C & 2.02 & 1.85 & 1.81 & 1.73 & 1.73 & 1.78 & 1.83 & 1.57 & 1.52 & 2.11 \\
\hline & $\mathrm{B}: \mathrm{C}$ & 1.92 & 1.48 & 1.48 & 1.62 & 1.62 & 1.83 & 1.31 & 1.48 & 1.85 & 1.65 \\
\hline \multirow[t]{3}{*}{ Multiple } & $A: B$ & 1.43 & 1.19 & 1.24 & 1.11 & 1.11 & 1.51 & 1.08 & 0.93 & 1.11 & 1.43 \\
\hline & A:C & 1.61 & 1.72 & 1.86 & 1.73 & 1.73 & 1.64 & 2.02 & 1.60 & 1.60 & 1.83 \\
\hline & B:C & 1.43 & 1.54 & 1.62 & 1.59 & 1.59 & 1.74 & 2.10 & 1.84 & 1.54 & 1.28 \\
\hline
\end{tabular}


Core types differ in proportion among the assemblages (Table 4.20). Flake cores, including side-struck or so called tranchet forms, are found only in the E29H1 assemblages, while both test cores (that is, cobbles with two or fewer flake removals) and multiple cores (discussed above) are more common in these assemblages compared to the two L1 assemblages. Multiple cores represent around 10 percent of all assemblages. In all assemblages, around half the cores retain more than 50 percent cortex (Table 4.20).

High proportions of cortex on the cores that remain at $\mathrm{E} 29 \mathrm{H} 1$ are significant given that no local flint sources are present on the Fayum north shore. Cobbles of stone moved into the north shore region were used to manufacture flakes, but these cores were abandoned without their full utility expended in the sense that more flakes could have been removed. Even those cores with multiple platforms retain quantities of cortex (Table 4.21). However, as noted above, experimental cobble working indicates that cores with cortex may still have lost significant surface area compared to the size of the cobble that was originally flaked.

Unifacial and bifacial cores with more than 50 percent cortex provide a means of estimating the size of the cobbles that were originally flaked. Bifacial cores were typically worked by removing an initial flake and then using the consequent flake scar as a platform. Both core types retain this morphology, with the bulk of the flake removals occurring on a single core surface. As discussed above, cores in the L1 assemblages indicate that flaking occurred through the shortest cobble axis, while the majority of the cores from the E29H1 assemblages were flaked along the longest cobble axis. For the L1 cores, flake removal leaves the dimensions
Table 4.19. Mean Complete Flake Length to Flake Width Ratios.

\begin{tabular}{|l|r|}
\hline & Length to Width Ratio \\
\hline E29H1 A & 1.30 \\
\hline E29H1 B & 1.94 \\
\hline E29H1 C & 1.78 \\
\hline E29H1 D & 1.60 \\
\hline E28H1 E & 1.85 \\
\hline E29H1 F & 1.34 \\
\hline E29H1 G & 1.51 \\
\hline L1T24 & 1.17 \\
\hline L1T5 & 1.21 \\
\hline XB11 & 1.17 \\
\hline
\end{tabular}

of the flake surface relatively unchanged, but the thickness reduces as measured through the core relative to the core surface from which flakes are removed. As indicated by the experimental core working results, it is possible to use this process to reconstruct the size of the cobbles used for cores by assuming that those with 50 percent or more cortex have lost a proportion of their core thickness. Length and width measurements, together with the thickness trebled, accounting for material removed by flaking using the estimate from experimental flaking above, can therefore be used to estimate the size of the original cobbles flaked using the formula for the volume of a scalene ellipsoid:

Volume $=4 / 3 \times \pi \times$ a-semiaxis $\times$ b-semiaxis $\mathrm{x}$ c-semiaxis

Table 4.20. Core Proportions with Proportion of Cortex Present by Assemblage.

\begin{tabular}{|l|r|r|r|r|r|r|r|r|r|r|}
\hline & E29H1A & & E29H1B & & E29H1C & E29H1D & E29H1E & \\
\hline $1-50 \%$ & 22 & $43.14 \%$ & 80 & $43.48 \%$ & 74 & $40.44 \%$ & 85 & $38.12 \%$ & 101 & $43.91 \%$ \\
\hline $51-99 \%$ & 28 & $54.90 \%$ & 99 & $53.80 \%$ & 106 & $58 \%$ & 135 & $60.54 \%$ & 127 & $55.22 \%$ \\
\hline None & 1 & $1.96 \%$ & 5 & $2.72 \%$ & 3 & $1.64 \%$ & 3 & $1.35 \%$ & 2 & $0.87 \%$ \\
\hline
\end{tabular}


Table 4.21. Cortex Proportion on Cores with Different Forms. E29H1

\begin{tabular}{|l|r|r|r|}
\hline Core Type & 1-50\% & 51-99\% & None \\
\hline Bidirectional & 50 & 29 & 2 \\
\hline Bifacial & 97 & 139 & 7 \\
\hline Flake & 30 & 12 & 3 \\
\hline Microblade & 5 & 1 & 0 \\
\hline Multiple & 146 & 53 & 3 \\
\hline Nuclear tool & 5 & 3 & 0 \\
\hline Test & 0 & 79 & 0 \\
\hline Tranchet & 0 & 1 & 0 \\
\hline Unifacial & 112 & 301 & 4 \\
\hline
\end{tabular}

L1 and XB11 Assemblages.

\begin{tabular}{|l|r|r|r|}
\hline Core Type & $1-50 \%$ & $50-99 \%$ & None \\
\hline Bidirectional & 3 & 5 & 1 \\
\hline Bifacial & 43 & 13 & 1 \\
\hline Microblade & 2 & 0 & 1 \\
\hline Multiple & 89 & 11 & 19 \\
\hline Test & 3 & 15 & 0 \\
\hline Tranchet & 5 & 0 & 0 \\
\hline Unifacial & 58 & 91 & 11 \\
\hline
\end{tabular}

Results were log-transformed since they were skewed, and the antilog of the mean was calculated.

For the E29H1 assemblages, length also remains relatively constant as flaking progresses for unifacial and bifacial cores. Because the orientation of the core measurements is relative to the longest remaining core flake scar, thickness also reduces for these cores as flaking progresses. Thus the same scalene ellipsoid volume formula is used, with the thickness dimension trebled to model original core size. Figure 4.15 shows the results for each of the analyzed assemblages. Cobble size estimates from the E29H1 E and F assemblages and from the L1T5 assemblage are close to one another and are higher than those from the other E29H1 assemblages. The largest cobble size estimates are for L1T24.

Figure 4.16 shows the ordered flake-to-core ratio for all assemblages recorded in L1 and E29H1. These were calculated by dividing the sum of all artifacts with a platform by the number of cores. The figure shows a more or less continuous distribution of flaketo-core ratio values from a low of around two flakes per core to a high of just under 11 flakes per core. A more refined measure is possible for assemblages that were fully analyzed, where the size of the original cobble, estimated in Figure 4.15, is used to relate the

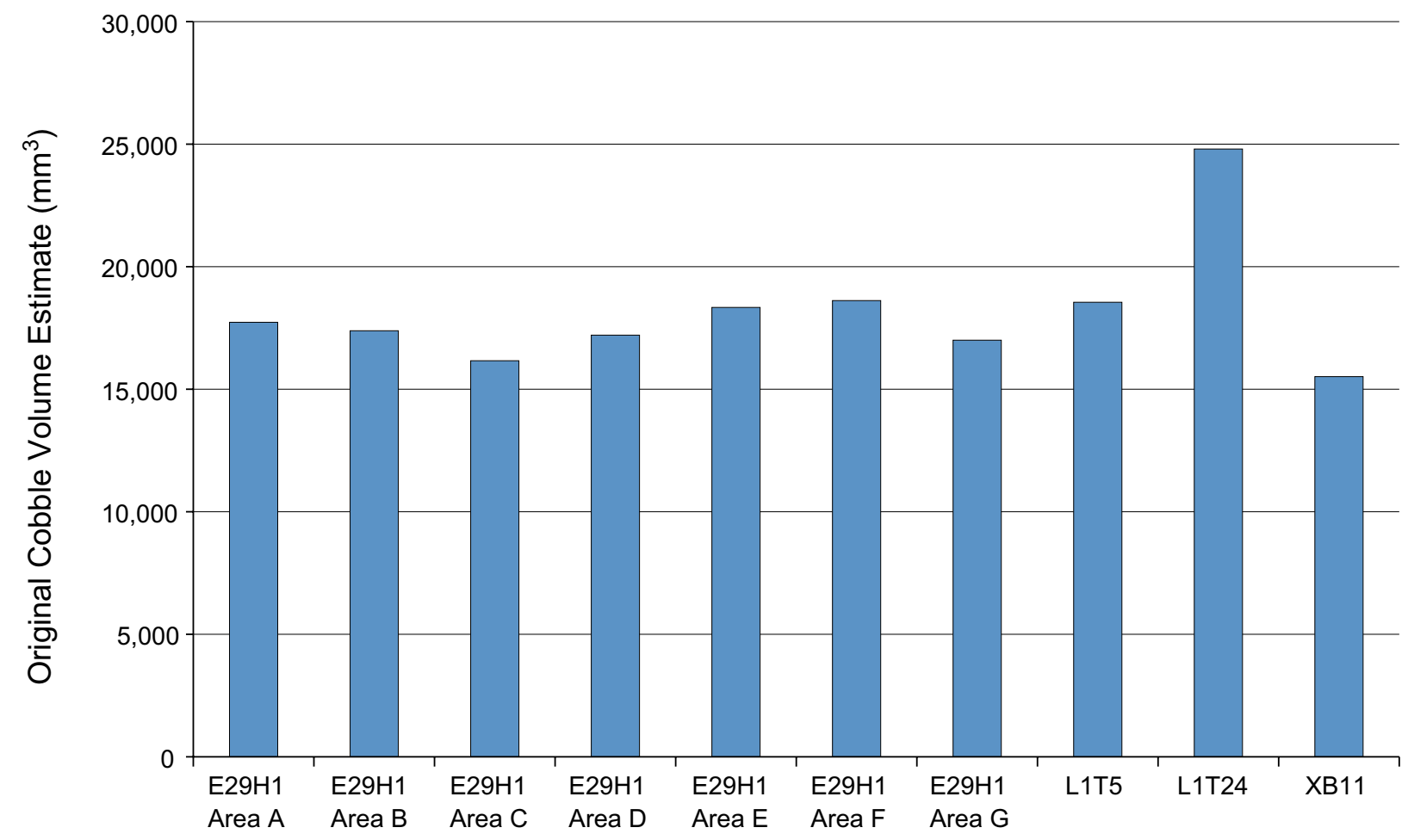

Figure 4.15. Original cobble volume estimates for analyzed assemblages in $\mathrm{mm}^{3}$. 
flake-to-core ratio values to the size of the cobble that was flaked (Barrett 2014). In Figure 4.17, values for the 10 analyzed assemblages are graphed, calculated by dividing the flake-to-core ratio by the estimated cobble volume. The numerator is multiplied by 10,000 to shift the range of values from 0 to 1.2. The same continuous distribution of values indicated in Figure 4.16 is indicated in this figure. However, comparing the results in Figure 4.17 to those in Figure 4.15 shows that the estimated size of the original cobbles does not correlate closely with the flake-to-core ratio values. For instance, E29H1 E and F have relatively large estimated cobble sizes but have very low corrected flake-to-core ratio values. The same is true for the L1T24 assemblage.

\section{Flakes}

It is interesting to compare the results in Figure 4.15 with the size of the complete flakes from the different assemblages (Table 4.22). Analysis of variance (ANOVA) results indicate significant differences among the means, while Bonferroni post hoc comparisons among the means indicate that the differences between the L1T5, L1T24, and E29H1 G assemblages, and to some degree the XB11 assemblages, and all other assemblages are significant, reflecting the relatively short complete flakes from these areas. E29H1 $\mathrm{E}$ is also significantly different from other assemblages, reflecting the relatively large mean size of flakes from this assemblage. ${ }^{9}$ The estimated cobble size based on the cores (Figure 4.15) is relatively larger for those assemblages with smaller mean complete flakes (Table 4.22). It might be imagined that larger flakes would come from the larger cobbles, but this is in fact not the case, suggesting that the flake-to-core ratio does indeed indicate higher levels of reduction in the L1T5, L1T24, and $\mathrm{E} 29 \mathrm{H} 1 \mathrm{G}$ assemblages.

It is interesting to consider why the mean flake lengths from L1T24, L1T5, and E29H1 G, and to some degree XB11, are smaller than the other E29H1 assemblages. The difference does not relate to the shifts in the sizes of artifacts with different proportions of cortex. Among the assemblages, those flakes that lack

9 Length $F=24.423 ; d f=9,6,364 ; p<0.001$. Width $F=59.583$; $d f=9,6,364 ; p<0.001$. Thickness $F=14.102 ; d f 9,6,364 ; p<$ 0.001 .

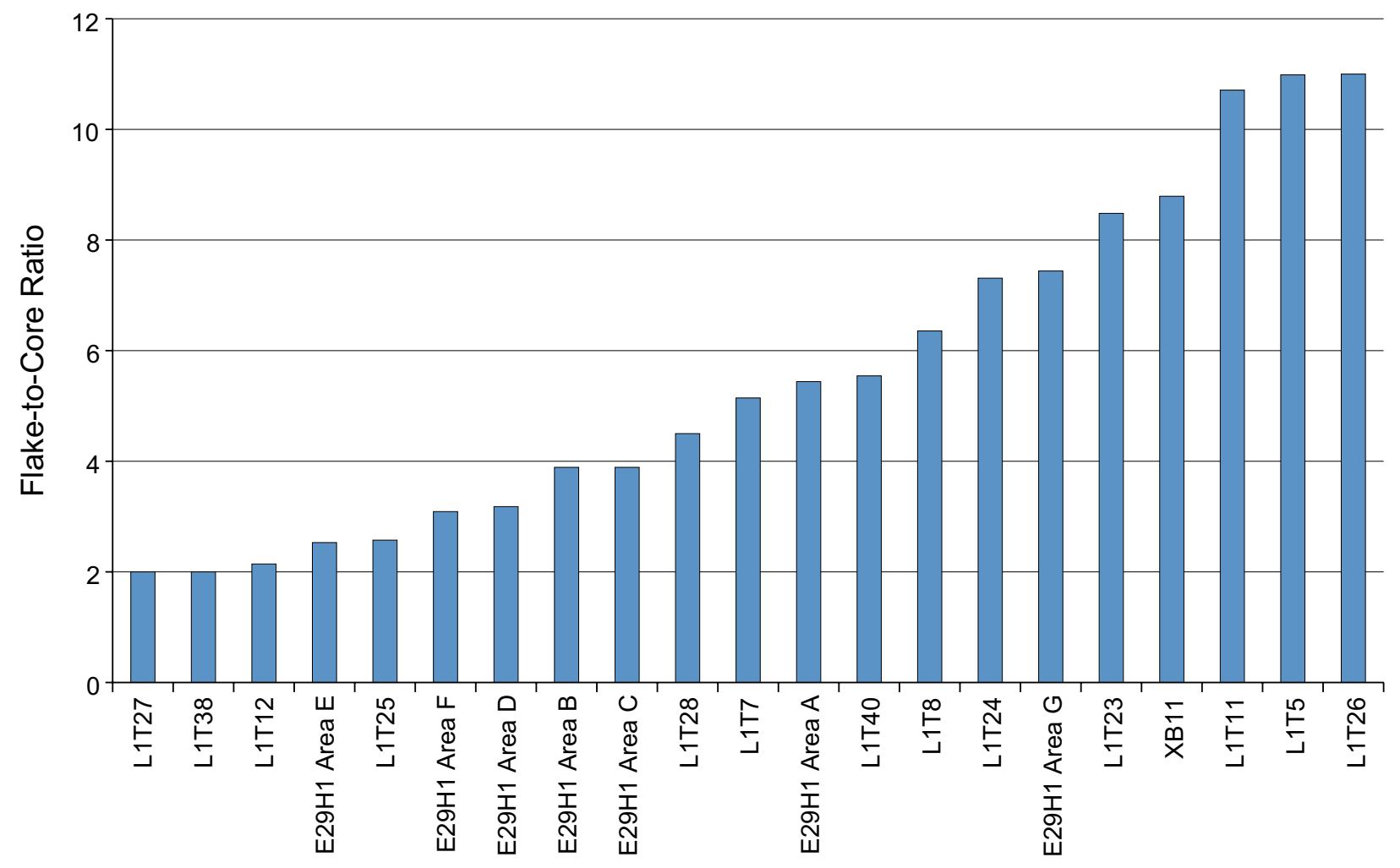

Figure 4.16. Flake-to-core ratios in order for all assemblages in L1 and E29H1. 


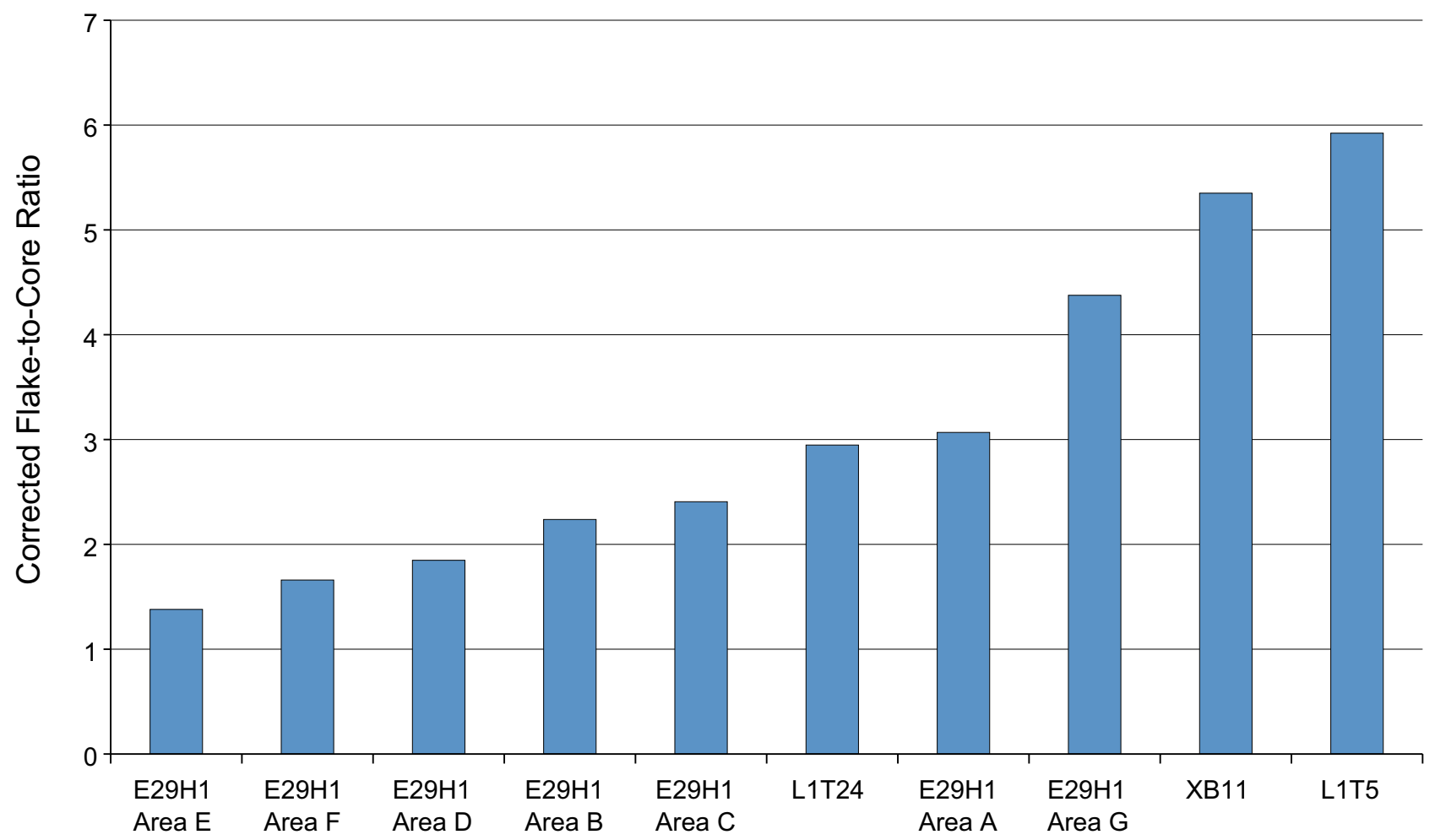

Figure 4.17. Ordered flake-to-core ratio divided by estimated cobble volume, calculated from unifacial and bifacial cores with more than 50 percent cortex (see text).

Table 4.22. Mean Complete Flake Dimensions for All Flakes and Those with Different Cortex Proportions.

\begin{tabular}{|c|c|c|c|c|c|c|c|}
\hline E29H1 A & $\mathbf{N}$ & Length Mean & SD & Width Mean & SD & Thickness Mean & SD \\
\hline $1-50 \%$ & 152 & 26.05 & 8.97 & 23.81 & 8.38 & 6.66 & 3.42 \\
\hline $51-99 \%$ & 71 & 25.11 & 7.47 & 22.93 & 8.64 & 7.76 & 2.69 \\
\hline $100 \%$ & 7 & 26.86 & 6.04 & 23.71 & 6.47 & 6.43 & 1.13 \\
\hline None & 29 & 26.76 & 7.68 & 22.28 & 8.28 & 5.66 & 2.53 \\
\hline Total & 259 & 25.89 & 8.35 & 23.39 & 8.37 & 6.85 & 3.15 \\
\hline \multicolumn{8}{|l|}{ E29H1 B } \\
\hline $1-50 \%$ & 323 & 25.20 & 7.49 & 15.72 & 5.34 & 5.32 & 2.55 \\
\hline $51-99 \%$ & 120 & 27.45 & 9.66 & 16.64 & 5.55 & 7.25 & 2.90 \\
\hline $100 \%$ & 67 & 24.82 & 7.03 & 16.99 & 4.85 & 7.27 & 3.22 \\
\hline None & 143 & 24.36 & 7.73 & 15.75 & 6.37 & 5.10 & 2.96 \\
\hline Total & 653 & 25.39 & 7.99 & 16.02 & 5.58 & 5.82 & 2.92 \\
\hline \multicolumn{8}{|l|}{ E29H1 C } \\
\hline $1-50 \%$ & 312 & 25.26 & 9.12 & 16.97 & 6.40 & 6.02 & 3.98 \\
\hline $51-99 \%$ & 194 & 26.99 & 8.89 & 17.68 & 6.19 & 6.93 & 3.13 \\
\hline $100 \%$ & 86 & 26.53 & 7.21 & 17.65 & 6.21 & 8.14 & 7.75 \\
\hline None & 67 & 24.15 & 8.17 & 15.13 & 5.73 & 4.31 & 2.36 \\
\hline Total & 659 & 25.82 & 8.76 & 17.08 & 6.28 & 6.39 & 4.44 \\
\hline
\end{tabular}




\begin{tabular}{|c|c|c|c|c|c|c|c|}
\hline E29H1 D & $\mathbf{N}$ & Length Mean & SD & Width Mean & SD & Thickness Mean & SD \\
\hline $1-50 \%$ & 285 & 24.99 & 8.48 & 17.68 & 5.06 & 5.83 & 2.28 \\
\hline $51-99 \%$ & 270 & 26.57 & 7.60 & 17.98 & 5.24 & 7.36 & 2.83 \\
\hline $100 \%$ & 83 & 25.23 & 5.89 & 19.60 & 4.36 & 8.49 & 3.24 \\
\hline None & 53 & 25.47 & 10.75 & 16.92 & 7.14 & 5.53 & 3.12 \\
\hline Total & 691 & 25.67 & 8.09 & 17.97 & 5.27 & 6.72 & 2.87 \\
\hline \multicolumn{8}{|l|}{ E29H1 E } \\
\hline $1-50 \%$ & 226 & 27.10 & 8.02 & 16.87 & 5.72 & 5.94 & 2.51 \\
\hline $51-99 \%$ & 171 & 29.13 & 8.36 & 17.48 & 5.79 & 7.12 & 3.01 \\
\hline $100 \%$ & 105 & 27.63 & 7.79 & 18.58 & 5.24 & 8.11 & 3.47 \\
\hline None & 43 & 28.37 & 7.70 & 16.51 & 6.52 & 4.88 & 2.12 \\
\hline Total & 545 & 27.94 & 8.09 & 17.36 & 5.74 & 6.65 & 3.01 \\
\hline \multicolumn{8}{|l|}{ E29H1 F } \\
\hline $1-50 \%$ & 207 & 25.80 & 7.00 & 22.54 & 6.78 & 6.93 & 2.66 \\
\hline $51-99 \%$ & 87 & 27.36 & 8.32 & 20.07 & 5.49 & 7.93 & 2.88 \\
\hline $100 \%$ & 17 & 22.41 & 6.08 & 20.65 & 4.92 & 7.00 & 2.72 \\
\hline None & 51 & 28.76 & 7.69 & 20.96 & 5.86 & 7.04 & 5.97 \\
\hline Total & 362 & 26.43 & 7.50 & 21.63 & 6.35 & 7.19 & 3.38 \\
\hline \multicolumn{8}{|l|}{$\mathrm{E} 29 \mathrm{H} 1 \mathrm{G}$} \\
\hline $1-50 \%$ & 308 & 23.71 & 6.47 & 18.23 & 5.47 & 5.39 & 2.03 \\
\hline $51-99 \%$ & 164 & 23.71 & 7.13 & 18.27 & 5.28 & 6.84 & 2.97 \\
\hline $100 \%$ & 24 & 22.29 & 6.52 & 18.54 & 5.80 & 8.63 & 4.92 \\
\hline None & 117 & 22.74 & 5.17 & 16.95 & 5.78 & 4.82 & 1.97 \\
\hline Total & 613 & 23.47 & 6.44 & 18.01 & 5.50 & 5.79 & 2.63 \\
\hline \multicolumn{8}{|l|}{ L1T5 } \\
\hline $10 \%$ & 104 & 22.06 & 6.59 & 19.88 & 5.78 & 5.59 & 2.10 \\
\hline $1-50 \%$ & 260 & 23.31 & 7.49 & 21.19 & 6.67 & 6.66 & 2.64 \\
\hline $50-99 \%$ & 132 & 23.98 & 8.56 & 20.55 & 6.52 & 7.39 & 2.99 \\
\hline $100 \%$ & 68 & 22.91 & 6.86 & 21.32 & 6.15 & 6.51 & 2.32 \\
\hline None & 106 & 22.02 & 7.03 & 19.23 & 6.44 & 5.23 & 2.49 \\
\hline Total & 670 & 23.00 & 7.47 & 20.56 & 6.44 & 6.39 & 2.68 \\
\hline \multicolumn{8}{|l|}{ L1T24 } \\
\hline $10 \%$ & 82 & 22.65 & 7.42 & 20.18 & 7.10 & 5.68 & 2.92 \\
\hline $1-50 \%$ & 262 & 22.97 & 7.61 & 22.27 & 7.86 & 6.60 & 2.87 \\
\hline $50-99 \%$ & 91 & 23.68 & 7.30 & 22.25 & 8.18 & 7.12 & 2.78 \\
\hline $100 \%$ & 29 & 25.17 & 8.47 & 21.76 & 7.36 & 7.21 & 2.92 \\
\hline None & 120 & 20.78 & 6.60 & 19.46 & 6.71 & 4.95 & 2.51 \\
\hline Total & 584 & 22.69 & 7.44 & 21.37 & 7.63 & 6.24 & 2.90 \\
\hline \multicolumn{8}{|l|}{ XB11 } \\
\hline $1-50 \%$ & 668 & 25.21 & 9.22 & 21.97 & 7.94 & 6.02 & 2.83 \\
\hline $50-99 \%$ & 204 & 25.88 & 9.50 & 21.87 & 8.18 & 7.31 & 4.41 \\
\hline $100 \%$ & 68 & 24.76 & 8.68 & 21.38 & 9.14 & 5.99 & 3.23 \\
\hline None & 298 & 21.96 & 8.59 & 17.71 & 7.38 & 4.49 & 2.59 \\
\hline Total & 1238 & 24.51 & 9.20 & 20.89 & 8.11 & 5.86 & 3.24 \\
\hline
\end{tabular}


cortex are significantly thinner than those that have dorsal cortex but are only sometimes significantly shorter. Flakes with cortex are significantly longer than those without for L1T24, E29H1 B, and E29H1 F. This likely reflects the way the flint cobbles were reduced. As discussed above, flint cobbles have the shape of scalene ellipsoids. This, combined with the relatively short and wide nature of the flakes indicated by the lack of difference between mean lengths and widths of complete flakes (Table 4.22), suggests that cores were struck along the c-semiaxis rather than down the longest a-semiaxis. Given the oval cross-section of scalene ellipsoid-shaped cobbles, flakes will tend to have dorsal cortex at all stages of core reduction. Only flakes struck from the center of the core will retain no cortex, and while these flakes do tend to have smaller mean dimensions than those with cortex, the differences are only significant for some of the assemblages (Phillipps 2012; Phillipps and Holdaway 2016). The dimension that shows the most consistent, significant difference is in fact flake thickness. Those flakes that lack cortex tend to be thinner than those where cortex is present. However, the mean differences are not large in any of the cases, and they seem to relate to subtle differences in the way cores were worked rather than to substantial shifts in reduction technology as core working progressed.

Cortex provides a means to assess the completeness of the assemblages, since if flaking occurred in situ, all flakes produced should be present in the assemblage. This can be assessed by comparing the surface area of cortex remaining in the assemblage against the surface area of cortex present on the cobbles that were originally flaked, expressed as the cortex ratio. The surface area of flakes and flake fragments (that is, proximal, distal, and medial flakes together with flake fragments) was calculated, and the product of maximum length times width was multiplied by the proportion of the artifact covered in cortex. (For example, flakes with 1 to 50 percent cortex had their surface areas multiplied by 0.25 .) For cores, the formula for a surface area of a scalene ellipsoid was used:

Surface area $=4 \pi\left[\left(a^{\mathrm{p}} b^{\mathrm{p}}+\mathrm{a}^{\mathrm{p}} \mathrm{C}^{\mathrm{p}}+\mathrm{b}^{\mathrm{p}} \mathcal{C}^{\mathrm{p}}\right) / 3\right]^{1 / \mathrm{p}}$, where $a, b$, and $c$ are the clast semiaxes while the constant $\mathrm{p}$ has a value of 1.6075 (Thomsen 2004)

The resulting values are approximations of the true surface areas with an error of 1.061 percent.
The observed cortical surface area is the summed cortical surface area from all flaked pieces together with the cortical surface remaining on the cores. The expected surface area calculation requires two variables: the surface area of the cobbles flaked and the number of these cobbles worked to form the assemblage.

The surface area of the original cobbles can be estimated from the dimensions of the remaining cores. This estimate assumes that each cobble was flaked to produce a single core, a reasonable assumption since the majority of the cores in the assemblage retain cortex (Table 4.21). As discussed above, cores were apparently worked by striking from one end of the cobble and continuing to work the core from a single platform until the core was abandoned. Given this, one core per cobble seems likely. The remaining surface of the core is therefore a fraction of the surface of the original core. Cores with a greater proportion of cortex are likely to represent a fraction that is closer to the original cobble size. Using this reasoning, these cores have lost volume, and therefore surface area, through flaking along one axis as the core was worked to produce flakes. In the same way the volume of cores was estimated in the discussion above using the formula for a scalene ellipsoid, dimensions can be used to calculate the surface area, with adjustments to the core semiaxis thickness measurement oriented in relation to the surface, with the longest core scar multiplied by three to account for lost volume following the experimental results discussed above.

Figure 4.18 shows the results of the cortex ratio calculations for each of the analyzed assemblages. Blue bars indicate the overall cortex ratio, while red and green bars indicate the relative contributions of cores and flakes to the ratio, respectively. Overall ratio results are higher for the L1 and XB11 assemblages and for E29H1 A, indicating that more cortical surface in these assemblages are present relative to those from the other E29H1 assemblages.

The flake-to-core ratio results shown in Figure 4.16 need to be interpreted in relation to the cortex ratio results. Removal of flakes will of course reduce the number of flakes and hence the value of the flake-tocore ratio. The assemblages from E29H1 have low flake-to-core ratios, consistent with results of the cortex ratio calculations. L1T24, however, has a relatively low flake-to-core ratio but a high cortex ratio. This suggests that relatively few flakes were removed from cores and that nearly all are present in the assemblage. 


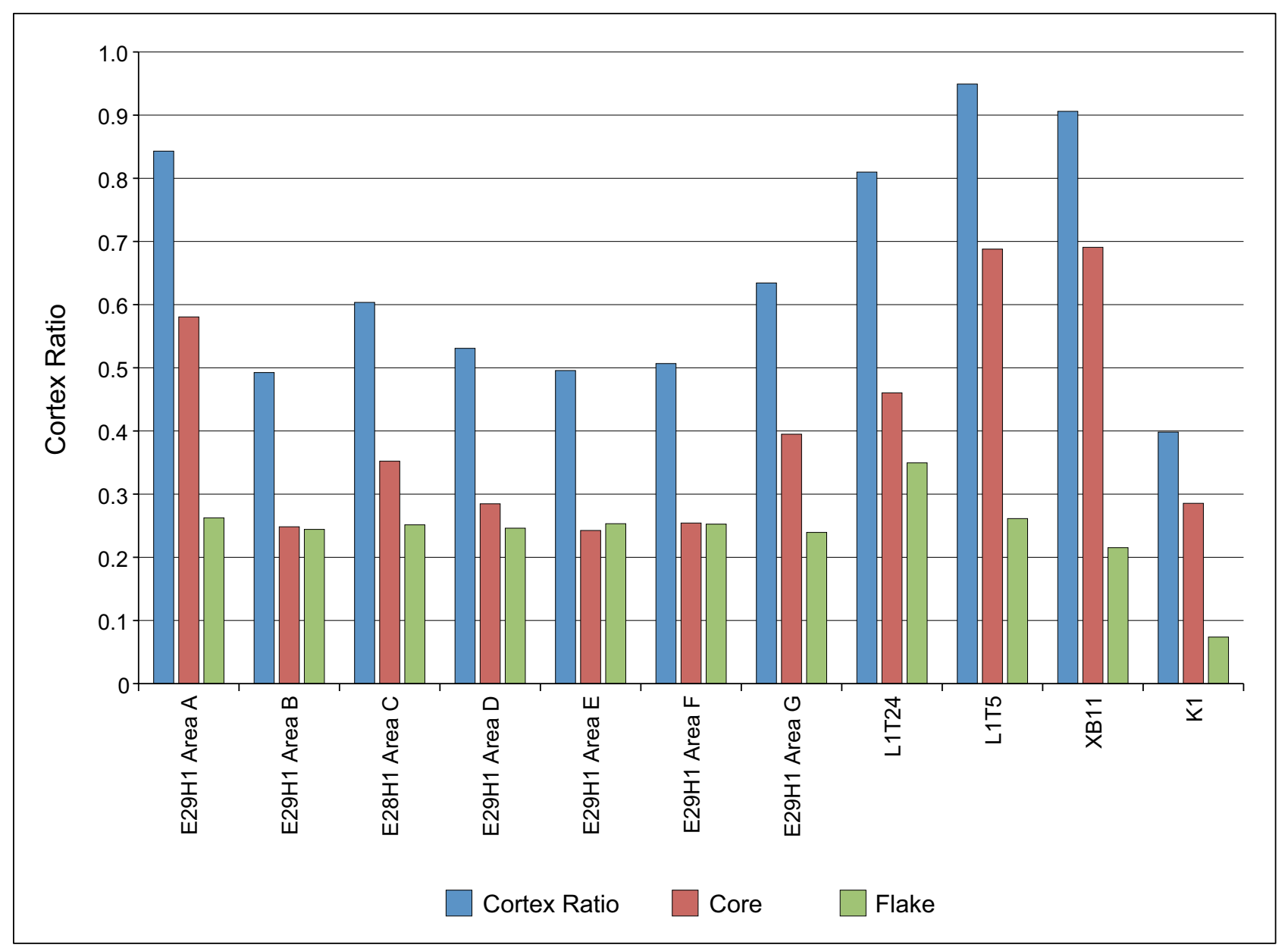

Figure 4.18. Cortex ratio results for analyzed assemblages. Core (red) and flake (green) components sum to form the overall ratio (blue).

A volume calculation helps demonstrate the difference between the E29H1 assemblages. Taken together, the total volume of the assemblages divided by the estimated cobble volume provides an indication of the number of cores that should be present, assuming that each cobble was flaked to produce a single core. This can be expressed as the volume ratio (Phillipps and Holdaway 2016), calculated by dividing the observed volume of flakes and cores by the expected volume based on the number of cores multiplied by the estimated cobble volume. Table 4.23 provides the volume ratio values for each of the assemblages.

Another way of expressing the volume ratio is to consider if there is sufficient volume in the assemblage (that is, the volume of all complete and fragmented flakes together with the volume of cores) to account for the number of cores observed. Table 4.23 provides
Table 4.23. Volume Ratio Values for E29H1 and L1 Assemblages.

\begin{tabular}{|l|r|r|r|}
\hline & \multicolumn{1}{|l|}{$\begin{array}{l}\text { Volume } \\
\text { Ratio }\end{array}$} & $\begin{array}{l}\text { Estimated } \\
\text { Number of } \\
\text { Cores }\end{array}$ & $\begin{array}{l}\text { Observed } \\
\text { Number of } \\
\text { Cores }\end{array}$ \\
\hline E29A & 1.93 & 98 & 51 \\
\hline E29B & 1.29 & 237 & 184 \\
\hline E29C & 1.22 & 225 & 184 \\
\hline E29D & 1.04 & 233 & 224 \\
\hline E29E & 0.81 & 186 & 230 \\
\hline E29F & 1.03 & 127 & 123 \\
\hline E29G & 1.36 & 119 & 87 \\
\hline All E29H1 & 1.12 & 1,218 & 1,083 \\
\hline L1T5 & 5.79 & 458 & 79 \\
\hline L1T24 & 4.67 & 753 & 93 \\
\hline XB11 & 4.42 & 435 & 213 \\
\hline
\end{tabular}


the observed number of cores in each of the assemblages as well as the estimated number of cores calculated by dividing the total volume of all flakes, fragments, and cores by the estimated cobble volume. For the $\mathrm{E} 29 \mathrm{H} 1$ assemblages, the observed volume indicates that slightly more cobbles were worked than accounted for by the observed number of cores. However, for the L1T5, L1T24, and XB11 assemblages, the difference is significantly larger, suggesting that the remaining volume is only a fraction of that required to account for the number of cores actually observed.

These results need to be considered in relation to the cortex ratio calculations discussed above. The E29H1 assemblages have relatively low values for the cortex ratio, indicating that there is insufficient cortical surface area present on the cores and flakes to account for the estimated cortical surface area of the cobbles. This suggests that flakes were removed from the assemblage, particularly those with cortex on their surfaces. Such flakes of course have volume, and removal of these flakes would remove this volume from the observed assemblage. However, the volume of flakes is relatively small compared to the cores; hence the difference between the estimated number of cores and the observed number of cores is also small. For all E29H1 assemblages, the estimated number for cores is 1,218 , whereas the observed number is 1,083 . In contrast, the differences in the observed versus the estimated number of cores for the two L1 and the XB11 assemblages are much larger. There is much less volume in the cores and flakes in these assemblages than that required for the volume of cobbles that were flaked. The cortex ratio values for these assemblages are high relative to the values from the E29H1 assemblages. This suggests that relatively few cortical flakes were removed compared to the numbers that exited the E29H1 assemblages. As noted, removal of flakes reduces cortical surface area but does not reduce volume to the same degree. In contrast, removing cores will remove much volume but relatively little surface area compared to the removal of flakes. The volume and cortex ratio results from the L1 and XB11 assemblages therefore suggest that cores were removed from these assemblages sufficient to deplete the volume of the assemblages that remains but not the cortical surface area. Cortex ratio values below 1 indicate that some cortex was removed but that much cortex remained in the assemblage as flakes removed from the cores before they were transported.
These results indicate a difference in the way stone volume was treated among the assemblages and therefore the how the potential utility of the stone was exploited. (The concept of utility is discussed in Holdaway and Douglass 2015.) For some assemblages, stone was removed as flakes, with the utility expressed as a high proportion of flake edge per unit volume. In other assemblages, utility was removed as volume, as cores with relatively low edge length. Of course, volume removed in this way provided the potential to create more flakes somewhere else, thus transforming volume into flake edge at some later time. As discussed elsewhere (Phillipps 2012; Phillipps and Holdaway 2016; and below), these two strategies may be used as proxies for understanding mobility of the people who made these artifacts.

The two strategies for dealing with the volume of flaked stone are apparent in the form of the flakes that remain in each of the assemblages studied (that is, those that were flaked but deposited rather than transported away). Figure 4.19 displays the analyzed assemblages plotted according to the values of mean complete flake platform thickness versus mean exterior platform angle. These measures are known experimentally to be important for assessing core reduction (Rezek et al. 2011). The two L1 assemblages (yellow) and the XB11 assemblage (red), all with relatively low angles, are separated from the E29H1 assemblages (blue) with high angles. The E29H1 and XB11 assemblages have relatively thin platforms while flakes from the L1 assemblages have relatively thick platforms. This confirms the results of the analyses presented above, indicating that differently shaped cobbles were reduced in different ways in the E29H1 and L1 assemblages, with XB11 having an intermediate position. In the E29H1 assemblages, long flakes relative to their widths were struck, while in the L1 assemblages, there was much less concern for transferring flake volume into flake edge (that is, relatively wide and thick flakes were struck).

\section{Tools}

Table 4.24 provides the number of tools in each of the analyzed assemblages. Backed blades are common in the E29H1 assemblages but less so in those from L1. Conversely, tools with bifacial retouch are more common in the L1 assemblages and less so in those from E29H1. Projectile points are present in nearly all assemblages, indicating that despite many decades of artifact collecting, projectile points are still found in surface assemblages (for example, Figure 3.14). The 


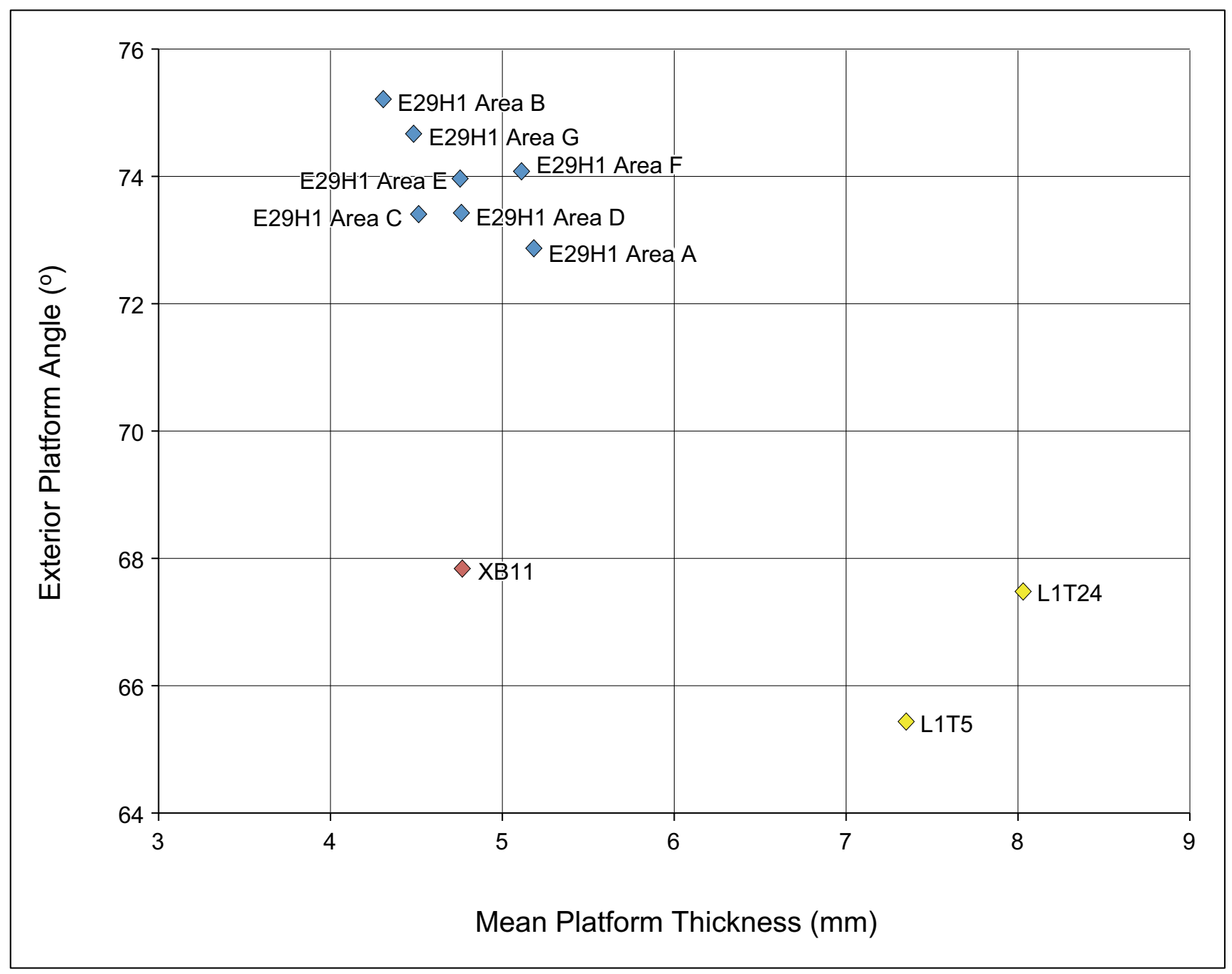

Figure 4.19. Mean platform thickness plotted against mean exterior platform angle for the analyzed assemblages. L1 assemblages: yellow icons. XB11 assemblage: red icon. E29H1 assemblages: blue icons.

rarest artifact types are axes and sickles. These types were a target of previous collectors, and it is possible that their low numbers are an outcome of intensive collection (see below). The most common tool types are denticulates, notches, and scrapers, which are present in all the assemblages studied.

The most common tool forms-scrapers, denticulates, and utilized flakes-are made on flake blanks that are larger than complete flakes from the combined assemblages from each of the areas studied. ${ }^{10}$ Analysis of

10 For example, E29 $\mathrm{H} 1$ complete flake length $25.70 \pm 8.02 \mathrm{~mm}$, complete tool length $32.52 \pm 10.45 \mathrm{~mm}$; L1 complete flake length $22.80 \pm 7.48 \mathrm{~mm}$, complete tool length $32.52 \pm 10.45$ $\mathrm{mm}$; XB11 complete flake length $24.22 \pm 9.08 \mathrm{~mm}$, complete tool length $28.16 \pm 18.59 \mathrm{~mm}$. variance indicates that there are significant differences among the lengths, widths, and thicknesses for complete flakes and tools from both sets of assemblages. ${ }^{11}$ Post hoc comparisons indicate that tools are larger than flakes in all dimensions. However, there are no differences in the dimensions of complete tools between the E29H1 and L1 assemblages. ${ }^{12}$ People retouched flake blanks that were among the larger of the examples produced, or they imported larger retouched flakes into the Fayum from manufacturing locations elsewhere.

11 Length $F=68.250 ; d f=5,6,538 ; p<0.001$. Width $F=68.644$; $d f=5,6,538 ; p<0.001$. Thickness $F=42.108 ; d f=5,6,538 ; p$ $<0.001$.

12 Length $F=1.457 ; d f=2,158 ; p=0.236$. Width $F=1.115 ; d f=$ 2,$158 ; p=0.330$. Thickness $F=2.006 ; d f=2,158 ; p=0.12$. 
Table 4.24. Tool Type Frequencies in the Analyzed Assemblages.

\begin{tabular}{|c|c|c|c|c|c|c|c|c|c|c|}
\hline & E29H1 A & E29H1 B & E29H1 C & E29H1 D & E29H1 E & E29H1 F & E29H1 G & L1T24 & L1T5 & XB11 \\
\hline Axe & 1 & & & & & & & & & 2 \\
\hline Backed blade & 1 & 11 & 15 & 6 & 11 & & 3 & & 1 & 5 \\
\hline Bifacial & & & 1 & & & 6 & & 16 & 10 & \\
\hline Denticulate & 8 & 4 & 4 & 2 & 5 & 7 & 6 & 14 & 11 & 72 \\
\hline Drill & & 1 & & & & & & 1 & & 6 \\
\hline Knife & & & 1 & & & & & & & 12 \\
\hline Notch & 11 & 5 & 2 & 2 & 3 & 4 & 2 & 6 & 2 & 15 \\
\hline Pebble & 18 & 4 & 4 & 1 & 3 & 5 & 6 & 3 & 2 & \\
\hline Projectile & 4 & 2 & 1 & & 2 & 2 & 1 & 1 & 1 & 10 \\
\hline Saw & 9 & 1 & & & & & & & & \\
\hline Scraper & 1 & 2 & 10 & 3 & 3 & 12 & 8 & 26 & 9 & 40 \\
\hline Sickle & 2 & & & & & & & & & 1 \\
\hline Utilized & & 4 & 5 & & 1 & 1 & 1 & 16 & 14 & 65 \\
\hline
\end{tabular}

However, the differences between the flake and tool dimensions are not sufficient to suggest that each category was manufactured from differently sized cobbles. Therefore it seems more likely that larger flakes were retouched to form tools. This explanation is likely for notched tools and scrapers. However, some of the L1 Basin projectile points have dimensions very much larger than the flakes that remain and were therefore likely manufactured at places outside the Fayum.

Typologically, the E29H1 assemblages can be distinguished from the analyzed L1 assemblages, with XB11 again in an intermediate position, on the basis of microblades and projectile point types. The E29H1 assemblages have small flaked arrow heads, while the L1 assemblages have larger, concave-based shaped points. However, both types are numerically rare, as are the sickles and axes. This could reflect the action of collectors as noted above. However, examples of projectiles were found in every assemblage analyzed, suggesting that either collectors were not particularly thorough or that previously buried artifacts are periodically exposed as aeolian sediments move around.

\section{Discussion}

Flakes, cores, and to a lesser degree retouched tools are abundant in the L Basin and E29H1 areas. Cobbles were imported into the Fayum, since there are no naturally occurring local sources of flint in the basin. While we remain unsure of the sources of this flint, material of different shapes was almost certainly used for the E29H1 and L1 assemblages, suggesting the use of different raw material sources. The cobbles were flaked in different ways, leading to the production of flakes that were relatively long and narrow in the E29H1 assemblages compared to the shorter and wider flakes manufactured in the L1 assemblages, an inference that is in line with qualitative observations on raw material forms (Shirai 2010). The L1 oval-shaped flint nodules were flaked across the b-semiaxis down the length of the c-semiaxis, while the cobble a-semiaxis was struck at E29H1. Cores were reduced to levels where a little under half of the original cortex remained. In all cases, cores were therefore abandoned with the potential to remove additional flakes; this despite the need to import material from elsewhere. The XB11 assemblage shows aspects of both the E29H1 and L1 assemblages.

The use of different forms of raw material may reflect the age of the deposits (Shirai 2010:309). Assemblages from E29H1 associated with hearth ages from the early Holocene have smaller cores than assemblages from farther east associated with more recent hearth ages. As noted above, cobble size can be related to the values of both the flake-to-core ratio and the cortex ratio. When sorted according to the values of these measures, a number of assemblages are grouped closely together, separate from the L1T5 assemblage. The L1T24, XB11, E29H1 A, and E29H1 G assemblages are similar to each other while also sharing some similarities with both the L1T5 assemblages and the other E29H1 assemblages. These similarities and differences are further discussed below. 
Most of the flakes produced in the L1 assemblages remained where they were struck, but flakes were removed from the E29H1 assemblages, at least partially accounting for the low flake-to-core ratio values for these assemblages. There is insufficient volume of stone in the L1 and XB11 assemblages to account for the flaking of as many cobbles as there are cores, suggesting the removal of volume by transporting cores elsewhere. In contrast, the assemblage volume for the E29H1 assemblages is sufficient to account for most of the cores that are actually present. This helps indicate the extent of flake removal that has occurred for the E29H1 assemblages. The flakes so removed were taken away from the area studied and not returned, since if they had been returned, the cortex surface area would have been incorporated into the analyzed assemblage and would have increased the cortex ratio back toward a value of 1 . The same can be said for the cores transported from the L1 and XB11 assemblages, with the proviso that they may have been transported relatively short distances to locations that we have not so far studied.

Retouched tools are rare in all the assemblages analyzed and are dominated by similar retouched forms, scrapers, denticulates, and utilized flakes. So-called typologically diagnostic forms can still be found among the extensive deposits of flakes and cores, but it is likely that more than a century of collecting has taken its toll and that retouched tool proportions can no longer be reliably calculated. Some of the collected tools have found their way into museum collections, but despite efforts to curate these collections, their significance is diminished away from the context in which they were found. Based on the flake tools that remain, it is possible to show that retouch was applied to flakes that are on average larger than those that lack retouch. Similarly sized flake blanks were retouched in both the E29H1 and L1 assemblages, with the exception of some larger projectile points that were potentially manufactured and brought into the Fayum from elsewhere.

At a gross level, the E29H1 assemblages suggest a different type of movement than the L1 assemblages, although the lithic analysis cannot be used to determine the absolute degree of difference this movement involved. What can be said is that those who transported the artifacts did so based on different concepts of flaked stone artifact utility. Most of the E29H1 assemblages suggest conversion of stone volume into flake edge through the production of flakes. In contrast, the L1 assemblages, the XB11 assemblage, and some of the E29H1 assemblages suggest movement of stone utility in the form of cores. Within the region considered, and to the degree that the sample analyzed can be generalized, those people responsible for the E29H1 assemblages moved flakes away from this location, where the flakes were likely made. Whatever uses to which these flakes were put, they were not returned to the immediate environs of E29H1. Thus we are able to conclude that activities at E29H1 required both the movement of material into the site, in the form of cortical cobbles, and its removal in the form of unretouched flakes. The loss of cortex from L1 and at XB11 occurred through the removal of cores, and any movement of flakes was more restricted than that at E29H1. Here raw material was also introduced, but as noted, it was moved in a form that suggests that people were accessing a different raw material source. The uses to which the L1 and XB11 flakes were put were more often local, leading to their abandonment close to the locations where they were made. As far as can be determined from lithic analysis, this suggests a different landscape use, one that changed from the earlier occupations represented by the E29H1 assemblages.

\section{Summary}

The area encompassing L Basin and E29H1 preserves significant archaeological deposits dating to the early to mid-Holocene. These are concentrated on areas with a surface made up of concentrated gravel and desert pavement irregularly covered by thin deposits of yellow sand, presumably sand that accumulated due to wind action. Effectively no vegetation obscures flaked stone artifacts, fragments of pottery, animal bones, grinding stones, and hearths, with the archaeological materials being obscured only in places by deep sand dune deposits and thinner aeolian sand sheets. Archaeological materials are most concentrated on erosive surfaces, due to the predominance of wind erosion in the region. However, there are indications that archaeological materials have not preserved equally in all places. Hearths and grinding stones are concentrated together within a band bounded by the 9-m and 14-m contours, but the distribution of grinding stones is wider than that of the hearths, suggesting that hearths have not preserved in all places. In a similar way, bone is not preserved in all locations. The poor correlation between flaked stone artifact and bone density suggests that archaeological materials were not all deposited uniformly but are at least partly the result of differential human behavior in the past. 
The archaeological deposits around E29H1 are more extensive than those recorded by earlier researchers, likely reflecting the intensity and duration of the current study. Those deposits near the location identified by Wendorf and Schild as E29H1 are higher in density than deposits identified here as L1 and XB11. This is true for the flaked stone artifacts but also the hearths. These are a little larger at E29H1 compared to the L1 examples, but not significantly so. Ages from the hearths span the first 4,000 years of the Holocene. Three periods of occupation are suggested. The oldest spans the period 9400 to $9000 \mathrm{cal} \mathrm{BP}$. A middle period has dates in the range 8300 to $8000 \mathrm{cal} \mathrm{BP}$, while the most recent hearths have dates that range from 7400 to $6800 \mathrm{cal}$ BP. How long each of these occupations lasted cannot yet be determined, although it would be wrong to interpret these age ranges directly as indicating occupation durations. Charcoal identified from the E29H1 hearths indicates tamarisk, with some samples indicating the use of thick branches or trunk wood.

The faunal material was collected from the surface in areas to the north and east of the E29H1 artifact distribution, with one additional area collected close to the concentration of hearths with early Holocene ages. With the exception of Trench 205, all trenches have a similar faunal composition, with fish-particularly clariid catfish, tilapia, and Nile perch-predominating. Soft-shell turtles are also common in the majority of trenches. Although mammal bones are never common, hare, gazelle, and hartebeest were identified, as well as a small number of sheep and goat bones. A radiocarbon date on one of these indicates that sheep bones are present around $7500 \mathrm{cal} \mathrm{BP}$, consistent with the most recent hearth ages from L1. The fauna from Trench 205, closest to the oldest $\mathrm{E} 29 \mathrm{H} 1$ hearths, is more fragmented than in the other trenches and has many fragments of small or large bovids, particularly teeth along with fish. Trench 205 also has a different shell assemblage than the other trenches, consistent with its earlier age. Fauna identified from the excavated hearths consists mostly of fish.

Flaked stone artifacts and animal bones are by far the most numerous archaeological remains in the area we studied. At least two forms of raw material were worked: smaller cobbles in the E29H1 assemblages and larger, flatter cobbles in some of the L1 and XB11 assemblages. The differently shaped cobbles were struck from different surfaces, leading to the production of differently shaped flakes. While those from the L1 assemblages are relatively short and wide, those from the E29H1 assemblages are longer and narrower. XB11 is intermediate between these assemblage groups. That said, the assemblages show a range of variation rather than a clear dichotomy in measures, as, for example, with the number of flakes per core. In all assemblages, many of the imported cobbles were abandoned with cortex remaining, indicating that the full potential for generating flakes was not realized. Retouched tools are never common, at least partly reflecting past collecting practices, and are uniformly made on the larger flakes available. Tool types differ in the types of projectiles present and in the presence of microblades but otherwise show similar tool type proportions.

There is a difference in the degree to which flakes were removed from the assemblages, with those from E29H1 showing lower cortex ratio values, and therefore increased flake removal, compared to the L1 assemblages studied. The L1 and XB11 assemblages show removal of cores rather than flakes. To some degree, these results correlate with the flake-to-core ratio results, suggesting perhaps that the continuous distribution of flake-to-core ratio values indicated for the assemblages that were not fully analyzed reflect a continuous distribution in the proportion of flakes or cores removed for use elsewhere.

It is tempting to suggest that some of the assemblages analyzed incorporate components of both an earlier Epipaleolithic and a more recent Neolithic. E29H1 A and G, together with the L1T24 and XB11 assemblages, are possible candidates, since, as noted above, values of the technological ratios, together with estimates of original cobble size, provide values intermediate between the other E29H1 assemblages and those from L1T5. However, intermediate values on their own need not reflect the mixture of chronologically distinct assemblages. As noted, there are at least three periods of occupation suggested by the radiocarbon determinations from hearths, so a simple dichotomy between an earlier Epipaleolithic occupation and a more recent Neolithic may not be indicated. Instead, it is possible to propose that the nature of use of the area studied changed from one that indicates high mobility but the deposition of relatively dense lithic assemblages to one where the density of artifacts abandoned was less and the nature of mobility different. The range of values for the various indices is equally interpretable as a continuous range of variability between these extremes, as it is as a result of admixture between two fundamentally different types of occupation. 
As discussed in chapter 2, previous flaked stone artifact analyses have focused on identifying typological similarities and differences that might be used to indicate the direction and timing of population movements. By design, such analyses find that assemblages are either similar to or different from others; there is no opportunity to investigate the nature of variability among assemblages. The result is a dichotomized vision of the past that also contrasts a reliance on wild animals with subsistence built around domestic plants and animals, and contrast a mobile lifestyle with one that is more sedentary. In the Fayum, such dichotomies have also been supported by what appeared to be shifts in lake levels, which provided an apparent chronostratigraphic basis for separating two distinct occupations. This difference in age was thought to indicate an early, mobile Epipaleolithic dependent on wild foods with a settled Neolithic dependent on domestic species. In other studies, the same dichotomy is proposed, this time based on a perceived difference between a forager versus logistic collector strategy (Shirai 2010). However, neither the flaked stone artifact analyses nor the results of the faunal analyses presented offer evidence for such a dichotomy; rather there is evidence for considerable variability among the assemblages from a relatively small region. As noted, the hearth chronology suggests that this variability was generated by multiple occupations dispersed over a prolonged period. Within these phases, the duration of which reflects the nature of the radiocarbon method, the archaeological record was no doubt generated by a small number of actual occupations that in themselves likely represented only a part of the lifeway of the individuals responsible for their formation. What went on during the different periods of occupation was likely constrained to some extent by the geographic location and consequent resource availability within the Fayum region. The faunal remains from all locations studied are, in the main, dominated by fish species. Lithic materials were always imported into the Fayum. However, within the boundaries of these constraints, there is plenty of variability in the material record, which likely reflects variability in behavior. It is far from clear how far this variability should be analyzed in the search for cultural behaviors characteristic of ethnically distinctive peoples or indeed whether it can be related to different socioeconomic strategies such as foraging and collecting.

Difficulties with the simple dichotomies between the Epipaleolithic and Neolithic have become increasingly apparent in eastern North Africa as more analyses are undertaken, and the results presented in this chapter add to the disquiet. The results of the analyses presented here do show differences-for instance, in the shape of the stone raw material worked and consequently the artifacts produced. But the analyses also show continuities in, for instance, the selection of larger flakes to form retouched tools such as scrapers, denticulates, and utilized flakes. More importantly, it seems less a case of the replacement of one lifestyle by another and more a case of the record reflecting a range of behaviors that changed through time, along with certain aspects of the socioeconomy that were retained for a considerable period.

These results indicate that we have only a portion of the total lifetime of an individual represented by the archaeological record we have studied to date. Much of the rest likely involved activities that occurred elsewhere. It is wrong, therefore, to conceive of the Fayum record as typical of a particular lifeway or "adaptation" or indeed as illustrative of the culture of a particular ethnic group. Typological approaches privilege central tendencies rather than variance, creating a vision of the past where people conform to cultural norms that span huge periods of time and space. While such an approach was perhaps acceptable when there was little data with which to work and therefore little opportunity to consider variability, the situation has changed, and simple labels such as Epipaleolithic and Neolithic have lost their utility. We need to situate analyses by considering variability at a number of different spatial and temporal scales (Bailey 1983). E29H1 and L1 provide a window into a period spanning more than 4,000 years. To understand the significance of the material, we need to compare it to other parts of the Fayum archaeological record, a task to which we now turn. 


\title{
5 \\ The K Basin Archaeological Record
}

\author{
Willeke Wendrich, Simon J. Holdaway, \\ Rebecca Phillipps, and Joshua J. Emmitt
}

The discoveries [of the $\mathrm{K}$ Pits] were the highlights of that year and aroused considerable interest in professional circles and columns in the press. For the wheat and barley were lowly forms of their kind, and opinions varied as to their original source-Palestine, or the Delta as a prehistoric extension of Palestine? [Caton-Thompson 1983:102].

T he area that surrounds Kom K (Figure 1.1) has in recent years been subject to considerable damage, as a result of both farming and construction associated with irrigation schemes. This has led to a great deal of surface disturbance, limiting ability to conduct the type of intensive survey described in chapter 4 . As well, subsurface deposits in a number of critical areas have been destroyed through earthworks. The key stratified deposit of Kom $\mathrm{K}$ is today protected, but the studies needed to contextualize these remains are truly an effort in salvage archaeology, as more and more of the surrounding area sees its archaeological potential destroyed through agriculture and associated development.

In this chapter we discuss the results of an intensive survey for surface flaked stone artifacts and pottery conducted to the north and west of Kom K. We also review studies of the Upper K Pits (Figure 1.1), subterranean basket-lined pits first excavated by Caton-Thompson and Gardner and unfortunately destroyed shortly after their rediscovery in 2004. The Lower K Pits, discovered and described by Caton-Thompson and Gardner (1934:52-54) but unfortunately also destroyed, are also reviewed. Despite the level of contemporary destruction, we were able to amass considerable data sets, which we contrast with the results of sites located farther to the west and discussed in chapter 4.

As a result of modern development, the topography of $\mathrm{K}$ Basin has changed significantly since the time of Caton-Thompson and Gardner's survey. The ground has been leveled and in places mounded, particularly as a result of irrigation canal construction. It is therefore difficult to understand the topographic relationships of different parts of the archaeological record in and around $\mathrm{K}$ Basin. At present, Kom $\mathrm{K}$ is an isolated stratified deposit forming a low rise covering the $16-\mathrm{m}$ to $18-\mathrm{m}$ contour bands, but it is unlikely that it was so isolated in the past. The K Pits that are now 
largely destroyed, either through excavation by CatonThompson and Gardner or through contemporary earthmoving, are situated to the north, on an area of high ground crossing the $27-\mathrm{m}$ to $31-\mathrm{m}$ contour bands, a region that is also considerably modified. To the north and particularly the west of this area, contemporary irrigated field systems crossing the $17-\mathrm{m}$ to $22-\mathrm{m}$ contour bands were constructed, but cultivation never occurred. Below we describe the results of our intensive survey of this area. Farther to the west and to the south and east of Kom K, previously disused irrigated fields have been cultivated in recent times. These fields prevented us from extending our survey area, as did in the now disused military area mentioned in chapter 4. However, in 2009 we did place one of the corridors discussed in chapter 3 along the western edge of $\mathrm{K}$ Basin, and we report on three transects that we analyzed in this chapter. Despite our best efforts, we have therefore a discontinuous survey area effectively separated from the L1 survey area discussed in the previous chapter. While not ideal, even with the gaps imposed on us by modern cultivation, our survey was extensive enough to provide an indication of differential landscape use across the eastern part of our north shore concession.

Detailed studies of the sediments on which the archaeological deposits rest were not completed at the time this book was written and will therefore be published separately. We did, however, make observations on the surface sediments in each of the transects recorded, using the same methods described in chapter 4. Results are therefore directly comparable between the two areas. Geomorphic observations on the sediments excavated at Kom $\mathrm{K}$ are discussed with the results of the excavation in chapter 6 . As noted above, the area surveyed (K1) covered the $16-\mathrm{m}$ to $26-\mathrm{m}$ contour bands. The K1 area is therefore at a higher elevation than the transects surveyed in the L1 survey area to the west.

\section{The K1 Survey Area}

A series of transects were laid out across the northern $\mathrm{K}$ Basin area. Each was around 2,000 $\mathrm{m}^{2}$, with four arms orientated north-south and east-west, similar to those described in chapter 4 . The surface geomorphology was mapped for each transect, and individual objects larger than $20 \mathrm{~mm}$ were located in three dimensions relative to a local datum established with a differential GPS. In all, in our K1 study area,
28 transects were recorded across three major modern field systems separated by large irrigation ditches. Three additional transects recorded in the $\mathrm{C} 4$ corridor are discussed below. Figure 5.1 shows the location of both sets of transects as well as the site of Kom K and the satellite-derived 5-m contour bands.

For each transect, different sediment types were traced using a self-tracking robotic total station. Objects, coded into the same forms discussed in chapter 4-flaked stone artifacts, bones, ostrich eggshells (OES), and pottery-were recorded for each transect. The data were analyzed using a GIS, permitting the relative density of objects on different surface types to be calculated (for example, Table 5.1). As was the case in the L1 study area, densities range in value depending on the object and sediment types.

In general, the $\mathrm{K} 1$ transects show object densities that are one to two orders of magnitude lower than those reported for the L1 transects. Flakes with platforms show the highest densities, but values do not exceed 0.55 flakes per $1 \mathrm{~m}^{2}$, and many densities are lower than one flake per $1,000 \mathrm{~m}^{2}$. Desert pavement shows the highest densities of objects among the sediment types, with windblown sand the next most densely populated surface. However, where densities of different objects can be compared between these surface types, no significant difference exists. ${ }^{1}$ These results are similar to those found in L1 Basin. Within individual transects, only transects K1T32 and K1T34 have multiple object types present in different sediment types. For K1T32, there is a significant difference in the density of different objects present on desert pavement and windblown sand. ${ }^{2}$ However, the result for a similar test for objects from K1T34 is not significant. ${ }^{3}$ These results suggest that it is possible to detect the influence of sediment type only in transects with a relatively large number and range of objects scattered across different surface types. This likely reflects that while objects were abandoned on the K1 Basin area we surveyed, those that remain occur in concentrations many times lower than those found in regions farther to the south and west.

\footnotetext{
1 Wilcoxon signed rank test standardized statistic $=-0.365 ; \mathrm{n}=4$; $p=0.715$, for K1T31, K1T32, K1T34, and K1T57 complete flake on desert pavement and windblown sand.

2 Wilcoxon signed rank test standardized statistic $=-2.366 ; \mathrm{n}=7$; $p=0.018$.

3 Wilcoxon signed rank test standardized statistic $=-1.461 ; \mathrm{n}=4$; $p=0.144$.
} 


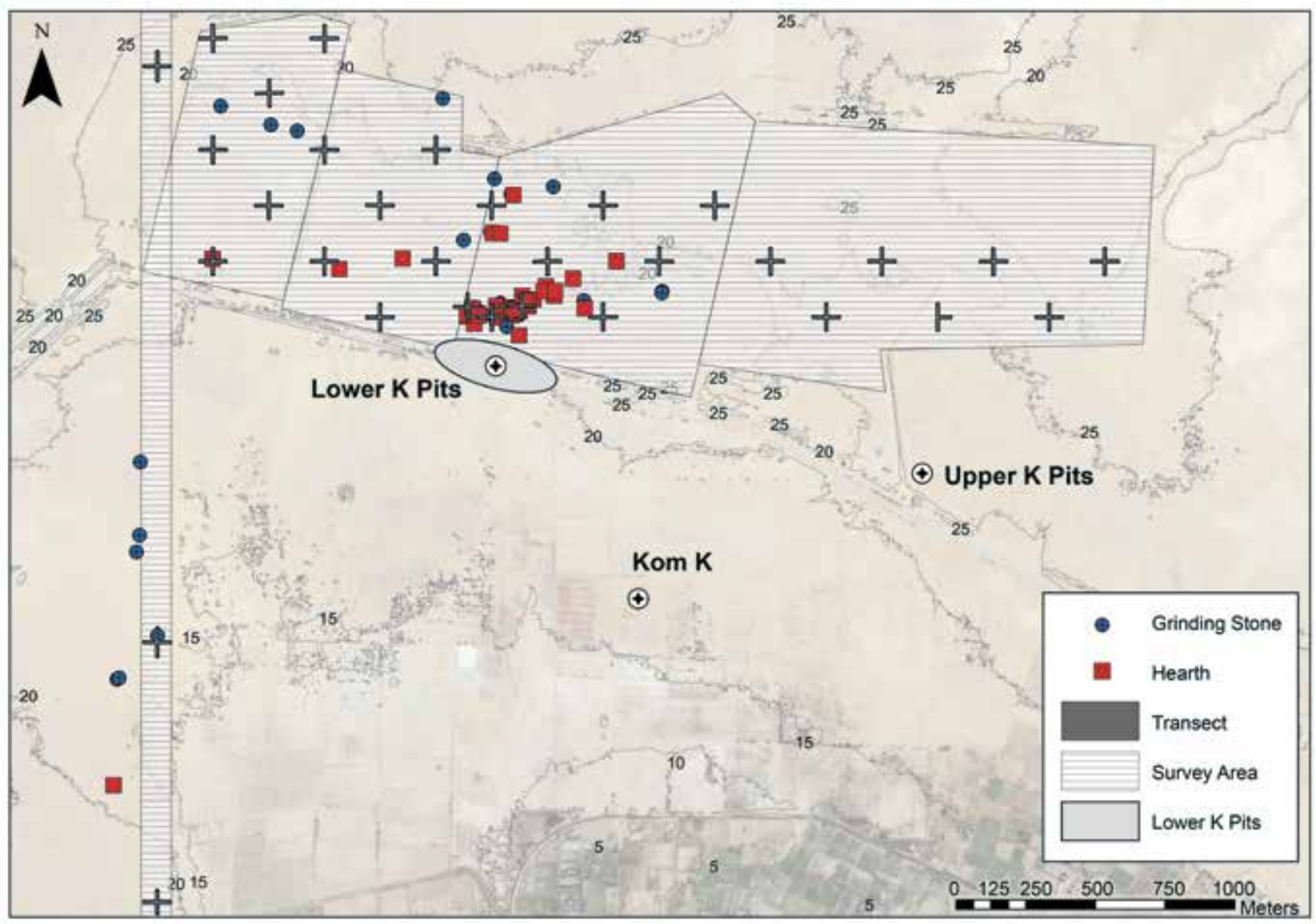

Figure 5.1. K Basin Corridor 4, K1, and C4 transects with the location of Kom K and the Upper K Pits and the probable location of the Lower K Pits. The distribution of K Basin hearths and grinding stones is also shown. Contour intervals are $5 \mathrm{~m}$.

Figure 5.2 uses tree-map plots to show the distribution of densities for all flake types on desert pavement, the sediment type with the highest object densities. Both platform flakes and broken flakes are concentrated in one part of the area studied, coincident with the highest density of hearths (discussed below). Flake densities are very low on the surrounding transects, including K1T68, located to the north of the Upper K Pits. However, the adjacent transect K1T67 is notable for having higher concentrations of flakes, although not broken flakes.

Six transects (K1T32, K1T34, K1T39, K1T57, K1T58, and K1T66) have densities of both platform flakes and cores on desert pavement surfaces, and there is a significant positive correlation between the densities of both objects using nonparametric tests. ${ }^{4}$ Bone is rare in the $\mathrm{K} 1$ transects, and there are insufficient

4 Correlations based on transects without extensions: Spearman's rho $=0.820 ; \mathrm{n}=6 ; p=0.046$. examples with which to test for a correlation with flaked stone artifacts.

Our survey covered more than 170 ha, but as discussed above, this was limited by agricultural and associated development along the boundaries in all four cardinal directions. Therefore we cannot be sure that further object concentrations do not occur beyond the area studied. However, it is notable that object concentrations do not occur in the neighborhood of the Upper K Pits, although there are artifacts on K1T69. It is possible, therefore, that the isolated object concentration that we did discover marks some form of northern limit for early to mid-Holocene material. We observed a scatter of objects from later periods, including a Roman bronze arrowhead from K1T32, but as in areas farther to the west, these items were rare in comparison to the number of flaked stone artifacts. The ceramics identified in the $\mathrm{K} 1$ transects were, in most cases, badly deteriorated, with few sherds identifiable at a diagnostic level (that is, 
Table 5.1. K1 Transect Object Density by Surface Type.

\begin{tabular}{|c|c|c|c|c|c|}
\hline K1T1 & Desert Pavement & Modern Intervention & Road & & \\
\hline Complete flake & 0.001 & 0 & 0 & & \\
\hline Broken flake & 0 & 0 & 0 & & \\
\hline Core & 0 & 0 & 0 & & \\
\hline Tool & 0 & 0 & 0 & & \\
\hline Ostrich eggshell & 0 & 0 & 0 & & \\
\hline Bone & 0 & 0 & 0 & & \\
\hline Pottery & 0 & 0 & 0 & & \\
\hline K1T2 & Desert Pavement & Modern Intervention & Vehicle Track & Water Erosion & \\
\hline Complete flake & 0 & 0 & 0 & 0 & \\
\hline Broken flake & 0 & 0 & 0 & 0 & \\
\hline Core & 0 & 0 & 0 & 0 & \\
\hline Tool & 0 & 0 & 0 & 0 & \\
\hline Ostrich eggshell & 0 & 0 & 0 & 0 & \\
\hline Bone & 0 & 0 & 0 & 0 & \\
\hline Pottery & 0 & 0 & 0 & 0 & \\
\hline K1T3 & Desert Pavement & Modern Intervention & Lake Sediment & & \\
\hline Complete flake & 0.001 & 0 & 0 & & \\
\hline Broken flake & 0 & 0 & 0 & & \\
\hline Core & 0 & 0 & 0 & & \\
\hline Tool & 0 & 0 & 0 & & \\
\hline Ostrich eggshell & 0 & 0 & 0 & & \\
\hline Bone & 0 & 0 & 0 & & \\
\hline Pottery & 0 & 0 & 0 & & \\
\hline K1T19 & Desert Pavement & Modern Intervention & & & \\
\hline Complete flake & 0 & 0 & & & \\
\hline Broken flake & 0 & 0 & & & \\
\hline Core & 0 & 0 & & & \\
\hline Tool & 0 & 0 & & & \\
\hline Ostrich eggshell & 0 & 0 & & & \\
\hline Bone & 0 & 0 & & & \\
\hline Pottery & 0 & 0 & & & \\
\hline K1T21 & Desert Pavement & Modern Intervention & Road & & \\
\hline Complete flake & 0.002 & 0.003 & 0 & & \\
\hline Broken flake & 0 & 0 & 0 & & \\
\hline Core & 0 & 0 & 0 & & \\
\hline Tool & 0 & 0 & 0 & & \\
\hline Ostrich eggshell & 0 & 0 & 0 & & \\
\hline Bone & 0 & 0 & 0 & & \\
\hline Pottery & 0 & 0 & 0 & & \\
\hline
\end{tabular}




\begin{tabular}{|c|c|c|c|c|c|}
\hline K1T22 & Modern Intervention & Windblown Sand & Road & & \\
\hline Complete flake & 0 & 0.002 & 0 & & \\
\hline Broken flake & 0 & 0.001 & 0 & & \\
\hline Core & 0 & 0 & 0 & & \\
\hline Tool & 0 & 0 & 0 & & \\
\hline Ostrich eggshell & 0 & 0.002 & 0 & & \\
\hline Bone & 0 & 0 & 0 & & \\
\hline Pottery & 0 & 0 & 0 & & \\
\hline K1T23 & Desert Pavement & Modern Intervention & Windblown Sand & Lake Sediment & \\
\hline Complete flake & 0 & 0 & 0.001 & 0 & \\
\hline Broken flake & 0 & 0 & 0 & 0 & \\
\hline Core & 0 & 0 & 0 & 0 & \\
\hline Tool & 0 & 0 & 0 & 0 & \\
\hline Ostrich eggshell & 0 & 0 & 0 & 0 & \\
\hline Bone & 0 & 0 & 0 & 0 & \\
\hline Pottery & 0 & 0 & 0 & 0 & \\
\hline K1T24 & Desert Pavement & Modern Intervention & Windblown Sand & Lake Sediment & Silty Sand \\
\hline Complete flake & 0 & 0 & 0 & 0 & 0 \\
\hline Broken flake & 0 & 0 & 0 & 0 & 0 \\
\hline Core & 0 & 0 & 0 & 0 & 0 \\
\hline Tool & 0 & 0 & 0.003 & 0 & 0 \\
\hline Ostrich eggshell & 0 & 0 & 0 & 0 & 0 \\
\hline Bone & 0 & 0 & 0 & 0 & 0 \\
\hline Pottery & 0 & 0 & 0 & 0 & 0 \\
\hline K1T25 & Desert Pavement & Modern Intervention & Windblown Sand & Outcrop & Road \\
\hline Complete flake & 0 & 0.007 & 0 & 0 & 0 \\
\hline Broken flake & 0 & 0 & 0 & 0 & 0 \\
\hline Core & 0 & 0 & 0 & 0 & 0 \\
\hline Tool & 0 & 0 & 0 & 0 & 0 \\
\hline Ostrich eggshell & 0 & 0 & 0 & 0 & 0 \\
\hline Bone & 0 & 0 & 0 & 0 & 0 \\
\hline Pottery & 0 & 0.007 & 0.008 & 0 & 0.020 \\
\hline K1T26 & Desert Pavement & Modern Intervention & Windblown Sand & Vehicle Track & \\
\hline Complete flake & 0 & 0 & 0 & 0.004 & \\
\hline Broken flake & 0.008 & 0 & 0 & 0 & \\
\hline Core & 0 & 0 & 0 & 0 & \\
\hline Tool & 0 & 0 & 0 & 0 & \\
\hline Ostrich eggshell & 0.031 & 0 & 0 & 0 & \\
\hline Bone & 0 & 0 & 0 & 0 & \\
\hline Pottery & 0 & 0 & 0 & 0 & \\
\hline
\end{tabular}


Table 5.1. K1 Transect Object Density by Surface Type. Continued

\begin{tabular}{|c|c|c|c|c|c|}
\hline K1T27 & Desert Pavement & Modern Intervention & Windblown Sand & Road & \\
\hline Complete flake & 0 & 0 & 0 & 0 & \\
\hline Broken flake & 0.001 & 0 & 0 & 0 & \\
\hline Core & 0 & 0 & 0 & 0 & \\
\hline Tool & 0 & 0 & 0 & 0 & \\
\hline Ostrich eggshell & 0 & 0 & 0 & 0 & \\
\hline Bone & 0 & 0 & 0 & 0 & \\
\hline Pottery & 0.001 & 0 & 0 & 0 & \\
\hline K1T30 & Desert Pavement & Modern Intervention & Road & & \\
\hline Complete flake & 0.005 & 0 & 0 & & \\
\hline Broken flake & 0.001 & 0 & 0 & & \\
\hline Core & 0 & 0 & 0 & & \\
\hline Tool & 0 & 0 & 0 & & \\
\hline Ostrich eggshell & 0 & 0 & 0 & & \\
\hline Bone & 0 & 0 & 0 & & \\
\hline Pottery & 0.001 & 0.001 & 0 & & \\
\hline K1T31 & Desert Pavement & Modern Intervention & Windblown Sand & Vegetation & \\
\hline Complete flake & 0.007 & 0 & 0.001 & 0 & \\
\hline Broken flake & 0.001 & 0 & 0 & 0 & \\
\hline Core & 0 & 0 & 0 & 0 & \\
\hline Tool & 0.002 & 0 & 0 & 0 & \\
\hline Ostrich eggshell & 0.001 & 0 & 0 & 0 & \\
\hline Bone & 0 & 0 & 0 & 0 & \\
\hline Pottery & 0.005 & 0 & 0 & 0 & \\
\hline K1T32 & Desert Pavement & Modern Intervention & Windblown Sand & Outcrop & \\
\hline Complete flake & 0.549 & 0 & 0.066 & 0 & \\
\hline Broken flake & 0.164 & 0 & 0.019 & 0 & \\
\hline Core & 0.066 & 0 & 0.007 & 0 & \\
\hline Tool & 0.011 & 0 & 0.002 & 0 & \\
\hline Ostrich eggshell & 0.005 & 0 & 0.002 & 0 & \\
\hline Bone & 0.164 & 0 & 0.001 & 0 & \\
\hline Pottery & 0.269 & 0 & 0.027 & 0 & \\
\hline K1T33 & Desert Pavement & Modern Intervention & Windblown Sand & Road & Vehicle Track \\
\hline Complete flake & 0 & 0 & 0 & 0 & 0 \\
\hline Broken flake & 0 & 0 & 0 & 0 & 0 \\
\hline Core & 0 & 0 & 0 & 0 & 0 \\
\hline Tool & 0 & 0 & 0 & 0 & 0 \\
\hline Ostrich eggshell & 0 & 0 & 0 & 0 & 0 \\
\hline Bone & 0 & 0 & 0 & 0 & 0 \\
\hline Pottery & 0.003 & 0 & 0.003 & 0 & 0 \\
\hline
\end{tabular}




\begin{tabular}{|c|c|c|c|c|c|}
\hline K1T34 & Desert Pavement & Windblown Sand & Outcrop & Vehicle Track & \\
\hline Complete flake & 0.015 & 0.078 & 0.012 & 0.040 & \\
\hline Broken flake & 0.020 & 0.026 & 0.024 & 0.013 & \\
\hline Core & 0.001 & 0.013 & 0 & 0.007 & \\
\hline Tool & 0.002 & 0 & 0 & 0 & \\
\hline Ostrich eggshell & 0 & 0 & 0 & 0 & \\
\hline Bone & 0 & 0 & 0 & 0 & \\
\hline Pottery & 0.004 & 0.003 & 0 & 0 & \\
\hline K1T35 & Desert Pavement & Modern Intervention & & & \\
\hline Complete flake & 0 & 0.001 & & & \\
\hline Broken flake & 0.003 & 0 & & & \\
\hline Core & 0 & 0 & & & \\
\hline Tool & 0 & 0 & & & \\
\hline Ostrich eggshell & 0 & 0 & & & \\
\hline Bone & 0 & 0 & & & \\
\hline Pottery & 0 & 0 & & & \\
\hline K1T37 & Desert Pavement & Modern Intervention & Windblown Sand & Vegetation & \\
\hline Complete flake & 0.005 & 0 & 0 & 0 & \\
\hline Broken flake & 0.001 & 0 & 0 & 0 & \\
\hline Core & 0 & 0 & 0 & 0 & \\
\hline Tool & 0 & 0 & 0 & 0 & \\
\hline Ostrich eggshell & 0 & 0 & 0 & 0 & \\
\hline Bone & 0 & 0 & 0 & 0 & \\
\hline Pottery & 0 & 0 & 0 & 0 & \\
\hline K1T39 & Desert Pavement & Modern Intervention & Windblown Sand & Road & Vehicle Track \\
\hline Complete flake & 0.029 & 0 & 0 & 0 & 0 \\
\hline Broken flake & 0.007 & 0 & 0 & 0 & 0 \\
\hline Core & 0.001 & 0 & 0 & 0 & 0 \\
\hline Tool & 0 & 0 & 0 & 0 & 0 \\
\hline Ostrich eggshell & 0 & 0 & 0 & 0 & 0 \\
\hline Bone & 0 & 0 & 0 & 0 & 0 \\
\hline Pottery & 0 & 0 & 0 & 0 & 0 \\
\hline K1T54 & Desert Pavement & Modern Intervention & Road & Vehicle Track & \\
\hline Complete flake & 0 & 0 & 0 & 0.002 & \\
\hline Broken flake & 0 & 0 & 0 & 0 & \\
\hline Core & 0 & 0 & 0 & 0 & \\
\hline Tool & 0 & 0 & 0 & 0 & \\
\hline Ostrich eggshell & 0 & 0 & 0 & 0 & \\
\hline Bone & 0 & 0 & 0 & 0 & \\
\hline Pottery & 0 & 0 & 0 & 0 & \\
\hline
\end{tabular}


Table 5.1. K1 Transect Object Density by Surface Type. Continued

\begin{tabular}{|c|c|c|c|c|c|}
\hline K1T55 & Desert Pavement & Modern Intervention & Road & & \\
\hline Complete flake & 0.001 & 0 & 0 & & \\
\hline Broken flake & 0.001 & 0 & 0 & & \\
\hline Core & 0 & 0 & 0 & & \\
\hline Tool & 0 & 0 & 0 & & \\
\hline Ostrich eggshell & 0 & 0 & 0 & & \\
\hline Bone & 0 & 0 & 0 & & \\
\hline Pottery & 0.001 & 0 & 0 & & \\
\hline K1T57 & Desert Pavement & Modern Intervention & Windblown Sand & Vehicle Track & \\
\hline Complete flake & 0.037 & 0 & 0.057 & 0.014 & \\
\hline Broken flake & 0.013 & 0 & 0.008 & 0 & \\
\hline Core & 0.008 & 0 & 0.006 & 0 & \\
\hline Tool & 0.003 & 0 & 0.002 & 0 & \\
\hline Ostrich eggshell & 0.008 & 0.005 & 0.004 & 0.007 & \\
\hline Bone & 0 & 0 & 0.003 & 0.007 & \\
\hline Pottery & 0.045 & 0 & 0.044 & 0.035 & \\
\hline K1T57E1 & Windblown Sand & & & & \\
\hline Complete flake & 0.066 & & & & \\
\hline Broken flake & 0.018 & & & & \\
\hline Core & 0.004 & & & & \\
\hline Tool & 0.002 & & & & \\
\hline Ostrich eggshell & 0 & & & & \\
\hline Bone & 0.002 & & & & \\
\hline Pottery & 0.055 & & & & \\
\hline K1T58 & Desert Pavement & Modern Intervention & Windblown Sand & Road & Vehicle Track \\
\hline Complete flake & 0.028 & 0 & 0 & 0 & 0 \\
\hline Broken flake & 0.008 & 0 & 0 & 0 & 0 \\
\hline Core & 0.005 & 0 & 0 & 0 & 0 \\
\hline Tool & 0 & 0 & 0 & 0 & 0 \\
\hline Ostrich eggshell & 0 & 0 & 0 & 0 & 0 \\
\hline Bone & 0 & 0 & 0 & 0 & 0 \\
\hline Pottery & 0.030 & 0 & 0 & 0 & 0 \\
\hline K1T58E1 & Desert Pavement & & & & \\
\hline Complete flake & 0.249 & & & & \\
\hline Broken flake & 0.075 & & & & \\
\hline Core & 0.028 & & & & \\
\hline Tool & 0.009 & & & & \\
\hline Ostrich eggshell & 0.059 & & & & \\
\hline Bone & 0 & & & & \\
\hline Pottery & 0.078 & & & & \\
\hline
\end{tabular}




\begin{tabular}{|c|c|c|c|c|c|}
\hline K1T65 & Desert Pavement & Modern Intervention & Windblown Sand & Outcrop & Road \\
\hline Complete flake & 0.002 & 0 & 0 & 0 & 0 \\
\hline Broken flake & 0 & 0 & 0 & 0 & 0 \\
\hline Core & 0 & 0 & 0 & 0 & 0 \\
\hline Tool & 0.001 & 0 & 0 & 0 & 0 \\
\hline Ostrich eggshell & 0 & 0 & 0 & 0 & 0 \\
\hline Bone & 0 & 0 & 0 & 0 & 0 \\
\hline Pottery & 0 & 0 & 0 & 0 & 0 \\
\hline K1T66 & Desert Pavement & Modern Intervention & Windblown Sand & Road & \\
\hline Complete flake & 0.001 & 0 & 0 & 0 & \\
\hline Broken flake & 0 & 0 & 0 & 0 & \\
\hline Core & 0.001 & 0 & 0 & 0 & \\
\hline Tool & 0 & 0 & 0 & 0 & \\
\hline Ostrich eggshell & 0 & 0 & 0 & 0 & \\
\hline Bone & 0 & 0 & 0 & 0 & \\
\hline Pottery & 0 & 0 & 0 & 0 & \\
\hline K1T67 & Desert Pavement & Modern Intervention & Outcrop & Silty Sand & \\
\hline Complete flake & 0.007 & 0 & 0 & 0 & \\
\hline Broken flake & 0 & 0 & 0 & 0 & \\
\hline Core & 0 & 0 & 0 & 0 & \\
\hline Tool & 0 & 0 & 0 & 0 & \\
\hline Ostrich eggshell & 0 & 0 & 0 & 0 & \\
\hline Bone & 0 & 0 & 0 & 0 & \\
\hline Pottery & 0 & 0 & 0 & 0 & \\
\hline K1T68 & Desert Pavement & Modern Intervention & Windblown Sand & Outcrop & \\
\hline Complete flake & 0 & 0 & 0 & 0 & \\
\hline Broken flake & 0 & 0 & 0 & 0 & \\
\hline Core & 0.004 & 0 & 0 & 0 & \\
\hline Tool & 0.001 & 0 & 0 & 0 & \\
\hline Ostrich eggshell & 0.018 & 0 & 0.002 & 0 & \\
\hline Bone & 0 & 0 & 0 & 0 & \\
\hline Pottery & 0 & 0 & 0 & 0 & \\
\hline
\end{tabular}

Note: All densities are expressed as object number per square meter. All surface types identified on each transect are indicated. However, transects where no objects were found have not been included.

rim or base fragments). Some of the sherds were typical of those identified in mid-Holocene contexts, such as at Kom $\mathrm{K}$ (see below), with sherds showing an oxidized surface, a reduced core, and chaff temper. All pieces were undecorated. Interspersed with the middle Holocene ceramics were ceramic sherds from later periods. These were in most cases unidentifiable sherds, often with salt encrustations, and were well fired compared to ceramics of the mid-Holocene. The greatest concentration of ceramics from the K1 area came from Transects K1T32, K1T57, and K1T58. In these transects, we found ceramics belonging to both middle Holocene and later periods. Over the K1 survey area, isolated scatters of Islamic pottery belonging to single vessels were identified outside of the K1 transects. 


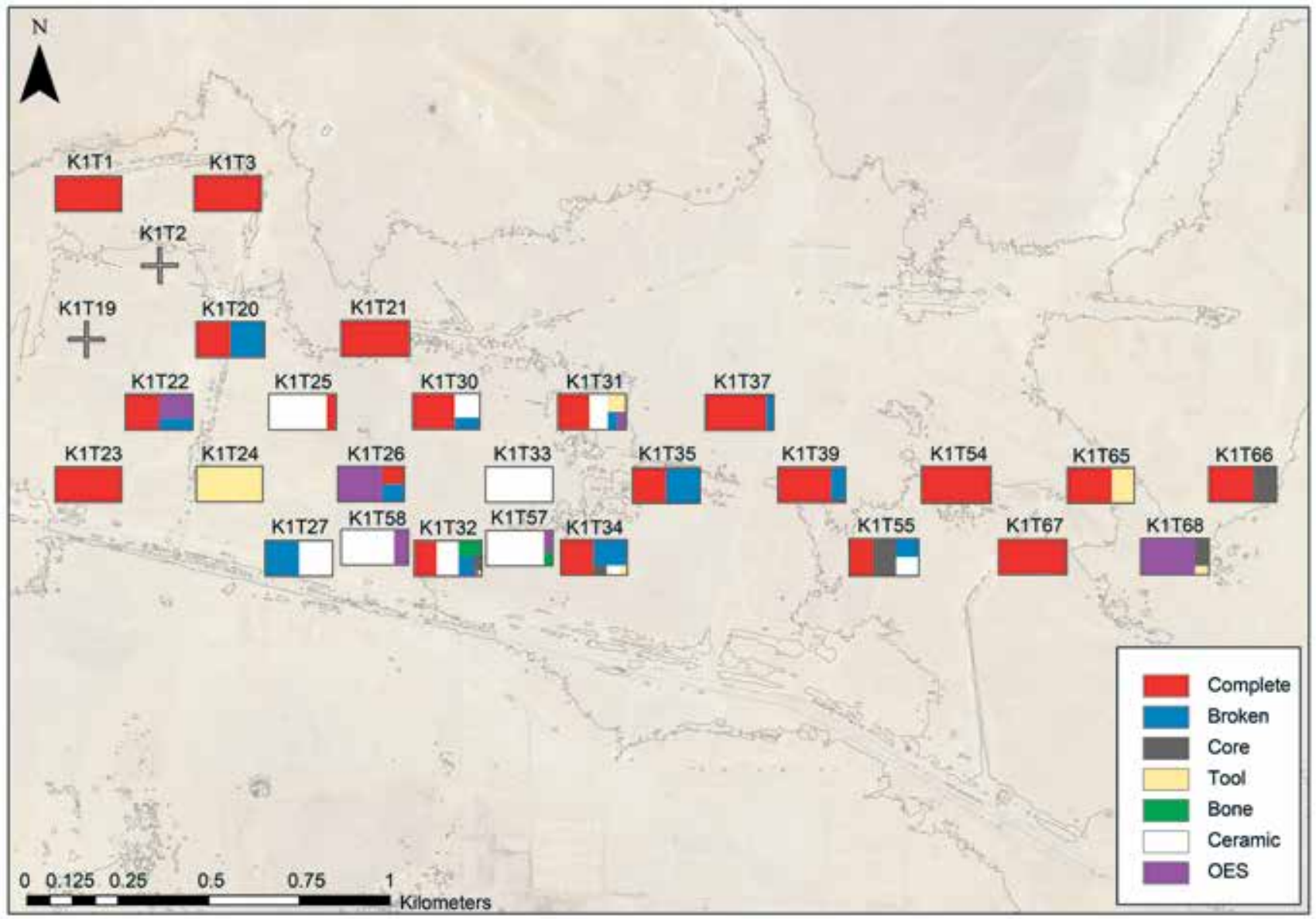

Figure 5.2. Tree-map (Jadeja and Shah 2015; Wang et al. 2015) plots showing the relative densities of complete flakes, broken flakes, cores, tools, bone, ostrich eggshell (OES), and ceramic in K1 transects on desert pavement.

\section{Hearths}

Forty-six hearths were identified in the area surveyed to the north of Kom K (Figure 5.1). However, many of these consisted of dispersed heat retainers distributed over large areas. The median number of fire-cracked rocks is 75 . However, some hearths have very large numbers of fire-cracked rocks, with K1H20 having approximately 1,500 (Figure 5.3). Fire-cracked rock numbers at $\mathrm{K} 1$ are well in excess of the median numbers recorded for the E29H1 and L1 hearths. However, as noted in chapter 4 , the number of fire-cracked rocks is a crude measure of hearth size. It is possible that multiple hearths were combined to form some of the larger estimates. The areal extent of the hearths with large numbers of fire-cracked rocks is also much larger than the estimates for the E29H1 and L1 hearths. The median hearth area is $14.35 \mathrm{~m}^{2}$. However, several hearths have much lower estimates. Dispersal of firecracked rocks to form large hearths is, in other parts of the world (e.g. Fanning et al. 2009), associated with hearth erosion, and it is possible that this is true for the K1 hearths. Certainly, the hearths that were excavated and provided sufficient charcoal for radiocarbon determinations were always smaller in size. The large excavated examples provided little remaining subsurface evidence of a hearth structure (Table 5.2).

Ages obtained for the K1 hearths are younger than those from the E29H1 hearths, but they overlap with hearth ages from L1. In addition, one hearth, K1H36, with an age of $6390 \pm 37 \mathrm{cal} \mathrm{BP}$, is younger than the hearths excavated farther west. Four of the hearths have ages around 7000 cal BP, while both $\mathrm{K} 1 \mathrm{H} 14$ and K1H17 have ages some centuries older, around 7600 cal BP. These two hearths are in turn a few centuries older than the majority of the L1 hearths and a few centuries younger than the hearths that lie to the east and west of $\mathrm{E} 29 \mathrm{H} 1$. As will be discussed later in this 


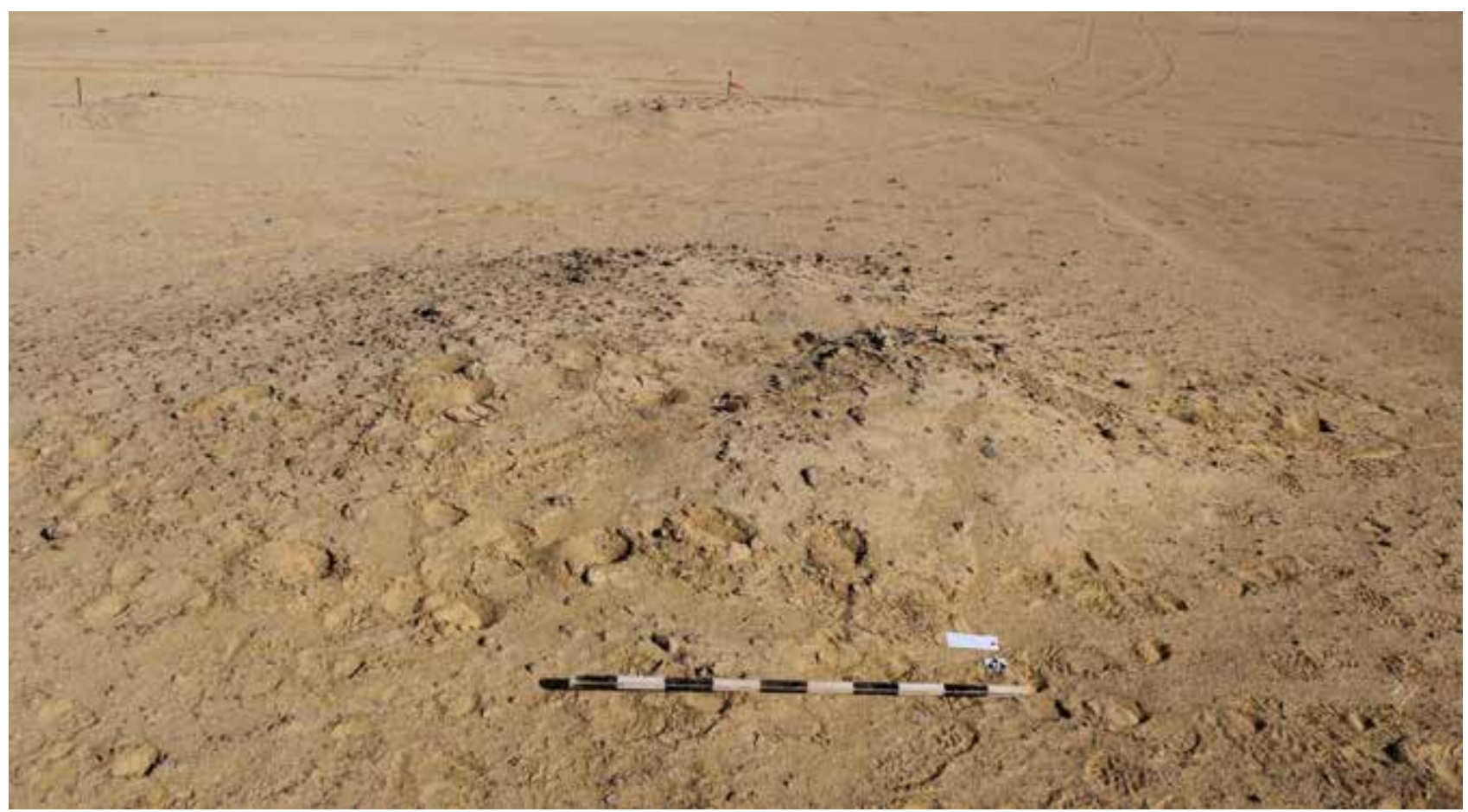

Figure 5.3. Hearth $\mathrm{K} 1 \mathrm{H} 20$ with in excess of 1,500 hearthstones.

chapter, with the exception of $\mathrm{K} 1 \mathrm{H} 36$, the $\mathrm{K} 1$ hearths are older than those dated at Kom K and the K Pits. Figure 5.4 shows the calibrated ages of the $\mathrm{K} 1$ hearths plotted against the IntCal13 calibration curve. The plot helps emphasize the existence of three groups of radiocarbon determinations.

Figure 5.5 illustrates the distribution of dated hearths together with the area surveyed. The dated hearths are clustered in the southeast corner of the surveyed area, immediately to the west of the location of the Upper K Pits excavated by Caton-Thompson and Gardner. Within this cluster of hearths, the two older hearths are located at the western edge, while more recent hearths are farther east. Survey was conducted immediately to the west and north of the Upper K Pits. However, no hearths were located. The northeastern-most hearth is $\mathrm{K} 1 \mathrm{H} 49$, with an age of $7126 \pm 65 \mathrm{cal} \mathrm{BP}$.

\section{Grinding Stones}

Figure 5.1 shows the distribution of the 19 grinding stones identified in the $\mathrm{K} 1$ area survey, while Table 5.3 summarizes the lithology and form. Overall grinding stone density is lower in the $\mathrm{K} 1$ area $(1.05$ grinding stones per ha) than in the L1/E29H1 area (1.73 grinding stones per ha). Most of the $\mathrm{K} 1$ grinding stones are manufactured from conglomerate or limestone and are most often oval in shape. Grinding stone surfaces are equally distributed between concave and flat. Grinding stones are found on surfaces that vary between $24 \mathrm{~m}$ and $18 \mathrm{~m}$ in elevation, higher than the bulk of those identified farther west. Half of the grinding stones are clustered close to the area with the highest concentration of hearths and flaked stone artifacts.

\section{Discussion}

Today Kom $\mathrm{K}$ is an isolated low hill surrounded by cultivated fields, but it is likely that in the past there were surface deposits of hearths, grinding stones, and artifacts within the immediate vicinity of the kom. We surveyed the one remaining area relatively close to Kom $\mathrm{K}$ where surfaces are still intact and found concentrations of features and objects but with fewer hearths, grinding stones, and objects in the surveyed K1 area compared to the dense deposits farther to the west discussed in chapter 4. Densities of objects are low across most of the area surveyed, with the exception of one area to the west of the Upper K Pits. Here, elevated concentrations of objects correlate with higher numbers of hearths and grinding stones. Most objects are found on desert pavement and windblown sand surfaces. 
Table 5.2. Excavated K1 Hearths That Provided Sufficient Charcoal for a Radiocarbon Determination.

\begin{tabular}{|c|c|c|}
\hline Hearth & Area & $\begin{array}{l}\text { Radiocarbon Determination } \\
\text { Oxcal 4.2.2 calibration (Bronk Ramsey 2009) plotted with atmospheric } \\
\text { data from Reimer et al. (2013). Error margins are } 68.2 \text { percent. }\end{array}$ \\
\hline K1H25 & $4.62 \mathrm{~m}^{2}$ & $\begin{array}{l}\text { UCAIMS } 121797 \\
6190 \pm 25 \mathrm{bp} \\
7048 \pm 48 \mathrm{cal} \mathrm{BP} \\
5135 \pm 48 \mathrm{cal} \mathrm{BCE}\end{array}$ \\
\hline K1H17 & $0.325 \mathrm{~m}^{2}$ & $\begin{array}{l}\text { UCIAMS } 121799 \\
6800 \pm 45 \mathrm{bp} \\
7640 \pm 34 \mathrm{cal} \mathrm{BP} \\
5691 \pm 34 \mathrm{cal} \mathrm{BCE}\end{array}$ \\
\hline K1H14 & $1.650 \mathrm{~m}^{2}$ & $\begin{array}{l}\text { UCAIMS } 122022 \\
6785 \pm 30 \mathrm{bp} \\
7631 \pm 24 \mathrm{cal} \mathrm{BP} \\
5682 \pm 24 \mathrm{cal} \mathrm{BCE}\end{array}$ \\
\hline K1H36 & no area recorded & $\begin{array}{l}\text { UCAIMS } 122023 \\
5620 \pm 20 \mathrm{bp} \\
6390 \pm 37 \mathrm{cal} \mathrm{BP} \\
4441 \pm 37 \mathrm{cal} \mathrm{BCE}\end{array}$ \\
\hline K1H38 & $1.010 \mathrm{~m}^{2}$ & $\begin{array}{l}\text { UCAIMS } 122025 \\
6160 \pm 20 \mathrm{bp} \\
7075 \pm 51 \mathrm{cal} \mathrm{BP} \\
5126 \pm 51 \mathrm{cal} \mathrm{BCE}\end{array}$ \\
\hline $\mathrm{K} 1 \mathrm{H} 40$ & no area recorded & $\begin{array}{l}\text { UCAIMS } 122026 \\
6170 \pm 20 \mathrm{bp} \\
7078 \pm 47 \mathrm{cal} \mathrm{BP} \\
5129 \pm 47 \mathrm{cal} \mathrm{BCE}\end{array}$ \\
\hline K1H47 & $0.951 \mathrm{~m}^{2}$ & $\begin{array}{l}\text { UCAIMS } 122027 \\
6150 \pm 60 \mathrm{bp} \\
7051 \pm 85 \mathrm{cal} \mathrm{BP} \\
5102 \pm 85 \mathrm{cal} \mathrm{BCE}\end{array}$ \\
\hline K1H48 & & $\begin{array}{l}\text { UCAIMS } 122028 \\
6185 \pm 20 \mathrm{bp} \\
7081 \pm 44 \mathrm{cal} \mathrm{BP} \\
5132 \pm 44 \mathrm{cal} \mathrm{BCE}\end{array}$ \\
\hline K1H49 & $2.217 \mathrm{~m}^{2}$ & $\begin{array}{l}\text { UCAIMS } 122029 \\
6220 \pm 20 \mathrm{bp} \\
7126 \pm 65 \mathrm{cal} \mathrm{BP} \\
5177 \pm 65 \mathrm{cal} \mathrm{BCE}\end{array}$ \\
\hline
\end{tabular}




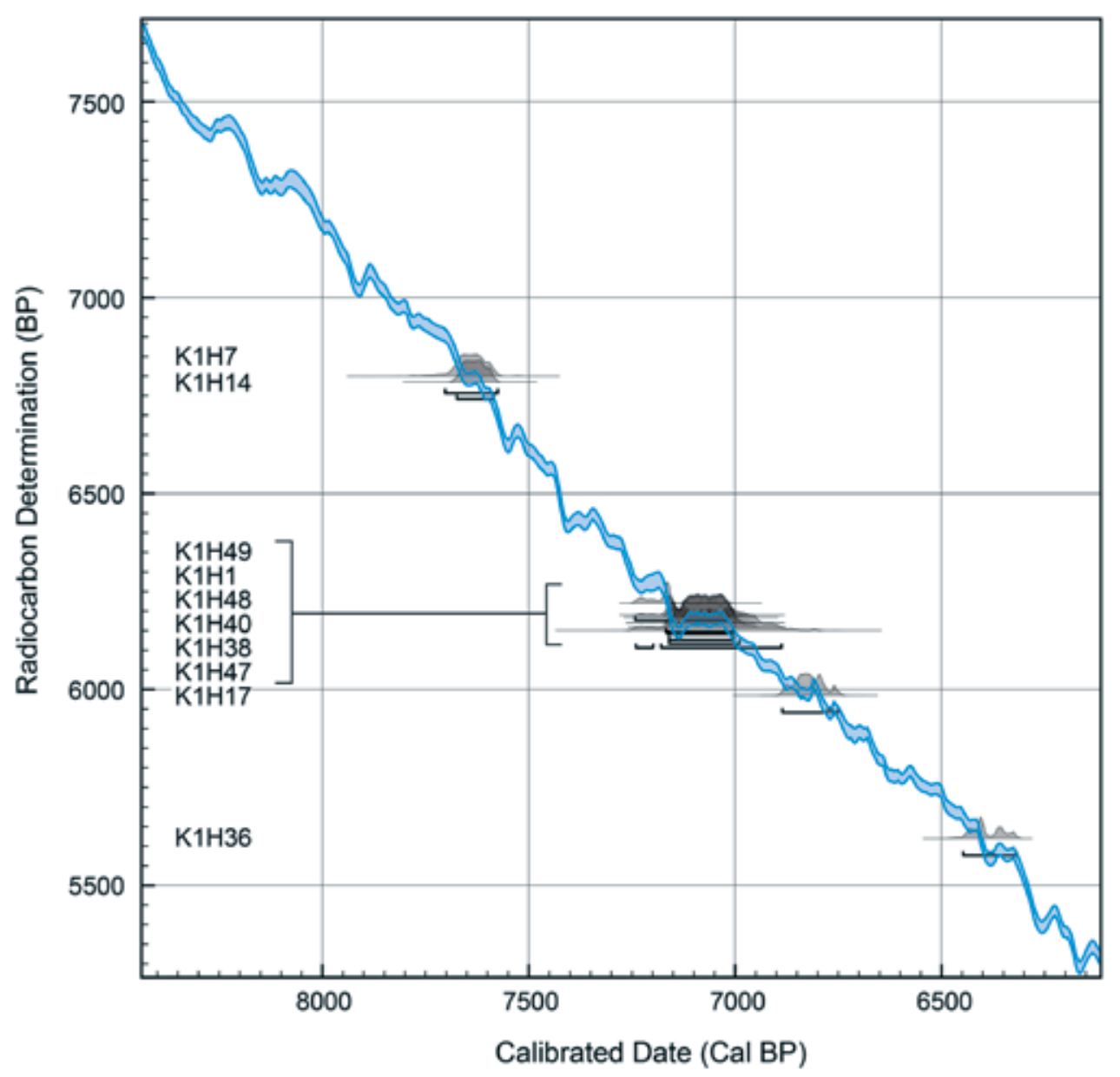

Figure 5.4. Calibrated ages from K1 hearths plotted against the IntCal13 calibration curve.

Table 5.3. K1 Grinding Stone Frequency by Raw Material and Form.

\begin{tabular}{|l|l|r|r|r|r|}
\hline Raw Material & Circular & Oval & Rectangular & Trapezoid & N/A \\
\hline Conglomerate & 1 & 4 & 1 & 2 & \\
\hline Granite & & & & 1 & 2 \\
\hline Limestone & & 5 & 1 & & \\
\hline Sandstone/conglomerate & & 1 & & & \\
\hline
\end{tabular}




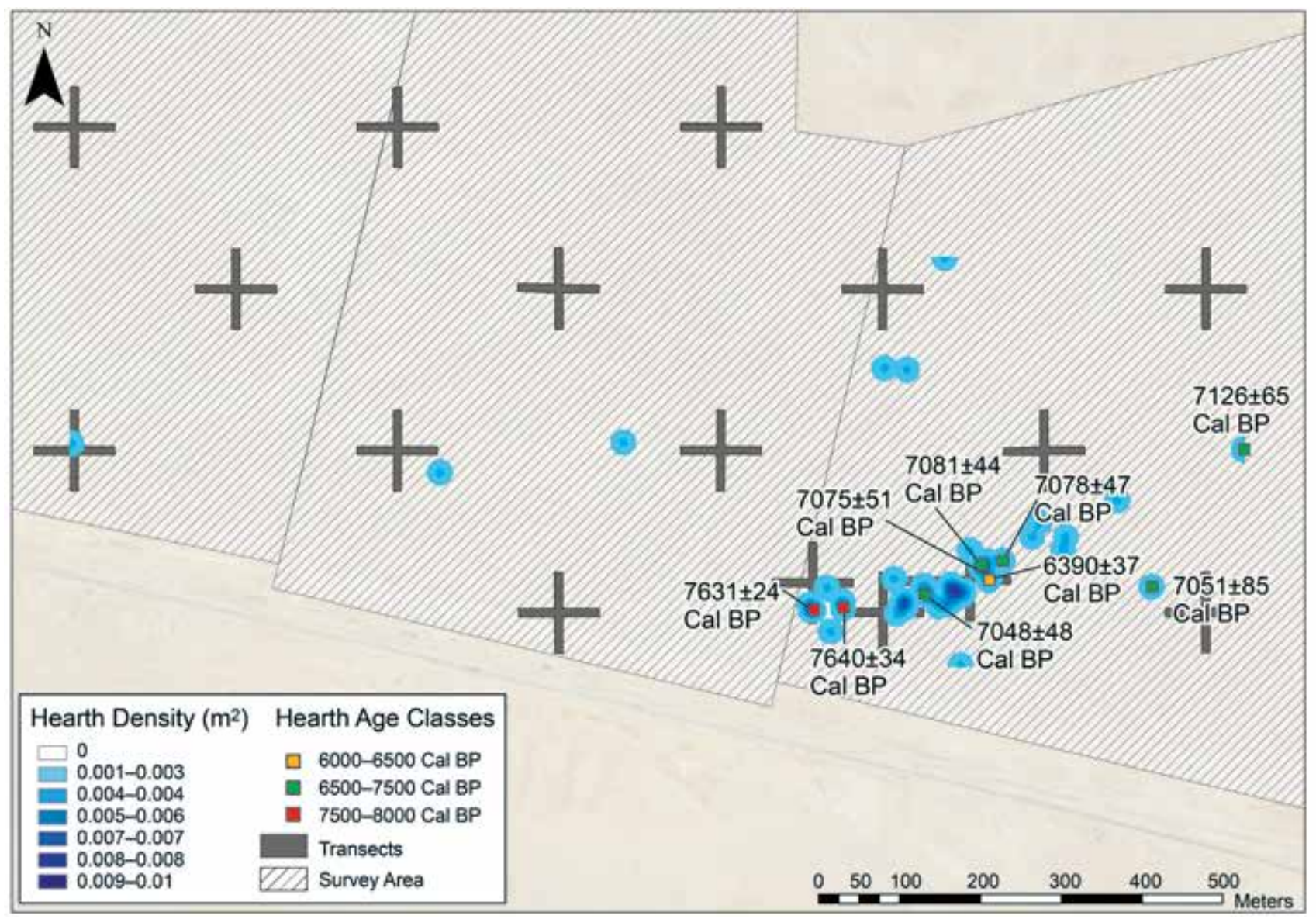

Figure 5.5. K1 hearth density $\left(\right.$ per $\left.\mathrm{m}^{2}\right)$ overlaid with dated hearths coded by age classes.

K1 hearths are much larger than the examples from L1, with very high numbers of fire-cracked rocks. This suggests that the $\mathrm{K} 1$ hearths have been significantly deflated. It is possible that the large hearths result from the combination of several smaller hearths that can no longer be individually distinguished. Excavated hearths that provided samples for radiocarbon dating returned a range of ages that overlap with those from L1 but are younger than the hearths excavated at E29H1. The oldest hearths are located farthest west in $\mathrm{K} 1$, although the small number of dated hearths makes this spatial pattern difficult to interpret.

Grinding stones are less abundant and therefore occur at lower densities than in the areas surveyed to the west. However, their forms, lithology, and surface shapes are comparable to the western examples. The spatial distribution shows that half the grinding stones identified are located within the area that also has higher concentrations of objects and hearths.

Although the areal extent of survey was limited by modern agricultural and light industrial development, it is possible that the $\mathrm{K} 1$ area surveyed represents the northern extent of early to mid-Holocene deposits. We were able to place a number of transects to the north and west of the Upper K Pits but failed to find quantities of objects. There are other low-lying valleys to the northeast of the area we surveyed, but like so much of the region around Kom K, these are currently developed for agriculture and therefore likely to have any surface record destroyed. The area where we were able to survey provides some context for the assemblages recovered from Kom K, and we turn to a one further area, running along the western border of $\mathrm{K} 1$, that also provides some indication of the previous extent of the surface archaeological record. 


\section{The C4 Corridor Survey}

As described in chapter 3, in 2009 we placed four corridors orientated north-south across the Fayum north shore and surveyed these north into the Fayum Basin (Figure 3.11). Corridor 4 was placed immediately to the east of the abandoned military structures between $\mathrm{L}$ and $\mathrm{K}$ Basins. This corridor ran across what CatonThompson refers to as Kom IV. We placed three transects along the parts of the corridor that sit along the edge of $\mathrm{K}$ Basin, as well as others farther north. We recorded objects on each of these transects as well as surveyed the corridor and its immediate vicinity for hearths and grinding stones. Since our work in 2009, the area has been further developed for agriculture, so the records we made during our 2009 survey are likely to be the only ones available.

Figure 5.1 shows the location of the transects along the corridor and the relationship between the corridor and the $\mathrm{K} 1$ transects discussed above. While the sample is small, it provides some indication of the archaeological record that likely covered the area between $\mathrm{K}$ and L Basins.

Caton-Thompson and Gardner (1934:73) describe what they named Kom IV as a limestone escarpment that they believed was close to the Neolithic lake edge. They comment that L Basin was used in Old Kingdom times, evidenced by flaked stone artifacts found on diatomaceous surfaces in the basin. They also note that the basin was irrigated in Ptolemaic times, and what are described as observation points were established on topographic high spots during this period.

Today Kom IV is considerably modified by modern terrace construction for agriculture on the southern slopes, and the eastern edge has been truncated by farm road construction (Figure 5.6). This has had an impact on the archaeological deposits, removing those to the south as well as potentially moving material from later periods (see below). Remnant deposits occur on the southern "toe" of one of the cut terraces to the south of the contemporary Kom IV extent, indicating the level of destruction. As Caton-Thompson and Gardner noted, Kom IV was used in historic periods and as reported below, we found evidence of Old Kingdom pottery in remnant deposits truncated by road construction along the eastern side. We recorded a large number of flaked stone artifacts in surface deposits across undisturbed surfaces near the southern edge of Kom IV. In addition, there are areas with very dense flaked stone artifact deposits on the top of Kom IV. However, beyond noting their presence, we were unable to analyze these further.

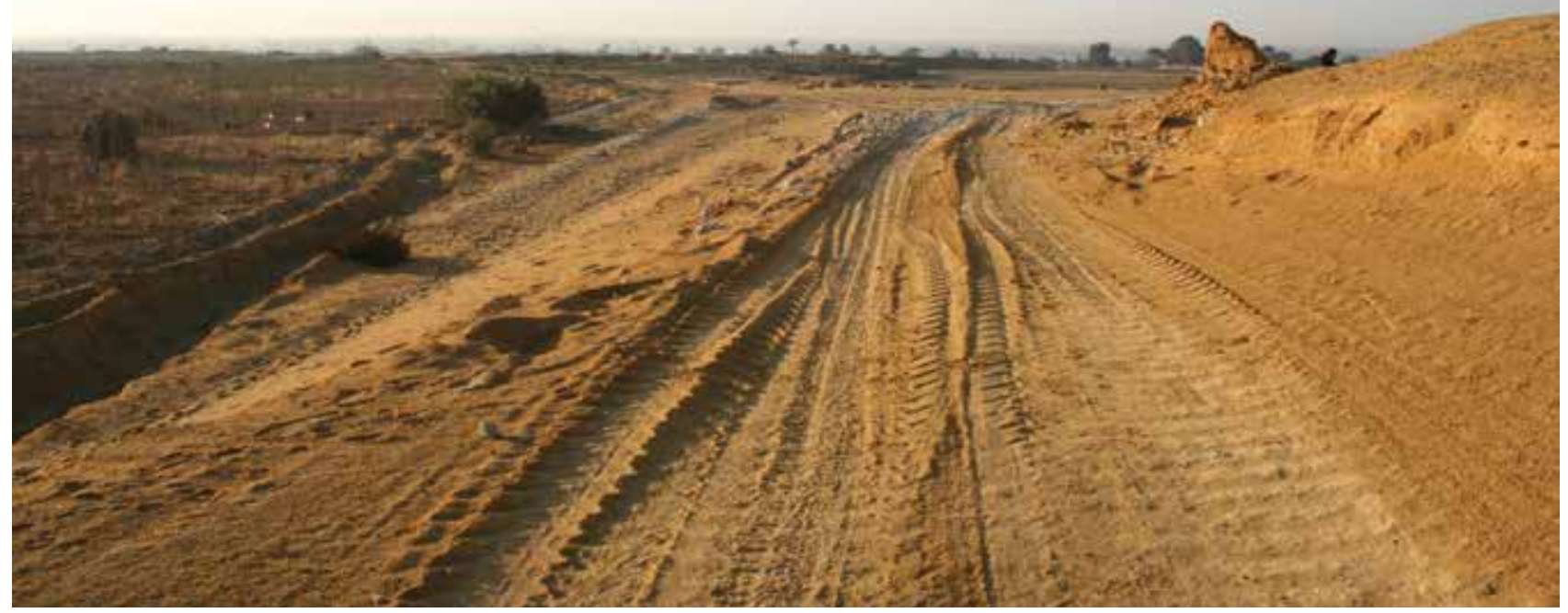

Figure 5.6. Kom IV identified by Caton-Thompson, eastern edge, looking southwest and showing the effect of modern-day development and the destruction of the Kom to the south. 
Corridor 4 extended north across Kom IV into $\mathrm{K}$ Basin proper, and we placed two transects-one on the northern edge of the kom (C4T10) and a second near the northern edge of the basin (C4T5).

\section{Object Density}

Table 3.4 provides a density measure for the objects identified in the three $\mathrm{C} 4$ transects surveyed along the edge of K Basin. Transects C4T5 and C4T10 have low object densities, close to those discussed above for the K1 transect. Transect C4T13, in contrast, has high object densities, more comparable to those found in L1 Basin. C4T13 was quite heavily disturbed by vehicles in places but retained very high densities of flaked stone artifacts where surfaces were intact. Because of the disturbance, only three arms of the transect were surveyed. Figure 5.7 shows the density of complete flake in C4T13, together with the identified sediment surfaces.
Transect C4T10 has objects only on desert pavement surfaces and is disturbed by vehicle tracks. C4T5 has objects on aeolian sand surfaces. It is located in an area that was prepared for modern-day irrigation agriculture but was not cultivated sufficiently to disturb surface objects at the time we undertook the survey.

\section{Hearths and Grinding Stones}

As described in chapter 3, a 100-m-wide corridor running north-south and connecting the transects was surveyed to locate hearths and grinding stones (Figure 5.1). Six grinding stones and a single hearth were located. Table 5.4 provides details of the lithology and shape of the grinding stones. The grinding stones are equally distributed among those with flat, concave, and convex cross-sections. The single hearth was partially buried, with 54 fire-cracked rocks present on the surface and dimensions of $1.24 \times 1.08 \mathrm{~m}$.

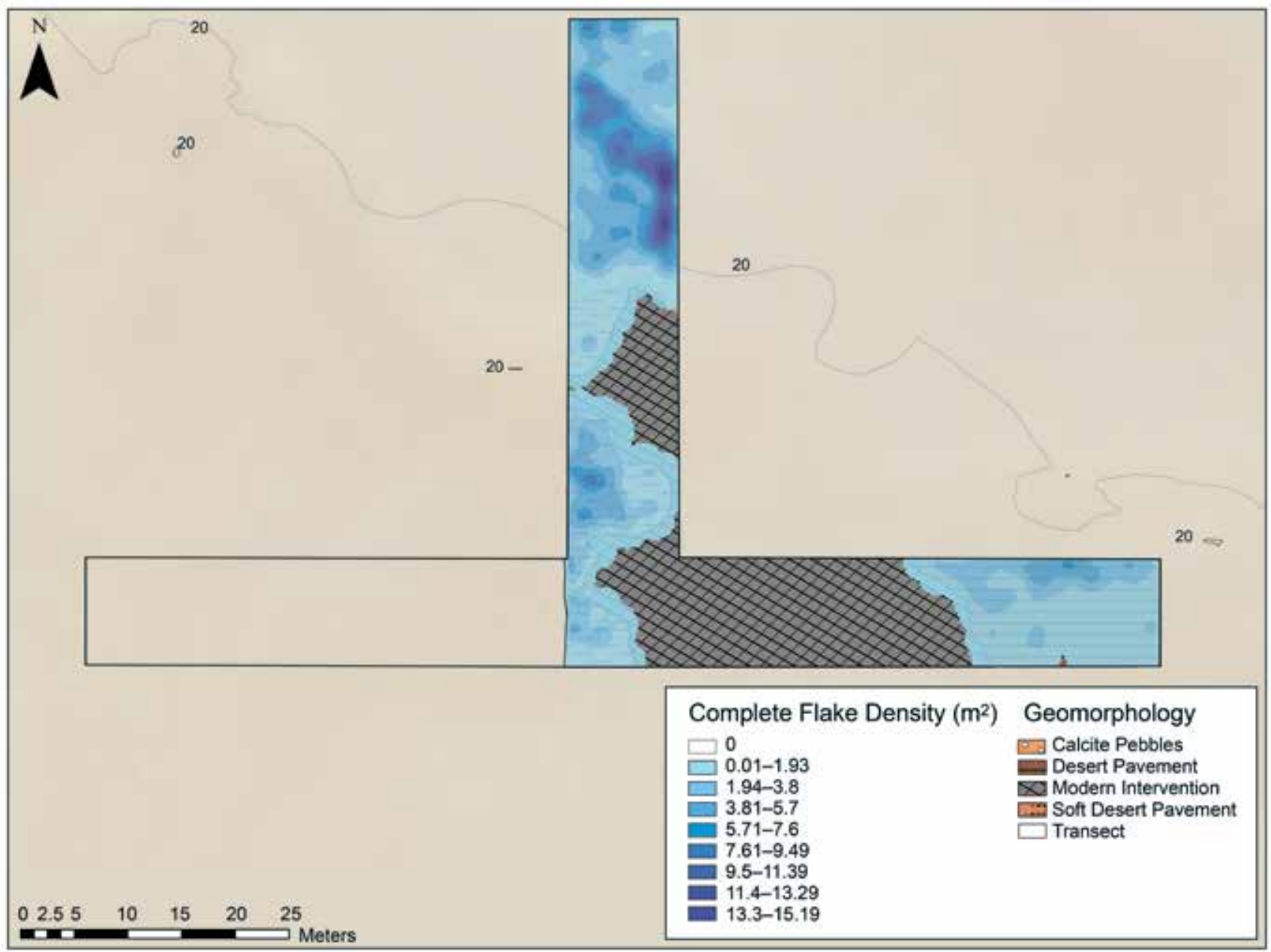

Figure 5.7. Identified C4T13 sediment surfaces overlaid with complete flake density $\left(\mathrm{m}^{2}\right)$. 
Table 5.4. Shape and Lithology of Grinding Stones Found in Corridor 4.

\begin{tabular}{|l|r|r|l|}
\hline \multicolumn{1}{|c|}{ Raw Material } & Circular & \multicolumn{1}{c|}{ Oval } & N/A \\
\hline Conglomerate & & & 3 \\
\hline Granite & & 1 & \\
\hline Sandstone & 2 & & \\
\hline
\end{tabular}

\section{Trench Excavations}

Three trenches were excavated either within or adjacent to C4T13. Trench 1 was excavated to retrieve part of the base of a ceramic vessel that was partially buried in an area with significant modern disturbance within C4T13. The vessel base is likely Old Kingdom in age and may be intrusive from elsewhere. Three unidentified bone fragments were found close to the ceramics.
Two further trenches were excavated to sample stratified deposits to the east of C4T13 to investigate objects found in the road cut section around the eastern edge of Kom IV (Figure 5.8). Trench 2 produced three flakes and four fragments of pottery, while Trench 3 produced one flake and eight fragments of pottery.

Both stratified sections were covered with sediments that were likely deposited as a result of construction of a farm track (Figure 5.6). Given the identification of Old Kingdom pottery in Trench 1, as well as the comments by Caton-Thompson and Gardner (1934) concerning later occupations adjacent to the Gebel, it is possible that these deposits are later than the mid-Holocene. Certainly the area has seen a great deal of activity, both ancient and modern, that complicates interpretation.

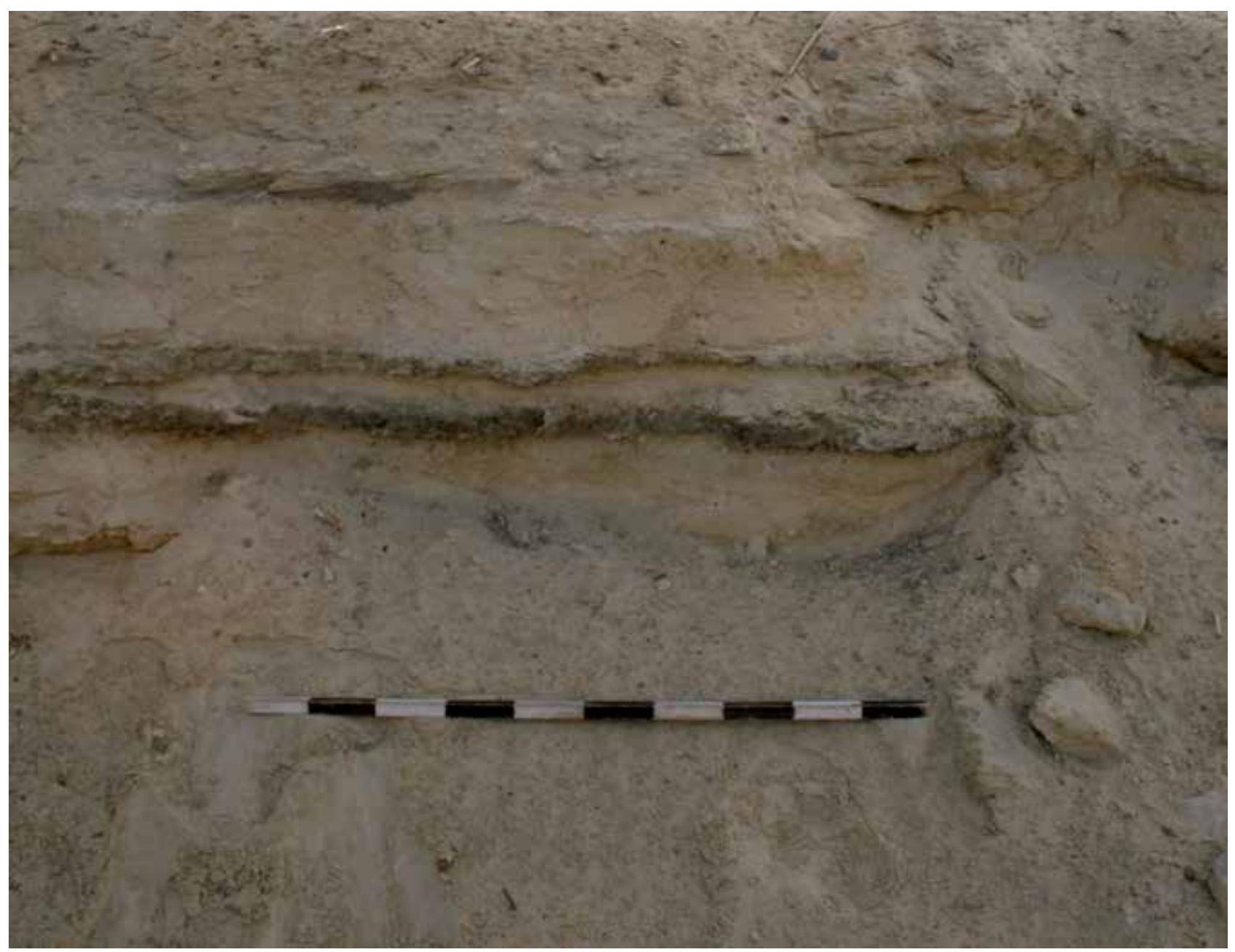

Figure 5.8. Stratified deposits to the east of C4T13, excavated as Trenches 2 and 3. 


\section{Discussion}

The C4 corridor was surveyed at the start of the project to determine the extent of archaeological deposits distributed across the Fayum north shore. The three transects reported here therefore provide only a limited sample of the surface archaeological deposits. Nevertheless, because of the extent of modern agricultural development, they are likely to be all that is available. The density of objects diminishes markedly northward along the corridor, with densities in the northern C4T5 transect similar to those in the $\mathrm{K} 1$ area. To the south, the C4T13 transect sampled what must have been a very dense area of flaked stone artifacts and pottery along the southern edge of CatonThompson and Gardner's Kom IV. Unfortunately, much of this area has been damaged by modern development, making the stratigraphic relationship between some of the objects found unclear. Our work suggests that the area from Kom IV west to L1 Basin may once have had significant concentrations of artifacts.
The corridor survey produced relatively few grinding stones and a single hearth, although it is important to note that the extent of the survey did not approach the areas covered in the L1 and K1 locations. Unfortunately, large, aerial survey to discover features was simply not possible given the level of destruction by modern development.

In the following discussions, the results of the flaked stone artifact surveys in the K1 and C4 areas are compared with the results from the $\mathrm{L}$ Basin area presented in chapter 4 .

\section{Flaked Stone Artifacts}

The analyses of the $\mathrm{K} 1$ assemblages follow the same format as those presented for the E29H1 and L1 assemblages in chapter 4. Most of the analyses are based on the measured sample of artifacts from $\mathrm{K} 1$ transects recorded in the densest area of objects. Comparisons are made to numbers of objects in the other transects and to the results from $\mathrm{L}$ Basin.

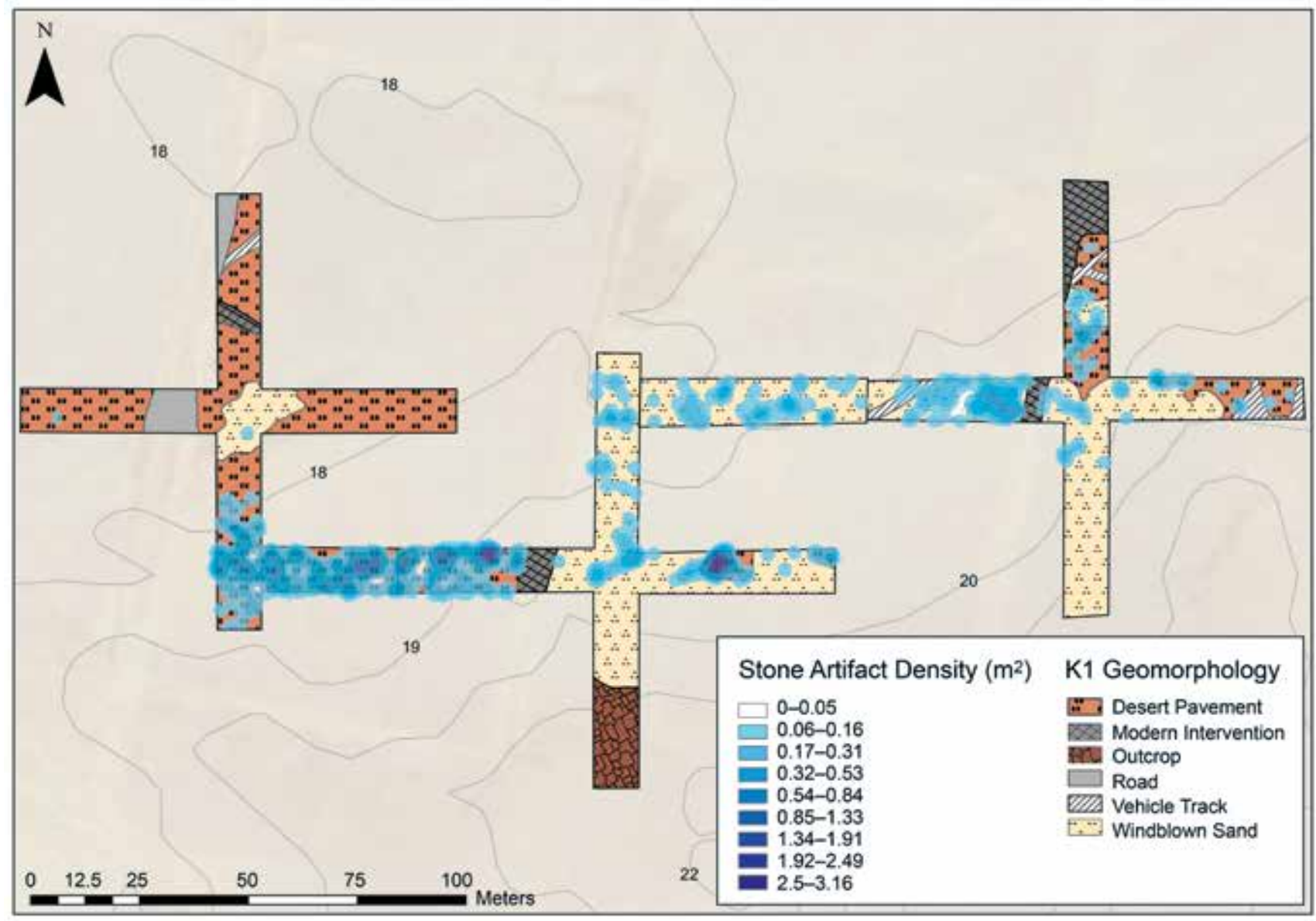

Figure 5.9. Transects and extensions used to obtain a sample of flaked stone artifacts to analyze in K1, with flaked stone artifact density. 
Figure 5.9 shows the transect configuration used to sample the $\mathrm{K} 1$ area for artifact analysis. Because overall the density of objects was low in K1, we extended the arms of the transects surveyed in the areas with the densest record to intersect one another, thereby recording artifacts over a larger area than that covered by individual transects. In total 656 flaked stone artifacts were recorded across the combined transects.

\section{Cores}

Table 5.5 provides the mean dimensions of the 45 cores recorded in the $\mathrm{K} 1$ transects. Cores are large compared to those from transects analyzed farther west. Comparisons of mean length, width, and thickness indicate significant differences among the E29H1, L1, and $\mathrm{K} 1$ assemblages, with post hoc Bonferroni tests indicating that the $\mathrm{K} 1$ assemblage is significantly larger than all other assemblages for all three dimensions ${ }^{5}$. For core scar length, the ANOVA result is also significant, ${ }^{6}$ with the $\mathrm{K} 1$ assemblage having a significantly larger mean core scar length than all assemblages except those from E29H1 A, E29H1 E, and XB11, the assemblages with the largest mean core scar lengths from the L1 survey area.

Table 5.6 shows the frequency and proportions of cores of different types in the $\mathrm{K} 1$ assemblages. Cores that have been rotated to bring multiple platforms into use are frequent, along with cores with only a single platform. Bifacial platforms with flakes removed from a single edge in two directions are also common. The proportion of multiple cores is similar to that found in the E29H1 assemblages A and G and is only slightly higher than that found in the two L1 transect assemblages analyzed.

Table 5.5. Flaked Stone Artifact Core Dimensions from the K1 Transects.

\begin{tabular}{|c|c|c|c|c|c|c|c|c|}
\hline & \multicolumn{2}{|c|}{$\begin{array}{l}\text { Maximum } \\
\text { Length } \\
(\mathrm{mm})\end{array}$} & \multicolumn{2}{|c|}{$\begin{array}{l}\text { Maximum } \\
\text { Width } \\
(\mathrm{mm})\end{array}$} & \multicolumn{2}{|c|}{$\begin{array}{l}\text { Maximum } \\
\text { Thickness } \\
\text { (mm) }\end{array}$} & \multicolumn{2}{|c|}{$\begin{array}{l}\text { Core Scar } \\
\text { Length } \\
(\mathrm{mm})\end{array}$} \\
\hline $\mathrm{n}$ & mean & SD & mean & SD & mean & SD & mean & SD \\
\hline 45 & 53.73 & 19.25 & 39.58 & 14.96 & 26.62 & 14.91 & 28.91 & 14.76 \\
\hline
\end{tabular}

5 ANOVA maximum length $F=22.312, d f=10,1,500, p<0.001$. Maximum width $F=14.298 ; d f=10,1,500 ; p<0.001$. Maximum thickness $F=16.767 ; d f=10,1,500 ; p<0.001$.

$6 F=8.257 ; d f=10,1,500 ; p<0.001$.
Table 5.6. K1 Flaked Stone Artifact Core Form Frequency and Proportion.

\begin{tabular}{|l|r|r|}
\hline Core Type & Frequency & Percentage \\
\hline Bidirectional & 2 & $4.44 \%$ \\
\hline Bifacial & 8 & $17.78 \%$ \\
\hline Multiple & 13 & $28.89 \%$ \\
\hline Nuclear tool & 1 & $2.22 \%$ \\
\hline Test & 7 & $15.56 \%$ \\
\hline Unifacial & 14 & $31.11 \%$ \\
\hline
\end{tabular}

Core dimensions oriented relative to the axis of the longest remaining core scar are also large in the $\mathrm{K} 1$ assemblage relative to those measured in the transects farther west (Figure 5.10). Mean core oriented length is larger than the equivalent mean width measurement, but the difference is small. The difference is larger for the L1 assemblages, and in this regard the K1 assemblage is closer to the E29H1 A and F assemblages.

These results suggest that before flaking, cobbles were shaped more like scalene ellipsoids than spheres. That is, the cobbles flaked to form the $\mathrm{K} 1$ cores were relatively thin in comparison to their lengths and widths (for example, Figure 3.14). Ratios of the oriented core axis dimensions indicate that the largest differences (in bold) occur for multiple and unifacial cores (Table 5.7). For these core types, the greatest difference occurs between the length and thickness measurements, while for bifacial cores the largest difference is between the width and thickness measurements. For all core forms, it is the thickness of the core that is most reduced during flaking (that is, the measure orthogonal to the plane formed by the core oriented length and width measurements with the orientation set relative to the largest remaining core flake scar). This pattern is similar to that recorded for the two L1 assemblages and E29H1 G, reported in chapter 4.

The complete flake length-to-width ratio indicates how blade-like the K1 assemblage flakes are. The ratio value for the $\mathrm{K} 1$ assemblage is 1.28 , which is lower than the E29H1 ratio values (although close to the values from E29H1 A and F) but higher than the values obtained for the two L1 assemblages and that from XB11. In other words, as far as can be determined from the flakes that remain in place, the $\mathrm{K} 1$ cores were used to produce flakes that were relatively wide compared to their lengths. Some of the assemblages from E29H1 produced flakes that were more blade-like in shape, while those from the $\mathrm{K} 1$ assemblages were even wider, relative to their length, than those from L1. 


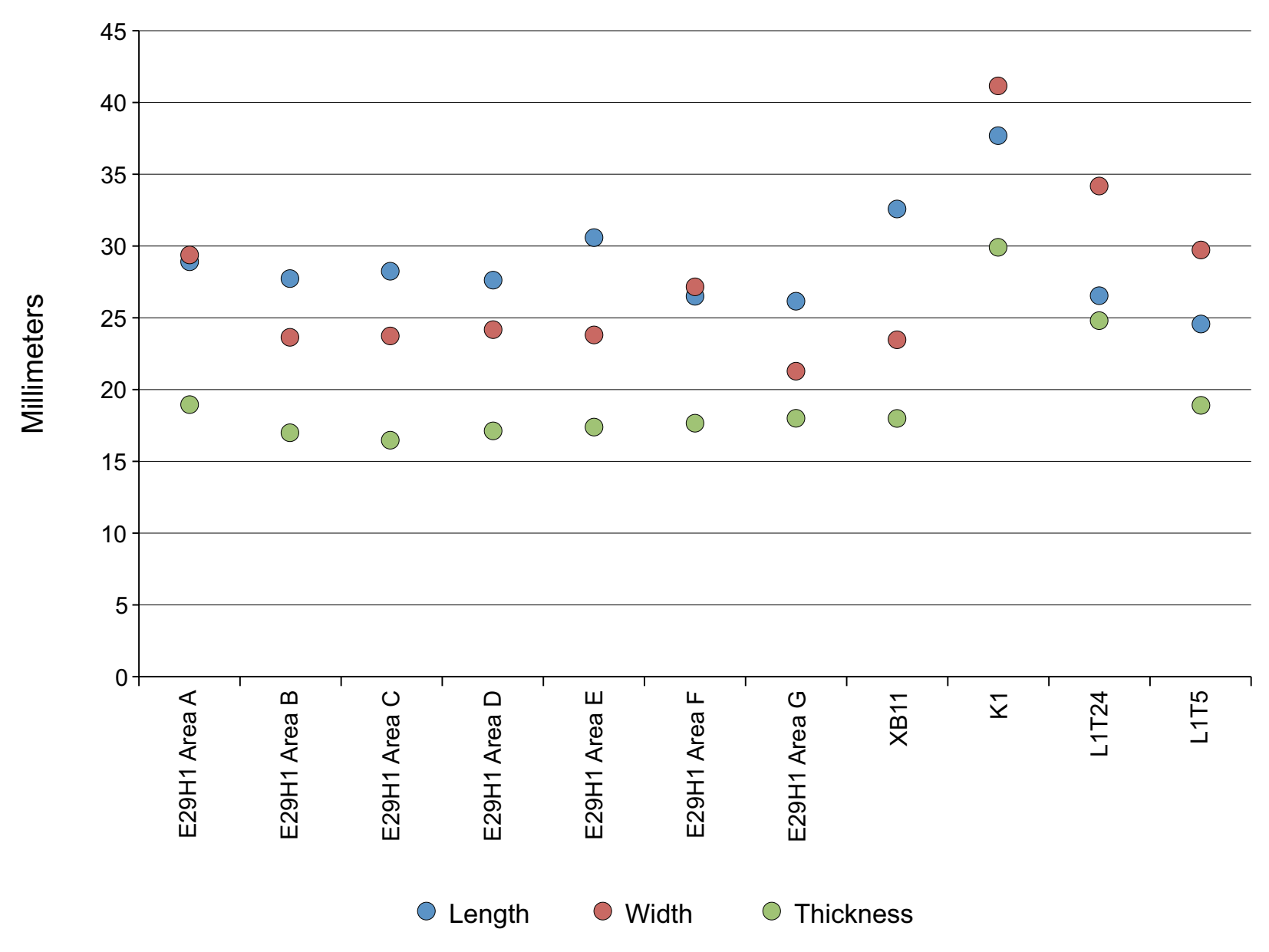

Figure 5.10. Mean K1 oriented core dimensions in comparison to the E29H1 and L1 assemblages.

Table 5.7. K1 Ratio of Mean Core Axis Oriented Dimensions for Core Types.

\begin{tabular}{|l|r|r|r|}
\hline Core Type & A:B & A:C & B:C \\
\hline Bifacial & 0.88 & 1.24 & 1.49 \\
\hline Multiple & 1.12 & 1.73 & 1.58 \\
\hline Unifacial & 1.15 & 1.76 & 1.71 \\
\hline
\end{tabular}

Note: The largest values are in bold.

Table 5.8 provides the number of cores of different types according to the proportion of cortex they retain. Cores with multiple platforms have less cortex than those flaked from a single platform or struck on a bifacial platform. For the three main core forms-bifacial, multiple and unifacial-the difference in frequency between cores with 1 to 50 percent and 51 to 99 percent cortex is significant. ${ }^{7}$ Only one core with multiple platforms contained no cortex. The distribution of cortex across core types is similar to that recorded for the E29H1, XB11, and L1 assemblages.

As discussed in chapter 4 , it is possible to use cores that retain cortex to estimate the size of cobbles originally flaked using the formula for the volume of a scalene ellipsoid. Following the discussion above, the oriented thickness measure is reduced most during reduction, so in the calculation of the cobble size estimate, this dimension is multiplied by 3 to reflect material removed during core flaking. For the K1 assemblage, the formula was applied to bifacial and unifacial cores with 50 to 99 percent cortex, and as with the cores analyzed in

7 Chi-square $=8.48 ; d f=2 ; p<0.014$. 
chapter 4 , the results were log-transformed to remove the effect of the resulting skewed distribution. The outcome of this calculation is an estimated cobble volume of $60,261.61 \mathrm{~mm}^{3}$, around three times the size of the cobble estimates for the E29H1, XB11, and L1 assemblages. The K1 cobble size estimate is based on only 14 cores, compared to much higher numbers recorded in the assemblages farther west. However, the magnitude of the difference strongly suggests that much larger cobbles were sourced for raw material by the people who left the assemblages at $\mathrm{K} 1$ than those who created the E29H1 and L1 assemblages.

Figure 5.11 shows the flake-to-core ratio calculated for transects with sufficient flakes with platforms and cores to allow calculation of the ratio. Two K1 transects are omitted from the figure (K1T39 complete flake 33, Core 1; K1T66 complete flake 2, Core 1), since limited numbers of flakes and cores led to extreme flake-tocore ratio values. Values of the transects that remain vary considerably, with that for C4T5 falling close to
Table 5.8. K1 Core Frequency by Core Type and Cortex Proportions.

\begin{tabular}{|l|r|r|l|}
\hline Core Type & $1-50 \%$ & $50-99 \%$ & None \\
\hline Bidirectional & 1 & 1 & \\
\hline Bifacial & 2 & 6 & \\
\hline Multiple & 10 & 2 & 1 \\
\hline Nuclear tool & & 1 & \\
\hline Test & & 7 & \\
\hline Unifacial & 5 & 9 & \\
\hline
\end{tabular}

the values from the E29H1 assemblages and those for the other transects falling within the range of values obtained from the L1 transects and E29H1 G.

The flake-to-core ratio for all analyzed artifacts from the $\mathrm{K} 1$ transects is 8.91 . If this is corrected for estimated cobble size, using the procedure described in chapter 4, following Barrett (2014), the corrected flake-to-core ratio has a value of 1.48 , within the range

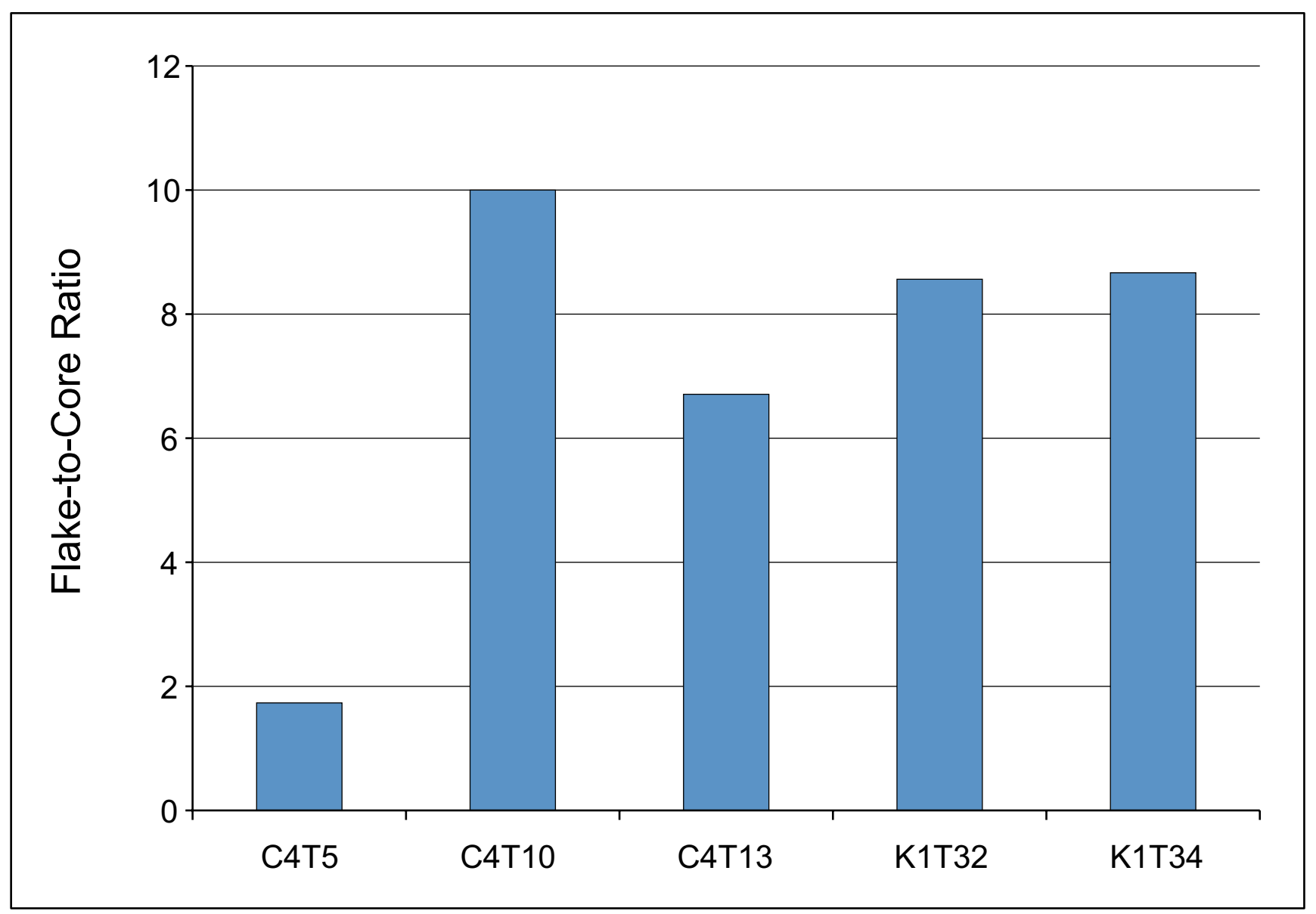

Figure 5.11. Flake-to-core ratio for the $\mathrm{K} 1$ and $\mathrm{C} 4$ transects, with sufficient flakes and cores to enable calculation. 
of the values calculated for the E29H1 assemblages but smaller than the values obtained for the L1 and XB11 assemblages. Thus, while cobbles flaked at K1 were large compared to those used in the other study areas, on average they were not flaked very intensively.

\section{Flakes}

Overall, complete flakes from the $\mathrm{K} 1$ assemblage are large, a result that is consistent with the size estimates for the cores discussed above (Table 5.9). Flakes with the most cortex are also the largest, with significant differences across all three dimensions among flake classes with different proportions of cortex. ${ }^{8}$ Post hoc tests indicate significant size differences between flakes with little or no cortex and those with higher proportions of cortex.

Comparisons with assemblages analyzed from transects farther west indicate significant differences in complete flake size among the assemblages for all three dimensions, ${ }^{9}$ with post hoc Bonferroni tests indicating that the $\mathrm{K} 1$ assemblages are significantly larger in length, width, and thickness than all other assemblages.

As discussed in chapter 4 , the calculation of a cortex ratio provides the means to assess the completeness of the assemblage by allowing an estimate of whether flakes have been removed after manufacture. As also described, the surface area of flakes and flake fragments from the $\mathrm{K} 1$ assemblage was calculated, and the product of maximum length was multiplied by width, which was multiplied in turn by the proportion of the artifact covered in cortex. For cores, the formula for the surface area of a scalene ellipsoid (chapter 4) was used. The observed cortical surface area was then calculated by summing the cortical surface area from all flaked pieces together with the cortical surface area remaining on the cores. The expected surface area calculation was based on the surface area of the cores, using those with more than 50 percent cortex remaining with a unifacial or bifacial form, as in the calculation of cobble volume, above, and then multiplying the result by the number of cores found in the K1 assemblage.

The result of these calculations for the $\mathrm{K} 1$ assemblage is a cortex ratio of 0.40 . This means there is just under half the expected cortical surface area available in the

8 ANOVA length $F=3.92 ; d f=4,339 ; p<0.001$. Width $F=$ 6.34; $d f=4,339 ; p<0.001$. Thickness $F=6.74 ; d f=4,339 ; p<$ 0.001 .

9 ANOVA length $F=27.258 ; d f=10,6,706 ; p<0.001$. Width $F=$ 53.983; $d f=10,6,706 ; p<0.001$. Thickness $F=4.729$; $d f=10$, 6,$706 ; p<0.001$.
$\mathrm{K} 1$ assemblage relative to that predicted based on the number of cores and an estimate of original cobble size. Approximately 30 percent of this cortical surface area exists on the 45 cores that were identified, while less than 10 percent remains on the complete flakes. The observed proportion of cortex remaining on the cores relative to the expected surface area of cortex is approximately the same as that for the cores from some of the E29H1 assemblages but lower than that on the L1 and XB11 cores. In addition, the volume of artifacts (that is, both cores and all flakes and fragments) accounts for 103 cobbles when in fact there were 45 cores found in the $\mathrm{K} 1$ assemblage. The volume ratio has a value of 2.28 , indicating that there is more volume present than can be accounted for by the number of cores. If each of the cores represents the flaking of a single cobble, then a significant number of cores must have been removed from the $\mathrm{K} 1$ assemblages. This result is similar to that found for the L1 assemblages, where the volume of material was also smaller than that required to account for the number of cobbles flaked, and it differs from the E29H1 assemblages, where the assemblage volume was almost sufficient to account for the cobbles flaked.

Figure 5.12 plots the mean platform thickness for complete flakes against the mean exterior platform angle for the $\mathrm{K} 1$ assemblage in comparison to values for the E29H1 and L1 assemblages. The remaining flakes in the $\mathrm{K} 1$ assemblage (that is, those that were not removed, as indicated by the cortex ratio values) are closest to those from the L1 assemblages (yellow icons in Figure 5.12) and are quite distinct from those from the E29H1 assemblages (blue icons).

\section{Tools}

Tools are rare in the $\mathrm{K} 1$ assemblage, and the number of tool types is also reduced compared to the western transect assemblages (Table 5.10). The most common tool type is the denticulate $(\mathrm{n}=10)$, in addition to which there is a single notch. Complete and distal scrapers are the next most common types, followed by tools with bifacial retouch. There was only a single fragmentary projectile point identified.

Although there are only a small number of complete tools, the mean size of the flakes on which retouch occurs is high compared to the dimensions of the unretouched flakes (tool length $45.75 \pm 11.39 \mathrm{~mm}$; width $37.13 \pm 6.69 \mathrm{~mm}$; thickness $14.63 \pm 4.78 \mathrm{~mm}$ ). Thus there is some evidence that larger flake blanks were selected for retouch. 
Table 5.9. K1 Complete Flake Dimensions by Cortex Proportion.

\begin{tabular}{|c|c|c|c|c|c|c|c|}
\hline \multirow{2}{*}{ K1 } & \multirow{2}{*}{$\mathbf{N}$} & $\begin{array}{l}\text { Length } \\
(\mathrm{mm})\end{array}$ & & $\begin{array}{l}\text { Width } \\
(\mathrm{mm})\end{array}$ & & $\begin{array}{l}\text { Thickness } \\
(\mathrm{mm})\end{array}$ & \\
\hline & & Mean & SD & Mean & SD & Mean & SD \\
\hline $10 \%$ & 47 & 24.55 & 7.32 & 23.36 & 8.54 & 6.23 & 2.96 \\
\hline $1-50 \%$ & 159 & 29.32 & 11.39 & 26.18 & 9.32 & 7.67 & 3.30 \\
\hline $51-99 \%$ & 62 & 31.21 & 11.47 & 27.50 & 12.62 & 9.23 & 4.44 \\
\hline $100 \%$ & 25 & 29.52 & 12.02 & 29.00 & 12.75 & 10.56 & 14.91 \\
\hline None & 51 & 25.41 & 9.62 & 19.67 & 8.39 & 5.43 & 2.37 \\
\hline Total & 344 & 28.44 & 10.92 & 25.27 & 10.35 & 7.63 & 5.30 \\
\hline
\end{tabular}

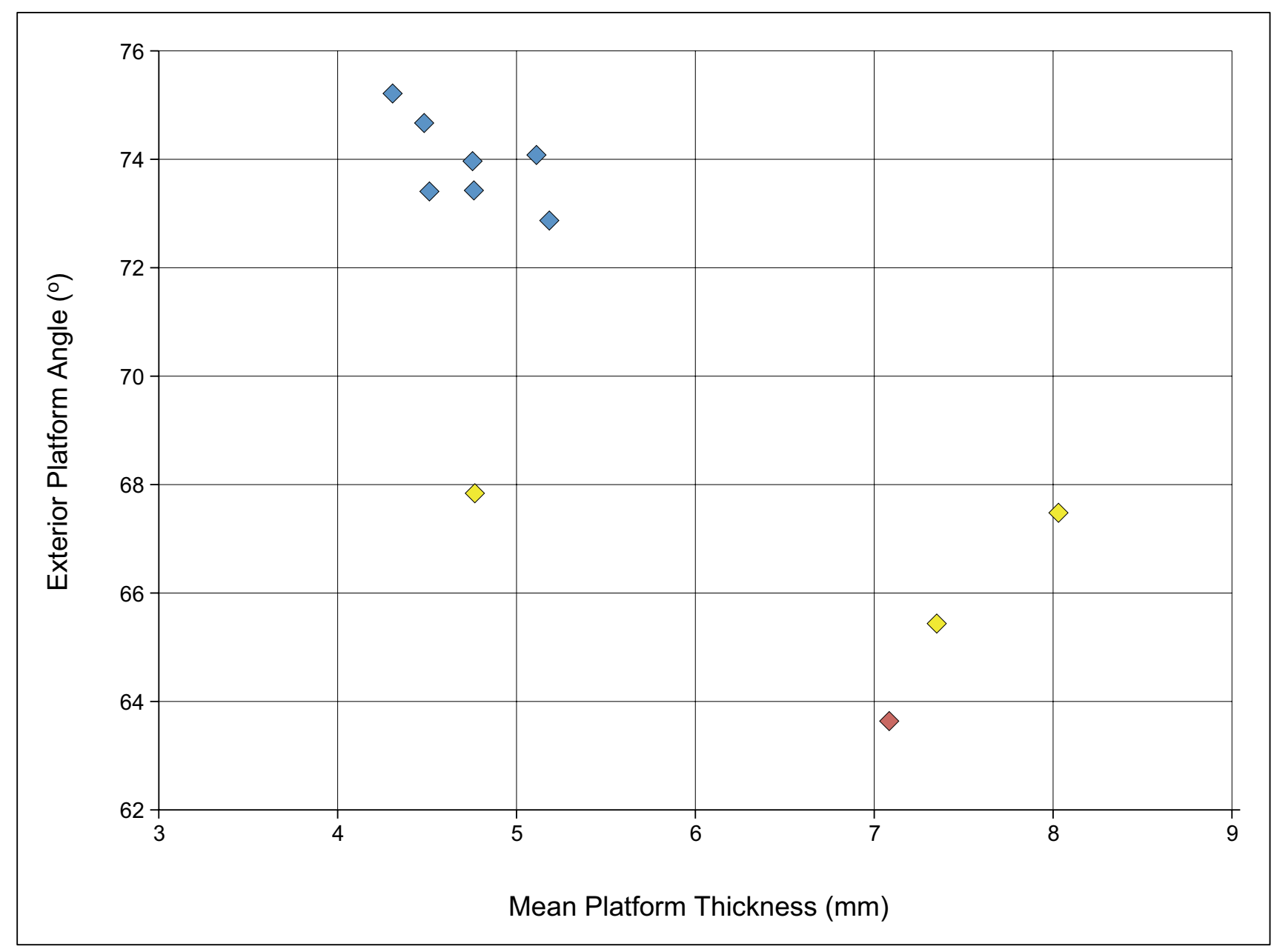

Figure 5.12. Mean complete flake platform thickness against mean exterior platform angle. Red icon: K1 assemblage; yellow icons: L1 and XB11 assemblages; blue icons: E29H1 assemblages. 
Table 5.10. K1 Tool Type Frequency by Flake Class.

\begin{tabular}{|l|r|r|r|r|}
\hline & \multicolumn{1}{|c|}{$\begin{array}{c}\text { Angular } \\
\text { Fragment }\end{array}$} & $\begin{array}{c}\text { Complete } \\
\text { Tool }\end{array}$ & \multicolumn{1}{c|}{$\begin{array}{c}\text { Distal } \\
\text { Tool }\end{array}$} & \multicolumn{1}{c|}{$\begin{array}{c}\text { Medial } \\
\text { Tool }\end{array}$} \\
\hline Bifacial & 1 & 2 & 2 & \\
\hline Denticulate & 3 & 3 & 3 & 1 \\
\hline Notch & & 1 & & \\
\hline Projectile & 1 & & & \\
\hline Scraper & & 3 & 4 & \\
\hline
\end{tabular}

\section{Discussion}

Although the K1 assemblage is relatively small, analysis results suggest that it was formed by flaking relatively larger cobbles than was the case in the $\mathrm{E} 29 \mathrm{H} 1$ and L1 assemblages. As a consequence, the cores are relatively large, and when the cortical cores flaked from single or bifacial platforms are used to estimate original cobble size, the results suggest that these were two to three times larger than those flaked elsewhere. These larger cobbles produced flakes with larger mean dimensions than those in other assemblages, but the cores were not completely reduced; cortex is found on the majority of the cores, with lower proportions on cores that have been rotated to engage multiple platforms.

Across the K Basin, the flake-to-core ratio results suggest the presence of some assemblages that are similar to the E29H1 assemblages (C4T5) and the L1 and XB11 assemblages (the $\mathrm{K} 1$ assemblages as well as the C4T10 and C4T13 assemblages).

Analysis of surface area and volume of the flakes and cores suggests that there is neither sufficient cortical surface area to accommodate the number of cobbles flaked nor a sufficient number of cores to account for the likely number of cobbles worked. These results suggest the removal of a relatively large number of cores. As discussed in chapter 4 , the cortex ratio and volume ratios need to be considered together (Phillipps and Holdaway 2016). For K1, loss of cortex probably reflects the removal of cores as much as it does the removal of flakes, particularly given the large volume of material present given the number of cores.

The flakes that remain are closest in platform size and exterior platform angle to those of the assemblages from the $\mathrm{L} 1$ transects, and this is also true for flake shape. The $\mathrm{K} 1$ flakes are relatively wide in relation to their length and do not have the blade-like dimensions of those from some of the E29H1 assemblages. Both the cortex and volume ratio values suggest removal of cores. However, in other ways, the $\mathrm{K} 1$ assemblage is unlike those analyzed from L1 and XB11. Certainly, the size of the cobbles used to form the $\mathrm{K} 1$ assemblage sets it apart from the others.

\section{Summary and Discussion of K1 Survey Area}

The K1 survey area is likely to have preserved a surface archaeological record that is more extensive than that evident today. Modern agricultural development continues to destroy the surface and with it the ability to understand the archaeological significance of $\mathrm{K} 1$. This is particularly unfortunate since there are stratified deposits at Kom IV (discussed above) and at Kom K (chapter 6). Nevertheless, some surfaces remain intact, and we were able to apply the methodology used in L1 to provide some understanding of how the basin was used. The deposits that remain are limited to an area north of Kom $\mathrm{K}$ and immediately east of the Upper K Pits, and a strip that runs along the western edge of the basin. Within these areas, the density of surface artifacts is lower than areas farther west. However, the same range of flaked stone artifacts, pottery fragments, animal bones, hearths, and grinding stones is present.

Radiocarbon determinations from hearths are limited to those in the north of Kom K, as well as those from Kom K itself, discussed in the next chapter. Results indicate three phases of occupation that match the more recent hearth ages found farther west, although the number of excavated hearths reported is relatively small. Hearth ages indicate occupation around $6500 \mathrm{cal}$ BP, $7000 \mathrm{cal} \mathrm{BP}$, and earlier than $7500 \mathrm{cal} \mathrm{BP}$. Like the results obtained from hearths farther west, the dates do not fall into two periods, an early and a late occupation, as is reported in the older literature.

Flaked stone artifacts are the most abundant form of material culture, and there were sufficient numbers of these in the $\mathrm{K} 1$ area to undertake comparative analyses with those found farther west. The raw material used at $\mathrm{K} 1$ was larger than that used in either the E29H1, L1, or XB11 assemblages, although as with the later assemblages, there is good evidence that cores were moved away from the $\mathrm{K} 1$ area. As a consequence of the size of the cobbles worked, flakes are large in the $\mathrm{K} 1$ assemblage. As discussed in chapter 4, differences in raw material form and removal of the products of cobble working suggest differences in the way utility was exploited by the people who spent time in different parts of the Fayum. As in other areas, at K1 material was imported and subsequently transported away after 
it had been partially worked (in this case as cores). The difference is that larger cobbles were sourced than in assemblages farther west. People were willing to move larger, and therefore heavier, cobbles and to produce larger flakes, but not in a form that maximized the cutting edge of these flakes, since they were still wide relative to their length.

This suggests a different strategy to those documented in chapter 4. However, rather than claim that this strategy is reflective of a different people than those who occupied areas farther west, it is important to reiterate that the material evidence we have at places like $\mathrm{K} 1$ represents the results of activities related to only part of the lifeways of the people responsible for their deposition. Therefore understanding the significance of the assemblage composition can come only by comparing it to artifacts found in other locations. For this reason, we report below on artifact assemblages obtained from our excavations at Kom K (chapter 6).

\section{Upper K Pits}

In 1925 geologist Elinor Gardner discovered the Upper K Pits (Caton-Thompson and Gardner 1934:46-54).
While Caton-Thompson was excavating at Kom K, Gardner set out to explore a high ridge, approximately $1 \mathrm{~km}$ to the north of Kom $\mathrm{K}$ at an elevation of $32 \mathrm{~m}$ asl, to determine its geological composition (Figure 5.1). She scraped away the desert pavement and found circular outlines that appeared to indicate pits. Subsequent investigation showed that the Upper $K$ Pits were characterized by excellent preservation of organic materials, which included wheat straw and grass basketry and wheat and barley grains, and thus provided information on the adoption of domesticated grain and the development of agriculture. Of the 67 features excavated by Caton-Thompson and Gardner (1934:41-42), 56 were identified as granaries or silos (Table 5.11). Of these, 42 were lined with "coiled straw" and four had imprints of matting (Figures 5.13 and 5.14). Seven of the pits contained remains of desiccated domesticated wheat and barley, and one held a piece of coarsely woven linen. CatonThompson and Gardner also explored an area to the west, where a group of similar pits were found. These Lower K Pits were less well preserved than the Upper $\mathrm{K}$ Pits group and are discussed below.

Table 5.11. Upper K Pits, Summary of Pits and Other Features from the Previous and Current Excavations.

\begin{tabular}{|c|c|c|}
\hline $\begin{array}{l}\text { Feature Number } \\
\text { and Reference }\end{array}$ & Dimensions & Description \\
\hline $\begin{array}{l}\text { Silo } 1 \text { (Plate xxv, } \\
1-2), \text { p. } 50\end{array}$ & $\begin{array}{l}\text { Diameter } 3 \mathrm{ft} .101 / 2 \mathrm{in} \text {. Depth } 2 \mathrm{ft} .7 \mathrm{in} . \\
(1.18 \times 1.18 \times 0.79 \mathrm{~m})\end{array}$ & $\begin{array}{l}\text { Lined. Contained also a loose circular straw mat (Plate } \\
\text { xxv, 3) at a depth of } 1 \mathrm{ft} .10 \mathrm{in} .(0.56 \mathrm{~m}) \text {. Lying in shelly } \\
\text { gravel that formed the infilling. No contents. }\end{array}$ \\
\hline Silo 2 , p. 50 & $\begin{array}{l}\text { Diameter } 3 \mathrm{ft} .41 / 2 \mathrm{in.} \text { Depth } 2 \mathrm{ft} .2 \text { in. } \\
(1.03 \times 1.03 \times 0.69 \mathrm{~m})\end{array}$ & Lined. Contents: tamarisk stake. \\
\hline Silo 3 , p. 50 & $\begin{array}{l}\text { Diameter about } 7 \mathrm{ft} .7 \text { in. A note indicates } \\
\text { that this is "a very doubtful measurement." } \\
\text { Depth } 3 \mathrm{ft} .(2.3 \times 2.3 \times 0.91 \mathrm{~m})\end{array}$ & Unlined. Contents: emmer spikelet. \\
\hline Silo 4, p. 50 & $\begin{array}{l}\text { Diameter } 3 \mathrm{ft} ., 41 / 2 \text { in. Distorted by “earth } \\
\text { pressure." Depth } 2 \mathrm{ft} .(1.05 \times 1.05 \times 0.61 \mathrm{~m})\end{array}$ & $\begin{array}{l}\text { Lined. Contained a loose straw lining similar to Silo } 1 \text { at } \\
10 \text { in. depth }(0.25 \mathrm{~m}) \text {, underlain and overlain by gravel } \\
\text { infilling. No contents. }\end{array}$ \\
\hline Silo 5 , p. 51 & $\begin{array}{l}\text { Diameter } 1 \mathrm{ft} .9 \text { in. Depth }+10 \text { in. } \\
(0.53 \times 0.53 \mathrm{~m} \mathrm{x}+0.25 \mathrm{~m})\end{array}$ & Lined. No contents. \\
\hline $\begin{array}{l}\text { Silo } 6 \text { (Plate } \\
\text { xxviii, 4), p. } 51\end{array}$ & $\begin{array}{l}\text { Diameter } 1 \mathrm{ft} .4 \text { in. }(0.41 \times 0.41 \mathrm{~m}) . \text { Laying } \\
\text { in gravel } 8 \text { in. }(0.20 \mathrm{~m}) \text { below the surface }\end{array}$ & $\begin{array}{l}\text { Now in Cairo. Platter or lid in woven grass. Found with } \\
\text { a spikelet of emmer. }\end{array}$ \\
\hline Silo 7, p. 51 & $\begin{array}{l}\text { Diameter } 1 \mathrm{ft} .6 \text { in. Depth }+9 \text { in. } \\
(0.46 \times 0.46 \times \text { approx. } 0.23 \mathrm{~m})\end{array}$ & Lined. Contents: ceramic bowl (Plate xvi, 8). \\
\hline Silo 8, p. 51 & $\begin{array}{l}\text { Diameter } 3 \mathrm{ft} . \text { Depth } 1 \mathrm{ft} .6 \text { in. } \\
(0.91 \times 0.91 \times 0.46 \mathrm{~m})\end{array}$ & $\begin{array}{l}\text { Sent to Newcastle. Lined, but only the floor could be } \\
\text { saved. No contents. }\end{array}$ \\
\hline Silo 9 , p. 51 & $\begin{array}{l}\text { Diameter } 3 \mathrm{ft} .2 \text { in. Depth } 1 \mathrm{ft} .9 \text { in. } \\
(0.96 \times 0.96 \times 0.53 \mathrm{~m})\end{array}$ & Lined. No contents. \\
\hline
\end{tabular}


Table 5.11. Upper K Pits, Summary of Pits and Other Features from the Previous and Current Excavations. Continued

\begin{tabular}{|c|c|c|}
\hline $\begin{array}{l}\text { Feature Number } \\
\text { and Reference }\end{array}$ & Dimensions & Description \\
\hline Silo 10, p. 51 & $\begin{array}{l}\text { Diameter top } 4 \mathrm{ft} .2 \text { in. Floor } 3 \mathrm{ft} \text {. Depth } 2 \\
\mathrm{ft} .11 \text { in. }(1.27 \times 1.27 \mathrm{~m} \text { at rim; } 0.91 \times 0.91 \\
\mathrm{m} \text { at bottom; depth } 0.89 \mathrm{~m})\end{array}$ & Lined. No contents. \\
\hline Silo 11, p. 51 & $\begin{array}{l}\text { Diameter } 2 \text { ft. }(0.61 \times 0.61 \mathrm{~m}) \text {; depth } \\
\text { uncertain }\end{array}$ & Lined. No contents. \\
\hline $\begin{array}{l}\text { Silo } 12 \text { (Plates } \\
\text { xxv, } 4 \text { and xxvii, } \\
3 \text { ), p. } 51\end{array}$ & $\begin{array}{l}\text { Diameter } 3 \mathrm{ft} .8 \text { in. Depth } 2 \mathrm{ft} .3 \text { in. } \\
(1.12 \times 1.12 \times 0.69 \mathrm{~m})\end{array}$ & Now in Hull. Lined. No contents. \\
\hline Silo 13 , p. 51 & $\begin{array}{l}\text { Diameter } 4 \mathrm{ft} .3 \text { in. Depth } 1 \mathrm{ft} .8 \text { in. } \\
(1.30 \times 1.30 \times 0.51 \mathrm{~m})\end{array}$ & $\begin{array}{l}\text { Lined. Contents: grain: mixed emmer and barley, about } \\
11 / 2 \text { pints ( } 0.85 \text { liter) of which were sifted out from the } \\
\text { basal infilling. A few charred corns and a fragment of } \\
\text { ostrich eggshell were mixed with it. }\end{array}$ \\
\hline Silo 14, p. 51 & $\begin{array}{l}\text { Diam. approx. } 2 \mathrm{ft} .5 \text { in. Depth }+9 \text { in. } \\
(0.74 \times 0.74 \times \text { approx. } 0.23 \mathrm{~m})\end{array}$ & $\begin{array}{l}\text { Bolton Museum. This specimen was in such derelict } \\
\text { condition by the time it reached Bolton that eventually, } \\
\text { owing to further disintegration by salt, it was destroyed. } \\
\text { Lined. A portion had become detached from the walls and } \\
\text { was doubled in. Contents: tamarisk stick, shaped (Plate } \\
\text { xxix, } 4 \text {; cf. sect. } 66) \text {; grain: mixed wheat and barley, about } \\
1 / 2 \text { pint ( } 0.28 \text { liters). }\end{array}$ \\
\hline Silo 15 , p. 51 & $\begin{array}{l}\text { Diameter } 1 \mathrm{ft} .61 / 2 \text { in. Depth }+6 \text { in. } \\
(0.47 \times 0.47 \times \text { approx. } 0.23 \mathrm{~m})\end{array}$ & Lined, but only a few wisps remained. No contents. \\
\hline $\begin{array}{l}\text { Silo } 16 \text { (Plate } \\
\text { xxv, } 5) \text {, p. } 51\end{array}$ & $\begin{array}{l}\text { Diameter } 5 \mathrm{ft} .2 \text { in. Depth } 2 \mathrm{ft} .8 \text { in } \\
(1.60 \times 1.60 \times 0.81 \mathrm{~m})\end{array}$ & $\begin{array}{l}\text { Lined. Contents: three tamarisk stems lying on, and } \\
\text { some inches above, floor level; a single valve of Spatha } \\
\text { (seen in photograph on infilling deposit) } 14 \text { in. }(0.36 \mathrm{~m}) \\
\text { above floor level; a fine sickle blade (cf. sect. } 63 \text { ) on floor } \\
\text { level. On the southeast stood a subsidiary silo (Plate xxv, } \\
5 \text {, foreground) at higher level. Diameter } 2 \mathrm{ft} .(0.61 \mathrm{~m}) \text {; } \\
\text { depth approx. } 1 \mathrm{ft} \text {. }(0.30 \mathrm{~m})(0.61 \times 0.61 \times 0.30 \mathrm{~m}) \text {. Its } \\
\text { lining touched that of the silo beside it (Plate xxv, } 5 \text {, left } \\
\text { foreground). Crumbling ceramic pot in badly fired coarse } \\
\text { brown-red ware; in it were a piece of linen (Plate xxviii, } \\
\text { 3), two pebbles, and a fish vertebra. }\end{array}$ \\
\hline Silo 17, p. 51 & $\begin{array}{l}\text { Diameter } 2 \mathrm{ft} .8 \text { in. Depth } 1 \mathrm{ft} .7 \text { in. } \\
(0.81 \times 0.81 \times 0.48 \mathrm{~m})\end{array}$ & Lined, but little remaining. No contents. \\
\hline $\begin{array}{l}\text { Silo } 18 \text { (Plate } \\
\text { xxv, 6), p. } 51\end{array}$ & $\begin{array}{l}\text { Diameter } 3 \mathrm{ft} .4 \text { in. Depth } 1 \mathrm{ft} .8 \text { in. } \\
(1.02 \times 1.02 \times 0.51 \mathrm{~m})\end{array}$ & $\begin{array}{l}\text { Went to Cairo. Lined and in very good condition. } \\
\text { Contents: tamarisk stem on the floor, Polygonum seeds } \\
\text { (knotgrass), and remains of unidentified fruit. }\end{array}$ \\
\hline $\begin{array}{l}\text { Silo } 19 \text { (Plate } \\
\text { xxvi, 6), p. } 51\end{array}$ & $\begin{array}{l}\text { Diameter } 2 \mathrm{ft} .6 \text { in. Depth } 1 \mathrm{ft} .6 \text { in. } \\
(0.76 \times 0.76 \times 0.46 \mathrm{~m})\end{array}$ & $\begin{array}{l}\text { Went to Ashmolean Museum. Lined; in good condition. } \\
\text { No contents. }\end{array}$ \\
\hline Silo 20, p. 51 & $\begin{array}{l}\text { Diameter } 3 \mathrm{ft} .10 \text { in. Depth }+1 \mathrm{ft} .1 \text { in. } \\
(1.17 \times 1.17 \mathrm{x}+0.33 \mathrm{~m})\end{array}$ & Lined but perished. No contents. \\
\hline Silo 21, p. 51 & $\begin{array}{l}\text { Diameter } 4 \mathrm{ft} .4 \text { in. Depth } 2 \mathrm{ft} .2 \text { in. } \\
(1.32 \times 1.32 \times 0.66 \mathrm{~m})\end{array}$ & $\begin{array}{l}\text { Lined, but the evidence of this rested with a perfect } \\
\text { impression on the floor. No contents. }\end{array}$ \\
\hline Silo 22, p. 51 & $\begin{array}{l}\text { Diameter } 3 \mathrm{ft} .1 \text { in. Depth } 3 \mathrm{ft} .3 \mathrm{in} . \\
(0.94 \times 0.94 \times 0.99 \mathrm{~m})\end{array}$ & $\begin{array}{l}\text { Unlined. Contents: a few spikelets of mixed emmer and } \\
\text { barley at } 3 \mathrm{ft} .3 \text { in. }(0.99 \mathrm{~m}) \text {, which is therefore taken as } \\
\text { floor level; small chips of tamarisk wood at } 2 \mathrm{ft} .(0.61 \mathrm{~m}) \\
\text { down. }\end{array}$ \\
\hline No. 23, p. 51 & & $\begin{array}{l}\text { A rough, large ceramic pot (Plate xix, } 42) \text { in completely } \\
\text { disintegrated condition, } 6 \text { in. }(0.15 \mathrm{~m}) \text { below surface. }\end{array}$ \\
\hline $\begin{array}{l}\text { Silo } 24 \text { (Plate } \\
\text { xxvii, I, } \\
\text { background), } \\
\text { p. } 51\end{array}$ & $\begin{array}{l}\text { Diameter } 3 \mathrm{ft} .7 \text { in. Depth } 1 \mathrm{ft} .7 \text { in. }(1.09 \mathrm{x} \\
1.09 \times 0.48 \mathrm{~m})\end{array}$ & $\begin{array}{l}\text { Lined. Bent in and broken on south side by external } \\
\text { pressure. Floor was also distorted and broken. No } \\
\text { contents. }\end{array}$ \\
\hline
\end{tabular}




\begin{tabular}{|c|c|c|}
\hline $\begin{array}{l}\text { Feature Number } \\
\text { and Reference }\end{array}$ & Dimensions & Description \\
\hline $\begin{array}{l}\text { No. } 25 \text { (Plate } \\
\text { xxvii, 1), p. } 51\end{array}$ & $\begin{array}{l}\text { Central diameter } 2 \mathrm{ft} . ; \text { remaining height } 9 \\
\text { in.; thickness approx. } 13 / 4 \text { in. }(0.61 \times 0.61 \\
\text { x } 0.23 \mathrm{~m})\end{array}$ & $\begin{array}{l}\text { Now at University College London. Basal half of a } \\
\text { large coarse ceramic pot. Red-brown ware; badly fired. } \\
\text { The pot, the raw edge of which was only } 4 \text { in. }(0.10 \mathrm{~m}) \\
\text { beneath the surface, stood on a base of plaited straw (cf. } \\
\text { sect. } 65 \text { ). }\end{array}$ \\
\hline Silo 26 , p. 51 & Diameter $1 \mathrm{ft} .11$ in. $(0.58 \times 0.58 \mathrm{~m})$ & Depth uncertain. Lined but fragmentary. No contents. \\
\hline Silo 27, p. 51 & $\begin{array}{l}\text { Diameter } 1 \mathrm{ft} .2 .5 \text { in. Depth } 9 \text { in. }(0.37 \mathrm{x} \\
0.37 \times 0.23 \mathrm{~m})\end{array}$ & Lined. No contents. \\
\hline $\begin{array}{l}\text { No. } 28 \text { (Plate } \\
\text { xxviii, 5), p. } 51\end{array}$ & Diameter 9 in. $(0.23 \times 0.23 \mathrm{~m})$ & $\begin{array}{l}\text { Shipped to Manchester Museum. Platter or lid in woven } \\
\text { grass. Lying in gravel approx. } 6 \text { in. }(0.15 \mathrm{~m}) \text { below } \\
\text { surface (cf. sect. 63). }\end{array}$ \\
\hline Silo 29 , p. 51 & & Lined but very fragmentary. No contents. \\
\hline $\begin{array}{l}\text { Silo } 30 \text { (Plate } \\
\text { xxvi, 7), p. } 51\end{array}$ & $\begin{array}{l}\text { Diameter } 1 \mathrm{ft} .8 .5 \text { in. Depth } 9 \text { in. }(0.52 \mathrm{x} \\
0.52 \times 0.23 \mathrm{~m})\end{array}$ & Now in British Museum. Lined. No contents. \\
\hline Silo 31, p. 51 & $\begin{array}{l}\text { Diameter } 2 \mathrm{ft} .5 \text { in. Depth } 1 \mathrm{ft} .9 \text { in. }(0.74 \mathrm{x} \\
0.74 \times 0.53 \mathrm{~m})\end{array}$ & Lined. No contents. \\
\hline Silo 32, p. 51 & $\begin{array}{l}\text { Diameter } 3 \mathrm{ft} .4 \text { in. Depth } 1 \mathrm{ft} .1 \text { in. }(1.02 \mathrm{x} \\
1.02 \times 0.33 \mathrm{~m})\end{array}$ & Lined. One side sagging. No contents. \\
\hline Silo 33, p. 51 & $\begin{array}{l}\text { Diameter } 3 \mathrm{ft} .3 .5 \text { in. Depth } 1 \mathrm{ft} .6 \text { in. }(1.00 \\
\mathrm{x} 1.00 \times 0.46 \mathrm{~m})\end{array}$ & $\begin{array}{l}\text { Lined. Contents: grain: about } 1 \text { pint ( } 0.57 \text { liter }) \\
\text { carbonized wheat and barley. Also pieces of charcoal; } \\
\text { possibly tamarisk. }\end{array}$ \\
\hline Silo 34, p. 51 & $\begin{array}{l}\text { Diameter } 3 \mathrm{ft} .8 .5 \text { in. Depth } 3 \mathrm{ft} .(1.13 \mathrm{x} \\
1.13 \times 0.90 \mathrm{~m})\end{array}$ & $\begin{array}{l}\text { Unlined. Contents: grain: about } 1.5 \text { pints }(0.85 \text { liter }) \\
\text { emmer and barley, sifted out from the bottom } 12 \text { in. } \\
(0.30 \mathrm{~m}) \text { shelly gravel infilling. }\end{array}$ \\
\hline Silo 35 , p. 51 & $\begin{array}{l}\text { Diameter } 2 \mathrm{ft} .1 \text { in. Depth } 1 \mathrm{ft} .(0.33 \times 0.33 \\
\mathrm{x} 0.30 \mathrm{~m})\end{array}$ & Lined but very fragmentary. No contents. \\
\hline $\begin{array}{l}\text { Silo } 36 \text { (Plate } \\
\text { xxvi, 3), p. } 51\end{array}$ & $\begin{array}{l}\text { Diameter } 3 \mathrm{ft} \text {. Depth } 1 \mathrm{ft} .10 \text { in. }(0.90 \mathrm{x} \\
0.90 \times 0.25 \mathrm{~m})\end{array}$ & $\begin{array}{l}\text { Lined and in good condition. Contents: tamarisk stem, } \\
1 \mathrm{ft} .8 \text { in. }(0.51 \mathrm{~m}) \text { long; a block of fossil wood, approx. } \\
8 \text { in. } \times 3.5 \text { in. }(0.20 \mathrm{~m} \times 0.09 \mathrm{~m}) \text {, apparently unworked. } \\
\text { This is unlikely to belong to the gravel infilling and was } \\
\text { probably artificially introduced. }\end{array}$ \\
\hline Silo 37, p. 51 & $\begin{array}{l}\text { Diameter } 3 \mathrm{ft} .3 \text { in. Depth } 2 \text { ft. } 1 \text { in. }(0.99 \times \\
0.99 \times 0.64 \mathrm{~m})\end{array}$ & Lined but disintegrated. No contents. \\
\hline Silo 38 , p. 51 & $\begin{array}{l}\text { Diameter } 3 \mathrm{ft} . \text { Depth } 3 \mathrm{ft} .7 \text { in. }(0.91 \times 0.91 \\
\mathrm{x} 1.09 \mathrm{~m})\end{array}$ & $\begin{array}{l}\text { Unlined. No contents. Depth measurement questioned by } \\
\text { Caton-Thompson. }\end{array}$ \\
\hline Silo 39, p. 51 & no dimensions recorded & Lined but trace only. No contents. \\
\hline Silo 40 , p. 51 & $\begin{array}{l}\text { Diameter } 3 \mathrm{ft} .2 \text { in. Depth }+10 \text { in. }(0.96 \mathrm{x} \\
0.96 \mathrm{x} \text { approx. } 0.25 \mathrm{~m})\end{array}$ & Lined but trace only. No contents. \\
\hline Silo 41, p. 51 & $\begin{array}{l}\text { Diameter } 1 \mathrm{ft} .10 \text { in. Depth } 1 \mathrm{ft} .2 \text { in. }(0.56 \\
\mathrm{x} 0.56 \times 0.36 \mathrm{~m})\end{array}$ & Lined, proved by gypseous impression only. No contents. \\
\hline Silo 42, p. 51 & $\begin{array}{l}\text { Diameter } 2 \mathrm{ft} .10 \text { in. Depth }+8 \text { in. }(0.86 \mathrm{x} \\
0.86 \mathrm{x} \text { approx. } 0.20 \mathrm{~m})\end{array}$ & Lined, proved by floor impression only. No contents. \\
\hline Silo 43, p. 51 & $\begin{array}{l}\text { Diameter } 4 \mathrm{ft} . \text { Depth } 2 \mathrm{ft} .6 \text { in. }(1.22 \times 1.22 \\
\mathrm{x} 0.76 \mathrm{~m})\end{array}$ & $\begin{array}{l}\text { Lined, proved by gypseous impression on floor. No } \\
\text { contents. }\end{array}$ \\
\hline Silo 44, p. 51 & no dimensions recorded & $\begin{array}{l}\text { Unlined and limits uncertain. Contents: grain: about } 2 \\
\text { pints }(1.14 \text { liter) of charred wheat and barley, mixed } \\
\text { with a few spikelets of uncharred corn; also fragments } \\
\text { of charcoal and unburned twigs. This was scattered } \\
\text { throughout the disturbed gravel between } 2 \mathrm{ft} \text {. and } 2 \mathrm{ft} .8 \\
\text { in. }(0.61-0.81 \mathrm{~m}) \text {. }\end{array}$ \\
\hline
\end{tabular}


Table 5.11. Upper K Pits, Summary of Pits and Other Features from the Previous and Current Excavations. Continued

\begin{tabular}{|c|c|c|}
\hline $\begin{array}{l}\text { Feature Number } \\
\text { and Reference }\end{array}$ & Dimensions & Description \\
\hline Silo 45 , p. 52 & $\begin{array}{l}\text { Diameter } 4 \mathrm{ft} .8 \text { in. Depth } 2 \mathrm{ft} .10 \text { in.? ( } 1.42 \\
\mathrm{x} 1.42 \times 0.86 \mathrm{~m} \text { ?) }\end{array}$ & No description. \\
\hline Silo 46, p. 52 & $\begin{array}{l}\text { Diameter } 2 \text { ft. } 8 \text { in. Depth } 2 \text { ft. }(0.81 \text { x } 0.81 \\
\mathrm{x} 0.60 \mathrm{~m})\end{array}$ & $\begin{array}{l}\text { Unlined. Contents: a few sherds of thick, badly fired } \\
\text { brown-red ware ceramics. }\end{array}$ \\
\hline Silo 47, p. 52 & $\begin{array}{l}\text { Diameter } 3 \mathrm{ft} .5 \text { in. Depth } 1 \text { ft. } 9 \text { in. }(1.04 \mathrm{x} \\
1.04 \times 0.53 \mathrm{~m})\end{array}$ & $\begin{array}{l}\text { Lined but reduced by disintegration to a ring in the floor } \\
\text { center only. Contents: small cracked ceramic bowl (Plate } \\
\text { xvi, } 7 \text {, and Plate xviii, 2) in red-brown rough ware with } \\
\text { darker rim. }\end{array}$ \\
\hline Silo 48, p. 52 & $\begin{array}{l}\text { Diameter } 3 \mathrm{ft} .7 \text { in. Depth } 3 \mathrm{ft} .(1.09 \times 1.09 \\
\text { x } 0.90 \mathrm{~m})\end{array}$ & $\begin{array}{l}\text { Lined but fragmentary. Contents: pair of Spatha valves } \\
\text { on floor; one perfect, the other with jagged edges as } \\
\text { though used. }\end{array}$ \\
\hline $\begin{array}{l}\text { No. } 49 \text { (Plate } \\
\text { xix, } 42 \text { ), p. } 52\end{array}$ & $\begin{array}{l}\text { Diameter at mouth } 14 \text { in.; height } 14 \text { in. } \\
(0.36 \times 0.36 \mathrm{~m}) \text {; central circumference } 3 \mathrm{ft} . \\
9 \text { in. }(1.14 \mathrm{~m}) \text {; thickness } 2 \text { in. }(0.05 \mathrm{~m})\end{array}$ & $\begin{array}{l}\text { Large pot in coarse red-brown ware, with black } \\
\text { crumbling core. Straight-rimmed type. Rim was just } \\
\text { beneath the surface. Ceramic pot was cracked and } \\
\text { crumbling and though waxed was not saved. }\end{array}$ \\
\hline $\begin{array}{l}\text { No. } 50 \text { (Plate } \\
\text { xxvi, 5), p. } 52\end{array}$ & no dimensions recorded & $\begin{array}{l}\text { Now in British Museum. Hole } 10 \text { to } 18 \text { in. }(0.25-0.46 \\
\text { m) below surface containing two wooden objects, bow } \\
\text { shaped and forked (cf. sect. } 66) \text {. }\end{array}$ \\
\hline $\begin{array}{l}\text { Silo } 51 \text { (Plate } \\
\text { xxvi, 1), p. } 52\end{array}$ & $\begin{array}{l}\text { Diameter } 2 \mathrm{ft} .10 \text { in., slightly sagging. } \\
\text { Depth } 1 \mathrm{ft} .5 \text { in. }(0.86 \times 0.86 \times 0.43 \mathrm{~m})\end{array}$ & $\begin{array}{l}\text { Lined. In fair condition. Contents: wooden sickle with } \\
\text { flint blade (now in British Museum) (Plate. xxviii, 2). } \\
\text { Also rim sherd of rough brown-red ware ceramic. }\end{array}$ \\
\hline Silo 52, p. 52 & $\begin{array}{l}\text { Diameter } 1 \mathrm{ft} .11 .5 \mathrm{in} . \text { Depth }+9 \text { in. }(0.60 \mathrm{x} \\
0.60 \mathrm{x} \text { approx. } 0.23 \mathrm{~m})\end{array}$ & $\begin{array}{l}\text { Lined but very fragmentary. Contents: wooden sickle } \\
\text { holder without blade (now in University College } \\
\text { London) (Plate xxviii, } 1 \text { ). }\end{array}$ \\
\hline Silo 53, p. 52 & $\begin{array}{l}\text { Diameter approx. } 5 \mathrm{ft} \text {. Depth about } 1 \mathrm{ft} .8 \\
\text { in. }(1.52 \times 1.52 \times 0.51 \mathrm{~m})\end{array}$ & $\begin{array}{l}\text { Unlined. Contents: wooden nabut (fighting stick) of } \\
\text { tamarisk wood, now in British Museum (Plate xxix, } \\
\text { 2); grinding stone of quartzite (cf. sect. 63); traces of } \\
\text { disintegrated leather or hide. }\end{array}$ \\
\hline No. 54 , p. 52 & Diameter 2 ft. 3 in. $(0.69 \times 0.69 \mathrm{~m})$ & $\begin{array}{l}\text { Circular straw matting, lying just covered and in very } \\
\text { bad condition. It was either the floor of a silo lining } \\
\text { ripped away from its sides or a separate mat, as in Silos } \\
1 \text { and } 3 \text {. } \\
\text { Disintegrated fragments of a big coarse ceramic pot with } \\
\text { coarse red fabric. }\end{array}$ \\
\hline $\begin{array}{l}\text { Silo } 55 \text { (Plate } \\
\text { xxvi, 2), p. } 52\end{array}$ & $\begin{array}{l}\text { Diameter } 2 \mathrm{ft} .5 \text { in. Depth }+10 \text { in. }(0.74 \mathrm{x} \\
0.74 \mathrm{x} \text { approx. } 0.25 \mathrm{~m})\end{array}$ & $\begin{array}{l}\text { Lined. Contents: fine woven oval basket (now in British } \\
\text { Museum) (Plate xxix, 1), standing on its base and tilted } \\
\text { to one side, with a stem of tamarisk. }\end{array}$ \\
\hline $\begin{array}{l}\text { No. } 56 \text { (Plate } \\
\text { xxvi, } 4 \text { ), p. } 52\end{array}$ & No dimensions recorded & $\begin{array}{l}\text { Hole containing a polished red ceramic pot with bulging } \\
\text { base and constricted top, resembling Plate xix, } 35 \text {, } \\
\text { with a dark red polished surface and traces of a straw } \\
\text { lid or carrier adhering; a plaited straw basket (now in } \\
\text { University College London), } 3 \text { in. }(0.08 \mathrm{~m}) \text { high, } 8 \text { in. } \\
(0.20 \mathrm{~m}) \text { below surface; two separate } S \text { patha valves. } \\
0.07 \text {-m-high basket, probably coiled, found } 0.20 \mathrm{~m} \\
\text { below the surface. }\end{array}$ \\
\hline Silo 57, p. 52 & $\begin{array}{l}\text { Diameter } 2 \mathrm{ft} .3 \text { in. Depth } 1 \mathrm{ft} .10 \text { in. }(0.69 \\
\mathrm{x} 0.69 \times 0.56 \mathrm{~m})\end{array}$ & No description recorded. \\
\hline No. 58 , p. 52 & No dimensions recorded & $\begin{array}{l}\text { Platter or lid in woven grass (now in Ashmolean } \\
\text { Museum), resembling Nos. } 6 \text { and } 28 \text {. Diameter } 12 \text { in. } \\
\text { Lying } 2 \mathrm{ft} .(0.60 \mathrm{~m}) \text { below surface in unconsolidated } \\
\text { gravel }(0.30 \times 0.30 \mathrm{~m}) \text {. }\end{array}$ \\
\hline
\end{tabular}




\begin{tabular}{|c|c|c|}
\hline $\begin{array}{l}\text { Feature Number } \\
\text { and Reference }\end{array}$ & Dimensions & Description \\
\hline Silo 59 , p. 52 & $\begin{array}{l}\text { Diameter } 1 \mathrm{ft} .9 \text { in. Depth }+12 \text { in. }(0.53 \mathrm{x} \\
0.53 \mathrm{x} \text { approx. } 0.30 \mathrm{~m})\end{array}$ & $\begin{array}{l}\text { Top is } 6 \text { in. }(0.15 \mathrm{~m}) \text { below surface. Lined, in good } \\
\text { condition. Contents: Polygonum (knotgrass) and Linum } \\
\text { (flax) seeds. }\end{array}$ \\
\hline $\begin{array}{l}\text { No. } 60 \text { (Plate } \\
\text { xvi, } 5 \text { and } 10) \\
\text { p. } 52\end{array}$ & & $\begin{array}{l}\text { Two small ceramic bowls (now in British Museum) in } \\
\text { hole in gravel, } 14 \text { in. }(0.36 \mathrm{~m}) \text { below surface, lying one } \\
\text { on top of the other. }\end{array}$ \\
\hline Silo 61, p. 52 & $\begin{array}{l}\text { Diameter } 1 \mathrm{ft} .6 \text { in. Depth } 2 \text { ft. } 6 \text { in. }(0.46 \mathrm{x} \\
0.46 \times 0.76 \mathrm{~m})\end{array}$ & $\begin{array}{l}\text { Lined. This narrow deep shape is comparable only to } \\
\text { No. } 62 \text {. Gravel infilling was particularly rocklike and } \\
\text { took a morning to chip out. No contents. }\end{array}$ \\
\hline Silo 62, p. 52 & $\begin{array}{l}\text { Diameter } 1 \text { ft. } 9 \text { in. Depth } 2 \text { ft. } 6 \text { in. }(0.53 \times x \\
0.53 \times 0.76 \mathrm{~m})\end{array}$ & Lined but very fragmentary. No contents. \\
\hline Silo 63, p. 52 & $\begin{array}{l}\text { Diameter } 2 \mathrm{ft} .3 \text { in. Depth } 1 \mathrm{ft} .4 \text { in. }(0.69 \mathrm{x} \\
0.69 \times 0.41 \mathrm{~m})\end{array}$ & $\begin{array}{l}\text { Lined. In good condition, but broken up in transport. } \\
\text { No contents. }\end{array}$ \\
\hline Silo 64, p. 52 & $\begin{array}{l}\text { Diameter } 3 \mathrm{ft} \text {. Depth } 2 \mathrm{ft} .(0.90 \times 0.90 \mathrm{x} \\
0.60 \mathrm{~m})\end{array}$ & $\begin{array}{l}\text { Found } 8 \text { in. }(0.20 \mathrm{~m}) \text { below surface. Lined but } \\
\text { fragmentary. Contents: small pot (Plate xvi, } 6) \text { and two } \\
\text { black polished sherds (Plate xvii, 14), all about } 6 \text { in. } \\
(0.15 \mathrm{~m}) \text { above base of silo. }\end{array}$ \\
\hline No. 65 , p. 52 & no dimensions recorded & $\begin{array}{l}\text { Three pieces of a small ceramic bowl, with no rim, in red } \\
\text { polished ware. In gravel } 14 \text { in. }(0.36 \mathrm{~m}) \text { below surface. }\end{array}$ \\
\hline Silo 66 , p. 52 & $\begin{array}{l}\text { Diameter } 2 \mathrm{ft} .6 \text { in. Depth }+9 \text { in. }(0.76 \mathrm{x} \\
0.76 \mathrm{x}+0.20 \mathrm{~m})\end{array}$ & Lined but fragmentary. No contents. \\
\hline $\begin{array}{l}\text { Silo } 67 \text { (Pit 67), } \\
\text { p. } 52 \\
\text { Trench UKP01 }\end{array}$ & $\begin{array}{l}\text { Diameter } 4 \text { ft. } 3 \text { in. Depth } 2 \text { ft. } 5 \text { in. } \\
\text { Diameter } 1.22 \times 1.04 \mathrm{~m} \text {, with smaller, } 0.89 \\
\text { x } 0.99 \text { m cut inside; depth } 0.74 \mathrm{~m}\end{array}$ & $\begin{array}{l}\text { Unlined. Separated from the rest on an outlying spur. } \\
\text { Contents: stake of unworked tamarisk stem, } 4 \mathrm{ft} .8 \mathrm{in} \text {. } \\
\text { long }(1.42 \mathrm{~m}) \text {, tilted across it. } \\
\text { Reexamination in } 2004 \text { showed that this pit is part of a } \\
\text { group of six pits surrounded by eight preparation areas. }\end{array}$ \\
\hline $\begin{array}{l}\text { Pit } 68 \\
\text { Trench UKP04 }\end{array}$ & Diameter $0.90 \times 0.90 \mathrm{~m}$; depth $0.60 \mathrm{~m}$ & $\begin{array}{l}\text { Well preserved. Basketry lined, with a } 0.20 \text {-m-thick lid. } \\
\text { Contents: shell scoop and two tamarisk sticks. }\end{array}$ \\
\hline $\begin{array}{l}\text { Pit } 69 \\
\text { Trench UKP09 }\end{array}$ & Diameter $1.00 \times 1.00$ m; depth 0.42 m & $\begin{array}{l}\text { Storage pit, unlined, with evidence of multiple uses, } \\
\text { including smaller cut in the bottom of the pit and cut in } \\
\text { the lid. Contents: Two shell scoops, a tamarisk stick, and } \\
\text { a large fragment of finely coiled basketry. }\end{array}$ \\
\hline $\begin{array}{l}\text { Feature } 70 \\
\text { Trench UKP09 } \\
\end{array}$ & Diameter $0.70 \times 0.50 \mathrm{~m}$; depth $0.22 \mathrm{~m}$ & Small, shelly gravel layer in natural depression. \\
\hline $\begin{array}{l}\text { Pit } 71 \\
\text { Trench UKP25 }\end{array}$ & Diameter $0.65 \times 0.60 \mathrm{~m}$; depth $0.26 \mathrm{~m}$ & $\begin{array}{l}\text { Basketry-lined pit with a ceramic vessel embedded in the } \\
\text { lid. }\end{array}$ \\
\hline $\begin{array}{l}\text { Feature } 72 \\
\text { Trench UKP05 } \\
\end{array}$ & Diameter $0.85 \times 0.70 \mathrm{~m}$; depth $0.07 \mathrm{~m}$ & $\begin{array}{l}\text { Preparation area for lid production in a shallow natural } \\
\text { depression. }\end{array}$ \\
\hline $\begin{array}{l}\text { Pit } 73 \\
\text { Trench UKP05 }\end{array}$ & Diameter $1.30 \times 1.20 \mathrm{~m}$; depth $0.40 \mathrm{~m}$ & $\begin{array}{l}\text { Large shallow pit with no basketry lining. A few coils of } \\
\text { a fine basket found in fill. }\end{array}$ \\
\hline $\begin{array}{l}\text { Feature } 74 \\
\text { Trench UKP06 }\end{array}$ & Diameter $1.00 \times 1.20 \mathrm{~m}$; depth $0.20-0.25 \mathrm{~m}$ & Preparation area for lid production. \\
\hline
\end{tabular}


Table 5.11. Upper K Pits, Summary of Pits and Other Features from the Previous and Current Excavations. Continued

\begin{tabular}{|c|c|c|}
\hline $\begin{array}{l}\text { Feature Number } \\
\text { and Reference }\end{array}$ & Dimensions & Description \\
\hline $\begin{array}{l}\text { Pit } 75 \\
\text { Trench UKP07 }\end{array}$ & Diameter $1.00 \times 0.90 \mathrm{~m}$; depth $0.35 \mathrm{~m}$ & $\begin{array}{l}\text { Shallow pit with no intact basketry lining, but many } \\
\text { straw fibers found in fill. }\end{array}$ \\
\hline $\begin{array}{l}\text { Feature } 76 \\
\text { Trench UKP17 }\end{array}$ & Diameter $0.75 \times 0.60 \mathrm{~m}$; depth $0.14 \mathrm{~m}$ & Mortar preparation area. \\
\hline $\begin{array}{l}\text { Feature } 77 \\
\text { Trench UKP14 } \\
\end{array}$ & Diameter $0.60 \times 0.60 \mathrm{~m}$; depth $0.15 \mathrm{~m}$ & Mortar preparation area in natural depression. \\
\hline $\begin{array}{l}\text { Feature } 78 \\
\text { Trench UKP04 }\end{array}$ & Diameter $0.50 \times 0.40 \mathrm{~m}$; depth $0.07 \mathrm{~m}$ & Mortar preparation area in natural depression. \\
\hline $\begin{array}{l}\text { Feature } 79 \\
\text { Trench UKP31 }\end{array}$ & Diameter $1.90 \times 1.90 \mathrm{~m}$; depth $0.15 \mathrm{~m}$ & $\begin{array}{l}\text { Large area, thought to be for mortar preparation, with } \\
\text { small gypsum and sand deposits forming gullies around } \\
\text { central compact area. Wheat chaff and one flaked stone } \\
\text { artifact found in compact area and adjacent deposits. }\end{array}$ \\
\hline $\begin{array}{l}\text { Feature } 80 \\
\text { Trench UKP30 }\end{array}$ & Diameter $1.60 \times 1.20 \mathrm{~m}$; depth $0.15 \mathrm{~m}$ & Mortar preparation area. \\
\hline $\begin{array}{l}\text { Pit } 81 \\
\text { Trench UKP01 }\end{array}$ & Diameter 1.00 x $0.99 \mathrm{~m}$ & Probable robber pit; not excavated. \\
\hline $\begin{array}{l}\text { Pit } 82 \\
\text { Trench UKP01 }\end{array}$ & Diameter 1.90 x 1.80 ; depth $0.70 \mathrm{~m}$ & Probable robber pit. No cultural materials. \\
\hline
\end{tabular}

Note: Pits are described as silos, while other finds are referred to only by number. Descriptions for the previously excavated pits and other finds are taken from Caton-Thompson and Gardner (1934), to which all page numbers refer. Plate numbers refer to photographs in the same volume. Museum locations are also taken from the 1934 publication. Dimensions are edited to give metric as well as the original imperial units.

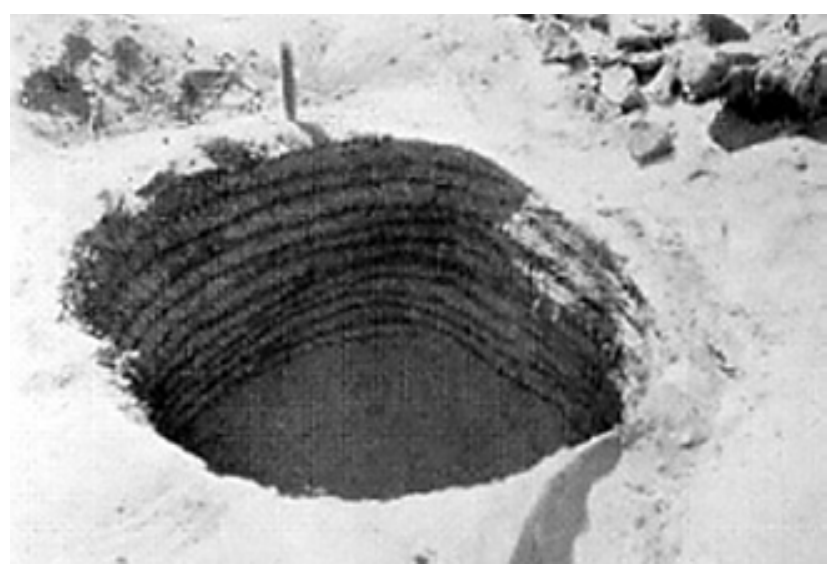

Figure 5.13. Basketry-lined Silo 12.

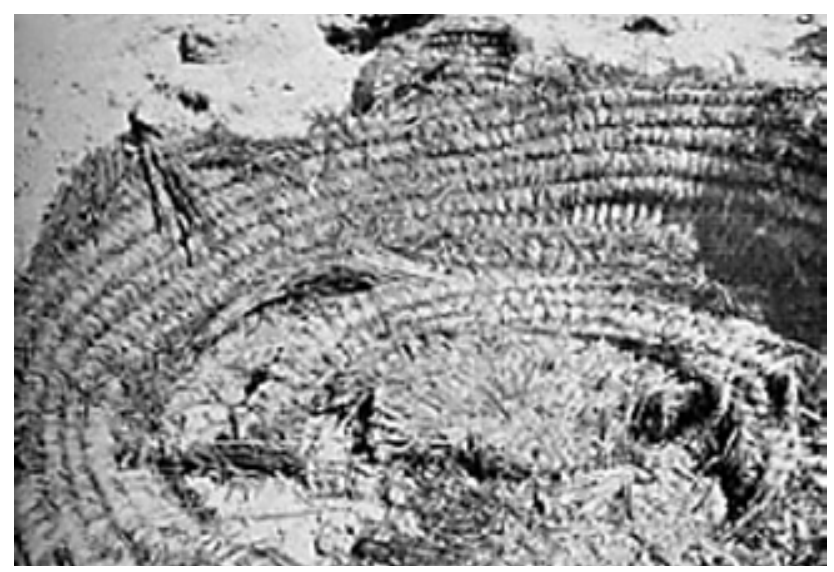

Figure 5.14. Basketry-lined Silo 1. 
Reexamination of the area in 2004 showed that a large portion-more than 5,200 feddan (2,184 ha)just north of Kom $\mathrm{K}$ was under development by the Egyptian Public Company for Land Reclamation, operating under a concession (No. 4490) granted in 1990. The Upper K Pits were cut through by a large north-south canal and were covered by adjacent dykes. Comparing our survey of the area with the map published in 1934, we estimated that this had destroyed approximately 15 pits, but the majority of the area was still intact (Figure 5.15; Wendrich and Cappers 2005). Negotiations with the Public Company for Land Reclamation resulted in permission to survey and fence off 15 feddan (6.3 ha) east of the canal. The company also allowed the team to excavate the area west of this major canal. However, in 2007 a large trough was excavated to a depth of approximately $20 \mathrm{~m}$, which completely destroyed the area east of the canal (Figure 5.16). The tertiary clay, of which most of the $\mathrm{K}$ ridge consists, was excavated and piled up around this hole, which was dug with drills and other large machinery. In 2014 a major freeway was built. It curves north of the K Pits area and south of the Kom W region, bringing further destruction to the fragile surface record.

Geologist Lewis Owen studied the cross-section of the modern canals dug through the Upper K ridge and described the $\mathrm{K}$ ridge as being built up of indurated tertiary shales and clays, with layers of evaporates, mostly

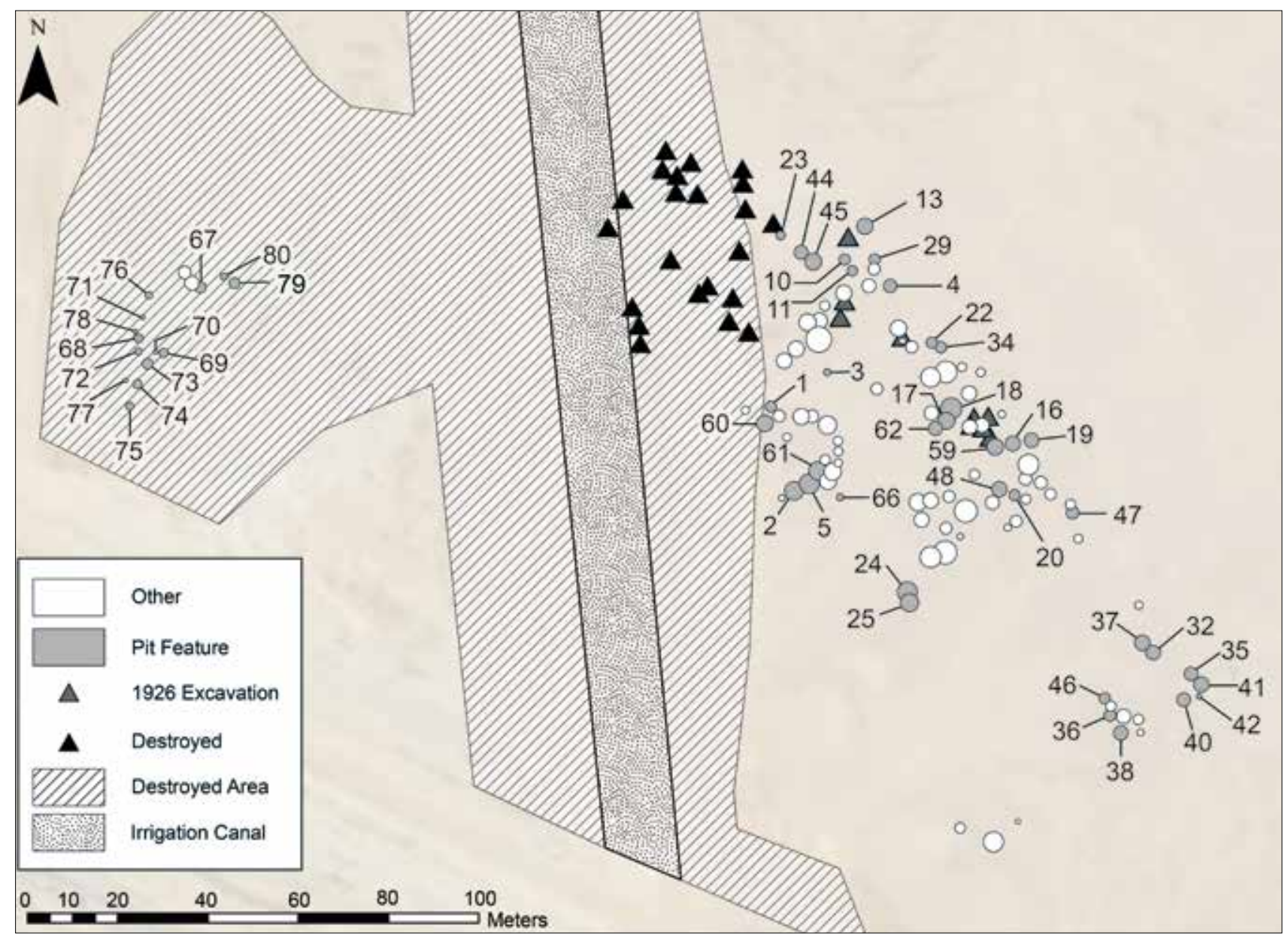

Figure 5.15. Distribution of the Upper K Pits identified by Caton-Thompson and Gardner in 1926 and additional pits discovered in 2004, as well as other features that may represent recent excavation. The pit numbers are those ascribed by Caton-Thompson and Gardner, the sequence of which was continued for pits discovered in 2004. The diameters of the circles give an indication of the relative sizes of the features. Triangles indicate the location of pit features that are now destroyed or where the size of a pit is not known. The area of the modern irrigation canal is outlined, together with the area disturbed by its construction and by looters. 


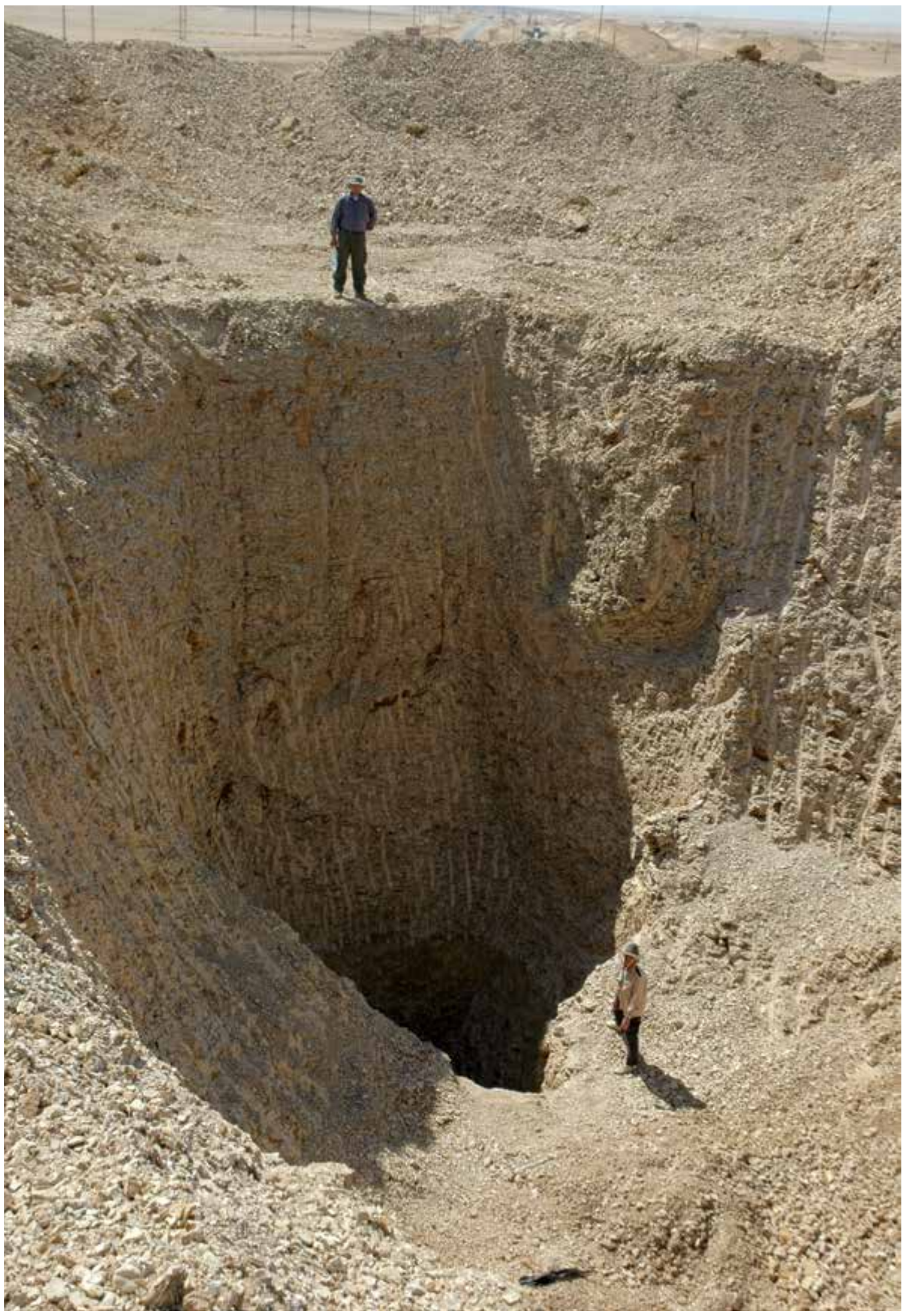

Figure 5.16. A mechanically excavated large trough that destroyed the newly discovered K Pits. 
gypsum. The desert pavement in this region consists of dense gravel on the surface, under which is a 20- to 50 -mm-thick layer of silty sand related to the deterioration of the top of the underlying bedrock.

Our survey of the eastern part of the K Pits area showed a large number of circular depressions. There were many more than the 67 pits excavated by CatonThompson and Gardner. Many of these additional pits are likely to be robber pits excavated after the 1920 s excavation team left the area.

Work in the Upper K Pits area west of the canal brought to light 13 new pits (Pits 68 to 80 and referred to as pits in Table 5.11 to distinguish them from the silos excavated by Caton-Thompson and Gardner). Six of them were probably storage pits, while eight others were shallow pits that investigation showed were used for mixing sand, salt, and water into a paste, probably to form a mortar with which the pits were closed (discussed below). The area around these pits was divided into $415 \times 5-m$ squares (Figures 5.17 and 5.18). Not all of these squares produced cultural remains; each square that did is discussed separately below, according to its trench number. Surface finds were rare, perhaps the result of the intensive work in the 1920s but also, as our surveys showed, because the areas to the north of the Upper K Pits have only sparse flakes and no hearths.

\section{Trench UKP01}

This square had indications of three adjacent concavities on the surface, one appearing in the 1934 report as the only pit on the western side of the $\mathrm{K}$ ridge (Pit 67) and two deeper hollows (Pits 81 and 82), which we surmised to be robber pits based on the results of the survey east of the canal.

Pit 81 , in the northwest corner of the square, consisted of a cut [01.0002] through the clayey sandstone bedrock [01.0008]. Only the top fill layer [01.0003] was excavated, and this consisted of the same material as the topsoil [01.0001] overlying it.

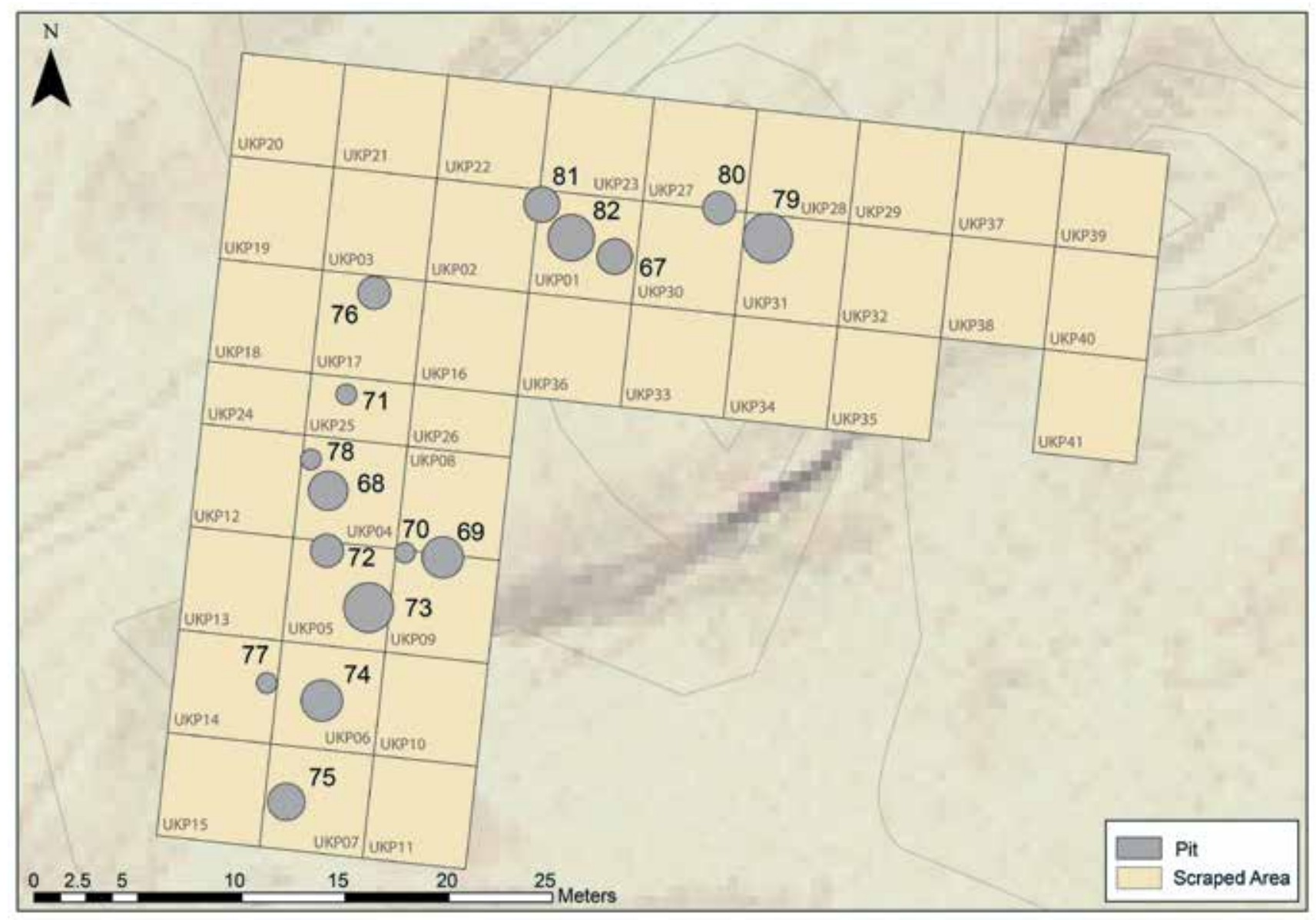

Figure 5.17. Schematic overview of the western Upper K Pits. 


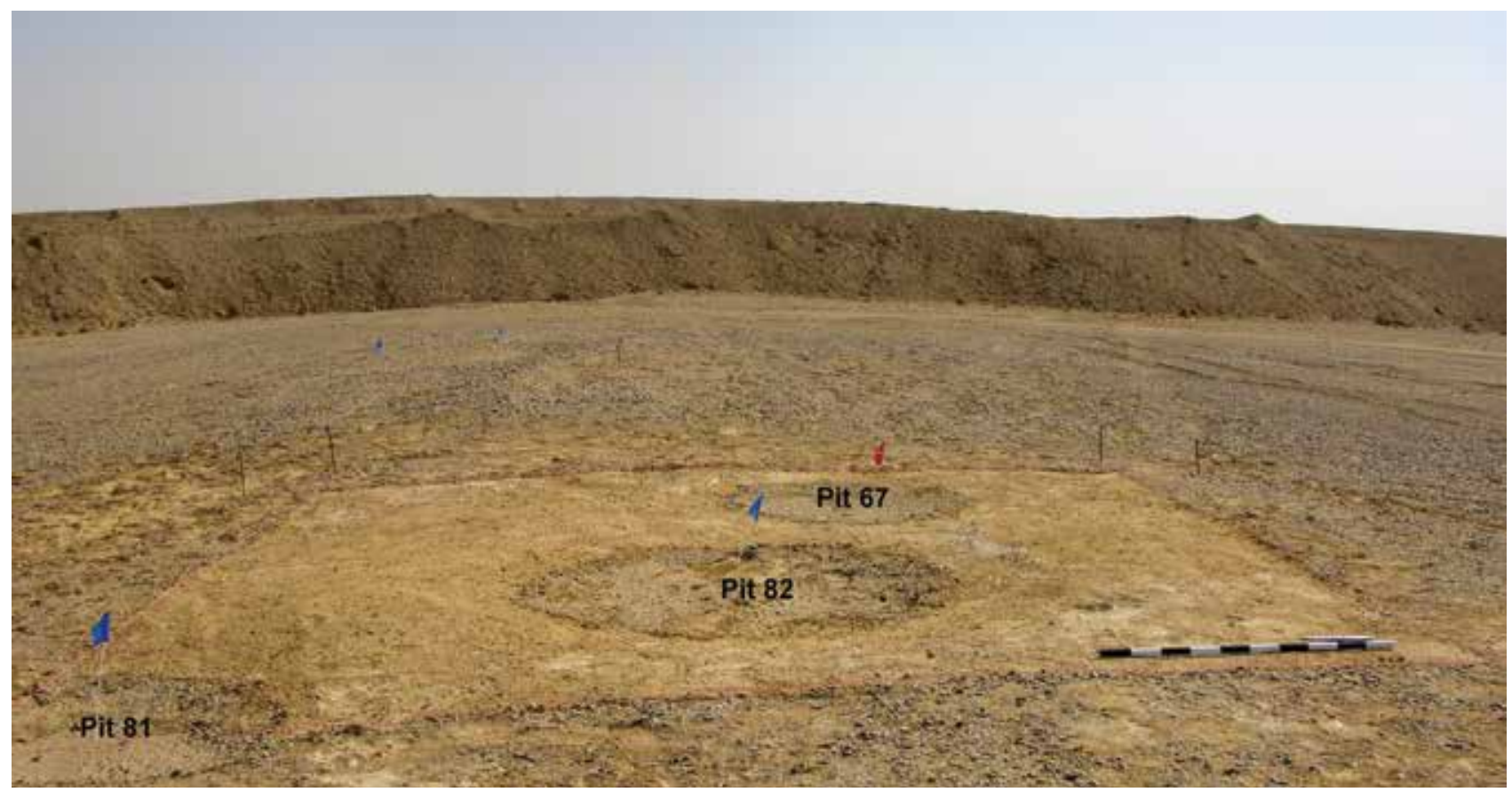

Figure 5.18. Trench UKP01.

Pit 82 , in the center of the trench, consisted of a large, irregular, circular cut [01.0004] of almost $2 \mathrm{~m}$ in diameter with six layers of fill, [01.0005], [01.0009], [01.0010], [01.0011], [01.0012], and [01.0013], identified by slight differences in compaction. Nothing was found in this pit except for natural inclusions, mostly pebbles, and the soil found inside was similar to the topsoil [01.0001]. Excavation showed that the pit had been cleared out but not backfilled. It confirmed the initial interpretation that both these features were indeed robber pits that did not have a relation to any ancient activities.

Pit 67 was previously excavated in 1925 and was identified by a cut [01.0006] in the bedrock [01.0008] forming an irregular round shape with a diameter varying from $1.22 \mathrm{~m}$ to $1.04 \mathrm{~m}$. Upon further excavation, however, it appeared that a slightly smaller cut [01.0019], measuring $0.89 \times 0.99 \mathrm{~m}$, penetrated the surrounding compacted silty sand bedrock [01.0008], as well as a lump of harder clayey bedrock that appeared in the side of the pit [01.0017] (Figure 5.19). The depth of this pit was $0.74 \mathrm{~m}$. The measurements taken in 2004 are smaller than the dimensions published in 1934, which record a diameter of 4 feet, 3 inches $(1.29 \mathrm{~m})$.

The pit was filled with loose sand and gray, yellow, and reddish pebbles, fewer in number, smaller, and less angular than those in the topsoil [01.0001]. The pit was $0.55 \mathrm{~m}$ deep. The base of the pit was cut into bedrock [01.0020], with a layer of decayed bedrock [01.0022] immediately above the pit base. Differences in firmness suggested the presence of four pit fill layers, [01.0014], [01.0015], [01.0016], and [01.0018], possibly indicating that the pit was only partially filled after initial excavation. No finds were retrieved from these fill layers. A cut in the side of the pit wall [01.0021] contained a small quantity of straw and was probably a mouse nest.

\section{Trench UKP04}

Within this $5 \times 5-\mathrm{m}$ trench, Pit 68 was identified by the presence of fibrous remains visible from the surface in and just under the desert pavement. These appeared to be part of a rim of a basket [04.0003] lowered into a cut [04.0002] in the bedrock [04.0007]. We were able to study the construction and context of such a storage pit in situ, the first time this had been done since the excavations in the 1920s. Pit 68 was round, with a diameter of $0.9 \mathrm{~m}$ and a depth of $0.6 \mathrm{~m}$. The basketry lining [04.0003] of the pit was mostly intact, except for a section to the west side where the fibers had decayed, forming a brown soil. The fibrous rim held back a layer of decayed bedrock that formed the topsoil [04.0001]. Within the outline of the basketry rim was 


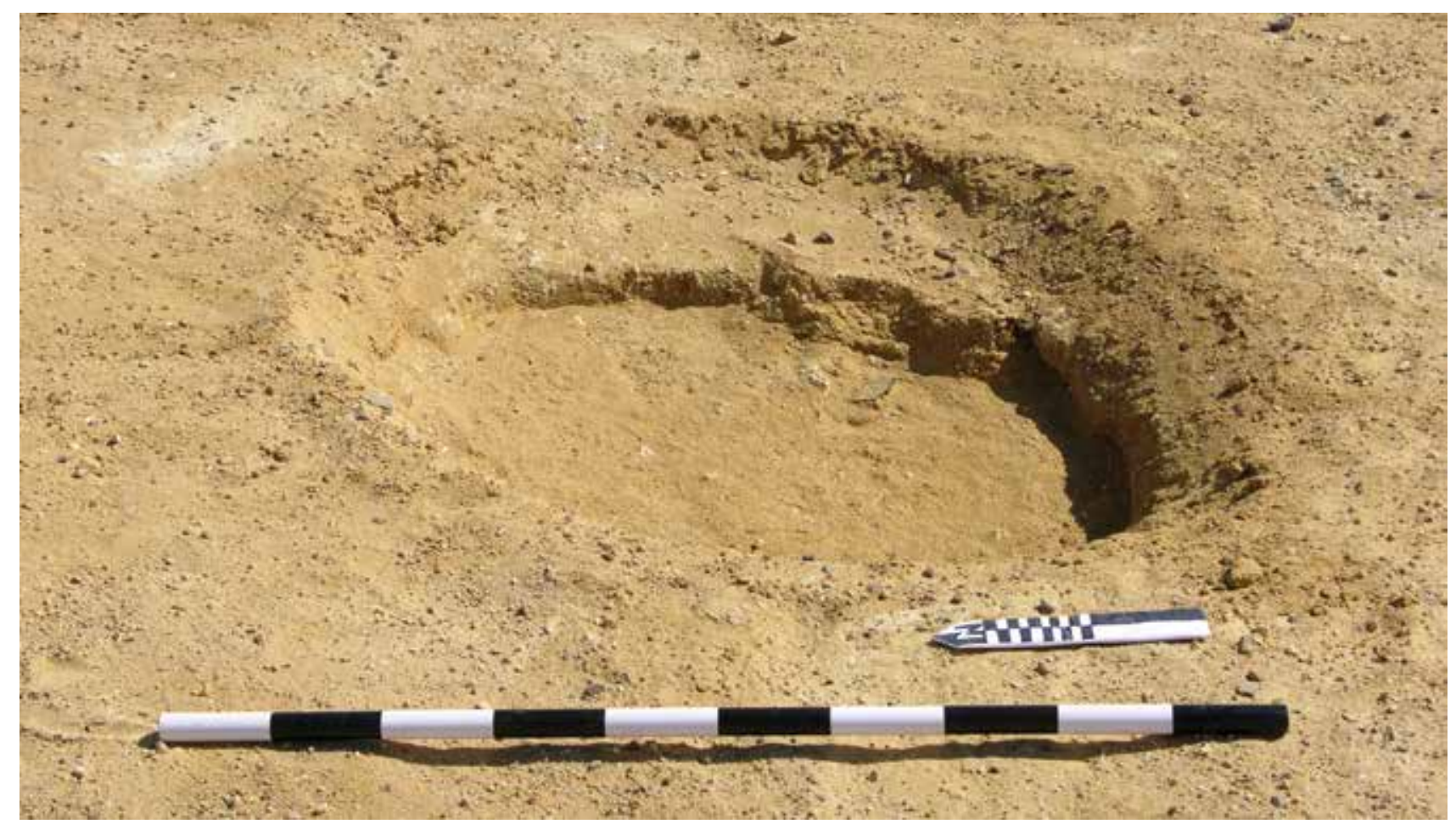

Figure 5.19. Pit 67 during reexcavation.

a well-sorted, silty, yellow-brown sand fill [04.0004] overlying an extremely hard deposit, later identified as a mortar lid [04.0006]. The sand layer that covered the lid was at the same level as a layer of soft decayed bedrock [04.0005] around the basketry rim but with a different color and composition. On the west side, a deposit [04.0013] of compact sand overlay the lid. The origin of this small deposit was unclear, but it may have acted to obscure the pit by moving sediment over the lid, thus preserving it from excavation by CatonThompson and Gardner.

The lid of Pit 68 [04.0006] consisted of a mixture of sand, salt, and crushed shell and was light gray in color (Figure 5.20). It was hard when exposed, but when put into water it dissolved. In their comments on the Upper K Pits, Caton-Thompson and Gardner wrote that some of the pits excavated had a very hard natural infill, which required chisels to break through (CatonThompson and Gardner 1934:43). Judging from the excavations in 2004, this infill was likely a purposely produced mortar lid, originally consisting of a moist mixture poured over the pits to seal them. When dry, this mixture formed a compact deposit about $10 \mathrm{~cm}$ thick, as discussed further below.
The rim of the basket on the west side of Pit 68 was pushed over so that fibers were embedded in the lid matrix. The cross-section of the lid showed the presence of two collapsed air bubbles, approximately $20 \times 30$ $\mathrm{mm}$, in the matrix of the lid material. This is consistent with a thick water-based deposit that subsequently dried.

Between cut [04.0002] and the basket [04.0003] was a light gray deposit with occasional iron staining [04.0014]. This may be the remains of a mud plaster that attached the basket to the wall of the pit. Half of the pit was initially excavated, to enable an overview of the contents in the section. In total, 10 layers of pit fill were identified, with these differing because of variation in compaction rather than composition. The topmost fill [04.0015] of Pit 68 had the same color as the lid [04.0006] but was much less compact. It contained a large percentage of crushed shell and was characterized by many air pockets. To the east, the lid did not completely reach the basketry rim and a soft sandy fill [04.0016] was present in the pit. This deposit had the same color but was not as compacted as the fill [04.0015]. The latter seems to be the result of moisture dripping or percolating down from the lid layer [04.0006] into a fill deposit below. This may suggest that the pit was closed off to preserve it for future use. 


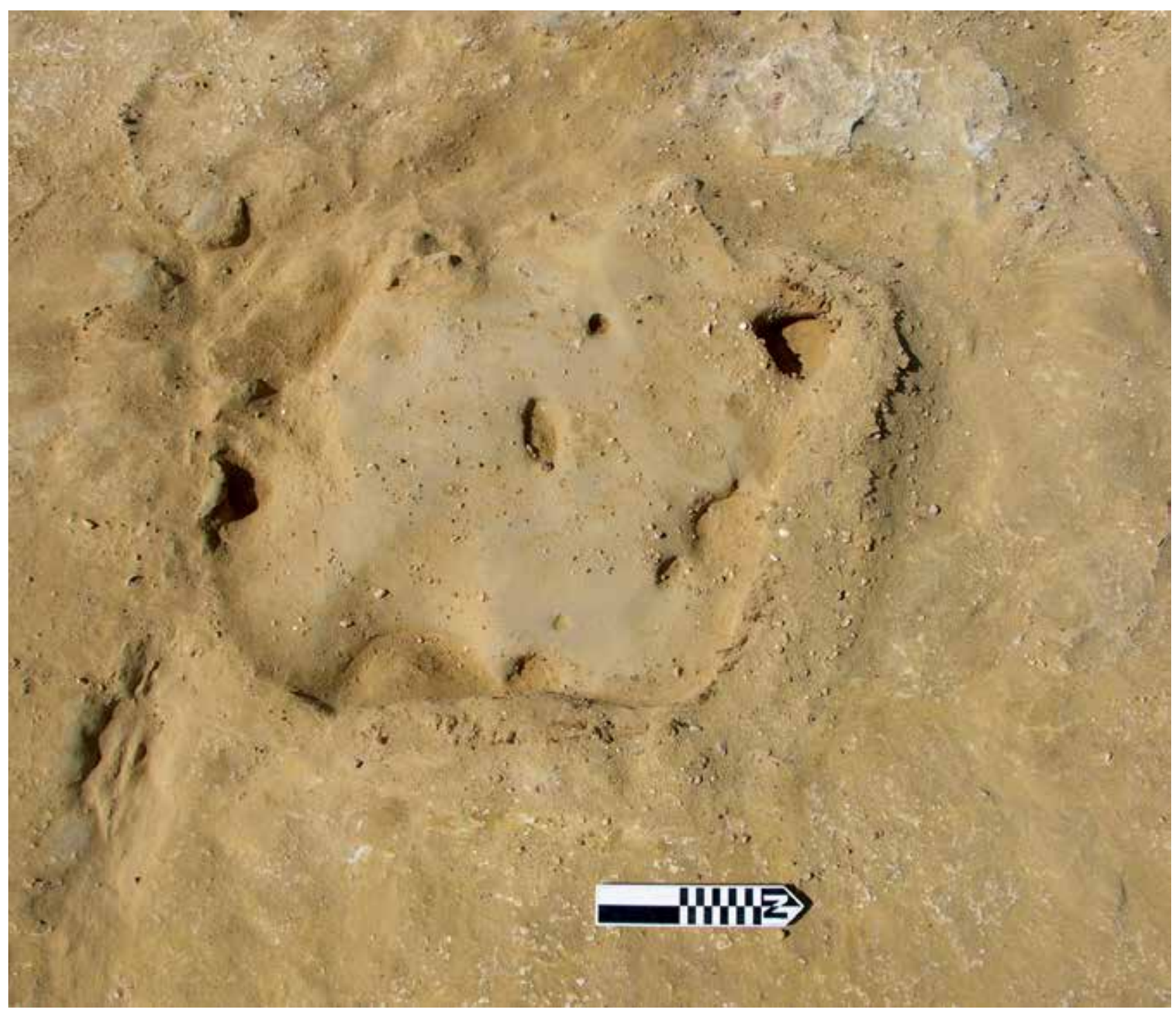

Figure 5.20. Lid of Pit 68 with the crushed shell deposit.

The soft sandy fill [04.0016] consisted of loose sand that may have trickled in between the edge of the lid and the basket. This loose sand was found above and between the compact lumps that made up the compacted fill [04.0015]. This second sandy fill ran from just underneath, alongside, and between the lid and the basket on the east side of the pit. The third layer of pit fill [04.0017] consisted of the same material as the layers above it, equally compact but containing more remains of the basketry lining. The entire unit was collected as a sample, analysis of which indicated that the plant materials were emmer wheat straw (Triticum turgidum subsp. dicoccon). Layers [04.0018] and
[04.0019] varied only in their degree of compaction, as did [04.0020], identified as loose sand. A large complete shell protruded from the compact layer below [04.0010]. Fill layer [04.0011] was again a loose sand layer, with a freshwater mussel shell from the family Iridinidae and tamarisk embedded together with more straw (Figure 5.21). ${ }^{10}$ These materials rested on a compact pit fill [04.0012] that covered the base of the basket, with the exception of the northeast corner, where

10 Caton-Thompson and Gardner identified large freshwater mussels as "Spatha shells," a term that is now replaced by Chambardia of the family Iridinidae, which includes Chambardia sp., Mutela sp., and Aspatharia sp. 
unit [04.0021] could be removed with a brush. Here a second tamarisk stick was found lying on the basket base (Figure 5.22).

Desiccated grains of both emmer wheat (Triticum turgidum subsp. dicoccon) and six-row hulled barley (Hordeum vulgare subsp. vulgare) were found in the fabric of the basket but not in any of the layers of pit fill. Many fragments of wheat straw, most likely from the coils of the basketry lining, were found mixed in with the fill, especially in layer [04.0017] and below.

In 2005 the other half of the pit fill was excavated, although half of the lid was left in situ (Figure 5.23). As in Pit 67, a small irregular hollow in the side of the pit wall, $10 \times 4 \mathrm{~cm}$ and approximately $4 \mathrm{~cm}$ deep, was loosely filled with clean straw [04.0023]. This also was probably a mouse nest, although no evidence for mouse droppings was found. The baskets and botanical remains are discussed in detail below.

Based on the excavated filled layers, a sequence of pit filling can be reconstructed. The empty, basket-lined pit, with a tamarisk stick lying at the bottom, was initially filled with approximately $5 \mathrm{~cm}$ of sand, to which a freshwater mussel shell and a second stick were added. Then the pit was filled up to its rim. There is no evidence for windblown sand layers except for some sand at the top of the fill, so

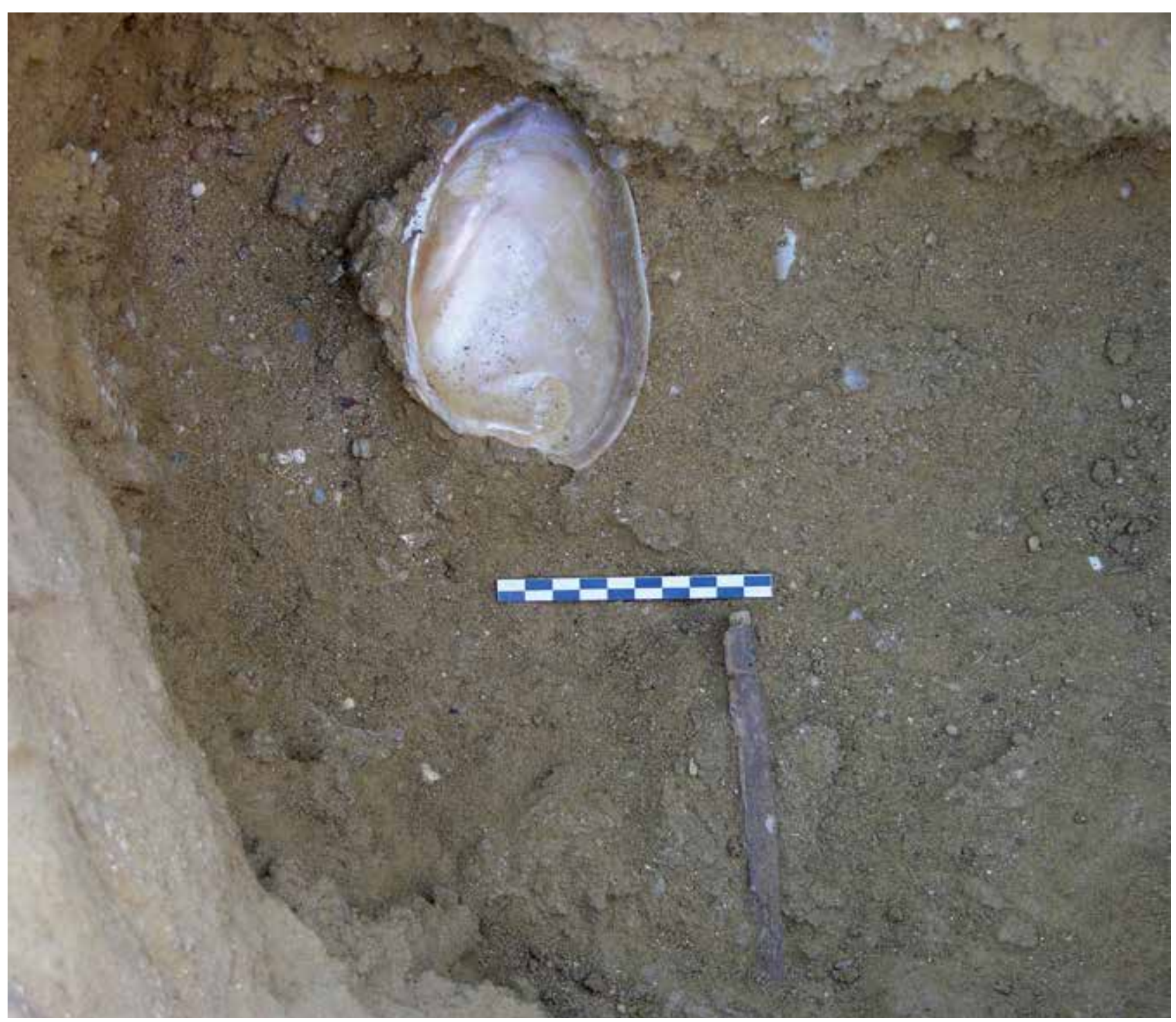

Figure 5.21. Pit 68; Chambardia sp. shell scoop and first of two tamarisk sticks. 


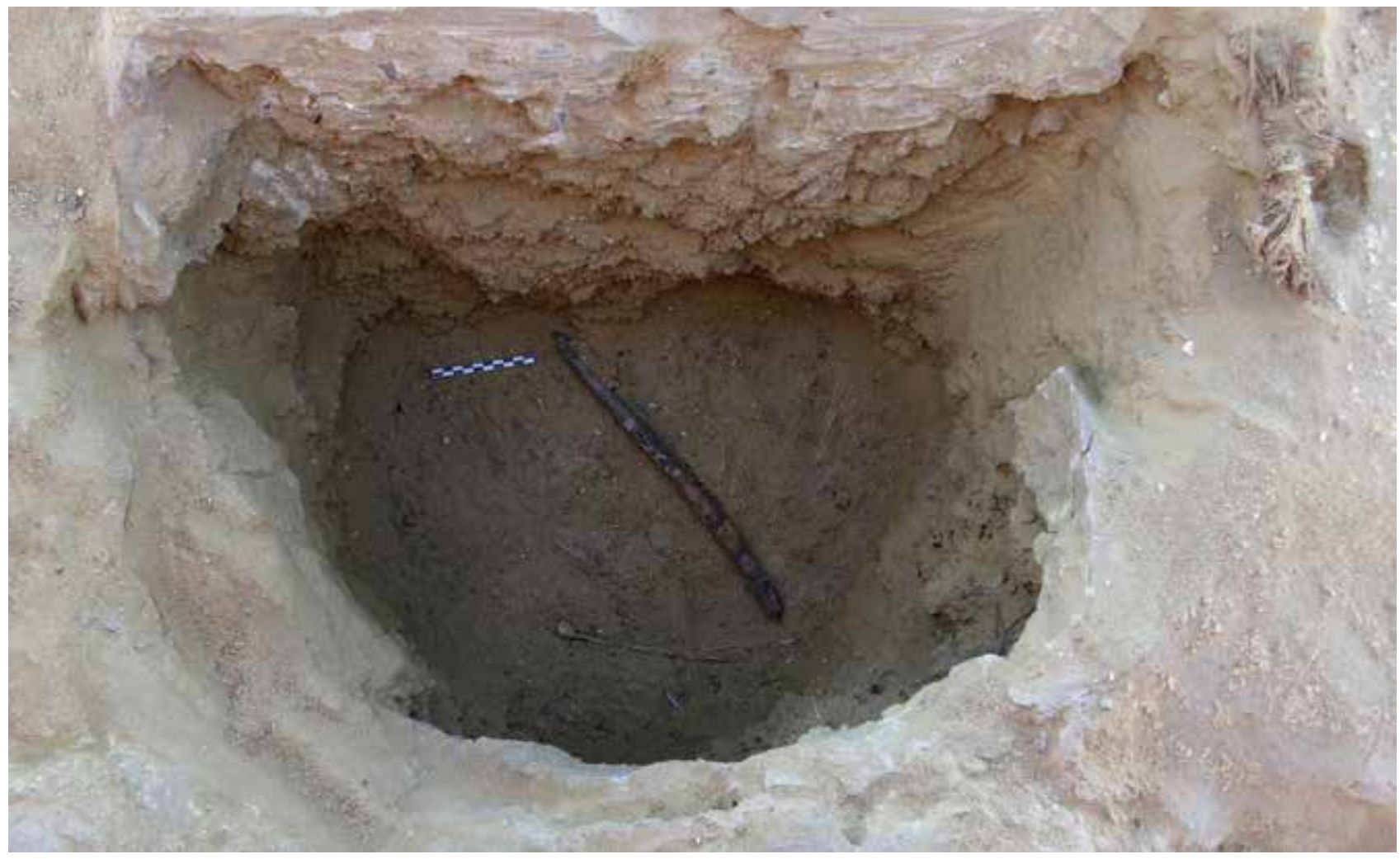

Figure 5.22. Pit 68; second tamarisk stick and base of basketry lining.

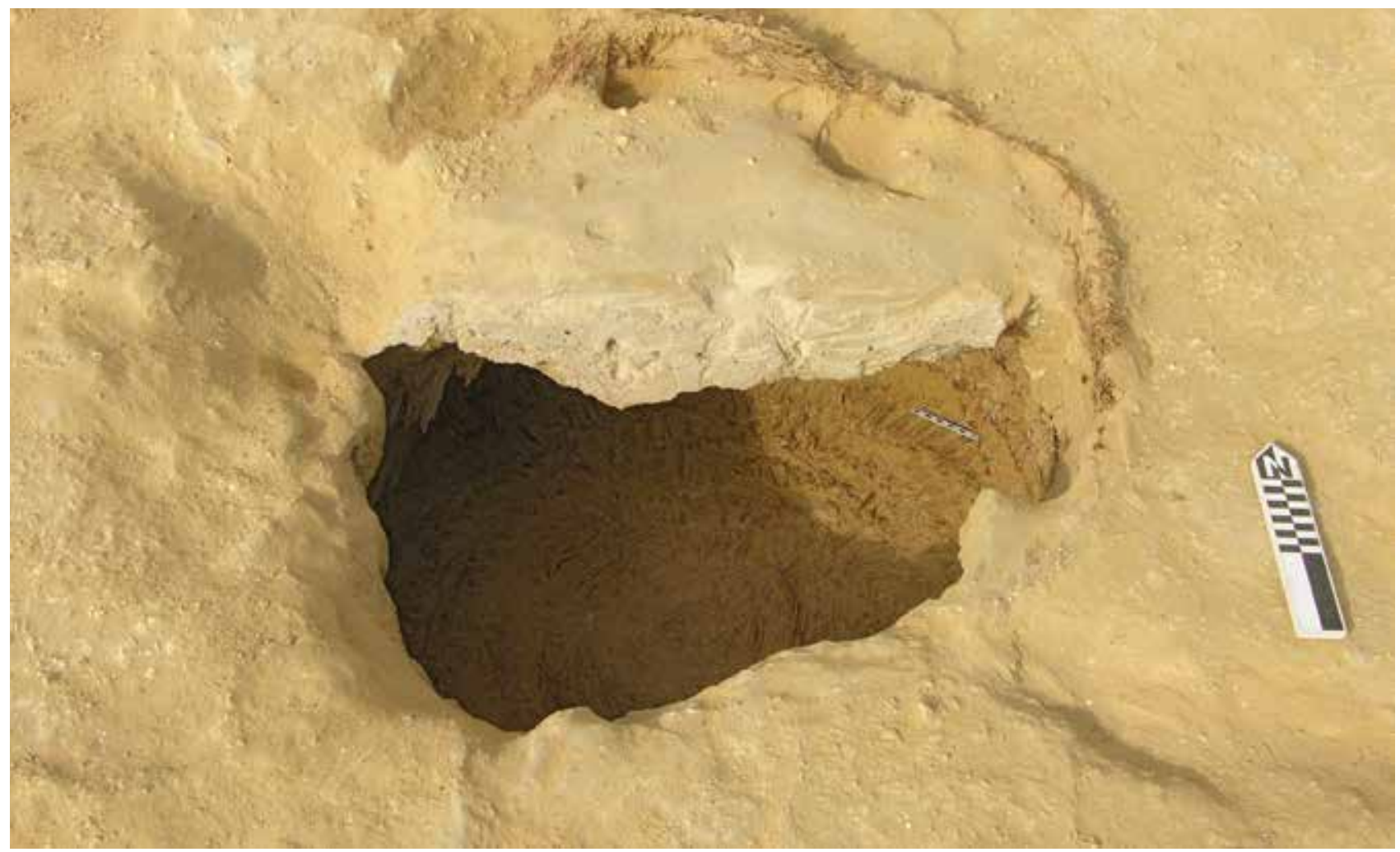

Figure 5.23. Pit 68; cross-section of lid and basket. 
infilling may have happened quickly, if not immediately, after deposition of the first stick, the shell, and the second stick. Since the only traces of wheat and barley were found in the fabric of the basket, it seems likely that the basketry-lined pit was empty but was not abandoned for good. Closure of the pit seems to indicate that it could have been opened, emptied out, and reused whenever required.

At a distance of $60 \mathrm{~cm}$ northwest of Pit 68, a natural depression in the bedrock was identified as a possible preparation area for the mortar mixture with which the storage pits were closed. This feature (78) consisted of a thin layer of shelly gravel [04.0008] that measured $0.48 \times 0.35 \mathrm{~m}$ and was only about $1 \mathrm{~cm}$ thick. It was overlaid by a coarse brown sandy sediment, mixed with gypsum, that measured $0.50 \times 0.40 \mathrm{~m}$, with a thickness of $5 \mathrm{~cm}$. In this area, and in the area immediately surrounding Pit 68, the soft sandy layer
[04.0005] found in the rest of the trench, directly overlying bedrock [04.0007], was missing, and it seems to have been removed in antiquity. Therefore the nature of the deposit is thought to be anthropogenic. Feature 78 differed from other depressions on the ridge identified as preparation areas because it did not have an actual layer of mortar material. As a consequence, its identification remains tentative.

\section{Trench UKP05}

Removal of the surface in Trench UKP05, immediately south of Trench 04, showed the outlines of two features (72 and 73). One of these consisted of loose and compacted shelly gravel in a 4-cm-deep irregular depression with a diameter of $0.80 \mathrm{~m}$ (Feature 72, units [05.0004] and [05.0005], Figures 5.24 and 5.25).

Pit 73, in the southeast quadrant of the trench, was $1.30 \times 1.20 \times 0.40 \mathrm{~m}$ deep, with a lid. It consisted of an

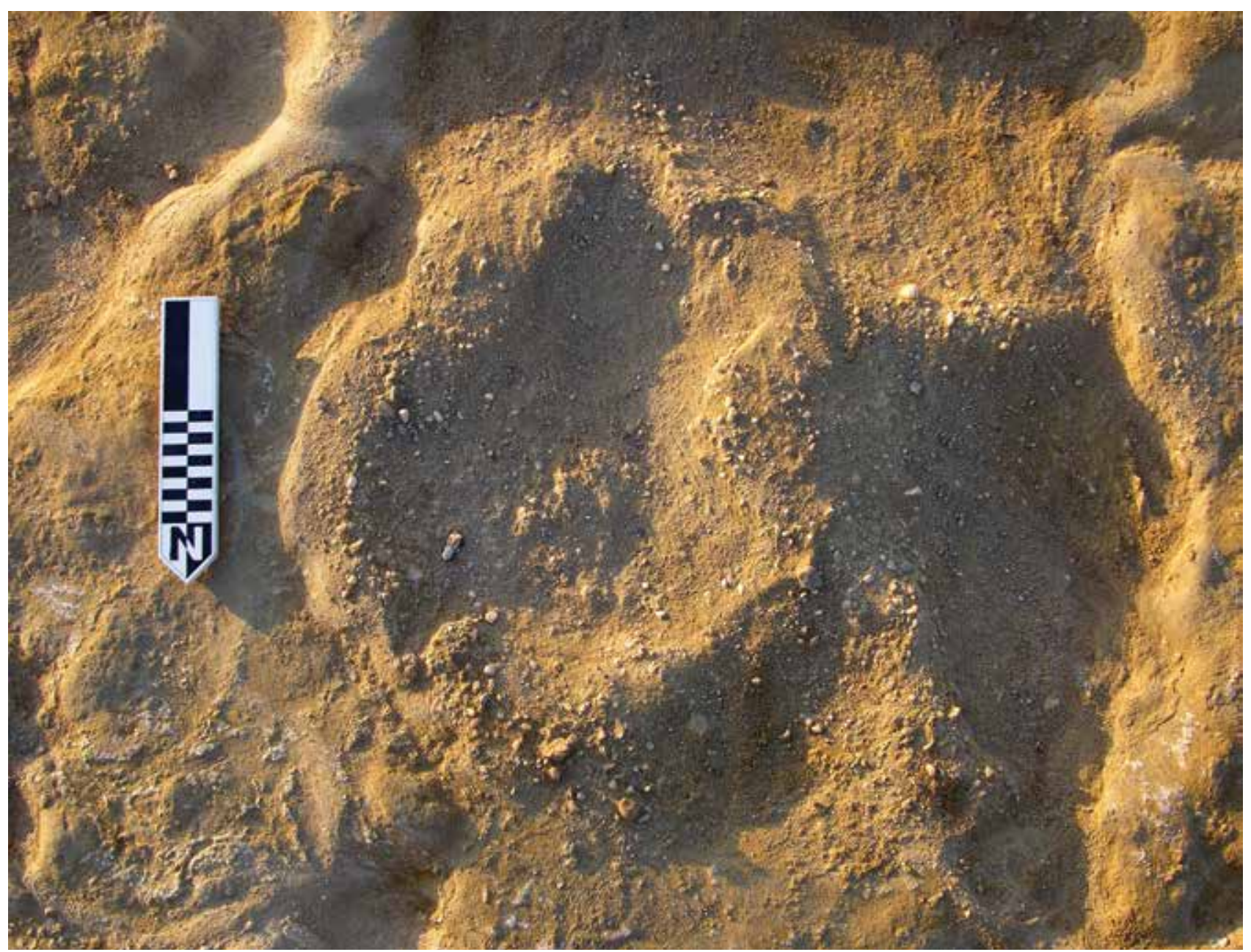

Figure 5.24. Feature 72, interpreted as a preparation area in UKP05. 


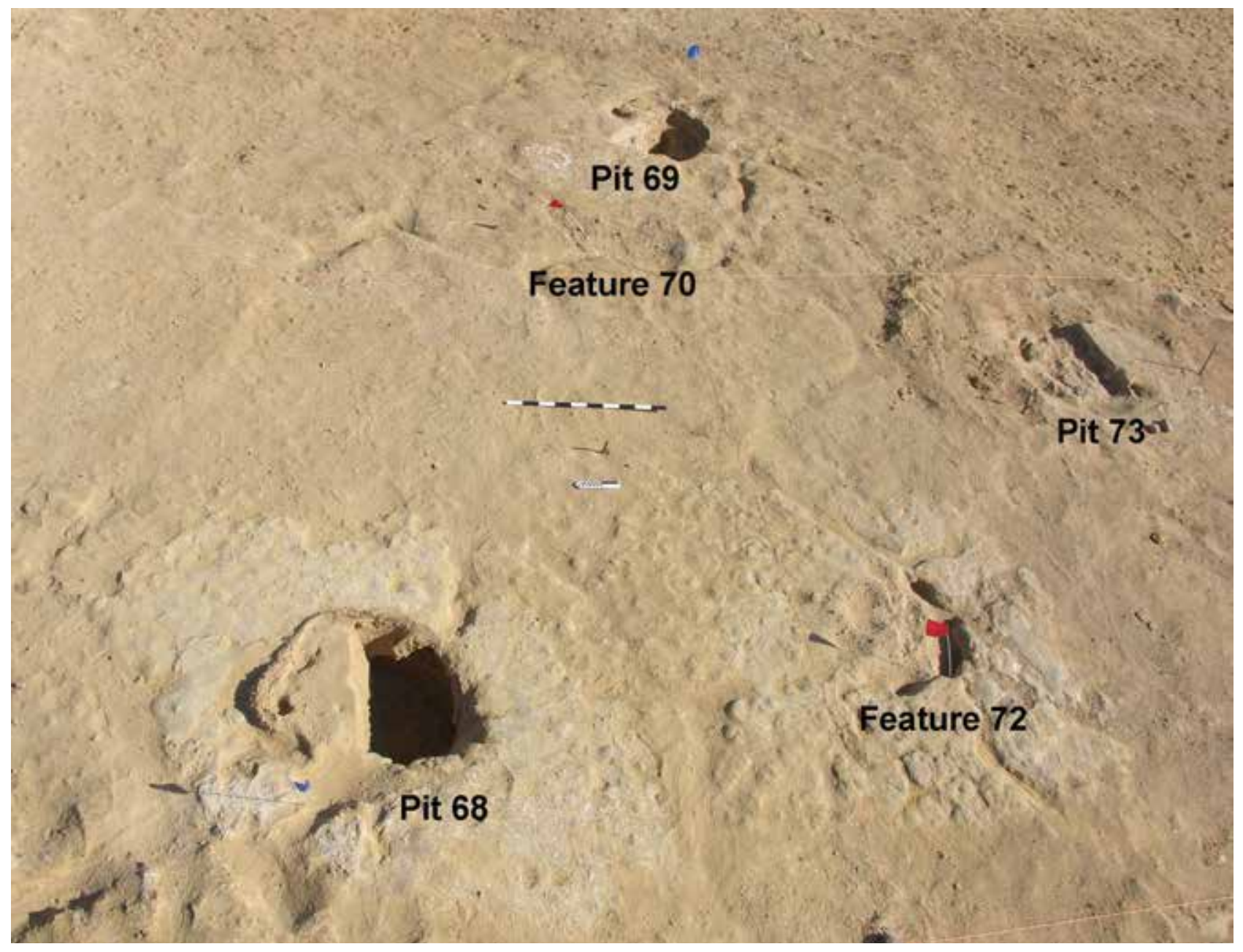

Figure 5.25. Overview of Pits 68, 69, and 73, with Features 70 and 72.

irregular round cut [05.0013] in soft, flaky, shale-like bedrock [05.0006]. The bedrock differed from that surrounding Pit 72 , where it was a soft silty sandstone [05.0002]. To the southeast of the pit, a deposit that consisted of coarse shelly sand with pebble inclusions [05.0007] could be distinguished.

The lid of Pit 73 [05.0009] was similar to that found with Pit 68 and consisted of a fine sand compact mortar with a yellowish-brown color. In areas around and between portions of the lid, a yellow-brown compact shelly gravel was found [05.0008]. It partially covered the lid and was similar to [05.0007] but was much more compact, perhaps because it was deposited over the lid [05.0009] while still wet.

The first fill underneath the lid [05.0010] was a coarse pebbly compact deposit of the same color, but much coarser in composition. On the east side, a loose yellow sandy fill [05.0014] could be discerned; it separated layer [05.0010] from a shelly fill [05.0011]. The latter was very similar in composition to [05.0008] but was separated from it by the lid, the top fill [05.0010], and the sandy fill [05.0014].

Below this was a compact layer [05.0012] underneath [05.0011]. In it were three tiny botanical fragments, consisting of strongly curved plant parts, indicating that they were the winders of a finely coiled basket. One of these was dark colored and thus an indicator that it was part of a decorated coiled basket, similar to a fragment found in Trench UKP09 [09.0005].

Similar to Pit 68, Pit 73 was abandoned, backfilled, and then closed with a lid, even though the cut of the pit was not as well defined and the pit was not basketry lined. 


\section{Trench UKP06}

After removal of the topsoil [06.0001], a sub-circular area of $1.00 \times 1.20 \mathrm{~m}$ was discovered. It was most probably a natural depression [06.0007] in the bedrock [06.0006], filled with a compact layer [06.0002] that contained no other cultural material (Figure 5.26). Underneath it was a second compact layer [06.0005], separated from the first by two yellowish sandy deposits [06.0003] and [06.0004]. The first was quite compacted, while the second consisted of loose sand with inclusions of pebbles, shell, a few small botanical fragments-including a long thin twig-and one unidentifiable bone fragment. The four layers that formed this feature filled a 0.20 -m- to 0.25 -m-deep depression, which may have been partly humanmade, but this was difficult to determine because the compact layer [06.0005] was hard to remove and separate from the bedrock. The two compact layers were different in composition. Unit [06.0002] had a fine and well-sorted matrix, while [06.0005] was coarser and contained many pebbles. Feature 74 is interpreted to be a preparation area for the production of mortar.

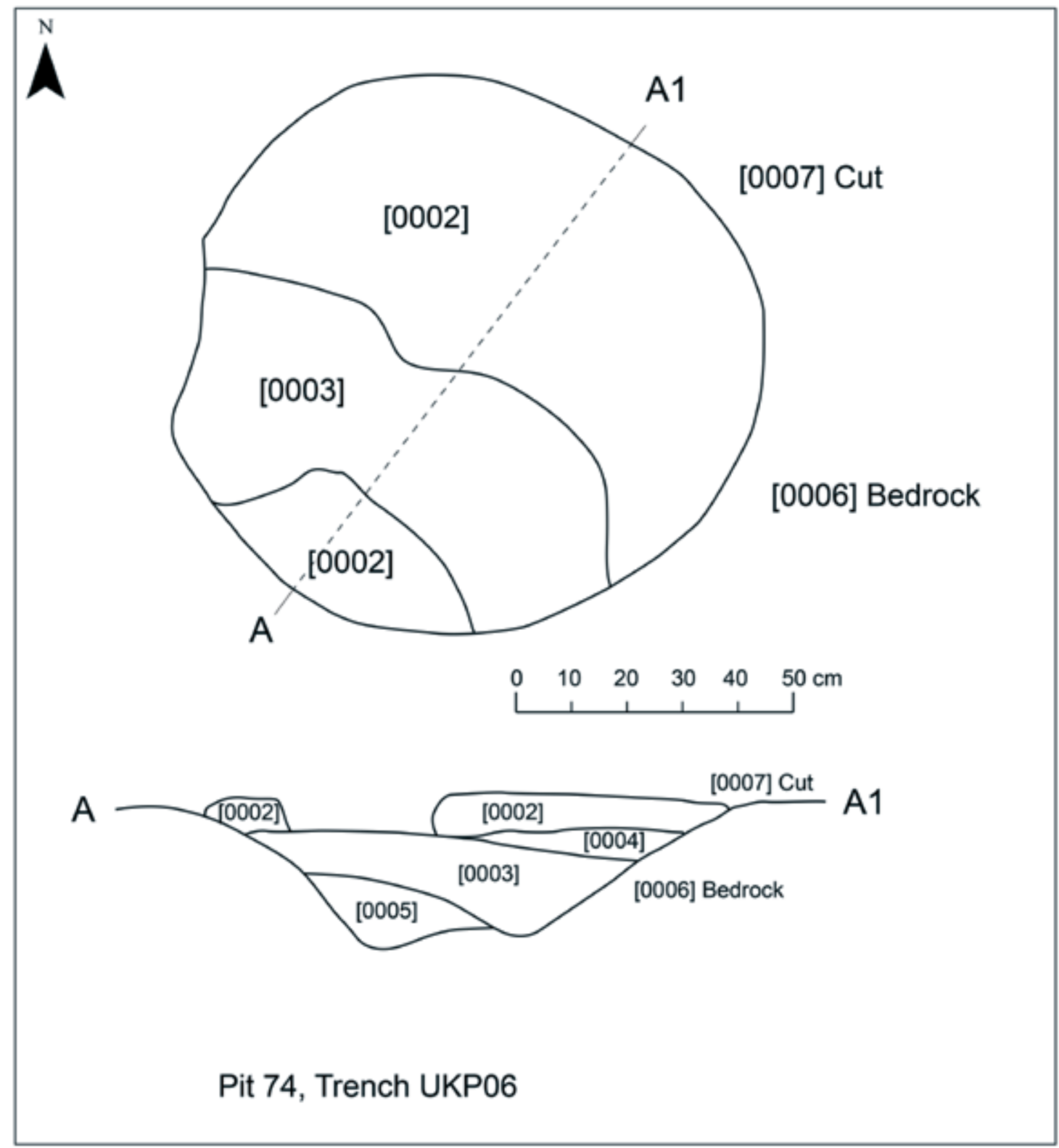

Figure 5.26. Feature 74 in UKP06, plan and cross-section. 


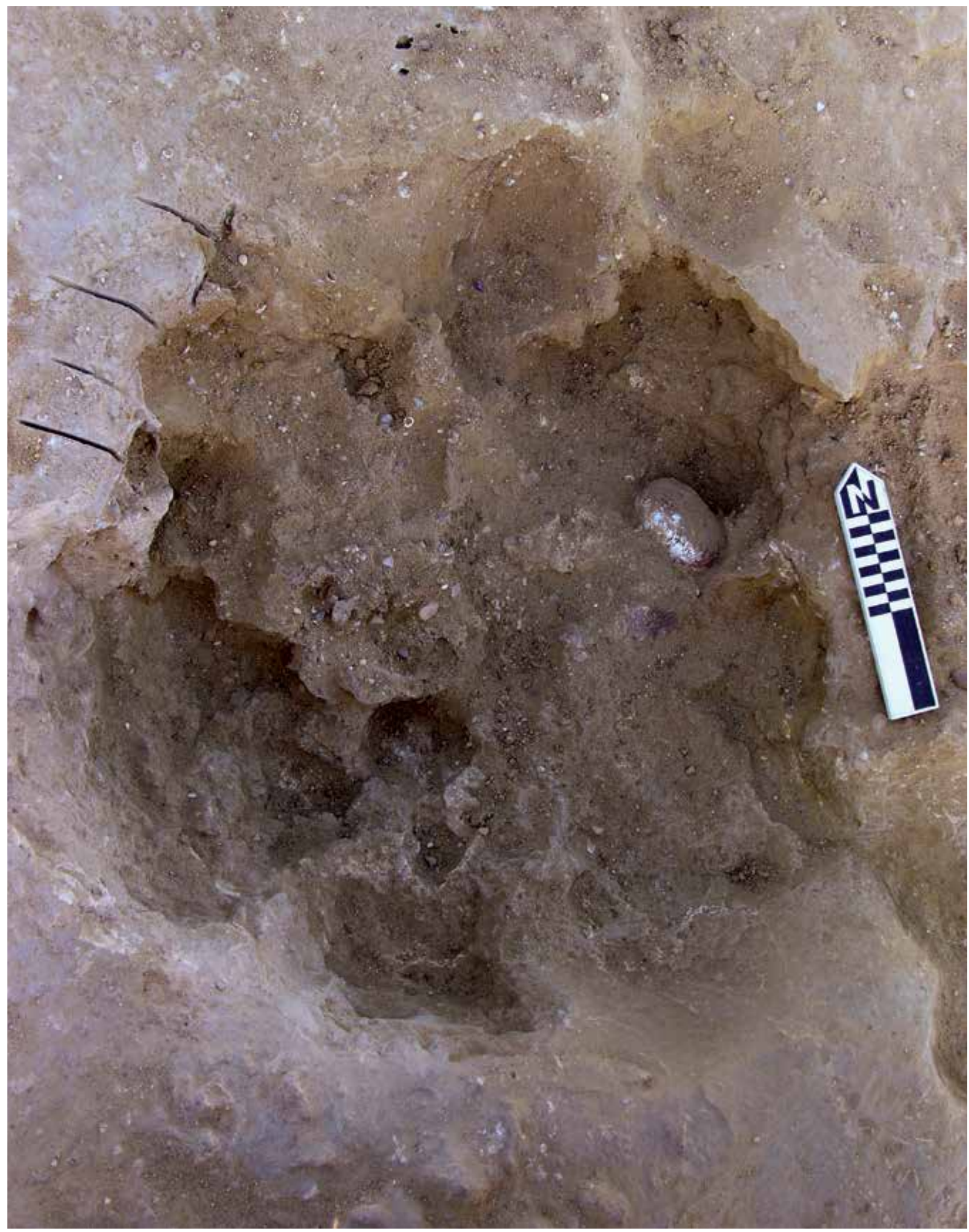

Figure 5.27. Pit 75 in Trench UKP07. 


\section{Trench UKP07}

Pit 75 was identified in Trench 07 by a $1.0 \times 0.9 \mathrm{~m}$ depression found on a slight slope. Excavation revealed many of the features associated with a backfilled storage pit (Figure 5.27). Although the cut [07.0011] was not deep $(0.35 \mathrm{~m})$ and formed an irregular circle sloping from northeast to southwest, the pit had a clearly distinguishable lid [07.0002] with a compact, wellsorted gray matrix (Figures 5.28 and 5.29). The six sandy fill units distinguished within the pit, [07.0003], [07.0004], [07.0005], [07.0007], [07.0008], and [07.0009], were characterized by different levels of compaction and all contained considerable quantities of straw fragments. Between the compact unit [07.0004] and the loose sandy unit [07.0005] was a small concentrated patch of straw approximately 0.10 $\mathrm{m} \times 0.12 \mathrm{~m}$ and $4 \mathrm{~cm}$ thick [07.0006] (Figure 5.29).
The pit was only $0.35 \mathrm{~m}$ deep. In one of the sandy pit fills [07.0009] a large shell was found; it was possibly used as a scoop. Two radiocarbon determinations on straw from this pit are discussed below.

\section{Trench UKP09}

While exploring the surface of Trench UKP09, we found a large fragment of a finely made coiled basket [09.0005] $2 \mathrm{~cm}$ underneath the desert pavement (Figure 5.30). The basket is discussed below. The stratigraphy of the sand layers underneath the topsoil [09.0001] consisted of a coarse, light reddish-yellow sand [09.0002] overlying a fine, light yellow sand layer [09.0003]. The sand inside the basket [09.0006] was similar to the reddish-yellow sand around it, although on the eastern side the basket was filled with a fine windblown sand [09.0007] similar to [09.0003]. The

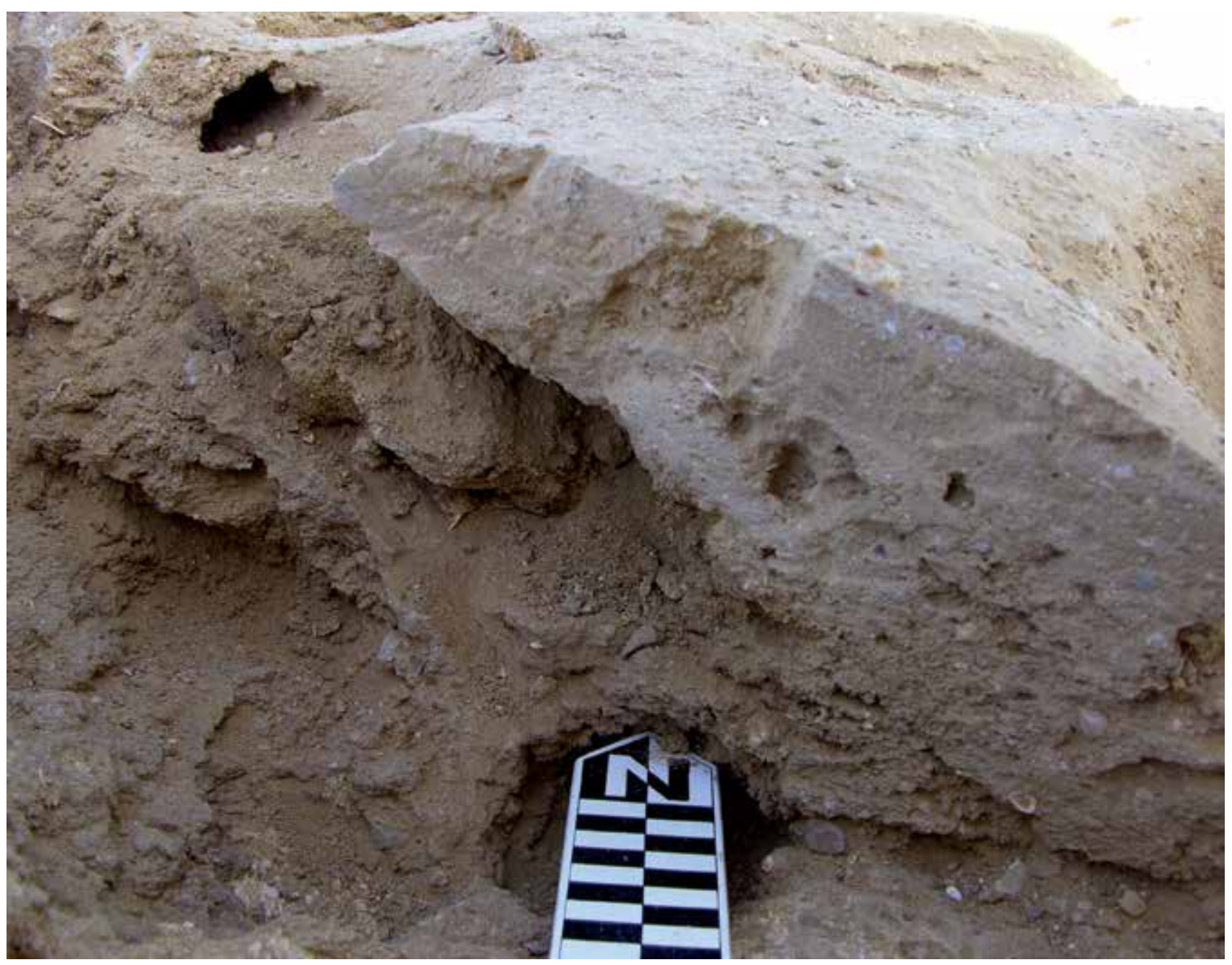

Figure 5.28. Cross-section of the lid of Pit 75. 


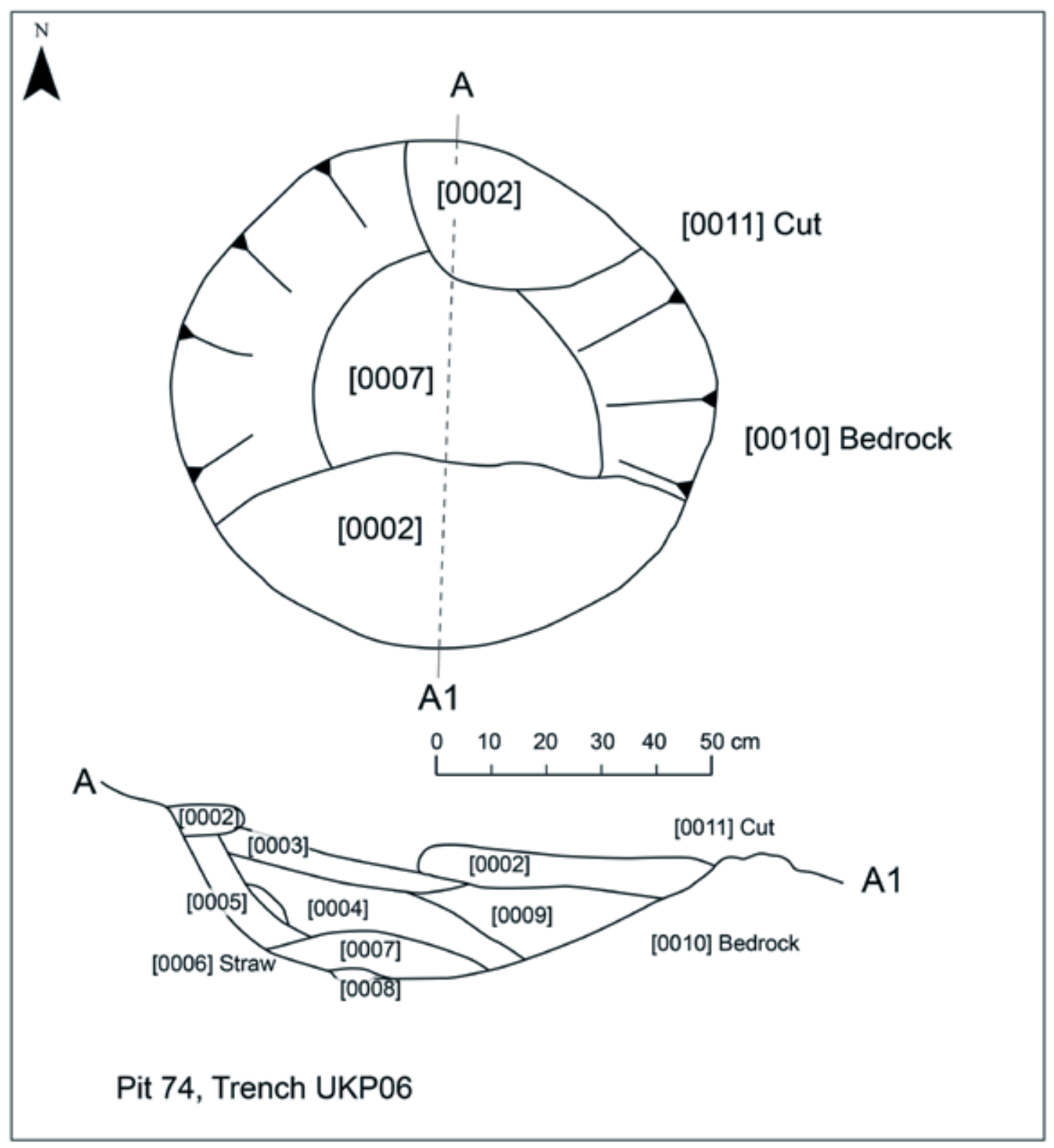

Figure 5.29. Plan and cross-section of Pit 75 in Trench UKP07.

basket content was sieved separately on a botanical sieve stack but yielded no organic materials and only a few naturally occurring pebbles.

The basket was positioned against the lid of Pit 69 , which was an irregular round cut in the bedrock [09.0011], approximately $1 \mathrm{~m}$ in diameter and $0.42 \mathrm{~m}$ deep. The lid [09.0004], a compacted, very pale brown circular feature, was in places up to 15 $\mathrm{cm}$ thick (Figures 5.31 and 5.32). It had been partly cut away in the past, with an irregular wedge-shaped section removed [09.0012]. This shallow cut did not go through the entire thickness of the lid and did not seem to have reached the upper pit fill. The purpose of this cut remains unclear, and it was filled with a coarse sandy deposit [09.0009] that included a shell and a tamarisk stick. After removal of the lid, the upper fill of the pit [09.0010] was found to consist of a poorly sorted coarse yellow-reddish sand, similar to [09.0009] but with a different consistency and a different ratio of sand and shells. On the south side 


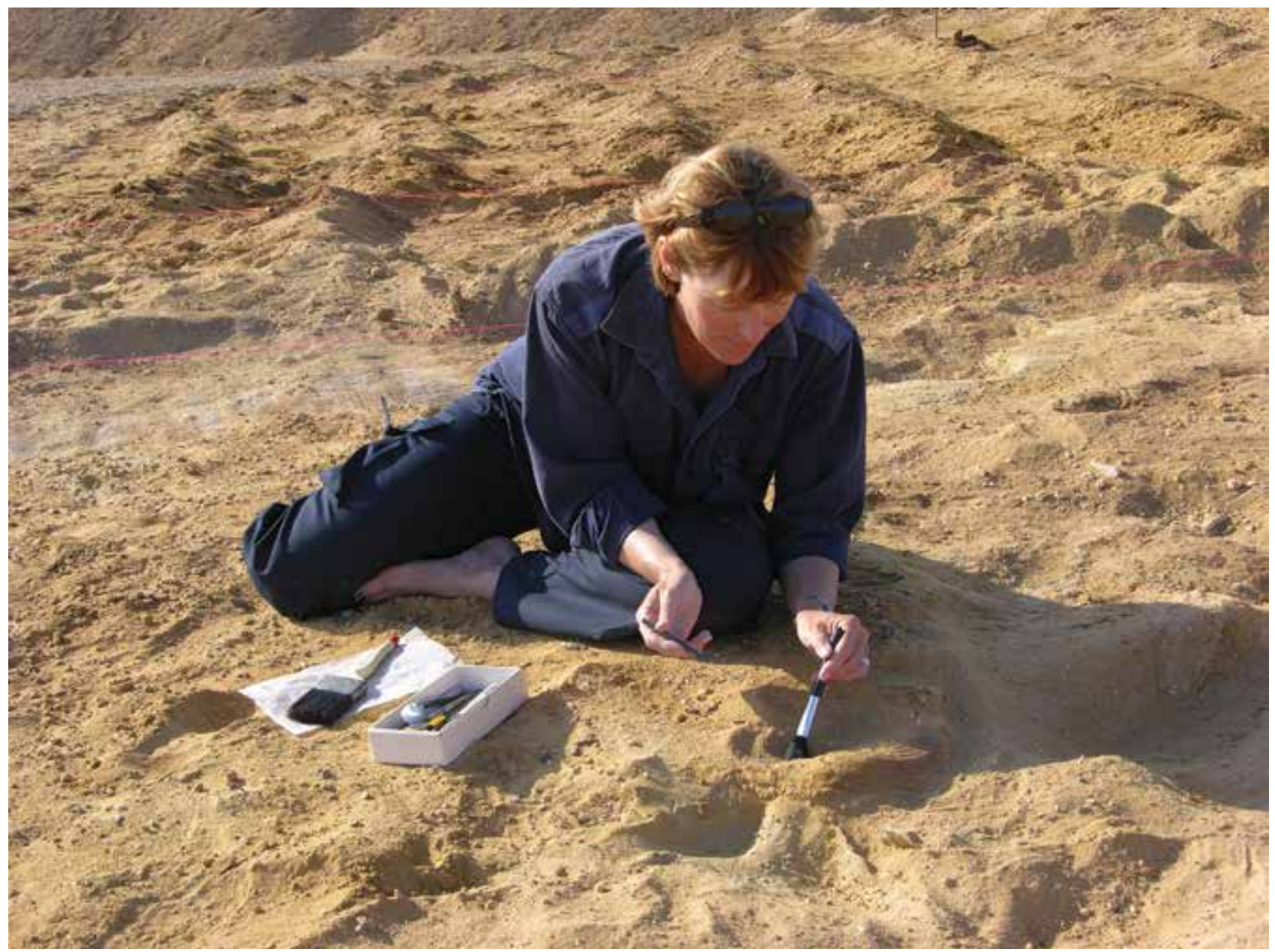

Figure 5.30. Surface of trench UKP09, with basket fragment in situ.

of the pit, we found fragments of a large shell, similar to that found in [09.0009]. The deposit had a high salt content. The lower pit fills differed slightly in the northwestern [09.0013] and southeastern [09.0014] halves of the pit. The latter fill was compacted but did not completely seal the layer below because loose sand and gravel had fallen through cracks in the deposit to the coarse shelly sand layer [09.0015] beneath. This formed the bottom fill of Pit 69, filling both cut [09.0011] and a smaller hole cut into the bottom of Pit 69 [09.0016]. One potsherd was found in this deposit.

In the northwest corner of Trench UKP09, bordering Trenches UKP04, UKP05, and UKP08, the outline of another feature was identified (Feature 70; Figure 5.33). This was oval in shape, measuring $0.5 \times 0.7 \mathrm{~m}$, and was found after the removal of several thin layers of sand underneath the desert pavement [09.0001]. A loose yellow silty sand with pebbles, including some shells [09.0017], lay above a mixed layer of brown to white silty sand [09.0018], revealing in turn an oval natural depression [09.0020] filled with very pale brown compact shelly gravel [09.0019]. Part of this gravel formed a compact rim in which straw fibers were embedded. The total depth from the top of the rim to the bedrock was $0.22 \mathrm{~m}$. The pit was interpreted as a mortar preparation pit.

\section{Trench UKP14}

Feature 77 in Trench 14 had a diameter of approximately $0.60 \mathrm{~m}$ and a depth of $0.15 \mathrm{~m}$ (Figure 5.17). The compact deposit in this feature [14.0002] was of a light brown-gray color. Like Feature 70, Feature 77 may show the use of natural depressions as mortar preparation areas, potentially in this case for the mortar used to close Pit 75 at different times. 


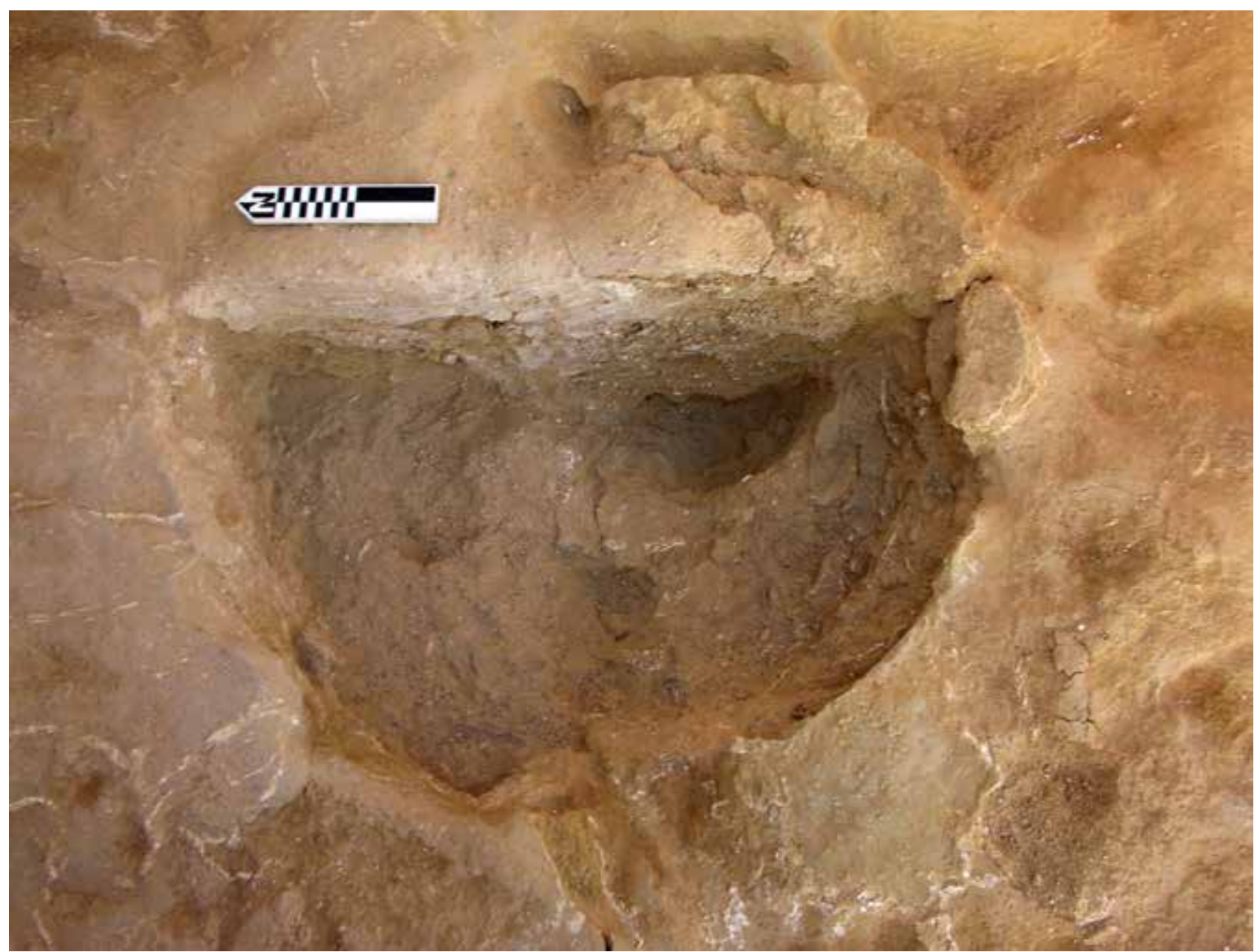

Figure 5.31. Pit 69 in Trench UKP09. Note the cut in the base of the pit.

\section{Trench UKP17}

In the northwest part of Trench 17, we uncovered a small sub-circular feature with dimensions of $0.75 \mathrm{x}$ $0.60 \mathrm{~m}$ and a depth of $0.14 \mathrm{~m}$. A layer of compacted, quite fine, light gray deposit [17.0002] was found in this natural depression [17.0003], labeled as Feature 76. This may also be a mortar preparation area.

\section{Trench UKP25}

Trench 25 is an area of $5 \times 3 \mathrm{~m}$, between UKP03 to the north and UKP04 to the south (Figure 5.17). The topsoil was similar in all three trenches, but unlike the trenches on the slope to the south, there were no layers of different sands and decayed bedrock. Instead, the upper deposits were directly overlying solid bedrock. The only exception was sand found collected in a depression northwest of Pit 71 [25.0003].
Pit 71 was identified as a dark gray-brown, irregular oval line standing out in the yellow sand, immediately after removal of topsoil [26.0001] revealing a badly preserved basketry rim (Figure 5.34). The lid [25.0006] of Pit 71 was very similar to that of Pit 68 , consisting of a light gray fine matrix with some small pebbles. The lid seemed to have collapsed in the middle, and a yellow-brown compacted sandy deposit [25.0007] was visible (Figure 5.35). The lid did not run over the basket rim but was deposited within the basket, forming the brown decomposed basketry outline just underneath the topsoil. A near-complete ceramic vessel was embedded in the lid. The fill beneath the lid [25.0008] was, however, similar to that in the area of collapse. No botanical remains were found apart from strands from the much deteriorated basketry lining. None of the winders of this coarse, coiled basket were preserved (Figure 5.36). The lowest pit fill [25.0009] was difficult to 


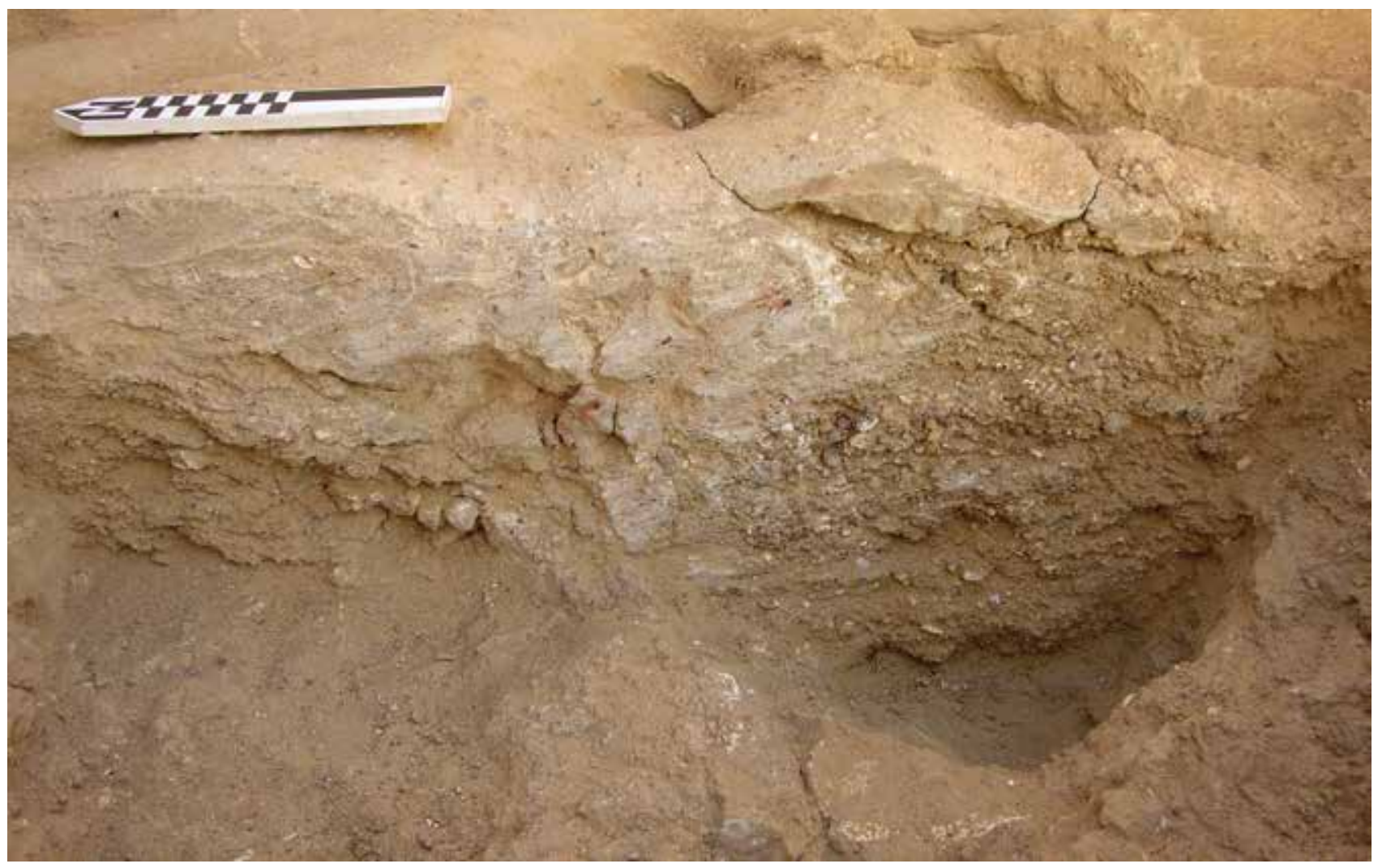

Figure 5.32. Cross-section of the fill of Pit 69 in Trench UKP09.

discern from the layer above but was a slightly different color and may have been a windblown sand fill prior to backfilling of the pit. The pit was an irregular oval, $0.65 \mathrm{x}$ $0.60 \mathrm{~m}$ at the rim, $0.36 \times 0.36 \mathrm{~m}$ at the bottom, and 0.26 $\mathrm{m}$ deep. Between the basket and the irregular bottom of the pit, a sandy layer [25.0010] was perhaps deposited before the basket was lowered into the depression.

\section{Trench UKP30}

Feature 80 was located approximately $5 \mathrm{~m}$ northeast of Pit 67. It consisted of a large concentration of compact material [30.0002] over an area of $1.6 \times 1.2 \mathrm{~m}$, with high shell content and bounded by sandy gypsum-rich deposits [30.0003]. It was similar in layout to Feature 79 in Trench UKP31 (see below), except that no cultural materials were found. A second compact layer [30.0005] was found beneath the first, separated by a compact sand layer [30.0004]. It was unclear whether or not deposit [30.0003] encircled the second compact layer. The second layer was $1.50 \times 1.25 \mathrm{~m}$, about the same size as the upper compact layer [30.0002]. The feature may represent another mortar preparation area.

\section{Trench UKP31}

In an area of $1.9 \times 1.9 \mathrm{~m}$, the smooth, gray-green, silty sandstone with gypsum veins that characterized the bedrock at the top of the ridge was covered by a mixture of compact deposits and deposits of gypsum-rich sand, enclosing narrow channels with traces of water runoff. Further exploration suggested that this was not a natural formation (Feature 79) (Figure 5.37). A compact layer with an irregular shape [31.0002] was found to overlay a sand layer [31.0004] over bedrock [31.0005], directly underneath the surface deposits [31.0001]. Unit [31.0003] seems to encircle and predate this compact layer. Straw and grain husks were embedded in the compact deposit but were also present in the sand layer underneath and in the sand and gypsum deposit that encircled the compact layer on the north and east side. The deposit may represent multiple events of preparation of the pit lid mortar. One chert flake was found embedded in the compact layer. The feature has more mixed deposits than the smaller features interpreted as mortar preparation areas, such as $70,72,74$, and 78 , perhaps indicating that it was used multiple times. 


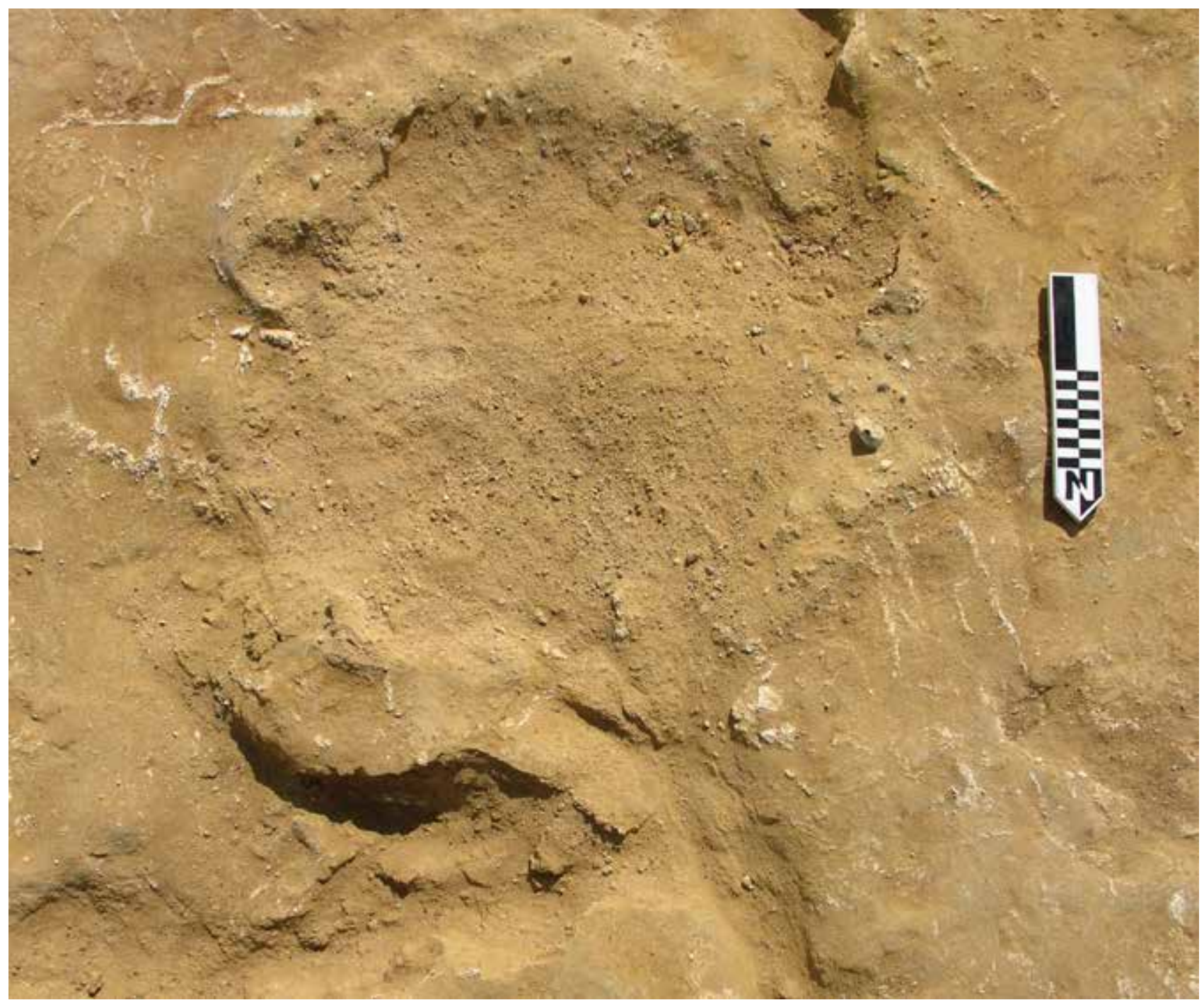

Figure 5.33. Feature 70 in Trench UKP09.

\section{Surface Finds}

Very few cultural remains were found in the topsoil, with four chert flakes (three in Trench UKP19 and one in Trench UKP37), two pottery body sherds (in Trenches UKP04 and UKP19), and three fragments of modern metal (in Trenches UKP11 and UKP18) identified.

\section{Basketry and Textiles}

One unique aspect of the Upper K Pits discovered in 1926, apart from evidence for domesticated wheat and barley, was the realization that the Fayum Neolithic basketry forms and techniques were very well developed and that basketry was used for several purposes. In addition, Caton-Thompson and Gardner
(1934) list one find of a textile (found in a pot near Pit 16), a coarse and loosely woven fragment of $Z$-spun linen. None of the photographs published in the 1934 volume have scales, and therefore it is often difficult to assess the dimensions and, in the case of the textile fragment, the fineness of the yarn and weave. The information that we could determine is summarized in Table 5.12, which lists the basketry finds from 1926, 2004, and 2005, with the recent finds discussed in more detail below.

As noted above, a large fragment of a finely made coiled basket was found $2 \mathrm{~cm}$ beneath the desert pavement in Trench UKP09 next to Pit 69 (Figure 5.38). It measured $2.70 \times 2.00 \times 0.07 \mathrm{~m}$. The side of the basket had 11 rows of coiling extant, and the bundle size was 


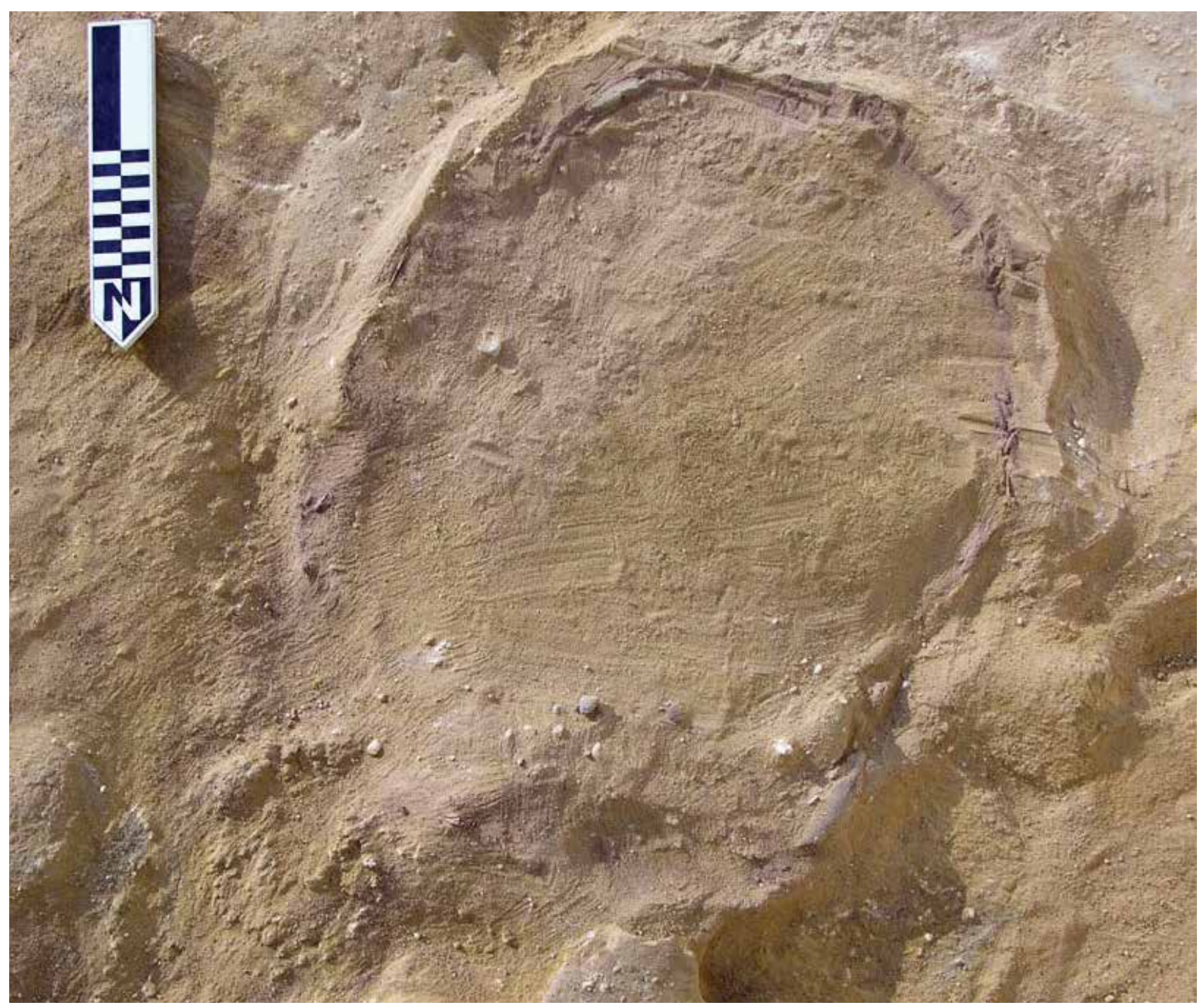

Figure 5.34. Discolored outline of the decayed basketry lining of Pit 71.

$9 \mathrm{~mm}$, although slightly flattened in places. The very narrow winders were between 1.5 and $2 \mathrm{~mm}$, with four winders per each $10 \mathrm{~mm}$ (Figures 5.39 and 5.40). The material of which this basket was made could not be determined with certainty. Macroscopically, both leaf and stem structures were visible in the bundle material, but the epidermis had deteriorated.

The appearance of the epidermis differs between the active winders (Wendrich 1991), which form the outside of the coils, and the passive elements, the bundle material protected by the winders, which completely cover it (Figure 5.41). Both seem to be made of the same plant material.

Otto Brinkkemper, archaeobotanist at the Cultural Heritage Agency of the Netherlands, analyzed the material using scanning electron microscopy (SEM) but did not succeed in determining the plant species due to deterioration of the material. It is clear, however, that neither the bundle (passive system) nor the winders (active system) are made of Triticum. This indicates that a different material was chosen for the fine basketry than for the coarse baskets lining the pits, which were made of straw of either wheat or barley.

The rim of the basket was made of dark-colored winders, which also formed a decorative pattern on the body of the basket. It is unclear what that pattern represented, but the decoration method is comparable to that of an oval basket found in 1926 in Pit 55, which is now in the British Museum (Figure 5.42). 


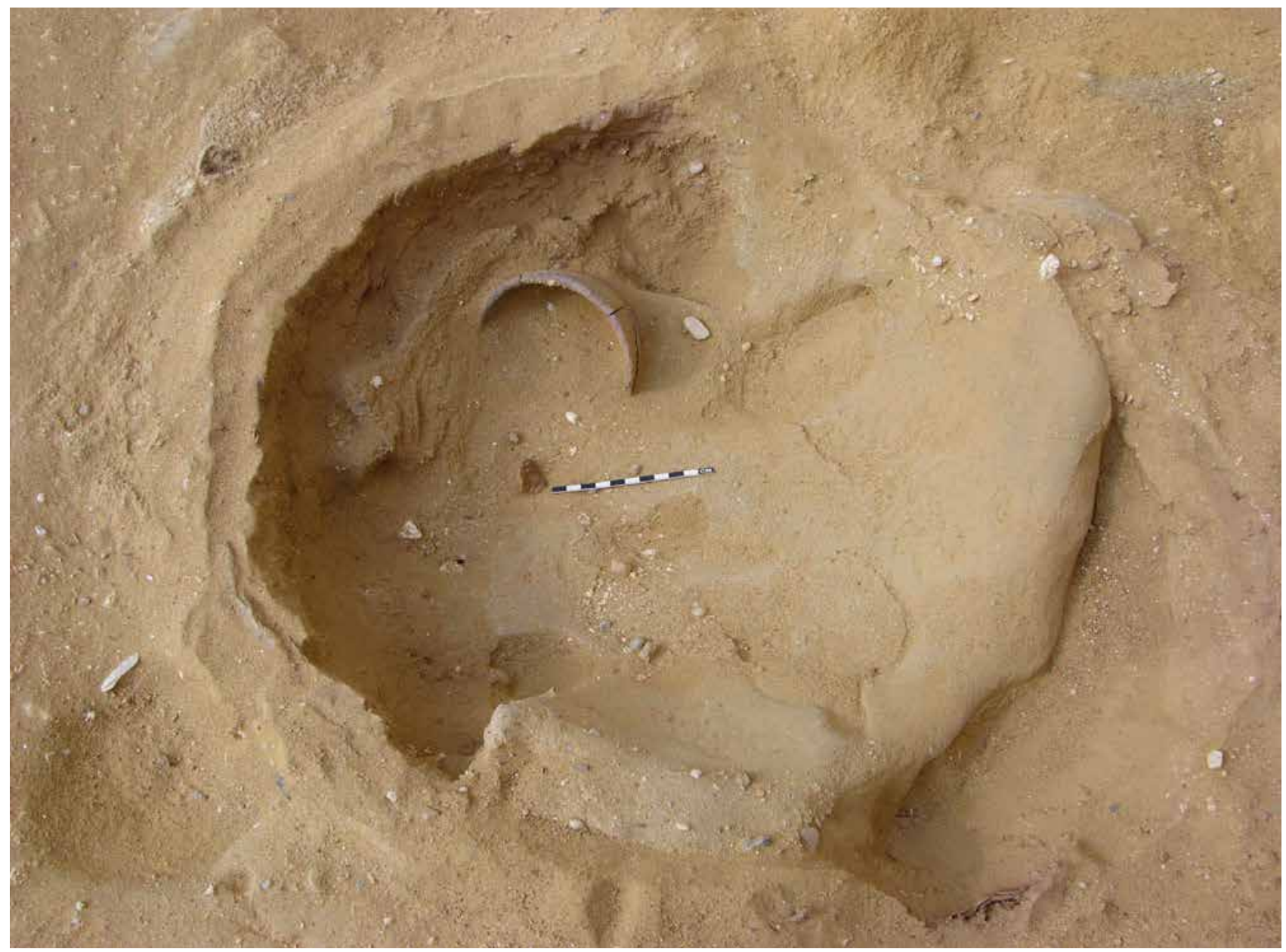

Figure 5.35. Lid of Pit 71 with an embedded, near-complete ceramic vessel.

The winders were stitched mostly in between those of the previous coil, picking up just a tiny bit of the bundle material. In general, this is the preferred technique for fine coiled basketry because a stitch through the winders or deep into the bundle material of the previous coil creates larger gaps in the pattern of the winders. The winders are straight on the outside of the basket but slightly slanted in a $\mathrm{Z}$ direction on the inside. The bundle material was inserted gradually, while the ends of the winders were worked into the coil, a technique used throughout Egyptian history until today (Wendrich 1999). This probably was a round open form of basket, shaped like a deep dish, with a diameter of $0.30 \mathrm{~m}$ and sides approximately $9 \mathrm{~cm}$ high. It would not have been very suitable as a scoop or a measuring basket but could have been used for winnowing.

Caton-Thompson and Gardner encountered a second, small, finely coiled object that was described as a "minute barrel shaped basket" (1934:44). This is now in the collection of the Petrie Museum in London.

The coiled mats found by Caton-Thompson in Pits 6, 28, and 58 (now in the Ashmolean Museum, Oxford) were made using the same coiling technique but were slightly less fine, with bundle sizes of an estimated 15 $\mathrm{mm}$ and winders of $7 \mathrm{~mm}$. In contrast to the fine basketry and the fine coiled mats, the baskets that lined the pits had a much more open structure in which the winders were widely spaced, so that the bundle material was visible, as in the basket that Caton-Thompson and Gardner removed from Pit 30 (now in the British Museum, Figure 5.43). In this basket, five rows of coiling were extant in the side of the basket, which measures $0.507 \mathrm{~m}$ in diameter, with a height of $2.60 \mathrm{~m}$ and a bundle size of $4.3 \mathrm{~mm}$, while the winders are approximately 15 to $20 \mathrm{~mm}$ wide and were spaced 20 to $30 \mathrm{~mm}$ apart. This is more widely spaced than the basketry lining of Pit 68, excavated in 2004 and 2005. 


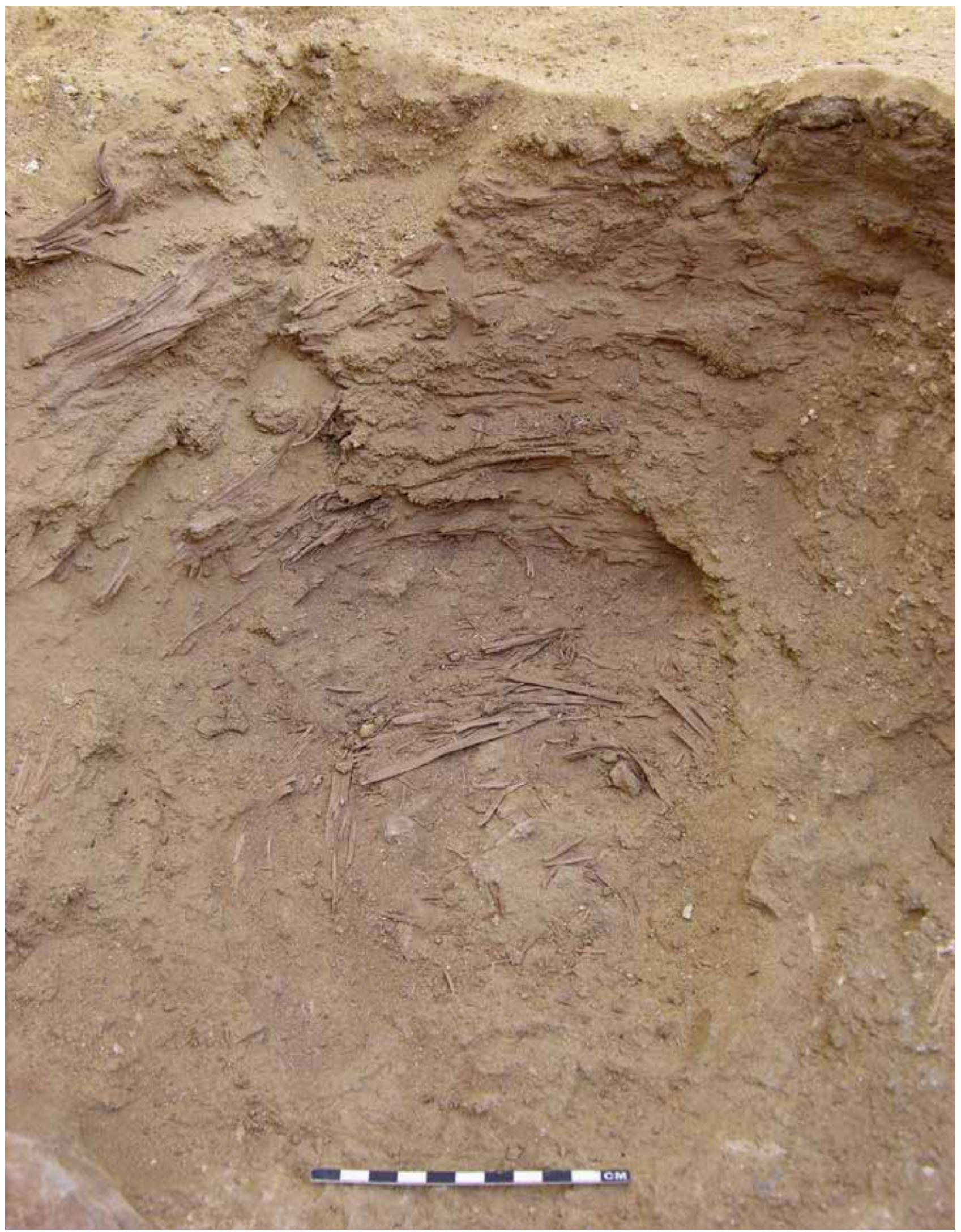

Figure 5.36. Remains of the badly preserved straw basketry lining of Pit 71 . 


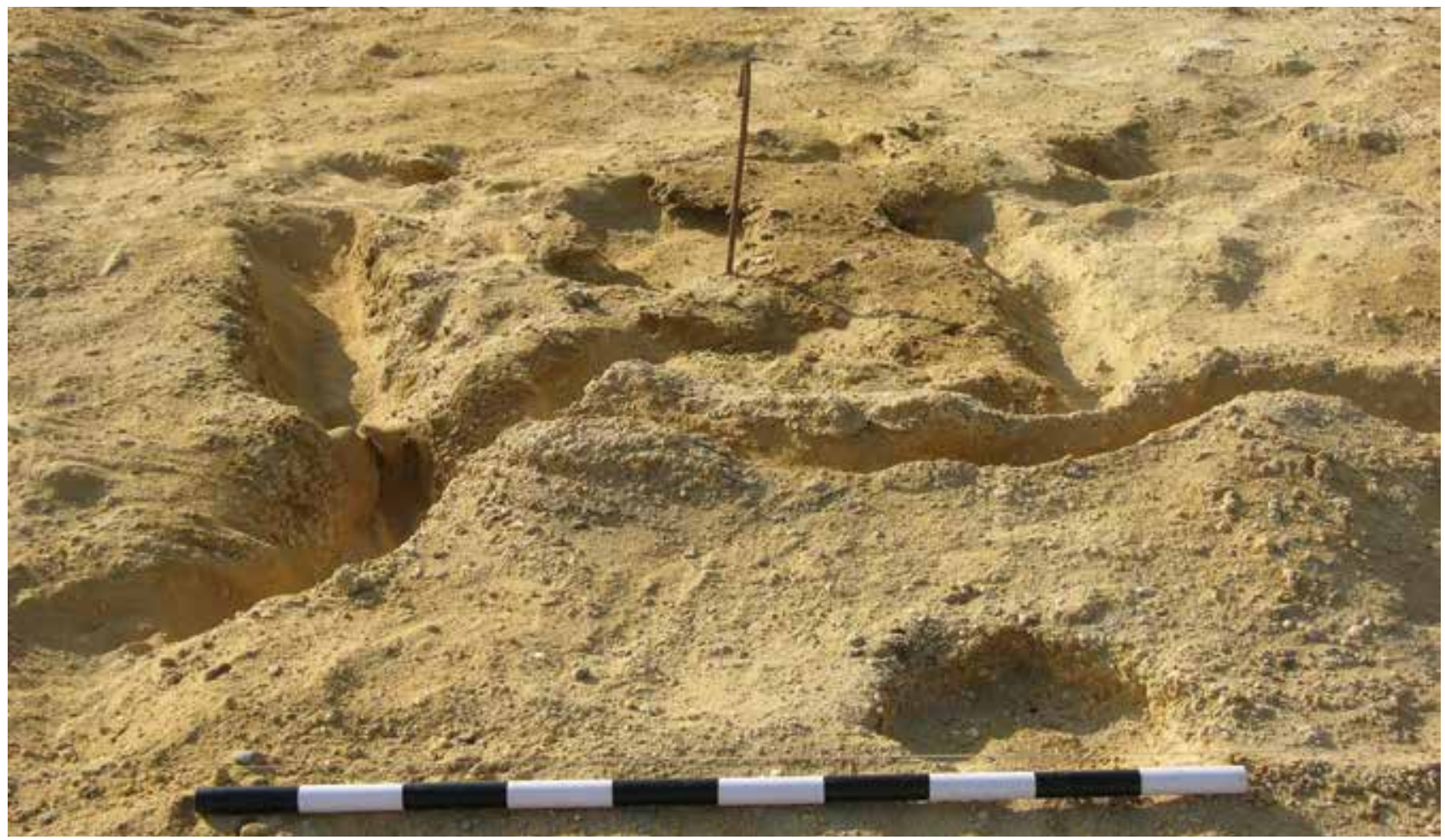

Figure 5.37. Feature 79 in Trench UKP31.

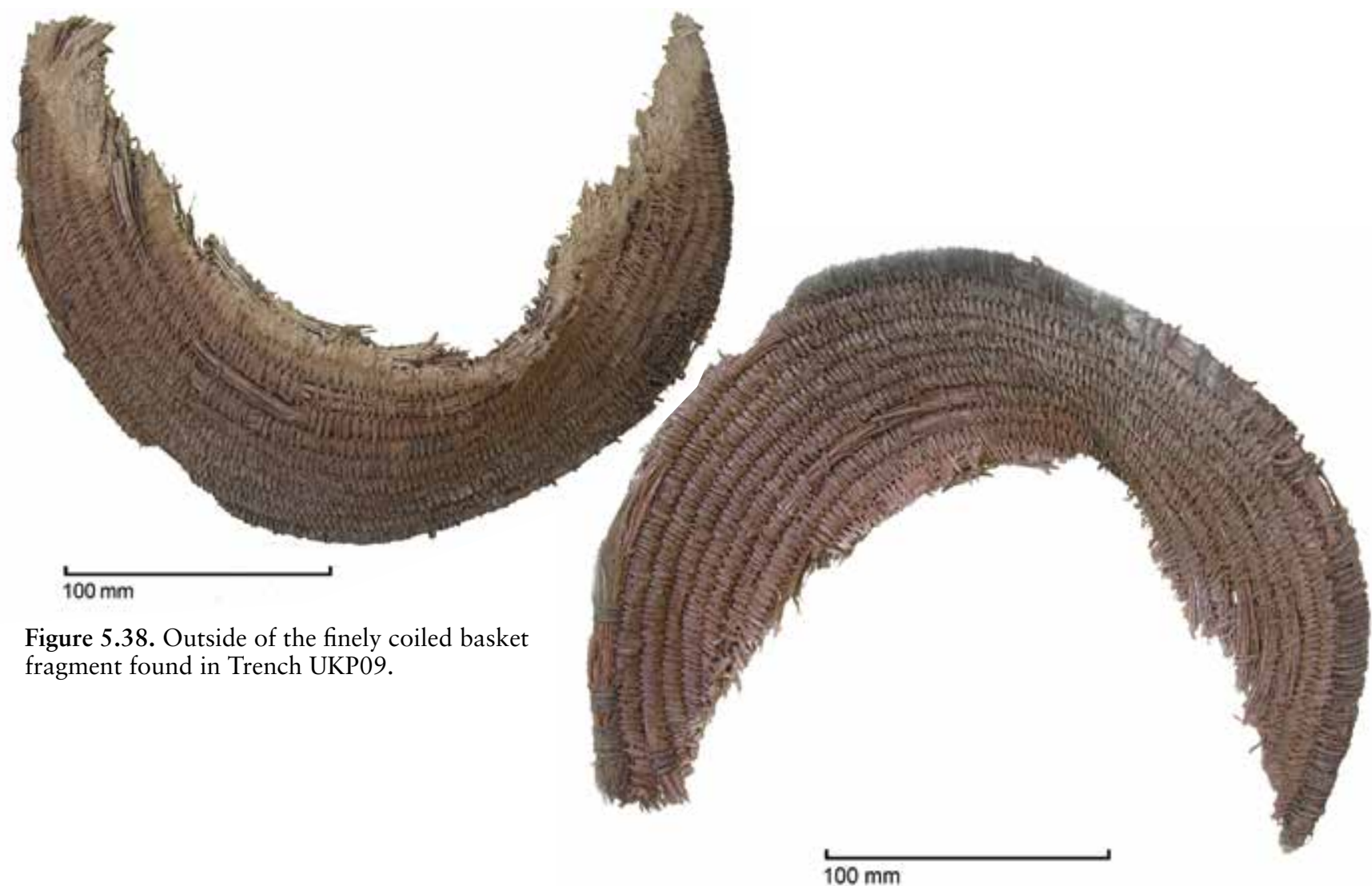

Figure 5.39. Inside of the finely coiled basket fragment found in Trench UKP09. 
Table 5.12. Basketry Finds from the K Pits from the 1926, 2004, and 2005 Excavations.

\begin{tabular}{|c|c|c|c|c|c|c|c|}
\hline $\begin{array}{l}\text { Pit } \\
\text { No. }\end{array}$ & Description & $\begin{array}{l}\text { Diameter } \\
\text { in } \mathrm{m}\end{array}$ & $\begin{array}{l}\text { Height } \\
\text { in } \mathrm{m}\end{array}$ & $\begin{array}{l}\text { Bundle/Winder } \\
\text { size in } \mathrm{cm}\end{array}$ & Material & $\begin{array}{l}\text { Present } \\
\text { Location }\end{array}$ & Photo \\
\hline 1 & Pit lining & 1.18 & 0.79 & & straw & & \\
\hline 1 & $\begin{array}{l}\text { Coiled matting, perhaps } \\
\text { different from pit lining } \\
\text { because it seems to have } \\
\text { separate materials used for } \\
\text { bundle and winder. }\end{array}$ & 1.15 & 0.05 & $5 / 2$ & straw & & $\begin{array}{r}\text { Plate } \mathrm{xxv}, \\
2,3\end{array}$ \\
\hline 2 & Pit lining & 1.03 & 0.69 & & straw & & \\
\hline 4 & Pit lining & 1.05 & 0.61 & & straw & & \\
\hline 5 & Pit lining & 0.53 & $\begin{array}{l}\text { Min. } \\
0.25\end{array}$ & & straw & & \\
\hline 6 & $\begin{array}{l}\text { Platter or lid in woven } \\
\text { grass. Finely coiled mat; } \\
\text { approximately } 20 \text { rows of } \\
\text { coiling. Bundle size smaller in } \\
\text { the center. }\end{array}$ & 0.41 & 0.02 & $2 / 1$ & grass & Cairo & $\begin{array}{r}\text { Plate } \\
\text { xxviii, } 4\end{array}$ \\
\hline 7 & Pit lining & 0.46 & 0.23 & & straw & & \\
\hline 8 & $\begin{array}{l}\text { Pit lining, coiled. } \\
\text { Approximately } 12 \text { rows of } \\
\text { coiling in base. }\end{array}$ & 0.91 & 0.07 & 7 & straw & Newcastle? & $\begin{array}{r}\text { Plate } \\
\text { xxvii, } 2\end{array}$ \\
\hline 9 & Pit lining & 0.96 & 0.53 & & straw & & \\
\hline 10 & $\begin{array}{l}\text { Pit lining. Tapering coiled } \\
\text { basket, from } 1.27 \text { to } 0.91 \mathrm{~m} \text { in } \\
\text { diameter. }\end{array}$ & 1.27 & 0.89 & & straw & & \\
\hline 11 & Pit lining & 0.61 & & & straw & & \\
\hline 12 & $\begin{array}{l}\text { Pit lining. Coiled basketry. } \\
\text { Approximately } 12 \text { rows of } \\
\text { coiling in the side. }\end{array}$ & 1.12 & 0.51 & 4 & straw & Hull? & $\begin{array}{r}\text { Plate } \\
\text { xxv, 4, } \\
\text { and Plate } \\
\text { xxvii, } 3\end{array}$ \\
\hline 13 & Pit lining & 1.30 & 0.51 & & straw & & \\
\hline 14 & Pit lining & 0.74 & 0.23 & & straw & & \\
\hline 15 & Pit lining & 0.47 & 0.23 & & straw & & \\
\hline $16 a$ & $\begin{array}{l}\text { Pit lining. Coiled basketry. } \\
\text { Approximately } 11 \text { rows of } \\
\text { coiling in the side. }\end{array}$ & 1.60 & 0.81 & 7 & straw & & $\begin{array}{r}\text { Plate } \mathrm{xxv}, \\
5\end{array}$ \\
\hline $16 b$ & $\begin{array}{l}\text { Pit lining. Coiled basketry. } \\
\text { Number of rows and size of } \\
\text { bundle cannot be determined } \\
\text { from } 1926 \text { photograph. }\end{array}$ & 0.61 & 0.30 & & straw & & Plate $x v, 5$ \\
\hline 17 & Pit lining & 0.81 & 0.48 & & straw & & \\
\hline 18 & $\begin{array}{l}\text { Pit lining. Coiled basketry. } \\
\text { Approximately } 11 \text { rows of } \\
\text { coiling in the side. }\end{array}$ & 1.02 & 0.51 & 4.5 & straw & Cairo & $\begin{array}{r}\text { Plate } \mathrm{xxv}, \\
6\end{array}$ \\
\hline
\end{tabular}


Table 5.12. Basketry Finds from the K Pits from the 1926, 2004, and 2005 Excavations. Continued

\begin{tabular}{|c|c|c|c|c|c|c|c|}
\hline $\begin{array}{l}\text { Pit } \\
\text { No. }\end{array}$ & Description & $\begin{array}{l}\text { Diameter } \\
\text { in } \mathrm{m}\end{array}$ & $\begin{array}{l}\text { Height } \\
\text { in } \mathbf{~}\end{array}$ & $\begin{array}{l}\text { Bundle/Winder } \\
\text { size in } \mathrm{cm}\end{array}$ & Material & $\begin{array}{l}\text { Present } \\
\text { Location }\end{array}$ & Photo \\
\hline 19 & $\begin{array}{l}\text { Pit lining. Coiled basketry. } \\
\text { Approximately eight rows of } \\
\text { coiling. }\end{array}$ & 0.76 & 0.46 & 6 & straw & $\begin{array}{l}\text { Ashmolean } \\
\text { Museum }\end{array}$ & $\begin{array}{r}\text { Plate } \\
\text { xxvi, } 6\end{array}$ \\
\hline 20 & Pit lining & 1.17 & 0.33 & & straw & & \\
\hline 21 & Pit lining; impression only & 1.32 & 0.66 & & & & \\
\hline 25 & $\begin{array}{l}\text { Platter underneath coarse } \\
\text { pot, probably coiled, but not } \\
\text { visible in the } 1926 \text { photo. }\end{array}$ & 0.61 & & & straw & $\begin{array}{r}\text { Petrie } \\
\text { Museum? }\end{array}$ & $\begin{array}{r}\text { Plate } \\
\text { xxvii, } 1\end{array}$ \\
\hline 28 & $\begin{array}{l}\text { Platter or lid in woven } \\
\text { grass. Finely coiled mat. } \\
\text { Approximately } 16 \text { rows of } \\
\text { coiling. }\end{array}$ & 0.23 & 0.015 & $1.5 / 0.7 ?$ & grass & Manchester? & $\begin{array}{r}\text { Plate } \\
\text { xxviii, } 5\end{array}$ \\
\hline 29 & Pit lining & 0.91 & 0.56 & & straw & & \\
\hline 30 & $\begin{array}{l}\text { Pit lining. Coiled basket; } \\
0.043 \mathrm{~m} \text { thick walls; made of } \\
\text { coarse straw coils; five rows of } \\
\text { coiling in the side. }\end{array}$ & 0.51 & 0.21 & 4.3 & & $\begin{array}{l}\text { British } \\
\text { Museum, } \\
\text { EA58695 }\end{array}$ & $\begin{array}{r}\text { Plate } \\
\text { xxvi, } 7\end{array}$ \\
\hline 31 & Pit lining & 0.74 & 0.53 & & straw & & \\
\hline 32 & Pit lining & 1.02 & 0.33 & & straw & & \\
\hline 33 & Pit lining & 1.00 & 0.46 & & straw & & \\
\hline 35 & Pit lining & 0.33 & 0.30 & & straw & & \\
\hline 36 & $\begin{array}{l}\text { Pit lining. Coiled basket; } \\
\text { coarse straw coils; similar to } \\
\text { Pit } 68 \text {. Approximately six } \\
\text { rows of coiling in the side. }\end{array}$ & 0.90 & 0.25 & 4 & straw & ? & $\begin{array}{r}\text { Plate } \\
\text { xxvi, } 3\end{array}$ \\
\hline 37 & Pit lining & 0.99 & 0.64 & & straw & & \\
\hline 39 & Pit lining & & & & & & \\
\hline 40 & Pit lining & 0.96 & 0.25 & & straw & & \\
\hline 41 & Pit lining; impression & 0.56 & 0.36 & & & & \\
\hline 42 & Pit lining; impression & 0.86 & 0.20 & & & & \\
\hline 43 & Pit lining; impression & 1.22 & 0.76 & & & & \\
\hline 47 & Pit lining base & 1.04 & 0.53 & & & & \\
\hline 48 & Pit lining & 1.09 & 0.90 & & & & \\
\hline 51 & $\begin{array}{l}\text { Pit lining. Coiled basket. } \\
\text { Approximately } 10 \text { rows of } \\
\text { coiling in the side. }\end{array}$ & 0.86 & 0.43 & 4 & straw & & $\begin{array}{r}\text { Plate } \\
\text { xxvi, } 1\end{array}$ \\
\hline 52 & Pit lining & 0.60 & 0.23 & & & & \\
\hline
\end{tabular}




\begin{tabular}{|c|c|c|c|c|c|c|c|}
\hline $\begin{array}{l}\text { Pit } \\
\text { No. }\end{array}$ & Description & $\begin{array}{l}\text { Diameter } \\
\text { in } \mathrm{m}\end{array}$ & $\begin{array}{l}\text { Height } \\
\text { in } \mathrm{m}\end{array}$ & $\begin{array}{l}\text { Bundle/Winder } \\
\text { size in } \mathrm{cm}\end{array}$ & Material & $\begin{array}{l}\text { Present } \\
\text { Location }\end{array}$ & Photo \\
\hline 54 & Mat or lining & 0.69 & 0.02 & & straw & & \\
\hline 55 & $\begin{array}{l}\text { Pit lining. Coiled basket. } \\
\text { Coarse straw coils. Similar to } \\
\text { pit } 68 .\end{array}$ & 0.74 & 0.05 & & straw & & $\begin{array}{r}\text { Plate } \\
\text { xxvi, } 2\end{array}$ \\
\hline 55 & $\begin{array}{l}\text { Finely coiled basket; oval. } \\
\text { Approximately seven rows } \\
\text { of coiling in the base and } 23 \\
\text { rows in the side. Decoration } \\
\text { with colored winders. }\end{array}$ & $0.42 \times 0.15$ & 0.13 & $0.55 / 0.1$ & grass & $\begin{array}{r}\text { British } \\
\text { Museum, } \\
\text { EA58696 }\end{array}$ & $\begin{array}{r}\text { Plate } \\
\text { xxvi, } 2\end{array}$ \\
\hline 56 & $\begin{array}{l}\text { Small bag-shaped basket, } \\
\text { described by Caton- } \\
\text { Thompson as "a minute } \\
\text { plaited straw basket." Only } \\
\text { the base and part of the sides } \\
\text { are left (approximately } 0.04 \mathrm{~m} \\
\text { in height). } \\
\text { Photo unclear, but it might be } \\
\text { twined. }\end{array}$ & 0.10 & 0.15 & $0.3 / 0.2$ & grass & $\begin{array}{r}\text { Petrie } \\
\text { Museum, } \\
\text { UC2941 }\end{array}$ & $\begin{array}{r}\text { Plate } \\
\text { xxvi, } 4\end{array}$ \\
\hline 58 & $\begin{array}{l}\text { Platter or lid in woven grass } \\
\text { forming a round coiled mat. }\end{array}$ & 0.30 & 0.01 & $1 / 0.5 ?$ & & $\begin{array}{r}\text { Ashmolean } \\
\text { Museum }\end{array}$ & \\
\hline 59 & Pit lining & 0.53 & 0.30 & & & & \\
\hline 61 & Pit lining & 0.46 & 0.76 & & & & \\
\hline 62 & Pit lining & 0.53 & 0.76 & & & & \\
\hline 63 & Pit lining & 0.69 & 0.41 & & & & \\
\hline 64 & Pit lining & 0.90 & 0.60 & & & & \\
\hline 66 & Pit lining & 0.76 & 0.20 & & & & \\
\hline 68 & $\begin{array}{l}\text { Pit lining; coiled basketry; } \\
\text { straw bundle with winders } \\
\text { taken from the bundle. Base } \\
\text { had eight and side had nine } \\
\text { rows of coiling. Winders are } \\
\text { closely spaced. }\end{array}$ & 0.90 & 0.60 & $4.5 / 3$ & & $\begin{array}{r}\text { destroyed by } \\
\text { looters }\end{array}$ & \\
\hline 69 & $\begin{array}{l}\text { Finely coiled basket with } \\
\text { colored winders, found } \\
\text { immediately outside Pit } 69 \text {. } \\
\text { No evidence for pit lining } \\
\text { found. }\end{array}$ & $0.27 \times 0.20$ & 0.07 & $0.9 / 0.1$ & & $\begin{array}{r}\text { URU storage } \\
\text { room }\end{array}$ & \\
\hline 71 & $\begin{array}{l}\text { Pit lining, coiled. Only } \\
\text { remains from bundle material } \\
\text { survived. Basket tapered } \\
\text { toward base. Base has five } \\
\text { rows of coiling extant, and } \\
\text { side has nine rows. None of } \\
\text { the winders survived. }\end{array}$ & $\begin{array}{r}\text { from } 0.65 \\
\times 0.60 \text { to } \\
0.36 \times 0.36\end{array}$ & 0.26 & & & $\begin{array}{r}\text { destroyed by } \\
\text { looters }\end{array}$ & \\
\hline 75 & $\begin{array}{l}\text { Straw inclusions suggest that } \\
\text { this pit was originally lined } \\
\text { with basketry. }\end{array}$ & $1.00 \times 0.90$ & 0.35 & 3 & & & \\
\hline
\end{tabular}

Note: For items recovered in 1926, locations are taken from Caton-Thompson and Gardner 1934, as are the photo numbers. 


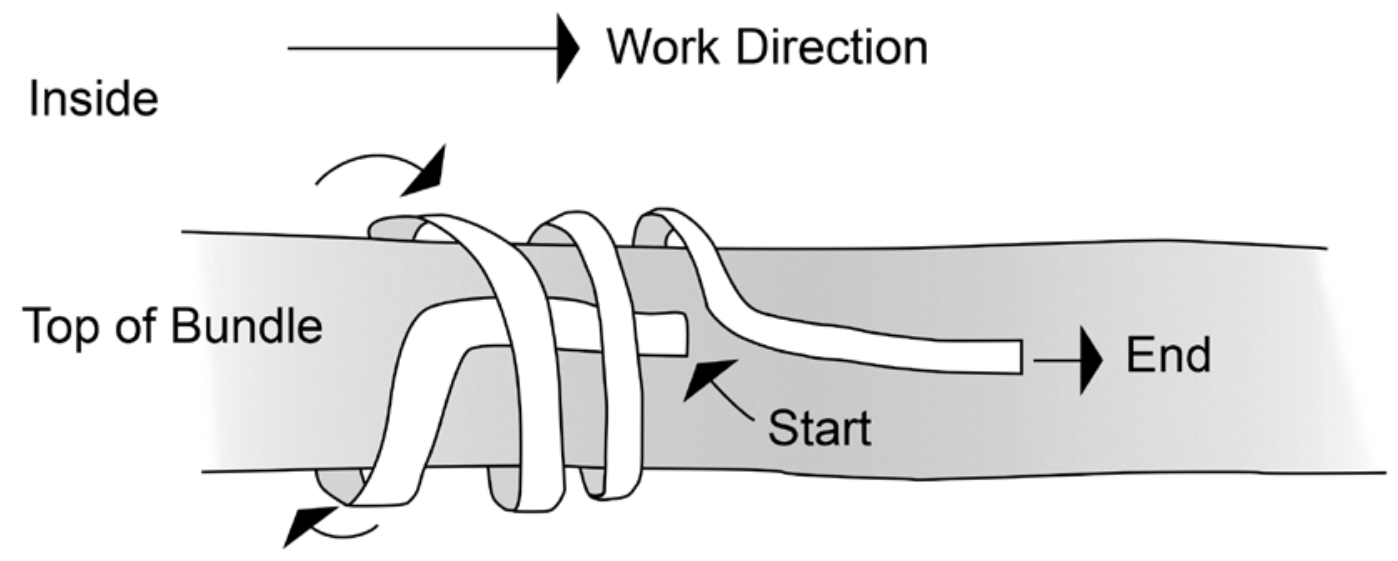

\section{Outside}

Figure 5.40. Construction drawing of the insertion of new grass winders in the finely coiled basketry; not to scale.

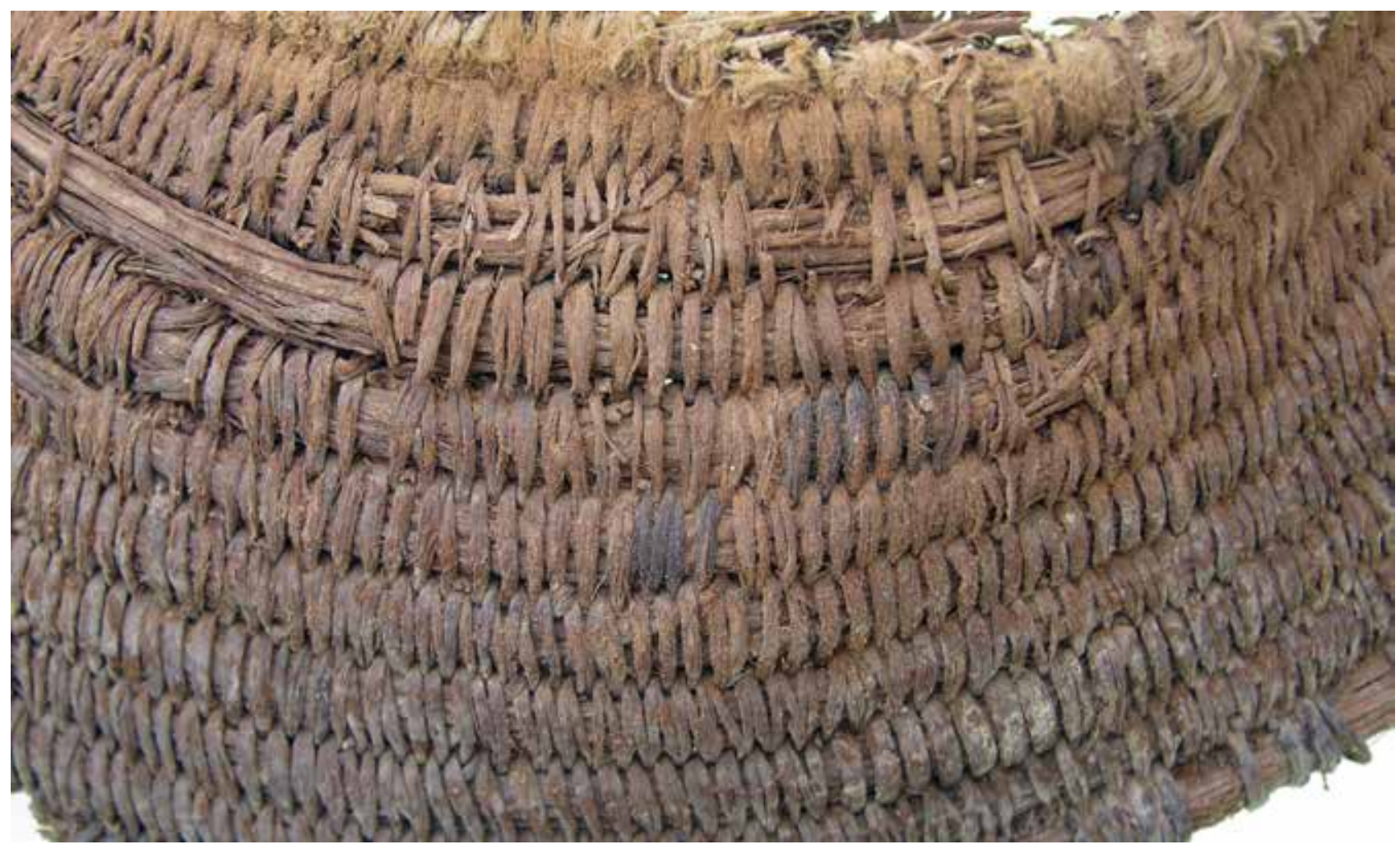

Figure 5.41. Close-up of the winders (active system) of a basket fragment. Note the darker color along the rim and selected colored winders in the fabric of the side of the basket. 


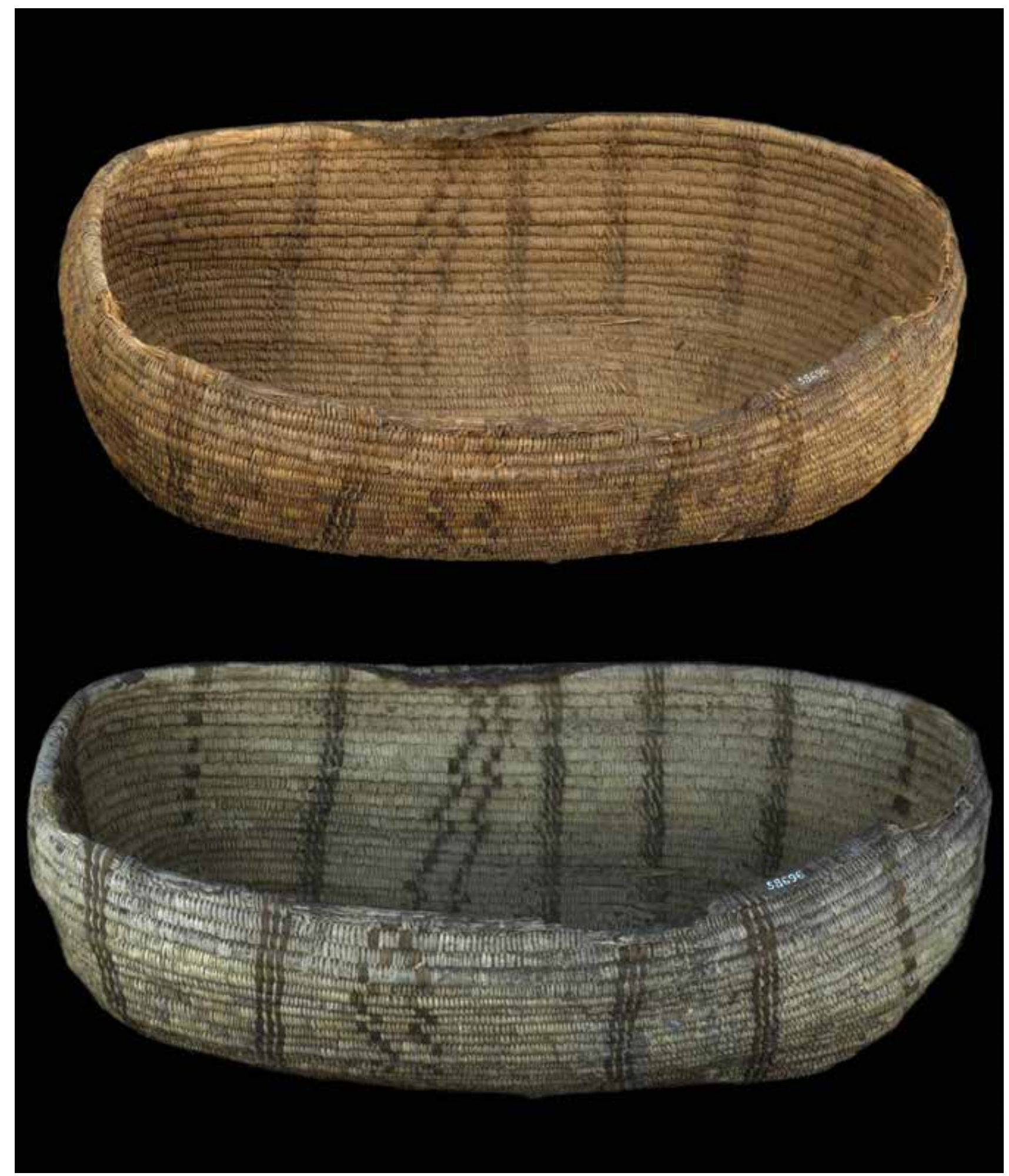

Figure 5.42. Finely coiled basket found in 1926; composite image showing the basket lit with an electronic flash (top) and ultraviolet lamps (bottom). (Accession no. EA58696, () Trustees of the British Museum) 
Pit 68 had a basketry lining [04.0003] with eight to nine rows of coiling in the base, which was $0.96 \mathrm{~m}$ in diameter (Figure 5.44). The bundles of straw, which were coiled from the center to the rim in a $G$ direction (that is, clockwise), were $40 \mathrm{~mm}$ wide near the center and $80 \mathrm{~mm}$ wide toward the edge of the base, where the coil gradually transitioned to the straight sides (Figures 5.45 and 5.46). The straw winders showed a technique not identified elsewhere in Egypt, in any period. The winders consisted of small bunches of three or four straw stems that were split off from the bundle material and used to stitch the rows of coiling together through the previous row of coiling. These straw winders were 10 to $30 \mathrm{~mm}$ in width and were spaced unevenly. In some sections, the stitches were close together, but there were irregular gaps between the winders. The side of the basket had nine rows of coiling over $0.48 \mathrm{~m}$, with an average coil diameter of 50 to $60 \mathrm{~mm}$.

On the north side of the rim of Pit 68 , the basketry projected out of the compact lid by $80 \mathrm{~mm}$. On the northwestern side, the top of the basket was folded inside under the weight of the compact layer. The outside of the basket was embedded in the compact layer, indicating that the lid material was poured onto the pit when it was not backfilled to the rim, pushing down the top of the basket. At the south and east sides, the basketry rim was not visible because it was covered in a compact layer that was separate from the lid. This likely indicates that there were multiple uses for the storage pit.

Apart from the straw fibers in Pit 75, which suggested that at one time Pit 75 may have had basketry, only Pit 71 was found with a lining in place. The basketry in this pit was in very bad condition. No winders could be discerned, but it was possible to make out that the base of the basket consisted of five rows of coiling with straw, while the sides had nine extant rows. The basket tapered out from a round bottom $(0.36 \mathrm{~m}$ in diameter $)$ to an oval rim $(0.65 \times 0.50 \mathrm{~m})$ over a height of $0.26 \mathrm{~m}$.

\section{Composition of the Lid Mortar}

Perhaps the most important result of the restudy of the Upper K Pits area was the realization that the pits were closed on purpose with a mud-like material, prepared in mostly natural depressions in the vicinity of

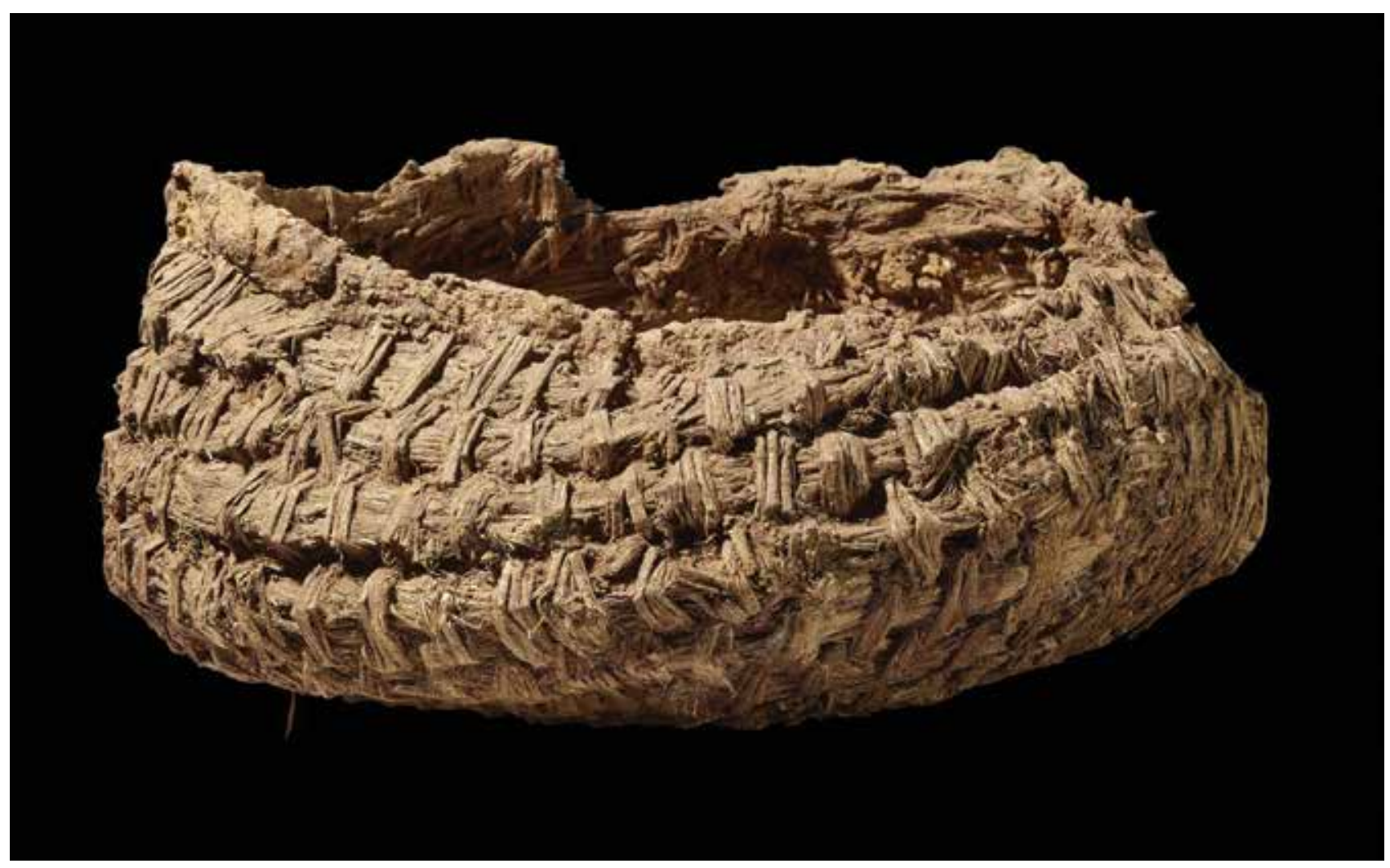

Figure 5.43. Lining of Pit 30, excavated by Caton-Thompson in 1926; now in the British Museum. (Accession no. EA58696, (c) Trustees of the British Museum) 


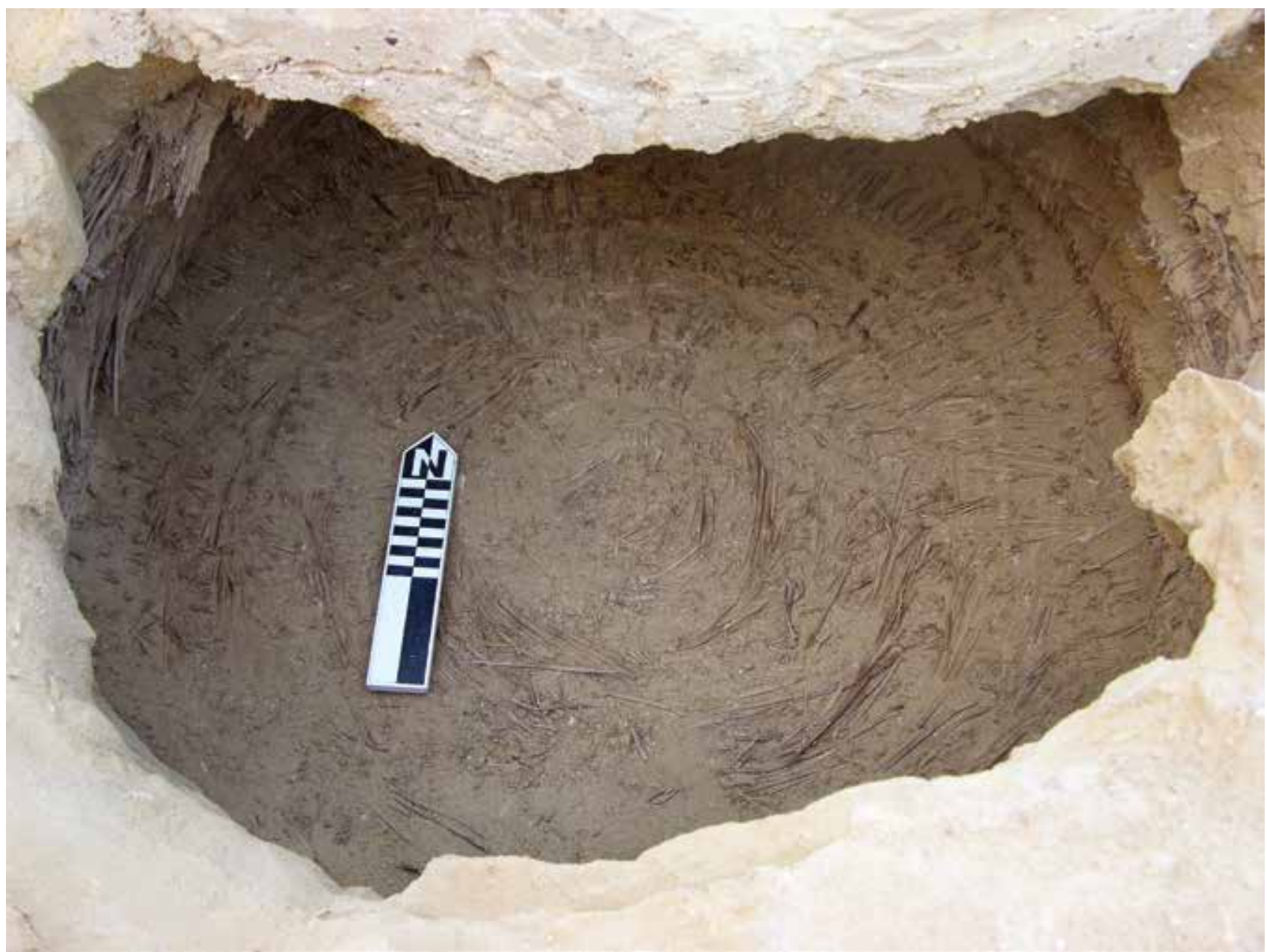

Figure 5.44. Basketry lining of Pit 68, excavated in 2004 and 2005.

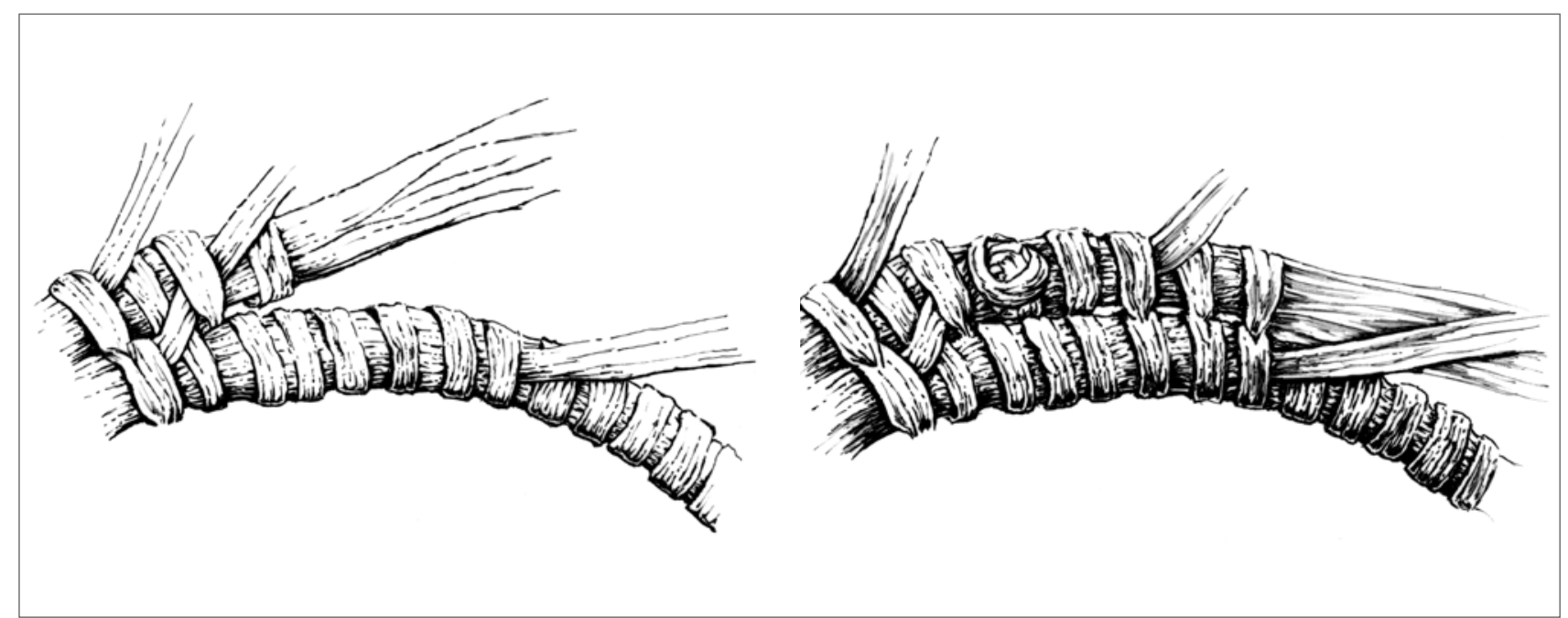

Figure 5.45. Construction drawing of how straw bundles are split off to form the winder that fastens the coils together; not to scale. 


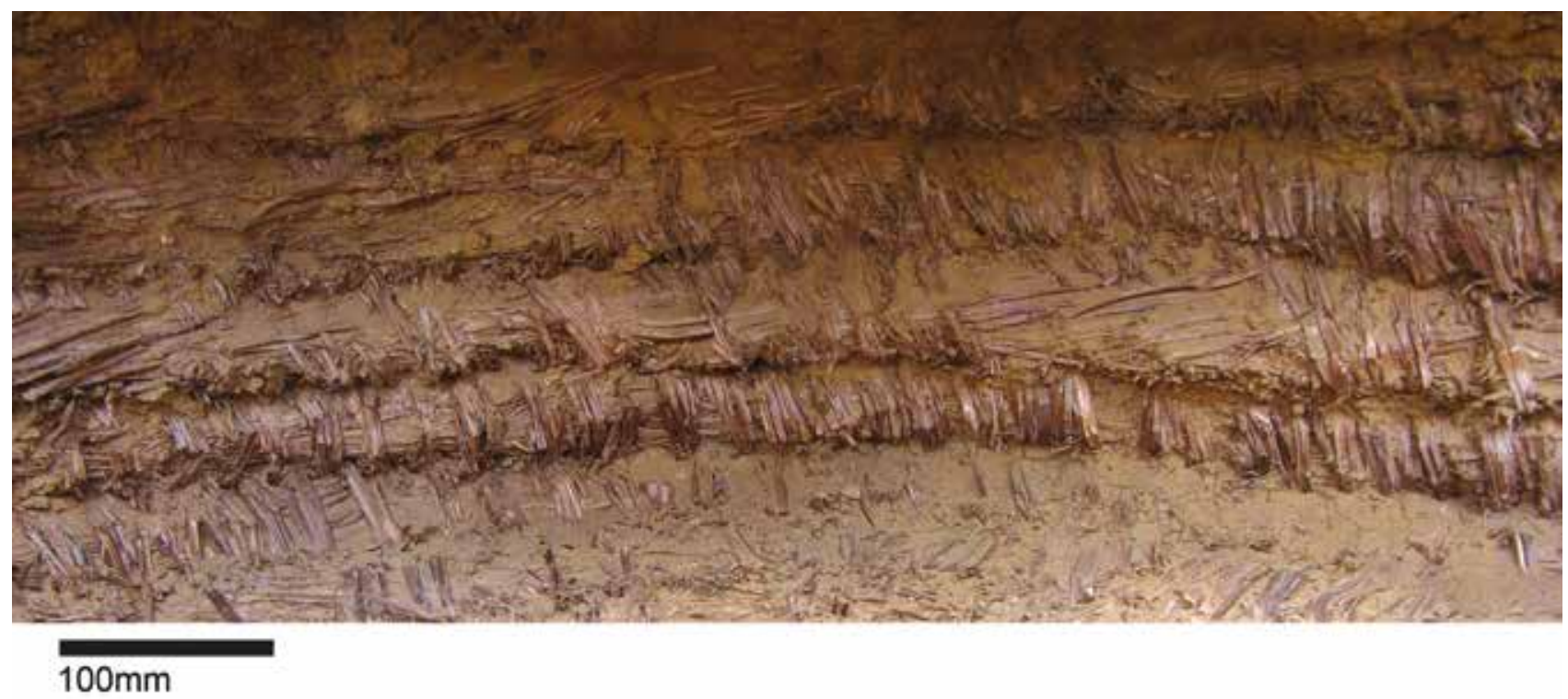

Figure 5.46. Detail of the straw coiled lining of Pit 68 [04.0003].

the excavated silos. A study of the mixture used for the lids of pits by Alexandra Winkels compared samples of three different lids, from Pits 69, 71, and 73.

The method entailed the following steps:

1. Microscopic examination and photographic documentation of the samples before treatment

2. Stereomicroscopy of cross-sections embedded in cyclododecane

3. Identification of the binding media and differentiation of mineral components by wet chemical staining methods and wet chemical analysis

4. Determination of the calcium carbonate content with a manometer

5. Determination of the matrix and mortar color with the Munsell rock or soil color chart

All three analyzed materials can be categorized as clay mortars. These mortars consist mainly of a matrix of yellowish-brown clay and embedded mineral aggregates of quartz sand and fine gravel in different grain sizes. The mortar matrix does not show the smooth texture of a fine homogeneous clay. Rather, under the stereomicroscope, a grainy to scaly consistency is indicated, with fine cohesive scales and grains or fine pats of clay between the mineral aggregates of the mortar.

These observations indicate that a kind of tafl, or inhomogeneous clay, was used as a binder for the mortar. Water causes clay to swell and makes it plastic. It can then be mixed with additional mineral aggregates, shaped, and applied as a mortar or spread over surfaces as a plaster. When soaked in water during mortar production, a homogeneous clay mass can be assembled. However, fine scales or small pats of clay within the matrices of the mortars indicate that in this case, the clay binder and the aggregates were mixed with water shortly before application.

The fine- to middle-grained mineral aggregates embedded in the matrices of the samples were mainly quartz sand and fine gravel, with grain sizes up to 6 to $8 \mathrm{~mm}$, with a few fine flint stone flakes and particles of other stone varieties, as well as shell inclusions in Pit 69 and plant material in Pit 71. The aggregates vary slightly among the different mortars. Compared to the other samples, Pit 71 shows a lower proportion of aggregates and thus a higher binder content (matrixto-binder ratio 70:30), whereas the matrix binder ratio is about 50:50 in the mortars from Pits 69 and 73. However, overall, all the mortars are similar in their composition and structure.

All three samples have low calcium carbonate content, between 2.5 percent (Pit 71) and 5 percent (Pit 73) (mass percent), and all have secondary gypsum deposits. These could originate from natural calcium carbonate and calcium sulfate in the clay material that functioned as a binder for the mortars. However, "they may also occur from secondary migration of salts from 
surrounding sediments and their accumulation within porous materials connected with periodic humidity, possibly caused by a rise of the groundwater level or occasional rainfall. The higher salt content is visible as salt crusts on edges of the mortar fragments on the samples from Pits 69 and 73. The recrystallization of salts may explain the compaction of the mortars at the time they were excavated, since they caused a structural stabilization of the original materials.

\section{Ceramics}

Ceramics were excavated in and around the Upper $\mathrm{K}$ Pits in 1926. Six large ceramic vessels were found by Caton-Thompson and Gardner (1934:44) in the Upper $\mathrm{K}$ Pits, but these did not survive removal from the site. They are described as similar in size and shape to a vessel that did survive from the excavation at Kom W (Caton-Thompson and Gardner 1934:Plate xviii, 1). This vessel is interpreted as a storage container, partly based on its size but also because it was set into a depression, suggesting that it was deliberately placed.
Some of the large vessels from the K Pits were found associated with basketry matting or lining, but in at least two cases the vessels were in pits without basketry. Based on their size and association with the pits, it is possible that, like the example from Kom W, the K Pit ceramic vessels also functioned as storage containers (Emmitt 2011). If this association is correct, then ceramic storage vessels are outnumbered by basketry-lined silos, which may suggest that they were used for the storage of different foods or goods from the basketry-lined pits. Another possibility is that the vessels found in pits were themselves being stored.

Additional ceramics were found by CatonThompson and Gardner, and because they were found only inside the pits, they were mostly complete rather than fragmentary sherds. Table 5.13 provides the volume, surface area, and range of shapes analysed. These vessels show a variety of shapes and volumes ranging from $592.35 \mathrm{~cm}^{3}$ to $5,537.06 \mathrm{~cm}^{3}$, with an average of $2,187.57 \mathrm{~cm}^{3}$, which might suggest that the vessels were used for a variety of functions (Table 5.13).

Table 5.13. Surface Areas $\left(\mathrm{cm}^{2}\right)$ and Volumes $\left(\mathrm{cm}^{3}\right)$ of the Upper K Pits Ceramic Vessels Organized by Shape, with Either a Restricted (R) or Unrestricted (U) Opening.

\begin{tabular}{|c|c|c|c|c|c|c|}
\hline & \multirow{2}{*}{$\begin{array}{c}\text { Cone } \\
\text { (Frustum) }\end{array}$} & \multicolumn{2}{|c|}{ Ellipsoid } & \multirow{2}{*}{$\frac{\text { Ovaloid }}{\text { R }}$} & \multirow{2}{*}{$\begin{array}{c}\text { Sphere } \\
\mathrm{R}\end{array}$} & \multirow[t]{2}{*}{ All } \\
\hline & & $\mathrm{R}$ & $\mathrm{U}$ & & & \\
\hline \multicolumn{7}{|l|}{ Surface Area $\left(\mathrm{cm}^{2}\right)$} \\
\hline $\mathrm{n}$ & 2 & 1 & 1 & 1 & 1 & 6 \\
\hline Mean & 745.64 & 341.80 & 532.68 & $1,539.47$ & 493.43 & 733.11 \\
\hline Standard deviation & 106.36 & - & - & - & - & 427.22 \\
\hline Minimum & 670.43 & - & - & - & - & 341.80 \\
\hline Maximum & 820.85 & - & - & - & - & $1,539.47$ \\
\hline \multicolumn{7}{|l|}{ Volume $\left(\mathrm{cm}^{3}\right)$} \\
\hline $\mathrm{n}$ & 2 & 1 & 1 & 1 & 1 & 6 \\
\hline Mean & $2,207.03$ & 592.35 & $1,139.74$ & $5,537.06$ & $1,412.20$ & $2,182.57$ \\
\hline Standard deviation & 837.11 & - & - & - & - & $1,798.01$ \\
\hline Minimum & $1,615.11$ & - & - & - & - & 592.35 \\
\hline Maximum & $2,798.96$ & - & - & - & - & $5,537.06$ \\
\hline
\end{tabular}




\section{Botanical Remains}

Excavation of the $\mathrm{K}$ Pits by Caton-Thompson and Gardner (1934) yielded 2,200 $\mathrm{ml}$ of emmer (Triticum turgidum subsp. dicoccon) grain kernels, $950 \mathrm{ml}$ of hulled barley (Hordeum vulgare) grain kernels, and some seeds of flax (Linum usitatissimum). A distinction between two-row and six-row barley was initially made based on the assumption that if six-row barley was present, two-thirds of the florets should be asymmetrical. However, it is now understood that in sub-fossil remains of hulled barley, the preservation of the chaff surrounding the grain kernel is often less than optimal. Therefore the assignment to a subspecies (that is, two row: distichon; six row: vulgare) is problematic (Cappers 2013). The absence of the twisted basal parts of the chaff results in an overrepresentation of symmetrical florets. Therefore, while the calculations made when the K Pits were first discovered resulted in the identification of both two-row and six-row barley (Caton-Thompson and Gardner 1934:48), this identification was based on floret asymmetry, and it is now assumed that all barley belonged to the subspecies vulgare (six-row barley).

New samples were obtained from the pits excavated in 2004 and 2005 (Schepers et al. 2006). Desiccated plant remains were found inside some pits and in the lids used for securing them. Small quantities of hulled barley were present; the largest concentration (more than 40 seeds) was in the best-preserved pit (Pit 68 in UKP04). Although the preservation did not allow for identification to the subspecies, it is likely that this is again six-row barley. This also fits with the sub-fossil record of Egypt in general, where six-row barley is the predominant subspecies from the Neolithic onward (Cappers and Neef 2012:406).

Whereas barley was represented by hulled grain kernels (florets), only empty spikelets and threshing remains were found from emmer. A small quantity of empty spikelets was present in Pit 68. The combination of barley grain and empty emmer spikelets in the same pit is surprising and may indicate that the barley belonged to the original pit fill, with the emmer spikelets deposited separately. It is also possible that the emmer spikelets were eaten by mice after the storage facility fell into disuse. Mice can isolate grain kernels without leaving gnawing marks on the chaff. However, while mouse nests were tentatively identified, no mouse droppings were found.
Threshing remains of emmer retrieved from the Upper K Pits were also encountered in the lids used for closing the pits. Their number is low, and it seems unlikely that such remains were added as a kind of temper. Instead they were likely unintentionally mixed into the mortar during production. The presence of threshing (dehulling) remains of emmer in the Upper $\mathrm{K}$ Pits indicates that dehulling was not only done at Kom $\mathrm{K}$ but also in the area where grain was stored.

\section{Dating}

Three radiocarbon determinations were obtained from two of the pits-two dates from Pit 75 and a single date from Pit 68 (Wendrich et al. 2010) (Table 5.14). Two older determinations were obtained from material collected by Caton-Thompson and Gardner, were dated by Libby (1952a, 1952b; Arnold and Libby 1951), and are included in Table 5.16, calibrated using the IntCal 13 curve.

The two radiocarbon determinations from Pit 75 both date the basket lining, so they effectively date the same event. The determination from Pit 68 returns a date that is slightly older than the dates from Pit 75, by around 200 years. Both of the Libby determinations have large errors and correspond to flat areas in the calibration curve (Figure 5.47), which reduces their precision. Both could represent older dates than Pits 75 and 68, with ages older than 6500 BP. However, neither date should be considered a precise age determination.

\section{Lower K Pits}

The location of the Lower K Pits is described as about "half a mile north of Kom K, and northwest of the [Upper K Pits]" (Caton-Thompson and Gardner 1934:53). This area is now partially underneath a modern canal and road, with the surrounding area disturbed by related construction work. No plan of the Lower K Pits was published by Caton-Thompson and Gardner, and we were unable to determine the exact location of the pits, although the probable area was searched in 2005 and again in 2012. Based on CatonThompson and Gardner's description, the Lower K Pits were located immediately south of K1. If this is the case, it is likely that the Lower K Pits were destroyed by the construction of the road and canal on the southern border of the K1 area, as shown in Figure 5.1.

Caton-Thompson and Gardner (1934) identified 109 silos at the Lower K Pits; 38 of them had evidence of reed or straw matting. The remaining 78 pits were 


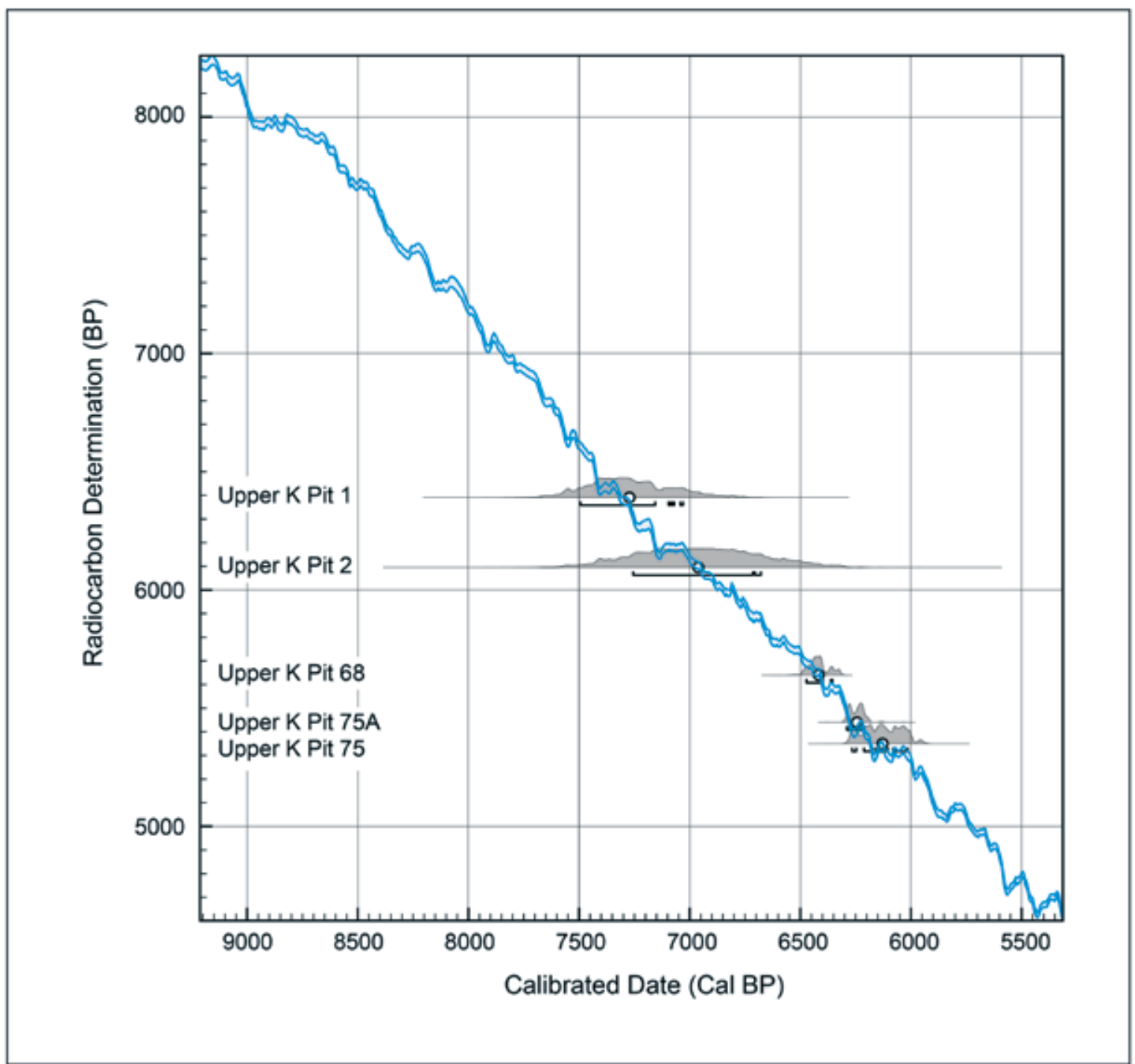

Figure 5.47. Radiocarbon determinations from Pit 68 and Pit 75 and the two older determinations from Libby (1952); plotted against the IntCal13 atmospheric data.

comparable in size to those with evidence of matting, leading Caton-Thompson and Gardner (1934:53) to suggest that these pits were also silos. The pits were filled in with lithic materials originally attributed to the Neolithic, which led Caton-Thompson and Gardner to suggest that they were dismantled during the Neolithic. The exact reason for any dismantlement is unknown, but it may have been to use the materials to furbish the Upper K Pits, although without any secure dates from the Lower K Pits, this interpretation is speculation. Another explanation is that the tops of the pits eroded and they were subsequently filled in by sediments that surrounded them.
Some of the pits had what Caton-Thompson and Gardner described as patches of mud and dung adhering to the walls. Presumably it was thought that this adhesive was used to hold a basketry lining in place. Twigs found in the walls of some pits were assumed to have a similar function (Caton-Thompson and Gardner 1934:53). However, it is possible that what CatonThompson and Gardner identified as unlined pits, with no evidence of any matting, was in fact similar to a form of clay vessel found in Kom $\mathrm{K}$ (described in chapter 6; Figure 6.10). The diameter of the clay vessel fits into the smallest category of silo identified by CatonThompson and Gardner at the Upper and Lower K Pits 
Table 5.14. Radiocarbon Determinations from K Pits.

\begin{tabular}{|c|c|}
\hline Pit number & Radiocarbon Determination Oxcal 4.2.2 calibration (Bronk Ramsey (2009)* \\
\hline $\begin{array}{l}\text { Pit } 75 \\
\text { [UKP 07.0002] } \\
\text { FY05 0789-fa basket lining }\end{array}$ & $\begin{array}{l}\mathrm{GrN}-30130 \\
5350 \pm 60 \mathrm{bp} \\
6129 \pm 85 \mathrm{cal} \mathrm{BP} \\
4180 \pm 85 \mathrm{cal} \mathrm{BCE}\end{array}$ \\
\hline $\begin{array}{l}\text { Pit } 75 \\
\text { [UKP 07.0002] } \\
\text { FY05 0789-fa basket lining }\end{array}$ & $\begin{array}{l}\text { GrA-31247 } \\
5440 \pm 35 \mathrm{bp} \\
6244 \pm 34 \mathrm{cal} \mathrm{BP} \\
4295 \pm 45 \mathrm{cal} \mathrm{BCE}\end{array}$ \\
\hline $\begin{array}{l}\text { Pit } 68 \\
\text { [UKP 04.0003] } \\
\text { FY05 0785-fa }\end{array}$ & $\begin{array}{l}\text { GrA-31248 } \\
5640 \pm 35 \mathrm{bp} \\
6416 \pm 45 \mathrm{cal} \mathrm{BP} \\
4467 \pm 45 \mathrm{cal} \mathrm{BCE}\end{array}$ \\
\hline Upper K1 & $\begin{array}{l}\mathrm{C}-550 / 551 \\
6391 \pm 180 \mathrm{bp} \\
7273 \pm 187 \mathrm{cal} \mathrm{BP} \\
5324 \pm 187 \mathrm{cal} \mathrm{BCE}\end{array}$ \\
\hline Upper K2 & $\begin{array}{l}\mathrm{C}-457 \\
6095 \pm 250 \mathrm{bp} \\
6961 \pm 273 \mathrm{cal} \mathrm{BP} \\
5012 \pm 273 \mathrm{cal} \mathrm{BCE}\end{array}$ \\
\hline
\end{tabular}

*Plotted with atmospheric data from Reimer et al. (2013).

(Table 5.15). Therefore, while it is possible that some of the silos from the Lower K Pits were indeed dismantled, it is also possible that some never had matting and had instead a lining of clay, mud, or dung.

Similar to the Upper K Pits, the Lower K Pits had evidence for the placement of ceramic vessels. Numerous sherds were identified from the pits, and these were described as similar to those from other Neolithic Fayum localities. They are described as containing chaff temper, poorly fired, and with thick black cores. Based upon Caton-Thompson and Gardner's illustrations (1934:Plates xviii-xx) and descriptions of several ceramic vessels from the Lower K Pits, it is probable that they were similar in form and manufacture to those identified at the Upper K Pits. However, beyond these illustrations, no further data are available, as none of the vessels survived excavation. In addition to the ceramics, Caton-Thompson and Gardner recorded one polished ax attributed to the Neolithic, gazelle horns, and several freshwater mussel shells within the pits.

\section{Summary and Discussion of the K Pits}

In 2004 it became apparent that the Lower K Pits had been completely destroyed by two irrigation canals excavated in the early 1990s. Part of the Upper K Pits area was destroyed at the same time by excavation of a north-south canal. However, our investigations west of this canal showed that some of the pits had survived, and excavation of these pits provided new insights in the use of the area for storage.

Of the 15 new areas investigated, three pits were found with basketry lining, two were unlined pits, eight were identified as preparation areas for mortar, and two were classified as robber pits, probably dug sometime after the 1926 expedition left the area. Unfortunately, in 2007 the entire area of the Upper K Pits was destroyed by large-scale destruction with mining machinery.

The geology of the top of the $\mathrm{K}$ ridge (Trenches UKP01 to UKP03 and UKP24 to UKP40) is characterized by a silty brown-green-gray sandstone with 
Table 5.15. Diameters of Features from the Lower K Pits Compared with Similar Features from the Upper K Pits (Caton-Thompson and Gardner 1934) and Kom K.

\begin{tabular}{|c|c|c|c|c|c|c|c|}
\hline Feet & $>7$ & $>6$ & $>5$ & $>4$ & $>3$ & $>2$ & $>1$ \\
\hline Centimeters & $>213.36$ & $>182.88$ & $>152.4$ & $>121.92$ & $>91.44$ & $>60.96$ & $>30.48$ \\
\hline Lower K Pits & 1 & & 7 & 17 & 60 & 15 & 3 \\
\hline Upper K Pits & & & 1 & 3 & 19 & 10 & 11 \\
\hline KK07 & & & & & & & 1 \\
\hline
\end{tabular}

gypsum veins running through, overlain by a thin layer $(5 \mathrm{~cm})$ of topsoil, covered by a desert pavement of medium-sized pebbles $(2$ to $4 \mathrm{~cm}$ ) over a thin layer of yellow-reddish silty sand. The area surrounding the pit in Figure 5.30 shows the typical appearance of the bedrock in this part of the ridge. The southern, downslope trenches have one or two sand layers overlying the bedrock, ranging from 10 to $20 \mathrm{~cm}$ in thickness. Toward the south, the compact silty sandstone cap gives way to a brown-green shale. The pits that we and CatonThompson and Gardner investigated were dug into this bedrock material.

The excavations undertaken in 2004 and 2005 showed that the Upper K Pits were used multiple times. Evidence for domesticated wheat and barley was mostly found in small quantities lost in the weave of the basketry lining of the pits. Only one pit, excavated in 1926, yielded a sizable quantity of grain. The rest were apparently emptied before being backfilled. There were few additional items found in the pits: one hafted sickle blade, several shell scoops, sticks, and a few baskets. One of these may have been a winnowing basket. It is therefore apparent that the latest phase of pit use was one of abandonment, and none of the excavated pits seem to have been in use for grain storage when they were abandoned. However, most were prepared for possible reuse. They were filled with sand and covered with a lid created from a mortar of water, clay, and quartz sand. Areas for the preparation of this mortar were found adjacent to the Upper K Pits. The high salt level of the mortar matrix resulted over time in a hardening of the lids into an indurated layer that would, however, dissolve again when put in water. In addition to the basket-lined storage pits, Caton-Thompson and Gardner found ceramic vessels that may also have served as storage devices. Alternatively or as well, it is possible that ceramic vessels were the items stored in the pits. Some of the Lower K Pits may have been lined with clay, mud, or dung as an alternative to basketry.

The construction of the basketry, as well as the linen found within one of the pits, illustrates a technology that was used to manufacture a number of different items using various techniques and different materials. One of the coiling styles has not been identified before in Egypt. Like the ceramic vessels, some of the smaller basketry items may have been left for storage in the pits.

The pits were ultimately abandoned. However, this final abandonment may have been with the intention to return, even if this intention was not fulfilled. If so, then the pits provide another material expression of mobility, albeit one that differs from the portable material culture discussed at the start of this chapter. Evidence that the pits were deliberately filled and sealed with mortar lids suggests that they acted as storage facilities that were reused. We cannot be certain how many of the pits were in use at one time, and there is the suggestion that some of the pits had their basketry linings removed, perhaps for use in other pits. Nevertheless, the close spatial relationship of the pits within the two locations suggests that people were aware of the presence of earlier pits, even if some of these were abandoned. The Upper K Pits that we studied had little portable material culture within the immediate vicinity. It is impossible to know what the area surrounding the Lower K Pits once contained. All we have is a comment by Caton-Thompson and Gardner that the Lower K Pit fills contained flaked stone artifacts. Our own surveys in the K1 area found concentrations of stone artifacts and hearths, but at some distance from the Upper K Pits. 
Combined, these findings may indicate that the K Pit locations acted as storage areas distinct from the location of other activities. If so, then the evidence for reuse suggests that these locations were returned to over some period of time, although we cannot be sure how long, and were used in largely similar ways. Whatever the duration and frequency of reuse, the existence of storage locations used in this way has implications for the settlement systems in which the Fayum played a part, a topic we consider in more detail in chapter 7 . Before this, however, we need to consider results from the excavation of a site that Caton-Thompson and Gardner investigated immediately before the discovery of the K Pits, Kom K. 


\section{6 \\ Kom K}

\section{Willeke Wendrich, Rebecca Phillipps, Simon J. Holdaway, Veerle Linseele, Joshua J. Emmitt, and John M. Marston}

The village débris was a mere skin, rarely exceeding 12 ins. in depth, which overlay a low dome of hardened sandrock deposit of the Pleistocene Lake. Interposed between the two was a salt-impregnated band of great hardness. The neolithic débris must be regarded, therefore, as the denuded remnants only of the settlement, whose original depth of deposit remains unknown [Caton-Thompson and Gardner 1934:38].

C aton-Thompson and Gardner report on their excavations at Kom $\mathrm{K}$ in chapter 8 of The Desert Fayum (1934:37-41) (Figure 1.1). The bulk of the text describes the artifacts that were recovered, with only a brief description of the excavation strategy and stratigraphy (1934:38). The site as a whole was described as an oval-shaped mound with dimensions of $400 \times 200$ feet $(122 \times 61 \mathrm{~m})$ and a depth of no more than 5 feet $(1.5 \mathrm{~m})$. Excavations proceeded using the same trenching technique as employed at Kom W. Five 9 -foot $(2.74-\mathrm{m})$ trenches were excavated across the site in an east-west direction. Caton-Thompson and Gardner report that work on the site was interrupted by the discovery of the K Pits (chapter 5) and that excavations were completed over an area measuring $180 \mathrm{x}$ 110 feet $(55 \times 33 \mathrm{~m})$.

Caton-Thompson and Gardner went on to report what they describe as "village debris"-that is, artifacts and faunal remains identified as Neolithic and thought to represent a settlement similar to that at Kom $\mathrm{W}$, rarely exceeding 12 inches $(0.30 \mathrm{~m})$ in depth. This material was on top of what was described as a hardened "sandrock" deposit, related, they suggested, to a Pleistocene lake. What was described as a "salt-impregnated band of great hardness" was stratified between the overlying artifact-rich deposit and the underlying "sandrock." Caton-Thompson and Gardner suggested that the overlying cultural materials were concentrated by deflation, "brought to the surface inch by inch by wind dispersal of the lighter elements" (1934:38).

Sixteen "sunk fire-holes," here referred to as hearths, were identified "scattered irregularly over the top of the mound only." These vary in size from " $3 \mathrm{ft} . \mathrm{x} 3 \mathrm{ft}$. 9 ins., to $1 \mathrm{ft} .6$ ins." $(0.91 \times 1.14 \mathrm{~m}$ to $0.46 \mathrm{~m})$ and are described as cut into the "sandrock" to a depth of 8 to 9 inches $(0.22 \mathrm{~m})$. The fill of these cuts consisted of material of a dark color with charcoal and burned earth. Ceramic sherds were found in two of the 
hearths, but the features were not otherwise associated with pottery vessels. They were interpreted as "cooking holes" (1934:38).

Caton-Thompson and Gardner commented that the salt-impregnated layer described above likely formed subsequent to the Neolithic occupation because the bases of what were described as "big cooking pots" were cemented in the layer such that they had to be chiseled out.

A number of authors referred to Kom $\mathrm{K}$ in their subsequent studies of the Fayum Neolithic, but no further work was carried out at the site before the excavations reported here. The site was incorporated into lands under contemporary cultivation before its rediscovery in 2005. At various times during the later decades of the twentieth century, the site was plowed and at one time used as a vineyard. Upon discovery, the site was still visible as a mound, much the way CatonThompson and Gardner described it, with the surface covered with concentrations of flaked stone artifacts, ceramic fragments, and faunal remains, and with the sediment discolored in places by charcoal and ash.

\section{Excavation Strategy}

The surface of Kom K at discovery was obviously disturbed, and the briefness of the report on CatonThompson and Gardner's work at the site made identification of intact materials difficult. The published plan of the excavations provided a general indication of where previous work was undertaken but was not detailed enough to enable accurate georegistration. For these reasons, two strategies were employed to further investigate the site. First, a systematic surface collection of flaked stone artifacts and ceramics was conducted using 99 squares with dimensions of 5 x $5 \mathrm{~m}$. All flaked stone artifact and ceramic material from the squares was collected, and an analysis of the flaked stone artifacts from a random selection of a quarter of these squares is reported below. The ceramics from Kom $\mathrm{K}$ are still under investigation and will be reported in a subsequent volume. Figure 6.1 shows the distribution of collection squares across the Kom $\mathrm{K}$ mound.

The second strategy involved the use of a paleomagnetic survey to locate concentrations of burned material that might indicate the presence of additional hearths like the 16 that Caton-Thompson and Gardner described. Figure 6.2 shows a map of the potential magnetic anomalies superimposed with $10-\mathrm{cm}$ contour lines to indicate the slope of the mound.
Figure 6.3 attempts to overlay the magnetic data with the plan of the five 9-foot-wide (2.74-m-wide) trenches excavated by Caton-Thompson and Gardner. Also indicated are the excavation trenches related to the work reported here. The correlations are not exact, because as noted, it was difficult to reconstruct both the location and the extent of Caton-Thompson and Gardner's original work. Our excavation trenches were located in areas with magnetic anomalies and at locations that would provide insight into the deposits at the upper, middle, and lower portions of the mound. The excavation units and stratigraphy for each of the excavated trenches are described in the next section.

A small $1 \times 1 \mathrm{~m}$ test trench (not reported here in detail) indicated that the magnetic anomalies did indeed relate in a general way to the location of hearths, confirmed by the excavation of an initial trench (KK02) in 2006. During the 2007 field season, further trenches were excavated at different heights across the kom. A total of six additional trenches were opened: two were excavated at the northern edge of the kom (KK04 and KK05), two in the center (KK07 and KK08), and two at the southern edge (KK03 and KK06), near KK02 (Figure 6.4). Excavations were also continued in $\mathrm{KK} 02$.

In the following, the stratigraphy of each of the trenches is described, together with a number of excavated hearth features. Hearth features were identified as roughly circular deposits of ash in plan, and once excavated they revealed a layer of reddened, oxidized sand below a basin-shaped cut that frequently contained ashy material. Discoloration of sand below the hearth relates to heat transferal and so is not part of the hearth structure itself (Aldeias et al. 2016). Results of radiocarbon determinations obtained from the hearths are also discussed. A number of sediment samples were obtained, both from the fill of features and from sediments into which the features were cut. These were investigated for charcoal and when present the charcoal was identified to species. Only one charcoal type was identified in the Kom K samples, while several other samples contained unidentifiable material that was clearly charcoal but too badly degraded or too small to permit the identification of its wood anatomy. The characteristics of this wood type that could be identified are indicated in Table 6.1.

The particular set of characters narrows the range of possible wood species to one: tamarisk (Tamarix sp.). 


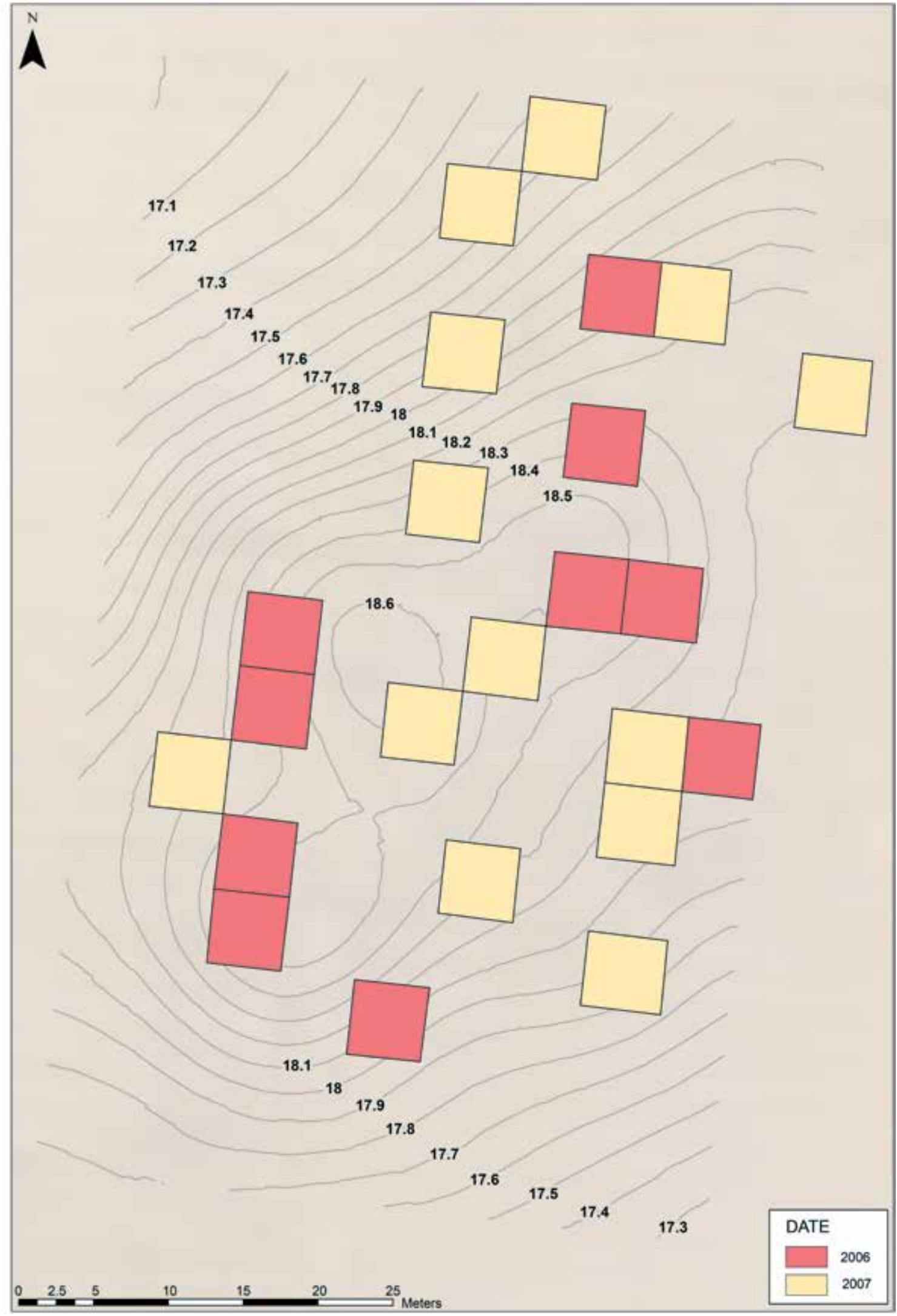

Figure 6.1. Kom K 5 x 5-m collection square locations with 10-cm contours showing the year of collection. 


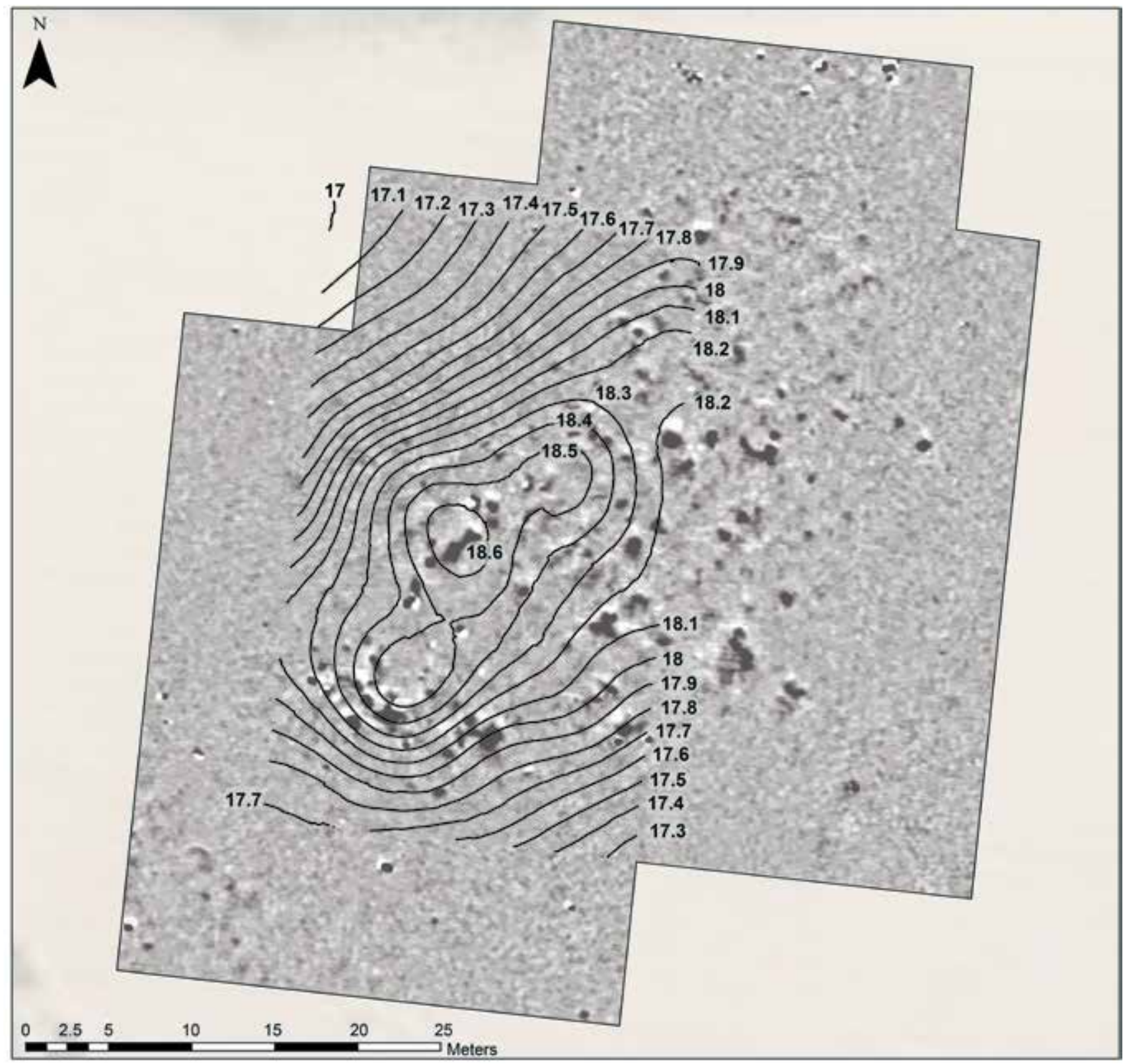

Figure 6.2. Magnetic anomaly survey of Kom K with 10-cm topographic contours superimposed.

Most archaeological samples appeared diffuse-porous, and tamarisk is ring- to semi-ring-porous. However, the archaeological samples consisted of fragments that were much smaller than a complete growth ring. The few archaeological fragments of very small stems with entire rings preserved do show a ring-porous arrangement. The characteristic very wide rays of tamarisk (10 to 20 seriate) were also not identified in the archaeological specimens, although this character is likely variable between species and environmental conditions. The radial section characters identified match published descriptions of tamarisk and modern reference materials. Generally, the archaeological material has fewer, smaller pores than modern comparative specimens, and the rays are narrower, closer together, and more frequent in the archaeological material. Whether this is a specific or environmental difference, or caused by carbonization processes, is unknown. 


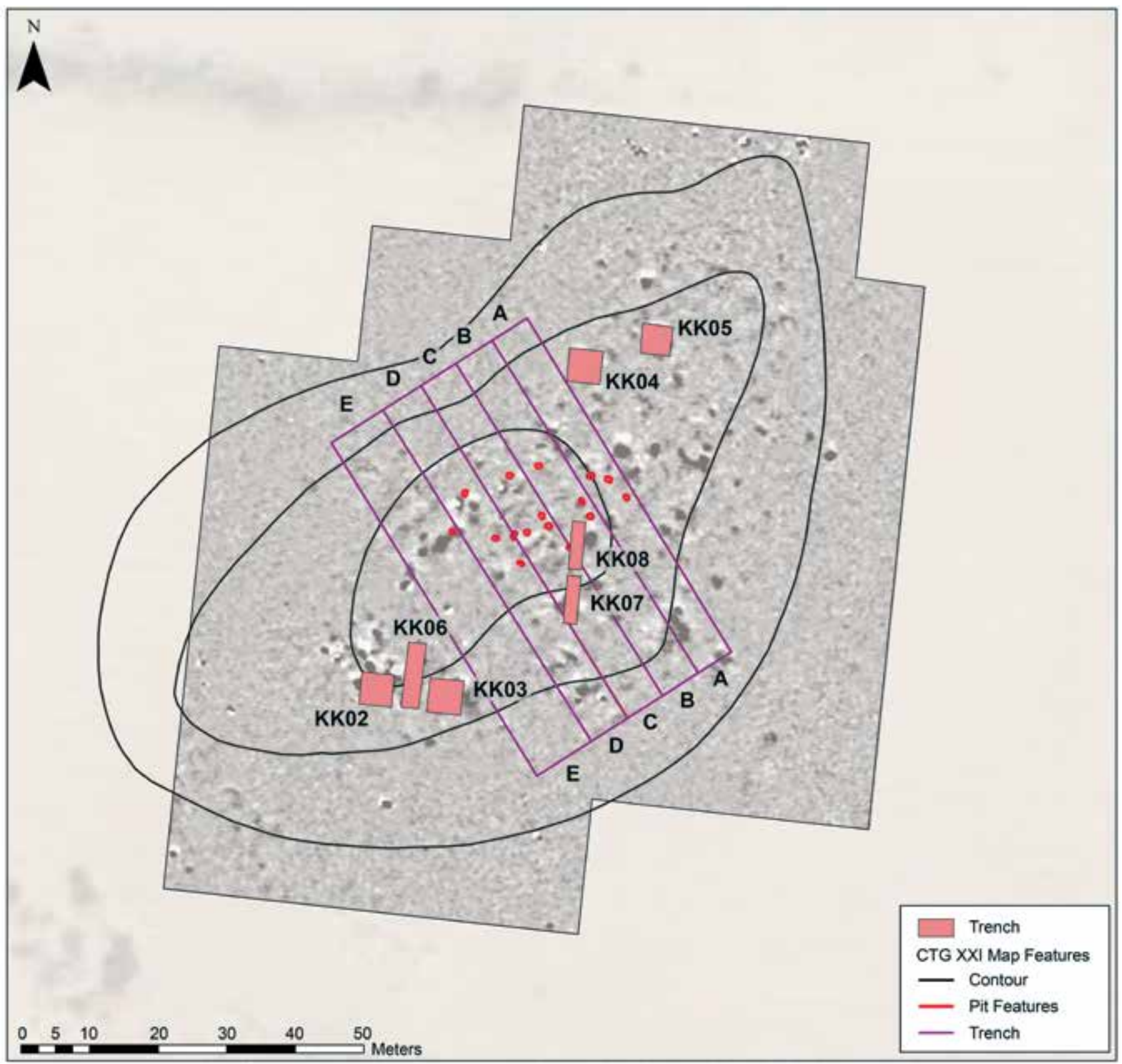

Figure 6.3. Excavation trenches overlaid onto a map of magnetic anomalies (dark areas) and the approximate location of Caton-Thompson and Gardner's excavations (E to A).

\section{KK02}

As noted, Trench KK02 was excavated over two seasons, with final dimensions of $5 \times 5 \mathrm{~m}$. The upper units of the trench, [02.0001], [02.0002], and [02.0003], were disturbed, with sediments likely mixed by plowing. Distinct plow lines were identified as cuts [02.0007] below the disturbed surface units. There were numbers of fragmented pieces of ceramic, bones, and flaked stone artifacts within the mixed sediments above the plow lines.
Below the plow line unit, excavation showed a number of ashy lenses (likely associated with hearth features, discussed below), as well as two small cuts [02.0021] (Hearth 101) truncated by the north baulk and [02.0022] (Hearth 102) truncated by the east baulk. These were in an area with massive ashy deposits, pointing at the presence of a number of hearths in the northeastern corner of the trench. A small hearth consisting of cut [02.0020] and sand fill [02.0019] (Hearth 103) overlay 


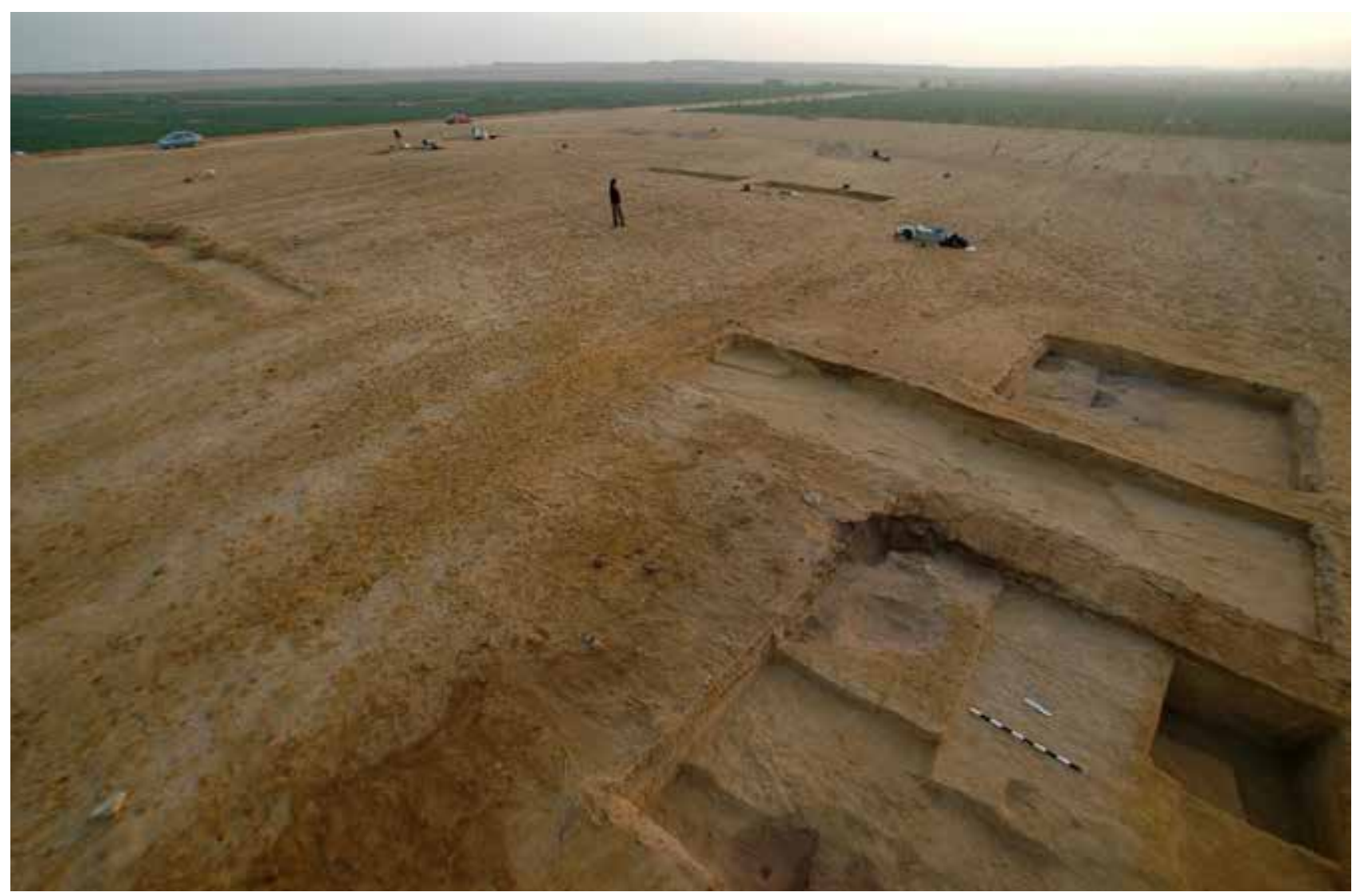

Figure 6.4. Overview of Kom K excavations with K ridge in the background.

Table 6.1. Characteristics of Wood Type Identification for Charcoal from Kom K Hearths.

\begin{tabular}{|l|l|}
\hline x-section & $\begin{array}{l}\text { Ring-porous (when ring visible), pores solitary, multi-seriate rays, characteristic radial fracture pattern } \\
\text { between single/double rows of pores, parenchyma occasionally visible as regularly spaced tangential bands. }\end{array}$ \\
\hline t-section & $6-10$ seriate rays, 20-30 cells high \\
\hline r-section & $\begin{array}{l}\text { Heterogeneous rays with one row upright marginal cells, no spiral thickenings seen, perforation plates } \\
\text { simple, vessel pits small and numerous. }\end{array}$ \\
\hline
\end{tabular}

a much larger hearth, consisting of cut [02.0027] and fill [02.0026] (Hearth 104), forming a red-oxidized layer of discolored sediment, underneath which was clean yellow sand. All four depressions included bone and ceramic fragments.

In the northwest quadrant of the trench, four additional depressions were identified: hearth cut [02.0055] with fill [02.0032] (Hearth 105); hearth cut [02.0057] with fill [02.0052] (hearth 106); hearth cut [02.0067] with fill [02.0062] (Hearth 108); and hearth cut [02.0069] with fill [02.0061] (Hearth 107) formed a series of superimposed set of hearths (Figure 6.5; Table 6.3). Hearth cut [02.0067] was the stratigraphically earliest and had a layer below the base of the hearth consisting of yellowish-red oxidized sand. A small $0.10 \times 0.10-\mathrm{m}$ hole was cut into the base of the feature [02.0064]. The fill of this hole [02.0065] was loose sand, in contrast to the compacted cut of the hearth, and contained plant material. Hearth cut [02.0067] (Hearth 108) was filled with fine yellowish-brown sand and contained charcoal as well as some bone [02.0062]. Charcoal samples from this fill (three samples totaling $2.8 \mathrm{~g}$ ) were identified as Tamarix. 


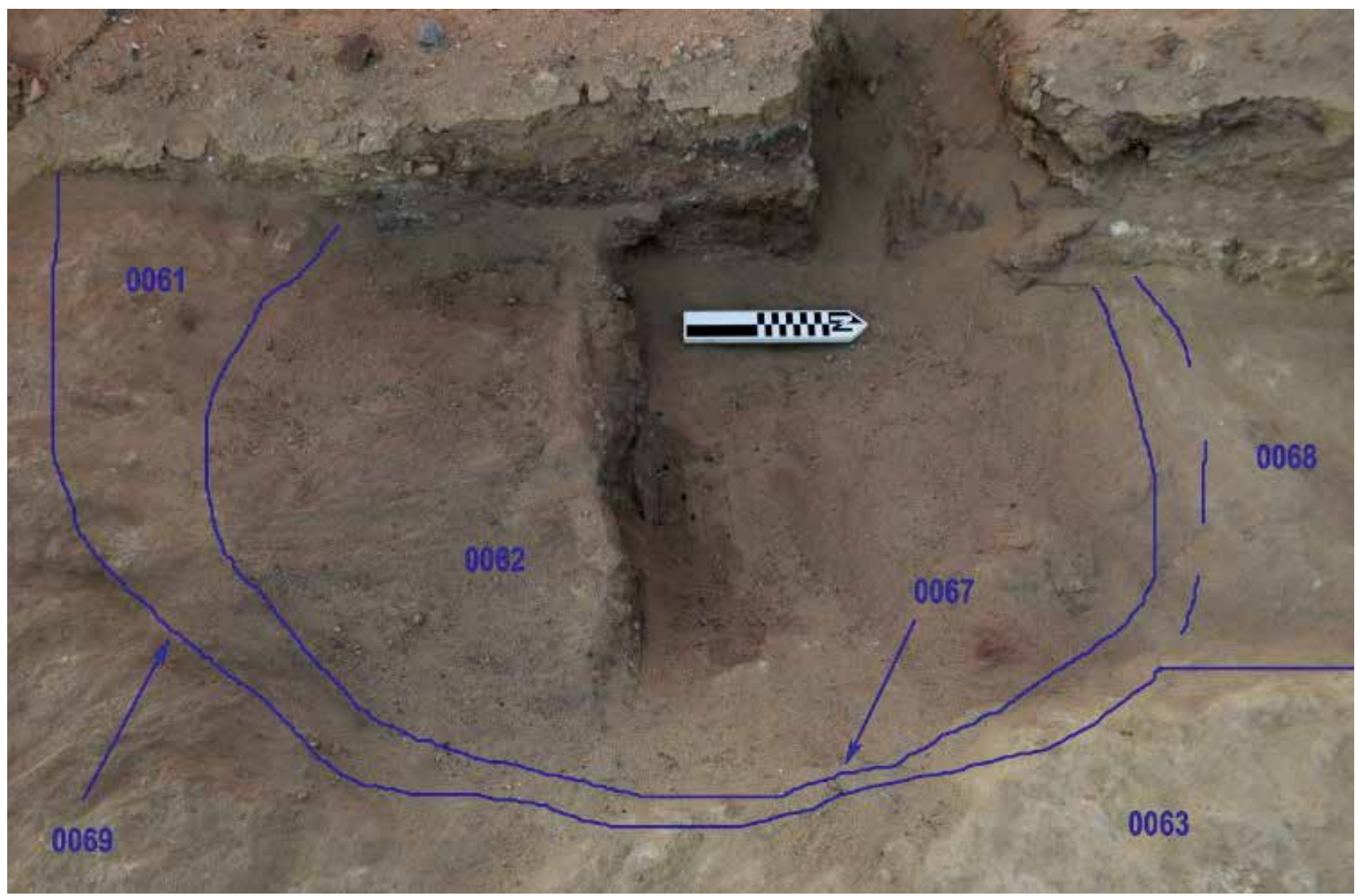

Figure 6.5. KK02 hearth set showing the extent of the excavation units. Hearth 107 was dug into Hearth 108, the earliest hearth in the northwest quadrant of the trench

The fill of hearth cut [02.0067] (Hearth 108) was capped by windblown, mottled, yellow-brown sand mixed with bone and ceramic. This hearth was reused, as indicated by a new cut and fill deposit, [02.0069] and [02.0061] (Hearth 107), stratified above cut [02.0067] with fill unit [02.0062] (Hearth 108). The later hearth was filled with dark reddish-brown, ashy sand and had a red oxidized layer beneath its base. A third hearth was subsequently formed in the same location with unit [02.0052] filling [02.0057] (Hearth 106), which cut into [02.0058], a mixed windblown sand deposit with a large amount of bone. A single charcoal sample from [02.0052] (0.2 g) was identified as Tamarix. The distinction between Hearth 106 with cut [02.0057] and fill [02.0052] and Hearth 107 with cut [02.0069] and fill [02.0061] was not as sharp as that between Hearth 107 and Heath 108, with cut [02.0067] and fill [02.0062]. In Hearth 106, cut [02.0057] was filled with [02.0052], a dark yellowish-brown sand and had the same red oxidized layer beneath the base as Hearth 107 (cut [02.0069] and fill [02.0061]). The fill had some charcoal and some small pieces of ceramic. A windblown deposit [02.0044] covered Hearth 106 and a fourth hearth depression consisting of cut [02.0055] and fill [02.0032] (Hearth 105) was cut into this deposit and the hearths below but was offset just to the north. Large numbers of objects were found both in the overlying unit [02.0044] and in the [02.0032] fill of cut [02.0055] (Hearth 105). These included ceramic sherds, many large pieces of bone, and charcoal fragments, together with patches of clay in [02.0044]. Two ashy deposits [02.0011] and [02.0012] may be associated with Hearth 105.

A radiocarbon sample was obtained from the fill [02.0062] of hearth cut [02.0067] (Hearth 108) (UCIAMS-45070). Table 6.2 lists the uncalibrated and calibrated radiocarbon ages in BP and BCE 
Table 6.2. Radiocarbon Determination for Charcoal from Kom K Hearths.

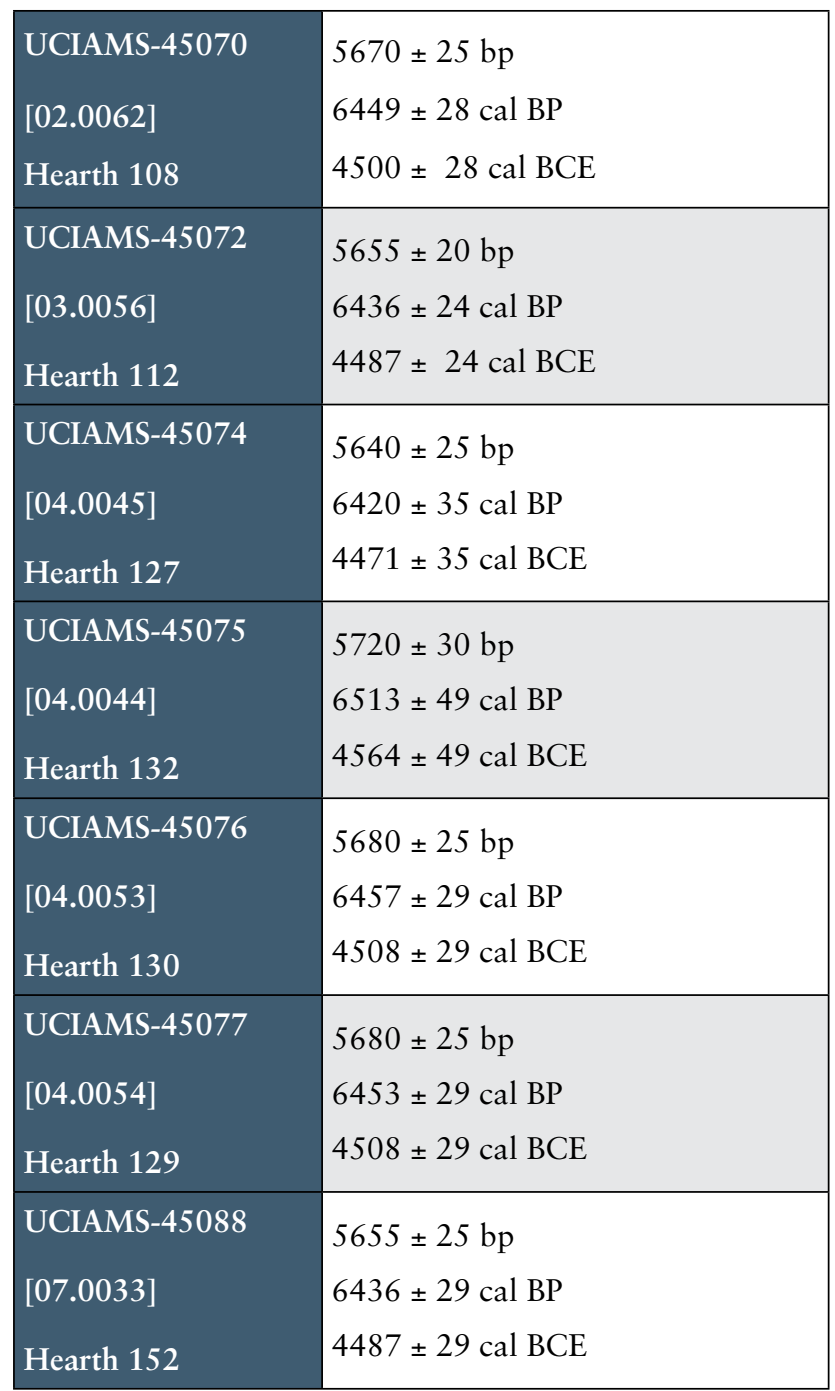

Note: CRA given as bp; calibrated results in $\mathrm{BP}$ and BCE using Oxcal 4.2.2 calibration (Bronk Ramsey 2009), plotted with atmospheric data from Reimer et al. (2013). Error margins are 68.2 percent.

Three other features were found in the northwest corner of KK02. One was a depression [02.0046] dug into unit [02.0044] and filled with [02.0045], which overlays Hearth 106 , and was identified by a change in compaction and color of the sand but did not otherwise contain material different from the surrounding matrix. It is possible that this unit represents another hearth, although this could not be determined during excavation. The second feature was a hearth, but it was shallow compared to the other features excavated in the unit, with a depth of only $0.03 \mathrm{~m}$. It was cut into the surface of unit [02.0044] and was recognized by a layer of red oxidized sand beneath the base of the hearth. Finally a small lens of ash-rich sand [02.0047] in depression [02.0048], which was also cut into unit [02.0044] was recognized only in the section. Charcoal obtained from [02.0044] sediment samples (five samples totaling less than $0.4 \mathrm{~g}$ ) was identified as Tamarix.

A probe, $2 \times 1.5 \mathrm{~m}$, was excavated in the southeast corner of the trench to a maximum depth of $0.76 \mathrm{~m}$ below the surface level in arbitrary $10-$ to $15-\mathrm{cm}$ units (Probe 93, consisting of units [02.0043], [02.0049], [02.0053], [02.0056], and [02.0060]). This exposed a number of windblown sand deposits with continuous, though sparse, cultural remains.

\section{KK03}

The modern agricultural activity described above damaged the upper parts of Trench KK03 in a similar manner to the disturbance recorded in KK02. Units [03.0009] and [03.0010] were formed by plowing, with the upper $0.1 \mathrm{~m}$ of the deposit likely disturbed. Below this, a surface with salt precipitates was identified [03.0006]. Modern plant growth continued past this level. Windblown sand, excavated as [03.0013], [03.0014], [03.0026], and [03.0027], was found below unit [03.0006], with a number of small evaporation cracks noted. Two of these, [03.0022] and [03.0024], had a circular profile suggesting the possibility of anthropogenic features. However, both were shallow (less than $0.05 \mathrm{~m}$ deep), and one was associated with modern plant roots. Charcoal was identified from units [03.0013], [03.0014], [03.0026], [03.0027], and [03.0028] as Tamarix. However, these samples may have been contaminated by modern root activity.

Below the windblown sediment, cultural material occurred in an ashy, gray-colored sand, [03.0028], [03.0029], and [03.0030], resting on a clay-dominated sediment that formed a hard surface, [03.0031], [03.0032], [03.0035], and [03.0040]. A set of hearth features was cut into this clay surface, with each of the hearths showing at least one secondary cut consistent with reuse. From the appearance of the surfaces, it seems possible that the clay layer into which the hearths were cut was anthropogenic in origin, but there are no independent means to assess this. No postholes were identified; nor were their impressions in the clay surface itself.

Four major hearth features were found in the northeast quadrant of the $5 \times 5-\mathrm{m}$ trench, and each of these consisted of a cluster of hearths, one dug into the other. Hearth Cluster A, the westernmost, consisted of three subsequent cuts with fills. The oldest one [03.0046] 
formed a sub-circular hearth with a diameter of $0.82 \mathrm{~m}$, with an ashy fill [03.0047] (Hearth 109). This was cut by a later hearth with a cut $0.91 \mathrm{~m}$ in diameter [03.0048] with ashy fill [03.0049] (Hearth 110), which also cut through the clay layer [03.0035] into which [03.0046] was cut. The topmost hearth was smaller, $0.61 \mathrm{~m}$ in diameter [03.0050] (Hearth 111), and cut into the two previous ones. It had a layer of windblown sand, which may indicate a period of abandonment or an attempt to clean out the hearth before reuse without recutting. Below this hearth was a layer of red oxidized sand [03.0052] and an ashy layer [03.0053] on top of the clean sand. A radiocarbon sample (UCIAMS-45072) was obtained from the ashy fill [03.0047] (Table 6.2).

The middle part of the cluster of three hearths (B) consisted of a 0.56-m cut [03.0055] with ashy fill [03.0056] (Hearth 112). This was cut through by a later hearth, formed by cut [03.0057], which was $0.50 \mathrm{~m}$ in diameter and had two fills: a red oxidized sand below the hearth [03.0058] and an ashy layer above [03.0059] (Hearth 113). Table 6.2 gives the age of a radiocarbon sample (UCIAMS-45073) obtained from ash deposit [03.0056].

The third cluster of hearth features $(\mathrm{C})$ was the easternmost cut into clay surface [03.0031], [03.0032], and [03.0040]. The hearth also shows evidence of reuse, with a new hearth cut [03.0063] (diameter approximately $0.50 \mathrm{~m}$; Hearth 115$)$ into a slightly smaller $(0.46 \mathrm{~m})$ older depression [03.0060], with oxidized fill below the hearth feature [03.0063] and an ash layer above [03.0064] (Hearth 115). The hearth, represented by cut [03.0060], contained an accumulation of windblown sand [03.0061] and ash [03.0062]. It was likely recut and reused multiple times, with cut [03.0080] and fill [03.0081] (Hearth 122) and cut [03.0082] and fill [03.0085] (Hearth 121) identified in the east baulk of the trench.

A fourth hearth cluster (D) was identifiable in the north trench wall and shows evidence for five distinct smaller hearths, all contained within the boundary of the earliest and deepest hearth cut. The original cut [03.0044] had an oxidized sand deposit below the base of the hearth [03.0066] overlaid by an ash deposit [06.0037] (Hearth 116). A subsequent cut was made to form a hearth in the western half of the depression [03.0067], with similar oxidized sand at its base [03.0068], below an ash layer [03.0069] (Hearth 117). In the eastern side of the original cut, a second, subsequent cut [03.0070], with the same reddened sand and ash fill below the hearth feature [03.0071] and [03.0072] was formed (Hearth 118). A small animal burrow or plant root hole [03.0038] was associated with this feature. Hearth cut [03.0070] contained two small hearths: cut [03.0073] on the western side and cut [03.0076] in the eastern part (Hearth 119). Cut [03.0073] was associated with a clay deposit at the base of the hearth [03.0074]. This clay lining contained oxidized sand [03.0075] and an ash deposit [03.0076]. Hearth cut [03.0077] (Hearth 120) did not have a clay layer fill, but as with most of the hearths, it was associated with an oxidized sand deposit [03.0078] and an ash deposit [03.0079]. A patch of unfired clay, $0.05 \mathrm{~m}$ thick, was found on the edge of the hearth features [03.0045]. It was different in composition from the clay layer in which the hearths were cut [03.0040] and was partially overlying it.

\section{KK04}

Excavation of KK04 revealed an initial shallow modified deposit, with plow cut marks [04.0002] evident below the surface layer and evidence of evaporation deposits [04.0003], [04.0014], and [04.0015]. Parts of [04.0015] were so hard that they had to be removed with an angle grinder, reminiscent of the deposits identified by CatonThompson and Gardner (1934:38).

Windblown sand deposits [04.0010], [04.0013], and [04.0034] occurred beneath the evaporation layer, cut into by a recent pit [04.0029] associated with modern fertilizer. Evidence of disturbance was also present in the windblown sand deposits [04.0041] and [04.0042] related to hearth features immediately below [04.0005], [04.0007], [04.0008], [04.0011], [04.0028], and [04.0033].

Cuts into the windblown sand deposits indicated three sets of hearth features with indications of multiple periods of use. In the northwest corner of the trench, the earliest hearth cut [04.0024] (Hearth 127) had dimensions of $1.18 \times 1.06 \mathrm{~m}$ and was cut into sand layer [04.0012]. There was a small depression at the base of this cut. Oxidized sand [04.0025] was present at the base of the cut, above which was an ash layer, [04.0032] and [04.0045]. Two subsequent cuts were made into the original cut [04.0022] and [04.0021] (Hearth 125), although the stratigraphically higher cut was truncated by recent agricultural disturbance. Sediment samples obtained from [04.0012] provided a total of 2.8 $\mathrm{g}$ of charcoal identifiable as Tamarix. A radiocarbon sample, UCIAMS-45074, was obtained from the fill [04.0045] (Hearth 127) (Table 6.2).

The hearth feature made up of cut [04.0052] and fill [04.0044] was represented by a single deposit, with no 
evidence for later reuse (Hearth 132). The cut formed a depression with dimensions of $1.26 \times 1.06 \mathrm{~m}$ and $0.18 \mathrm{~m}$ deep. Table 6.2 shows radiocarbon sample UCIAMS-45075, obtained from fill [04.0044]. Two charcoal samples (totaling less than $0.2 \mathrm{~g}$ ) were identified from ash layer [04.0044] as Tamarix.

The third set of features contained hearths with heat retainers (Figures 6.6 and 6.7). The initial cut [04.0057] was sub-circular in shape and contained several distinguishable ashy fill deposits (Hearth 130). Two cylindrical pit features were also cut into the base of [04.0057]. Both of the pit features and the hearth cut were filled with the same deposit [04.0053]. Subsequent use led to the deposition of unit [04.0054] and to the formation of a circular pit [04.0055] cut into the lower deposits and filled with an organic rich ash [04.0051] (Hearth 129). Two small, sterile windblown sand lenses, [04.0060] and [04.0048], were found on top of this ash deposit.

A soft ash deposit including charcoal, shell, bone, and botanical inclusions [04.0038] was stratified above the windblown sand lenses and indicated a further episode of use, followed by a period of abandonment that left a sterile windblown deposit between some areas of the hearth fill and the stone lining [04.0037] (Figure 6.7). Charcoal fragments were present beneath the hearthstones [04.0039]. The hearth (Hearth 130) was covered by poorly sorted, sub-rounded, fine and coarse sand grains and silt [05.0036] and numerous pieces of charcoal, ceramic, bone, shell, and some calcium carbonate (from evaporation).

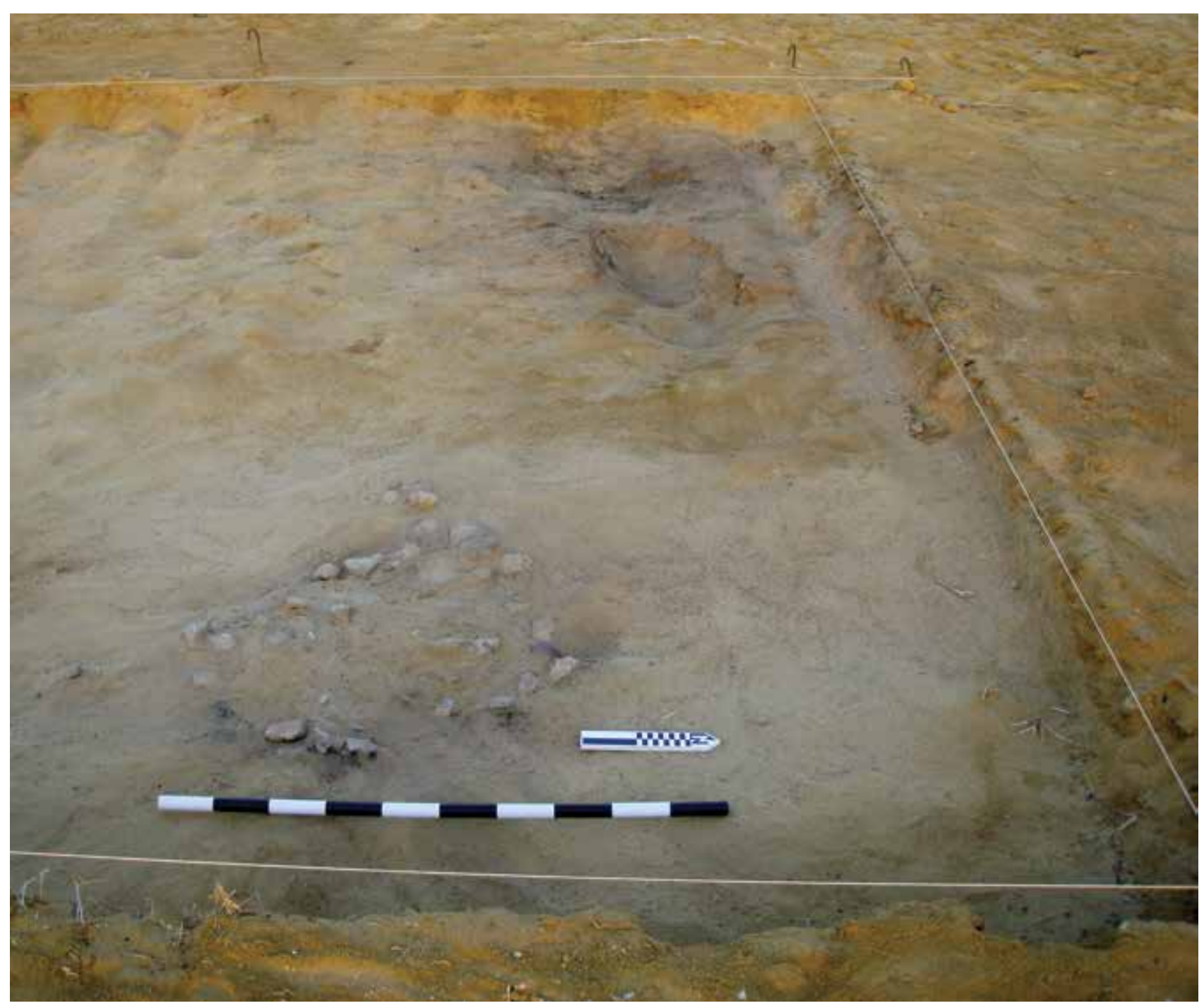

Figure 6.6. Northern part of Trench KK04 with the heat retainers of Hearth 130 in the foreground. 


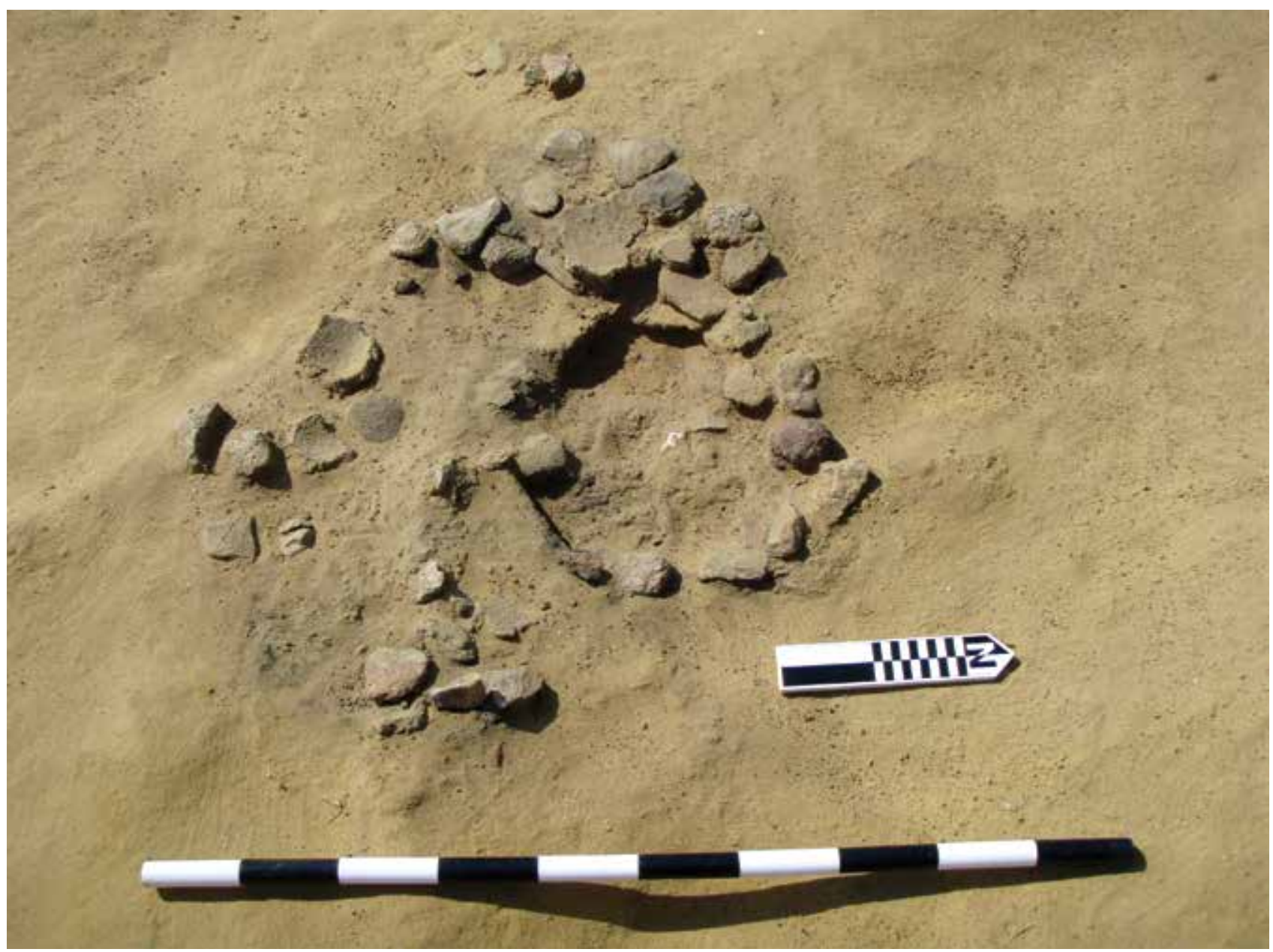

Figure 6.7. KK04 Hearth 130, a large hearth with stone and ceramic heat retainers.

Another cut [04.0019] may represent an additional hearth (Hearth 124). However, it lacked oxidized sediment below the base, indicating that heat was not sufficiently localized to discolor the sand. It was filled with sand and charcoal remains, [04.0006] and [04.0020], and was truncated by recent agricultural activity.

Two radiocarbon samples were obtained from the sequence of hearths. The first, UCIAMS-45076, was obtained from the [04.0053] hearth fill (Hearth 130; $6457 \pm 29 \mathrm{cal} \mathrm{BP}, 4508 \pm 29 \mathrm{cal} \mathrm{BCE})$. The second, UCIAMS-45077, was obtained from the fill [04.0054] of a hearth (Hearth 129) cut into the [04.0053] deposit $(6453 \pm 29$ cal BP, $4504 \pm 29$ cal BCE) (Table 6.2). As discussed further below, both ages are indistinguishable.

Two additional pit features were present. One may be a hearth and is recorded as such in Table 6.3. Unit [04.0017], measuring $0.58 \times 0.22 \mathrm{~m}$, with a depth of $0.11 \mathrm{~m}$, was cut into [04.0006] and [04.0020] and was filled with a silty sand containing botanical and charcoal remains [04.0018] (Hearth 123). The second, [04.0046], was $0.60 \mathrm{~m}$ in diameter and $0.07 \mathrm{~m}$ deep, cut into an accumulated sand deposit [04.0061] and filled with sterile windblown sand [04.0041]. The function of both these pits is unknown.

The windblown sand deposits into which the hearths were cut continued below the hearth features themselves. They consisted of fairly well-sorted, medium to coarse sand and silt.

A small area of $2 \times 1.5 \mathrm{~m}$ was excavated in the southwest corner of KK04 and showed the presence of additional occupation deposits beneath [04.0012], the mottled sand layer that covered most of the trench. Unit [04.0066] was an earlier windblown sand layer, beneath [04.0012], and a bone-rich layer with very coarse, subrounded, poorly sorted sand and silt. A circular pit feature [04.0064] was cut into [04.0066] and was filled 
with [04.0065], consisting of ash, sandy silt, and some cultural material: ceramic, bone, and charcoal (Hearth 131). Unit [04.0070] was a deep, irregular cut identified in windblown deposits [04.0071] and [04.0072]. It was filled by [04.0069], a medium and coarse, poorly sorted sand and silt and may reflect rodent activity since the cut was sub-circular on the surface but irregular throughout. Charcoal was obtained from [04.0069] and [04.0071] but may be contaminated by the rodent activity and so was not submitted for dating.

\section{KK05}

Plow cuts were found beneath the surface sediments in KK05 and likely reflect disturbance of the upper levels of the trench. Evaporation deposits associated with [05.0009] and cracks in the sediments, cuts [05.0011] and [05.0012], were also identified, together with significant root masses, likely from modern cultivation.

Removal of the disturbed sediments exposed [05.0013] in the southern half of the trench, a clean yellow sand into which were cut a number of depressions associated with hearths. These cuts were associated with oxidized, red-colored sands below the base of the cut depressions. As is the case in the trenches discussed above, the hearths showed evidence for multiple episodes of use. Charcoal was identified in samples from [05.0013] but may be contaminated. In general, the hearths in this trench were less defined, occurring within large areas of ashy, mottled sands.

Cut [05.0022] represented the lowest hearth depression (Hearth 133) and was filled with [05.0016] and [05.0017], a dark grayish-brown, mottled ash deposit with soft, poorly sorted, fine silty sand. This deposit was cut by [05.0027] and contained [05.0028], a pale brown mottled, yellowish-brown, soft, poorly sorted, medium silty sand that included flaked stone artifacts, ceramic, and bone. However, the deposit lacked evidence for oxidized sand below the hearth feature or charcoal in the hearth fill (Hearth 135). Unit [05.0028] was capped by a second ash deposit [05.0018], which also included ceramic, bone, and flaked stone artifacts together with shell.

Three hearths were found in the northwestern quadrant of the trench. Cut [05.0036] with fill [05.0037] (Hearth 136) is the northernmost and is adjacent to ashy fill [05.0043], for which no cut could be identified. Underneath these were a large ashy area and a truncated hearth [05.0053]. A small circular ash deposit was identified [05.0014]. It was not associated with a cut and had a depth of $0.04 \mathrm{~m}$.
Beneath the hearth depressions in the south of the trench, a windblown deposit [05.0032], consisting of brownish-yellow, compact, poorly sorted, fine siltysand, extended across the entire trench area. The unit in turn rested above a darker, ash-rich deposit [05.0033], indicating the presence of a hearth cut [05.0036], which was filled with an ash deposit consisting of dark grayish-brown, soft, poorly sorted, fine silty sand [05.0035] (Hearth 136). Units [05.0032] and [05.0033] provided small quantities (less than $0.2 \mathrm{~g}$ ) of charcoal identified as Tamarix.

This hearth was cut into windblown sand, [05.0045] and [05.0044], an ash deposit composed of dark gray, compact, poorly sorted, silty sand with inclusions of ceramic, bone, shell, flaked stone artifacts, beads, and ostrich eggshell (Hearth 139). The sediment beneath this [05.0060] consisted of clean yellow sand with inclusions of ceramic, shell, bone, flaked stone artifacts, and ostrich eggshell.

Cut [05.0066], a small, shallow depression, was cut into [05.0060]. It was tentatively identified as a posthole. It was filled by [05.0063], an ashy, poorly sorted, fine silty sand with a more mottled silty sand [05.0062] above, which may have derived from hearth activity. A windblown deposit [05.0056], composed of yellowish-brown, firm, poorly sorted, fine silty sand with inclusions of ceramic, bone, shell, and flaked stone artifacts, was distinguished from [05.0045], in which [05.0046] was cut, forming a circular depression with a rounded bottom. It was filled with a light gray ashy deposit with lumps of light yellow clay. A secondary hollow [05.0058] was excavated in the clay fill of the first (Figure 6.8). It was filled with a light, olive brown, compact, poorly sorted, fine silty sand [05.0059] with ceramic, shell, bone, and flaked stone artifact inclusions. A similar depression was cut into this fill [05.0057]. It had similar dark gray fill with light yellow clay lumps [05.0047]. This fill contained a large number of bone fragments. A small quantity of charcoal $(0.1 \mathrm{~g})$ was identified as Tamarix from [05.0048]. Finally, [05.0055] formed a depression that cut through the clay lining. These hollows were approximately 0.50 $\mathrm{m}$ in diameter and were 0.20 to $0.30 \mathrm{~m}$ deep. There is no clear evidence for their function.

A separate depression, [05.0053], was cut into the [05.0045] sediments in the southern area of the trench associated with a second, smaller cut [05.0050].

No charcoal samples were submitted for radiocarbon dating from the fill or these features. 


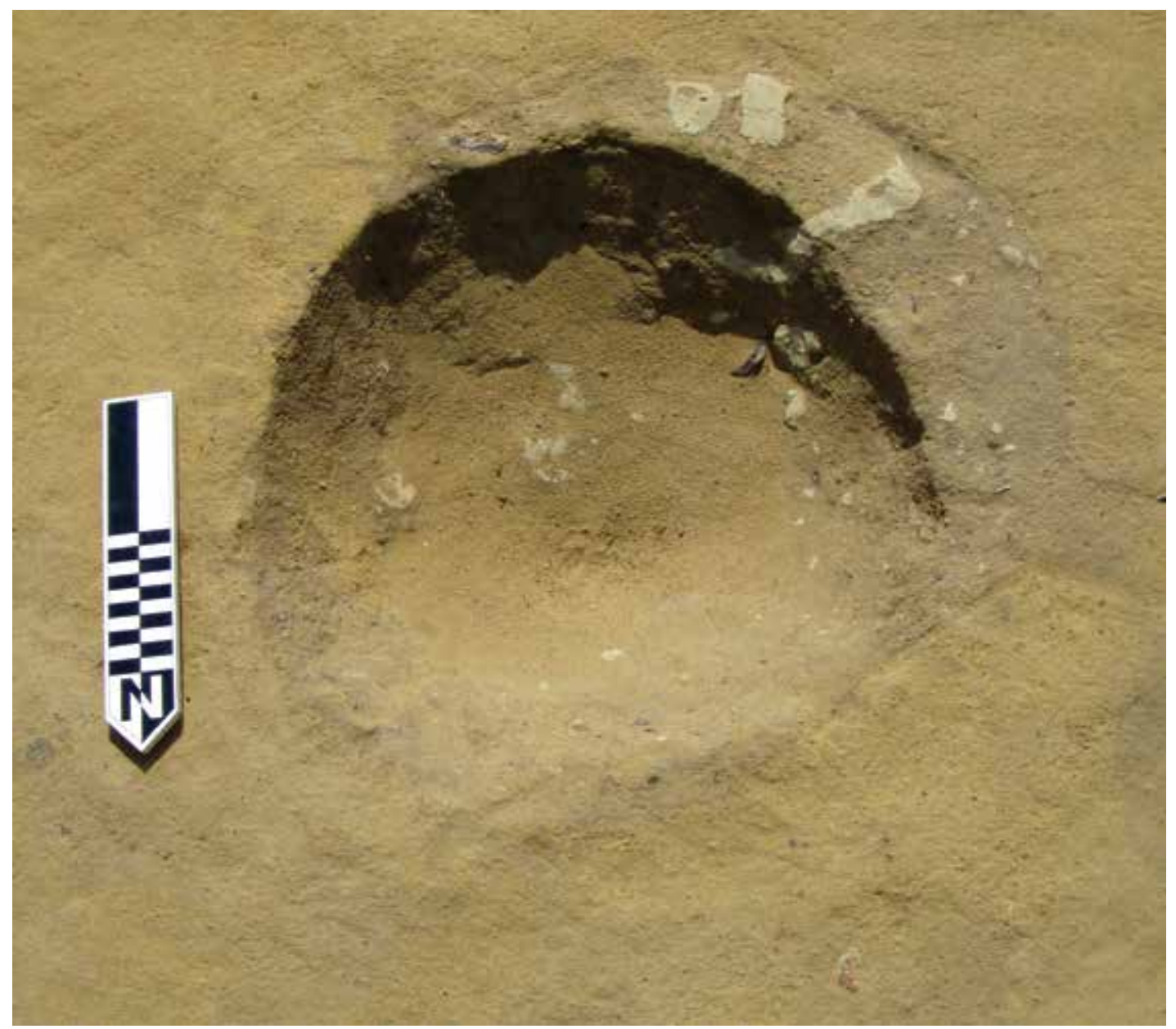

Figure 6.8. KK05, one of two circular pits with rounded bottoms; cut [05.0058] in fill [05.0048] of earlier cut [05.0046].

\section{KK06}

Excavation of the trench revealed the expected plow damage to the surface levels and a modern ditch running in a north-south direction along the eastern side of the trench, around which excavation continued (Figure 6.9). A layer compacted by evaporates, most likely salt [06.0009] and associated sediment cracks, [06.0012] and [06.0013], was identified. It is possible that hearth depressions once existed in the disturbed sediments, but these could not be defined as cuts.

Beneath the compacted layer [06.0009], three units, [06.0014], [06.0015], and [06.0016], were composed of windblown sand mixed with quantities of ash and bone. A possible pit feature [09.0017] of approximately $0.3 \times 0.36 \mathrm{~m}$ was cut into [06.0014], but it could not be fully defined because of the presence of the desiccation cracks, [06.0012] and [06.0013], described above.
On the west side of the trench, two depressions were found to have clay linings: [06.0021] with lining [06.0020] and [06.0023] with lining [06.0022]. The clay used for the linings showed finger scrapes, indicating that they were human-made. A hearth cut [06.0019] and fill [06.0018] (Hearth 140) were found at the eastern edge of the trench. Multiple episodes of reuse of this hearth were identified by a stratified series of cuts, which could be recognized only in the section due to the softness of the ashy deposits (Hearths 141 and 143). Sediment samples from these units produced charcoal totaling $0.9 \mathrm{~g}$, identified as Tamarix.

Beneath the pit features, sandy layers [06.0029], [06.0030], and [06.0031] contained quantities of animal bone but no additional features. Excavation proceeded on either side of the modern trench [06.0005], removing units composed of equivalent sediments until a large clay deposit, [06.0040] and 
[06.0044], was revealed. A single hearth depression, cut [06.0039] with fill [06.0038] (Hearth 142), was cut into this clay layer. Two ash deposits, [06.0032] and [06.0034], were likely associated with the hearth cut. Charcoal samples from these units with a combined weight of $4.3 \mathrm{~g}$ were identified as Tamarix. No charcoal samples were submitted for radiocarbon dating from the fill or these features.

\section{KK07}

Plow lines were identified $0.15 \mathrm{~m}$ beneath the surface in this trench, but, as was indicated in the other trenches, there was disturbance by modern agricultural activity below these features, to a depth of approximately $0.3 \mathrm{~m}$ in the north of the trench and $0.49 \mathrm{~m}$ in the south. Beneath the disturbed sediments, [07.0005] consisted of a thick layer of soft, relatively fine windblown sand, with ceramic, charcoal, faunal bone, fish bone, and shell, and flaked stone artifact inclusions. A large amount of botanical material was also present in the form of plant roots related to modern agricultural activity. This unit extended across the northern two-thirds of the trench $(7 \times 2 \mathrm{~m})$ and was approximately $0.57 \mathrm{~m}$ thick. The only visible occupation activity underneath the plowed sediment within [07.0005] were units [07.0006], [07.0010], [07.0011], and [07.0012].

In the north of the trench, an unfired clay vessel [07.0010] was found. It measured $0.36 \times 0.35 \mathrm{~m}$ and was $0.04 \mathrm{~m}$ thick. The vessel was placed in a cut [07.0012], with both units separated by a thin fill of sand [07.0011] (Figure 6.10). The fill of the unfired clay vessel [07.0006] consisted of medium sand, pinkish in color, with inclusions of charcoal, flaked stone artifacts, and botanical remains, in addition to large pieces of animal bone and ceramic fragments. A natural windblown sand fill layer under the clay vessel [07.0011] confirmed that it represented a vessel rather than a clay lining, as the deposit was formed around the pot and within the cut. Some of the cracks at the bottom of the pot were not filled with sand and therefore occurred after the deposition of [07.0011]. Unit [07.0011] was not found under the clay vessel, so the windblown sand was deposited after the pot was placed in cut [07.0012].

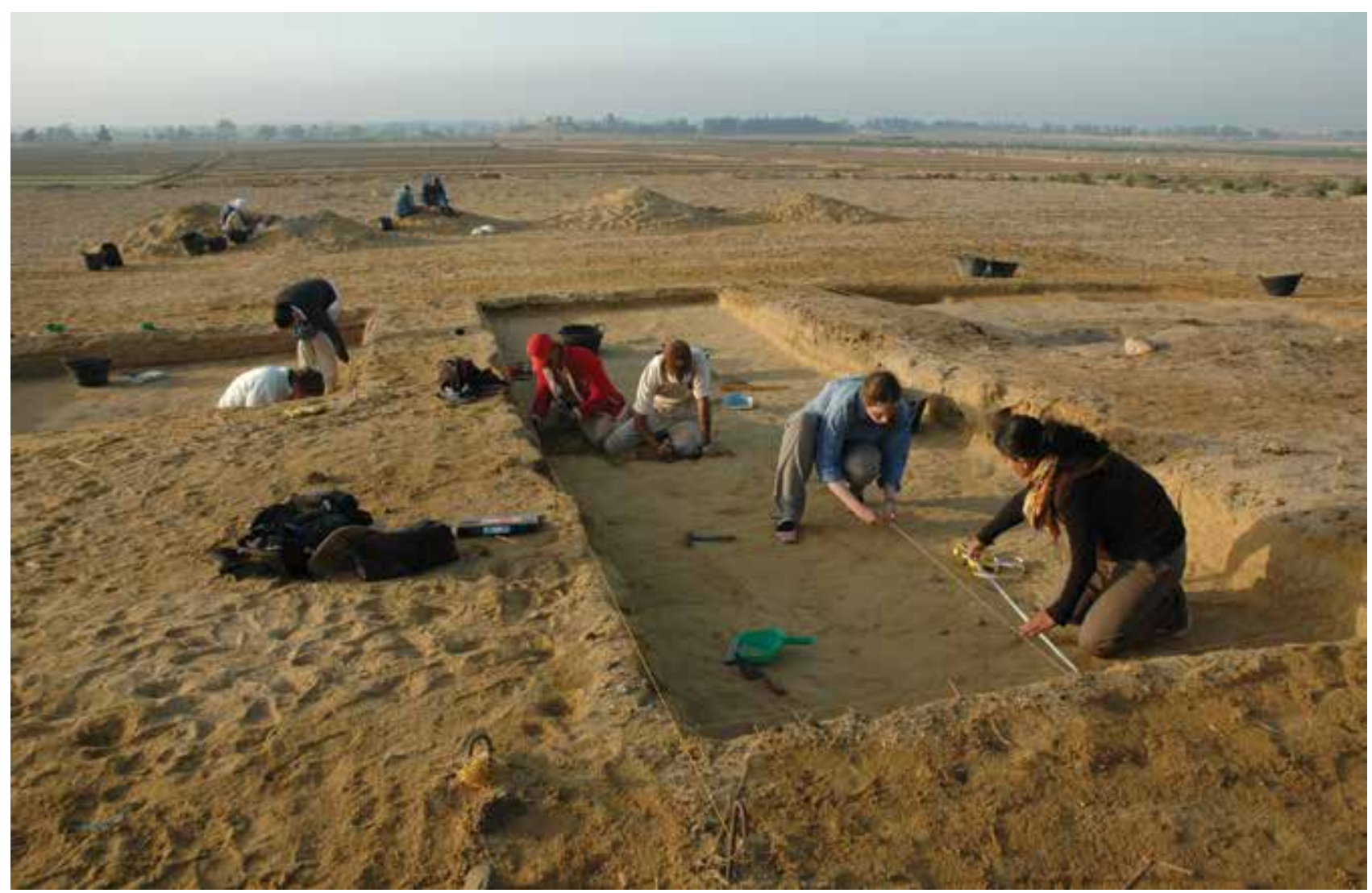

Figure 6.9. Overview of KK06, looking south. 


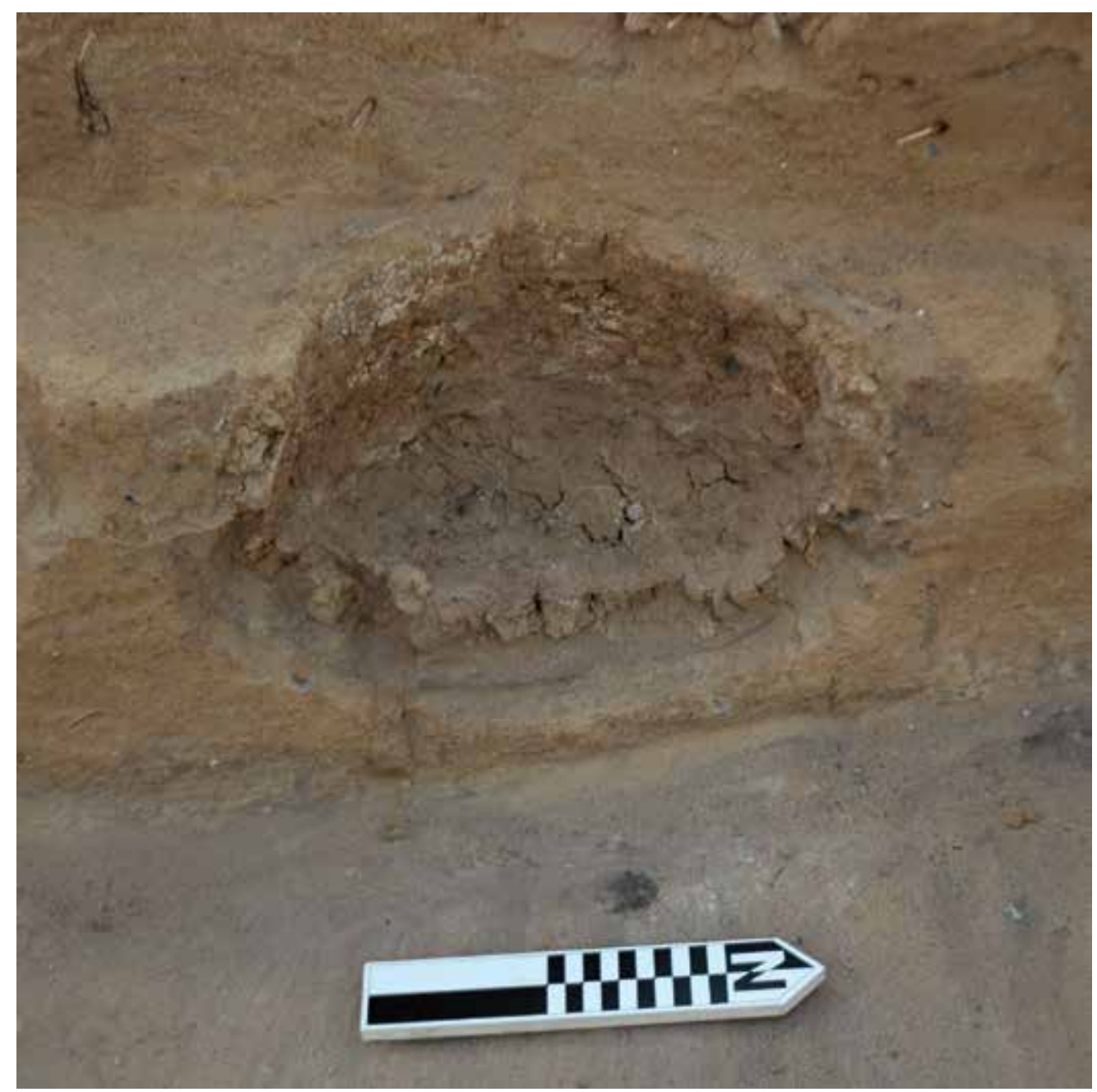

Figure 6.10. KK07 half section of excavated unfired clay vessel.

Underneath [07.0005], a number of sediment layers and features related to hearth activity (Figures 6.11 and 6.12) were found. The hearths were small and shallow and were cut into windblown sand deposits. Hearth use events were divided with lenses of windblown sand, some mixed with ash and other material from the hearth features. Sediment from such units often radiated some distance from hearth features and in some cases overlapped with other hearth features, making the boundaries between features quite diffuse. These units also contained a large amount of faunal material.

Unit [07.0007] indicated a change from the overlying windblown sand [07.0005] and consisted of dark ashy sand with inclusions of unworked stones (natural), botanical remains, ceramic fragments, bone, flaked stone artifacts, coarse sand, charcoal, and ash. It likely reflected ash scatter from a hearth, and it extended 4.9 $\mathrm{x} 2 \mathrm{~m}$ in the central and southern portion of the trench, with a thickness of $0.1 \mathrm{~m}$. Unit [07.0008] was found underlying this ash scatter and was definable as a hearth cut [07.0027] (Hearth 144), which extended under the western baulk of the trench. Fill deposits [07.0018] and [07.0024] consisted of oxidized sandy silt with ash and charcoal with flaked stone artifacts, ceramic fragments, and botanical remains as inclusions. The deposit itself was relatively thin $(0.6 \mathrm{~m})$ but represented only the most recent example of substantial hearth activity in this portion of the trench. The boundary of the unit was very diffuse and blended into the surrounding matrix. Directly underlying hearth cut [07.0008] were two units, [07.0014] and [07.0015], both associated with the hearth feature. They consisted of compact, oxidized sand, suggesting that the hearth was formed on a windblown sand surface. Unit [07.0014] had dimensions 


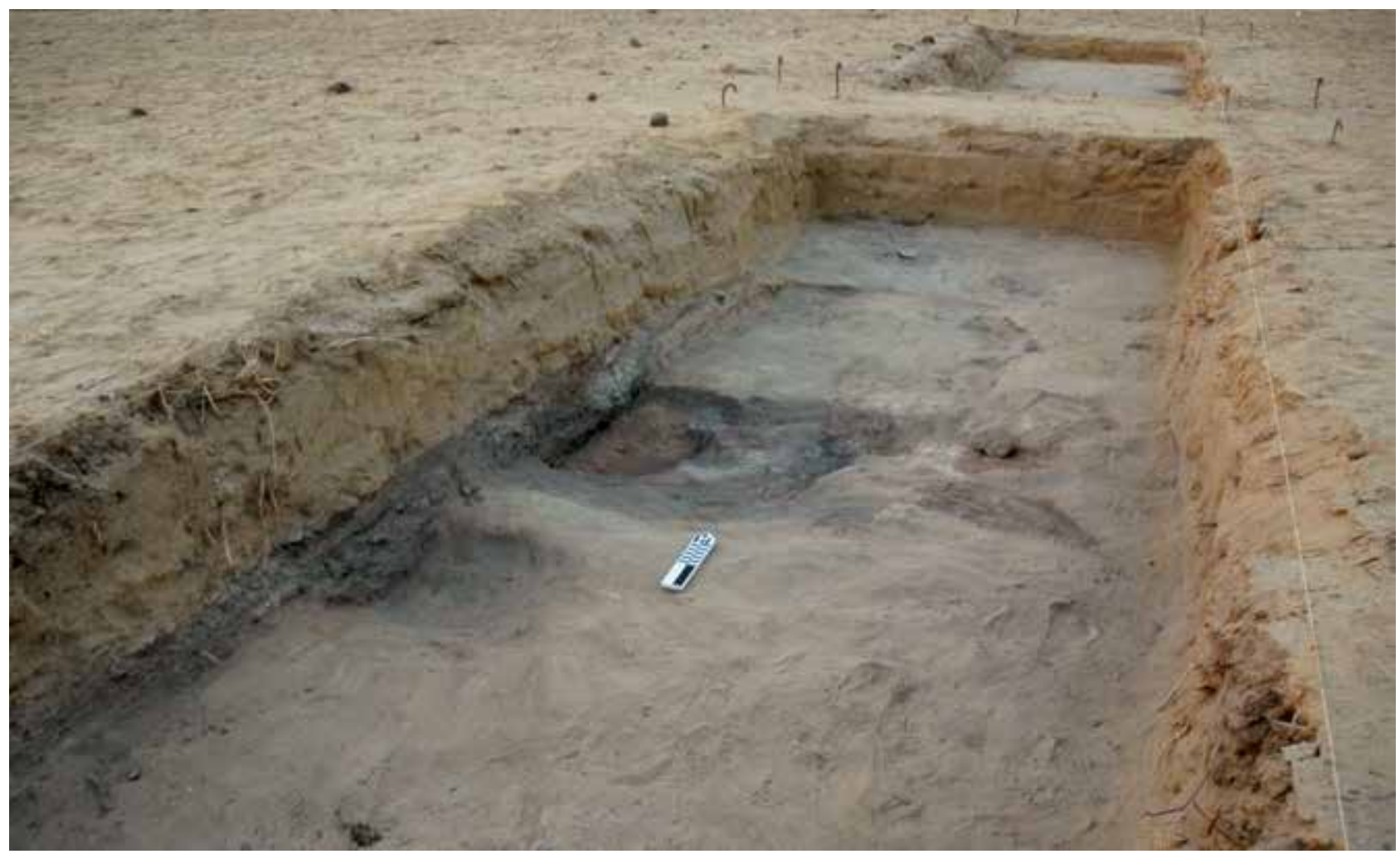

Figure 6.11. KK07 (front) and KK08 (back), looking north; hearth activity to the west side of the trench underneath sand layer [07.0005].

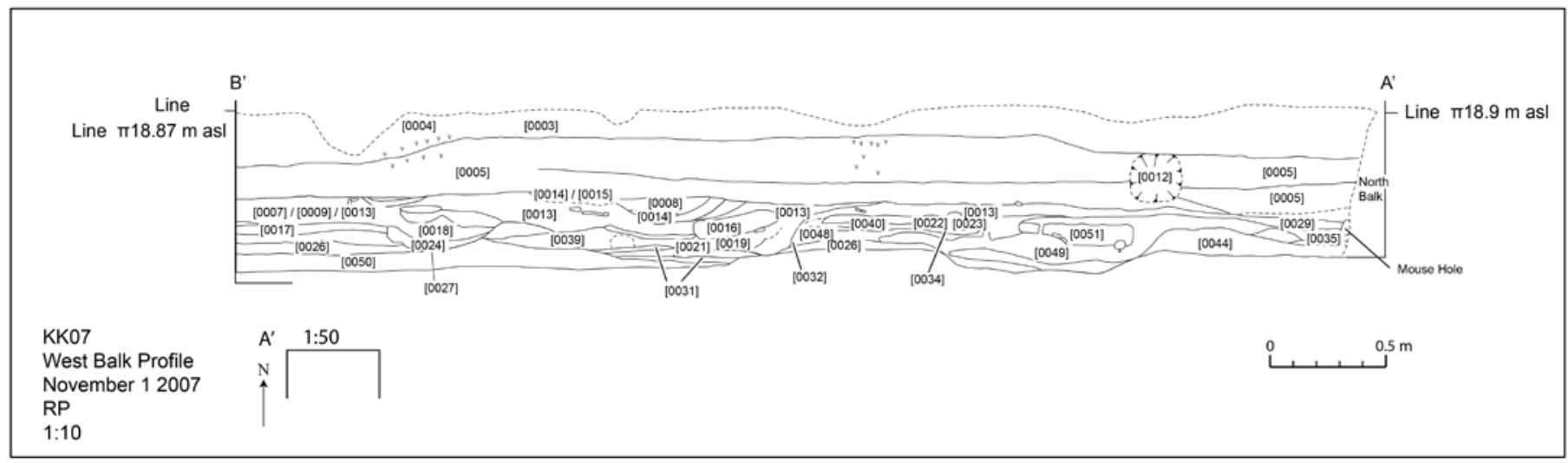

Figure 6.12. KK07 western profile of excavation trench.

of $0.77 \times 0.58 \mathrm{~m}$ and was $0.02 \mathrm{~m}$ thick, while unit [07.0015] was $0.38 \times 0.37 \mathrm{~m}$ and $0.09 \mathrm{~m}$ thick. Unit [07.0015] contained inclusions of coarse sand, charcoal, botanicals, and ceramic fragments.

A deposit of loose, ashy, windblown sand [07.0009] consisted of the same material as [07.0007], the deposit overlaying the hearths, while [07.0009] was the deposit in which the hearths were cut. Another ashy, sand layer [07.0013] was found underlying [07.0009] but was of the same composition and was spatially concentrated in the center of the trench, the major area of hearth activity. A sample from this unit produced $0.2 \mathrm{~g}$ of charcoal identified as Tamarix. Unit [07.0013] extended under hearth cut [07.0008] and was concentrated around a 
large hearth feature (discussed below). The unit consisted of ashy sand, similar to [07.0007], [07.0009], and [07.0017], with inclusions of charcoal, bone, and ceramic. Unit [07.0013] included pieces of compacted, silty clay.

A deposit of loose, ashy, windblown sand [07.0017] underlay the southern and eastern portions of [07.0013], with a similar composition to [07.0007], [07.0009], and [07.0013]. It contained inclusions of ceramic, bone, flaked stone artifacts, shell, botanicals, and coarse sand.

An additional ash scatter [07.0025] was associated with [07.0017]. It appeared in the southern portion of the trench and may represent a filled depression in the underlying compacted windblown sand deposit. It included charcoal, ceramic, bone, and flaked stone artifacts.

A second set of hearths, including ashy deposits [07.0018], occurred in the south-central area of the trench underlying [07.0013] (Hearth 145). This hearth set was truncated by the western baulk of the trench and lay immediately to the south of the third hearth feature set (discussed below). Unit [07.0018] was $0.6 \mathrm{x}$ $0.47 \mathrm{~m}$ and $0.04 \mathrm{~m}$ thick. The matrix contained inclusions of bone, flaked stone artifacts, and ceramic. The removal of this unit exposed an underlying deposit [07.0024] of oxidized sand, likely formed beneath the hearth, with dimensions $0.53 \times 0.44 \mathrm{~m}$ and 0.04 $\mathrm{m}$ thick. It had inclusions of charcoal and botanical remains. Unit [07.0027] was the cut into underlying windblown sand deposit [07.0026], in which the ash deposits [07.0018] and [07.0024] were situated. Unit [07.0026] produced a charcoal sample of $0.2 \mathrm{~g}$ identifiable as Tamarix.

The third hearth set, with ash deposit [07.0019] underlying [07.0013], was also truncated by the western baulk of the trench (Hearth 146). It was $0.75 \mathrm{x}$ $0.28 \mathrm{~m}$ and $0.43 \mathrm{~m}$ thick and formed part of the main hearth feature in the center of the trench, north of Hearth 145. The cut for this hearth deposit [07.0032] was $1.11 \times 0.97 \mathrm{~m}$ and approximately $0.19 \mathrm{~m}$ deep. The cut was filled by [07.0019], [07.0021], [07.0028], and [07.0031].

Unit [07.0019] contained inclusions of bone, charcoal, and coarse sand and was deeper, at $0.43 \mathrm{~m}$, than other hearth deposits. Unit [07.0020] was an area of compact ashy silt, similar to [07.0016]. It measured $0.57 \times 0.3 \mathrm{~m}$ and was $0.41 \mathrm{~m}$ thick. Unit [07.0021] was an area of firm, ashy, silty sand with inclusions of charcoal, flaked stone artifacts, bone, and ceramic. The unit was closely associated with Hearths 144 and 146. Unit [07.0028] was defined as a deposit of windblown sand mixed with ash and oxidized sand, situated at the base of cut [07.0032], and was $0.79 \times 0.49 \mathrm{~m}$ and 0.09 $\mathrm{m}$ thick. Inclusions consisted of coarse sand, flaked stone artifacts, and bone, but ceramics were absent. Unit [07.0031] was a deposit of oxidized windblown sand below the central hearth feature (Hearth 146). The unit directly underlay hearth units [07.0019] and [07.0021] (Hearth 146), and the hearth feature rested on windblown sand. Inclusions consisted of charcoal, bone, ceramic fragments, and flaked stone artifacts.

An additional patch of ash was found on the edge of the central hearth feature, [07.0039]. This unit was most likely a remnant of [07.0021] (Hearth 146) that was left unexcavated. Inclusions consisted of charcoal, bone, shell, flaked stone artifacts, and ceramic. Unit [07.0039] was $1.11 \times 0.18 \mathrm{~m}$ and $0.115 \mathrm{~m}$ thick. Unit [07.0041] was part of the same deposit but was separated from it as a dense concentration of ash and charcoal, although no clear hearth was definable. Inclusions consisted of bone, charcoal, and flaked stone artifacts. Unit [07.0048] was also an ash patch associated with the central hearth feature, and like [07.0039] it was probably an extension of [07.0021] (Hearth 146) but was not completely excavated. This unit was $0.43 \mathrm{x}$ $0.4 \mathrm{~m}$ and $0.045 \mathrm{~m}$ thick. Unit [07.0048] consisted of fine sand and ash with inclusions of ceramic, bone, and flaked stone artifacts.

A fourth hearth deposit, [07.0033] (Hearth 152), underlay a deposit of windblown sand and ash [07.0029], which itself underlay [07.0022] and [07.0023]. This hearth was small $(0.35 \times 0.25 \mathrm{~m}$ and $0.07 \mathrm{~m}$ deep $)$ and was initially thought to be a charcoal and ash concentration within [07.0029], although its circular morphology and high concentration of charcoal warranted a separate unit designation. The unit was not situated in any discernible cut, as with other hearths in the trench, and no clear structure was visible.

A radiocarbon determination, UCIAMS-45088, was obtained from a sample obtained from the [07.0033] fill, although as described above, the integrity of the feature remains in doubt, so the age must be viewed with caution (Table 6.2).

The fifth hearth set in Trench KK07, [07.0034] (Hearth 147), underlay [07.0029] and was partially truncated by the western baulk of the trench. Deposit [07.0034] was $1.2 \times 0.54 \mathrm{~m}$ and $0.08 \mathrm{~m}$ thick. The matrix consisted of 
ash and silty sand with inclusions of charcoal, coarse sand, large clay chunks, bone, shell, ceramic, and flaked stone artifacts. Cut [07.0037] for this hearth feature was cut into [07.0040] and [07.0049].

The sixth hearth, ashy deposit [07.0049] (Hearth 151), rested beneath windblown sand deposit [07.0034] and hearth cut [07.0037] and represented the first phase of hearth use in this area. The hearth was partially truncated by the western baulk of the trench. The cut [07.0052] with fill [07.0049] had dimensions of $1.13 \mathrm{x}$ $0.58 \mathrm{~m}$ and was $0.06 \mathrm{~m}$ thick. The matrix consisted of ash and fine sand with inclusions of charcoal, ceramic, shell, bone, and flaked stone artifacts. This hearth was cut into the underlying windblown sand deposit [07.0044].

The hearth deposit [07.0035] (Hearth 148) was located to the north of [07.0034] and was stratigraphically below [07.0029]. This hearth was truncated by both the west baulk and the north baulk of the trench and the cut [07.0038] and fill [07.0044] was $0.63 \times 0.23$ $\mathrm{m}$ and $0.05 \mathrm{~m}$ thick. The matrix consisted of ash and silty sand with charcoal and bone inclusions.

Deposit [07.0043] was the eighth and oldest hearth deposit identified in KK07 (Hearth 149) and was found beneath [07.0042]. The matrix consisted of ash and oxidized sand. Unit [07.0043] was $0.61 \times 0.49 \mathrm{~m}$ and $0.055 \mathrm{~m}$ thick. Unit [07.0045] cut the underlying windblown sand deposit [07.0044], in addition to also partially cutting an underlying ash deposit [07.0046].

An ashy sand deposit [07.0046] underlying [07.0042] and [07.0043] was truncated by the eastern baulk of the trench. This unit was $1.45 \times 0.94 \mathrm{~m}$ and $0.05 \mathrm{~m}$ thick. The deposit may indicate the presence of another hearth concealed outside the trench. Inclusions consisted of bone, ceramic fragments, flaked stone artifacts, and shell. A sand lens, [07.0045], partially covered [07.0046] and was discovered only during the excavation of [07.0046]. It consisted of windblown sand with inclusions of ceramic, flaked stone artifacts, and bone and was $0.5 \times 0.39 \mathrm{~m}$ and $0.005 \mathrm{~m}$ thick. This sand lens may represent the abandonment of this section of the trench before the construction of hearth [07.0043/07.0045] (Hearth 149).

A series of units were found beneath the hearth features discussed above and were only partially excavated. A compact windblown sand layer with inclusions of charcoal, bone, ceramic fragments, and flaked stone artifacts [07.0026] extended $5.25 \times 1.98 \mathrm{~m}$ across the central and southern portion of the trench and was $0.23 \mathrm{~m}$ thick. It was the southern extension of [07.0022] in the north of the trench, although the same degree of salt encrustations was not observable in both units. The connection between [07.0022] and [07.0026] suggested that the northern hearth feature predated those found in the center and southern portions of the trench. Unit [07.0026] was cut by [07.0027] and [07.0032], but unit [07.0022] overlay units [07.0023], [07.0029], [07.0030], [07.0033], [07.0034], [07.0035], [07.0037], and [07.0038]. A compact windblown sand deposit in the north of the trench [07.0044] contained inclusions of bone, ceramic fragments, charcoal, flaked stone artifacts, and shell and extended $2.1 \times 2 \mathrm{~m}$ across the trench and was 0.06 $\mathrm{m}$ thick. It was similar in composition to [07.0026], [07.0040], and [07.0050] and most likely represented the same depositional event. Unit [07.0044] was cut by hearth features in the northern section of the trench (for example, [07.0038], [07.0045], and associated deposits).

A deposit of compact aeolian sand in the central and southern portion of the trench [07.0050] extended $4.83 \times 2 \mathrm{~m}$. It was similar in composition to [07.0026], [07.0040], and [07.0044] and probably represented the same deposition event as [07.0044]. This unit was not completely excavated (to a depth of only $0.34 \mathrm{~m}$ ) and represented the arbitrary end of the excavation.

\section{KK08}

The upper $0.15 \mathrm{~m}$ of deposit from this unit were removed to reveal plow cut marks approximately $0.1 \mathrm{~m}$ thick. Below the cut marks, [08.0003] included material mixed by cultivation with inclusions of flaked stone artifacts, ceramic, bone, charcoal, shell, and botanicals (from modern-day agriculture). A contemporary irrigation ditch was identified running east-west across the southern part of the trench, $0.5 \mathrm{~m}$ wide and $0.06 \mathrm{~m}$ deep. A desiccation crack with a length of $1.3 \mathrm{~m}$ ran across the southern end of the trench.

A firm windblown sand deposit [08.0006] was cut by the desiccation crack and irrigation ditch discussed above. The unit contained inclusions of flaked stone artifacts, ceramic fragments, bone, shell, and botanical remains (from modern-day agriculture). Unit [08.0003] extended across the whole trench $(7 \times 2 \mathrm{~m})$ and was excavated to a depth of $0.25 \mathrm{~m}$, although this is not the base of the unit.

Cut into the underlying windblown sand deposit [08.0006] was a hearth [08.0008] (Hearth 153) with fill [08.0007]. The matrix consisted of ash above a 
layer of oxidized sand, and the unit was $0.3 \times 0.2 \mathrm{~m}$. It was truncated by the eastern baulk of KK08. It is possible that the hearth feature was not fully excavated when work ceased in KK08.

\section{Discussion}

The seven trenches excavated across the Kom $\mathrm{K}$ show a remarkably similar stratigraphy. Recent agricultural activity has obviously damaged near-surface deposits, but this damage is largely shallow and easily identified by the presence of both plow marks and layers indurated by evaporates. Significantly, CatonThompson and Gardner did not excavate Kom K as thoroughly as Kom W, because beneath the damaged zone there remain large numbers of in situ hearth features similar to the 16 they identified on the surface of the kom during their excavations. The stratigraphy of the site is deeper and more complex than Caton-Thompson and Gardner reported, possibly because they did not penetrate the salt-rich, compact deposit reported as separating what is described as a deflation deposit from the basement "sandrock." The multiple examples of intercut hearths reported above indicate that the deposit was not as uniformly deflated as Caton-Thompson and Gardner believed, although it is also clear that the upper deposits at the kom have been badly damaged, certainly by modern agriculture but also by the Caton-Thompson and Gardner excavation, to a degree that is no longer possible to determine.

Numbers of hearth features, often with evidence for reuse, dominate the stratigraphic record in all the trenches we excavated (Table 6.3). The majority of these hearths were excavated into windblown sand deposits, with the exception of hearths in KK03, which were cut into a clay surface. In addition to the hearths, there is only limited evidence for other anthropogenic features. Small depressions may indicate postholes, but these are shallow, and even assuming that they do represent such features, none form patterns that indicate the presence of structures. The clay surface in KK03 may have been deliberately laid, but there is no independent evidence that this is so. Clay deposits associated with hearths occur in a number of the trenches. In Trench KK05, there were two round-bottomed pits, one of which had evidence of being recut, filled with a mixture of ashy sand and light yellow lumps of clay, while in Trench KK06 there were two clay-lined depressions, perhaps associated with nearby hearths.
The sand layers that make up most of the kom show a differentiation between ashy and yellow sandy areas, probably the result of raking ashes out of the hearths. The sand areas may have been windblown, but in some cases they seem to be deliberate deposits. These sandy areas are not necessarily "clean." In Trench KK06, for example, they were littered with animal bone.

The hearths themselves come in a variety of forms, although their shapes have often been considerably changed through reuse (Table 6.3). The majority are depressions created in sand layers into which fires were formed. In many cases, the depressions seem to have been reused, with material excavated out and new hearth cuts formed. The process of reuse may well explain the mixed ashy deposits common in all the trenches. Only in KK04 is there evidence for the use of stone heat retainers in the form of concentrations of burned rock and ceramic sherds associated with the hearths. Fire-cracked rock is present in the other trenches but is dispersed rather than concentrated within the hearth features.

Hearths are associated with bone, both fish and nonfish, but as noted, because so many of the hearths have been reused, it is difficult to definitively associate cooking with all of them as Caton-Thompson and Gardner suggested. Certainly, the ash deposits often contain quantities of bone that likely originated as fire pit rakeouts, but in Trench KK06, the large quantities of animal bone were mostly found in yellow sand layers.

Episodes of hearth reuse are partly indicated by sequences of cut and fill into existing depressions but also by periods of windblown sediment deposition. In many instances, the same depression was reused, but there are also multiple cuts that are stratigraphically contemporary. Windblown sediments can deposit in a matter of moments, so it is difficult to draw conclusions concerning duration of occupation on the basis of the sediment stratigraphy alone. However, reuse of the same depression at least indicates that earlier hearths were visible to those who remade the fireplaces.

In KK06 the structure of the hearths was not uniform. Apart from the shallow concave hearths, KK06 had one deeply cut hearth (Hearth 140). In KK07 there was evidence of burning siliceous materials, but the use of heat retainers was not clearly demonstrated. Materials such as flint, chalcedony, and chert are not suitable for use as heat retainers, and the burning of these materials may be incidental to hearth use. Rakeout from hearth features made up the majority of the 
Table 6.3. Feature Numbers and Units for Hearths Excavated at Kom K Discussed in Text.

\begin{tabular}{|c|c|c|c|}
\hline Hearth & $\begin{array}{l}\text { Units } \\
\text { Cut } \\
\text { Fill(s) }\end{array}$ & Size in $\mathbf{m}$ & Depth in $\mathbf{m}$ \\
\hline \multicolumn{4}{|c|}{ Trench KK02 } \\
\hline 101 & $\begin{array}{l}{[02.0021] \text { cut }} \\
{[02.0017],[02.0018],[02.0019] \text { fill }}\end{array}$ & $0.55 \times 0.27$ & \\
\hline 102 & $\begin{array}{l}{[02.0022] \text { cut }} \\
{[02.0023] \text { fill }}\end{array}$ & $0.30 \times 0.20$ & 0.05 \\
\hline 103 & $\begin{array}{l}{[02.0020] \text { cut }} \\
{[02.0019] \text { fill }}\end{array}$ & $0.25 \times 0.25$ & 0.03 \\
\hline 104 & $\begin{array}{l}{[02.0027] \text { cut }} \\
{[02.0026] \text { fill }}\end{array}$ & $0.64 \times 0.90$ & 0.06 \\
\hline 105 & $\begin{array}{l}{[02.0055] \text { cut }} \\
{[02.0032] \text { fill }}\end{array}$ & $0.63 \times 0.67$ & 0.06 \\
\hline 106 & $\begin{array}{l}{[02.0057] \text { cut }} \\
{[02.0052] \text { fill }}\end{array}$ & $0.90 \times 1.25$ & 0.10 \\
\hline 107 & $\begin{array}{l}{[02.0069] \text { cut }} \\
{[02.0061] \text { fill }}\end{array}$ & $0.70 \times 0.90$ & 0.10 \\
\hline 108 & $\begin{array}{l}{[02.0067] \text { cut }} \\
{[02.0062] \text { fill }}\end{array}$ & $0.80 \times 1.30$ & 0.10 \\
\hline \multicolumn{4}{|c|}{ Trench KK 03} \\
\hline 109 & $\begin{array}{l}{[03.0046] \text { cut }} \\
{[03.0047] \text { fill }}\end{array}$ & $0.85 \times 0.85$ & 0.17 \\
\hline 110 & $\begin{array}{l}\text { [03.0048] cut } \\
{[03.0049] \text { fill }}\end{array}$ & $0.91 \times 0.91$ & 0.12 \\
\hline 111 & $\begin{array}{l}{[03.0050] \text { cut }} \\
{[03.0051] \text { fill }} \\
{[03.0052] \text { fill }} \\
{[03.0053] \text { fill }}\end{array}$ & $0.61 \times 0.61$ & 0.11 \\
\hline 112 & $\begin{array}{l}{[03.0055] \text { cut }} \\
{[03.0056] \text { fill }}\end{array}$ & $0.56 \times 0.56$ & 0.12 \\
\hline 113 & $\begin{array}{l}{[03.0057] \text { cut }} \\
{[03.0058] \text { fill }} \\
{[03.0059] \text { fill }}\end{array}$ & $0.46 \times 0.46$ & 0.09 \\
\hline 114 & $\begin{array}{l}{[03.0060] \text { cut }} \\
{[03.0061] \text { fill }} \\
{[03.0062] \text { fill }}\end{array}$ & $0.50 \times 0.50$ & 0.08 \\
\hline
\end{tabular}




\begin{tabular}{|c|c|c|c|}
\hline Hearth & $\begin{array}{l}\text { Units } \\
\text { Cut } \\
\text { Fill(s) }\end{array}$ & Size in $\mathbf{m}$ & Depth in $\mathrm{m}$ \\
\hline 115 & $\begin{array}{l}{[03.0063] \text { cut }} \\
{[03.0064] \text { fill }}\end{array}$ & $0.40 \times 0.40$ & 0.06 \\
\hline 116 & $\begin{array}{l}{[03.0044] \text { cut }} \\
{[03.0037] \text { fill }}\end{array}$ & $1.25 \times 0.70$ & 0.23 \\
\hline 117 & $\begin{array}{l}{[03.0067] \text { cut }} \\
{[03.0068] \text { fill }} \\
{[03.0069] \text { fill }}\end{array}$ & $0.60 \times 0.60$ & 0.20 \\
\hline 118 & $\begin{array}{l}{[03.0070] \text { cut }} \\
{[03.0071] \text { fill }} \\
{[03.0072] \text { fill }}\end{array}$ & $0.40 \times 0.40$ & 0.08 \\
\hline 119 & $\begin{array}{l}{[03.0073] \text { cut }} \\
{[03.0074] \text { fill }} \\
{[03.0075] \text { fill }} \\
{[03.0076] \text { fill }}\end{array}$ & $0.30 \times 0.30$ & 0.10 \\
\hline 120 & $\begin{array}{l}{[03.0077] \text { cut }} \\
{[03.0078] \text { fill }} \\
{[03.0079] \text { fill }}\end{array}$ & $0.30 \times 0.30$ & 0.08 \\
\hline 121 & $\begin{array}{l}{[03.0082] \text { cut }} \\
{[03.0085] \text { fill }}\end{array}$ & $0.60 \times 0.60$ & 0.10 \\
\hline 122 & $\begin{array}{l}{[03.0080] \text { cut }} \\
{[03.0081] \text { fill }}\end{array}$ & $0.25 \times 0.25$ & 0.20 \\
\hline \multicolumn{4}{|c|}{ Trench KK04 } \\
\hline 123 & $\begin{array}{l}\text { [04.0017] cut } \\
{[04.0018] \text { fill }}\end{array}$ & $0.58 \times 0.22$ & 0.11 \\
\hline 124 & $\begin{array}{l}{[04.0019] \text { cut }} \\
{[04.0006] \text { fill }} \\
{[04.0020] \text { fill }}\end{array}$ & $0.22 \times 0.22$ & 0.07 \\
\hline 125 & $\begin{array}{l}{[04.0021] \text { cut }} \\
{[04.0005] \text { fill }}\end{array}$ & $0.38 \times 0.34$ & 0.03 \\
\hline 127 & $\begin{array}{l}{[04.0024] \text { cut }} \\
{[04.0025] \text { fill }} \\
{[04.0045] \text { fill }}\end{array}$ & $1.18 \times 1.06$ & 0.09 \\
\hline 128 & $\begin{array}{l}{[04.0026] \text { cut }} \\
{[04.0027] \text { fill }}\end{array}$ & $0.36 \times 0.34$ & 0.12 \\
\hline
\end{tabular}


Table 6.3. Feature Numbers and Units for Hearths Excavated at Kom K Discussed in Text. Continued

\begin{tabular}{|c|c|c|c|}
\hline Hearth & $\begin{array}{l}\text { Units } \\
\text { Cut } \\
\text { Fill(s) }\end{array}$ & Size in $\mathbf{m}$ & Depth in $\mathbf{m}$ \\
\hline 129 & $\begin{array}{l}{[04.0055] \text { cut }} \\
{[04.0051] \text { fill }}\end{array}$ & $0.21 \times 0.20$ & 0.05 \\
\hline 130 & $\begin{array}{l}{[04.0057] \text { cut }} \\
{[04.0037] \text { fill }} \\
{[04.0038] \text { fill }} \\
{[04.0039] \text { fill }} \\
{[04.0047] \text { fill }} \\
{[04.0048] \text { fill }} \\
{[04.0053] \text { fill }}\end{array}$ & $0.84 \times 0.79$ & 0.19 \\
\hline 131 & $\begin{array}{l}{[04.0064] \text { cut }} \\
{[04.0065] \text { fill }}\end{array}$ & $0.76 \times 0.74$ & 0.08 \\
\hline 132 & $\begin{array}{l}{[04.0052] \text { cut }} \\
{[04.0044] \text { fill }}\end{array}$ & $1.26 \times 1.06$ & 0.18 \\
\hline \multicolumn{4}{|c|}{ Trench KK05 } \\
\hline 133 & $\begin{array}{l}{[05.0022] \text { cut }} \\
{[05.0016] \text { fill }} \\
{[05.0017] \text { fill }}\end{array}$ & $0.80 \times 0.54$ & 0.10 \\
\hline 135 & $\begin{array}{l}{[05.0027] \text { cut }} \\
{[05.0028] \text { fill }}\end{array}$ & $1.00 \times 1.00$ & 0.10 \\
\hline 136 & $\begin{array}{l}{[05.0036] \text { cut }} \\
{[05.0037] \text { fill }}\end{array}$ & $0.50 \times 0.45$ & 0.05 \\
\hline 139 & $\begin{array}{l}{[05.0045] \text { fill }} \\
{[05.0044] \text { fill }}\end{array}$ & $0.50 \times 0.50$ & 0.10 \\
\hline \multicolumn{4}{|c|}{ Trench KK06 } \\
\hline 140 & $\begin{array}{l}{[06.0019] \text { cut }} \\
{[06.0018] \text { fill }}\end{array}$ & $0.65 \times 0.40$ & 0.40 \\
\hline 141 & $\begin{array}{l}{[06.0037] \text { cut }} \\
{[06.0041] \text { fill }}\end{array}$ & $0.90 \times 0.60$ & 0.09 \\
\hline 142 & $\begin{array}{l}{[06.0038] \text { cut }} \\
{[06.0039] \text { fill }}\end{array}$ & $0.35 \times 0.36$ & 0.03 \\
\hline 143 & $\begin{array}{l}{[06.0054] \text { cut }} \\
{[06.0053] \text { fill }} \\
{[06.0055] \text { fill }}\end{array}$ & $0.50 \times 0.46$ & 0.09 \\
\hline
\end{tabular}




\begin{tabular}{|c|c|c|c|}
\hline Hearth & $\begin{array}{l}\text { Units } \\
\text { Cut } \\
\text { Fill(s) }\end{array}$ & Size in $\mathbf{m}$ & Depth in $\mathrm{m}$ \\
\hline \multicolumn{4}{|c|}{ Trench KK07 } \\
\hline 144 & [07.0008] fill & $0.38 \times 0.37$ & 0.09 \\
\hline 145 & $\begin{array}{l}{[07.0027] \text { cut }} \\
{[07.0018] \text { fill }} \\
{[07.0024] \text { fill }}\end{array}$ & $0.60 \times 0.47$ & 0.04 \\
\hline 146 & $\begin{array}{l}{[07.0032] \text { cut }} \\
{[07.0019] \text { fill }} \\
{[07.0020] \text { fill }} \\
{[07.0021] \text { fill }} \\
{[07.0031] \text { fill }}\end{array}$ & $1.11 \times 0.97$ & 0.19 \\
\hline 147 & $\begin{array}{l}{[07.0037] \text { cut }} \\
{[07.0034] \text { fill }}\end{array}$ & $1.23 \times 0.54$ & 0.08 \\
\hline 148 & $\begin{array}{l}{[07.0038] \text { cut }} \\
{[07.0035] \text { fill }}\end{array}$ & $0.63 \times 0.23$ & 0.05 \\
\hline 149 & $\begin{array}{l}{[07.0045] \text { cut }} \\
{[07.0043] \text { fill }}\end{array}$ & $0.61 \times 0.49$ & 0.055 \\
\hline 150 & [07.0046] fill & $1.45 \times 0.94$ & 0.05 \\
\hline 151 & $\begin{array}{l}{[07.0052] \text { cut }} \\
{[07.0049] \text { fill }}\end{array}$ & $1.13 \times 0.58$ & 0.06 \\
\hline 152 & [07.0033] fill & $0.35 \times 0.25$ & 0.07 \\
\hline \multicolumn{4}{|c|}{ Trench KK08 } \\
\hline 153 & $\begin{array}{l}{[08.0008] \text { cut }} \\
{[08.0007] \text { fill }}\end{array}$ & $0.30 \times 0.20$ & unexcavated \\
\hline
\end{tabular}

remaining deposits, creating a general matrix of ashy, silty sand, mixed with bone. The homogeneity of the deposits made discerning distinct episodes of use difficult, and these deposits likely represent more use and reuse phases than the stratigraphy can identify. As described above, 54 discrete hearth feature sets were recorded during the course of excavation. All hearths were constructed on windblown sand surfaces, with the exception of those constructed on earlier hearth features: Hearth Clusters A, B, C, and D in Trench KK03 and Hearth 144 over Hearth 146; Hearth 149 over Hearth 150. All these hearths were separated from their earlier counterparts by intervening sand or sand/ash layers and so were considered discrete. This may indicate temporary abandonment and subsequent reuse of this area of the site, although of course it is not possible to determine the time between the episodes of use and reuse. All the charcoal identified was Tamarix, 
but ovicaprid dung was also found in a hearth context, namely in ashy hearth fill [07.0019] (Hearth 146), and was possibly used as fuel (see below).

Figure 6.13 combines the probability plots for the dated hearths from all the trenches. Hearth ages most probably fall in the period 6600 to $6400 \mathrm{cal} \mathrm{BP}$. However, the calibration curve at this period means that the probability distributions for the age determinations are quite wide. The hearths could relate to a closely timed set of events within the fifth millennium BCE, or they may be spread across several centuries. Unfortunately, the stratigraphy at the site provides little opportunity to increase the certainty of the age of occupation. Four radiocarbon determinations were obtained for hearths from KK04, but these come from three sets of hearths that may have been cut from the same surface. Thus Hearth 127 has a calibrated date of $6420 \pm 35 \mathrm{cal} \mathrm{BP}$ and adjacent Hearth 128 has a date of $6513 \pm 49 \mathrm{cal} \mathrm{BP}$, while there are two dates from the 130 and 129 hearth set of $6457 \pm 29$ $\mathrm{cal} \mathrm{BP}$ and $6453 \pm 29 \mathrm{cal} \mathrm{BP}$. The determination from Hearth 128 may be somewhat older than the others, as indicated in Figure 6.13, but as noted, the probability curves also mean that the hearths might be very close to one another in age.

Excavation trench placement was guided to some degree by the magnetic survey and therefore targeted locations with magnetic anomalies that might indicate the presence of hearth features. However, the placement

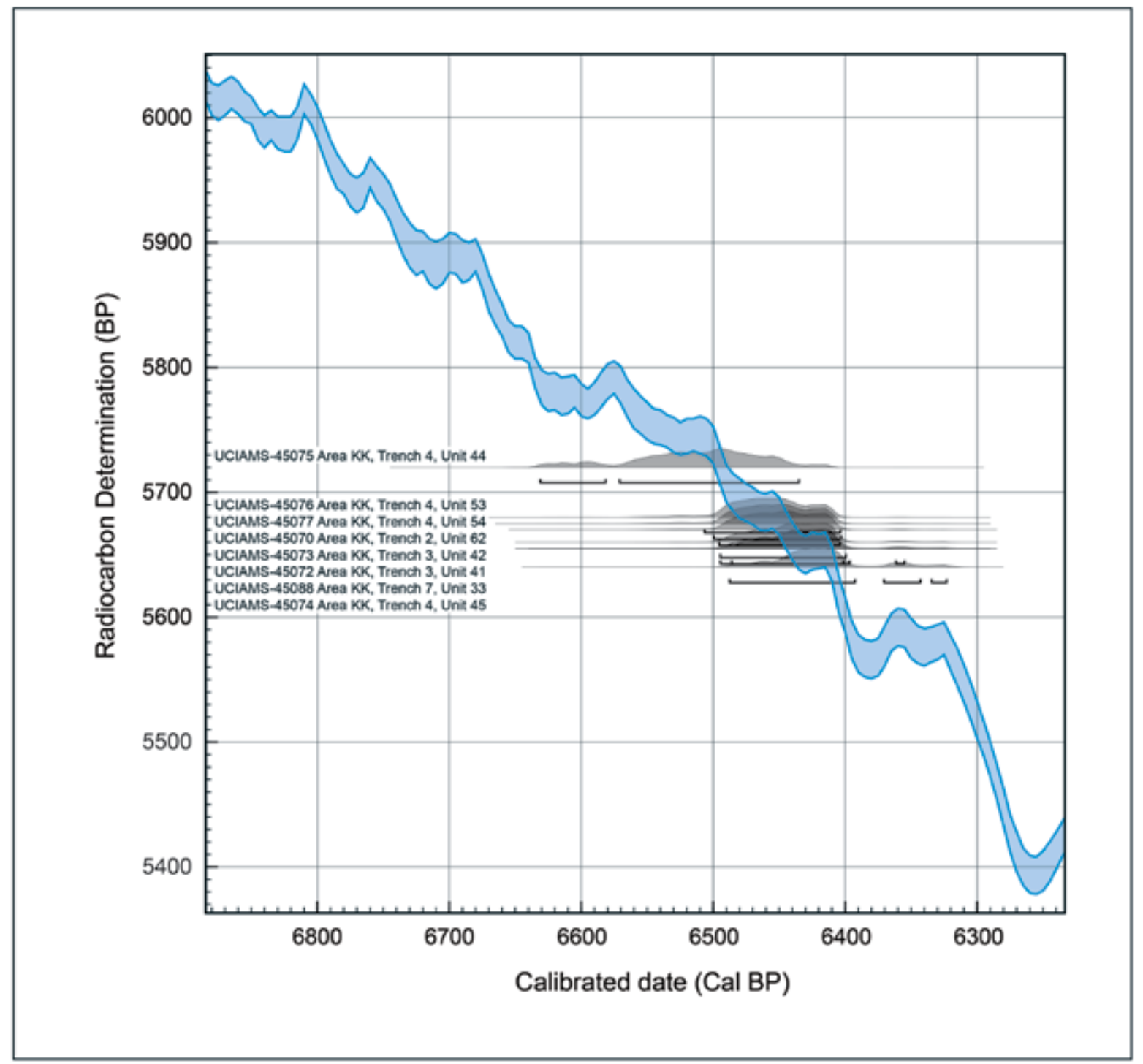

Figure 6.13. Radiocarbon determinations from the Kom $\mathrm{K}$ hearths plotted against the IntCal13 calibration curve. 
of the trenches was also intended to ensure that areas with different topographic elevations were targeted. This helps mitigate the problem of sampling an area as large as Kom $\mathrm{K}$ through excavation. There is always the chance that the excavation strategy led to the selection of areas where only a particular set of features was found. However, in this case trenches were located in the highest and lowest parts of the Kom as well as at intermediate positions. Based on the sample of areas excavated, different areas of the Kom show quite similar patterns of use.

Charcoal identification suggests that tamarisk was the only wood used as fuel in the hearths. Six species of tamarisk grow in Egypt today. Generally, tamarisk is a shrub or tree 1 to $3 \mathrm{~m}$ in height, although Tamarix aphylla grows to $12 \mathrm{~m}$ (Boulos 2000:127). Native to North Africa and Southwest and Central Asia, Tamarix is a xerophilous and halophilous genus that often occurs as a locally dominant shrub. It can grow densely along watercourses, swamps, and salt marshes. Boulos (2000:127) notes that Tamarix aphylla is widely cultivated in modern Egypt for timber and shade, and as a windbreak. It is the dominant native shrub in sandy and saline areas of Egypt and is frequent in the Nile Valley and across the desert areas of the region (Newton 2005).

The presence of Tamarix species in the vicinity of Kom $\mathrm{K}$ during the mid-Holocene period is not surprising; nor is its frequency in archaeological deposits unusual. In her analysis of wood charcoal from two third-millennium BCE sites in the central Nile Valley, Newton found that Tamarix was the dominant wood type, making up more than 60 percent of all identified wood charcoal fragments (2005:359). Tamarix grows spontaneously in the Fayum today, in both dry and irrigated areas. It is a hardwood that burns well and produces high-quality fuel charcoal, which is still sold commercially in Fayum City.

Caton-Thompson and Gardner (1934) interpreted Kom $\mathrm{K}$ as a village, and to a greater or lesser degree, their interpretation has been repeated in the literature ever since. Obviously, the excavations reported here do not provide evidence for the range and concentration of features that would support such an interpretation. Kom $\mathrm{K}$ does indicate concentrated activity connected with the repeated use of hearths in multiple places in the site. However, this activity did not leave traces of features in addition to the hearth remains. To understand the range of activities that occurred at Kom $\mathrm{K}$ we need to turn to a consideration of the material culture and fauna.

\section{Material Culture}

The excavations undertaken by Caton-Thompson and Gardner were sufficiently extensive to produce a range of material culture types. In The Desert Fayum, 28 axes are recorded; the majority are made from flint. Caton-Thompson and Gardner remarked on evidence for reworking of the axes, including one example that was used as a hammerstone. In addition to the axes, 10 sickle blades were recovered; two of them were noted as retaining silica polish. Seven concave-based bifacial points were found together with a single tanged bifacial point. Caton-Thompson and Gardner commented on the presence of this type within the in situ deposit and the presence of multiple other examples on the surface (1934:38-40).

The presence of pallets was also recorded, with two examples illustrated, both made from white limestone. A range of bone and shell artifacts was recovered, including bone points and green feldspar beads. A number of shells were recorded as having perforations. The presence of Spatha shell scoops was also recorded.

Seven large ceramic cooking pots were reported, with one example described as standing below the surface. Decorated sherds were limited to what were described as peaked rims, four sherds with red-polished ware, and a single black-polished sherd.

The range of material culture types was not matched in the modern excavations, but we were able to accumulate large collections of flaked stone artifacts and ceramics together with faunal remains and limited botanical remains. In the following we report on analyses conducted on the flaked stone artifacts, fauna, and flora and make some preliminary observations about the ceramics and personal ornaments.

\section{Surface Collection and Analysis}

As noted above, a surface survey was conducted in 2006. The intention of the survey was to collect surface artifacts, including flaked stone artifacts and ceramics, due to their high numbers visible on the surface as a result of modern agricultural activity. A grid was placed over the surface to define $5 \times 5-\mathrm{m}$ squares (Figure 6.14). Flaked stone artifacts found on the surface were completely collected in a total of 99 squares. This resulted in the collection of approximately 50,000 artifacts. To sample the Kom $\mathrm{K}$ artifacts for analysis, 25 percent of the squares were selected at random. As a result, 8,574 artifacts from Kom $\mathrm{K}$ were analyzed (Table 6.4). 
Table 6.4. Stone Artifact Frequency by Technological Category and Artifact Density from Kom K Surface Sample Squares.

\begin{tabular}{|c|c|c|c|c|c|c|c|c|c|c|}
\hline $\begin{array}{l}\text { Surface } \\
\text { Collection }\end{array}$ & $\begin{array}{l}\text { Total } \\
\text { Artifact } \\
\text { (n) }\end{array}$ & $\begin{array}{l}\text { Total per } \\
\text { Square m }\end{array}$ & $\begin{array}{l}\text { Complete } \\
\text { Flake }\end{array}$ & $\begin{array}{l}\text { Complete } \\
\text { Flake per } \\
\text { Square m }\end{array}$ & Broken & $\begin{array}{l}\text { Broken } \\
\text { Artifacts } \\
\text { per Square m }\end{array}$ & Tool & $\begin{array}{l}\text { Tools per } \\
\text { Square m }\end{array}$ & Core & $\begin{array}{l}\text { Cores per } \\
\text { Square m }\end{array}$ \\
\hline 20011 & 353 & 14.12 & 194 & 7.76 & 101 & 4.04 & 8 & 0.32 & 31 & 1.24 \\
\hline 20018 & 869 & 34.76 & 560 & 22.4 & 247 & 9.88 & 15 & 0.6 & 47 & 1.88 \\
\hline 20022 & 603 & 24.12 & 363 & 14.52 & 172 & 6.88 & 19 & 0.76 & 34 & 1.36 \\
\hline 20025 & 304 & 12.16 & 191 & 7.64 & 61 & 2.44 & 8 & 0.32 & 16 & 0.64 \\
\hline 20027 & 130 & 5.2 & 67 & 2.68 & 42 & 1.68 & 4 & 0.16 & 12 & 0.48 \\
\hline 20029 & 112 & 4.48 & 63 & 2.52 & 38 & 1.52 & 3 & 0.12 & 4 & 0.16 \\
\hline 20051 & 699 & 27.96 & 439 & 17.56 & 235 & 9.4 & 11 & 0.44 & 13 & 0.52 \\
\hline 20052 & 921 & 36.84 & 628 & 25.12 & 223 & 8.92 & 34 & 1.36 & 34 & 1.36 \\
\hline 20055 & 201 & 8.04 & 130 & 5.2 & 42 & 1.68 & 16 & 0.64 & 13 & 0.52 \\
\hline 20056 & 428 & 17.12 & 287 & 11.48 & 98 & 3.92 & 16 & 0.64 & 27 & 1.08 \\
\hline 20074 & 445 & 17.8 & 267 & 10.68 & 121 & 4.84 & 15 & 0.6 & 25 & 1 \\
\hline 20099 & 276 & 11.04 & 178 & 7.12 & 75 & 3 & 11 & 0.44 & 11 & 0.44 \\
\hline 20101 & 274 & 10.96 & 184 & 7.36 & 57 & 2.28 & 23 & 0.92 & 10 & 0.4 \\
\hline 20103 & 379 & 15.16 & 281 & 11.24 & 68 & 2.72 & 12 & 0.48 & 18 & 0.72 \\
\hline 20115 & 76 & 3.04 & 47 & 1.88 & 24 & 0.96 & 2 & 0.08 & 2 & 0.08 \\
\hline 20117 & 256 & 10.24 & 146 & 5.84 & 67 & 2.68 & 12 & 0.48 & 19 & 0.76 \\
\hline 20118 & 211 & 8.44 & 122 & 4.88 & 56 & 2.24 & 4 & 0.16 & 15 & 0.6 \\
\hline 20120 & 613 & 24.52 & 413 & 16.52 & 153 & 6.12 & 21 & 0.84 & 25 & 1 \\
\hline 20124 & 505 & 20.2 & 269 & 10.76 & 163 & 6.52 & 23 & 0.92 & 33 & 1.32 \\
\hline 20137 & 186 & 7.44 & 125 & 5 & 38 & 1.52 & 14 & 0.56 & 9 & 0.36 \\
\hline 20155 & 733 & 29.32 & 427 & 17.08 & 196 & 7.84 & 15 & 0.6 & 32 & 1.28 \\
\hline Total & 8,574 & 16.33 & 5,381 & 10.25 & 2,277 & 4.34 & 286 & 0.54 & 430 & 0.82 \\
\hline
\end{tabular}

\section{Density of Artifacts from Kom K}

The distribution of artifacts across the surface of Kom $\mathrm{K}$ is the result of a combination of the discard of the past occupation, including Caton-Thompson and Gardner's excavations in the 1920s, and plowing for agriculture that began in the later part of the twentieth century and continued intermittently thereafter. The density of artifacts on the surface is therefore the product of a number of processes that occurred at different times. This said, there are differences in density that may relate to the subsurface remains.

Table 6.4 provides artifact counts and densities for the squares that were analyzed. There is a significant correlation in the frequency of artifacts between all combinations, complete and broken flakes, cores and tools. However, there are some differences in density high points among the artifact categories, with complete and broken flakes having a slightly different distribution to cores and tools. There is also a tendency for high densities of flaked stone artifacts to fall outside the peaks in the magnetic survey, suggesting some remnant spatial patterning despite the high level of surface disturbance. Although these results are necessarily coarse grained, given the level of disturbance, they are consistent with the stratigraphic evidence that indicates multiple episodes of hearth reuse at the same location. The distribution of flaked stone artifacts suggests that they were distributed close to, but not always directly on top of, areas where concentrated hearths are found. A slight tendency for flakes and cores to be spatially separated is investigated in the flaked stone artifact analysis reported below. 


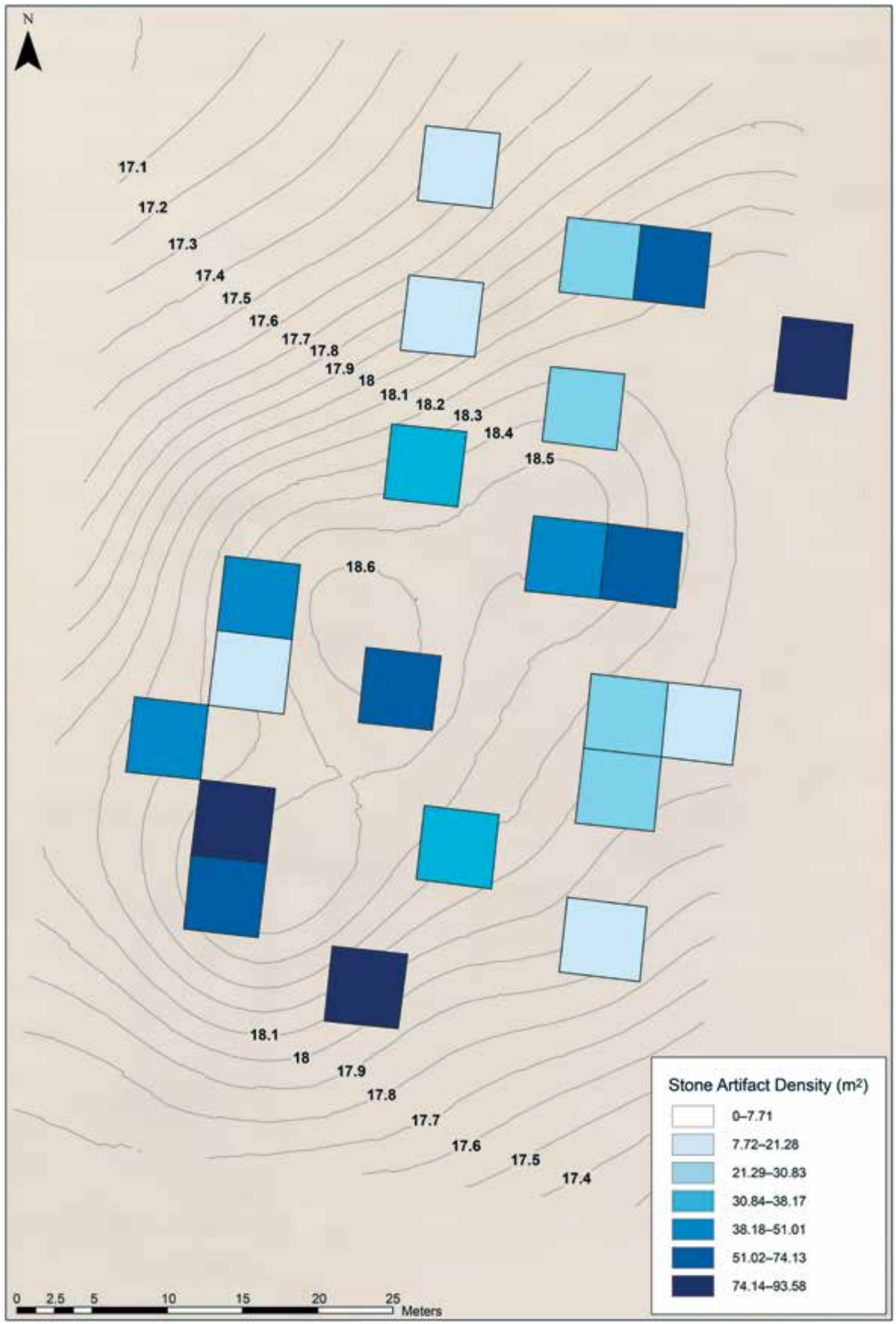

Figure 6.14. Stone artifact density for collection squares where artifacts were recorded. 


\section{Flaked Stone Artifacts}

The following presents the results of an analysis of both the Kom K surface collection and the excavated flaked stone artifacts. The results of an analysis of approximately 25 percent of the surface flaked stone artifacts collected are reported here, together with the results of the analysis of flaked stone artifacts from excavated Trenches KK05, KK07, and KK08. All the artifacts collected from KK07 were analyzed, while subsamples were analyzed from KK05 and KK08.

In KK05, units [05.0016], [05.0026], and [05.0044] were analyzed, with the selection of these units based on the abundance of flaked stone artifacts (Table 6.5). As described above, [05.0016] was as an ash deposit consisting of yellowish-brown, firm, poorly sorted, coarse sand. This deposit contained ceramic, flaked stone artifacts, bone, and shell and represented a lens within a windblown sand deposit. Unit [05.0026] was an ash deposit of yellowish-brown silt-sand containing charcoal, ceramic, shell, bone, and flaked stone artifacts. Unit [05.0044] was described as an ash deposit of dark gray, compact, silt-sand. This unit contained ceramic, bone, shell, flaked stone artifacts, beads, ostrich shell, and burned clay.

Only artifacts from [08.0006] were analyzed from KK08 (Table 6.6). This unit was defined as a firm windblown sand deposit with inclusions of flaked stone artifacts, ceramic, bone, shell, and modern plant material. At the time of excavation, this unit was considered to be part of Caton-Thompson and Gardner's backfill, later disturbed by agricultural activity. The unit was not completely excavated.

Table 6.5. Frequency of Analyzed Stone Artifacts by Technological Class Analyzed from Kom K Trench KK05.

\begin{tabular}{|l|r|r|r|r|r|}
\hline KK05 & & & & & \\
\hline Unit & Total & Complete & Broken & \multicolumn{1}{|l|}{ Tool } & \multicolumn{1}{l|}{ Core } \\
\hline$[05.0016]$ & 70 & 22 & 18 & 2 & 1 \\
\hline$[05.0026]$ & 78 & 17 & 27 & 0 & 2 \\
\hline$[05.0044]$ & 53 & 23 & 27 & 2 & 0 \\
\hline
\end{tabular}

Table 6.6. Frequency of Analyzed Stone Artifacts by Technological Class from Kom K Trench KK08.

\begin{tabular}{|l|c|r|r|r|r|}
\hline KK08 & & & & & \\
\hline Unit & Total & Complete & Broken & Tool & Core \\
\hline$[08.0006]$ & 1,039 & 570 & 393 & 18 & 30 \\
\hline
\end{tabular}

The assemblage of flaked stone artifacts from KK07 was completely analyzed. Artifacts were found in the 31 units discussed in detail above. The trench consisted of a series of hearth features, generally small and shallow, cut into windblown sand deposits, some intercutting, with intermittent lenses of windblown sand.

\section{Artifact Density}

As Table 6.7 indicates, unit [07.0005] contained a larger number of flaked stone artifacts $(1,510)$ than the other units, although this may be explained by the large volume of this unit. The upper layers, [07.0001], [07.0002], [07.0004], [07.0005], and [07.0013], have large numbers of artifacts compared to those lower in the trench. Hearth units have fewer artifacts than units that lack these features, a result suggested by the distribution of the surface flaked stone artifacts reported above.

\section{Cores}

More than 500 cores were analyzed from the surface and excavated assemblages at Kom K (Table 6.8). The majority of these are multiple cores-that is, cores that have been rotated to initiate multiple platforms-and unifacial cores, where only one platform was struck. There are smaller proportions of bifacial cores, where the core surface was prepared by removing flakes before further flakes were removed from a surface orthogonal to the platform, and test cores, where only a single flake was removed. Comparing the four most abundant core types (bifacal, multiple, test, and unifacial) between the surface and excavated assemblage shows no significant difference. ${ }^{1}$ These core forms were also the most common in the L1 Basin and E29H1 assemblages (chapter 4).

Table 6.9 provides dimensions for cores from both the surface and excavated assemblages. Size comparisons between the excavated and surface assemblages for each core type indicate no significant differences for bifacial and unifacial cores, but the multiple cores in the surface assemblage are longer and thicker and have larger flakes scars than those from the excavated assemblage (Table 6.9). Core maximum dimensions from the Kom K surface and excavated assemblages combined, compared to the dimensions of all unifacial, bifacial, and multiple cores, fall approximately midway between the small core dimensions in the E29H1 assemblage and the larger core maximum dimensions in the L1 and K1

$\overline{1 \text { Chi-square }=5.12 ;} d f=3 ; p=0.164$. 
Table 6.7. Frequency of Analyzed Stone artifacts by Technological Class from Kom K Trench KK07.

\begin{tabular}{|c|c|c|c|c|c|}
\hline KK07 & & & & & \\
\hline Unit & Total $\mathbf{n}$ & Complete & Broken & Tool & Core \\
\hline 0001 & 370 & 159 & 105 & 23 & 26 \\
\hline 0003 & 252 & 89 & 77 & 6 & 9 \\
\hline 0004 & 190 & 94 & 57 & 7 & 6 \\
\hline 0005 & 1,510 & 593 & 501 & 19 & 33 \\
\hline 0007 & 63 & 25 & 30 & 1 & 2 \\
\hline 0009 & 29 & 4 & 7 & 0 & 2 \\
\hline 0013 & 588 & 116 & 180 & 1 & 3 \\
\hline 0014 & 6 & 4 & 0 & 0 & 0 \\
\hline 0015 & 17 & 3 & 4 & 0 & 0 \\
\hline 0016 & 10 & 3 & 1 & 0 & 0 \\
\hline 0017 & 193 & 48 & 45 & 0 & 3 \\
\hline 0019 & 3 & 0 & 0 & 0 & 0 \\
\hline 0020 & 5 & 1 & 1 & 0 & 0 \\
\hline 0021 & 47 & 12 & 12 & 0 & 0 \\
\hline 0022 & 28 & 7 & 10 & 0 & 0 \\
\hline 0023 & 71 & 12 & 26 & 0 & 0 \\
\hline 0025 & 19 & 5 & 8 & 0 & 0 \\
\hline 0026 & 143 & 18 & 52 & 0 & 5 \\
\hline 0028 & 6 & 0 & 3 & 0 & 0 \\
\hline 0029 & 17 & 1 & 7 & 0 & 0 \\
\hline 0030 & 9 & 1 & 1 & 0 & 0 \\
\hline 0034 & 16 & 4 & 5 & 0 & 0 \\
\hline 0039 & 9 & 0 & 2 & 0 & 0 \\
\hline 0040 & 34 & 7 & 13 & 0 & 0 \\
\hline 0042 & 14 & 1 & 8 & 0 & 0 \\
\hline 0044 & 39 & 12 & 12 & 0 & 2 \\
\hline 0046 & 15 & 2 & 3 & 0 & 0 \\
\hline 0047 & 2 & 2 & 0 & 0 & 0 \\
\hline 0048 & 5 & 0 & 1 & 0 & 0 \\
\hline 0049 & 2 & 0 & 0 & 0 & 0 \\
\hline $\begin{array}{l}\text { Frequency of analyzed stone artifacts by technological } \\
\text { class from Kom K Trench } 0050\end{array}$ & 83 & 13 & 21 & 0 & 0 \\
\hline
\end{tabular}


Table 6.8. Core Type Frequencies for Kom K Analyzed Assemblages.

\begin{tabular}{|c|c|c|c|c|c|c|}
\hline Core Type & $\begin{array}{l}\text { Kom K Surface } \\
\text { (n) }\end{array}$ & $\begin{array}{l}\text { Kom K Surface } \\
(\%)\end{array}$ & $\begin{array}{l}\text { Kom K Excavated } \\
\text { (n) }\end{array}$ & $\begin{array}{l}\text { Kom K Excavated } \\
(\%)\end{array}$ & $\begin{array}{l}\text { All Kom K } \\
\text { (n) }\end{array}$ & $\begin{array}{l}\text { All Kom K } \\
(\%)\end{array}$ \\
\hline Bidirectional & 4 & 0.97 & 5 & 4.03 & 9 & 1.67 \\
\hline Bifacial & 68 & 16.43 & 16 & 12.90 & 84 & 15.61 \\
\hline Microblade & 0 & 0 & 1 & 0.81 & 1 & 0.19 \\
\hline Multiple & 180 & 43.48 & 44 & 35.48 & 224 & 41.64 \\
\hline Nuclear tool & 1 & 0.24 & 0 & 0 & 1 & 0.19 \\
\hline Test & 48 & 11.59 & 22 & 17.74 & 70 & 13.01 \\
\hline Tranchet & 1 & 0.24 & 0 & 0 & 1 & 0.19 \\
\hline Unifacial & 112 & 27.05 & 36 & 29.03 & 148 & 27.51 \\
\hline Total & 414 & 100 & 124 & 100 & 538 & 100 \\
\hline
\end{tabular}

Note: Totals are for all surface and excavated cores.

Table 6.9. Core Type Mean Dimensions (with Standard Deviations) for Analyzed Kom K Assemblages.

\begin{tabular}{|c|c|c|c|c|}
\hline Coretype & $\begin{array}{l}\text { Kom K Average Max } \\
\text { Length }(\mathrm{mm})\end{array}$ & $\begin{array}{l}\text { Kom K Average Max } \\
\text { Width }(\mathrm{mm})\end{array}$ & $\begin{array}{l}\text { Kom K Average Max } \\
\text { Thickness (mm) }\end{array}$ & $\begin{array}{l}\text { Kom K Average Core Scar } \\
\text { Length }(\mathrm{mm})\end{array}$ \\
\hline Bidirectional & $39.90 \pm 6.2$ & $28.75 \pm 3.7$ & $20.23 \pm 3.1$ & $23.18 \pm 7.1$ \\
\hline Bifacial & $38.17 \pm 7.5$ & $28.50 \pm 8.5$ & $18.28 \pm 5.6$ & $23.95 \pm 7.0$ \\
\hline Microblade & 25.00 & 18.00 & 23.00 & 22.00 \\
\hline Multiple & $34.88 \pm 7.8$ & $26.71 \pm 6.1$ & $19.62 \pm 5.1$ & $23.29 \pm 5.9$ \\
\hline Test & $34.96 \pm 9.0$ & $25.24 \pm 6.6$ & $17.44 \pm 5.4$ & $21.66 \pm 7.4$ \\
\hline Tranchet & 28.00 & 21.00 & 7.00 & 22.00 \\
\hline Unifacial & $36.80 \pm 8.1$ & $26.68 \pm 6.8$ & $19.14 \pm 5.4$ & $22.19 \pm 6.0$ \\
\hline All & $33.96 \pm 7.7$ & $24.98 \pm 6.4$ & $17.82 \pm 4.9$ & $22.61 \pm 6.7$ \\
\hline
\end{tabular}

Note: Means are for all surface and excavated cores.

assemblages (chapters 4 and 5). ANOVA is significant for the three core maximum dimensions and the core scar length squared, and the post hoc Bonferroni comparisons show that the Kom $\mathrm{K}$ cores are significantly different from E29H1, L1, and K1 cores in all dimensions but are different from XB11 cores only in their maximum thickness and core scar length (Table 6.10).

The highest values for the ratios of the core dimensions measured relative to the longest flake scar are those for the ratio of the length to thickness. This is true for all three of the most common core types (Table 6.11). As discussed in chapters 4 and 5, this suggests that thickness reduces relative to length, indicating the production of relatively short, wide flakes. In this sense, the Kom $\mathrm{K}$ assemblages are closer to those analyzed from L1 and $\mathrm{K} 1$ and they differ from the majority of the E29H1 assemblages. Consistent with this interpretation, the mean length of cores oriented relative to the core scar for the Kom K surface assemblage is shorter than the mean width (Figure 5.10). For the excavated assemblage, the mean oriented length and mean oriented width are very close. Complete flake dimensions illustrate how flake shape is not elongated. The ratio of the complete flake length to width oriented relative to the platform for the surface assemblage is 1.14 , while 
that for the excavated assemblage is 1.11 . These values are comparable to those calculated for the L1 assemblages and those from XB11 but are lower than the value (1.28) reported above for K1.

The majority of cores from both of the Kom K assemblages retain cortex (Table 6.12). This includes multiple cores that despite being rotated to use multiple platforms include a large number of examples with more than 50 percent cortex. Following the discussion in chapters 4 and 5 and the results of the comparison of the ratio of the oriented core dimensions outlined above, the same approach can be used to calculate the size of the cobbles worked at Kom $\mathrm{K}$ based on size estimates derived from the worked cores. Length and width measurements, together with the thickness trebled to account for material removed by flaking, using the estimate from experimental flaking, are used to estimate the size of the original cobbles flaked using the formula for the volume of a scalene ellipsoid. As in previous analyses, the results are log-transformed since they are skewed, and the anti-log of the mean is calculated.

Results of the calculations suggest that original cobble size for the Kom $\mathrm{K}$ excavated assemblage was $28,676.81 \mathrm{~mm}^{3}$ while that for the excavated assemblage was $21,586.10 \mathrm{~mm}^{3}$. Both estimates are considerably smaller than the estimate for the K1

Table 6.10. T-test Comparisons between the Three Most Common Core Forms in the Surface and Excavated Assemblages.

\begin{tabular}{|l|l|l|l|l|}
\hline & & t & df & $\begin{array}{l}\text { Sig. } \\
\text { (2-tailed) }\end{array}$ \\
\hline Bifacial & maximum length $(\mathrm{mm})$ & 0.928 & 82 & 0.356 \\
\hline & maximum width $(\mathrm{mm})$ & 0.478 & 82 & 0.634 \\
\hline & maximum thickness $(\mathrm{mm})$ & 1.160 & 82 & 0.249 \\
\hline & core scar length $(\mathrm{mm})$ & 0.611 & 82 & 0.543 \\
\hline Multiple & maximum length $(\mathrm{mm})$ & 2.006 & 220 & 0.046 \\
\hline & maximum width $(\mathrm{mm})$ & 1.584 & 220 & 0.115 \\
\hline & maximum thickness $(\mathrm{mm})$ & 2.137 & 220 & 0.034 \\
\hline & core scar length $(\mathrm{mm})$ & 2.265 & 220 & 0.025 \\
\hline & maximum length $(\mathrm{mm})$ & 1.745 & 144 & 0.083 \\
\hline & maximum width $(\mathrm{mm})$ & 1.801 & 144 & 0.074 \\
\hline & maximum thickness $(\mathrm{mm})$ & 0.967 & 144 & 0.335 \\
\hline & core scar length $(\mathrm{mm})$ & 1.762 & 144 & 0.080 \\
\hline
\end{tabular}

Note: Dimensions for multiple core maximum length and thickness and core scar length show significant differences. assemblages discussed in chapter 5. However, both are at the higher end of the range for cobbles from the L1 and E29H1 assemblages reported in chapter 4. Thus, based on these estimates, the Kom K assemblages were flaked from raw material that differs in dimensions from that worked at E29H1, confirming the impressions formed by earlier researchers (Shirai 2010).

The uncorrected flake-to-core ratios for the Kom $\mathrm{K}$ surface and excavated assemblages, calculated by dividing the sum of all artifacts with a platform by the number of cores, are 7.96 and 16.35 , respectively. However, if the flake-to-core ratios are corrected using estimates of the size of the cobbles that were flaked, the value of the ratios reduces to 2.78 for the surface assemblage and 7.58 for the excavated assemblage. Both these values are considerably higher than that for the K1 assemblage (1.48) reported above. The value for the surface assemblage is at the upper end of the range of values for the E29H1 assemblages, but the value for the excavated assemblage is the highest yet reported, comparable to (although higher than) the values reported for XB11 and L1T5.

\section{Flakes}

As noted above, complete flake length-to-width ratio values suggest that flakes are relatively square, which is consistent with the shape of the flint nodules used and the likely flaking strategy employed (scenario two, as discussed in chapter 4; Figure 4.13). If the ratios are calculated for flakes with differing cortex proportions, results suggest that slightly longer flakes were removed during the middle stages of reduction, when flakes retained some cortex on their dorsal surfaces (Table 6.13). Complete flakes from excavated contexts are smaller than those from surface contexts.

The cortex ratio for Kom $\mathrm{K}$ surface artifacts is 0.7

Table 6.11. Core Type Axial Dimension Ratios for Kom K Surface and Excavated Assemblages for the Most Common Worked Core Types.

\begin{tabular}{|l|c|c|c|c|c|c|}
\hline $\begin{array}{l}\text { Kom K } \\
\text { Surface }\end{array}$ & & & \multicolumn{4}{|l|}{$\begin{array}{l}\text { Kom K } \\
\text { Excavated }\end{array}$} \\
\hline Core type & $\mathrm{a}: \mathrm{b}$ & $\mathrm{a}: \mathrm{c}$ & $\mathrm{b}: \mathrm{c}$ & $\mathrm{a}: \mathrm{b}$ & $\mathrm{a}: \mathrm{c}$ & $\mathrm{b}: \mathrm{c}$ \\
\hline Bifacial & 1.39 & $\mathbf{2 . 1 3}$ & 1.56 & 1.38 & $\mathbf{2 . 3 1}$ & 1.72 \\
\hline Multiple & 1.34 & $\mathbf{1 . 8 3}$ & 1.38 & 1.31 & $\mathbf{1 . 8 8}$ & 1.46 \\
\hline Unifacial & 1.42 & $\mathbf{2 . 0 6}$ & 1.49 & 1.43 & $\mathbf{1 . 9 6}$ & 1.40 \\
\hline
\end{tabular}

Note: The largest values are in bold. 
(Phillipps 2012), and the combined cortex ratio for all $\mathrm{Kom} \mathrm{K}$ material is 0.8 (0.77). These values are closer to those from the L1 assemblages and XB11 than they are to values for the E29H1 assemblages. The cortex ratio for $\mathrm{K} 1$ is much lower, indicating a significant loss of surface area, probably in the form of the transport of cores, as argued above. The volume ratio values for the Kom $\mathrm{K}$ surface and excavated assemblages also indicate core transport. If the total volume of the whole assemblage - that is, the volume of the cores added to the volume of all the flaked artifacts-is compared to the volume estimated on the basis of the number of cores found and the estimated volume of the cobbles, the resulting ratio has a value of 2.23 for the surface assemblage and 2.93 for the excavated assemblage. Stating this another way, there is sufficient volume to account for 921 cores in the surface assemblage, whereas only 414 cores were in fact found. The values for the excavated assemblage are 363 cores expected and 124 cores observed. These values for the volume ratio indicate some core movement but at much lower levels than evident for L1 assemblages, where the values were twice as high, but the Kom $\mathrm{K}$ values are very close to the value for the K1 assemblage (2.28).

Figure 6.15 updates Figure 5.12 with two additional green icons for the Kom $\mathrm{K}$ assemblages. These form a line that separates the E29H1 assemblages (the blue icons) and the yellow XB11 and L1 assemblages. The Kom K flakes have higher platform angles than the later assemblages and have platform widths closer to the value of XB11.

\section{Tools}

Tools are more common in the Kom $\mathrm{K}$ assemblage than in the rest of the $\mathrm{K} 1$ area. Table 6.14 includes

Table 6.12. Core Type Frequencies by Cortex Proportion for Surface and Excavated Assemblages.

\begin{tabular}{|l|l|r|r|l|r|r|r|r|r|}
\hline & Surface & \multicolumn{3}{|l|}{ Excavation } & \multicolumn{3}{|c|}{ Both } & \\
\hline Core type & None & $1-50 \%$ & $50-99 \%$ & None & $1-50 \%$ & $50-99 \%$ & None & $1-50 \%$ & $50-99 \%$ \\
\hline Bidirectional & 1 & 2 & 1 & 0 & 0 & 5 & 1 & 2 & 6 \\
\hline Bifacial & & 40 & 28 & & 11 & 5 & & 51 & 33 \\
\hline Microblade & & & & & & & 1 & & 1 \\
\hline Multiple & 23 & 138 & 19 & 3 & 31 & 10 & 26 & 169 & 188 \\
\hline Test & & 4 & 44 & & 6 & 16 & & 10 & 60 \\
\hline Tranchet & & 1 & & & & & & 1 & 76 \\
\hline Unifacial & 3 & 50 & 59 & & 16 & 20 & 3 & \\
\hline
\end{tabular}

Table 6.13. Complete Flake Dimensions with Different Proportions of Dorsal Cortex.

\begin{tabular}{|l|r|r|r|r|r|r|r|r|}
\hline & \multicolumn{2}{|l|}{ Surface } & & & Excavation & & \\
\hline Cortex & $\begin{array}{l}\text { Flake } \\
\text { (n) }\end{array}$ & $\begin{array}{l}\text { Flake Length } \\
\text { Mean (mm) }\end{array}$ & $\begin{array}{l}\text { Flake Width } \\
\text { Mean (mm) }\end{array}$ & Ratio & Flake (n) & $\begin{array}{l}\text { Flake Length } \\
\text { Mean (mm) }\end{array}$ & $\begin{array}{l}\text { Flake Width } \\
\text { Mean (mm) }\end{array}$ & Ratio \\
\hline Complete & 234 & 22.75 & 19.76 & 1.22 & 111 & 20.91 & 17.66 & 1.25 \\
\hline $50-99 \%$ & 706 & 24.86 & 20.37 & 1.3 & 204 & 21.71 & 18.12 & 1.28 \\
\hline $1-50 \%$ & 2,940 & 22.64 & 20.01 & 1.23 & 898 & 19.57 & 17.97 & 1.17 \\
\hline None & 1,081 & 19.38 & 17.35 & 1.2 & 442 & 15.90 & 14.81 & 1.17 \\
\hline
\end{tabular}




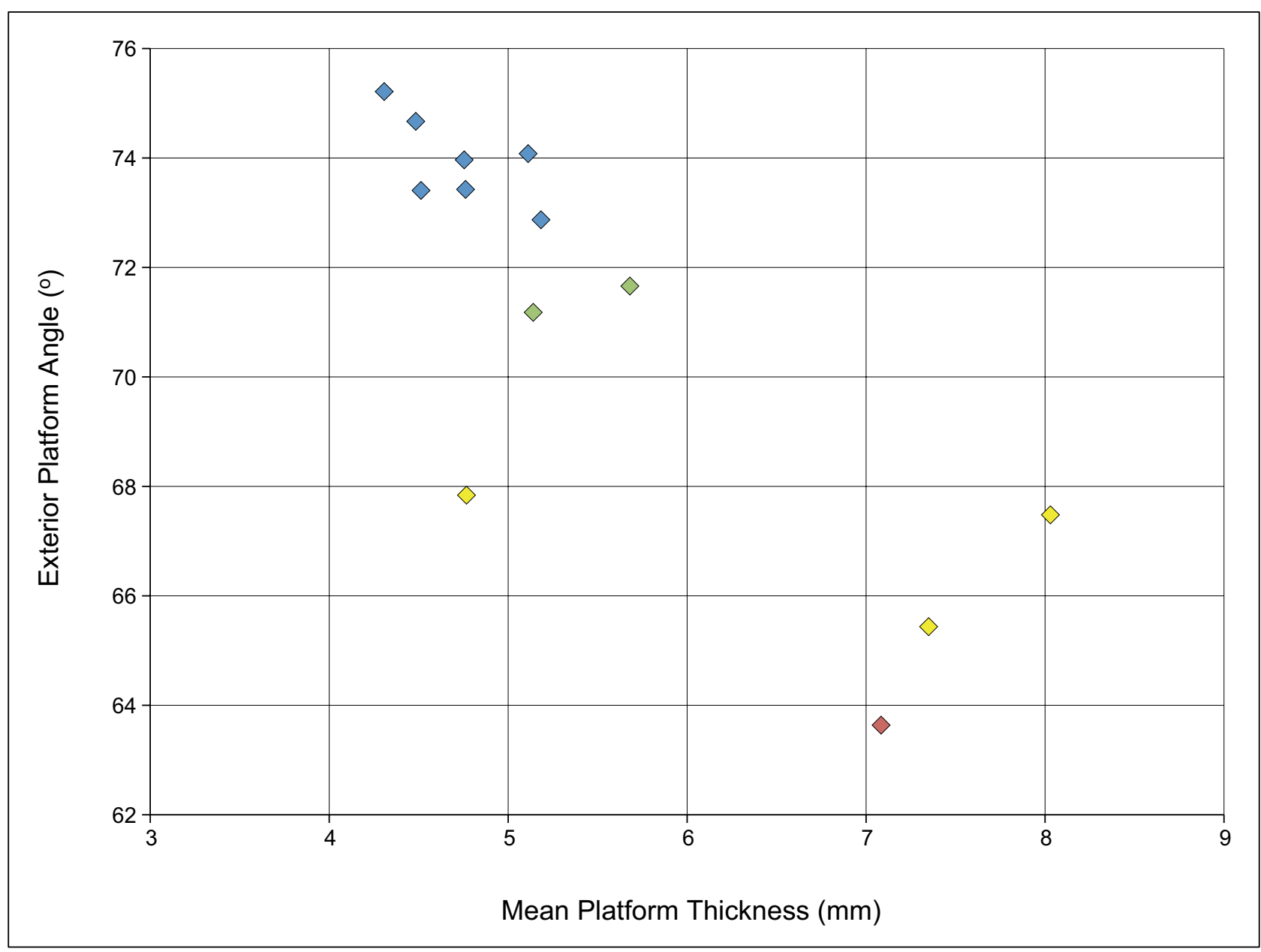

Figure 6.15. Mean complete flake platform thickness against mean exterior platform angle. Green icon: Kom K surface and excavated assemblage; red icon: K1 assemblage; yellow icons: L1 and XB11 assemblages; blue icons: E29H1 assemblages.

all tools from surface and excavated contexts. Denticulates are the most common tool type, after which utilized flakes, scrapers, and notches outnumber bifacial tool types, including sickles. While Caton-Thompson and Gardner recorded bifacial points at Kom K (1934:39), none have been recorded in the material collected and analyzed by this project to date.

As observed for the other assemblages, average complete tool size for Kom $\mathrm{K}$ is large compared to flake size, which may suggest that the largest flake blanks are selected for retouch. The average sizes for excavated tools (length $=26.34 \pm 6.9 \mathrm{~mm}$, width $=$ $22.95 \pm 5.69 \mathrm{~mm}$, thickness $=8.56 \pm 2.04 \mathrm{~mm})$ and surface collected tools (length $=36.06 \pm 8.2 \mathrm{~mm}$, width $=25.83 \pm 7.1 \mathrm{~mm}$, thickness $=10.69 \pm 3.7$ $\mathrm{mm}$ ) conform to this pattern.

\section{Discussion}

The artifacts analyzed from Kom $\mathrm{K}$ are distributed across the deflated and disturbed surface of the site, and those that form the excavated sample come from a smaller number of locations, as described at the beginning of this chapter. Cores occur in the same proportions in the two assemblages and, apart from multiple cores, are of similar size. Core dimensions fall midway between those from E29H1 and from L Basin. The relative dimensions of the cores suggest that those from Kom $\mathrm{K}$ were flaked in a similar way to those from $\mathrm{L}$ Basin but not those from E29H1. Estimates of original cobble dimensions based on the cores are large compared to the assemblages farther west but not nearly as large as the estimates for $\mathrm{K} 1$. The results suggest that assemblages from different parts of the Fayum were created by people who made use of differently sized 
Table 6.14. Retouched Tools from Kom K from Surface and Excavated Contexts Combined.

\begin{tabular}{|c|c|c|c|c|c|c|c|}
\hline Tool Type & $\begin{array}{l}\text { Angular } \\
\text { Fragment }\end{array}$ & $\begin{array}{l}\text { Complete } \\
\text { Split Tool }\end{array}$ & $\begin{array}{l}\text { Complete } \\
\text { Tool }\end{array}$ & Distal Tool & Medial Tool & $\begin{array}{l}\text { Proximal } \\
\text { Tool }\end{array}$ & $\begin{array}{l}\text { Proximal Split } \\
\text { Tool }\end{array}$ \\
\hline Axe & & & 1 & & & & \\
\hline Bifacial & 4 & & 1 & & 3 & & \\
\hline Denticulate & 41 & 3 & 116 & 4 & & 9 & 1 \\
\hline Drill & & & & 1 & & & \\
\hline Notch & 11 & 6 & 30 & 4 & 1 & 3 & \\
\hline Pebble-backed & & & 3 & & & & \\
\hline Scraper & 11 & 1 & 32 & 1 & 2 & 1 & \\
\hline Sickle & & & & 2 & & & \\
\hline Utilized & 18 & 5 & 41 & 3 & & 3 & 2 \\
\hline
\end{tabular}

raw material. This would be consistent with the cobbles coming from different sources.

Given the radiocarbon chronology for Kom K, differences between the surface and excavated assemblages may represent changes through time. The overall result of the cortex ratio when compared to the rest of the K1 area suggests that very little cortex was lost from Kom K. The volume ratio similarly suggests less loss of volume in comparison to Kom W (Phillipps 2012). These results together may suggest that much of the raw material used at Kom $\mathrm{K}$ was reduced and discarded in place. Any material that was transported away was likely in the form of cores, with proportionately less surface and greater volume than flakes.

Flakes struck at Kom $\mathrm{K}$ were square in outline and are smaller in the excavated assemblage than those from the surface. It is possible that this reflects post-depositional disturbance on the surface of the site that differentially removed some of the smaller flakes, hence increasing the mean dimensions of those that remain, although there is no direct indication of how this occurred. The flakes have platform thickness and exterior platform angle measurements that place them in an intermediate position between the flakes from the E29H1 assemblages and those from L1 Basin. They are closest to the assemblage from K1. Tools are more common at Kom $\mathrm{K}$ than in the other E29H1 assemblages, but not the point and sickle forms that Caton-Thompson and Gardner recorded. It seems likely that for the surface assemblage, this reflects a long history of collection. However, it is harder to use this explanation for the excavated assemblages. Evidently these forms were not common in the areas that were excavated. As we discuss below, the distinctive aspects of the Kom $\mathrm{K}$ flaked stone artifact assemblages are best understood in relation to the history of occupation of the site as inferred from both the superimposed hearth features, as well as other forms of material culture, and the fauna, to which we turn shortly.

\section{Ceramics}

The ceramics from Kom $\mathrm{K}$ are generally considered similar to those from Kom W and the Upper K Pits, based on Caton-Thompson and Gardner's description. Ceramics were collected from surface sample squares in the same way as lithics, although a different sample of squares was analyzed (Figure 6.16). There is no apparent pattern to the density of the ceramics selected from these squares. Excavations have not uncovered any complete ceramic vessels since those found by CatonThompson, but numerous sherds were identified during the current excavations. In total, 1,335 ceramic sherds were uncovered from Trenches KK02 to KK08 on Kom K. A small number of sherds with incised decoration were found (Figure 6.17). Decoration does not exist on any of the surviving vessels from Caton-Thompson and Gardner's excavations.

A large number of sherds were found in the Trench KK02 [02.0023] hearth fill, perhaps suggesting that the hearth was filled with a broken vessel or that sherds were used as heat retainers in the hearth. Fills of other hearths (for example, [04.0036]) also contained high numbers of sherds but with no difference in matrix from the deposit units stratigraphically above. These might also indicate that sherds were used to fill hearth depressions or that sherds were deliberately used in construction of the hearths, but it is also possible that 


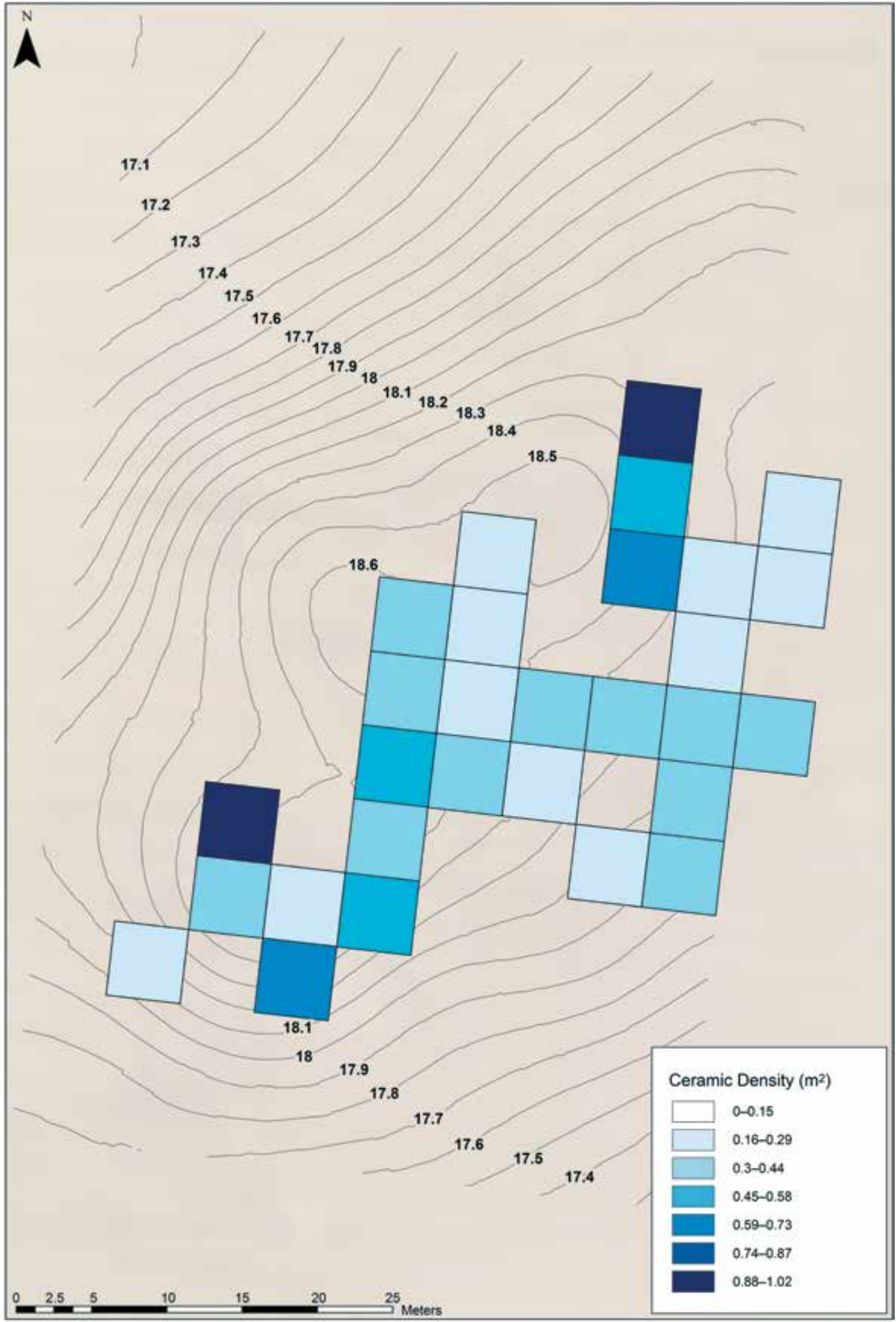

Figure 6.16. Density of ceramics recorded in analyzed Kom K sample squares. 


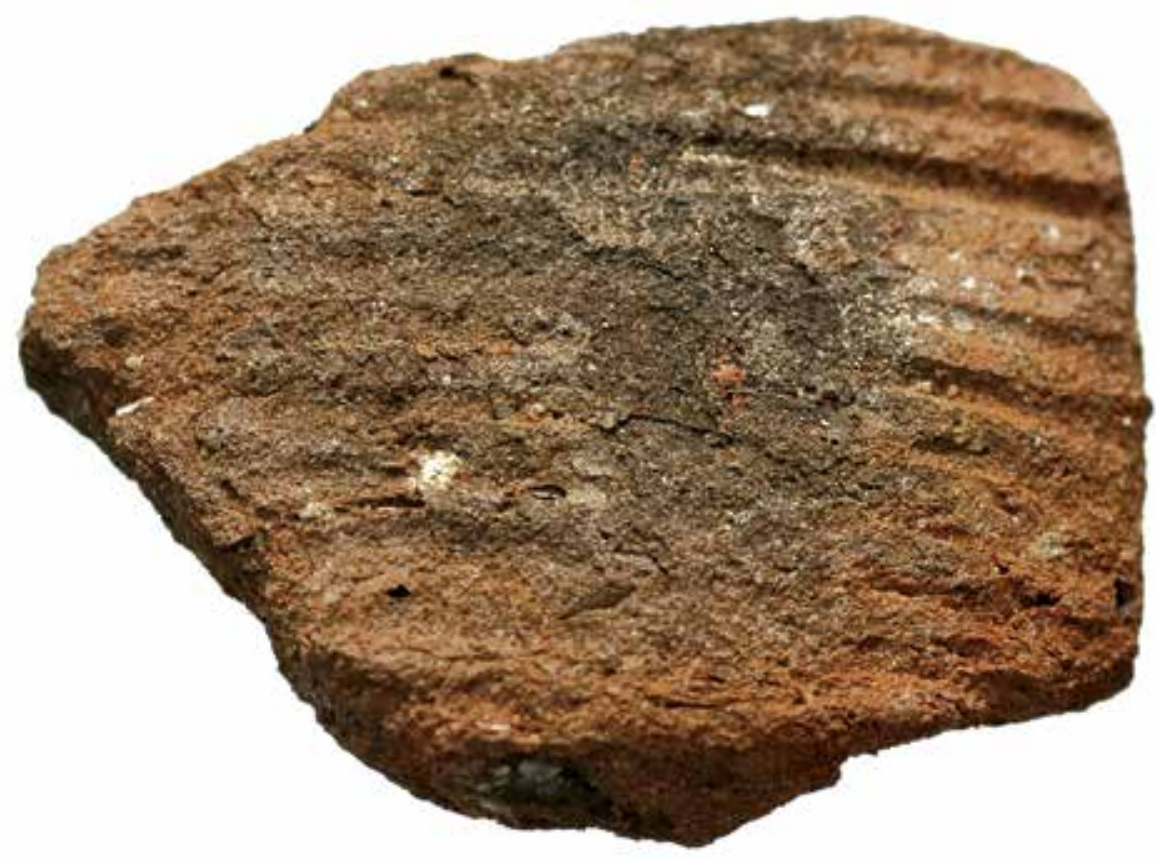

Figure 6.17. Kom K sherd with parallel incised line decoration.

$3 \mathrm{~cm}$

they were combined into pit fills from adjacent deposits. Some of the cuts identified at Kom K may be pit features originally used to support ceramic vessels, as is suggested for Kom W (Emmitt 2011), although further research is required to support this. Based on results from the trenches excavated, the majority of cuts are associated with fills that suggest they represent hearths. Additional studies of Kom K ceramics will be reported in a future volume.

\section{Personal Ornaments}

Eleven small, simple beads made from pieces of ostrich eggshell were found in KK05 and KK07 (each with four beads), while single beads were found in KK04, KK06, and KK08. The beads are slightly convex in shape and range between 1.8 and $2 \mathrm{~mm}$ thick. Most beads are between 7 and $9 \mathrm{~mm}$ in length. Some larger pieces (up to $16 \mathrm{~mm}$ in length) may be fragments of pendants or vessels rather than beads. Two of the larger pieces from trench KK07 have perforated holes.

Most beads are angular or irregular in shape, with a smaller number having an oval or circular form (Figures
6.18 and 6.19). The edges were shaped by abrasion, probably with the help of a smooth grinding stone. Under the microscope, the worked edges of most beads show abrasion traces that run perpendicular or oblique to the edge. Almost all beads have chipped edges. These are old fractures, and it is not clear whether they were left unfinished by the bead-maker or whether the edges broke at a later date. At least some beads were subject to minimal shaping efforts only, with a hole drilled into a roughly chipped bead blank without any traces of further abrasion or polishing.

In most cases, the hole was drilled from the ventral surface of the shell fragment. When the drill had almost penetrated the shell, the remaining shell broke away, leaving chipped edges around the hole on the dorsal surface. A smaller number of beads show evidence of additional drilling from the dorsal surface. In one case, two holes were drilled from the dorsal surface. Most drill holes have a conical cross-section, with a larger diameter (2.6 to $4.5 \mathrm{~mm}$ ) on the ventral than on the dorsal surface (1.5 to $2.1 \mathrm{~mm}$ ). In two cases, the drill left concentric scratches around the drill hole in an 


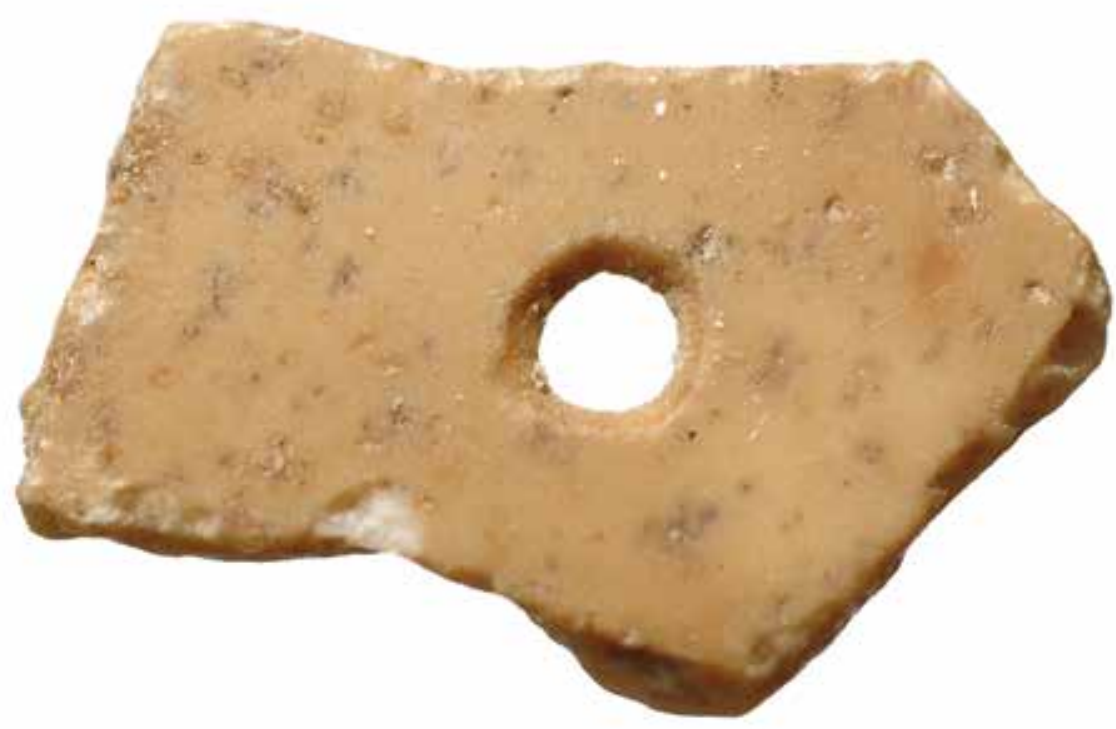

Figure 6.18. Incomplete ostrich eggshell bead.

\section{$2 \mathrm{~cm}$}
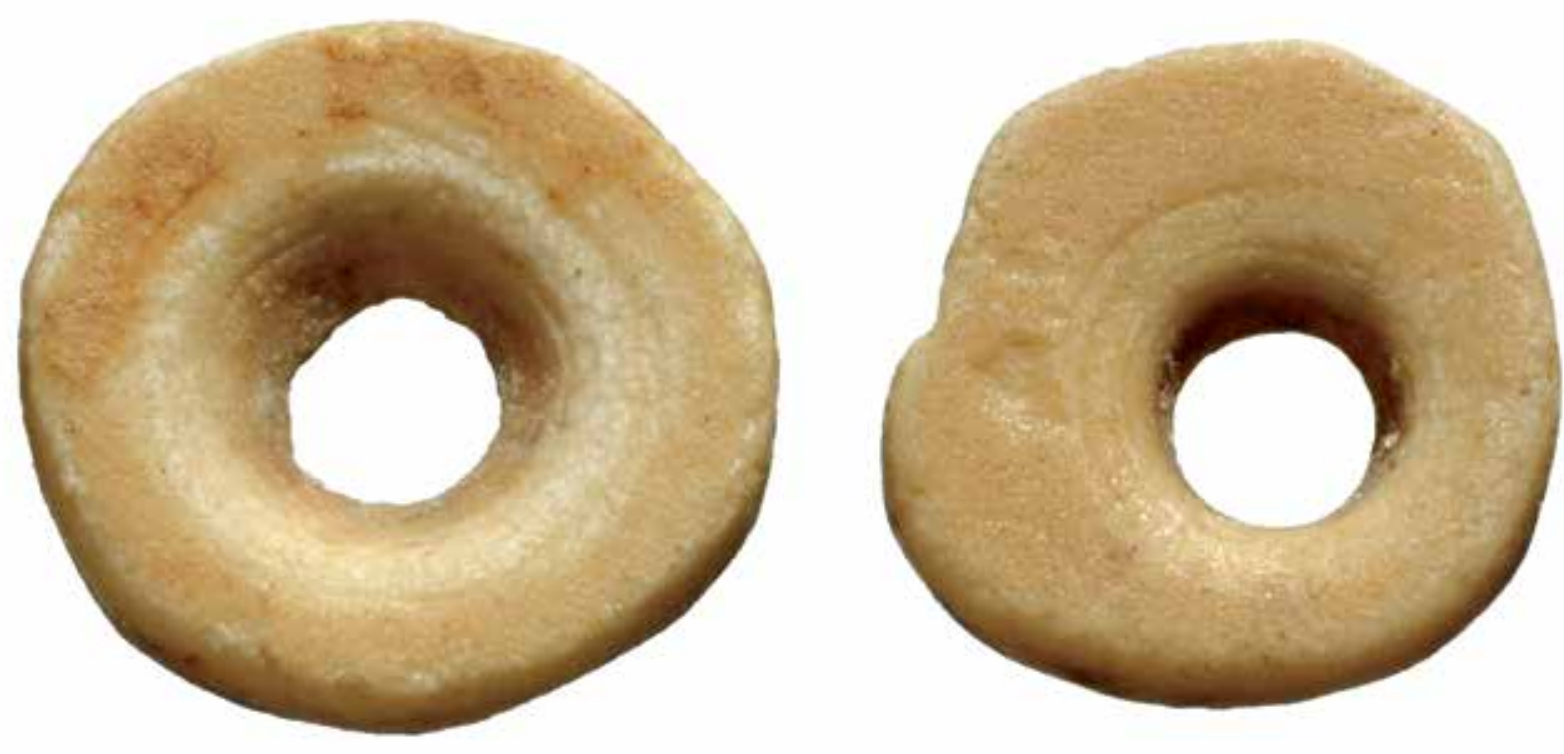

Figure 6.19. Complete ostrich eggshell beads.

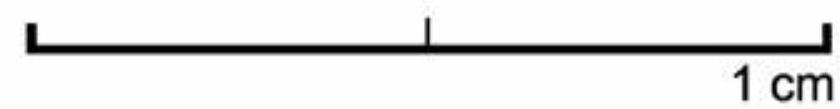


area up to $6 \mathrm{~mm}$ in diameter. In two beads, the drill bit seems to have shifted sideways during the drilling, resulting in a stepped drill-hole section. In two other cases, the drill hole has an almost cylindrical section.

One tubular bead made from animal bone was found in KK05 (Figure 6.20). The bead was made from a dark brown thin mammal bone of unknown species. The length of the bead is $11 \mathrm{~mm}$ and the diameter is $4 \mathrm{~mm}$. The bone was not pierced and may have been strung using the medullary cavity. The bone was cut obliquely at both ends. The outside surface of the bone and the cut edges are smooth and shiny.

Two small stone beads were found in KK02 and KK05. The first is an oval disk-shaped bead made from a gray-black stone, possibly limestone, about $6.5 \mathrm{~mm}$ in diameter and $1.2 \mathrm{~mm}$ thick. The stone is fine grained and seems to naturally split in thin layers. One surface was polished into a shiny surface, while the other surface was left dull. The edges were abraded, creating facets. The bead was drilled in the center from two sides, creating a biconical 2-mm-wide hole. The second bead is an irregularly shaped angular flat piece of light beige-gray, fine-grained stone, also possibly limestone, $9 \mathrm{~mm}$ in length by $7 \mathrm{~mm}$ wide and $1.5 \mathrm{~mm}$ thick. All edges are roughly chipped. A

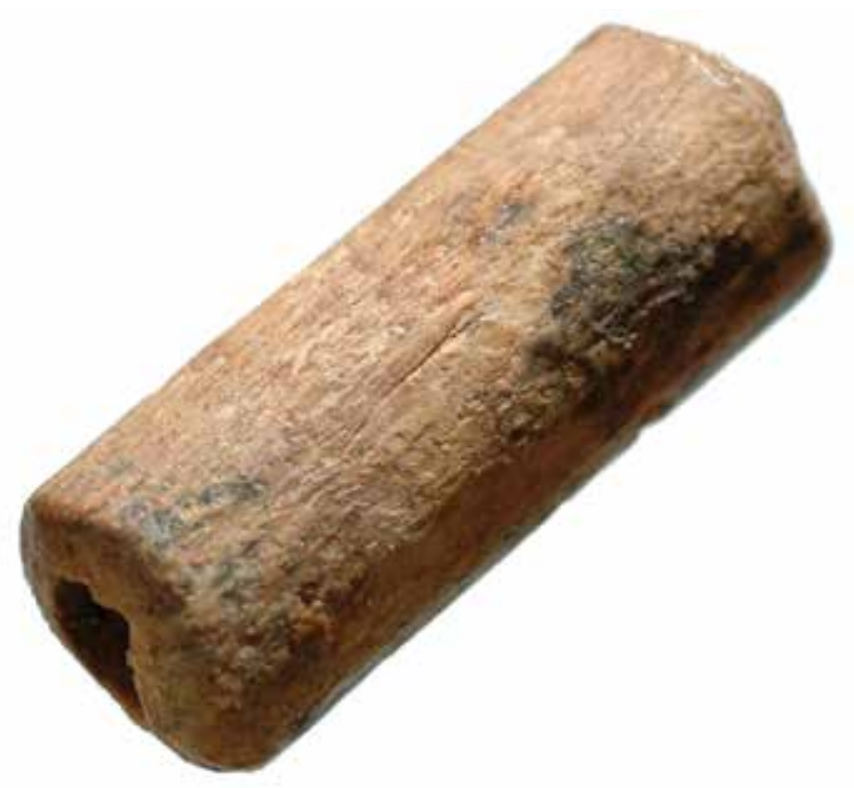

L

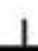

hole was drilled from two sides, creating a biconical 1-mm-wide hole. The drilling left traces of 2 to $3 \mathrm{~mm}$ in diameter on both sides around the hole.

Two fragments of what may be bone rings were found in KK04 and KK07 (Figure 6.21). Both were made from the humerus of an ovicaprid or other similarly sized animal. The first fragment is a partially preserved ring, $19 \mathrm{~mm}$ in diameter and 4 to $6 \mathrm{~mm}$ thick. It was made by sawing a slice from a longer bone. One side was completely sawn through, while the other side was partly sawn and partly broken. The sawing resulted in oblique sides, creating a trapezoidal section of the ring. The surfaces were not smoothed further or worked.

The second fragment comes from the upper part of a humerus and is $41 \mathrm{~mm}$ long, $18 \mathrm{~mm}$ wide, and $2 \mathrm{~mm}$ thick. The artifact is eroded and sawn at one end, leaving a slightly oblique incision. Two other incisions run parallel to this cut, at distances of $6 \mathrm{~mm}$ from each other. It may be that the bone was used as a blank from which bone rings were cut but the bone broke before the last two rings could be sawn off.

In KK03 was found a fragment from a bracelet or a large ring, made from a marine shell of unknown species. The fragment is $58 \mathrm{~mm}$ long, $9 \mathrm{~mm}$ wide, and $5 \mathrm{~mm}$ thick. The ring form was cut from a larger shell. The ventral surface of the ring shows the spiral structure of the ventral surface of the shell. No traces of sawing, abrasion, or other working are visible. If the original shell was circular, the outer diameter of the ring would be $75 \mathrm{~mm}$.

In KK05 was found a partly damaged tool made from a half shell of the freshwater bivalve Aspatharia sp. The whole outer edge of the shell is abraded through, changing the original shape and creating a rim with a square section. Under the microscope, faint scratches are visible on the flat side of the rim, perpendicular to it, but not on the ventral or dorsal surface of the shell.

Three small pieces of worked ostrich eggshell were found in KK04, KK07, and KK08 (Figure 6.22). All are small, angular pieces, each with one or more abraded straight edges. The two largest pieces are approximately $38 \times 25 \mathrm{~mm}$ in size. The straight edges show traces of abrasion perpendicular to the edge. It is possible that these fragments once belonged to an ostrich eggshell vessel.

Figure 6.20. Bone bead. 


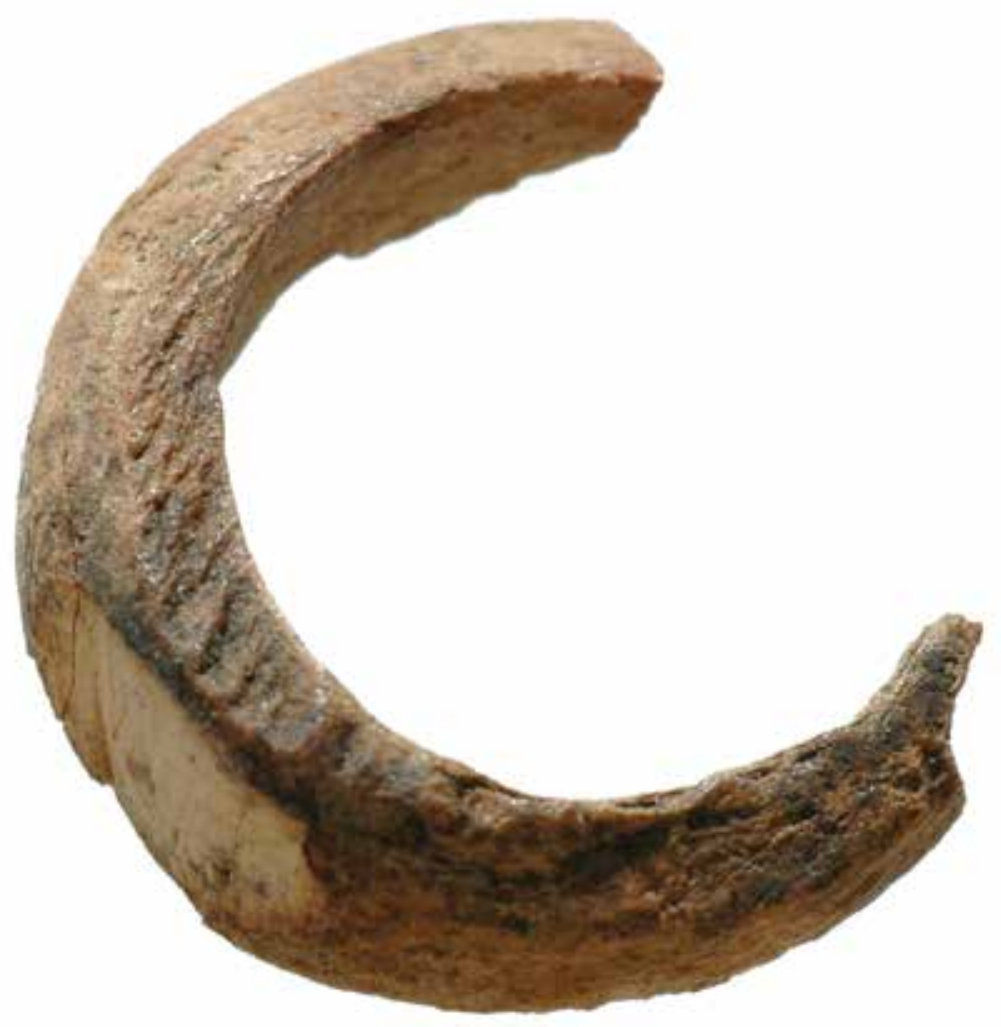

Figure 6.21. One of two fragmentary bone rings found in Kom K.
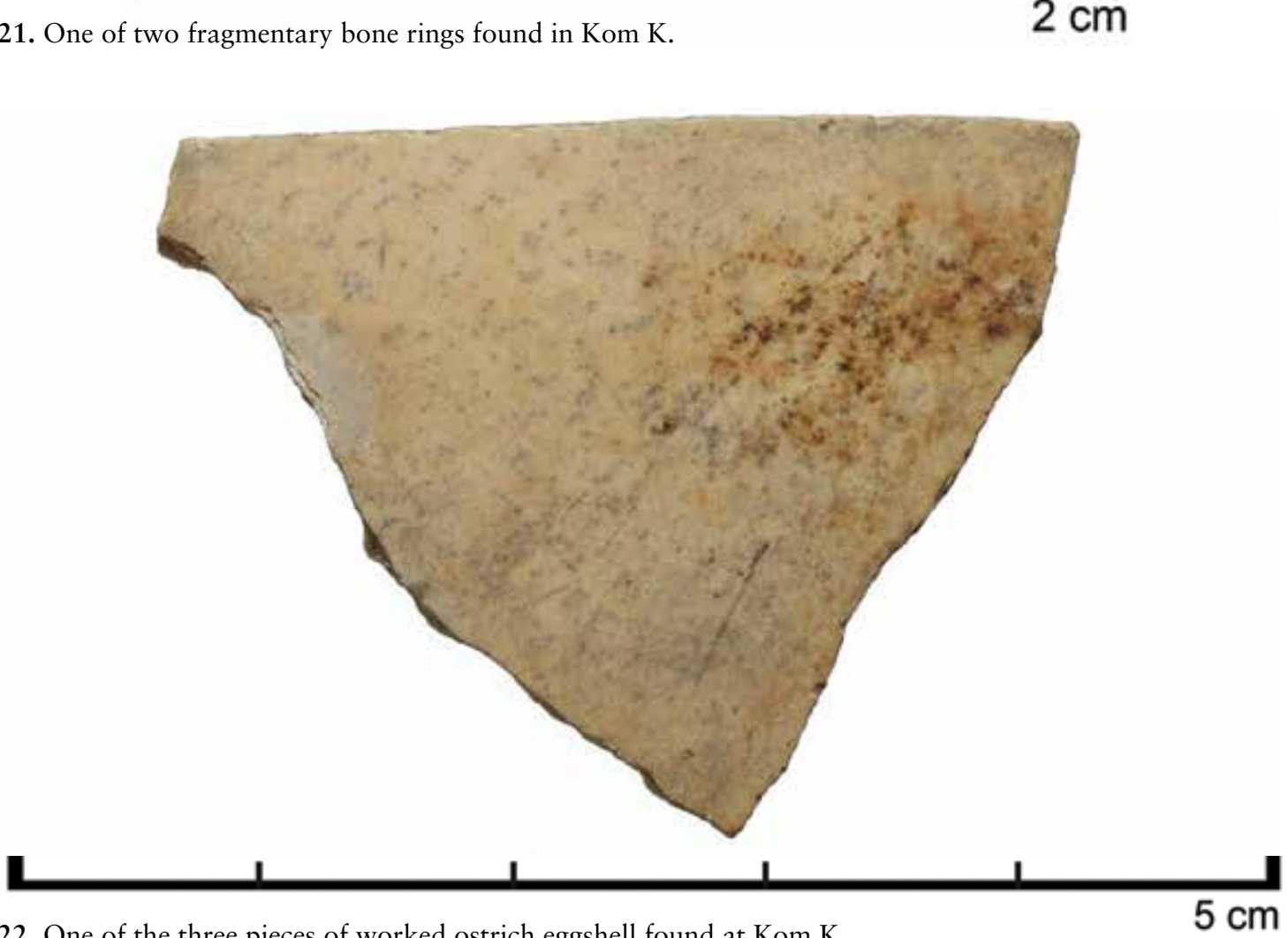

Figure 6.22. One of the three pieces of worked ostrich eggshell found at Kom K. 


\section{Faunal Remains}

The fauna from Kom $\mathrm{K}$ and methods used in the study of these assemblages are reported in Linseele et al. (2014). Archaeological deposits at Kom K were systematically dry-sieved in the field through 2-mm meshes, and finds were picked out in the field. The meshes were fine enough to guarantee that most of the animal bones were collected (cf. Payne 1972). Bulk sediment samples were taken from several units, mostly hearths. These were sieved through 5-mm, 2-mm, and 1-mm meshes, mainly to retrieve archaeobotanical remains. Animal remains were picked out from the sieve residues ( $5 \mathrm{~mm}$ and $2 \mathrm{~mm}$ only). The faunal composition of these samples suggests that the sampling in the field results in minimal loss (Table 6.15).

Approximately 150,000 animal remains, mainly shells, bones, and teeth, were collected and studied from Kom K. This is by far the largest faunal sample for early- to mid-Holocene contexts in the Fayum. Excavated units from Kom K directly associated with the hearths discussed above were not particularly rich in faunal remains. It is rather in the ashy, sand layers with which the hearths are associated where most of the bones were found. The preservation of the faunal remains from Kom $\mathrm{K}$ was poor, however. As shown below, they are less effected by preservation than surface samples, where mainly hard, compact bones were preserved. Fragmentation of the remains is common and apparent in the large numbers of bovid teeth splinters (Linseele et al. 2014). The uneven appearance of the bones suggests exposure and reburial, processes that are not unexpected given the stratigraphic contexts of the hearths discussed above.

For Kom K, 10.6 percent of the bone remains were identifiable, meaning that the skeletal element was determined and the specimen was attributable to an animal taxon below class level (Table 6.15). In comparison, identification rates for E29H1 vary according to the area but were usually around 30 percent (chapter 4 ).

A part of the fauna from Kom $\mathrm{K}$ is probably intrusive, notably the numerous small shells but also various groups of small vertebrates (Table 6.15). The small shells include several species of freshwater gastropods, mainly represented by Cleopatra bulimoides, as well as freshwater bivalves, mainly Corbicula. In addition to the freshwater shells, there were also fossil shells and shark teeth, which must have come from marine deposits within the vicinity. The small vertebrate species, such as rodents and lizards, probably lived and died at Kom K around the time people were present.
With these exceptions, the majority of the faunal remains recovered likely represent food refuse. The faunal remains are discussed by larger animal group, in order of increasing numbers of identified remains: marine shells, birds, wild terrestrial animals, domesticated animals, and fish and turtles.

Only a few species of marine shells were recorded. Nerita sp. can be found only in the Red Sea, but all other taxa also occur in the Mediterranean (Poppe and Goto 1991; Sharabati and Sharabati 1984). Bird bones are not common, but this may be due to taphonomic reasons. Predominantly aquatic taxa were recorded, including ducks, water rail, and coot. Wild mammals, among which are cat, fox, hare, hippopotamus, Dorcas gazelle, and hartebeest, represent less than 1 percent of the identified vertebrate fauna. The presence of aurochs could not be confirmed. The two measurable cattle bones from Kom $\mathrm{K}$ fall in the range of domesticated cattle. Another hunted species is the monitor lizard. The terrestrial, wild game indicates an environment that is not unlike the present one, although the sample sizes involved are too small to draw definitive conclusions.

All major domesticated species present at other Lower Egyptian mid-Holocene sites, including dog, pig, sheep, goat, and cattle, were probably present at Kom K. Making a distinction between the domestic forms and their wild relatives is not always possible on a purely osteological basis. However, in the case of the ovicaprines as well as the cattle, all available measurements are congruent with the domesticated forms, which are smaller than the wild ones. No measurements could be taken on the pig bones, but the absence of wild pigs in the older assemblages suggests that they are most likely from domesticates.

A canid metapodal from Kom $\mathrm{K}$ showed cut marks on its distal end and on the diaphysis. These marks are likely linked with skinning. Throughout the different trenches at Kom K, domesticates are present in similar proportions (Figure 6.23). In Trench KK06, small bovid teeth fragments are more frequent that elsewhere, but overall bones of caprines are the most numerous. In total at Kom K, 15 sheep bones were identified, compared to seven goat bones.

Sheep are grazers while goats are browsers that can live on a more varied diet. Of the domesticated species, data on ages at death could be recorded only for caprines. They indicate that the animals died young; about half did not reach the age of 13 to 16 months. Domesticated pig is present in the assemblage. 


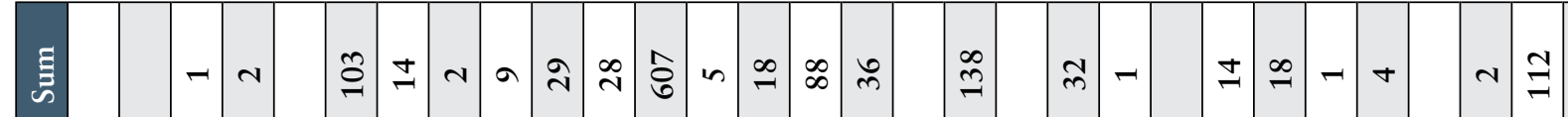
$a, \cdot-r)=0$

w

is

$-1 \cdot 1 \cdot 1 \cdot-4$

f

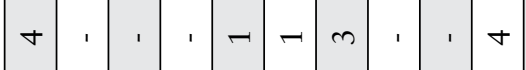

$-$

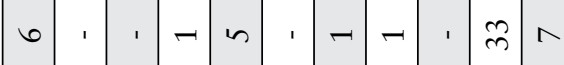

$-1-1 \infty$

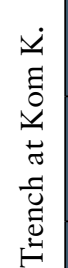

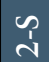

$\checkmark$

ᄂ

\begin{tabular}{|l|l|l|l|}
\hline & 1 & \\
\hline
\end{tabular}

'

$\cdot-$

$\widehat{\overline{\hat{n}}}$

密

:

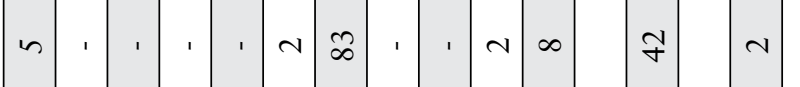

:

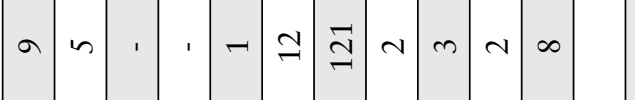

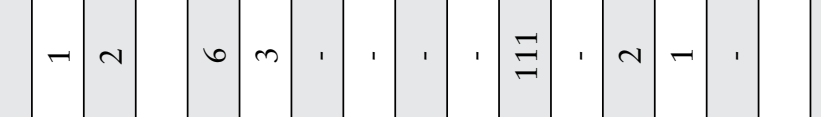

$\stackrel{\infty}{\infty}$ in $-r$

$+\left[\begin{array}{ll|lll}0 & 0 & 0 & n & n\end{array}\right.$

in $\operatorname{in}$

$n+$

in

宽

峁

言

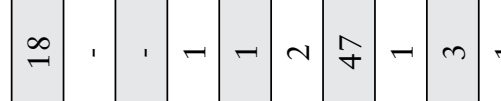

$\because-$

$n e-4$

กิ

荧

ฮี

ت

능

$\stackrel{4}{3}$

$\dot{m}$

ำ

䨔 
Wendrich • Phillipps • Holdaway • Linseele • Emmitt • Marston

ह) co is. 6

i $\underset{+}{+}-$

m 2

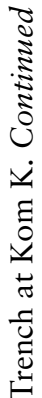

20

$\widehat{\overline{\mathrm{n}}}$
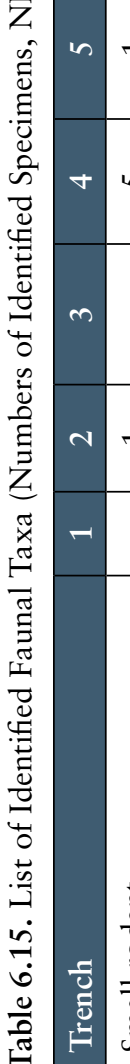

$-\infty-$

$-a m$

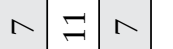

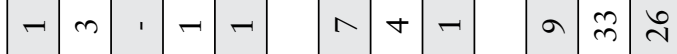

으ำ 은

$\stackrel{\circ}{\circ}$ ก

n一

in

$-$

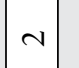

$-\infty m$

๑

$6 m$

के $n$

- $1 . m$. m n m

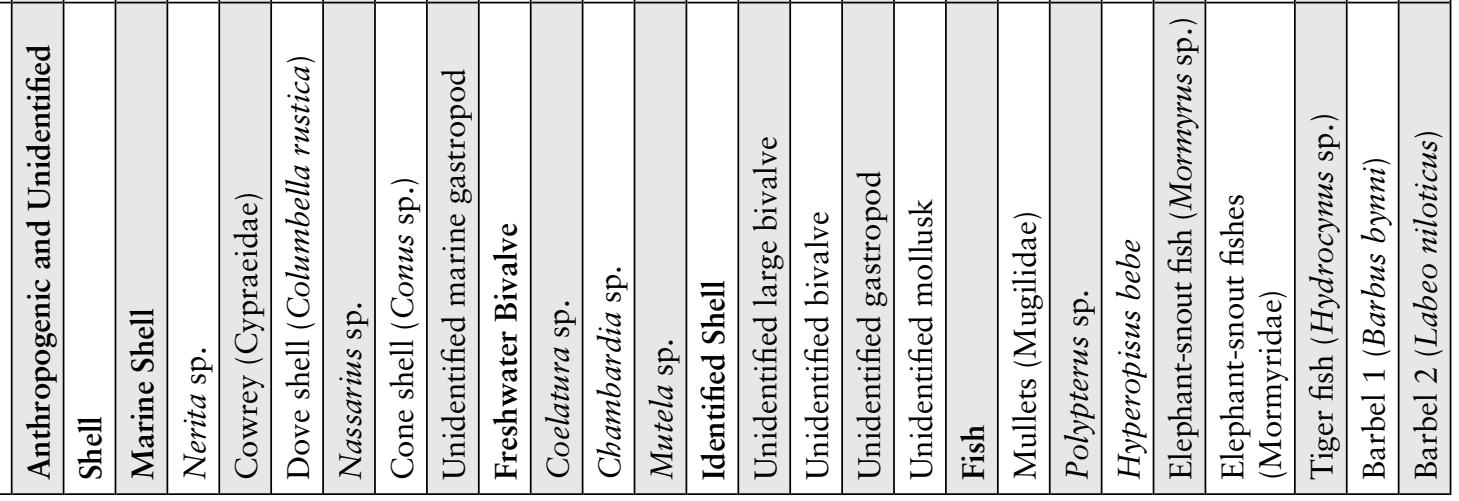




\begin{tabular}{|c|c|c|c|c|c|c|c|c|c|c|c|c|c|c|c|c|c|c|c|c|c|c|c|c|c|c|c|c|c|}
\hline हే & $\Lambda$ & $\stackrel{m}{m}$ & $\mathrm{~N}$ & - & ి & $\neg$ & 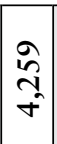 & - & $\infty$ & 우 & $\begin{array}{c}\underset{ \pm}{\sim} \\
\stackrel{\sim}{\sim}\end{array}$ & $\begin{array}{l}n \\
\tilde{n} \\
-1\end{array}$ & 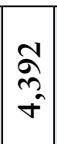 & 워 & $\begin{array}{c}\infty \\
\underset{\nabla}{\sigma} \\
\dot{m}\end{array}$ & $\begin{array}{l}n \\
n \\
7 \\
\sigma\end{array}$ & & $\nabla$ & $\widehat{へ}$ & $\bar{m}$ & & 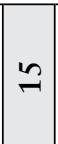 & - & - & $\stackrel{6}{1}$ & -1 & ఉ & $\stackrel{\infty}{\sim}$ & $\infty$ \\
\hline$\alpha$ & & ' & I & ' & I & I & ' & ' & ' & 1 & $N$ & $m$ & ' & ' & in & $\approx$ & & ' & 1 & 0 & & ' & ' & ' & ' & ' & 0 & ' & ' \\
\hline P & ' & $\neg$ & ' & ' & ' & ' & $\Lambda$ & ' & ' & I & $a$ & $\approx$ & $\stackrel{\infty}{\rightarrow}$ & ' & 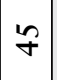 & $\widehat{\curvearrowright}$ & & ' & ' & 0 & & ' & 1 & ' & ' & ' & 0 & 1 & 1 \\
\hline b & ' & I & I & 1 & ' & ' & ' & I & ' & ' & - & ' & $m$ & ' & $\nabla$ & $\mathcal{N}$ & & I & ' & 0 & & 1 & 1 & I & 1 & ' & 0 & ' & 1 \\
\hline 4 & & I & ' & ' & ' & ' & $n$ & ' & ' & 1 & $\infty$ & $\infty$ & $a$ & ' & $\vec{m}$ & $\stackrel{\infty}{\infty}$ & & ' & ' & 0 & & ' & ' & ' & 1 & ' & 0 & ' & . \\
\hline f & & $m$ & 1 & ' & - & ' & $\stackrel{ \pm}{\sim}$ & ' & ' & ' & -1 & $\nabla$ & $\approx$ & ' & $\stackrel{m}{\sim}$ & $\stackrel{2}{R}$ & & ' & ' & 0 & & ' & 1 & ' & 1 & ' & 0 & ' & - \\
\hline $\begin{array}{l}n \\
m\end{array}$ & ' & $\stackrel{6}{\models}$ & I & ' & ' & - & 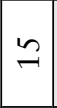 & ' & ' & ' & ชె & $\infty$ & ㄴ. & ' & $\stackrel{\infty}{\sim}$ & $\begin{array}{c}\hat{\lambda} \\
\\
-1\end{array}$ & & ' & $\neg$ & - & & ' & ' & ' & ' & ' & 0 & 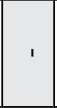 & 1 \\
\hline $\begin{array}{l}n \\
\sim \\
\sim\end{array}$ & & ' & ' & ' & I & ' & ' & ' & ' & 1 & ' & -1 & $N$ & ' & $m$ & $\infty$ & & ' & ' & 0 & & ' & ' & ' & ' & ' & 0 & ' & ' \\
\hline$\infty$ & -1 & $a$ & ' & ' & I & ' & $\stackrel{\circ}{\sim}$ & ' & $\neg$ & 1 & $\stackrel{\mathcal{F}}{+}$ & 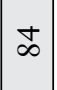 & ন & $\nabla$ & $\begin{array}{l}n \\
\infty \\
m\end{array}$ & $\stackrel{6}{2}$ & & ' & $N$ & $N$ & & - & 1 & ' & ' & ' & - & -1 & $\hat{n}$ \\
\hline$\Lambda$ & $\sim$ & $\overleftarrow{b}$ & $v$ & ' & $n$ & ' & 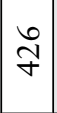 & ' & $\cong$ & $\approx$ & gั & $\stackrel{\circ}{m}$ & 亩 & $N$ & $\begin{array}{l} \pm \\
\\
0 \\
\text { í }\end{array}$ & $\begin{array}{c}1 \\
\infty \\
0 \\
\approx \\
=\end{array}$ & & $\neg$ & $\neg$ & $\mathrm{N}$ & & - & ' & I & $\nabla$ & ' & in & in & $\stackrel{\sim}{\sim}$ \\
\hline 6 & & $\stackrel{\infty}{\sim}$ & ' & ' & 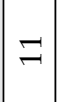 & ' & $\stackrel{n}{n}$ & ' & $\stackrel{m}{=}$ & $\infty$ & $\stackrel{\mathbb{N}}{\mathrm{N}}$ & $\begin{array}{l}\stackrel{0}{0} \\
\stackrel{2}{n}\end{array}$ & ్ㅡ & ' & $\begin{array}{l}\infty \\
\underset{\sim}{\sim} \\
\sim\end{array}$ & 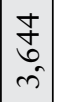 & & $\neg$ & $m$ & $\nabla$ & & 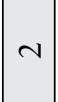 & ' & 1 & $n$ & ' & in & $N$ & $\stackrel{+}{m}$ \\
\hline in & $N$ & $\stackrel{n}{n}$ & ' & ' & $\sigma$ & ' & 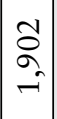 & $\neg$ & $\stackrel{\infty}{\infty}$ & - & $\tilde{a}$ & $\vec{\varpi}$ & $\left|\begin{array}{c}\mathcal{N} \\
\infty \\
\sim \\
\sim\end{array}\right|$ & $=$ & $\begin{array}{l}\hat{s} \\
\stackrel{+}{+} \\
\text { in }\end{array}$ & $\stackrel{\infty}{\sigma}$ & & ' & 6 & 6 & & $\Lambda$ & - & ' & - & ' & $\sigma$ & $m$ & $\overline{6}$ \\
\hline$\forall$ & - & ปี & ' & ' & - & ' & ㅊ & ' & in & ' & 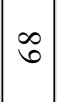 & $\stackrel{\sim}{ \pm}$ & i & ' & $\stackrel{\substack{\infty \\
=}}{=}$ & $\mid \begin{array}{l}6 \\
\infty \\
\wedge \\
\sigma^{2}\end{array}$ & & $\neg$ & $m$ & $\nabla$ & & ' & ' & ' & $\neg$ & ' & - & $m$ & $\stackrel{\infty}{\sim}$ \\
\hline$m$ & - & in & ' & I & I & ' & $\frac{a}{6}$ & ' & $\nabla$ & $\nabla$ & $\underset{\forall}{\stackrel{7}{\checkmark}}$ & $\stackrel{\tilde{\sim}}{\tilde{v}}$ & 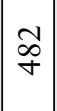 & N & $\begin{array}{l} \pm \\
\infty \\
- \\
-1\end{array}$ & $\begin{array}{l}0 \\
m \\
n \\
\sigma\end{array}$ & & $\neg$ & in & 6 & & $\nabla$ & 1 & $\neg$ & $n$ & -1 & $\sigma$ & $\Lambda$ & $\stackrel{\infty}{=}$ \\
\hline$N$ & ' & $\stackrel{2}{\rightarrow}$ & ' & $\neg$ & ' & ' & $\stackrel{\infty}{\sim}$ & ' & - & I & ষ & $\stackrel{0}{0}$ & $\stackrel{\curvearrowright}{\sim}$ & - & $\begin{array}{l}2 \\
\text { in }\end{array}$ & $\begin{array}{l}n \\
\infty \\
\infty \\
-1\end{array}$ & & ' & 6 & 6 & & ' & ' & I & $\nabla$ & ' & $\nabla$ & 6 & 6 \\
\hline- & 1 & $\stackrel{0}{-}$ & ' & ' & $n$ & ' & $\underset{v}{v}$ & ' & $\nabla$ & 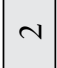 & $\stackrel{6}{-}$ & N & $\widetilde{\sigma}$ & ' & $\stackrel{n}{ \pm}$ & $\infty$ & & ' & ' & 0 & & ' & ' & I & ' & ' & 0 & - & ' \\
\hline 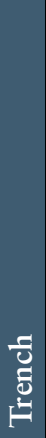 & $\begin{array}{c}\dot{2} \\
\infty \\
\dot{8} \\
\dot{0} \\
-1\end{array}$ & 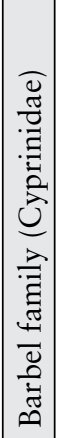 & 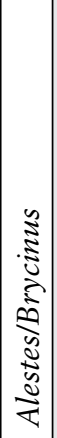 & 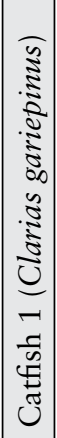 & 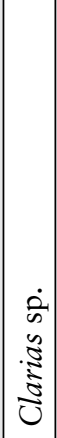 & 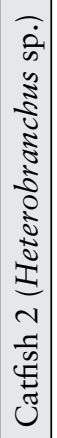 & 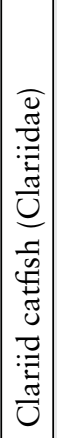 & 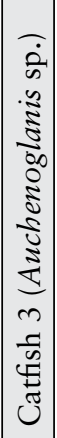 & 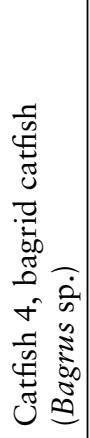 & 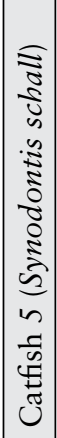 & 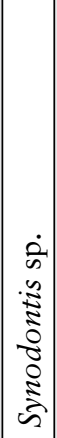 & 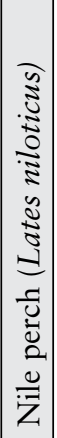 & 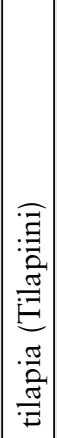 & 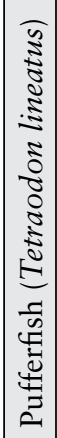 & $\mid \begin{array}{c}\frac{1}{5} \\
0 \\
0 \\
0 \\
0 \\
0 \\
0 \\
0 \\
0 \\
0\end{array}$ & 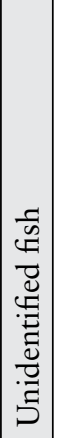 & 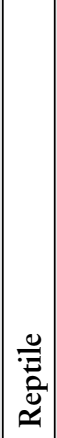 & 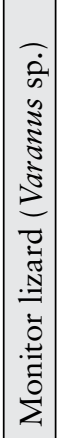 & 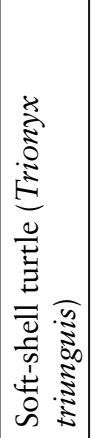 & 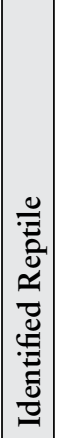 & . & 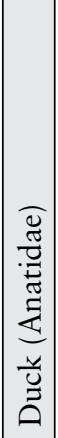 & 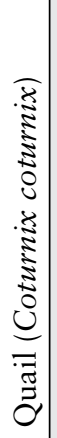 & 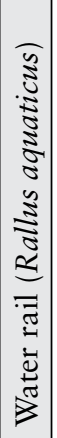 & 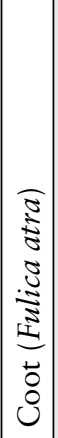 & 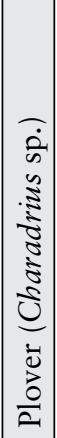 & 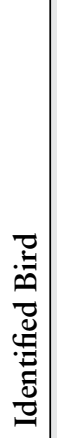 & 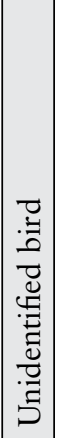 & 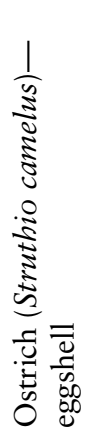 \\
\hline
\end{tabular}


Wendrich • Phillipps • Holdaway • Linseele • Emmitt • Marston

\begin{tabular}{|c|c|c|c|c|c|c|c|c|c|c|c|c|c|c|c|c|c|c|c|c|c|c|c|c|c|}
\hline 豞 & & & N & 0 & -1 & $\nabla$ & $\nabla$ & $\nabla$ & $m$ & $\Lambda$ & & $\stackrel{0}{-1}$ & \pm & $\Lambda$ & $\underset{\infty}{\mathfrak{y}}$ & \pm & & ఫु & $\vec{\nabla}$ & $\begin{array}{l}0 \\
2 \\
n \\
2 \\
n\end{array}$ & $\begin{array}{l}\infty \\
\sim \\
\gamma\end{array}$ & $\approx$ & $\begin{array}{l}0 \\
m \\
\infty \\
m \\
m\end{array}$ & 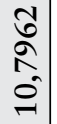 & $\begin{array}{l}n \\
\infty \\
m\end{array}$ \\
\hline $\begin{array}{l}\infty \\
\infty \\
\infty\end{array}$ & & & ' & ' & ' & ' & ' & ' & ' & ' & ' & ' & ' & ' & ' & ' & 1 & ' & 1 & ' & ' & ' & 0 & $\stackrel{\infty}{\wedge}$ & ' \\
\hline$\stackrel{n}{n}$ & & & ' & ' & 1 & ' & ' & ' & ' & ' & ' & ' & 1 & ' & $\neg$ & ' & ' & ' & 1 & $\neg$ & $\nabla$ & ' & 6 & $\begin{array}{l}\infty \\
\stackrel{\infty}{\sim}\end{array}$ & $\begin{array}{l}\stackrel{m}{m} \\
\stackrel{m}{m}\end{array}$ \\
\hline b̧ & & & 1 & ' & ' & ' & ' & ' & ' & 1 & 1 & ' & 1 & 1 & 1 & ' & ' & ' & 1 & ' & ' & ' & 0 & $\underset{\infty}{\varpi}$ & ' \\
\hline n & & & ' & ' & ' & ' & ' & ' & ' & ' & 1 & ' & 1 & ' & ' & ' & ' & ' & 1 & 1 & ' & I & 0 & $\hat{\imath}$ & ' \\
\hline f & & & ' & ' & 1 & ' & ' & ' & ' & ' & ' & ' & 1 & ' & ' & ' & 1 & ' & 1 & 1 & $\neg$ & ' & - & in & ' \\
\hline m & & & ' & ' & ' & ' & ' & ' & ' & ' & ' & ' & 1 & ' & ' & ' & ' & ' & ' & $\stackrel{\infty}{\sim}$ & $\exists$ & ' & $\hat{m}$ & $\begin{array}{l}\mathfrak{T} \\
\text { f }\end{array}$ & ' \\
\hline d̂ & & & ' & ' & 1 & ' & ' & ' & ' & ' & ' & ' & 1 & ' & ' & $\neg$ & ' & ' & 1 & $\neg$ & ' & ' & $N$ & ๓े & ' \\
\hline$\infty$ & & & ' & ' & ' & ' & ' & ' & ' & ' & ' & ' & - & ' & 6 & ' & ' & $m$ & $m$ & $\stackrel{3}{0}$ & 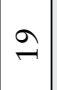 & ' & $\stackrel{\infty}{\rightleftharpoons}$ & $\begin{array}{l}0 \\
\stackrel{\hbar}{a} \\
m\end{array}$ & ' \\
\hline$\Lambda$ & & & $\neg$ & I & $\neg$ & $\neg$ & $n$ & $\neg$ & ' & ' & 1 & - & $\nabla$ & $v$ & $\underset{v}{\sigma}$ & $\nabla$ & ' & $a$ & 6 & ồ & જิ & $n$ & 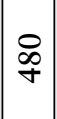 & $\begin{array}{l}0 \\
+ \\
\infty \\
0 \\
-\end{array}$ & $\stackrel{\vartheta}{\forall}$ \\
\hline 6 & & & ' & ' & ' & - & ' & $\neg$ & ' & $m$ & ' & $\neg$ & ' & $m$ & $\stackrel{m}{n}$ & $N$ & ' & $\stackrel{\nabla}{\sim}$ & - & $\begin{array}{c}0 \\
1 \\
0 \\
-1\end{array}$ & $\stackrel{ \pm}{\sim}$ & $\nabla$ & $\begin{array}{l}\tilde{n} \\
\stackrel{n}{n}\end{array}$ & $\begin{array}{l}\hat{\Omega} \\
\tilde{\sigma} \\
\hat{\jmath}\end{array}$ & ' \\
\hline in & & & - & ' & ' & ' & - & - & $\neg$ & - & 1 & N & $N$ & ' & $\hat{\sigma}$ & $\neg$ & ' & $\underset{+}{ \pm}$ & $n$ & $\begin{array}{l}0 \\
\text { ? } \\
\text { n. }\end{array}$ & $尺$ & $\nabla$ & $\begin{array}{l}\nabla \\
\text { in }\end{array}$ & $\begin{array}{l}\tilde{n} \\
\tilde{n} \\
\tilde{v}\end{array}$ & ' \\
\hline$\nabla$ & & & ' & ' & 1 & ' & ' & ' & N & - & ' & N & 1 & ' & $i n$ & ' & ' & - & ' & $\begin{array}{l}n \\
\infty\end{array}$ & ㄱ & N & 옹 & $\begin{array}{l}\vec{V} \\
\sigma_{2}\end{array}$ & 1 \\
\hline$m$ & & & ' & ' & ' & $\neg$ & ' & $\neg$ & ' & $\neg$ & ' & $N$ & in & ' & $\hat{n}$ & 6 & ' & $\stackrel{ }{-}$ & $\Lambda$ & $\begin{array}{l}\tilde{b} \\
\tilde{\nu}\end{array}$ & ஓ & $n$ & 年 & $\begin{array}{c}\infty \\
\vec{\sim} \\
\vec{\sim}\end{array}$ & ' \\
\hline$N$ & & & ' & ' & ' & - & 1 & ' & ' & - & 1 & $N$ & $v$ & $v$ & in & ' & ' & $m$ & - & $\stackrel{+}{\sim}$ & $\approx$ & $\Lambda$ & $\stackrel{\infty}{\sim}$ & $\begin{array}{l}\infty \\
\infty \\
\\
\infty\end{array}$ & $N$ \\
\hline- & & & ' & ' & 1 & 1 & ' & ' & ' & ' & 1 & ' & 1 & ' & $\stackrel{n}{\sim}$ & ' & 1 & ' & ' & ' & $\Lambda$ & ' & 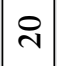 & $\begin{array}{l}\infty \\
\sim \\
\sim\end{array}$ & ' \\
\hline 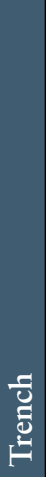 & 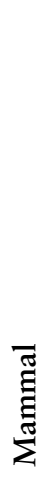 & 룰 & 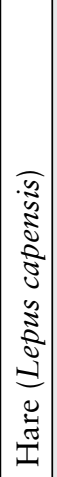 & 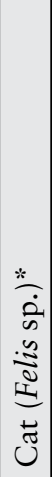 & 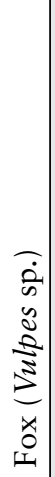 & 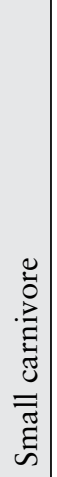 & 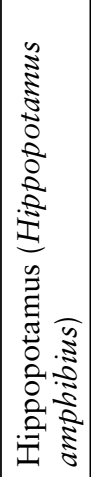 & 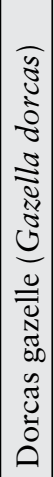 & 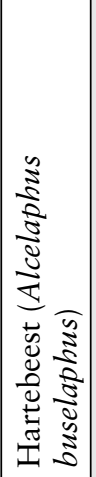 & 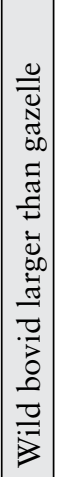 & 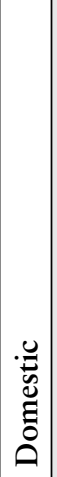 & 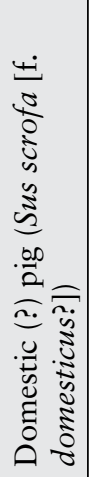 & 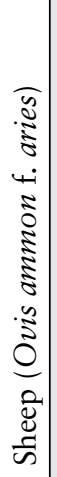 & 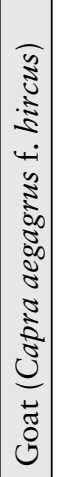 & 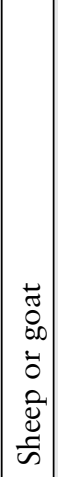 & 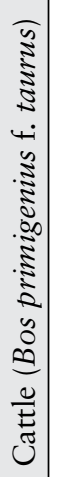 & 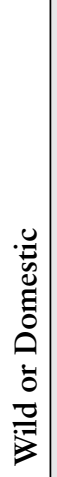 & 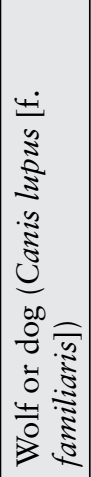 & 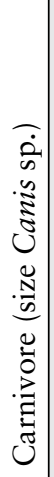 & 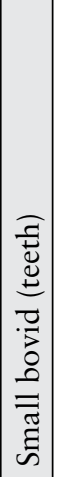 & 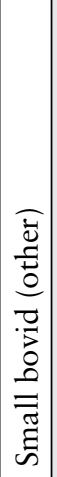 & 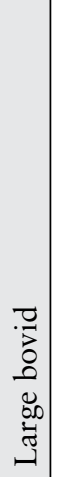 & 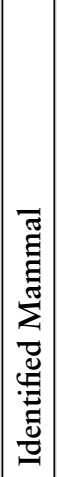 & 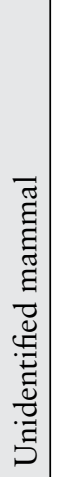 & 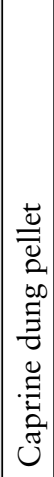 \\
\hline
\end{tabular}




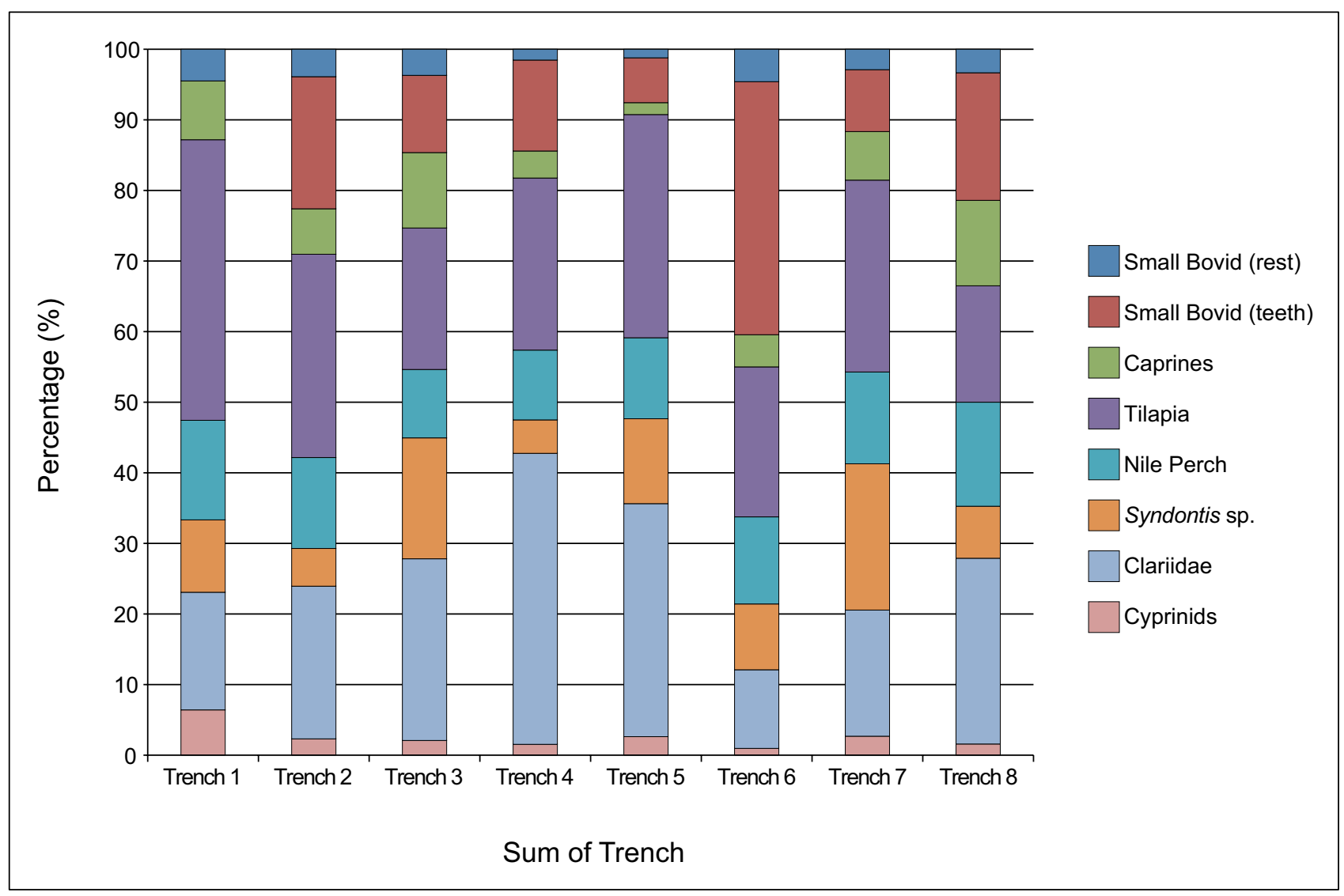

Figure 6.23. Faunal proportions based on NISP from excavated trenches at Kom K.

While pigs are numerous at some mid-Holocene and Predynastic sites in the Nile Valley, they have not been attested elsewhere from the Egyptian deserts before the arrival of the Romans (Van Neer 1997).

In a 5-liter sample of sediment from Trench 7 (KK07), unit [07.0019], comprising the fill of Hearth 145 , a set of around 100 pellets from caprids was found (cf. Walker 1996) (Figure 6.24). The size, shape, and weight of a sample of 25 specimens enabled gazelles to be excluded, since the feces of these animals are clearly smaller and lighter than those that were recovered and smaller are than those of the other possible candidates: sheep, goat, ibex, and Barbary sheep (Linseele et al. 2010; Riemer et al. 2008). Because they were found associated with the hearths, and since no other small bovids are represented in the bone remains, they probably belong to domestic caprines. The fact that most of the dung was recovered from hearth contexts may mean that the pellets were used as fuel, a practice for which both ethnographic and archaeological parallels exist (Miller and Smart 1984).
Fish and soft-shell turtles are the most common group among the faunal remains. They are discussed together as they were presumably caught in the same environments with similar techniques and represent 76 percent of all identified remains. Some types of bones and taxa preserve better than others in stratified contexts, even if the precise effects are difficult to estimate. The species composition of the fish is similar throughout the different trenches. Clariidae, especially their heads, have hard, compact bones that preserve well and are also easy to recognize from small fragments, while, as noted, cyprinids have more fragile bones. The aquatic fauna from the early- to mid-Holocene sites in the Fayum is rich and diverse, especially in comparison to the present-day species in Lake Qarun, which are all adapted to the saline conditions that characterize the lake today (Van Zon and Jeannes 1992). The rich spectrum of freshwater species can only have existed due to connections to the Nile that occurred at various times during the early to mid-Holocene. 


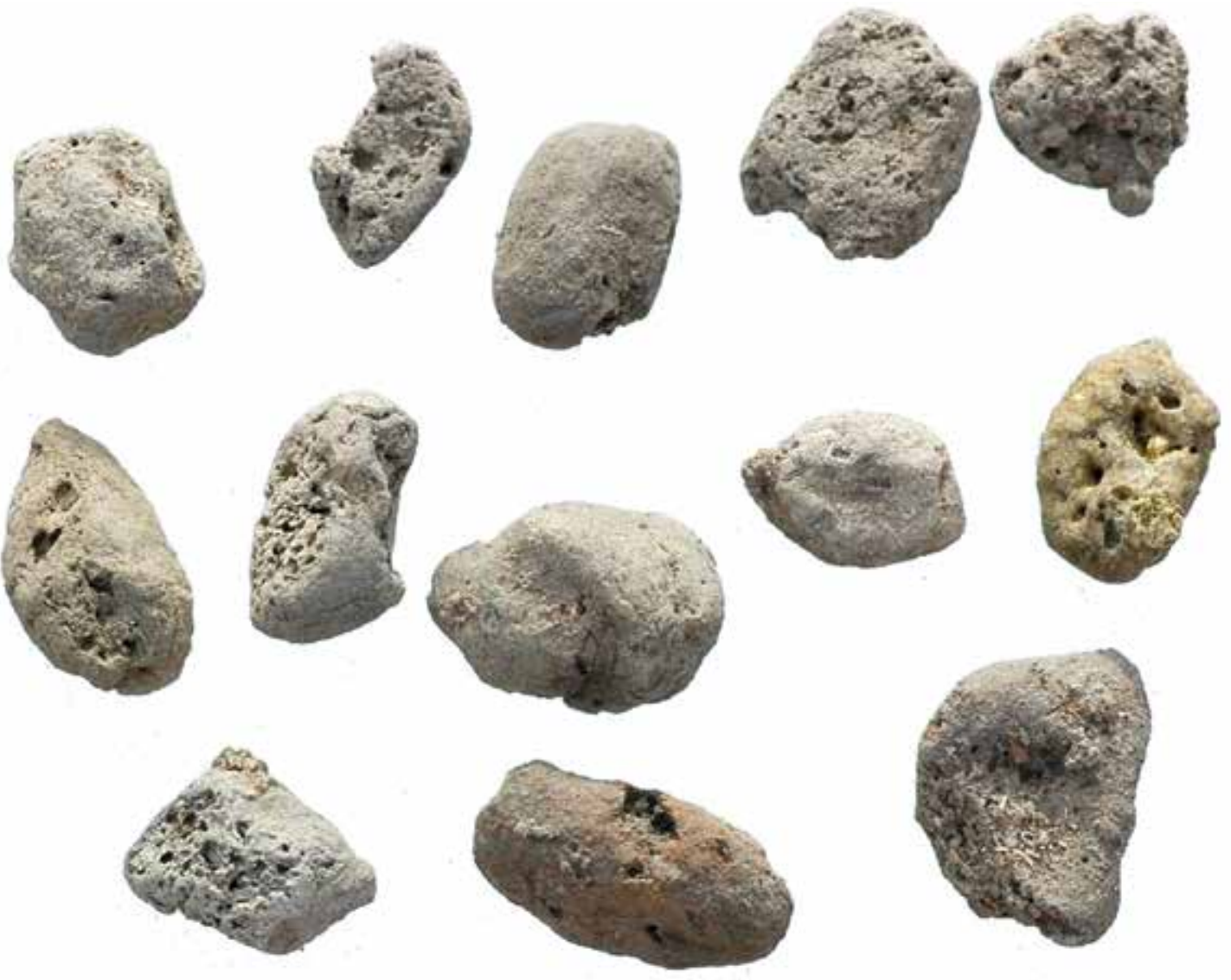

Figure 6.24. Caprid pellets from Kom K hearth [07.0019].

The fish remains indicate that different types of aquatic habitats were exploited (Van Neer 2004). Most of the fish came from shallow waters, especially represented by clariid catfish and tilapia but also the cyprinids. Other species are more typical of well-oxygenated waters: tiger fish, bagrid catfish, Synodontis catfish, and Nile perch. The reconstructed standard lengths for Nile perch are relatively small, mostly between 30 and $60 \mathrm{~cm}$ (Table 6.16), which suggests that fishing was not done very far offshore (Beadle 1981; Tobor 1974). Few fish remains indicate marshy, vegetated aquatic environments, with these represented only by the genus Polypterus. Clariid catfish and tilapia, the two most common fish, are predominantly represented by large, adult specimens, indicating that fishing mainly took place in the spawning season, when these taxa are known to concentrate and are easy to catch. The spawning season may have corresponded to the Nile flood season. In addition to fish and turtles, large freshwater bivalves are present. Coelatura sp., Spathopsis sp. and/or Chambardia sp., and Mutela sp. were identified. 
Table 6.16. Nile Perch Sizes from Kom K in cm.

\begin{tabular}{|lr|r|r|r|r|r|r|r|}
\hline Trench & 1 & 2 & 3 & 4 & 5 & 6 & 7 & 8 \\
\hline $10-20$ & 0 & 0 & 0 & 0 & 0 & 2 & 0 & 0 \\
\hline $20-30$ & 0 & 0 & 2 & 2 & 9 & 24 & 17 & 0 \\
\hline $30-40$ & 7 & 13 & 28 & 31 & 190 & 81 & 68 & 2 \\
\hline $40-50$ & 7 & 40 & 50 & 39 & 147 & 55 & 68 & 21 \\
\hline $50-60$ & 0 & 10 & 35 & 7 & 21 & 26 & 25 & 9 \\
\hline $60-70$ & 1 & 0 & 3 & 3 & 4 & 7 & 5 & 0 \\
\hline $70-80$ & 0 & 0 & 1 & 0 & 2 & 1 & 3 & 0 \\
\hline $80-90$ & 0 & 0 & 0 & 0 & 0 & 1 & 0 & 0 \\
\hline $90-100$ & 0 & 0 & 0 & 0 & 1 & 0 & 0 & 1 \\
\hline \pm 100 or & 0 & 0 & 1 & 2 & 5 & 3 & 2 & 1 \\
larger & & & & & & & & \\
\hline
\end{tabular}

\section{Discussion}

Aquatic fauna is predominant, and these remains indicate an orientation to resources from the shallow water environments, although some deepwater species are also present. The faunal composition is consistent with seasonal exploitation of resources, particularly fish, possibly during the Nile flood season. Both observations may relate to the nature of $\mathrm{K}$ Basin, which, as discussed in chapter 3, was likely relatively shallow compared to the basins farther west. For at least part of the year, people using Kom K likely made use of seasonally abundant shallow water fish species.

Sheep are grazers, while goats are browsers that can live on a more varied diet. Caprines are indicated by young individuals and were perhaps abundant enough to provide dung for fuel. However, only a small number of bones were recovered during excavation. The different trenches at Kom $\mathrm{K}$ all show a similar range of species.

\section{Botanical Remains}

Carbonized kernels and threshing remains of emmer were found in Kom $\mathrm{K}$ in Hearth 105, unit [02.0032], but no barley remains were found. Threshing remains of emmer consist of rachis fragments with the basal part of the glumes, the "spikelet forks" and "glume bases" discussed in archaeobotanical publications. Although both cereals are hulled, threshing remains differ in their morphology, and hence the chance of becoming part of the local archaeological record is differentiated as well. In hulled barley, the large lemma (lower bract enclosing the grass floret) and palea (upper bract of the floret of a grass) remain tightly attached to the grain kernel, and it is most likely that such hulled grain kernels (florets) are ground as such. The lemma and palea may become fragmented as well, and large pieces are easily removed by sieving. The spine awns and glumes, as well as the small fragments of barley, are light and are blown away easily during winnowing. This explains why such fragments are sometimes underrepresented in the archaeological record.

In emmer, on the other hand, each spikelet usually consists of two grain kernels as well as large pieces of the rachis and chaff (that is, two glumes, two lemmas, and two paleas). Therefore, dehulling is necessary as a step in crop processing to get rid of the large quantity of inedible parts. Dehulling is done prior to food processing and will produce large quantities of relatively large pieces of the rachis and the chaff. These are more correctly referred to as "dehulling remains" rather than "threshing remains," and it is these that were found at Kom K.

\section{Summary and Discussion}

The Kom K excavation revealed a largely similar stratigraphy in locations from the top, middle, and base of the mound. Although recent agricultural activity damaged the upper layers, those below show a large number of hearth features often showing evidence of reuse. Most of these hearths were created as depressions cut into windblown sand, although for one set of hearths, these were cut into a clay surface. The hearths vary in shape, often as a result of repeated use. Heat retainers are rare, with examples found only in the hearths from KK04. Radiocarbon ages indicate that the hearths were created in the period 6600 to $6400 \mathrm{cal} \mathrm{BP}$, although the nature of the calibration curve at this time limits the degree to which the chronology can be refined. People used tamarisk for fuel. However, they also used dung, most likely from caprids.

Associated with the hearths are large numbers of flaked stone artifacts, the analysis of which indicates that raw material brought to the location was worked in place. Movement of material away was largely limited to the movement of cores. Ceramics were found at the site, but not as complete vessels. A very small number of the studied sherds showed decoration, something previously unknown for this region and time period. A single example of an unfired clay vessel is different from the more generally encountered clay pit linings. 
Bone and ostrich eggshell were worked, probably at the site, as indicated by unfinished ostrich eggshell beads, as well as ostrich eggshell worked fragments. Most of the worked bone and ostrich eggshell was likely used as personal ornaments, including finger rings and beads. A fragment of a shell bracelet from a marine species was found, presumably discarded because it was broken.

All the major domesticated species found at other mid-Holocene Lower Egyptian sites are present, including dog, pig, sheep, goat, and cattle. Caprines dominate the domestic animal assemblages, and within these, sheep are more common than goat, although neither is represented by abundant remains. The aquatic fauna is rich and diverse, especially in comparison to the present-day species in Lake Qarun, which are all adapted to the saline conditions that characterize the lake today. Most of the fish come from shallow waters, especially represented by clariid catfish and tilapia but also including the cyprinids. Although people had access to domestic animals, fish was clearly an important resource.

Evidence for the crop assemblage in the Upper $\mathrm{K}$ Pits and Kom $\mathrm{K}$ is limited to hulled barley and emmer. Pulses are absent. However, these are often less prominent in archaeological assemblages and might become visible only if a substantial number of samples are analyzed. The combination of hulled barley and emmer is typical in Egyptian archaeological sites right up until the Greco-Roman period (332 BCE to $395 \mathrm{CE}$ ), when hulled emmer is replaced by the naked hard wheat (Triticum turgidum subsp. durum). Hulled barley and emmer are ideal cereals in a subsistence economy as they require relatively little labor to process, though dehulling is necessary for emmer, and they also provide fuel. The storage of emmer takes more space in comparison with naked wheat, but the presence of chaff protects the yield, and a reduction of storage capacity may not be problematic within a domestic economy. The only evidence of naked wheat comes from El Omari, which has been identified as club wheat (Triticum aestivum subsp. compactum) by Helbaek
(1955). It is possible, however, that the grain kernels belong instead to a multi-seeded landrace of hard wheat (Triticum turgidum subsp. durum). If species were selected for use from a range of available crops, it is possible that hulled barley and emmer were preferred because of their attractiveness for subsistence (Cappers 2013). Because dehulling of barley is not necessary, its ease of processing gives it the potential to form a major part of the human diet. Any additional uses, for instance in beer production or as fodder, need to be considered in relation to its relative ease of processing (Cappers et al. 2014).

Despite discussions in the older literature that group Kom K and Kom W together and interpret these locations as villages, detailed excavation indicates that Kom $\mathrm{K}$ lacks the range of features that would support interpretation of the site as a village. Although we do not discuss Kom W in this volume, we know enough about the remains from the site to indicate that the structure and composition of Kom K and Kom W are not similar. Kom $\mathrm{K}$ preserves a large number of superimposed hearths together with numbers of flaked stone artifacts, some ceramics, botanical remains from one hearth, and fish, as well as a limited number of domestic animal bones. Activities at the location involved multiple episodes of hearth use, and while we cannot be certain what each individual hearth was used for, it is probably safe to assume that they were used for cooking wild and domestic animals as well as seeds. Aeolian sand deposits within the hearths indicate periods when individual hearths were abandoned, but we cannot be sure of the length of time between episodes of hearth reuse. Although radiocarbon ages from the hearths indicate a relatively short duration of use, the nature of the calibration curve in the mid-Holocene means that hearth use could have spanned some centuries or a much shorter period, or some combination of both. As we comment in the following chapter, Kom $\mathrm{K}$ is important not as a "type" site for the Fayum mid-Holocene occupation but because it provides a window into one aspect of place use at one particular period in the history of the Fayum north shore settlement system. 


\title{
The Desert Fayum Reinvestigated: The Evidence Considered
}

\author{
Simon J. Holdaway, Willeke Wendrich, and Rebecca Phillipps
}

The method of inquiry (...) needed to be framed on anthropogeographical lines, (...which), invited a fresh method of treatment which would endeavour to do justice to those conditions in relation to man's reactions to them, as well as to use old lake-levels as stratigraphical agents of archaeological control [Caton-Thompson and Gardner 1934:1].

\begin{abstract}
A s discussed in chapter 2, a great deal of the debate concerning the origins of the Neolithic in Egypt has focused on two themes: why did it develop and where did it come from? Both these have been foci for research in the Fayum, with environmental change involving increasing desiccation seen to drive the movement of people. These people, bearing particular artifact types, came from the eastern Sahara toward the Nile Valley, while different people, with domestic plants and animals together with other aspects of the Neolithic package, came from Southwest Asia, either via the Mediterranean coast or the Red Sea. However, the issue with these and other large-scale models that seek to answer "The Origins of the Neolithic in Egypt" question is that they necessarily average out a great deal of regional variability in favor of a generalized explanation. At one level this is of course the point of model building. However, in the Egyptian case, this form of large-scale model
\end{abstract}

generation has focused discussion on a very limited set of observations in the generalized models. A focus on certain artifact forms thought to be diagnostic of the movement of people has not been accompanied by a theoretical discussion indicating why projectile points, for instance, should in fact be so diagnostic of a particular people, particularly since the inferred patterns of movement are not reflected in other forms of material culture, such as ceramics (Warfe 2003).

In this volume we seek to provide an alternative to the construction of generalized models by focusing closely on a detailed study of an archaeological landscape at multiple temporal and spatial scales from one region. Rather than beginning with a conceptual model of Neolithic lifeways, we instead consider first and foremost the nature of archaeological and related evidence from the Fayum. We consider how this can be used to reconstruct social structure, economic practice, settlement pattern, and response to 
both large-scale and small-scale climatic and environmental changes. We do this by suggesting an expansion of Smith's (2001) notion of low-level food production as a way of conceptualizing the variability that may be expected. In this chapter, we summarize the results obtained to date. We begin by discussing whether the early to mid-Holocene Fayum archaeological record is best conceived of as a series of independent archaeological sites.

\section{The Fayum Archaeological Record as a Landscape}

The Fayum region has seen a long history of archaeological research focusing largely on a small number of archaeological sites. However, despite this emphasis, archaeological site definition in the Fayum north shore is not as simple as it might first appear. Stratified sites like Kom K are present, but the stratigraphic structure of this site is complex and not as easily translatable into functional categories like villages, hamlets, or campsites as the literature in the past once suggested. The site is largely composed of windblown sand deposits mixed with cultural material in the form of portable artifacts, fauna, and burned material, including charcoal derived from hearths. Sets of superimposed hearths can be defined by careful excavation, and it is likely that hearth reuse has removed some of the evidence for even more hearth construction events than those we were able to identify. Furthermore, the site lacks evidence that might indicate the presence of other types of structures.

Reuse of hearths gives an indication that Kom $\mathrm{K}$ was occupied repeatedly, but the radiocarbon determinations we obtained give only a general indication of the time span of this reuse. As discussed in chapter 6 , the radiocarbon calibration curve precludes a precise understanding of occupation duration, as does the way different sets of hearths were cut into a common surface. Despite careful stratigraphic excavation, the nature of the windblown sediment accumulation at Kom $\mathrm{K}$ means that the hearths could have been created over a very short period of time or alternatively one that spanned some centuries, or for that matter some combination of both, with periods of short-term occupation separated by longer periods of abandonment.

The Upper K Pits represent another example of a distinct archaeological site now unfortunately destroyed by contemporary development. On the basis of the pits that were excavated (chapter 5), we know a great deal about their use as storage devices. Some of the large ceramic vessels found in the pits were likely used for storage, while some of the smaller vessels could have been for grain preparation (for example, threshing) or cooking and were stored in the pits when not needed. The fill contents indicate that most of the pits were abandoned, with indications of the original fill of wheat (Triticum turgidum subsp. dicoccon), barley (Hordeum vulgare subsp. vulgare), and seeds of Polygonum and Linum mostly present as remains hidden in the fabric of the basketry lining. Several of the pits were, nevertheless, carefully closed off with compact mortar lids, which were prepared in shallow depressions near the storage pits. From the configuration of the pits it is clear that these were reused, probably multiple times. The finds of hafted sickle blades and finely coiled basketry as well as the ceramics, in addition to other portable material types, indicate that the pits were used for storage of implements as well as crop yields. The Lower K Pits and several of the dilapidated ones identified by Caton-Thompson and Gardner in the Upper K Pits make it likely that upon abandonment, the pits may have been actively decommissioned by removing the basketry lining. We also know, within the precision of the radiocarbon dates that we obtained, rather than the older Libby determinations, that they were constructed at approximately the same time as the hearths at Kom $\mathrm{K}$, but as noted above, the precision provided by the radiocarbon calibration curve does not allow us to state definitively that these two sites were contemporary (we make further comments on this below) or that all the pits were created at the same time. The precision of the dates that we do have is commensurate with the location having been repeatedly reused (Figure 7.1).

Because the Upper K Pits were cut and fill features, the boundary of the site where they occurred was relatively easy to define, as was the spatial distribution of the pit features themselves. We can reconstruct the extent of both the Lower and Upper K Pits based partly on our own survey and also from observations made by Caton-Thompson and Gardner in their published work. However, detailed description and dating is possible only for the pits we were able to excavate. Thus, while we can define the extent and content of the site, we can date only a small sample of the features that were once present. It is the same situation at Kom K, except that we were able to investigate areas at the top, middle, and bottom of the Kom K mound. Thus 
to some degree we can comment on the chronology of the depositional history of the site, recognizing how the near-surface deposits have been damaged by recent cultivation and that the excavation continued until the density of cultural material was very low rather than absent. We also used subsurface survey to target concentrations of hearths, thereby giving us some idea of both the extent and the proportion of the deposits we sampled. However, it is also true that the extent of the site was initially determined by the topographic elevation of the kom, and it is only over this area that we placed the squares for surface sampling. The extent of the site is therefore something that we as archaeologists have determined rather than indicative of a spatial entity that existed in the past.

The significance of this observation becomes clear when we consider the surface archaeological materials discussed in chapters 4 and 5 . Stratified deposits in fact make up only a small proportion of the archaeological record of the Fayum north shore. The majority of the record consists of surface scatters of flaked stone artifacts, ceramics, faunal material, and the remains of heat retainer hearths. These archaeological materials are much harder to identify as sites since their boundaries cannot be identified on the basis of either cut and fill features or topographic changes. This was made abundantly clear to us in the early stages of the project when we began reconnaissance of our study area and attempted pedestrian "site survey." The result was an impressive collection of "dots on maps" but with little understanding about what each dot might represent or indeed whether adjacent dots were in any way comparable. The solution was to develop the approach based on transects, as described in chapter 3 , inspired by the Mediterranean archaeological literature (e.g., Bintliff 2000; Cherry 2003), where similar issues of site definition were encountered.

As the results presented in chapters 4 and 5 indicate, surface deposits are ubiquitous in the Fayum north shore but differ in interesting ways in density and composition. The corridor surveys undertaken north into the Fayum Basin indicated very low densities of portable artifacts at distances away from the lake basins that Caton-Thompson and Gardner identified. The majority of the archaeological record is indeed concentrated a few hundred meters north of these basins, just as they originally observed. However, within this artifact-rich zone, there is considerably more spatial variability in the surface record than that observed by many who worked in the Fayum subsequent to CatonThompson and Gardner. Thus our intensive recording at and around the site identified as E29H1 by Wendorf and Schild demonstrated a more extensive record than they were able to document given the time they had available in the field. Importantly, this more extensive record was shown to extend to elevations well below those they originally considered likely for the Epipaleolithic deposits in the Fayum North shore.

Apart from the extensive deposits of portable artifacts and fauna (discussed further below), we identified numerous heat retainer hearths, a sample of which we were able to date with radiocarbon. The age determinations returned provide a different view of the chronology of occupation of the Fayum north shore compared to that provided by earlier studies. Hearth ages to some degree fill the gap between what was thought to be a dichotomous occupation with an earlier Epipaleolithic separated in time by a period of abandonment before the later Neolithic. The results indicate that "stratified sites" like Kom K reflect only a limited record of occupation in the Fayum. A great deal more occupation occurred but is preserved only in the form of a surface archaeological record.

To conclude, archaeological site definition in the Fayum is problematic as a result of how the archaeological record formed and is preserved, an observation that is similar to the comments made originally by Wendorf and Schild (1976:215) when discussing Kom W. Stratified sites occur, but their composition indicates that they are not so different from the majority of the archaeological deposits that occur on the surface. When all deposits are considered, rather than just those that fit more easily into the conventional category of archaeological site, significant adjustments are needed to our understanding of the history of occupation of the region.

\section{Early to Mid-Holocene Archaeological Chronology}

The radiocarbon determinations we obtained from hearth features in the Fayum certainly indicate times when people were present and constructed hearths that survived, but we need to consider the nature of the samples obtained carefully before using these dates to construct a chronology of occupation phases. As illustrated in chapters 4,5 , and 6 , different sampling locations indicate occupation at different times in the early to mid-Holocene. 
We predominantly sampled hearth features to obtain samples for radiocarbon analysis since we reasoned that these features provided a structure that ensured a clear relationship between past human behavior and the age obtained. The construction of the hearth and burning of the material within it provide the link between the behavioral events that created the charcoal and the age obtained from this charcoal (Phillips 2013). Charcoal analysis suggests the use of relatively short-lived plants, so there is likely to be a negligible effect from inbuilt age. The only exceptions to the use of hearths as the source for radiocarbon samples were the ages obtained from material in Upper K Pits 68 and 75 . However, both these structures were not unlike the hearths in the sense that they indicated a clear relationship between the sample obtained and the behavior we intended to date. The material dated (wheat straw) was also unlikely to have a significant inbuilt age.

In addition to the radiocarbon determinations we obtained, the sample can be expanded somewhat by considering dates reported in previous studies. In the following we consider radiocarbon dates using only those obtained from features like hearths and pits, as discussed above. The calibrated radiocarbon data set from the Fayum area includes all the determinations reported from E29H1, L1, K1, Kom K, and the K Pits. Where more than one determination was obtained from the same hearth, these were averaged. The combined data set represents 42 determinations (Figure 7.1).

Plotting the calibrated age median midpoint for each radiocarbon sample by site produces the graph illustrated in Figure 7.1. Shown in this format, the degree to which the available radiocarbon ages for the area of the Fayum reported here reflects that work undertaken at individual localities is evident. As noted above, the addition of new radiocarbon determinations changes the previously reported general trend of pronounced activity in the first half of the early Holocene (circa 9200 to 9000 cal BP) followed by a period of abandonment and then reoccupation during the mid-Holocene (circa 6550 to $6100 \mathrm{cal} \mathrm{BP}$ ) (Hassan 1986; Wendorf and Schild 1976). There are

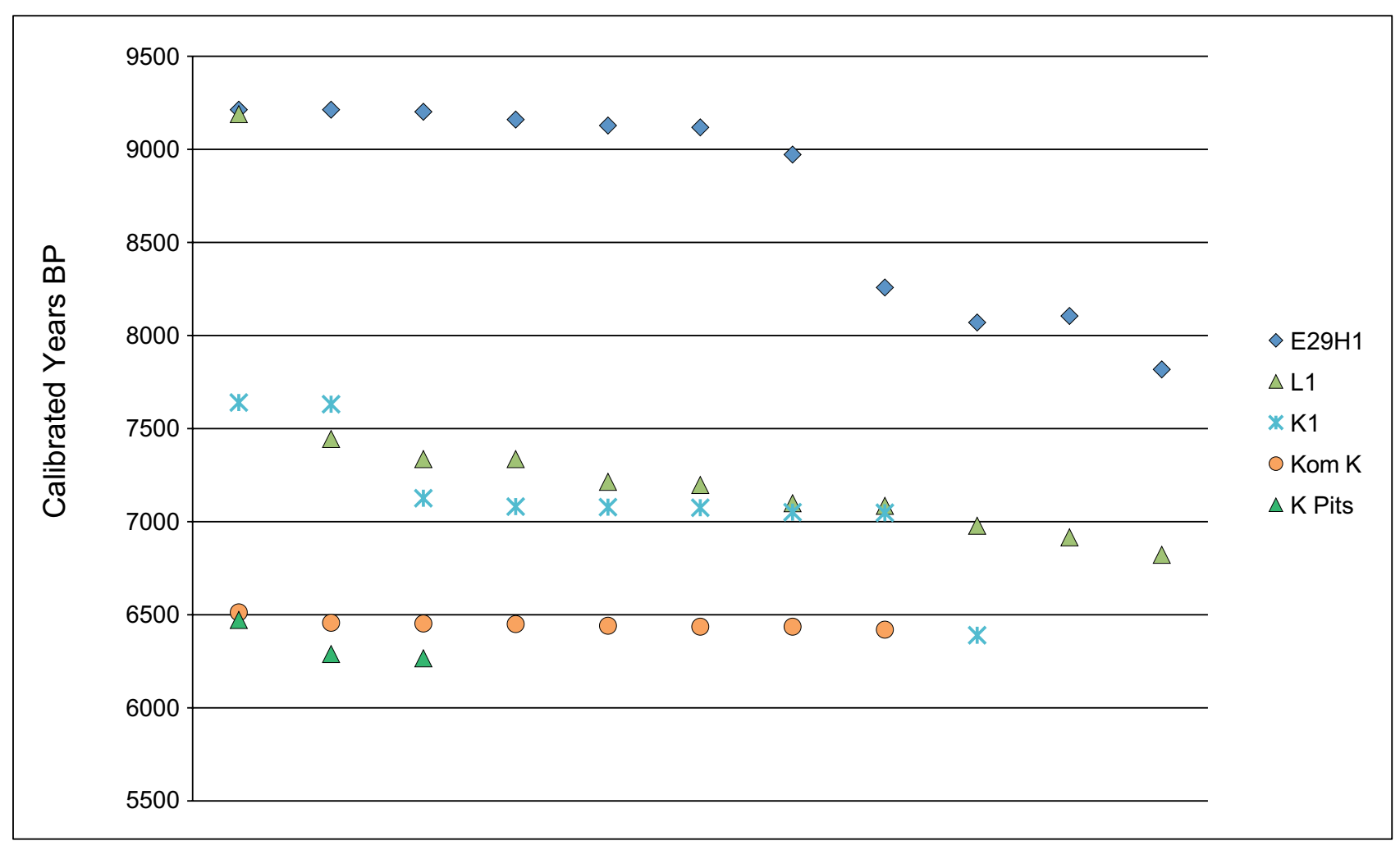

Figure 7.1. Radiocarbon determinations from hearths from sample locations in the Fayum with ages between 10,000 and $5500 \mathrm{cal} \mathrm{BP}$. 
now a number of ages from the latter part of the early Holocene (from circa 8500 to 7500 cal BP). However, there are still significant gaps in the distribution of ages throughout the early to mid-Holocene when dates from the Fayum are viewed together.

Only three of the seven sites have ages dated to the first half of the early Holocene. The largest number come from E29H1 ( $\mathrm{n}=11)$ and a single determination from the L1 area. Calibrated determinations from all other study locations fall between the late early Holocene and the early mid-Holocene. Three hearth dates from E29H1 occur in the period 8500 to 7500 cal BP. A number of hearths from K1 and L1 have ages within the range 7600 to 6700 cal BP. Hearths from Kom $\mathrm{K}$ and the Upper K Pits have ages that range from 6500 to $5700 \mathrm{cal} \mathrm{BP}$.

Identification of an early mid-Holocene hiatus in occupation, reported by both Hassan (1986) and Wendorf et al. (2001), was based on an interpretation of the low numbers of determinations dated to this time compared to the larger numbers of dates both before and after this period. However, this hiatus reflected the number of samples dated from particular sites rather than the actual absence of people (Phillips 2013). Despite a significant increase in the number of dated hearths reported in this volume, samples still cluster in age by site, meaning that the frequency of age determinations at any particular point in time is a product of site-specific sampling and preservation. Intensive study at $\mathrm{E} 29 \mathrm{H} 1$, for instance, has increased the resolution of hearth use at this site and therefore has an impact on the apparent intensity of occupation. This effect is particularly noticeable if a summed probability distribution is used to provide an indication of the regional chronology. In such a plot (Figure 7.2), fluctuations that at first sight seem to show periods of more and less intensive occupation are apparent. These fluctuations actually reflect different intensities of site-specific sampling (Phillips 2013). Equally, the intensive study and sampling at Kom K, due to excavation of hearths at this location, contributes to the appearance of occupation intensity during the mid-Holocene. While Kom K was a location where a number of hearths were constructed, hearth survey in the K1 and L1 areas suggests that both taphonomic processes and visibility impact our understanding of occupation intensity.

It is sobering to conclude that despite what may appear to be intensive study of the Fayum in comparison to other parts of the eastern Sahara, the chronology of this region continues to be site and research project dependent. Among the calibrated radiocarbon samples from the Fayum are a number of identical or nearly identical conventional radiocarbon determinations (that is, with the same or very similar mean and standard error). This also has an impact on the apparent likelihood of activity occurring during a particular time period, as indicated in Figure 7.2 (for example, between 6500 and 6300 cal BP) (Phillips 2013). This effect is enhanced in instances where the shape of the calibration curve produces calibrated results that have similar probability distributions.

All these points need to be considered when comparing the chronology of occupation in the Fayum north shore with other regions. The regional summed probability distribution for the entire Lower Nile Valley calibrated radiocarbon data set, for instance, seemingly indicates a high level of variability during the early Holocene compared to the relatively continuous spread of age determinations at the end of this period and throughout the early mid-Holocene. However, this likely reflects the way radiocarbon determinations were obtained. Comparing the Fayum chronology with those from other regions must therefore be approached cautiously, lest the accumulated record of a series of sites be treated as though it were a regional pattern (Phillips 2013).

The survey and excavation strategy described in chapters 4, 5, and 6 applied a common sampling design to the analysis of landscapes without privileging either buried or surface deposits. The survey was also spatially extensive, as much as the limits imposed by modern-day cultivation and development allowed. Within these limits we are able to indicate how different parts of the Fayum north shore were used at different times. What we can conclude on the basis of the results obtained to date is that the Fayum saw a greater degree of use than previously envisaged. Gaps in the chronology still exist, but they consist of a number of shorter episodes rather than the large hiatus indicated in the older literature. There is a general trend for older ages to occur in the western edge of our study area, associated with E29H1, and more recent ages to occur in the east, in the $\mathrm{K} 1$ area and at Kom $\mathrm{K}$ and the K Pits. Within the limits imposed by our sampling strategy and places where we were able to find extant hearths to study, 


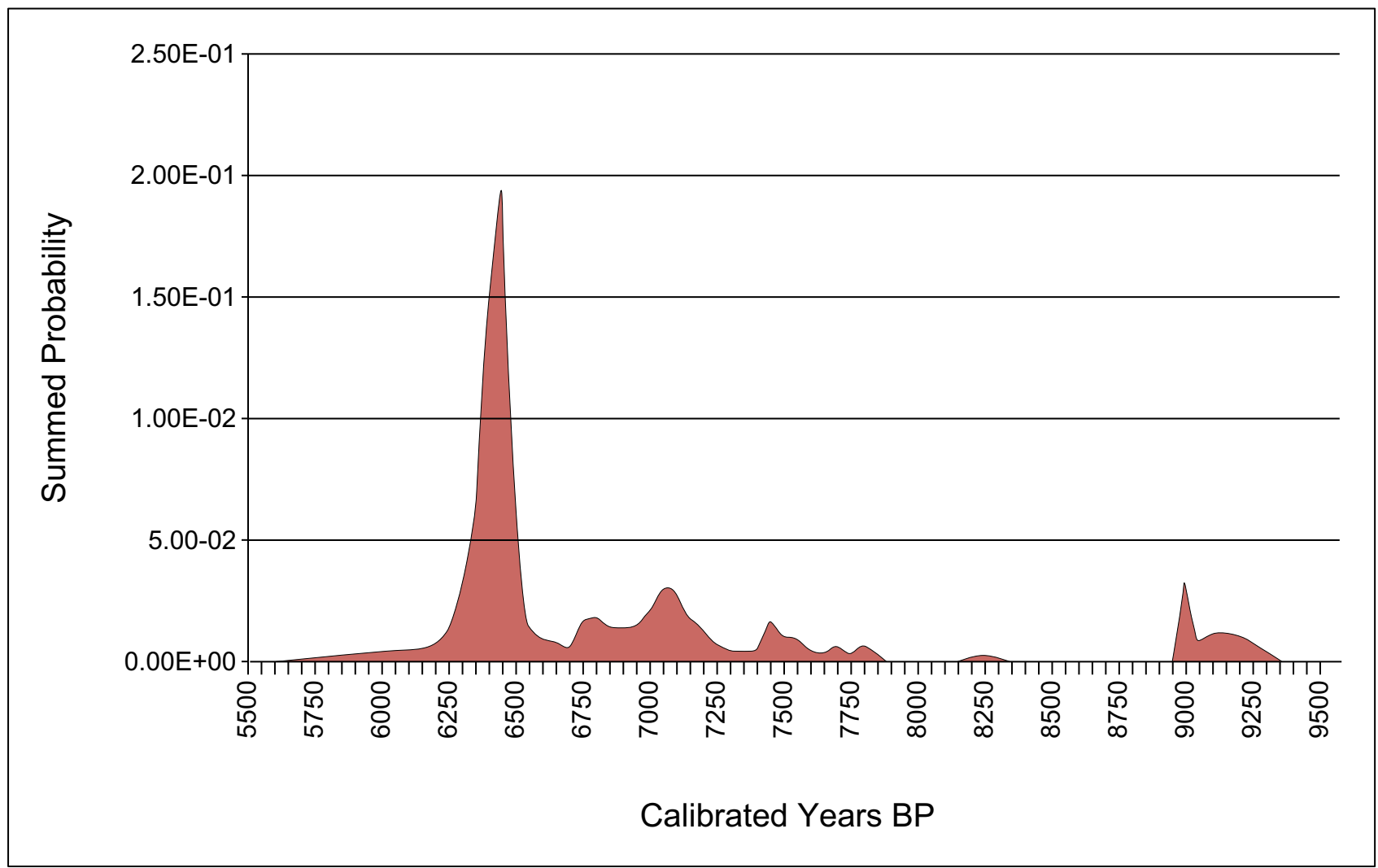

Figure 7.2. Summed probability plot for radiocarbon determinations from the Fayum north shore. The shape of the plot reflects the numbers of determinations obtained from different locations.

this trend shows relatively limited overprinting of later material on earlier remains. That is, the range in ages at any one location represents a relatively short period of time. We return to the significance of this observation below.

The dating of the appearance of domesticated species is complicated by this revised picture of occupation of the Fayum discussed above. Past interpretations of the occupation chronology used the pre- to mid-Holocene hiatus as a convenient time of departure from a socioeconomy based on hunting and gathering wild plants and animals to one based on domesticated plants and animals, as part of the introduction of the Neolithic package. Typological divisions also contributed to the dichotomy between the Epipaleolithic and the Neolithic. However, with the disappearance of the hiatus, owing to the contribution of more radiocarbon determinations, it becomes more difficult to draw the line for the beginning of the use of domesticates in the Fayum. In terms of absolute evidence for the presence of domesticated plants and animals, as discussed in chapter 6, Kom $\mathrm{K}$ and the $\mathrm{K}$ Pits contain evidence for domesticated cereal species originating from Southwest Asia. There are also faunal remains from locations within E29H1 that include domestic animals, notably domestic caprines. However, preservation in the form of stratified deposits is favorable for botanical and faunal remains but is less so in the majority of the archaeological record in the Fayum. As we discuss below, the previous emphasis on a distinction between "cultural phases" is perhaps less important in view of the continuity in some aspects of use of landscape in the Fayum during the early to mid-Holocene. A more productive line of enquiry is therefore to examine the evidence for change beyond typology and how might we understand change, both temporal and spatial, in the intraregional environmental context of the Fayum. Our results can then be compared to evidence for spatially and temporally changing socioeconomy elsewhere in Egypt during the early to mid-Holocene (e.g., Wengrow et al. 2014) (chapter 8). 


\section{Paleoenvironmental History of the Early to Mid-Holocene Fayum Basin}

It was clear enough to Caton-Thompson and Gardner that the history of human occupation of the Fayum Basin was closely connected to the history of Lake Qarun, as it is presently known, or Lake Moeris to use its ancient name. A great deal of effort has been directed at understanding the relationship between past occupation within the vicinity of the lake and changes that occurred in the paleo-lake levels. However, to truly understand this relationship, lake level changes need to be dated independently from the archaeological record. At issue is the relationship between the places people occupied and the location of these places in relation to the lake. Analysis of the faunal remains indicates that people were making extensive use of fish, obtained from ancient Lake Moeris but also from shallow lacustrine environments likely typical of the eastern lake basins. Therefore it is reasonable to suggest that people were economically engaged with the lake and adjacent lake basins. But whether this can be used to infer that occupations match changes in lake levels, and therefore that these changes can be dated using charcoal from the hearths associated with these occupations, is a different story. As we indicated in chapter 3, the topography of the north shore is not steep. The majority of archaeological materials are found within a relatively narrow topographic band north of the lake basins that CatonThompson and Gardner identified. This means that people might have occupied places close to the lake basins without these occupations matching any changes in actual lake levels other than those associated with seasonal shifts in inward and outward water flow associated with the Nile flood.

It is important to consider what is meant by lake level change. Since the ancient Lake Moeris was likely at times connected to the Nile, it was prone to changes in level as a consequence of the Nile flood cycle. The relationship between occupation in the Fayum and the Nile flood is itself the subject of considerable debate. On the one hand, Hassan $(1984,1997)$ links periods of occupation with high Nile levels and periods of abandonment with times when Nile levels were lower. On the other hand, Williams (2009:11) suggests that it was times of lower rather than higher Nile flow that were conducive to agriculture on the Nile floodplain since this allowed swampy ground to drain. This uncertainty in the impact of river levels on occupation and subsistence applies equally to the Fayum. It is not possible to simply assume that people reacted in a particular way or indeed reacted uniformly though time to the level of the lake. It is also important to consider the different forms of possible interaction. Most discussion involves lake level changes and potential areas for growing domesticates, but any lake level changes might also have an impact on the availability of fish. As our faunal analysis results indicate, fish was an important economic resource.

As we noted at the start of this chapter, the adoption of agriculture in Egypt is often linked to changes in environment, particularly the shifts toward greater aridity that occurred toward the mid-Holocene. The difficulty with applying such arguments to the Fayum is that while there was indeed a shift to greater aridity with the southward movement of the ITCZ, environmental change at the local level, within the Fayum Basin itself, was likely the result of the interaction of a number of regional shifts in climate. As we argued in chapter 3 and elsewhere (Phillipps et al. 2012), there is evidence that the mid-Holocene Fayum was influenced by the southward movement of the Mediterranean winter rains, particularly during the period 6700 to $5800 \mathrm{cal} \mathrm{BP}$, associated with moister conditions. This time may have provided what Williams (2009) refers to as a period of "geological opportunism" in the Fayum, meaning that the use of plants and animals occurred when it was environmentally possible for this to occur. Cappers (2013) makes a similar observation when he suggests that water and nutrients form ecological constraints on the use of cereals.

One of the difficulties in demonstrating an environmental explanation for periods of occupation in the Fayum is the problem of separating the behavioral pattern indicated by the presence or absence of radiocarbon dates at a particular period from the patterns that relate to the preservation of hearths and the sampling bias in obtaining determinations. Despite the Fayum now having a large number of radiocarbon dates from secure locations we are only beginning to develop a spatial and temporal understanding of landscape use through hearth construction. There is an apparent trend in the age of hearths from E29H1 in the west to Kom K in the east, but this trend needs to be explored across larger areas of the north shore. This is only possible, however, if the places that do indeed have archaeological materials (and equally those that do not) remain relatively undisturbed. Unfortunately, in our investigations we seem to be fighting a losing battle, as the level of destruction as a result of modern development has increased exponentially in recent years (Figure 7.3). 


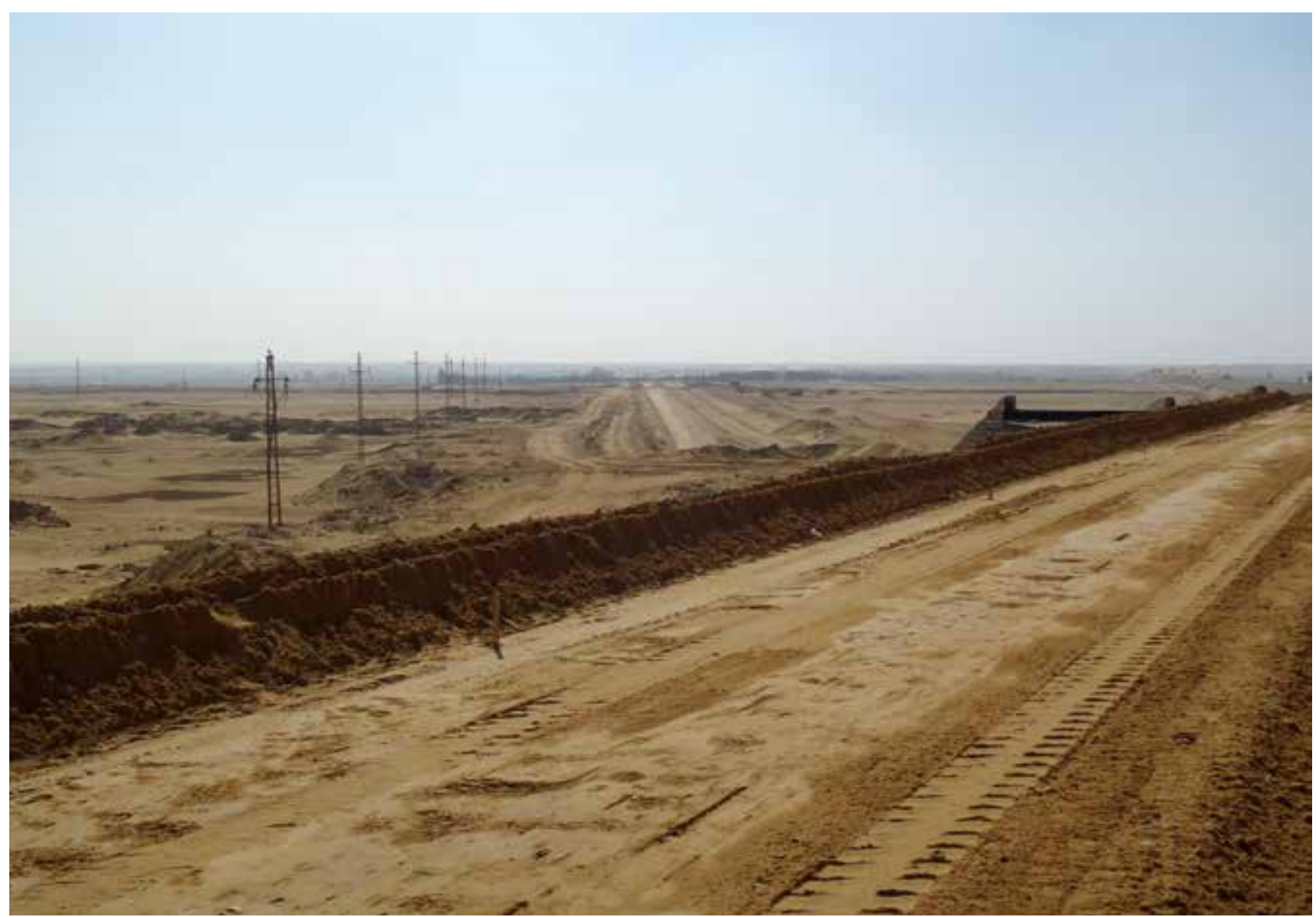

Figure 7.3. North of L Basin showing recent destruction as of December 2014.

It is instructive to consider the change our work has made in the extent of the Fayum chronology. As noted above, we have begun to fill in the hiatus between the early and mid-Holocene proposed in earlier studies. However, we have made relatively little impact on the age at which the archaeological materials ceased to be deposited, estimated to be circa 6200 to $6000 \mathrm{cal} \mathrm{BP}$ by Wenke (2009). Although some of our age determinations are slightly more recent than this age range, the date of apparent abandonment of the Fayum remains more or less intact. This date may correlate with age estimates for an increase in the Nile floods, as Williams (2009) suggests, the result of greater summer precipitation in Ethiopia at around $6100 \mathrm{cal} \mathrm{BP}$, and somewhat counterintuitively, a decrease in Nile sediment loads, in addition to a decline in winter rainfall. We may therefore have a rather more secure correlation between abandonment and environmental change at the end of this period of occupation of the basin than we do at any other time period. However, we also need to consider alternative explanations that do not involve domestic crops to explain abandonment of the Fayum Basin. Fish continued to be the predominant faunal material throughout the period from the early to mid-Holocene when the Fayum was occupied. Changes in lake levels, including any connection to the Nile that had an impact on fish abundance in the lake and the lake basins, might also be responsible for significant changes in human use of the Fayum region.

For earlier periods such a clear correlation is lacking. Therefore, rather than seek a specific environmental causal change, it may be more useful to consider evidence for environmental changes in general. As noted, the movement of the ITCZ south increased broad regional desiccation levels, while in the north, including the Fayum, winter rain may have increased seasonal moisture levels. Nile flood levels were fluctuating. Thus, from the early mid-Holocene on, there was considerable evidence for environmental change. We have a number of dated hearths from this period 
from L1, K1, and Kom K, as well as dates from the $\mathrm{K}$ Pits. We have much more intermittent evidence for occupation before this period, possibly a reflection of preservation as well as different periods and perhaps types of occupation. While we cannot define phases of occupation and abandonment for the whole period, we can make comparisons between different locations on the north shore that apparently preserve occupation deposits dating to different time periods. This allows us to make some observations concerning correlations between the general nature of environmental change and patterns of settlement.

The way large-scale climatic change manifests at the local environmental level is also important. This relates environment to the ways people engaged with landscape, but also the preservation of the remains of their occupation. Topographic variability between basins, while not extreme, may provide opportunities for particular plant and animal communities and human exploitation of those communities. Equally, topographic variability may have played a role in agricultural potential and perhaps changes in the availability of fish. In effect, "geological opportunism" may occur at the local scale as well as at the regional scale that Williams (2009) discusses.

\section{The Nature of Settlement in the Fayum}

Other than the heat retainer hearths and the Upper $\mathrm{K}$ Pits, we did not find features that relate to structures in the areas we investigated. However, the absence of such features does not lead directly to inferences about settlement because their absence can be used neither as a marker of the lack of permanency of occupation nor as a measure of only small settlement size. Permanent structures were at times constructed by peoples who were highly mobile, and equally, people who were relatively sedentary made structures that do not survive archaeologically. Relatively large settlements that left few features might have existed (Barnard and Wendrich 2008; Boyd 2006; Edwards 1989).

Mobility, as described in chapter 2, is often discussed in analogical terms as a concept rather than as a reflection of actual evidence for movement (Phillipps and Holdaway 2016). This is particularly true for the early Neolithic in Egypt, where mobility most often refers to the migrations of people or the transfer of ideas, indicated by the presence of artifact types and/ or domestic species. In addition, settlement pattern is often characterized by extension based on the economy or environmental context (Phillipps et al. 2016). In the analyses presented here, we sought to consider actual evidence for movement and separated this from the concept of mobility, as Close (2000) originally suggested. The advantage in making this distinction is that it provides the ability to document landscape use. All peoples were to some degree mobile, just as they were to some degree sedentary. Archaeological remains indicate occupation of places within a landscape, but it is reasonable to assume that people made use of parts of the landscape where archaeological materials either have not survived or where they do survive but have not been studied. By investigating how objects moved based on analyses of these objects, archaeologists are able to reconstruct the spatial extent of landscape use beyond the spatial distribution of the objects themselves. In this sense, studying mobility is a means to an end rather than an end in itself. Using a variety of analyses to measure movement into and away from the Fayum, we were able to draw inferences about the population of activities that were undertaken at many different places in the landscape. These inferences are therefore based on using what is preserved to talk about activities that are not directly represented rather than working from the assumption that the archaeological record is a direct reflection of all aspects of past lifeways that occurred at one place.

While the range of features preserved is limited, the range of portable artifacts is wider, and many of these are preserved even in surface deposits. People reduced stone cobbles to manufacture artifacts in a variety of places across the Fayum north shore. We analyzed the resulting abundant flaked stone artifacts using techniques that allowed us to determine the shape and size of the raw material worked and whether or not the assemblages reflected complete discard at one location or the movement of artifacts to other locations. We were able to show that assemblages associated spatially with hearths of different ages indicated the movement of flaked stone artifacts in different forms. We used artifact movement as a proxy for human movement and from this made inferences about the nature of mobility and thereby landscape use at different times in the history of occupation of the Fayum.

Flint is not found naturally in the Fayum Basin, so raw material for flaking was moved into the area from elsewhere. Cobbles of flint were treated differently at different times and estimates of original cobble size 
differ among the assemblages studied. Cores from all the areas frequently retain some cortex and therefore could have been flaked further, but in many cases they were abandoned before this occurred. Despite this, it is likely that significant numbers of flakes were moved to other locations. Quantification of shape and size of flakes and cores also shows variability across the landscape. At E29H1, cores were often worked along their longest axis, leading to long, relatively narrow flakes. In contrast, cobbles were worked across their shortest axis in the L1, K1, and Kom K assemblages, leading to the production of relatively short, thick flakes. Comparing the size estimates for the original cobble with the numbers of flakes in the assemblages shows a range of values. In the E29H1 assemblages we find the smallest number of flakes relative to the original cobble volume. This measure is matched by values for the cortex ratio, which calculates the relative proportion of cortex remaining in the assemblage compared to that expected given the size and frequency of the cobbles knapped. Values of the cortex ratio are lower for the E29H1 assemblages compared to those from $\mathrm{L} 1, \mathrm{~K} 1$, and Kom K, indicating the removal of a larger number of flakes relative to the numbers of cores that were worked at E29H1. The low flake-to-core ratio at E29H1 therefore likely reflects the removal of flakes.

In contrast, the L1 and XB11 assemblages show many fewer cores than should be present given the total volume of the flaked artifacts found. This suggests that cores were moved from these locations, leaving behind the flakes. Differences in assemblage composition therefore indicate changes in the way the available volume of the cobbles was used at different times in the Fayum. In the earlier E29H1 assemblages, this volume was utilized in the form of flakes, with these being removed. In the later L1 and XB11 sites, as well as those from Kom K, this volume was utilized in the form of cores, with these transported. The different ways that stone volume was reduced explains differences in the shape of flakes abandoned in different parts of the Fayum.

Cobbles flaked in the $\mathrm{K} 1$ area were larger than those worked in the other study locations, and as a result, the flakes produced were also larger in this area. However, a comparison of the volume of the assemblage to the number of cores suggests that cores were removed from the assemblage in a way similar to the L1 and XB11 assemblages. The size of the cobbles flaked at Kom $\mathrm{K}$ fits between the larger cobbles flaked at $\mathrm{K} 1$ and the smaller cobbles worked at L1 and E29H1. Cores were likely moved from the Kom $\mathrm{K}$ assemblages (both excavated and collected from the surface) since there were insufficient cores remaining to account for the assemblage volume. Cortex ratio results suggest the removal of cores and some flakes, with the remaining flakes being removed in proportions that fall between the L1 and XB11 assemblages and those from E29H1.

Movement therefore characterized all periods of occupation in the Fayum for which we have evidence. Differences in the estimated size of the cobbles suggest that raw material was sourced from different locations at different times. The oldest of the sampling areas we studied, E29H1, has some of the densest artifact deposits but ironically contains evidence for two-way movement. Not only were stone cobbles introduced to the location but substantial quantities of flakes were removed. This emphasizes how the E29H1 site was simply one place within a wider settlement system. People knapped stone at this location and created the hearths that we were able to date. They left faunal material that indicates the exploitation of fish and, to some degree, wild terrestrial animals. Their activities left large quantities of worked stone that are today distributed across a substantial area. Yet despite the size of E29H1, as far as we can determine, the location is surrounded by assemblages that indicate the use of differently sized raw material worked in a different manner. Hearths in the surrounding areas also date to later periods. Unless other early locations have not survived, a scenario perhaps indicated by the sediment analysis at E29H1, the activity that left a record within the Fayum dated to the period immediately after the beginning of the Holocene was restricted to a small number of localities. We know of only one other location at the western edge of the Fayum north shore that contains an assemblage similar in appearance to E29H1. It is Z1 (Caton-Thompson and Gardner 1934:79), which equals E29G1 reported by Wendorf and Schild (1976:162)

Evidence for the recent use of the E29H1 location is sparse. During the extensive survey we conducted before the surface deposits were destroyed, we identified only a handful of historic items (likely Romanperiod pottery and Islamic coins) mixed with tens of thousands of flaked stone artifacts and faunal remains. In fact, the apparent lack of mixing of materials from different periods at individual locations is one of the striking features of the north shore record in general. 
Moving east from the E29H1 deposit, hearth ages are more recent. The evidence for flake removal is replaced by evidence for the movement of cores. Raw material indicates the use of different sources, with the relatively large size of the cobbles from the $\mathrm{K} 1$ area being distinct from those found at Kom $\mathrm{K}$ and the L1 area locations, all of which are different than the likely shape of material worked at E29H1. Cores rather than flakes were moved from the eastern locations, suggesting some form of mobility, although with a different method of exploiting stone raw material utility than earlier occupations. Where these cores might have moved to remains uncertain, although in a separate study Phillipps (2012) showed that core movement is also high away from Kom W. We intend to expand this study and report the result in a subsequent volume. Cores were removed from the L1 area assemblage, from the Kom $\mathrm{K}$ excavated and surface assemblages, and from the K1 area. However, there is the possibility that these cores were not moved great distances. Unfortunately, we are hampered in demonstrating this by contemporary development that has destroyed the region surrounding Kom $\mathrm{K}$ and the area between this site and the L1 study area. However, we hope to pursue this question further in future studies of surfaces around Kom W that remain (at least for the present) intact.

The hearth features at Kom $\mathrm{K}$ indicate repeated usage of the same location over some period of time that cannot have spanned multiple centuries but could have occurred over many decades or a small number of seasons. We know that people cooked food at this location, including making use of domestic grains, and that they abandoned flaked stone artifacts and ceramics. Animal dung was used as fuel. They left at least one clay vessel in place, suggesting that it was left for a reason, potentially to store something. It is possible that part of the reason cores are missing from both the Kom $\mathrm{K}$ surface and excavated assemblages is that people collected cores that were not fully expended and moved them to other locations. This process could also, of course, explain the other assemblages where we found relative core depletion, such as XB11.

Kom $\mathrm{K}$ retained stratified deposits, but the contents of these deposits do not differ substantially from the surface materials we studied in the areas that surround Kom K, with the caveat of the limits imposed by modern development discussed above. The areas we studied indicate the remains of hearth construction, the exploitation of fish, and the use of pottery.
Grindstones occur in more locations than, for instance, the ceramics. Their extensive distribution is consistent with their use throughout much of the history of occupation in the Fayum. Their presence need not relate solely to the presence of domestic grains. As indicated in other parts of eastern North Africa, grinding equipment is present before evidence for the use of domestic grain (Holmes 1989; Warfe 2003). Acknowledging the lack of age determinations for the grindstones, their presence associated with deposits from E29H1 and L1 moving east to Kom K and north to $\mathrm{K} 1$ supports the notion that they were potentially in use throughout much of the early to mid-Holocene in the Fayum. The Upper K Pits provide direct evidence for the use of domestic grain, since these structures were likely constructed for the storage of grain within basket-lined pits as well as unlined pits and potentially ceramic vessels.

Interpreting the evidence from the Lower $\mathrm{K}$ Pits is an exercise in forensic rather than salvage archaeology. Almost all the evidence is now destroyed, and we were not even able to relocate the Lower Pits, which are now likely destroyed or buried beneath contemporary irrigation works. From what can be reconstructed from the published sources, some of the Lower K Pits were lined with basketry while others were not. The sizes of the pits varied considerably, with the smallest not much larger than some of the intact ceramic vessels that Caton-Thompson and Gardner retrieved, although none of those from the Lower K Pits have survived. It is possible that the smallest depressions from the Lower K Pits were used to hold clay vessels like that excavated in Kom $\mathrm{K}$ or indeed fired vessels like those Caton-Thompson and Gardner did retrieve intact from Kom W (Emmitt 2011). If so, and assuming that the pits and vessels were related to storage, then placing storage facilities at different points in the landscape was part of the Fayum settlement system during at least some periods of occupation of the region.

The K Pits are spatially restricted to only two localities, frustratingly now both largely destroyed. We surveyed as much as we were able in the area to the west and north of the Upper K Pits and found no evidence that these features were once more extensively distributed. This may simply be a function of site preservation and modern development, but as best as we can determine, they do not exist in other locations. Ceramics, on the other hand, are more extensively distributed. The preservation of ceramics 
is dependent upon their context, with intact examples found in stratified deposits, while those in surface scatters are often heavily eroded. There is at least circumstantial evidence that some of the ceramics reflect storage activities, although it is yet to be seen if these forms exist beyond the Upper and Lower $\mathrm{K}$ Pit or kom assemblages (Emmitt 2011). If so, then the landscape may have seen a larger number of storage locations than the Upper and Lower K Pit evidence alone indicates.

It is also true, however, that surface deposits of flaked stone artifacts are more ubiquitous than deposits that contain both flaked stone artifacts and ceramics. This reflects preservation to a degree but even so, the ubiquity of the flaked stone artifacts indicates spatially extensive activity within the band of a few hundred meters north of the basins that Caton-Thompson and Gardner identified. Putting these sets of evidence together, and considering the Fayum north shore from a landscape rather than site-based perspective, we get a vision of people moving into, out of, and across a landscape rather than settling within it. Seen from this perspective, the spatially extensive evidence for grinding stones makes sense if these artifacts were left in place as "site furniture" (Binford 1979), to be reused by people as and when they returned.

In sum, our research suggests aspects of two distinct settlement systems that occurred at different times. Early in the Holocene we have evidence for concentrated, although temporary, activity at one location. Later in time we have evidence for different and spatially varied forms of movement and settlement. We do not have evidence for a village- or even hamlet-based settlement. Instead we have extensive deposits made up of relatively short-lived features and a material culture that indicates movement into, away from, and within the region. However, combined with this we have evidence for dispersed locations with concentrated evidence for storage. We do not, therefore, have a simple mobile-versus-sedentary dichotomy. Instead we have evidence that people were involved in a variety of activities that included movement in a complex set of ways. It is into these settlement contexts that we need to place the socioeconomic evidence that we have for the Fayum at different times.

It is tempting to equate the different settlement systems with major phases of occupation and to link these to Epipaleolithic and Neolithic economies, but this temptation must be resisted. As we discussed in reference to the radiocarbon determinations from the hearths, what we can say relates to what has preserved and what we have studied. To a degree we can understand how people made use of a landscape by understanding how artifacts were moved around this landscape. We need to extend our survey beyond the immediate area of the $\mathrm{L}$ and $\mathrm{X}$ Basins to gain a better understanding of where material might have been moved to before drawing firm conclusions about the significance of changes in the nature of the settlement system in the Fayum. We also need to understand more about the reasons we have temporal gaps in the record. Are these evidence of a lack of people in the Fayum, do they relate to movement of people to other places along the north shore, or are they an artifact of preservation or sampling? To some degree these questions are addressed in chapter 8 , where we consider results of the Fayum study in their wider regional context.

\section{Socioeconomy of the Early to Mid-Holocene Fayum}

A number of authors have commented on the continuing tendency to propose a fundamental separation between the socioeconomy of hunter-gatherers and agriculturalists. However, in the Fayum there are as much data that could be used to argue for continuity as there are data that indicate a difference. The faunal deposits available for study pose a number of issues. Preservation differs between locations, particularly between the surface locations and those that are buried. The larger, robust bones are differentially preserved in the surface contexts, while the buried deposits to a degree preserve a wider range of anatomical parts. The effects of differential preservation in surface collections are especially clear among the aquatic taxa, with the Tilapia and Cyprinids probably underrepresented because their bones are fragile. The hard and sturdy bones of the soft-shell turtles, on the other hand, preserve well. Interpretations of the faunal assemblages also need to take account of the contexts in which the deposits are found. To some degree, comparison of the surface and buried faunal assemblages allows the influence of differential preservation to be controlled for.

How people processed and disposed of bone will have a marked impact on bone survival in the archaeological record, and disposal in the past was of course affected by the nature of the activities undertaken at 
different times and places. It is also often assumed that the first appearance of faunal materials in the archaeological record marks the first use of these species, but of course this is not necessarily true. More so for fauna than for some types of portable artifacts, we have very limited samples from the Fayum from a small number of locations, and this necessarily places limits on the conclusions that can be drawn.

Recent examination of faunal remains from the Fayum suggests that the use of fish dominated socioeconomic practice in all periods of occupation (Linseele et al. 2014, 2016). Given the proximity to the lake, this is perhaps not surprising. However, even in the context where domesticated animals can be identified, for instance at Kom $\mathrm{K}$, their presence is not overwhelming, whereas fish predominate. This perhaps reflects the minor role played by domesticated animals in the Fayum, which is likely linked to the nature of settlement.

The bird fauna from Kom $\mathrm{K}$ are low in number but relatively rich in taxa, with coot the most common. In Egypt today, coots are most abundant from mid-September to early April. Among the other bird taxa, ducks are probably winter visitors as well. Tilapia and clariid catfish are represented at Kom $\mathrm{K}$ by large, sexually mature specimens, with average standard lengths of about $30 \mathrm{~cm}$ and $60 \mathrm{~cm}$, respectively, and were therefore likely captured when spawning in shallow waters during periods when the lake basins were flooded from August to September (Linseele et al. 2014). As discussed in chapter 3, topographic evidence suggests that the eastern basins were shallow and lacked steep shorelines. These basins may have received water from the lake only during periods when the Nile flooded. At other times, they may have been rain-fed via wadi systems. Brewer (1987, 1989a) analyzed growth rings on the pectoral spines of clariid catfish and correlated growth phases in these with periods of high temperatures. The conclusion from this study, that fishing was mainly practiced at times with temperatures similar to those of modern Egypt's late spring or early summer, as well as at times with temperatures equivalent to those later in the summer, is compatible with the hypothesis that some of the fishing activity happened mainly in the late summer months based on the predominance of adult spawning fish (Linseele et al. 2014) in the shallow-water microenvironments provided by the eastern basins.
Variability characterized the environmental context of the region. Topographic variability likely influenced the impact of fluctuations in lake level, whether they occurred annually or over broader time scales. Water depth, size, and energy (that is, wind action) variability between the basins identified by CatonThompson and Gardner likely affected the types of vegetation and fauna found both around the basins and within them. This may be significant in relation to the resources available to inhabitants of the northern shore, for as noted above, particular fish species have well-documented habitats that differ, including during seasonal spawning. Vegetation may additionally have facilitated or at times limited access to the lake and basin shores, and this was also likely variable in different parts of the north shore.

Evidence for wheat and barley storage is present in the Fayum. However, it is not clear whether these locations were created for the storage of seed crop or surplus to be used after the grains were harvested. What is clear is that the storage pits were accessed multiple times. Not all pits were constructed in the same way; nor were they the same size. Furthermore, CatonThompson and Gardner (1934:53) suggested that basketry was removed from some of the Lower K Pits, which suggests abandonment of those particular pits and potentially the reuse of the baskets derived from them elsewhere. Other examples of storage pits are discussed in chapter 8 .

How were people processing wheat and barley? As at other locations throughout Egypt where early evidence for wheat and barley is present, the means by which these species were exploited is yet to be determined (see chapter 8).

Equally uncertain are the locations in which people cultivated wheat and barley, if these crops were indeed grown in the Fayum at all. The use of wheat straw in the Upper K Pits basketry and the presence of sickles lend credence to the idea of local cultivation. Previous research proposed the use of the lake margin to cultivate cereals, with the annually receding floodwaters providing irrigated sediment and potentially also fresh sediment via the Nile inundation. If the lake inundated annually, with a rise of between 2.5 and $4 \mathrm{~m}$ as has been proposed (Ball 1939; also Wenke et al. 1988), given the topographic variability of the lake basins, it is important to consider what the local impact of such a rise might be. If a midpoint of Ball's estimate is taken (that is, $3 \mathrm{~m}$ ), approximately $235.7 \mathrm{~km}^{2}$ would become 
available upon water recession across the north shore. $\mathrm{L}, \mathrm{K}$, and $\mathrm{X}$ basins are the largest and shallowest of the lake basins along the north shore and would have been affected most by lake level changes (Figure 3.6). This may have provided a suitable environment for lakeshore agriculture.

In addition to the impact of seasonal lake level fluctuations, the influence of rainfall and possibly the natural channels provided by wadis for soil irrigation needs to be considered. The strongest support for this influence comes in the form of abandonment of the region, which, as noted above, is supported by the current radiocarbon determination corpus. If lake edge agriculture was viable and productive, why was it not maintained beyond $6000 \mathrm{BP}$, when, according to Wendorf and Schild (1976) and Hassan (1986), the lake level was relatively high? Maybe, as Williams (2009) suggests, from this date the lake was too high and as a consequence regions close to the lake were not conducive to cultivation. This of course depends on the relationship between crop growing and the lake edge, something for which we lack information, and as discussed above, the impact may potentially relate to fish rather than crops. Where people moved after they left the Fayum around $6000 \mathrm{BP}$ is unknown, but it is possible they moved into the Nile Valley proper. This has obvious implications for the development of agricultural practice, since they likely encountered an environment that was somewhat different than that of the Fayum.

\section{Cultural Groups in the Fayum}

Various authors have commented on the abandonment of the Fayum at dates of around $6000 \mathrm{cal} \mathrm{BP}$, and dates from hearths reported here have not greatly changed this age. However, the notion of abandonment needs to be considered carefully in relation to the evidence for movement discussed above. Abandonment and indeed movement are scale-dependent phenomena. In one sense, the Fayum was often abandoned and then reoccupied, since both the hearth-based chronology and analysis of the flaked stone artifacts indicate movement away from, as well as into, the region. Abandonment at $6000 \mathrm{cal}$ BP was simply one more episode in people leaving. It is important to acknowledge that at a different temporal scale, even this last abandonment was of course not final, since the Fayum north shore was again reoccupied, possibly during the Old Kingdom and certainly during the Greco-Roman period. At around $6000 \mathrm{cal} \mathrm{BP}$ or a little later, people left and simply did not return. Unless they died out, for which we have no evidence, they must have gone somewhere else. This presumably occurred because wherever they ended up suited these people better than the Fayum. As discussed above, this may have implications for agriculture. It is obvious, therefore, that we cannot determine why the Fayum was abandoned by studying the Fayum itself. The push and pull factors can be identified only by considering the wider context of movement across multiple landscapes.

We can, however, consider the implications of the repeated occupation of the Fayum as shown by the evidence for movement. First, it is clear that any models that seek to trace the movement of culturally distinct peoples either from the eastern Sahara or from Southwest Asia must take into account the level of movement indicated by the early to mid-Holocene Fayum record. It is not a question of a single movement by one group of people who brought with them a particular artifact suite or indeed a package of domestic plants and animals. Movement seems to feature throughout the history of the region. Second, when domestic plants and animals were added to the economic repertoire of the Fayum people, they were accommodated into a system that involved people moving around (at least minimally to gain stone raw material). These people were also establishing storage locations, possibly at multiple places across the region. Domestic plants and animals bring with them shifts in an economic system that can be characterized by greater flexibility but also, paradoxically, by greater constraints.

The mix of domestic plants and animals in the Fayum is intriguing. Several components (for example, wheat and barley) seem to lessen the ability to move, either because of the need to be present when grains are planted and harvested and the associated need to store the harvest, or the presence of species that placed limitations on movement (Linseele et al. 2014). Domestic animals in general require access to water, and this is a constraint on where and when people were able to move. The flexibility comes from the ability to have a "larder on the hoof," so to speak, and also to amass a dependable supply of food that can be used to tide over times when other resources are not available (Marshall and Hildebrand 2002). The temptation is of course to see the use of domesticates as inevitable, since it led to the development of more complex forms of social organization in 
later times. It is often associated with a fundamental change in the relationship between people and the environment. The need to care for plants and animals is, for example, seen as hampering the ability to move. However, these associations need to be challenged. For the Fayum, despite the introduction of domesticate species, there are many indications of mobility occurring at different spatial and temporal scales.

Rather than see the introduction of domesticates as a wholesale replacement of one lifeway with another, it is important to consider what sets of conditions coincided about the time the various domestic species begin to appear in the Fayum record. As discussed above, paleoenvironmental evidence indicates shifts in both winter rains and levels of the Nile flood immediately before and then during the period for which we have the best evidence for the presence of domestic animals. We have a single dated caprid bone from E29H1 that indicates the presence of this domestic species before 7000 BP (Linseele et al. 2016). The largest assemblage of domestic fauna and domestic cereals comes from Kom K, associated with ages more recent than $6600 \mathrm{cal}$ BP obtained from numerous intercut hearths, but otherwise we have no evidence of permanent structures. Fish remains are common in the Kom $\mathrm{K}$ fauna. We also have evidence for core movement at Kom K and the surrounding locations. People who inhabited these locations were mobile, but unlike the earlier levels of mobility, they were using a different economic strategy when dealing with stone. Although we do not as yet have direct evidence, it is tempting to interpret the movement of cores as a form of scavenging of materials (McDonald 1991) already brought into the Fayum by earlier, although not necessarily much earlier, inhabitants. If so, then in addition to the contingencies required by the use of domestic species, and the need to develop storage facilities, people changed both their economic exploitation of raw material and their use of distant raw material sources.

In the same areas where we find evidence for the use of these new raw materials, we also find ostrich eggshell, often in the form of perforated beads (Figure 7.4). Ceramics show a similar distribution to ostrich eggshell (Figure 7.5). The small numbers of ceramic fragments from E29H1 are likely to be Greco-Roman rather than Neolithic in age. Although we did not always find additional examples, Caton-Thompson and Gardner in their Kom $\mathrm{K}$ excavations found a larger array of artifacts, including pallets, a range of bone and shell artifacts including bone points, and green feldspar beads, as well as perforated shells. The flaked stone artifact inventory included ground stone axes and sickle blades.

Combined with the evidence for changes in stone raw material economy, storage, domesticated plants and animals, the presence of ostrich eggshell beads and ceramics as well as adzes, sickle blades, bone, and shell artifacts suggest a changing relationship with the environment and also potentially relationships with other groups of people in ways that Wengrow et al. (2014) have documented for other parts of Egypt. Although we have only limited indications of external contacts, our work in the Fayum has provided evidence in the form of shells from the Red Sea and the Mediterranean (Caton-Thompson and Gardner 1934; Linseele et al. 2014) in addition to evidence for the transport of stone raw material from outside the Fayum. Eventually, these new interrelationships led people to center their activities at places outside the Fayum, and our study region was, as noted above, not reoccupied again until the Old Kingdom and GrecoRoman periods.

From this perspective, there was nothing inevitable about the adoption of domestic species in the Fayum, but their adoption does correlate with other economic and environmental changes as well as shifts in the nature of mobility. Therefore, rather than discuss the Fayum evidence as the development of the "Neolithic in Lower Egypt," with the implication that the Neolithic "arrived" as a package of traits and an integrated system that was primed to give rise to the décrue system of cultivation that characterized later Dynastic Egypt, we have evidence for the use of domestic animals, mainly sheep and goat, with much more limited evidence for pig and cattle, in a system where people were moving material culture around at least within the Fayum if not farther afield. Part of this system later involved using domesticated wheat and barley, although the evidence for this use is restricted to basket-lined storage devices and limited use in the Kom $\mathrm{K}$ hearths, together with the presence of sickles. If the presence of ceramics also indicates storage, then storage places were distributed at a number of locations across the landscape. People still made extensive use of fish caught, probably seasonally, from the lake basins as well as the lake itself. However, their use of wild terrestrial animals was limited since earlier times. 


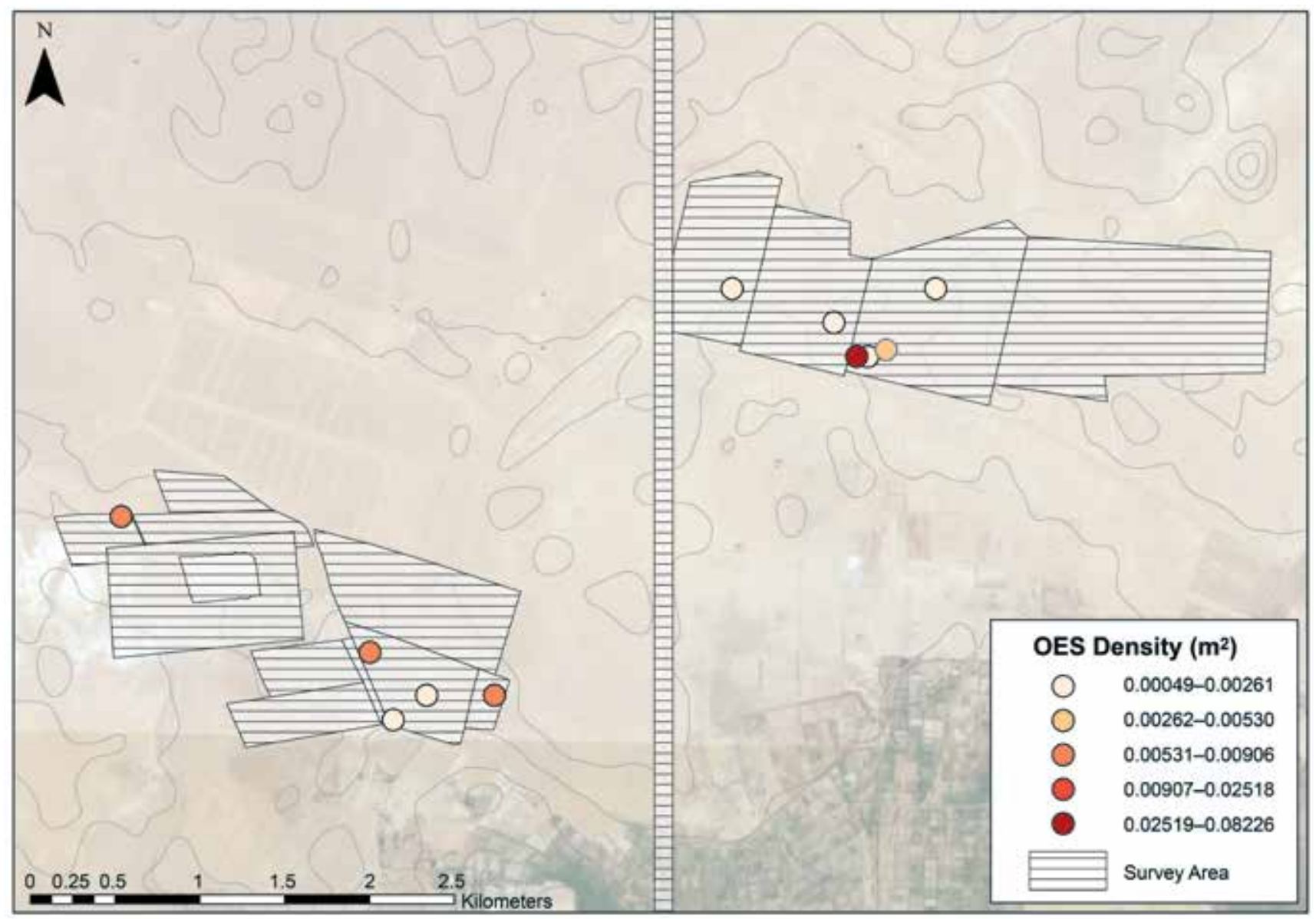

Figure 7.4. Ostrich eggshell density $\left(\mathrm{m}^{2}\right)$ in transects from all areas with corridors and survey areas.

Such a system could have been introduced by people migrating to the Fayum from elsewhere, but combining the different aspects of movement, storage, and wild and domestic plant and animal use results in a picture for which there are few analogs. As emphasized above, the Fayum likely records only one aspect of the settlement systems that characterized the people who used the region (Lower Egypt), since we have good evidence for their movement to places elsewhere. The complexity of the behavior evidenced in the archaeological record therefore poses a challenge for culture historical interpretations of the Fayum. It is not that people did not move and bring with them new material culture and economic pursuits; it is rather the likelihood that the extent of such movement will not be apparent from a limited range of archaeological sites from one region. Regional comparisons of material culture types fail to show simple regional sequences (e.g., Warfe 2003). If other locations show the type of evidence for movement that we have found in the Fayum (discussed in chapter 8), then the failure of the culture historical approach that Warfe (2003) demonstrates is to be expected.

\section{Adoption of Domestic Species in the Fayum}

One of the key questions that has driven debate on the origins of the Egyptian Neolithic is why aspects of the Neolithic package appear so late in comparison to adjacent Southwest Asia. The answer to this question relies on dating the earliest evidence for the appearance of domestic plants and animals. This is never an easy task, since what this really means is dating the earliest evidence for domestic species that are both present and preserved. As we have repeated in a number of points in the discussion above, preservation has likely played a significant role in what we have available today to study from the Fayum, and as also noted a number of 


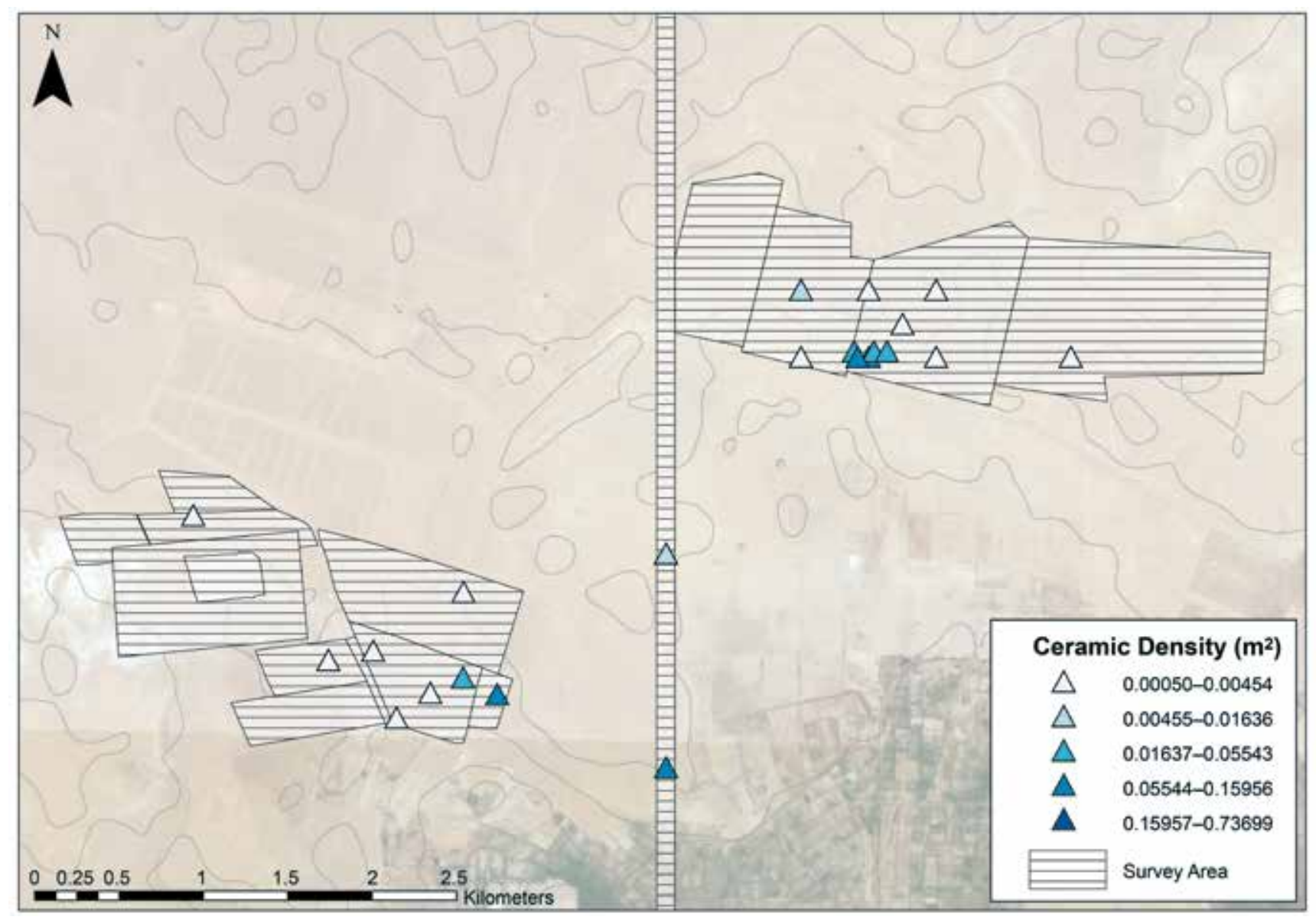

Figure 7.5. Ceramic density $\left(\mathrm{m}^{2}\right)$ in transects from all areas with corridors and survey areas.

times, the Fayum preserves only certain aspects of the wider settlement system in which the inhabitants were engaged. What gets preserved depends on both how this system operated and what role the Fayum played within it.

We have a limited number of bones from potential domesticates from E29H1, with the oldest date circa $7000 \mathrm{cal} \mathrm{BP}$, and more numerous bones for domestic animals coming from Kom $\mathrm{K}$ dated somewhat later. The most abundant evidence for domestic grains comes from the Upper K Pits. As noted, age determinations for these sites fall around 6500 to $6300 \mathrm{cal} \mathrm{BP}$, although precision is limited by the calibration curve. Flaked stone artifact assemblages from Kom $\mathrm{K}$ share similarities with those from L1, particularly in the economics of raw material exploitation and movement. It is possible, therefore, that aspects of the settlement system associated with the use of domestic species later in time were operating as early as 7400 to $6800 \mathrm{cal} \mathrm{BP}$, and this may relate to domestic animals initially, with domestic plants incorporated later. If so, the age range coincides more closely with ages for the circum-Mediterranean dispersal of some aspects of the Neolithic package, specifically Southwest Asian domesticated species (Zeder 2008).

If the Neolithic in the Fayum gave rise to the Predynastic agricultural system, it is easy to slip into the position that the Fayum system should show "proto" aspects of the later system. However, the logic of this argument runs against, not with, Time's Arrow; what people did earlier in time cannot be based on what has yet to happen (Finlayson 2013). There is also a tendency to assume that once domestic species were adopted, they were both successful and came to dominate the economy. In fact, neither position finds support archaeologically (Rowley-Conway and Layton 2011). Use of domestic species was not always initially successful, as evidenced by its adoption and then 
abandonment in Sweden; nor need either domestic animals or domestic crops dominate the economy, as shown in Smith's (2006) discussion of low-level food production from the Mississippi Basin. Evidence for the relatively rapid appearance of domestic species coincident with the arrival of new peoples, arguably by boat, is apparent for parts of Europe (Rowley-Conway and Layton 2011), but other than the timing of shifts in the settlement noted above, there is no evidence in the Fayum for the type of Neolithic colonization the European evidence implies. Instead, there is evidence for a pattern of movement, storage, and domestic species exploitation in association with a continued use of fish resources. This looks less like a "package" and more like the variable association of economic behaviors that Finlayson (2013) has suggested should characterize the Neolithic.

The answer to the question of why the Neolithic package arrived so late may indeed be that it never arrived at all. Domestic species were certainly sourced from elsewhere, but they did not form part of a uniform package of economic pursuits, ways of living, and associated material culture. Instead, as we have outlined in detail above, domestic species were combined into a variety of socioeconomic systems that involved considerable mobility as well as the exploitation of seasonally abundant fish. Storage technologies were either adopted or invented but seem to have been incorporated into a mobile lifeway. People reorganized themselves, as seen in such things as the different economic exploitation of stone raw material and the implied changes in temporality involved in the use of domestic species, both animals and perhaps later plants. There are also changes suggested by the appearance of new forms of material culture, like the beads and perhaps the pallets. Ceramics also appear in the record, but we need the results of ongoing studies to more clearly determine what role they played. There are certainly hints that part of this role was storage, suggesting a spatially extensive system that involved both mobility and fixed place use (Emmitt 2011). Finally, we need to acknowledge that the Fayum north shore was likely only one part of a more spatially extensive system. If so, this has implications for how contemporary sites should be assessed. They may well have been occupied at periods by different peoples than those who spent time in the Fayum, but how much such differences should be emphasized, given the evidence for mobility that the Fayum record suggests, needs to be considered carefully. Early socioeconomies in the Fayum, Nile Valley, and Nile Delta may well have been produced by groups of people moving among these places, who made adjustments to the local environments they encountered (see chapter 8 ).

Ultimately the socioeconomic basis for use of the Fayum ceased to be viable for reasons that may not relate to the Fayum at all. People moved and changed their socioeconomy, developing the décrue system of agriculture, and from this economic base instituting the changes that we know as early state formation in Egypt. The key to understanding the Fayum may therefore not be as a model for the origins of later systems but as an example of just how flexible people can be when availing themselves of the opportunity to use domestic plants and animals in combination with wild resources. Of course, from the perspective of low-level food production societies (Smith 2001), this should come as no surprise. Rather than the Neolithic as a package, we now know that the early to mid-Holocene sees the development of a fascinating variety of forms of human plant and animal interactions. The Fayum adds one more example to the mix.

\section{Summary}

The Fayum preserves, or now unfortunately in many cases once preserved, a range of stratified and surface sites. To understand the landscape archaeology of the region, all sites need to be considered together in relation to the distribution in age of material culture, fauna, features, and organic remains, but also the depositional contexts in which material is found. In many instances, it is as important to understand where materials are not found as it is to understand where they are found. The relatively large number of radiocarbon determinations obtained for the Fayum in comparison to other parts of the eastern Sahara provides an indication of the chronology of human occupation of the region but one that continues to be site and research project dependent. This said, the hiatus between an older Epipaleolithic and more recent Neolithic is now less apparent. This in turn makes it more difficult to draw a line for the beginning of the use of domesticates in the Fayum.

Rather than change related to the arrival of domestic plants and animals, two settlement systems that occurred at different times are indicated. Early in the Holocene, evidence indicates concentrated, although 
temporary, activity at one or more locations. Later in time there is evidence for different and spatially varied forms of movement and settlement. Deposits are made up of relatively short-lived features and a material culture that indicates movement into, away from, and within the region. Combined with this, there are dispersed locations with concentrated evidence for storage. People were evidently involved in a variety of activities that included movement in a complex set of ways.

There was nothing inevitable about the adoption of domestic species in the Fayum, but their adoption does correlate with other local economic and environmental changes, as well as shifts in the nature of mobility. Variability between the lake edge basins likely supported different plant and animal communities and may have played a role in agricultural potential and the availability of fish. There is evidence for the use of domestic animals, mainly sheep and goat, with limited evidence for pig and cattle, in a system where people were moving material culture around, at least within the Fayum if not farther afield. Part of this system later involved using domesticated wheat and barley. If the presence of ceramics also indicates storage, then storage places were distributed at a number of locations across the landscape. Such a combination of movement, storage, and wild and domestic plant and animal use results in a system of settlement for which there are few analogs. 


\section{8 The Desert Fayum Reinvestigated:
The Fayum in Context} Simon J. Holdaway, Rebecca Phillipps, and Willeke Wendrich

W hen seeking data against which to compare the results presented in this volume, it quickly becomes apparent just how much the current understanding of the Neolithic in the Nile Valley and Delta of Egypt has suffered from a lack of detailed study that not only documents the nature of archaeological remains without typological bias but also considers such occupations within their wider landscape contexts. Typically, investigations in Egypt assume a later Dynastic model of settlement when conducting excavation, and more rarely survey. As a consequence, the research has focused on the largest in situ deposits available for study. The emphasis is put on single locations, and specific aspects of material culture are considered as holding "cultural" information. This results in a number of assumptions about the adoption of domesticated plants and animals, changes in population-level movement, type of settlement pattern, shifts in economic practice, and form of social organization.

The archaeological record of the early to mid-Holocene in Egypt is unique when compared to that of Southwest Asia. However, considered relative to its local context, the record is consistent with other parts of North Africa (Wengrow et al. 2014). The study of settlement in the Neolithic in any part of the world, but particularly in Southwest Asia, suffers from the problems encountered when the presence of permanent, surviving structures is considered indicative of minimal or no mobility. In Southwest Asia, this reduces the understanding of Neolithic settlements to a single "village" model. While there has been criticism of using houses, or built structures interpreted as houses, to define settlement pattern categories (e.g., Boyd 2006), permanent structures continue to be used as a proxy for occupation duration in discussions of the Egyptian Neolithic (Wengrow 2006). Salvatori (2012), for example, in commenting on the ways sedentism can be assessed, notes problems in the archaeological identification of places occupied continuously versus places that were simply returned to frequently. While it may seem self-evident to use permanence of structures to indicate permanence of settlement, the presence of house remains is no guarantee that any associated occupation was also permanent. As many archaeologists know too well, the longevity of structures often outlives the longevity of human association with them.

Agriculture is often linked to particular lifeways involving sedentism, but as our research and the research of many others have shown, the use of agriculture and mobility of people are not fundamentally linked (e.g., Edwards 1989; Ingold 1985). Smith (2001) proposed low-level food production as a way of conceptualizing variable use of domesticates, and in an earlier study we suggested how this might be extended to other aspects of human behavior, including mobility (Holdaway et al. 2010). 
From this discussion it is evident why it is so important to consider the specific archaeological record at different locations throughout the Nile Valley, the Delta, and the Egyptian Western Desert rather than assume that object typology can be used to define uniform regional cultural entities with similar socioeconomies. The Fayum illustrates the importance of a landscape approach at multiple temporal and regional scales. Whereas in the past, occupation of the Fayum was considered to be chronologically limited to two periods, as was summarized in chapter 7 , the history of occupation now appears to be more complex. Understanding the formation of the archaeological record is critical to determining the context of data that is preserved. Unfortunately, there are very few examples in Egypt where the formation of the archaeological record is studied explicitly and sufficiently. It is likely, therefore, that more variability that has not been accounted for, either by previous studies or in the current suite of regional syntheses, exists beyond the Fayum. Here we review the extent of this variability by considering evidence, taken from Phillipps et al. (2016), for settlement in Egypt in places comparable to the Fayum. We follow with a similar comparison for socioeconomy from Holdaway and Phillipps (2017).

\section{Settlement beyond the Fayum The Nile Delta Sites}

Two sites, Saiis and Merimde Beni Salama, provide evidence for Neolithic occupation of the Nile Delta. The deeply buried Neolithic deposits at Sais provided faunal remains, pottery, and flaked stone artifacts. The earliest Neolithic phase, Saïs I, has a suggested age of 7000 to $6800 \mathrm{BP}$, with the later Neolithic phase dated to 6500 to $6300 \mathrm{BP}$. However, these ages are based on typological comparisons of artifacts rather than radiocarbon determinations (Wilson 2006:83). Pig bones are the most commonly identified faunal material, with smaller numbers of cattle, sheep, and goat bones (Wilson and Gilbert 2003), in addition to a dense fish-bone layer (Wilson et al. 2014). Ceramics in a range of shapes and sizes indicate similarities with those from the earliest phases from Merimde Beni Salama (Wilson and Gilbert 2003; Wilson et al. 2014). When analyzed using the same methods applied in the Fayum (Phillipps 2012), flaked stone artifacts indicate the removal of elements of the assemblage, particularly flakes in contrast to cores or tools. Retouched stone tools are typologically associated with the later Neolithic layers at Merimde Beni Salama
(Wilson and Gilbert 2003). Fish species and sizes, the majority of which are clariid catfish with smaller numbers of tilapia (Linseele et al. 2014), suggest that people were present at different seasons. The site is spatially extensive but was sampled only by limited excavations. Typological links with other sites exist, but the nature of these links remains speculative.

Merimde Beni Salama is located in the western Nile Delta and dates between 6800 and 6400 cal BP (Hassan 1988:142). The site is also spatially extensive, with occupation probably moving because of changes in an adjacent branch of the Nile. Initial Neolithic features suggest the presence of roofed structures, while later in time are the remains of small circular huts (Eiwanger 1979). Partially subterranean pits lined with clay and basketry are reported but without evidence for grain (Hassan 1988). Shallow, circular depressions, with coiled matting attached to the sides, are interpreted as threshing floors (Hassan 1988). The most recent occupation, dated to $6400 \mathrm{BP}$ (Hassan 1985:95), contains semisubterranean oval structures with aboveground mudbrick foundations (Hassan 1980). Baskets set into the ground were probably used for grain storage, with some found sitting in clay-lined pits (Eiwanger 1978; Midant-Reynes 2000:116). Domestic emmer wheat (Hassan 1988), six-rowed barley, lentils and peas, wild sedges, and legumes are reported (Zohary and Hopf 2000:219). Faunal remains from the site include sheep, goat, cattle, pig, and fish (Hawass et al. 1988).

Flaked stone artifact and ceramic typological comparisons suggest connections with sites in the Fayum and with Haua Fteah (McBurney 1960). Ceramics similar in form to those found at Maadi are also found in a burial to the north of the site (Badawi 1980). Projectile points have been linked typologically to the PPNB of Southwest Asia (11,700 to 8400 BP; Kuijt and GoringMorris 2002:362), particularly with the presence of Helwan points (Eiwanger 1979:Figure 4; Kuijt and Goring-Morris 2002:Figure 11). The later Neolithic phases include bifacial tools, projectile points, and ground adzes very similar in form to those found in the Fayum (Eiwanger 1979:Figure 10).

In sum, Merimde Beni Salama has a limited number of features that probably represent houses and storage structures. Changes in the river flow at both Merimde and Sais likely led to changes in the areas occupied. Faunal material is similar at both sites, as are the flaked stone artifacts. Material culture suggests links with the Fayum and may show links to sites in the Levant. 


\section{Nile Valley Sites}

El Omari, located south of Cairo, is dated to 6435 to $5670 \mathrm{BP}$ (Hassan 1985:Table 1; Mortensen 1992). More than 100 semisubterranean circular pit features are recorded, with basketry- and clay-lined grain storage pits (Debono and Mortensen 1990; Hassan 1980). Cultivated six-row barley, einkorn, and emmer wheat are present (Wetterstrom 1993), and Mortensen (1999) reports the use of flax for textiles. Wild grasses were also used for basketry. Faunal remains consist of pig, cattle, sheep, goat, fish, and waterfowl (Hayes 1965; Mortensen 1999). Typological connections between El Omari and the Fayum are claimed based on bifacial flaked stone tools and similarities in ceramic manufacturing techniques and forms, but there is no direct evidence of interaction.

The North Spur of Hemamieh, excavated by CatonThompson in 1924 and 1925, is located near the modern town of Hemamieh in Middle Egypt. Hut circles are described (Holmes and Friedman 1994), with walls made of mud and limestone chips (Brunton and CatonThompson 1928:82). The structures were in most cases considered too small for use as dwellings, and storage is suggested based on the presence of sheep and goat dung (Brunton and Caton-Thompson 1928:82; Holmes and Friedman 1994), although they may alternatively represent animal pens. Hearths are scattered throughout the site, away from the potential huts. Ceramics were found in pit features together with freshwater mussel shells (Brunton and Caton-Thompson 1928:44), and emmer wheat is present in small quantities (Brunton and Caton-Thompson 1928:77). Sheep, goat (both identified by the presence of dung), and pig are present in the faunal assemblage. Barley, as well as several weed species, is recorded by Holmes and Friedman (1994).

Based on radiocarbon dates, Mahgar Dendera, located $60 \mathrm{~km}$ north of Luxor, was occupied from 6400 to 5700 cal BP (Hendrickx et al. 2001:89). Twenty hearths were reportedly associated with a small number of postholes, along with 11 storage pits (Hendrickx et al. 2001:24). Ceramic pots found at the site are interpreted as storage jars, and faunal remains indicate a dominance of fish in addition to some herd animals (Linseele et al. 2009). The seasonality of resources is used to suggest intermittent occupation (Hendrickx et al. 2001:102).

The Nile Valley sites are more varied than those in the Delta region, with varying numbers of structures thought to be dwellings and storage facilities. Domestic animal species are present, together with grain, but at El Omari and at Mahgar Dendera, fish remains are prominent.

\section{Desert Oases Sites}

Based on radiocarbon determinations, Nabta Playa and surrounding areas were occupied intermittently from the early to mid-Holocene, circa 10,800 BP until 6000 BP (Wendorf and Schild 1998:97), during periods when precipitation provided standing water (Banks 1989; Nicoll 2001; Wendorf and Expedition Members 1977). Archaeological deposits contain flaked stone artifacts, pottery, faunal remains, ostrich eggshell, firecracked rocks, and hearths with evidence for huts, storage pits, and wells at site E-75-6 (Królik and Schild 2001; Wendorf and Schild 2002; Wendorf et al. 2001). Typological analysis of flaked stone artifacts indicates similarities to other Epipaleolithic assemblages, although through time some of the geometric microlith types disappear (Close 2001:71). Early ceramic types are associated with those of the Khartoum Neolithic (Close and Wendorf 2001:68), and later types with the A-Group (Banks 1980:301; Gatto 2006) and Badarian (Nelson et al. 2002:542). Analysis of flaked stone artifacts from E-75-8 (Close 2001) indicates heightened levels of mobility during the middle Holocene period, associated with the later Neolithic phases (Phillipps 2012). Early evidence for domestic cattle at the site is disputed (see below), with sheep and goat present during later periods. Botanical remains at E-75-6, for example, suggest the exploitation of wild sorghum, sedges, legumes, and other wild grass seeds, tubers, and fruit (Wasylikowa 2001; Wasylikowa et al. 1997; Wetterstrom 1993).

Early archaeological remains at Dakhleh fall into three cultural phases: Masara (Epipalaeolithic, $10,300$ to $8500 \mathrm{BP})$, Bashendi A (8400 to 7650 $\mathrm{BP}$ ), and Bashendi B (7400 to $5800 \mathrm{BP}$ ) (McDonald 2009:8). During the Masara period, flaked stone artifact scatters, grinding stones, and hearths are present (McDonald 1998:129), together with round or oval and crescent-shaped stone structures (McDonald 2009:11). No botanical remains are documented, but faunal remains include hartebeest, gazelle, hare, birds, and turtles (McDonald 1998:131). Bashendi A remains include flaked stone artifacts, pottery, grinding stones, and hearths (McDonald 2009:10). Features include slab structures, stone clusters, and small bins associated with evidence of grinding, suggesting repeated use (McDonald 2009:22-23). Faunal remains include 
gazelle, hartebeest, fox, hare, and bird but no domesticated species (McDonald 1998:132, 2009). Botanical remains include wild sorghum and millet. Bashendi B archaeological remains are more dispersed, consisting of surface scatters of artifacts and stone structures (McDonald 2009:27). Faunal remains include abundant evidence of domestic cattle and goat $(\mathrm{McD}$ onald 1998:134).

Like other oases in the Egyptian portion of the eastern Sahara, Farafra represents a permanent water source (Barich 1993, 2004, 2014a). Archaeological remains dating to between 7200 and $6000 \mathrm{BP}$ are concentrated around ephemeral lakes such as Bahr Playa and Hidden Valley Playa. Stone-lined heaths, pits, and potholes are reported (Barich et al. 2012; Barich and Lucarini 2008). Floral and faunal remains point to a combination of plant harvesting and sheep/goat pastoralism (Barich and Lucarini 2008). In the Hidden Valley Playa, circular stone huts are reported, with hearths dating to between 7300 and 6200 BP (Barich 2014b; Barich and Hassan 2000:13; Barich and Lucarini 2002). The hearths included remains of morphologically wild sorghum seeds and fruits (Barakat and Fahmy 1999; Fahmy 2014; Lucarini 2007). The area of Sheikh el Obeiyid, west of Hidden Valley, contains a number of stone slab-lined structures interpreted as dwellings (Hamdan and Lucarini 2013).

To summarize, the desert sites unsurprisingly lack evidence for fish exploitation, but in many cases this is replaced by the exploitation of wild species. Later in time, faunal remains indicate the presence of domestic species. Wild plants were used and habitation and storage structures are indicated.

\section{Discussion}

Neolithic deposits occur at a number of locations outside the Fayum Basin, but for a variety of reasons these have been only partially sampled. Structures exist in some locations, but it is difficult to determine how these structures relate to one another and how their presence relates to shifts in local environment. Faunal records suffer from the same problem. The contextual studies that would indicate how these faunal materials were deposited are largely lacking. With some exceptions, studies of portable material culture are typologically based, and while they indicate potential relationships among sites, analyses to investigate the form these relationships might take are lacking. We know that people used locations along the Nile Valley and
Delta, sometimes creating structures, sometimes utilizing domestic animals and plants, and exploiting fish resources. We know that they returned to these places at different seasons, over periods that span many centuries. We know that people moved around to some degree and stored resources at some locations, presumably with the intention of returning. Sometimes their time at locations involved creating structures in which they probably lived.

More is known about the spatially extensive Western Desert sites, with occupation related not only to climatic fluctuations but also to local environmental contexts, including resource availability. Mobility, or lack thereof, is inferred through a variety of means, including reconstructions of subsistence resources, the presence of structural features, and the movement of material culture. Subsistence reconstructions suggest a reliance on domesticated animals, although this is somewhat spatially and temporally variable. Wild plants and animals were exploited, and as noted above, in contrast to the Nile Valley, fish are obviously absent.

\section{Socioeconomy beyond the Fayum Pastoralism}

Evidence for the early domestication of animals, particularly cattle, has dominated archaeological accounts for many years, suggesting that the first African food producers were herders rather than farmers (Close 1996; Gautier 1980, 1987, 2001, 2002; Marshal and Hildebrand 2002; Smith 1992; Wendorf and Schild 1980, 1984, 1994, 1998; Wendorf et al. 1976). Domestic animals, it is argued, provided a predictable, mobile food supply in regions of Northeast Africa that were experiencing considerable environmental variability in the early to mid-Holocene (Marshall and Hilderbrand 2002). However, the archaeological basis for this from the early Holocene relies on faunal remains identified as domestic cattle from Nabta Playa-Bir Kiseiba, and this evidence is controversial (de Lernia 2013). More convincing evidence for domestic cattle is found considerably later in time, around $8000 \mathrm{BP}$, at Nabta PlayaBir Kiseiba. At other Egyptian Western Desert sites, remains of bovids are not often abundant or not clearly domestic (Linseele et al. 2014). At Djara, for example, bovid remains could not be identified further to species. At Dakhleh, cattle bones are reported, but without details of the number and type of remains. The Wadi Bakht remains amount to five cattle tooth fragments and some unidentified bovid bones. No cattle were found at 
Farafra, and only at Kharga Oasis are cattle remains relatively abundant. In the Nile Valley sites, cattle remains are found from the Fayum, as described in this volume, and at Merimde and El Omari, as noted above, but the numbers are not substantial.

In contrast to cattle, the caprine remains are more abundant, and the evidence for early use is more secure (Linseele et al. 2014). Nabta Playa-Bir Kiseiba has reliable dates circa 7200 BP for caprine remains (Gautier 2001), and similarly aged finds come from Sodmein Cave circa 7100 to 7000 cal BP (Vermeersch et al. 1994), while Dakhleh (McDonald 1998) and Farafra Oases (Barich 2002) both have remains with circa 7000 to $6900 \mathrm{cal}$ BP dates. These dates match the unfused sheep radius from the site of E29H1 in the Fayum reported here, and the E29H1 age is similar to dated deposits with caprines from QS XI/81 and QSIX/81 near Qasr el-Sagha on the northwestern edge of the Fayum (Linseele et al. 2016).

Farther south, caprines, mainly in the form of sheep, are found at Nabta Playa in the Middle (8100 to 7400 BP) and Late Neolithic (7400 to $6650 \mathrm{BP}$ ), where they are more numerous than cattle remains. Caprines are present at Farafra Oasis and are reported from the Bashendi A period (Linseele et al. 2014). At Kharga Oasis remains are abundant at KS43, dated to 6800 to 6400 BP (Lesur et al. 2011).

In the Lower Nile and Delta sites, caprines outnumber cattle, However, there is considerable variability in the relative proportions of the different domesticated species. At Merimde, in the earliest levels, sheep and goat predominate, followed by cattle and pig, while in higher levels, pig is more common. Among the caprines, sheep greatly outnumber goat. In contrast, the earliest levels at Saïs show the predominance of cattle and pig rather than caprines (Bertini and Ikram 2014). The situation is similar at El Omari.

Summarizing this evidence, two separate phases of use are suggested (Linseele et al. 2014). With an early Holocene domestication of cattle now thought to be unlikely, the earliest phase of domestic species use can be dated to the eighth millennium BP, during which sheep, goat, and cattle are present, all introduced from Southwest Asia. However, remains of these species come from only a small number of locations, Nabta Playa-Bir Kiseiba, the Fayum, and some of the desert oases sites. This places severe limits on the degree to which pathways for introduction can be reconstructed (di Lernia 2013). And at none of these locations do domestic species represent the majority of the fauna. Wild game predominates in the desert sites, while fish are dominant in sites close to water bodies.

\section{Early Domestic Plant Use}

The earliest evidence for domestic plant use in Northeast Africa comes from dental calculus samples from burials in the R12 cemetery site in northern Sudan, which provided wheat and barley chaff phytoliths. Although a specific species identification is not determined, it is likely that the phytoliths come from emmer wheat (Triticum turgidum subsp. dicoccon) and hulled barley (Hordeum vulgare), species that are present in the Fayum and in Merimde farther north (Madella et al. 2014). Burials from R12 and the related cemetery site of Ghaba indicate that people also exploited mixed stands of wild savanna grasses.

Barley is present at Merimde and El Omari, with six-row barley identified at Merimde but with no subspecies identification possible at El Omari. Emmer wheat (Triticum turgidum subsp. dicoccon) is present at both Merimde and El Omari, and Cappers (2013) confirms the presence of Triticum aestivum subsp. compactum (club wheat) at El Omari. The Western Desert sites do not contain evidence for domestic cereals, but like the Sudanese cemetery sites and Nabta Playa discussed above, there is evidence for use of wild grasses. Intensive use of wild plants is inferred from the presence of grinding stones in numerous Western Desert sites, as well as in the Fayum, in contexts older than those where domestic grains are found. Considering the evidence for domestic plants and animals together, the presence of domestic grains is matched by an increase in the abundance of domestic animals, particularly pigs, in the Lower Egyptian Nile Valley sites. Among the Western Desert sites, where evidence for both domesticates is sparse, this combination is present only at Kharga Oasis.

\section{Nondomestic Animal Exploitation}

Fish are present in high numbers at Merimde, where they represent 11.5 percent of the identified remains in Level I and up to 45 percent in later levels. As noted above, the Saïs fish midden is dominated by catfish and tilapia, while at El Omari the fauna also contains a large number of fish, mainly consisting of deepwater species. Fish are replaced by nondomestic land animals away from major water sources. At Djara, for example, desert antelopes (addax, oryx, and gazelle) and many 
unidentifiable bovids are found. Kharga Oasis shows a dominance of gazelle accompanied by hare, Barbary sheep, and ostrich. The same is true at Nabta Playa, although the proportion of hunted animals decreases over time. At Sodmein Cave, hunted animals include cat, rock dassie (Procavia capensis), and gazelle. Although the specifics of the faunal assemblages show variation, the overall pattern is one where locally available animals are the primary prey targets. When domesticates become available, they form an addition to, rather than a replacement for, the nondomestic species. The shift to the incorporation of domestic species-animals or plants-can therefore not be attributed to their arrival, since these species were present for a considerable period during which nondomestic species continued to be significant. As the use of domestic grains in the Fayum indicates, technologies like basket-lined storage pits for grain and sickles for harvest continued in parallel with wild species exploitation. Archaeologically, we do not see a marked impact of invention or technological innovation. Therefore, rather than search for one primary cause for the shift to a dependency on domestic species, causation is better investigated by considering a series of interacting processes, both cultural and natural, that altered the dependencies between people and the range of resources they exploited.

\section{Horizontal Tells in the Egyptian Neolithic}

In Egypt, Wengrow (2006:83) describes the Neolithic and early Predynastic occupation as a move to "complexity without villages." In contrast to the development of Mesopotamian tells, with their extensive vertical stratigraphic depth from prolonged occupation of spatially discrete locations, Predynastic occupations in the Egyptian Nile Valley "were for the most part light and ephemeral ... human activity ... defined by lateral spreading of cultural material along a horizontal axis."

It is possible that both Merimde Beni Salama and El Omari indicate a dispersed rather than a concentrated settlement form, while the best evidence for this way of living is found in the Fayum. The results of the analyses of portable material culture presented in this volume, as well as evidence from storage structures and the excavation of stratified sites and hearths, provide insights into a nature of movement that lacks parallels in village-like occupation in Southwest Asia. While it is true that the Fayum lacks the cemeteries that Wengrow et al. (2014) suggest were the focal points in Upper
Egypt, given the level of landscape change in Lower Egypt, together with the relatively few areas that have been studied intensively from a landscape perspective, it is possible that such sites have been destroyed or are as yet to be found. Burials are largely absent from the Fayum, but as in other areas, this does not necessarily mean they never existed. The few documented occupations attributed to the mid-Holocene period in Lower Egypt outlined show that the archaeological record of nonburial remains includes similar features and portable material culture. As discussed, aspects of economy are very similar, especially with regard to the abundance of fish in the faunal assemblages. As emphasized a number of times, the archaeological record documents what survived, not simply what was present. There are substantial areas of the Fayum north shore that are no longer accessible and where any archaeological record is either buried or destroyed. Moreover, there are areas that have not been investigated. Given this, there remains the possibility that the lack of burials is a "false negative."

Hassan (1988) noted that the nature of Neolithic remains in Egypt suggests small communities living in short-lived settlements. Explanations for this are often environmental. For instance, as Hassan (1988:154) suggests, "Large individual settlements may have been unlikely to develop because of the narrowness of the floodplain in the region," in this case the Badari region of Middle Egypt. Even later in the prehistoric sequence, there is little evidence for substantial architecture aside from organic structures and the introduction of mud huts. Data from Naqada and later phases at Hemamieh "suggests that up to about 3600 B.C., the pattern of settlements consists of small, dispersed communities with small huts or shelters associated with storage pits, animal enclosures, and refuse areas (Hassan 1988:155).”

And as Wenke (1989:142) notes: For most of the fourth millennium BC, the majority of Egyptians lived in small communities of oval huts, with a transition to communities of interlocking mudbrick rectangular buildings coming only quite late.”

Comparing early Predynastic settlements with those identified in Neolithic sites, the differences between the Neolithic and later times may be more in degree rather than in kind. Thus it may be that storage or repeated occupations left less of a material trace in Egypt than in some places in Southwest Asia because settlement in Egypt shifted spatially and more frequently through time. 
In reviewing the Egyptian evidence, there are a number of factors that would have affected the ability to continuously occupy a spatially restricted location. In the case of Merimde and Saï, for example, the shifting river had an impact on the location of settlement. In Western Desert sites like Dakhleh and Nabta Playa, shifts in local environmental conditions may be responsible for shifts in the places occupied at different times. The same may be true of the Fayum. Here local environmental conditions, like the changing water levels in the lake basins, necessitated the movement of settlement. This need not have involved inundation by rising lake waters, and evidence from our excavations indicates it probably did not, but it might have reflected the lack of accessible locations from which to exploit spawning fish, the inability to repeatedly cultivate the same tract of land if rain-fed agriculture was practiced, and/or the absence of fresh sediment, which required a shift in the position of "fields." There may equally be social or other economic reasons why settlement shifted, and of course any of these reasons may have changed over time.

One of the lessons to be drawn from the Fayum, and from Neolithic settlement pattern studies in those areas with extensive surface deposits, is the size of the regions used by Neolithic peoples. Despite the extent of landscape investigations in the Fayum, it is likely that at any one period we have remains from only a part of any settlement pattern. People were able to move over remarkably large areas, so as archaeologists we must resist the tendency to see one site as central to any settlement pattern. In the Nile Valley, early settlements were probably as spatially extensive as areas like the Fayum or indeed those in the Western Desert. People could and probably did move over significant distances. This needs to be kept in mind when interpreting sites that today for a variety of reasons are limited in their spatial extent or in the extent to which they can be investigated.

If settlement shifts are at least partly reflective of changes in local environments, the question is why people chose to engage in agriculture in the north of Egypt only during this period and not before (and in the case of the Fayum, not in the period after 6000 BP). As we have outlined, Williams (2009) discusses the concept of geological opportunism, whereby occupants of a particular location may choose to engage with agriculture when it is geologically or environmentally appropriate to do so. This may be linked to the mid-Holocene increase in winter rainfall (Phillipps et al. 2012), but it also relates to the variable environmental and socioeconomic conditions under which people chose to engage in low-level food production (Holdaway et al. 2010). If we can overcome the assumption that the use of domesticates is inevitably better than the exploitation of wild resources, the question of why domestic species were used can be answered by noting the particular set of historically contingent conditions found in different parts of Egypt. It is then clear that while displaying some degree of local variability, the use of domestic species also displays similarities up and down the Nile. Southwest Asian domesticates were eventually adopted but were incorporated into a socioeconomy and settlement pattern that was African, not Southwest Asian. Given the difference between the regions, this should come as no great surprise.

\section{Epipaleolithic and Neolithic}

The Fayum was occupied throughout the Holocene, and the nature of that occupation likely changed through time. However, a simple dichotomy between Epipaleolithic and Neolithic can no longer be maintained. We have shown that there are clear differences in raw material availability and selection; in reduction strategy, projectile points, and artifact movement. In some cases, changes in the composition of faunal assemblages may appear to create a strong contrast between the two periods. However, we have also shown that there is a remarkable continuity in the use of wild resources. The chronology of these changes may differ, so that adoption and adaptation did not occur at the same time as part of the transferal of a "cultural package." Furthermore, this research highlights the problem of single elements (such as ceramic or tool types) identifying "cultural" change or "cultural" contact. What do typological similarities indicate, especially if they are not consistent across different artifact groups?

Rather than focus on the cultural origins of the people who inhabited the Fayum during the Holocene, or attempt to model why they adopted some domesticated plants and animals into their economy and not others, we have used the archaeological record from the Fayum to address alternative questions. These relate to the formation of the archaeological record, its visibility and age in particular, and the degree to which this record varies through time and across space. Intensive targeted investigations of this record situated within a landscape allow for a more detailed understanding of 
how the relationship between people and environment changed over time. Results of flaked stone artifact studies suggest that the nature of mobility shifted considerably throughout the Holocene, likely in response to changes in environment but also changes in social structure and resource use. The results seen in the Fayum emphasize the importance of such studies when discussing changes in subsistence strategy and human environment interrelationships elsewhere.

Where did the Neolithic come from? Was the Fayum influenced by Southwest Asian ideas or by those derived from people in the desert? Is the Fayum more like Upper or Lower Egypt, or the eastern Sahara? What caused the late arrival of the Neolithic into Lower Egypt? Many of the current models that seek to explain the Fayum and Northeast Africa more generally involve attempts to answer these types of questions. Maps trace the movement of things or ideas between sites that are often separated by considerable distances. Spheres of influence are mapped, and regions with evidence for different types or degrees of influence are described. Artifact form is assessed as emblematic of the ethnicity of particular groups whose movements can be traced accurately by the things they used, while what we mostly find are the things they discarded. All too frequently, the outcome is some form of dichotomy that contrasts sites (in Egypt) with influence from Southwest Asia or from the desert to the west, or alternatively influence from the south as distinct from influence from the north. The results derived from small excavations, at single sites, cannot be taken as emblematic of particular peoples moving long distances from their origins in distant places.

For the Fayum and related sites in Egypt, we therefore propose no such model. As documented here, the results of our research suggest variability across the landscape, with different archaeological records preserved in different places dating to different times. There is no evidence that this variability was produced by the arrival of a particular people bringing with them a package of things and/or ideas. We have evidence that material preserved in the Fayum is part of a wider settlement system and therefore not interpretable as the lifeway of a particular people. Because we have looked extensively and found a variable record, we look with some skepticism on models derived from spatially or temporally limited records that claim to show the presence of a particular people or cultural influence derived from a particular people. Cultural identity certainly existed in the past, as it does today, but we know from contemporary studies that cultural identification is a complex business not reducible to the use of a particular form of material culture. It is dependent on how people interact together in particular settings. Archaeologists should concentrate a great deal more of their effort on understanding the nature of these settings rather than on constructing models that selectively make use of a limited array of data types. Future research should focus less on deterministic environmental drivers for changes in socioeconomy and focus instead on ideas such as shared cultural or social identity as suggested by Wengrow et al. (2014:170):

The question will inevitably, and rightly, be asked: what kind of historical entity is the "primary pastoral community"? Clearly it is inconceivable that communities throughout the entire length of the Nile Valley, a distance of c. $1800 \mathrm{~km}$, shared anything approaching a conscious social identity (e.g. of the sort that could be articulated in tribal or ethnic terms) during the fifth millennium BC. Instead, what came to be shared across this extensive region were the materials and practices-including, and perhaps especially, modes of ritual practice-out of which more local contrasts and group identities were constructed. It may be precisely the maintenance of local differences within a shared social milieu that gave rise simultaneously to such geographically expansive uniformities and, within them, to the kind of internal variations observed in ceramic assemblages and other traditional markers of archaeological "cultures."

In the Fayum we have an example of the local contrasts to which Wengrow and colleagues refer. Such a concept accounts for the variability found in the Fayum archaeological record when compared to that from other locations more effectively than does a notion of the importation of a specific suite of behaviors. It is also much more in line with recent theory on material remains that emphasizes the importance of context and meaning (e.g., Hodder 2012).

We have talked about changes and continuities in aspects of the socioeconomies of the people who left material for us to study in the Fayum. We have highlighted how what we can see in the Fayum differs 
from what we can see in the Nile Valley proper in later times but is similar to a number of near contemporary Neolithic sites in the Nile Valley, the Delta region, and the Western Desert. While there is a great deal more to say about this topic, it is important to reiterate that what we can determine from the Fayum was not the forerunner for Predynastic Egypt. People acted the way that they did because of the particular contexts in which they found themselves and because of the historical relationships that developed with others and with the environment. Our Fayum material illustrates the changing relationships between people and place from the early to mid-Holocene, but it does not document an evolution in these relationships that would lead to a particular outcome when people moved to the Nile Valley. Evolution is a form of description looking back to how a set of properties allowed a group to survive or thrive rather than an inevitable outcome or improvement.

We end therefore not with a behavioral model that describes what happened in the Fayum. We cannot answer the seemingly simple question "Where did they come from and where did they go?" This is not because the record is inadequate but because this is the wrong question to ask. There is no "they," and as a consequence, there is no place to which the "they" came and went. The emphasis should be on documenting how places were used in different ways at different times so that we can begin to appreciate the complex diversity in the way people interacted with materials, plants, and animals in the low-level food production societies that Smith (2001) posits. Based on recorded data, we contribute to the anthropological discussion of human existence, which to us is more informative and rewarding than attempts to identify the cultural influence of hypothetical cultural groups.

Gertrude Caton-Thompson recognized that the Fayum archaeological record required a methodological revision. She saw the importance of geoarchaeological work in relation to understanding the anthropogenic remains. Throughout our work we have developed an ongoing respect for her forward thinking, originality of approach, precise and detailed recording, and thoroughness of reporting. She and Elinor Gardner spent the time and effort needed to begin to understand the complicated archaeological record of the Fayum. Building on this legacy, we have presented an approach to the study of early and mid-Holocene Egyptian archaeology that considers the complexity of the archaeological record and presents avenues of further enquiry rather than unsupported models of explanation. Most importantly, the work is based on three important elements: spending substantial time in the field to enable thorough recording; rethinking method in relation to our research objectives and the record at hand; and theorizing our findings. 


\section{References Cited}

Aldeias, V., H. L. Dibble, D. Sandgathe, P. Goldberg, and S. McPherron

2016 How Heat Alters Underlying Deposits and Implications for Archaeological Fire Features: A Controlled Experiment. Journal of Archaeological Science 67:64-79.

Aleem, A. A.

1958 A Taxonomic and Paleoecological Investigation of the Diatom Flora of the Extinct Fayoum Lake (Upper Egypt). Systematic Part. Bulletin of the Faculty of Science Alexandria University 2(1):99-138.

Arnold, J. R., and W. F. Libby

1951 Radiocarbon Dates. Science 113:111.

Arz, H. W., F. Lamy, J. Patzold, P. J. Muller, and M. Prins

2003 Mediterranean Moisture Source for an EarlyHolocene Humid Period in the Northern Red Sea. Science 300:118-121.

Badawi, A.

1980 Beigabengräber aus Merimde. Mitteilungen des Deutschen Archäologischen Instituts 36:70-76.

Bailey, G. N.

1983 Concepts of Time in Quaternary Prehistory. Annual Review of Anthropology 12:165-192.

2006 Time's Arrow: The Measurement and Theory of Archaeological Time. Antiquity 80(309):717-720.

2008 Time Perspectivism: Origins and Consequences. In Time in Archaeology, edited by S. J. Holdaway and L. Wandsnider, pp. 13-30. University of Utah Press, Salt Lake City.

Ball, J.

1939 Contributions to the Geography of Egypt. Government Press, Bulaq, Egypt.

Banks, K. M.

1980 Ceramics of the Western Desert. In Prehistory of the Eastern Sahara, edited by F. Wendorf and R. Schild, pp. 299-316. Academic Press, New York.
1989 The Appearance and Spread of Cattle-keeping in Saharan North Africa. In Late Prehistory of the Nile Basin and the Sahara, edited by L. Krzyzaniak and M. Kobusiewicz, pp. 57-60. Poznań Archaeological Museum, Poznań, Poland.

Barakat, H., and A. G. Fahmy

1999 Wild Grasses as "Neolithic" Food Resources in the Eastern Sahara: A Review of the Evidence from Egypt. In The Exploitation of Plant Resources in Ancient Africa, edited by M. van der Veen, pp. 33-46. Kluwer Academic/Plenum Publishers, New York.

Bard, K. A.

2008 An Introduction to the Archaeology of Ancient Egypt. Blackwell, Oxford.

Barich, B.

1993 Culture and Environment between the Sahara and the Nile in the Early Mid-Holocene. In Environmental Change and Human Culture in the Nile Basin and Northern Africa until the Second Millennium $B C$, edited by L. Krzyzaniak, M. Kobusiewicz and J. Alexander, pp. 171-183. Poznań Archaeological Museum, Poznań, Poland.

2002 Cultural Responses to Climatic Changes in North Africa: Beginning and Spread of Pastoralism in the Sahara. In Droughts, Food, and Culture: Ecological Change and Food Security in Africa's Later Prehistory, edited by F. A. Hassan, pp. 111122. Kluwer Academic/Plenum Publishers, New York.

2004 Archaeological Research in the Farafra Oasis (Egypt): Contribution to the Study of Early Cultivation in the Eastern Sahara. In Combining the Past and the Present: Archaeological Perspectives on Society, edited by T. Oestigaard, N. Anfinset, and T. Saetersdal, pp. 143-147. British Archaeological Reports, Oxford. 
2014a Farafra Oasis and the Archaeological Project. In From Lake to Sand: The Archaeology of Farafra Oasis, Western Desert, Egypt, edited by B. E. Barich, G. Lucarini, M. A. Hamdan, and F. A. Hassan, pp. 3-21. All'Insegna del Giglio, Florence.

2014b Hidden Valley: A 7,000-Year-Old Village in Wadi el Obeiyid. In From Lake to Sand: The Archaeology of Farafra Oasis, Western Desert, Egypt, edited by B. E. Barich, G. Lucarini, M. A. Hamdan, and F. A. Hassan, pp. 167-207. All'Insegna del Giglio, Florence.

Barich, B., and F. Hassan

2000 A Stratified Sequence from Wadi El Obeiyed Farafra: New Data on Subsistence and Chronology of the Egyptian Western Desert. In Recent Research into the Stone Age of Northeastern Africa, edited by L. Krzyzaniak, K. Kroeper and M. Kobusiewicz, pp. 11-20. Poznań Archaeological Museum, Poznań, Poland.

Barich, B., and G. Lucarini

2002 Archaeology of Farafra Oasis (Western Desert, Egypt): A Survey of the Most Recent Research. Archeo-Nil 12:101-108.

2008 The Nile Valley Seen from the Oases: The Contribution of Farafra. In Egypt at Its Origins 2, edited by B. Midant-Reynes and Y. Tristant, pp. 569-584. Peeters, Leuven.

Barich, B., G. Lucarini, M. Gallinaro, and M. Hamdan

2012 Sheikh/Bir El Obeiyid: Evidence of Sedentism in the Northern Farafra Depression (Western Desert, Egypt). In Prehistory of Northeastern Africa: New Ideas and Discoveries, edited by J. Kabacińsk, M. Chłodnicki, and M. Kobusiewicz, pp. 255-278. Poznań Archaeological Museum, Poznań, Poland.

Barnard, H., and W. Wendrich (editors)

2008 The Archaeology of Mobility: Old World and New World Nomadism. Cotsen Institute of Archaeology Press, Los Angeles.

Barrett, M.

2014 Flake to Core Ratios and Human Mobility: An Investigation of Stone Artefact Assemblages from Egypt and Australia. Unpublished master's thesis, Department of Anthropology, University of Auckland.

Bar-Yosef, O.

1998 The Natufian Culture in the Levant, Threshold to the Origins of Agriculture. Evolutionary Anthropology 6:159-177.

2002 Early Egypt and the Agricultural Dispersals. In Magical Practices and Ritual in the Near

Eastern Neolithic, edited by H. G. K. Gebel, B. Dahl

Hermansen, and C. Hoffmann Jensen, pp. 49-65. Ex Oriente, Berlin.

Baumgartel, E.

1955 The Cultures of Prehistoric Egypt. Oxford University Press, London.
Beadle, L. C.

1981 The Inland Waters of Tropical Africa. Longman, London.

Bertini, L., and S. Ikram

2014 Animal Bones. In Sais II: The Prehistoric Period, edited by P. Wilson, G. Gilbert, and G. Tassie, pp. 135-141. Egypt Exploration Society, London.

Binford, L. R.

1979 Organization and Formation Processes: Looking at Curated Technologies. Journal of Anthropological Research 35:255-273.

Bintliff, J. L.

2000 The Concepts of "Site" and "Offsite" Archaeology in Surface Artefact Survey. In Non-Destructive Techniques Applied to Landscape Archaeology, edited by M. Pasquinucci and F. Trement, pp. 200215. Oxbow Books, Oxford.

Bond, G., W. Showers, M. Cheseby, R. Lotti, P. Almasi, P. deMenocal, P. Priore, H. Cullen, I. Hajdas, and G. Bonani

1997 A Pervasive Millennial-Scale Cycle in North Atlantic Holocene and Glacial Climates. Science 278:1257-1266.

Boulos, S. T.

2000 Flora of Egypt: 2. Geraniaceae-Boraginaceae. Al Hadara Publishing, Cairo.

Bouquet, C.

1990. Insulaires et riverains du lac Tchad. L'Harmattan, Paris.

Boyd, B.

2006 On "Sedentism" in the Later Epipalaeolithic (Natufian) Levant. World Archaeology 38(2):164-178.

Braidwood, R.

1960. The Agricultural Revolution. W. H. Freeman, San Francisco.

Brewer, D. J.

1987 Seasonality in the Prehistoric Faiyum Based on the Incremental Growth Structures of the Nile Catfish (Pisces: Clarias). Journal of Archaeological Science 14:459-472.

1989a A Model for Resource Exploitation in the Prehistoric Fayum. In Late Prehistory of the Nile Basin and the Sahara, edited by L. Krzyzaniak and M. Kobusiewicz, pp. 127-138. Poznań Archaeological Museum, Poznań, Poland.

1989b Fishermen, Hunters, and Herders: Zooarchaeology in the Fayum, Egypt (ca. 8200-5000 bp). BAR International, Oxford.

1991 Temperatures in Predynastic Egypt Inferred from the Remains of the Nile Perch. World Archaeology 22(3):288-303.

Bronk Ramsey, C.

2001 Development of the Radiocarbon Calibration Program. Radiocarbon 43(2A):355-363. 
2009 Bayesian Analysis of Radiocarbon Dates. Radiocarbon 51(1):337-360.

Brown, A. G.

2008 Geoarchaeology, the Four Dimensional (4D) Fluvial Matrix and Climatic Causality. Geomorphology 101:278-297.

Brunton, G., and G. Caton-Thompson

1928 The Badarian Civilisation and Predynastic Remains Near Badari. British School of Archaeology in Egypt, London.

Bryson, R. A.

1992 A Macrophysical Model of the Holocene Intertropical Convergence and Jetstream Positions and Rainfall for the Saharan Region. Meteorology and Atmospheric Physics 47:247-258.

Bubenzer, O., and H. Riemer

2007 Holocene Climatic Change and Human Settlement between the Central Sahara and the Nile Valley: Archaeological and Geomorphological Results. Geoarchaeology 22(6):607-620.

Butzer, K. W.

1971 Environment and Archaeology: An Ecological Approach to Prehistory. 2nd ed. Aldine-Atherton, Chicago.

1976 Early Hydraulic Civilization in Egypt: A Study in Cultural Ecology. University of Chicago Press, Chicago.

1982 Archaeology as Human Ecology: Method and Theory for a Contextual Approach. Cambridge University Press, Cambridge.

2011 Geoarchaeology, Climate Change, Sustainability: A Mediterranean Perspective. In Geoarchaeology, Climate Change, and Sustainability, edited by A. G. Brown, L. S. Basell, and K. W. Butzer, pp. 1-14. Special Paper 476. Geological Society of America, Boulder.

Cagle, A.

1995 University of Washington 1994 Fayum Project. Electronic document, https://www.academia. edu/31023758/Narce.docx (accessed January 2017).

Cappers, R. T. J.

2006 The Reconstruction of Agricultural Practices in Ancient Egypt: An Ethnoarchaeobotanical Approach. Palaeohistoria 47/48:429-446.

2013 Modelling Cereal Selection in Neolithic Egypt: An Evaluation of Economic Criteria. In Neolithisation in Northeastern Africa, edited by N. Shirai, pp. 109-120. Ex Oriente, Berlin.

Cappers, R. T. J., and R. Neef

2012 Handbook of Plant Palaeoecology. Barkhuis, Groningen, Netherlands.

Cappers, R. T. J., F. B. J. Heinrich, S. R. Kaaijk, F. Fantone, J. C. Darnell, and C. Manassa
2014 Barley Revisited: Production of Barley Bread in Umm Mawagir (Kharga Oasis, Egypt). In Current Research in Egyptology 2013, Proceedings of the Fourteenth Annual Symposium, edited by K. Accetta, R. Fellinger, P. Lourenço Gonçalves, S. Musselwhite, and W. P. van Pelt, pp. 49-63. Oxbow Books, Oxford.

Caton-Thompson, G.

1926a The Neolithic Industry of the Northern Fayum Desert. Journal of the Royal Anthropological Institute 56:309-323.

1926b The Recent Geology and Neolithic Industry of the Southern Fayum Desert. Journal of the Royal Anthropological Institute 56:301-308.

1927 Explorations in the Northern Fayum. Antiquity 1:326-340.

1928 Recent Excavations in the Fayum. Man 28:109-113.

1952 Kharga Oasis in Prehistory. Athlone Press, London.

1983 Mixed Memoirs. Paradigm Press, Gateshead, UK.

Caton-Thompson G., and E. W. Gardner

1929 Recent Work on the Problem of Lake Moeris. Geographical Journal 73:20-58.

1934 The Desert Fayum. Royal Anthropological Institute of Great Britain and Ireland, London.

Caton-Thompson, G., E. W. Gardner, and S. Huzzayim

1936 Lake Moeris: Reinvestigations and Some Comments. Journal of the Royal Anthropological Institute 61:301-308.

1937 Lake Moeris: Reinvestigations and Some Comments. Bulletin de l'Institut d'Egypte 19:243-303.

Cherry, J. F.

2003 Archaeology beyond the Site: Regional Survey and Its Future. In Theory and Practice in Mediterranean Archaeology: Old World and New World Perspectives, edited by J. K. Papadopoulos and R. M. Leventhal, pp. 137-159. Cotsen Institute of Archaeology Press, Los Angeles.

Childe, V. G.

1956 [1936] Man Makes Himself. Watts, London.

Chmielewska, W.

1968 Early and Middle Paleolithic Sites Near Arkin, Sudan. In The Prehistory of Nubia, edited by F. Wendorf, pp. 110-147. Fort Burgwin Research Center and Southern Methodist University Press, Dallas.

Clark, R. H., and A. J. Schofield

1991 By Experiment and Calibration: An Integrated Approach to Archaeology of the Ploughsoil. In Interpreting Artefact Scatters: Contributions to Ploughzone Archaeology, edited by A. J. Schofield, pp. 93-106. Oxbow, Oxford.

Close, A. E.

1978 The Identification of Style in Lithic Artefacts. World Archaeology 10(2):223-237.

1996 Carry That Weight: The Use and Transportation of Stone Tools. Current Anthropology 37(3):545-553. 
1999 Distance and Decay: An Uneasy Relationship. Antiquity 73:24-32.

2000 Reconstructing Movement in Prehistory. Journal of Archaeological Method and Theory 7(1):49-77.

2001 Site E-75-8: Additional Excavation of the Seventh Millennium Site at Nabta Playa. In Holocene Settlement of the Egyptian Sahara: 1. The Archaeology of Nabta Playa, edited by F. Wendorf and R. Schild, pp. 352-385. Kluwer Academic/ Plenum Publishers, New York.

2002. Sinai, Sahara, Sahel: The Introduction of Domestic Caprines into Africa. In Tides of the Desert: Contributions to the Archaeology and Environmental History of Africa in Honour of Rudolph Kuper, edited by Jennerstrasse 8, pp. 459-470. HeinrichBarth-Institute, Cologne.

Close, A. E., and F. Wendorf

2001 Site E-77-7 Revisited: The Early Neolithic of El Adam Type at El Gebal El Beid Playa. In Holocene Settlement of the Egyptian Sahara: 1. The Archaeology of Nabta Playa, edited by F. Wendorf and R. Schild, pp. 57-70. Kluwer Academic/Plenum Publishers, New York.

Cruz-Uribe, E. D.

1992 The Lake of Moeris: A Reprise. In Life in a Multi-Cultural Society: Egypt from Cambyses to Constantine and Beyond, edited by J. H. Johnson, pp. 63-66. Oriental Institute of the University of Chicago, Chicago.

Davoli, P.

1998 L'archeologia urbana nel Fayyum di eta ellenistica e romana. Generoso Procaccini, Naples.

Debono, F., and B. Mortensen

1990 El Omari: A Neolithic Settlement and Other Sites in the Vicinity of Wadi Hof, Helwan. Verlad Philipp Von Zabern, Mainz am Rhein.

Derda, T.

2006 Administration of the Fayum under Roman Rule. Journal of Juristic Papyrology Supplement 7.

di Lernia, S.

2013 The Emergence and Spread of Herding in Northern Africa: A Critical Reappraisal. In The Oxford Handbook of African Archaeology, edited by $\mathrm{P}$. Mitchell and P. J. Lane, pp. 527-540. Oxford University Press, Oxford.

Douglass, M. J., S. J. Holdaway, P. C. Fanning, and J. J. Shiner

2008 An Assessment and Archaeological Application of Cortex Measurement in Lithic Assemblages. American Antiquity 73:513-526.

Dunnell, R. C.

1989 Aspects of the Application of Evolutionary Theory in Archaeology. In Archaeological Thought in America, edited by C. C. Lamberg-Karlovsky, pp. 35-49. Cambridge University Press, New York.
Ebert, J. I.

1992 Distributional Archaeology. University of New Mexico Press, Albuquerque.

Edwards, P. C.

1989 Problems of Recognizing Earliest Sedentism: The Natufian Example. Journal of Mediterranean Archaeology 2(1):5-48.

Eiwanger, J.

1978 Erster Vorbericht über die Wiederaufnahme der Grabungen in der neolithischen Siedlung MerimdeBenisalame. Mitteilungen des Deutschen Archäologischen Instituts, Kairo, 34:33-42.

1979 Zweiter Vorbericht über die Wiederaufnahme der Grabungen in der neolithischen Siedlung MerimdeBenisalâme, Mitteilungen des Deutschen Archäologischen Instituts, Kairo, 35:23-57..

1984 Merimde-Benisalame I: Die Funde der Urschicht. Verlag Philipp von Zabern, Mainz am Rhein.

1988 Merimde-Benisalame II: Die Funde der mittleren Merimdekultur. Verlag Philipp von Zabern, Mainz am Rhein.

1992 Merimde-Benisalame: Die Funde der jungeren Merimdekultur. Verlag Philipp von Zabern, Mainz am Rhein.

Elliot Smith, G.

1932 In the Beginning: The Origin of Civilization. Watts, London.

Emmitt, J. J.

2011 Investigating Ceramics from the Neolithic Occupation of Kom W, Fayum, Egypt. Unpublished master's thesis, Department of Anthropology, University of Auckland.

Fahmy, A. G.

2014 Plant Food Resources at Hidden Valley, Farafra Oasis. In From Lake to Sand: The Archaeology of Farafra Oasis, edited by B. E. Barich, G. Lucarini, M. A. Hamdan, and F. A. Hassan, pp. 333-344. All'Insegna del Giglio, Florence.

Fahn, A., E. Werker, and P. Bass

1986 Wood Anatomy and Identification of Trees and Shrubs from Israel and Adjacent Regions. Israel Academy of Sciences and Humanities, Jerusalem.

Fanning, P., and S. J. Holdaway

2001 Stone Artifact Scatters in Western NSW, Australia: Geomorphic Controls on Artifact Size. Geoarchaeology 16:667-686.

2004 Stone Artifact Exposure and Visibility at Open Sites in Western New South Wales, Australia: A Geomorphic Framework for Survey and Analysis. Journal of Field Archaeology 29(3/4):255-271.

Fanning, P., S. J. Holdaway, and R. Phillipps

2009 Heat Retainer Hearth Identification as a Component of Archaeological Survey in Western NSW, Australia. In New Directions in Archaeological Science, edited by A. Fairbairn and S. O'Connor, pp. 13-24. ANU Press, Canberra. 
Fanning, P., S. J. Holdaway, and E. Rhodes

2008 A New Geoarchaeology of Aboriginal Artefact Deposits in Western NSW, Australia: Establishing Spatial and Temporal Geomorphic Controls on the Surface Archaeological Record. Geomorphology 101:524-532.

Finlayson, B.

2004 Island Colonization, Insularity or Mainstream? In Neolithic Revolution: New Perspectives on Southwest Asia in Light of Recent Discoveries on Cyprus, edited by E. Peltenburg and A. Wasse, pp. 15-22. Oxbow Books, Oxford.

2013 Imposing the Neolithic on the Past. Levant 45(2):133-148.

Flower, R. J., K. Keatings, M. Hamdan, F. A. Hassan, J. F. Boyle, K. Yamada, and Y. Yasuda

2012 The Structure and Significance of Early Holocene Laminated Lake Sediments in the Faiyum Depression (Egypt) with Special Reference to Diatoms. Diatom Research 27(3):127-140.

Flower, R. J., C. Stickley, N. L. Rose, S. Peglar, A. A. Fathi, and P. G. Appleby

2006 Environmental Changes at the Desert Margin: An Assessment of Recent Paleolimnological Records in Lake Qarun, Middle Egypt. Journal of Paleolimnology 35(1):1-24.

Galili, E., R. Rosen, A. Gopher, and L. Kolska-Horwitz

2002 The Emergence and Dispersion of the Eastern Mediterranean Fishing Village: Evidence from Submerged Neolithic Settlements off the Carmel Coast, Israel. Journal of Mediterranean Archaeology 15(2):167-198.

Galili, E., M. Weinstein-Evron, I. Hershkovitz, A. Gopher, M. E. Kislev, O. Lernau, 1. Kolska-Horwitz, and H. Lernau

1993 Atlit-Yam: A Prehistoric Site on the Sea Floor off the Israeli Coast. Journal of Field Archaeology 20(2):133-157.

Gallant, T. W.

1986 "Background Noise" and Site Definition: A Contribution to Survey Methodology. Journal of Field Archaeology 13(4):403-418.

Gardiner, A. H., and H. I. Bell

1943 The Name of Lake Moeris. Journal of Egyptian Archaeology, Vol. 29, 37-50.

Gatto, M.

2006 The Nubian A-Group: A Reassessment. Archeo-Nil 16:61-76.

Gautier, A.

1980 Contributions to the Archaeozoology of Egypt. In Prehistory of the Eastern Sahara, edited by F. Wendorf and R. Schild, pp. 317-344. Academic Press, New York.

1987 Prehistoric Men and Cattle in North Africa: A Dearth of Data and a Surfeit of Models. In
Prehistory of Arid North Africa: Essays in Honor of Fred Wendorf, edited by A. E. Close, pp. 163-188. Southern Methodist University Press, Dallas.

2001 The Early to Late Neolithic Archaeofaunas from Nabta and Bir Kiseiba. In Holocene Settlement of the Eastern Sahara: 1. The Archaeology of Nabta Playa, edited by F. Wendorf and R. Schild, pp. 609-635. Kluwer Academic/Plenum Publishers, New York.

2002 The Evidence for the Earliest Livestock in North Africa: Or Adventures with Large Bovids, Ovicaprids, Dogs, and Pigs. In Droughts, Food, and Culture: Ecological Change and Food Security in Africa's Later Prehistory, edited by F. A. Hassan, pp. 195-208. Kluwer Academic/Plenum Publishers, New York.

Geb, M.

2000 Factors Favouring Precipitation in North Africa: Seen from the Viewpoint of Present-Day Climatology. Global and Planetary Change 26:85-96.

Ginter, V. B., W. Heflik, J. K. Kozlowski, and J. Sliwa

1980 Excavations in the Region of Qasr el-Sagha, 1979: Contributions to the Holocene Geology, the Predynastic and Dynastic Settlements in the Northern Fayum Desert. Mitteilungen des Deutschen Archäologischen Instituts, Kairo 36:105-169.

Gkiastra, M., T. Russell, S. Hennan, and J. Steele

2003 Neolithic Transition in Europe: The Radiocarbon Record Revisited. Antiquity 77:45-62.

Glennie, K. W.

2005 The Desert of Southeast Arabia. Gulf PetroLink, Bahrain.

Gopher, A., and R. Gophna

1993 Cultures of the Eighth and Seventh Millennia BP in the Southern Levant: A Review for the 1990s. Journal of World Prehistory 7(3):297-353.

Goring-Morris, N.

1993 From Foraging to Herding in the Negev and the Sinai: The Early to Late Neolithic Transition. Paléorient 19(1):65-89.

Haaland, R.

1984 Continuity and Discontinuity: How to Account for a Two Thousand Years Gap in the Cultural History of the Khartoum Nile Environment. Norwegian Archaeological Review 17(1):39-51.

Hamdan, M., and G. Lucarini

2013 Holocene Paleoenvironmental, Paleoclimatic and Geoarchaeological Significance of the Sheikh El-Obeiyid Area (Farafra Oasis, Egypt). Quaternary International 302:154-168.

Hamdan, M., M. I. A. Ibrahim, M. A. Shiha, R. J. Flower, F. A. Hassan, S. A. M. Eltelet

2016 An Exploratory Early and Middle Holocene Sedimentary Record with Palynoforms and Diatoms from Faiyum Lake, Egypt. Quaternary International 410:30-42. 
Hassan, F. A.

1978 Archaeological Explorations of the Siwa Oasis Region, Egypt. Current Anthropology 19(1):146-148.

1980 Prehistoric Settlements along the Main Nile. In The Sahara and the Nile, edited by M. A. J. Williams and H. Faure, pp. 421-450. Balkema, Rotterdam.

1984 Environment and Subsistence in Predynastic Egypt. In From Hunters to Farmers: The Causes and Consequences of Food Production in Africa, edited by J. D. Clark and S. A. Brandt, pp. 57-64. University of California Press, Berkeley.

1985 Radiocarbon Chronology of Neolithic and Predynastic Sites in Upper Egypt and the Delta. African Archaeology Review 3:95-116.

1986 Holocene Lakes and Prehistoric Settlements of the Western Faiyum, Egypt. Journal of Archaeological Science 13:483-501.

1988 The Predynastic of Egypt. Journal of World Prehistory 2(2):135-185.

1997 Holocene Palaeoclimates of Africa. African Archaeological Review 14(4):213-230.

2002 Palaeoclimate, Food, and Culture Change in Africa: An Overview. In Droughts, Food, and Culture: Ecological Change and Food Security in Africa's Later Prehistory, edited by F. A. Hassan, pp. 11-26. Kluwer Academic/Plenum Publishers, New York.

Hassan, F., B. Barich, M. Mahmoud, and M. A. Hamdan

2001 Holocene Playa Deposits of Farafra Oasis, Egypt, and Their Palaeoclimatic and Geoarchaeological Significance. Geoarchaeology 16(1):29-46.

Hassan, F. A., M. A. Hamdan, R. Flower, and K. Keatings

2012 Oxygen and Carbon Isotopic Records in Holocene Freshwater Mollusc Shells from the Faiyum Paleolakes, Egypt: Paleoenvironmental and Paleoclimatic Implications. Quaternary International 266:175-187.

Hassan, F., G. Tassie, R. Flower, M. Hughes, and M. Hamdan

2006 Modelling Environmental and Settlement Change in the Fayum. Egyptian Archaeology 29:37-40.

Hawass, Z., F. A. Hassan, and A. Gautier

1988 Chronology, Sediments, and Subsistence at Merimda Beni Salama. Journal of Egyptian Archaeology 74:31-38.

Hayes, W. C.

1965 Most Ancient Egypt. University of Chicago Press, Chicago.

Haynes, C. V.

2001 Geochronology and Climate Change of the Pleistocene-Holocene Transition in the Darb el Arba'in Desert, Eastern Sahara. Geoarchaeology 16(1):119-141.

Hays, T. R.

1975 Neolithic Settlement of the Sahara as It Relates to the Nile Valley. In Problems in Prehistory: North
Africa and the Levant, edited by F. Wendorf and A. E. Marks, pp. 193-206. SMU Press, Dallas.

Helbaek, H.

1955 Ancient Egyptian Wheats. Proceedings of the Prehistoric Society 21:93-95.

Hendrickx, S., B. Midant-Reynes, and W. Van Neer

2001 Maghar Dendera 2 (Haute Egypte), un site d'occupation Badarien. Leuven University Press, Leuven, Belgium.

Hodder, I.

2012 Entangled: An Archaeology of the Relationships between Humans and Things. Wiley-Blackwell, Malden, Massachusetts.

Holdaway, S. J., and M. J. Douglass

2012 A Twenty-First Century Archaeology of Stone Artifacts. Journal of Archaeological Method and Theory 19(1):101-131.

2015 Use beyond Manufacture: Non-Flint Stone Artifacts from Fowlers Gap, Australia. Lithic Technology 40(2):94-111.

Holdaway, S. J., and P. Fanning

2008 Developing a Landscape History as Part of a Survey Strategy: A Critique of Current Settlement System Approaches Based on Case Studies from Western New South Wales, Australia. Journal of Archaeological Method and Theory 15:167-189.

2014 Geoarchaeology of Aboriginal Landscapes in Semiarid Australia. CSIRO Publishing, Clayton, Australia.

Holdaway, S. J., and R. S. Phillipps

2017 Human Environmental Interrelationships and the Origins of Agriculture in Egypt and Sudan. Oxford Research Encyclopedia of Environmental Science. Electronic document, in press.

Holdaway, S. J., and N. Stern

2004 A Record in Stone: The Study of Australia's Flaked Stone Artefacts. Museum Victoria, Melbourne.

Holdaway, S. J., and L. Wandsnider

2008 Time in Archaeology: An Introduction. In Time in Archaeology: Time Perspectivism Revisited, edited by S.J. Holdaway and L. Wandsnider, pp. 1-12. University of Utah Press, Salt Lake City.

Holdaway, S. J., W. Wendrich, and R. S. Phillipps

2010 Identifying Low-level Food Producers: Detecting Mobility from Lithics. Antiquity 84(232):185-194.

Holmes, D. L.

1989 The Predynastic Lithic Industries of Upper Egypt: A Comparative Study of the Lithic Traditions of Badari, Nagada and Hierakonopolis. British Archaeological Reports, Oxford.

Holmes, D. L., and R. F. Friedman

1994 Survey and Test Excavations in the Badari Region, Egypt. Proceedings of the Prehistoric Society 60:105-142. 
Hugget, R. J.

2007 Fundamentals of Geomorphology. Routledge, New York.

Ingold, Timothy

1985 The Significance of Storage in Hunting Societies. In Les Techniques de conservation des grains à long terme: Leur rôle dans la dynamique des systèmes de cultures et des sociétés, edited by Marceau Gast, François Sigaut, and Corinne Beutler, pp. 33-45. Éditions du Centre National de la Recherche Scientifique, Paris.

InsideWood

2004 onward Inside Wood. Available at http://insidewood. lib.ncsu.edu/search.

Jadeja, M., and K. Shah

2015 Tree-map: A Visualization Tool for Large Data. SIGIR Workshop on Graph Search and Beyond '15 Santiago, Chile. Electronic document, http:// ceur-ws.org/Vol-1393/ (accessed September 2016).

Jelinek, A. J.

1976 Form, Function, and Style in Lithic Analysis. In Cultural Change and Continuity: Essays in Honor of James Bennett Griffin, edited by C. E. Cleland, pp. 19-33. Academic Press, London.

Kelly, R. L.

1992 Mobility/Sedentism: Concepts, Archaeological Measures, and Effects. Annual Review of Anthropology 21:43-66.

Kindermann, K.

2003 Investigations of the Mid-Holocene Settlement of Djara (Aby Muhariq Plateau, Western Desert of Egypt). In Cultural Markers in the Later Prehistory of Northeastern Africa and Recent Research, edited by L. Krzyżaniak, K. Kroeper, and M. Kobusiewicz, pp. 51-72. Poznań Archaeological Museum, Poznań, Poland.

2004 Djara: Excavations and Surveys of the 1998-2002 Seasons. Archeo-Nil 14:31-50.

Kindermann, K., O. Bubenzer, S. Nussbaum, H. Riemer, F. Darius, N. Pölath, and U. Smettan

2006 Palaeoenvironment and Holocene Land Use of Djara, Western Desert of Egypt. Quaternary Science Reviews 25:1619-1637.

Koopman, A., S. J. Kluiving, W. Wendrich, R. T. J. Cappers, and S. J. Holdaway

2010 Lake Dynamics and Human Occupation during the Early and Mid-Holocene, Fayum Basin, Egypt. IGBA Report 2010-09. Institute for Geoand Bioarchaeology, VU University, Amsterdam.

Koopman, A., S. Kluiving, S. J. Holdaway, and W. Wendrich

2016 The Effects of Holocene Landscape Changes on the Formation of the Archaeological Record in the Fayum Basin, Egypt. Geoarchaeology 31(1):17-33.
Kozlowski, J. K.

1983 Qasr el-Sagha 1980, Contributions to the Holocene Geology, the Predynastic and Dynastic Settlements in the Northern Fayum Desert. Nakladem Uniwersytetu Jagiellonskiego, Warsaw.

Kozlowski, J. K., and B. Ginter

1989 The Fayum Neolithic in the Light of New Discoveries. In Late Prehistory of the Nile Basin and the Sahara, edited by L. Krzyżaniak and M. Kobusiewicz, pp. 157179. Poznań Archaeological Museum, Poznań, Poland.

1993 Holocene Changes in the Fayum: Lake Moeris and the Evolution of Climate in Northeastern Africa. In Environmental Change and Human Culture in the Nile Basin and Northern Africa until the Second Millennium $B C$, edited by L. Krzyzaniak, M. Kobusiewicz, and J. Alexander, pp. 327-336. Poznań Archaeological Museum, Poznań, Poland.

Królik, H., and R. Schild

2001 Site E-75-6: An El Nabta and Al Jerar Village. In Holocene Settlement of the Eastern Sahara: 1. The Archaeology of Nabta Playa, edited by F. Wendorf and R. Schild, pp. 111-146. Kluwer Academic/Plenum Publishers, New York.

Kröpelin, S., D. Verschuren, A. M. Lezine, H. Eggermont, C. Cocquyt, P. Francus, J.-P Cazet, M. Fagot, B. Rumes, J. M. Russell, F. Darius, D. J. Conley, M. Schuster, H. von Suchodoletz, and D. R. Engstrom

2008 Climate-Driven Ecosystem Succession in the Sahara: The Past 6,000 Years. Science 320:765-768.

Kuhn, S. L.

2004 Upper Palaeolithic Raw Material Economies at Ucagizli Cave, Turkey. Journal of Anthropological Archaeology 23:431-448.

Kuijt, I., and N. Goring-Morris

2002 Foraging, Farming, and Social Complexity in the PrePottery Neolithic of the Southern Levant: A Review and Synthesis. Journal of World Prehistory 16(4):361-440.

Kuper, R.

2006 After 5,000 BC: The Libyan Desert in Transition. Comptes Rendus Palevol 5:409-419.

Kuper, R., and S. Kröpelin

2006 Climate-Controlled Holocene Occupation in the Sahara: Motor of Africa's Evolution. Science 313:803-807.

Kvamme, K. L.

1998 Spatial Structure in Mass Debitage Scatters. In Surface Archaeology, edited by A. P. Sullivan, pp. 127-141. University of New Mexico, Albuquerque.

Lesur, J., F. Briois, B. Midant-Reynes, and M. Wuttman

2011 Domesticates and Wild Game in the Egyptian Western Desert at the end of the Fifth Millennium BC: The Fauna from KS 43, Kharga Oasis. In People and Animals in Holocene Africa: Recent Advances in Archaeozoology, edited by H. Jousse and J. Lesur, pp. 59-74. Africa Magna Verlag, Frankfurt-am-Main. 
Libby, W. F.

1952a Radiocarbon Dates II. Science 114:291.

1952b Radiocarbon Dating. University of Chicago Press, Chicago.

Lindstädter, J., and S. Kröpelin

2004 Wadi Bakht Revisited: Holocene Climate Change and Prehistoric Occupation in the Gilf Kebir Region of the Eastern Sahara, SW Egypt. Geoarchaeology 19(8):753-778.

Linseele, V., S. J. Holdaway, and W. Wendrich

2016 The Earliest Phase of Introduction of Southwest Asian Domesticated Animals into Africa. New Evidence from the Fayum Oasis in Egypt and Its Implications. Quaternary International 412(B):11-21.

Linseele, V., E. Marinova, W. Van Neer, and P. Vermeersch

2010 Sites with Holocene Dung Deposits in the Eastern Desert of Egypt: Visited by Herders? Journal of Arid Environments 74:818-828.

Linseele, V., W. Van Neer, and R. Friedman

2009 Special Animals from a Special Place? The Fauna from HK29A at Predynastic Hierakonpolis. Journal of the American Research Center in Egypt 45:105-136.

Linseele, V., W. Van Neer, S. Thys, R. Phillipps, R. T. J. Cappers, W. Wendrich, and S. Holdaway

2014 New Archaeological Data from the Fayum "Neolithic" with a Critical Assessment of the Evidence for Early Stock Keeping in Egypt. PLoS ONE 9(10):e108517.

Lucarini, G.

2007 The Use and Exploitation of Sorghum and Wild Plants in the Hidden Valley Village (Farafra Oasis, Egypt). In Archaeology of Early Northeastern Africa, edited by K. Kroeper, M. Chlodnicki, and M. Kobusiewicz, pp. 463-478. Poznań Archaeological Museum, Poznań, Poland.

Lucas, G.

2012 Understanding the Archaeological Record. Cambridge University Press, Cambridge.

Madella, M., J. J. Garcia-Granero, W. A. Out, P. Ryan, and D. Usai

2014 Microbotanical Evidence of Domestic Cereals in Africa 7000 Years Ago. PLoS ONE 9(10):e110177.

Marks, A. E.

1970 Preceramic Sites, Vol. 2. Scandinavian University Books, Copenhagen.

Marshall, F., and E. Hildebrand

2002 Cattle before Crops: The Beginnings of Food Production in Africa. Journal of World Prehistory 16(2):99-143.

McBurney, C. B. M.

1960 The Stone Age of North Africa. Penguin Books, Harmondsworth, UK.

1967 The Hana Fteah (Cyrenaica) and the Stone Age of the South-east Mediterranean. Cambridge University Press, Cambridge.
McCorriston, J.

2006 Breaking the Rain Barrier and the Tropical Spread of Near Eastern Agriculture into Southern Arabia. In Behavioral Ecology and the Transition to Agriculture, edited by D. J. Kennett and B. Winterhalder, pp. 217-264. University of California Press, Berkeley.

McDonald, M. M. A.

1982 Dakhleh Oasis Project: Third Preliminary Report on the Lithic Industries in the Dakhleh Oasis. Journal of the Society for the Study of Egyptian Antiquities 12:115-138.

1991 Technological Organization and Sedentism in the Epipalaeolithic of Dakhel Oasis, Egypt. African Archaeological Review 9:81-109.

1998 Early African Pastoralism: View from Dakhleh Oasis (South Central Egypt). Journal of Anthropological Archaeology 17:124-142.

2001 The Late Prehistoric Radiocarbon Chronology for Dakhleh Oasis within the Wider Environmental and Cultural Setting of the Western Desert of Egypt. In The Oasis Papers 1: Proceedings of the First International Symposium of the Dakbleh Oasis Project, edited by M. Marlow, pp. 26-42. Oxbow, Oxford.

2009 Increased Sedentism in the Central Oases of the Egyptian Western Desert in the Early to MidHolocene: Evidence from the Peripheries. African Archaeological Review 26(1):3-43.

Mehringer, P. J., K. L. Petersen, and F. A. Hassan

1979 A Pollen Record from Birket Qarun and the Recent History of the Fayum. Quaternary Research 11(2):238-256.

Midant-Reynes, B.

1992 Prehistoire de l'Egypte: des premiers hommes aux premiers pharaons. A. Colin, Paris.

2000 The Prehistory of Egypt, from the First Egyptians to the First Pharaohs. Blackwell, Oxford.

Miller, N., and T. Smart

1984 Intentional Burning of Dung as Fuel: A Mechanism for the Incorporation of Charred Seeds into the Archaeological Record. Journal of Ethnobiology $4: 15-28$.

Mortensen, B.

1992 Carbon-14 Dates from El Omari. In The Followers of Horus: Studies Dedicated to Michael Allen Hoffman, edited by R. F. Friedman and B. Adams, pp. 173174. Monograph 20. Oxbow, Oxford.

1999 El-Omari. In Encyclopedia of the Archaeology of Ancient Egypt, edited by K. A. Bard, pp. 592-594. Routledge, New York.

Murray, T.

1999 A Return to the "Pompeii Premise." In Time and Archaeology, edited by T. Murray, pp. 8-27. Routledge, London. 
Museum of London Archaeological Service

1994 Archaeological Site Manual. Museum of London, London.

Mussi, M., I. Caneva, and A. Zarattini

1984 More on the Terminal Paleolithic of the Fayum Depression. In Origin and Early Development of Food-Producing Cultures in North-Eastern Africa, edited by L. Krzyzaniak and M. Kobusiewicz, pp. 185-191. Poznań Archaeological Museum, Poznań, Poland.

Nasoordeen, S. M.

2009 Grindstones from E29H1 (Fayum, Egypt) as a Component of Eastern Saharan, Early Holocene Archaeological Assemblages. Unpublished master's thesis, Department of Anthropology, University of Auckland.

Nelson, K., M. C. Gatto, F. Jesse, and M. N. Zedeno (editors)

2002 Holocene Settlement of the Egyptian Sahara, Vol. 2. Kluwer Academic/Plenum Publishers, New York.

Neumann, K.

1989 Zur Vegetationsgeschichte der Ostsahara im Holozan Holzkohlen aus prahistorischen Fundstellen. In Forschungen zur Umweitgeschichte der Ostsahara, edited by R. Kuper, pp. 13-181. Heinrich-BarthInstitut, Cologne.

Neumann, K., W. Schoch, P. Détienne, F. H. Schweingruber, and H. G. Richter

2001 Woods of the Sahara and the Sahel: An Anatomical Atlas. Paul Haupt, Bern.

Newton, C.

2005 Upper Egypt: Vegetation at the Beginning of the Third Millennium BC Inferred from Charcoal Analysis at Adaima and Elkab. Journal of Archaeological Science 32:355-367.

Nichols, G.

2006 Sedimentology and Stratigraphy. Blackwell Science, Oxford.

Nicoll, $\mathrm{K}$.

2001 Radiocarbon Chronologies for Prehistoric Human Occupation and Hydroclimatic Change in Egypt and Northern Sudan. Geoarchaeology 16(1):47-64.

Olsen, B.

2010 In Defense of Things: Archaeology and the Ontology of Objects. Altamira Press, Lanham, Maryland.

Olsson, I. U.

1986 Radiometric Dating. In Handbook of Holocene Palaeoecology and Palaeohydrology, edited by B.

E. Berglund and M. Ralska-Jasiewiczowa, pp. 273312. Wiley, New York.

Parsons, A. J.

1988 Hillslope Form. Routledge, London.

Payne, S.

1972 Partial Recovery and Sample Bias: The Results of Some Sieving Experiments. In Papers in Economic
Prehistory, edited by E. S. Higgs, pp. 49-64. Cambridge University Press, Cambridge.

Pazdur, M. F.

1983 Radiocarbon Dating of Organic Samples. In Qasr el-Sagha 1980, Contributions to the Holocene Geology, the Predynastic and Dynastic Settlements in the Northern Fayum Desert, edited by J. K. Kozlowski, pp.114-117. Nakladem Uniwersytetu Perles, C. Jagiellonskiego, Warsaw.

2001 The Early Neolithic in Greece. Cambridge University Press, Cambridge.

Perrot, J.

1966 Le gisement Natufien de Mallaha (Eynan) Israel. L'Anthropologie 70:437-484.

Perry, W. J.

1923 The Children of the Sun: A Study in the Early History of Civilization. Methuen, London.

Petrie, W. F.

1939 The Making of Egypt, Vol. 61. Sheldon Press, London.

Phillipps, R., and S. J. Holdaway

2016 Estimating Core Number in Assemblages: Core Movement and Mobility in the Holocene in the Fayum, Egypt. Journal of Archaeological Method and Theory 23:520-540.

Phillipps, R., S. J. Holdaway, W. Wendrich, and R. T. J. Cappers

2012 Mid-Holocene Occupation of Egypt and Global Climatic Change. Quaternary International 251:64-76.

Phillipps, R., S. J. Holdaway, J. Emmitt, and W. Wendrich

2016 Variability in the Neolithic Settlement Patterns of the Egyptian Nile Valley. African Archaeological Review 33:277-295.

Phillipps, R. S.

2006 Neolithic Surface Stone Artefact Assemblages from the Fayum, Egypt. Unpublished master's thesis, Department of Classics and Ancient History, University of Auckland.

2012 Documenting Socio-economic Variability in the Egyptian Neolithic through Stone Artefact Analysis. Unpublished $\mathrm{PhD}$ thesis, Department of Anthropology, University of Auckland.

Phillips, J. L.

1972 North Africa, the Nile Valley, and the Problem of the Late Paleolithic. Current Anthropology 13(5):587-590.

Phillips, N.

2013 Assessing the Temporal Foundations of SupraRegional Models for Early to Mid-Holocene ClimateCultural Change, Northeast Africa. Unpublished master's thesis, Department of Anthropology, University of Auckland. 
Poppe, G. T., and Y. Goto

1991 European Seashell. Verlag Christa Hemmen, Wiesbaden. Pumpelly, R.

1908 Explorations in Turkestan: Expeditions of 1904: Prehistoric Civilizations of Anau: Origins, Growth, and Influence of Environment. Carnegie Institution of Washington, Washington, D.C.

Redman, C. L., and P. Watson

1970 Systematic, Intensive Survey Collection. American Antiquity 35:279-291.

Reimer, P. J., E. Bard, A. Bayliss, J. W. Beck, P. G. Blackwell, C. Bronk Ramsey, P. M. Grootes, T. P. Guilderson, H. Haflidason, I. Hajdas, C. Hatte, T. J. Heaton, D. L. Hoffmann, A. G. Hogg, K. A. Hughen, K. F. Kaiser, B. Kromer, S. W. Manning, M. Niu, R. W. Reimer, D. A. Richards, E. M. Scott, J. R. Southon, R. A. Staff, C. S. M. Turney, and J. van der Plicht

2013 IntCal13 and Marine13 Radiocarbon Age Calibration Curves 0-50,000 Years cal BP. Radiocarbon 55(4):1887-1969.

Rezek, Z., S. C. H. Lin, R. P. Iovita, and H. L. Dibble

2011 The Relative Effects of Core Surface Morphology on Flake Shape and Other Attributes. Journal of Archaeological Science 38:1346-1359.

Richerson, P. J., R. Boyd, and R. L. Bettinger

2001 Was Agriculture Impossible during the Pleistocene but Mandatory during the Holocene? A Climate Change Riemer, H. Hypothesis. American Antiquity 66(3):387-411.

2007 When Hunters Started Herding: Pastro-Foragers and the Complexity of Holocene Economic Change in the Western Desert of Egypt. In Aridity, Change, and Conflict in Africa, edited by M. Bollig, O. Bubenzer, R. Vogelsang, and H. Wotzka, pp. 105-144. HeinrichBarth Institute, Cologne.

Riemer, H., N. Pollath, S. Nussbaum, I. Teubner, and H. Berke

2008 El Kharafish. A Sheikh Muftah Desert Camp Site between the Oasis and the Nile. In Egypt at Its Origins 2, edited by B. Midant-Reynes and Y. Tristant, pp. 585-608. Peeters, Leuven, Belgium.

Rowley-Conway, P., and R. Layton

2011 Foraging and Farming as Niche Construction: Stable and Unstable Adaptations. Philosophical Transactions of Royal Society B: Biological Sciences 366(1566):849-862.

Runnels, C.

2003 The Origins of the Greek Neolithic. In The Widening Harvest, edited by A. Ammerman and P. Biagi, pp. 121-132. Archaeological Institute of America, Boston. Salvatori, S.

2012 Disclosing Archaeological Complexity of the Khartoum Mesolithic: New Data at the Site and Regional Level. African Archaeological Review 29:399-472.
Sandford, K. S., and A. J. Arkell

1929 Paleolithic Man and the Nile-Faiyum Divide: A Study of the Region During Pliocene and Pleistocene Times, Vol. 1. University of Chicago Oriental Institute Publications, Chicago.

Schepers, M., R. T. J. Cappers, and I. Heijen

2006 Neolitische graanopslag in de Fayum. English summary. Paleo-aktueel 17:55-62.

Schick, K. D.

1986 Stone Age Sites in the Making: Experiments in the Formation and Transformation of Archaeological Occurrences. British Archaeological Reports, Oxford.

Schild, R., M. Chmielewska, and H. Wieckowska

1968 The Arkinian and Shamarkian Industries. In The Prehistory of Nubia, edited by F. Wendorf, pp. 651767. Fort Burgwin Research Center and Southern Methodist University Press, Dallas.

Schild, R., and F. Wendorf

1975 New Explorations in the Egyptian Sahara. In Problems in Prehistory: North Africa and the Levant, edited by F. Wendorf and A. E. Marks, pp. 65-112. SMU Press, Dallas.

Schoch, W, I. Heller, F. H. Schweingruber, and F. Kienast

2004 Wood Anatomy of Central European Species. Available at http://www.woodanatomy.ch.

Schofield, A. J.

1991 Artefact Distributions as Activity Areas: Examples from South-east Hampshire. In Interpreting Artefact Scatters: Contributions to Ploughzone Archaeology, edited by A. J. Schofield, pp. 117-128. Oxbow Books, Oxford.

Schweingruber, F. H.

1990 Anatomy of European Woods. Haupt, Stuttgart.

Shackley, M. L.

1978 The Behaviour of Artefacts as Sedimentary Particles in a Fluviatile Environment. Archaeometry 20(1):55-61.

Sharabati, D., and I. A. Sharabati

1984 Red Sea Shells. KPI Limited, London.

Shirai, N.

2005 Bifacial Technology, Socioeconomic Competition, and Early Farming and Herding in the Fayum. In Current Research in Egyptology 2003: Proceedings of the Fourth Annual Symposium, edited by K. Piquette and S. Love, pp. 135-147. Oxbow Books, Oxford.

2006 Supra-Regional Concepts in Near Eastern Neolithisation from a Viewpoint of Egyptian Neolithic Research. Paléorient 32(2):7-21.

2010 The Archaeology of the First Farmer-Herders in Egypt: New Insights into the Fayum Epipalaeolithic and Neolithic. Leiden University Press, Leiden.

Smith, A. B.

1992 Origins and Spread of Pastoralism in Africa. Annual Review of Anthropology 21:125-141. 
Smith, B. D.

2001 Low-Level Food Production. Journal of Archaeological Research 9(1):1-43.

2006 Eastern North America as an Independent Center of Plant Domestication. Proceedings of the National Academy of Sciences 103(33):12223-12228.

Tachikawa, T., M. Kaku, A. Iwasaki, D. Gesch, M. Oimoen, Z. Zhang, J. Danielson, T. Krieger, B. Curtis, J. Haase, M. Abrams, R. Crippen, and C. Carabajal

2011 ASTER Global Digital Elevation Model Version 2Summary of Validation Results. Electronic document, http://www.jspacesystems.or.jp/ersdac/GDEM/ver2Validation/Summary_GDEM2_validation_report_final. pdf (accessed February 2015).

Talbot, M. R., and P. A. Allen

1996 Lakes. In Sedimentary Environments: Processes, Facies, and Stratigraphy, edited by H. G. Reading, pp. 83-124. Blackwell Science, Chichester, UK.

Thompsen, K.

2004 Numericana. Electronic document, http://www.numericana.com/answer/ellipsoid.htm\#thomsen (accessed January 2017).

Thompson, D. J.

1999 Irrigation and Drainage in the Early Ptolemaic Fayyum. In Agriculture in Egypt: From Pharaonic to Modern Times, edited by A. K. Bowman and E. Rogan, pp. 107-122. Oxford University Press, Oxford.

Tixier, J.

1963 Typologie de l'Épipaléolithique du Maghreb. Arts et Métiers Graphiques, Paris.

Tobor, J. G.

1974 A Contribution to the Study of Nile Perch, Lates niloticus in Lake Chad. Occasional Paper 17:1-23. Federal Ministry of Information Printing Division, Lagos.

Turq, A., W. Roebroeks, L. Bourguignon, and J Faivre

2013 The Fragmented Character of Middle Palaeolithic Stone Tool Technology. Journal of Human Evolution 65:641-655.

Van Neer, W.

1997 Archaeozoological Data on the Food Provisioning of Roman Settlements in the Eastern Desert of Egypt. ArchaeoZoologia 9:137-154.

2004 Evolution of Prehistoric Fishing in the Egyptian Nile Valley. Journal of African Archaeology 2:251-269.

Van Zon, H. C. J., and K. W. Jeannes

1992 Fauna. In Environmental Profile Fayoum Governorate, Egypt, edited by H. C. J. Van Zon and K. W. Jeannes, pp. 20-24. Euroconsult/Darwish Consulting Engineers, Arnhem, Netherlands.

Vermeersch, P.

1984 Subsistence Activities on the Late Palaeolithic Sites of Elkab (Upper Egypt). In Origin and Early Development of Food-Producing Cultures in North-East Africa, edited by L. Krzyzaniak and M. Kobusiewicz, pp.
137-142. Poznań Archaeological Museum, Poznań, Poland.

2006 La vallée du Nil et le Sahara oriental: une population préhistorique fluctuante sous l'effet des variations climatiques. Comptes Rendus Palevol, 5:255-262.

Vermeersch, P., P. Van Peer, J. Moeyersons, and W. Van Neer

1994 Sodmein Cave Site, Red Sea Mountains (Egypt). Sahara 6:31-40.

Vita-Finzi, C.

1978 Archaeological Sites in Their Setting. Thames and Hudson, London.

Walker, C.

1996 Signs of the Wild. Struik Publisher, Cape Town.

Wang, L., G. Wang, and C. Alexander

2015 Big Data and Visualization: Methods, Challenges, and Technology Progress. Digital Technologies 1:33-38.

Warfe, A. R.

2003 Cultural Origins of the Egyptian Neolithic and Predynastic: An Evaluation of the Evidence from the Dakhleh Oasis (South Central Egypt). African Archaeological Review 20(4):175-202.

Wasylikowa, K.

2001 Site E-75-6: Vegetation and Subsistence of the Early Neolithic at Nabta Playa, Egypt, Reconstructed from Charred Plant Remains. In Holocene Settlement of the Egyptian Sahara: 1. The Archaeology of Nabta Playa, edited by F. Wendorf and R. Schild, pp. 544-591. Kluwer Academic/Plenum Publishers, New York.

Wasylikowa, K., J. Mitka, F. Wendorf, and R. Schild

1997 Exploitation of Wild Plants by Early Neolithic HunterGatherers of the Western Desert, Egypt: Nabta Playa as a Case Study. Antiquity 71(274):932-941.

Wendorf, F.

1968 Summary of Nubian Prehistory. In The Prehistory of Nubia, edited by F. Wendorf, pp. 791-953. Fort Burgwin Research Center and Southern Methodist University Press, Dallas.

Wendorf, F., A. E. Close, and R. Schild

2001 Site E-91-1: An El Jerar Phase Locality at Nabta Playa: 1. The 1991 and 1997 Excavations. In Holocene Settlement of the Egyptian Sahara: 1. The Archaeology of Nabta Playa, edited by F. Wendorf, and R. Schild, pp. 147-183. Kluwer Academic/ Plenum Publishers, New York.

Wendorf, F., and R. Schild

1976 Prehistory of the Nile Valley. Academic Press, New York.

1980 Prehistory of the Sahara. Academic Press, New York.

1984 The Emergence of Food Production in the Egyptian Sahara. In From Hunters to Farmers: The Causes and Consequences of Food Production in Africa, edited by J. D. Clark and S. A. Brandt, pp. 93-101. University of California Press, Berkeley. 
1994 Are the Early Holocene Cattle in the Eastern Sahara Domestic or Wild? Evolutionary Anthropology 3:118-128.

1998 Nabta Playa and Its Role in Northeastern African Prehistory. Journal of Anthropological Archaeology 17:97-123.

2001 Combined Prehistoric Expedition's Radiocarbon Dates Associated with Neolithic Occupations in the Southern Western Desert of Egypt. In Holocene Settlement of the Egyptian Sahara: 1. The Archaeology of Nabta Playa, edited by F. Wendorf and R. Schild, pp. 51-56. Kluwer Academic/Plenum Publishers, New York.

2002 The Role of Storage in the Neolithic of the Egyptian Sahara. In Tides of the Desert: Contributions to the Archaeology and Environmental History of Africa in Honour of Rudolph Kuper, edited by Jennerstrasse 8, pp. 41-49. Heinrich Barth Institute, Cologne.

Wendorf, F., R. Schild, R. Said, C. V. Haynes, A. Gautier, and M. Kobusiewicz

1976 The Prehistory of the Egyptian Sahara. Science 193(4248):103-114.

Wendorf, F., and the Members of the Combined Prehistoric Expedition

1977 Late Pleistocene and Recent Climatic Changes in the Egyptian Sahara. Geographical Journal 143(2):211-234.

Wendrich, W.

1991 Who Is Afraid of Basketry? A Guide to Recording Basketry and Cordage for Archaeologists and Ethnographers. CNWS 6. Research School for Asian, African, and Amerindian Studies, Leiden.

1999 The World According to Basketry: An Ethnoarchaeological Interpretation of Basketry Production in Egypt. CNWS 83. Research School for Asian, African, and Amerindian Studies, Leiden.

Wendrich, W., and R. Cappers

2005 Egypt's Earliest Granaries: Evidence from the Fayum. Egyptian Archaeology 27:12-15.

Wendrich, W., R. E. Taylor, and J. Southon

2010 Dating Stratified Settlement Sites at Kom K and Kom W: Fifth Millenium BCE Radiocarbon Ages for the Fayum Neolithic. Nuclear Instruments and Methods in Physics Research B 268:999-1002.

Wengrow, D.

2003 Landscapes of Knowledge, Idioms of Power: The African Foundations of Ancient Egyptian Civilization Reconsidered. In Ancient Egypt in Africa, edited by D. O'Connor and A. Reid, pp. 121-135. UCL Press, London.

2006 The Archaeology of Early Egypt: Social Transformations in North-East Africa 10,000 to 2650 BC. Cambridge University Press, Cambridge.
Wengrow, D., M. Dee, S. Foster, A. Stevenson, and C. Bronk Ramsey

2014 Cultural Convergence in the Neolithic of the Nile Valley: A Prehistoric Perspective on Egypt's Place in Africa. Antiquity 88:95-111.

Wenke, R. J.

1984 Early Agriculture in the Southern Fayum Depression: Some Test Survey Results and Research Implications. In Origin and Early Development of Food-Producing Cultures in North-Eastern Africa, edited by L. Krzyzaniak and M. Kobusiewicz, pp. 193-198. Poznań Archaeological Museum, Poznań, Poland.

1989 Egypt: Origins of Complex Societies. Annual Review of Anthropology 18:129-155.

2009 The Ancient Egyptian State: The Origins of Egyptian Culture (c. 8000-2000 BC). Cambridge University Press, Cambridge.

Wenke, R. J., C. E. Buck, J. Hanley, M. Lane, J. E. Long, and R. Redding

1983 The Fayyum Archaeological Project: Preliminary Report of the 1981 Season. Newsletter of the American Research Center in Egypt 122:25-40.

Wenke, R. J., and M. Casini

1989 The Epipaleolithic-Neolithic Transition in Egypt's Fayum Depression. In Late Prehistory of the Nile Basin and the Sahara, edited by L. Krzyzaniak and M. Kobusiewicz, pp. 139-155. Pozna Archaeological Museum, Pozna , Poland.

Wenke, R. J., J. E. Long, and P. E. Buck

1988 Epipaleolithic and Neolithic Subsistence and Settlement in the Fayyum Oasis of Egypt. Journal of Field Archaeology 15:29-51.

Wetterstrom, W.

1993 Foraging and Farming in Egypt: The Transition from Hunting and Gathering to Horticulture in the Nile Valley. In The Archaeology of Africa: Food, Metals and Towns, edited by T. Shaw, P. Sinclair, B. Andah, and A. Okpoko, pp. 165-226. Routledge, London.

Wheeler, E. A.

2011 InsideWood: A Web Resource for Hardwood Anatomy. IAWA Journal 32:199-211.

Williams, M. A. J.

2009 Late Pleistocene and Holocene Environments in the Nile Basin. Global and Planetary Change 69:1-15.

Williams, M. A. J., F. M. Williams, G. A. T. Duller, R. N. Munro, O. A. M. El Tom, T. T. Barrows, M. Macklin, J. Woodward, M. R. Talbot, D. Haberlah, and J. Fluin

2010 Late Quaternary Floods and Droughts in the Nile Valley, Sudan: New Evidence from Optically Stimulated Luminescence and AMS Radiocarbon Dating. Quaternary Science Reviews 29:1116-1137. 
Wilson, P.

2006 Prehistoric Settlement in the Western Delta: A Regional and Local View from Sais (Sa el-Hagar). Journal of Egyptian Archaeology 92:75-126.

Wilson, P., and G. Gilbert

2003 The Prehistoric Period at Sais (Sa el Hagar). ArchaeoNil 13:65-72.

Wilson, P., G. Gilbert, and G. Tassie

2014 Sais II: The Prehistoric Period. Egypt Exploration Society, London.

Zahran, M. A., and A. J. Willis 2009 The Vegetation of Egypt. Springer, Netherlands.
Zeder, M. A.

2008 Domestication and Early Agriculture in the Mediterranean Basin: Origins, Diffusion, and Impact. Proceedings of the National Academy of Sciences of the United States of America 105(33):11597-11604.

2009 The Neolithic Macro-(R)evolution: Macroevolutionary Theory and the Study of Culture Change. Journal of Archaeological Research 17(1):1-63.

Zohary, D., and M. Hopf

2000 Domestication of Plants in the Old World: The Origin and Spread of Cultivated Plants in West Asia, Europe, and the Nile Valley. Oxford University Press, Oxford. 


\section{Index}

Note: Page numbers in bold italics indicate illustrations or tables.

abandonment, 10, 24-25, 31, 51, 53, 75, 79, 82, 85, 90, $94,95,163,212-216,219-226,230$

acacia, 30

adzes, 227, 234

aeolian deposits, 57, 59, 65, 182

agriculture, 14-15, 25-26, 28-29, 225-226, 229-230, 233-234. See also pastoralism

Alcelaphus buselaphus (hartebeest), 75, 77, 78, 96, 204, 208, 235-236

Alestes sp., 76, 207

Alopochen aegyptiaca (Egyptian goose), 77

Amaranthaceae, 29

Anatidae (duck), 77, 204, 207, 225

animal exploitation, 237-238

animal remains. See faunal remains

Anser anser (goose), 77

antelope, 78, 237-238

Area A, 20, 21, 21, 22, 79, 80, 81

Area C, 21, 21, 22, 80, 81

Area E, 21, 21, 79, 80, 81

artifact density, 30-31, 34-36, 37, 38-41

in $\mathrm{K}$ Basin, 100

at Kom K, 190, 190.191, 192

in L Basin, 52, 59-65, 60-64

ashy deposit, 49, 166, 169, 171, 172, 173, 174, 176, 177, 180-183, 187, 188, 204

Aspatharia sp (freshwater bivalve), 134, 202

ASTER GDEM (advanced space-borne thermal emission and reflection radiometer global digital elevation model), 26

Auchenoglanis (catfish), 207

aurochs, 77, 204, 208

axes, 46, 93, 94, 94, 189, 227

backed bladelets, 14, 15, 92, 94

Badarian, 11, 13, 235 bagrid catfish, 78, 79, 207

Bagrus (catfish), 78, 79, 207

Bahr el-Yusuf, 2

Bahr Playa, 236

Barbary sheep, 209, 238. See also sheep

barbel family (Cyprinidae), 76, 78, 206, 207, 209, 212, 224

Barbus bynni (barbel), 206

barley, 124, 125, 135, 146, 160, 211, 212, 225, 227, 235, 237

Bashendi A, 235, 237

Bashendi B, 235, 236

basket, 123, 126, 127, 138, 141-142, 143, 144, 146-159, 147, 148, 150, 151-155, 151-158, 156, 158, 214, 234

basket-lined pits, 127, 128, 136, 151-153, 156, 157, 158, 214

basketry, 47, 214, 225, 235

Batrachia, 76, 205

bats, 75,76

beads, 47, 189, 192, 200, 201, 202, 212, 227, 230

Bellamya unicolor (freshwater snail), 76, 78, 205

bifacial, 94

bifacial crescent, 44

bifacial platforms, 13, 117, 118, 122

bifacial points, 189, 197

bifacial stemmed point, 44

bird eggshell, 76. See also ostrich eggshell

birds, 75, 77, 78, 79, 204, 205, 207, 225, 235, 236

Bir Kiseiba, 236, 237

Bithyniidae, 205

bivalves, 76, 78, 202, 204, 205, 206

Blue Nile, 24

bone artifacts, at L Basin, 60-62, 64

bone beads, 202

bone count density, 47

bone density, 58, 64, 95 
bone points, 189, 227

bone preservation, 47,78

bone processing and disposition, 224-225

bone ring, 202, 203

Bos primigenius taurus, 208

Bos taurus. See cattle

bovids, 208, 209. See also cattle

bracelet, 202, 212

bronze arrowhead, 101

brown sands (BS), 22

Brycinus sp. (freshwater fish), 76, 207

Bufonidae, 76, 205

Bulinus (freshwater snail), 78, 79, 205

Bulinus truncates (freshwater snail), 76, 78

burials, 12, 204, 234, 237, 238

C4 corridor, 113-122, 113-122

Caelatura aegyptiaca, 78

canid, 204. See also dog

Canis lupus, 77, 208. See also dog

Capparaceae, 30

Capra aegagrus hircus, 77, 208. See also goats

caprids, 11, 75, 209, 210

caprines, 209, 212

cat, 204, 208

catfish, 28, 75, 76, 78, 79, 207, 210, 225, 234

cattail, 30

cattle, 204, 208, 212, 235, 236-237

ceramic, 47

categories, 34

density, 229

at Hemamieh, 235

at K Basin, 101, 102-108, 124, 125, 126, 127, 144, 148, 159, 159, 161-162, 223-224

at Kom K, 169, 178, 179, 189, 198-200, 199, 200

at L Basin, 60-62, 64

at Mahgar Dendera, 235

at Merimde Beni Salama, 234

role of, 230

in Sahara vs. Nile Valley, 11

Chambardia sp. (mussel), 206, 210. See also mussel

Charadrius (plover), 207

charcoal, 47-48, 58, 66, 125, 166, 170, 174, 176, 180

Chenopodiaceae, 29, 30, 66

Chiroptera (bats), 76

chronology, 215-218, 216, 218. See also radiocarbon determinations

chrono-stratigraphy, 17, 18, 19, 22, 30, 49, 52, 97

Ciconiidae, 77

Clarias gariepinus (catfish), 76, 207

Clariidae (airbreathing catfish), 207, 209, 209

clay surface, 172, 173, 183, 211

Cleopatra bulimoides (freshwater snail), 76, 78, 78, 79, 204, 205

climate, 10, 219, 220. See also paleoenvironmental history; winter rains

cobble, 44, 79-80, 80, 82, 90, 122, 195, 221-222

Coelatura (freshwater bivalve), 206, 210
Coelatura aegyptiaca (freshwater bivalve), 76

coiled basket, 123, 126, 127, 138, 141, 144, 146, 148, 150, $151-155,156,158,214,234$

coins, 222

Columbella rustica (dove shell), 206

complete assemblages, 43, 45

complex white sands and silts (CWSS), 22

cone shell, 206

Conus (cone shell), 206

coot, 77, 204, 207, 225

Corbicula, 204

Corbicula consobrina (freshwater bivalve), 76, 78, 205

core reduction, 46, 82, 90, 92

cores, 38-41, 44, 44

at K Basin, 102-108, 117-119, 117-120

at Kom K, 192-195, 193, 194, 195, 196

at L Basin, 60-62, 64, 79-87, 80-86, 118

core scar length, 79, 80, 90, 117, 194, 195

corn, 125

cortex, 82, 83, 85, 85, 86, 90, 91, 195, 196

cortex ratio, 90, 91, 92, 94, 95, 96, 119, 120, 121, 122, 195-196, 198, 222

Corvus corax (common raven), 77

Coturnix coturnix (quail), 207

cowrey, 206

cultural groups, 226-228, 227, 229, 240

Cyperaceae (sedges), 30

Cypraeidae, 206

Cyprinidae (barbels), 76, 78, 207, 209, 212, 224

Dakhleh, 13, 235-236, 237, 239

debitage analysis, 15

décrue system, 25, 227, 230

dehulling, 160, 211, 212

denticulates, 93, 94, 95, 97, 120, 122, 197, 198

Desert Fayum, The (Caton-Thompson \& Gardner), 165, 189

diatomites, 20, 22, 31, 53, 55

differential visibility, 34, 59, 63

digital elevation model, 26

digital surface models (DSM), 19, 20, 21, 22, 26

Dimai, 3

Djara, 236, 237-238

dog, 204, 208, 212

domestic species adoption, 228-230

Dorcas gazelle, 77, 78, 204, 208

dove shell, 206

drill, 94

duck, 77, 204, 207, 225

E29G1, 2, 19, 21, 23, 23

E29G2, 2

E29G3, 2, 21

E29G5, 2

E29G6, 2

E29H1, 2, 3, 4, 19, 21-22, 23, 23, 29, 31, 43, 47, 215, 217, 222-223, 229, 237. See also L Basin

E29H2, 2, 23, 29 
E-75-6, 235

E-75-8, 235

early mid-Holocene, 217, 220

eastern Sahara, 7, 10-12, 15, 24, 213, 217, 226, 230, 236, 240

eggshell, 60-62, 76, 77, 78, 100, 102-108, 201, 202, 203, $205,207,228$

einkorn, 235

elephant-snout fishes, 76, 206

El Omari, 13, 212, 235, 237, 238

el-Qarah el-Hamra, 2, 2-3

emmer wheat, 124, 134, 160, 211, 212, 234, 237

environment, 10, 24-26, 219-221

Epipaleolithic period, 10, 14, 15, 24, 52, 96-97, 215, 218, $224,230,235,239-240$

Euphorbiacae, 30

excavation, 48-49, 115, 115, 166-189, 167-172, 174, 175, 177-180, 185-188

Fabaceae, 30

Faidherbia, 30

Farafra, 236, 237

faunal remains, 47

at Dakhleh, 235

at El Omari, 235

in $\mathrm{K}$ basin, 160

in Kom K, 204-210, 205-211, 227

in L Basin, 53, 74-79, 75-79, 96

at Merimde Beni Salama, 234

Fayum A, 14, 20, 42

Fayum B, 14, 20, 42

Fayum north shore, 1, 46, 49, 51, 52-53, 53, 65, 73, 79, $85,113,116,212,214,215,217,218,219,221,222$, $225,226,238$

feldspar beads, 189, 227

Felis (cat), 208. See also cat

fish, 76, 207, 209, 210, 225, 227, 230, 234, 237

flaked stone artifacts, 13, 15, 34, 38-41, 43-46, 44, 226

at K Basin, 100, 116, 116-117, 122, 128

at Kom K, 169, 190, 192, 192

at L Basin, 54, 57-58, 59, 64, 79-95, 80, 81, 83-89, 91, 93, 94

at Merimde Beni Salama, 234

mobility and, 221, 240

at Nabta Playa, 235

flaked stone artifact utility, 95

flake removal, 83, 85, 95, 96, 223

flakes, 37, 43-44

at K Basin, 102-108, 114, 120, 121

at Kom K, 195-196, 197

at L Basin, 59, 60-62, 87-92, 88, 89, 94-95

utilized, 93, 95, 97, 197

flake-to-core ratio, $37,42,86-87,88,90-91,119,119$, 119-120, 195

flaking scenarios, 80-82

flaking strategy, 82-85, 195

flax, 127, 160, 235

flint, 15, 44, 46, 74, 79, 80, 85, 90, 94, 126, 158, 183, 189, $195,221-222$ food production. See agriculture

formational perspective, 6

fox, 204, 208, 236

frogs, 75, 76, 78, 205

Fulica atra (coot), 77, 204, 207, 225

Gazella dorcas, 77, 208

gazelle, 77, 78, 204, 208, 237-238

geological opportunism, 25, 29, 219, 221, 239

geomorphology, 3-4, 6, 1621, 26, 31, 51-54, 54-57, 56-58, 100

Ghaba, 237

goats, 75, 77, 204, 208, 209, 209, 212, 235

goose, 77

grass, 15, 30, 123-127, 151-154, 211, 235, 237

gray hard silts (GHS), 22

grinding stone density, 42, 42, 74, 112

grinding stones, 15, 36, 46, 46, 223, 235

at $\mathrm{K}$ basin, 101, 109, 114, 115

at L Basin, 73, 74

Gyraulus costulatus (freshwater snail), 76, 78, 205

Haloxylon salicornicum, 29, 66

hare, 77, 204, 208

Harris matrix, 48

hartebeest, 78, 204, 208

hartebeest (Alcelaphus buselaphus), 75, 77, 78, 96, 204, 208, 235-236

Haua Fteah, 14, 234

Hawara, 2

hearths, 36, 42, 42, 47, 47, 48, 58

erosion of, 71

at Hidden Valley Playa, 236

at K Basin, 101, 108-109, 109, 110-112, 114

at Kom K, 165-166, 169-171, 170, 171, 172-183, 174, 175, 180, 184-187, 187-188, 198-200, 214

at L Basin, 65-73, 66-72, 74

near-surface, $6,47-48,73,183$

heat retainer, $47,47,52,66,68,71,108,174,174,175,198$, $211,215,221$

Helicidae (terrestrial snails), 76, 78, 205

Helwan point, 234

Hemamieh, 235, 238

Heterobranchus (catfish), 207

Hidden Valley Playa, 236

hillslope model, 26, 27

hippopotamus, 77, 78, 204, 208

Hordeum vulgare subsp. distichion (two-row barley), 160, 237

Hordeum vulgare subsp. vulgare (six-row barley), 160

horizontal tell, 238-239

hut circles, 235

Hydrocynus, 206

Hyperopisus bebe (elephant fish), 206

ibex, 209

Inter-Tropical Convergence Zone (ITCZ), 10, 24, 26, 219, 220

Iridinidae (freshwater mussels), 134 
K1 Survey Area, 100-112, 101-112

Karanis, 1, 3

K Basin, 27, 28, 29, 29, 43, 45

archaeological record, 99-164, 101-159, 161-163

artifact density, 100

basketry at, 146-159, 147, 150, 151-158

C4 corridor at, 113-122, 113-122

ceramic at, 101, 102-108, 124, 125, 126, 127, 144, 148, $159,159,161-162,223-224$

cores at, 102-108, 117-119, 117-120

flaked stone artifacts at, 100, 116, 116-117, 122, 128

flakes at, 102-108, 114, 121

grinding stones at, 101, 109, 115

hearths at, 101, 108-109, 109, 110-112, 114

tools at, 102-108, 120

UKP01 in, 131-132, 132, 133

UKP04 in, 132-137, 134-136

UKP05 in, 137, 137-138

UKP06 in, 139, 139

UKP07 in, 140, 141, 142

UKP09 in, 141-143, 143, 145, 146

UKP14 in, 143

UKP17 in, 144

UKP25 in, 144-145

UKP30 in, 145

UKP31 in, 145

Kharga Oasis, 237, 238

Khartoum Neolithic, 12, 13, 235

knife, 94

Kom Aushim, 2, 3, 4

Kom IV, 2, 2, 113, 113

Kom K, 3, 4-5, 23, 23, 27, 29, 45, 47, 48-49, 99-100. See also $\mathrm{K}$ Basin

artifact density at, 190, 192

botanical remains at, 211

ceramic at, 169, 178, 179, 189, 198-200, 199, 200

cores at, 192-195, 193, 194, 195, 196, 197

excavation at, 166-189, 167-172, 174, 175, 177-180, 185-188

faunal remains at, 204-210, 205-211, 227

flaked stone artifacts at, 169, 190, 192, 192

flakes at, 195-196

hearths at, 165-166, 169-171, 170, 171, 172-183, 174, 175, 180, 184-187, 187-188, 198-200, 214

Kom W and, 212

material culture at, 189

personal ornaments at, 200-202, 201-203

surface collection and analysis at, 189, 190

tools at, 190, 193, 196-197, 198

Kom W, 4, 22, 23, 23, 27, 45, 212, 223

$\mathrm{K}$ ridge, 129, 131, 162, 170

Labeo, 207

Labeo niloticus (barbel), 206

lacustrine marls, 22

lacustrine units, 56, 59

lake basins, 17-24, 18, 21, 23, 26-30, 27-29, 225-226

lake edge sediments, 17, 18, 49
Lake Moeris, 16, 23, 26, 219

Lake Qarun, 1, 3, 15, 17, 18, 22, 24, 209

landscape approach, 15-16, 43-49, 44, 46, 47, 214-215

Lanistes carinatus (freshwater snail), 205

Lates niloticus (Nile perch), 28, 76, 207. See also Nile perch

L Basin, 2, 27, 28, 29, 29, 43, 45, 48-49, 220, 224

archaeological record, 51-97, 53-64, 66-72, 74-81, $83-89,91,93,94$

artifact density, 52

boundaries, 52

cores at, 79-87, 80-86, 118

faunal remains, 53, 96

faunal remains in, 53, 74-79, 75-79

flaked stone artifacts at, 54, 57-58, 59, 79-95, 80, 81, $83-89,91,93,94$

flakes, 87-92, 88, 89

geomorphology, 54-57, 56-58

grinding stones at, 73,74

hearths at, 65-73, 66-72, 74

object density at, 59-65, 60-64

object distribution at, 52-54, 53, 54

tools at, 60-62, 92-94, 93, 94

topography, 54, 55, 56

Lepus capensis (Cape hare), 77, 208

Linum usitatissimum (flax), 127, 160

lizards, 75, 76, 204, 205, 207

Lower K Pits, 2, 99, 101, 160-164, 163, 223, 225. See also $\mathrm{K}$ Basin

low-level food producers, 12, 13, 214, 230, 233, 239, 241

Lymnaea natalensis (freshwater snail), 76

Maadi, 234

magnetic survey, 166, 168, 169

Mahgar Dendera, 235

Masara, 235

material culture. See also ceramic; flaked stone artifacts

analysis, 13-15

at Kom K, 189

landscape approach to, 43-49, 44, 46, 47

Melanoides tuberculata (freshwater snail), 76, 78, 79, 205

Merimde Beni Salama, 11, 234, 237, 238

microblades, 44, 81, 86, 94, 96, 194, 196

mid-Holocene, 3, 5, 7, 10, 11, 13, 14, 16, 17, 24, 28, 95, $101,107,112,115,189,204,209,212,215-220$, $224,226,233,235,236,238,239,241$

Mississippi Basin, 230

mobility, 5, 12, 13, 15, 45, 92, 96, 163, 221, 240

monitor lizard, 207

Mormyridae, 76

Mormyrus (elephant snout fish), 76, 206

mortar mixture, 137, 158

Mugilidae (mullets), 76, 206

mullets, 76, 206

multiple cores, 80, 82, 85, 117, 192, 195, 197

mussel, 134, 135, 135, 189

Mutela (mussel), 206, 210 
Nabta Playa, 11, 15, 235, 236, 237, 238, 239

Naqada I, 9, 238

Nassarius (marine snail), 206

$\mathrm{N}$ Basin, 27, 28, 29

near-surface hearth, 6, 47-48, 73, 183

Negev, 11

Neolithic culture, 11, 227, 240

Neolithic package, 9, 11, 213, 218, 228, 229, 230

Neolithic period, 10, 12, 218, 224, 239-240

Nerita (marine snail), 204, 206

Nile Delta, 10, 11, 230, 234

Nile-Fayum divide, 1

Nile flood, 24, 25, 219, 220, 225, 227

Nile flow, 29, 219

Nile inundation, 28, 49, 225

Nile perch, 76, 207, 209, 210, 211

Nile perch (Lates niloticus), 28, 75

Nile Valley, 2, 10, 11, 230, 235, 241

non-site material, 30

North Atlantic Bond Events, 24

notches, 93, 94, 94, 120, 122, 197, 198

oasis hypothesis, 10

origins, 10-12

ostrich eggshell, 60-62, 77, 78, 100, 102-108, 201, 202, 203, 207, 228

Ouchtata backing, 14

ovicaprid dung, 188, 208, 209, 210

Ovis ammon (mountain sheep), 77, 208. See also sheep

paleoenvironmental history, 10, 24-26, 219-221

paleomagnetic survey, 166, 168, 169

paleo-Moeris lake, 20

paleoshoreline, $17,26,32$

palimpsest, 16

pallets, 227, 230

Passeriformes (birds), 76, 205

passerines, 75

pastoralism, 5, 12, 236-237, 240

personal ornaments, 47, 49, 200-202, 201-203. See also beads

Phragmites australis (reeds), 29, 66

pig, 204, 208, 209, 234, 235

Pila ovata (freshwater snail), 21

pit feature

at El Omari, 235

at $\mathrm{K}$ Basin, 129

at Kom K, 174, 175-176, 177, 177

at Merimde Beni Salama, 234

Planorbis planorbis (freshwater snail), 205

Pleistocene lake level, 20

plover, 207

Poaceae, 30

Podiceps cristatus (great crested grebe), 77

Polygonum (buckweeds and knotweeds), 124, 127, 214

Polypterus (freshwater fish), 206, 210

postholes, 172, 176, 183, 235

pottery. See ceramic
PPNA, 11

PPNB, 11, 234

pre-Moeris deposits, 20, 22

prime mover, 9

Procavia capensis (rock hyrax/dassie), 238

processual archaeology, 5, 14

projectile points, $11,13,14,15,20,92,94,95,120,213$, 234, 239

pufferfish, 76, 78, 207

Qaret Rusas, 2, 3

Qasr el-Sagha, 1, 2, 3, 15, 22

quail, 207

radiocarbon determinations, 10, 18, 20-22, 48, 66, 67-71, $69,75,78-79,110,111,122,160,161,162,171$, $172,175,188,188,216,216,216-217,218,235$

rail, 77, 204, 207

Rallidae, 77

Rallus aquaticus, 207

raven, 77

Red Sea, 11, 24, 204, 213, 227

reduction sequence, $15,46,80$

reeds (Phragmites australis), 29, 66

Ricinus, 30

rock dassie, 238

root casts, 31, 32

Saïs, 234

Salicaceae, 30

Salix, 30

sand layers, 31-32, 32

sandrock deposit, 165, 183

Sarcocornia sp., 29

saw, 94

scalene ellipsoids, 82, 85, 86, 90, 117, 118, 120, 195

scrapers, 15, 93, 94, 95, 97, 120, 122, 197, 198

sedge, 30, 234, 235

Serpentes, 205. See also snakes

settlement pattern, 12, 16, 213, 221-224, 239

settlement systems, 7, 25, 30, 45, 164, 212, 222, 223, 224, 228-230, 229, 240

shark tooth, 76, 204, 205

sheep, 75, 77, 204, 208, 209, 212, 235

Sheikh el Obeiyid, 236

shell, 76, 204, 205, 206, 227

shell scoop, 127, 135, 163, 189

sickle blades, 15, 94, 126, 189, 214, 227

Sinai, 11

single platform, 90, 117, 118

snail, 76, 205

snakes, 75, 76, 205

social structure, 12, 213, 240

socioeconomy, 3, 7, 10, 11, 12, 13, 15, 16, 97, 218, 224226, 236-238

Sodmein Cave, 237, 238

soft-shell turtle, 75, 207, 209

sorghum, 235, 236 
Southwest Asia, 14, 15, 16, 25, 213, 218, 226, 228, 229, $233,234,237,238,239,240$

Spatha, 124, 189

Spathopsis (freshwater bivalve), 210

stone artifact analysis, 16, 45, 97, 190

stone artifact backing, 14

stone artifacts, at L Basin, 63. See also flaked stone artifacts stone beads, 202

storage containers, 159

stork, 77

stratigraphy, 56, 57, 166. See also chrono-stratigraphy; geomorphology

straw, wheat, 123, 134, 135, 216, 225

straw basket, 126

straw matting, 126, 160

Struthio camelus (ostrich), 207. See also ostrich eggshell

Suaeda, 30

sub-fossil plant remains, 48, 160

Sudan, 12, 237

surface area, 85, 90, 92, 95, 120, 122, 159, 159, 196

surface sediment, 31-32, 32, 33, 35, 36, 100, 176

survey, 30-43, 32-42, 37

Sus scrofa (domestic pig), 208. See also pig

Sweden, 230

Synodontis catfish, 75, 76, 79, 207, 209, 210

Synodontis schall (upside-down catfish), 207

tamarisk, 29, 124, 125, 126, 136, 166, 168, 170, 171, 172, $174,176,178,180,187,189$

Tamarix aphylla, 189

Tamarix nilotica, 29

temper, 47, 107, 160, 162

Tetraodon fahaka (Nile pufferfish), 76, 78

Tetraodon lineatus, 76, 207

textiles, 47, 146, 235

Theodoxus niloticus (freshwater snail), 205

tiger fish, 206, 210

tilapia, 75, 76, 78, 79, 207, 209, 210, 225, 234

Tixier typology, 14

toads, 75, 76, 78, 205

tools, 38-41

at K Basin, 102-108, 120

at Kom K, 190, 193, 196-197, 198

at L Basin, 60-62, 92-94, 93, 94 transect survey units, 31, 32, 32, 36

Trench 205, 75, 78, 96

Trionyx triunguis (African softshell turtle), 75, 77, 207

Triticum turgidum subsp. compactum (club wheat), 212, 237

Triticum turgidum subsp. dicoccon (emmer wheat), 124, 134, 160, 211, 212, 234, 237

Triticum turgidum subsp. durum, 212

tubular bead, 202

turtle, 75, 77, 207, 209

Typhaceae (cattail), 30

typology, 14, 218, 234

U Basin, 27, 28, 29, 29

unifacial reduction, 80

Upper K Pits, 2, 4, 48-49, 99, 109, 122-131, 122-131, 163, 163, 214, 223-224, 229. See also K Basin

Upper Nile Valley, 12

utilized flakes, 93, 95, 97, 197

Valvata nilotica (freshwater snail), 76, 76, 78, 205

Varanus (monitor lizard), 207

vegetation, 10, 19, 28, 29-30, 32, 40, 49, 56, 104, 105, 225

village, $2,5,11,43,165,189,212,233,238$

volume ratio, 91, 91-92, 120, 122, 196, 198

Vulpes (fox), 208, 236

Wadi Bakht, 236-237

water movement, 19, 29

wells, 235

wheat, 124, 125, 134, 135, 146, 160, 211, 212, 225, 227, $234,235,237$

wheat straw, 123, 134, 135, 216, 225

White Nile, 24

wild bovids, 208

winders, 138, 144, 147-148, 151-154, 156, 157

winter rains, 10, 24, 25, 29, 219, 220, 227, 239

wolf, 208

$\mathrm{XB11}, 2,31,32,64,87,222,223$

X Basin, 4, 27, 27-28, 28, 29, 29, 43, 224

Z Basin, 3-4, 26, 27, 28, 29, 29

Zootecus insularis (freshwater snail), 205 


\title{
UCLA COTSEN INSTITUTE OF ARCHAEOLOGY PRESS
}

\author{
MONUMENTA ARCHAEOLOGICA
}

Volume 39

Volume 38

Volume 37

Volume 36

Volume 35

Volume 34

Volume 33

Volume 32

Volume 31

Volume 30

Volume 29

Volume 28

Volume 27

Volume 26

Volume 24

Volume 23

Volume 22

Volume 21
The Desert Fayum Reinvestigated: The Early to Mid-Holocene Landscape Archaeology of the Fayum North Shore, Egypt, edited by Simon J. Holdaway and Willeke Wendrich The Archaeology of Grotta Scaloria: Ritual in Neolithic Southeast Italy, edited by Ernestine S. Elster, Eugenia Isetti, John Robb, and Antonella Traverso

A Bronze Age Landscape in the Russian Steppes: The Samara Valley Project, edited by David W. Anthony, Dorcas R. Brown, Aleksandr A. Khokhlov, Pavel F. Kuznetsov, and Oleg D. Mochalov

Rural Archaeology in Early Urban Northern Mesopotamia: Excavations at Tell al Raqa'i, edited by Glenn M. Schwartz

New Insights into the Iron Age Archaeology of Edom, Southern Jordan, by Thomas E. Levy, Mohammad Najjar, and Erez Ben-Yosef

The Excavation of the Prehistoric Burial Tumulus at Lofkënd, Albania, by John K. Papadopoulos, Sarah P. Morris, Lorenc Bejko, and Lynne A. Schepartz

Formative Lifeways in Central Tlaxcala: Volume 1: Excavations, Ceramics, and Chronology, edited by Richard G. Lesure

Integrating Çatalhöyük: The 2000-2008 Seasons (Çatal Research Project Volume 10), edited by Ian Hodder

Substantive Technologies at Çatalhöyük: Reports from the 2000-2008 Seasons (Çatal Research Project Volume 9), edited by Ian Hodder

Humans and Landscapes of Çatalhöyük (Çatal Research Project Volume 8), edited by Ian Hodder

Çatalhöyük Excavations: The 2000-2008 Seasons (Çatal Research Project Volume 7), edited by Ian Hodder

Light and Shadow: Isolation and Interaction in the Shala Valley of Northern Albania, edited by Michael L. Galaty, Ols Lafe, Wayne E. Lee, and Zamir Tafilica

Last House on the Hill: BACH Area Reports from Çatalhöyük, Turkey, edited by Ruth Tringham and Mirjana Stevanovi

The History and Archaeology of Jaffa I, edited by Martin Peilstöcker and Aaron A. Burke

The Early Iron Age Cemetery at Torone, by John K. Papadopoulos

The Plain of Phaistos: Cycles of Social Complexity in the Mesara Region of Crete, by L. Vance Watrous, Despoina Hadzi-Vallianou, and Harriet Blitzer

K'axob: Ritual, Work, and Family in an Ancient Maya Village, edited by Patricia A. McAnany

The Sydney Cyprus Survey Project: Social Approaches to Regional Archaeological Survey, by Michael Given and A. Bernard Knapp 
Volume 20

Volume 19

Volume 18

Volume 17

Volume 16

Volume 15

Volume 14

Volume 13

Volume 12

Volume 11

Volume 10

Volume 9

Volume 8

Volume 7

Volume 6

Volume 5

Volume 4

Volume 3

Volume 2

Volume 1
Prehistoric Sitagroi: Excavations in Northeast Greece 1968-1970. Volume 2. Final Report, edited by Ernestine S. Elster and Colin Renfrew

Archaeology of Solvieux, An Upper Paleolithic Open Air Site in France, by James Sackett Down by the Station: Los Angeles Chinatown, 1880-1933, by Roberta S. Greenwood Rock Art of Easter Island: Symbols of Power, Prayers to the Gods, by Georgia Lee Landscape Archaeology as Long-Term History: Northern Keos in the Cycladic Islands, by J. E. Cherry, J. L. Davis, and E. Mantzourani

Selevac: A Neolithic Village in Yugoslavia, edited by Ruth Tringham and Dusan Krstic

Achilleion: A Neolithic Settlement in Thessaly, Greece, 6400-5600 B.C., by Marija Gimbutas, Shan Winn, and Daniel Shimabuku

Excavations at Sitagroi: A Prehistoric Village in Northeast Greece. Volume 1. By Colin Renfrew, Marija Gimbutas, and Ernestine S. Elster

Petroglyphs in the Guianas and Adjacent Areas of Brazil and Venezuela: An Inventory with a Comprehensive Bibliography of South American and Antillean Petroglyphs, by C. N. Dubelaar

Chinese Archaeological Abstracts 4, edited by Albert E. Dien, Jeffrey K. Riegel, and Nancy T. Price

Chinese Archaeological Abstracts 3, edited by Albert E. Dien, Jeffrey K. Riegel, and Nancy T. Price

Chinese Archaeological Abstracts 2, edited by Albert E. Dien, Jeffrey K. Riegel, and Nancy T. Price

Kolmakovskiy Redoubt: The Ethnoarchaeology of a Russian Fort in Alaska, by Wendell Oswalt

Prehistoric Trails of the Atacama: Archaeology of Northern Chile, by Clement Meighan and D. L. True

Chinese Archaeological Abstracts, by Richard C. Rudolph

The Stone and Plaster Sculpture: Excavations at Dura Europos, by Susan B. Downey

The Transition to Mycenaean, by Sarah and Jeremy Rutter

The Marine Thiasos in Greek Sculpture, by Steven Lattimore

The Archaeology of Amapa, Nayarit, edited by Clement W. Meighan

Neolithic Macedonia as Reflected by Excavations at Anza, Southeast Yugoslavia, edited by Marija Gimbutas 


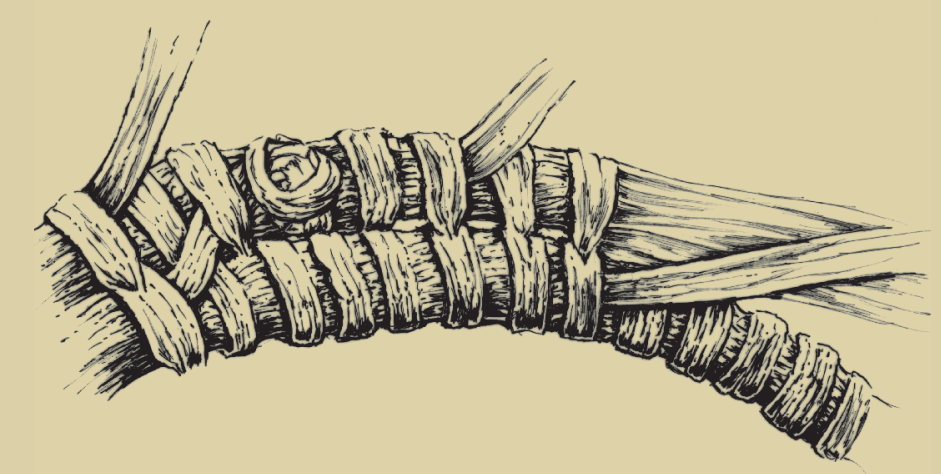




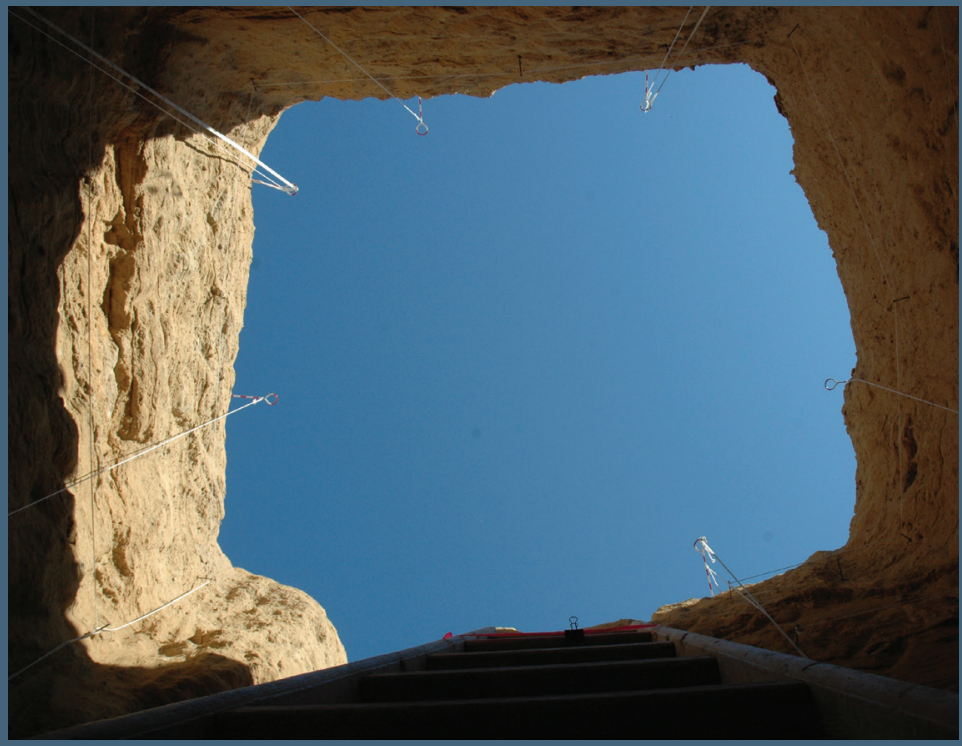

he Neolithic in Egypt is thought to have arrived via diffusion from an origin in southwest Asia. In this volume, the authors advocate an alternative approach to understanding the development of food production in Egypt based on the results of new fieldwork in the Fayum. They present a detailed study of the Fayum archaeological landscape using an expanded version of low-level food production to organize observations concerning paleoenvironment, socioeconomy, settlement, and mobility. While domestic plants and animals were indeed introduced to the Fayum from elsewhere, when a number of aspects of the archaeological record are compared, a settlement system is suggested that has no obvious analogues with the Neolithic in southwest Asia. The results obtained from the Fayum are used to assess other contemporary sites in Egypt.

A landmark publication for Egyptian prehistory and for the general understanding of cultural and environmental change in North Africa and the Mediterranean.

David Wengrow, Professor of Comparative Archaeology

UCL Institute of Archaeology

This book results from a remarkable international collaboration that brings together archaeological and geoarchaeological data to provide a new landscape understanding of the early to mid-Holocene in the Desert Fayum. The results are of great significance, demonstrating a distinct regional character to the adoption of farming and substantiating the wider evidence for a polycentric development of the Neolithic in the Middle East. This is interdisciplinary archaeology at its best.

Ian Hodder, Dunlevie Family Professor Department of Anthropology, Stanford University

\section{Contributors}

\begin{tabular}{llll}
\hline Joshua J. Emmitt & Annelies Koopman & John M. Marston & Rebecca Ramsay \\
Simon J. Holdaway & Veerle Linseele & Rebecca S. Phillipps & Willeke Wendrich
\end{tabular}

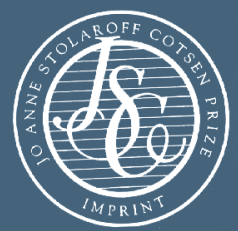

Monumenta ARChaeologica 39 